\title{
International Journal for \\ Computational Civil and Structural Engineering
}

(МежАународный журнац по расчету гражАанских и строительных конструкций)

Volume 12, Issue 4

\section{EXECUTIVE EDITOR}

Vladimir I. Travush, Professor,

Vice-President of Russian Academy

of Architecture and Construction Sciences;

24, Ulitsa Bolshaya Dmitrovka, 107031, Moscow, Russia

\section{EDITOR-IN-CHIEF}

Vladimir N. Sidorov, Professor

Department of Advanced Mathematics and Structural Mechanics,

Moscow Institute of Architecture (State Academy); 11/4, Building 4, Ulitsa Rozhdestvenka, Moscow, 107031, Russia

\section{EDITORIAL DIRECTOR}

Valery I. Telichenko, Professor,

The First Vice-President of Russian Academy

of Architecture and Construction Sciences;

24, Ulitsa Bolshaya Dmitrovka, 107031, Moscow, Russia

\section{MANAGING EDITOR}

Nadezhda S. Nikitina, Professor

Director of ASV Publishing House;

26, Yaroslavskoe Shosse, 129337 Moscow, Russia

\section{TECHNICAL EDITOR}

Taymuraz B. Kaytukov, Associate Professor Research \& Educational Center

of Computational Simulation, National Research

Moscow State University of Civil Engineering;

26, Yaroslavskoe Shosse, 129337 Moscow, Russia

\section{ASSOCIATE EDITORS}

Pavel A. Akimov, Professor

Executive Scientific Secretary of Russian Academy

of Architecture and Construction Sciences;

Scientific Research Center "STADYO";

National Research Moscow State University

of Civil Engineering;

24, Ul. Bolshaya Dmitrovka, 107031, Moscow, Russia

Alexander M. Belostotsky, Professor

Scientific Research Center "STADYO";

National Research Moscow State University

of Civil Engineering;

8th Floor, 18, ul. Tretya Yamskogo Polya,

125040, Moscow, Russia

Vladimir Belsky, Ph.D.

Dassault Systèmes Simulia;

1301 Atwood Ave Suite 101W

02919 Johnston, RI, United States

Mikhail Belyi, Professor

Dassault Systèmes Simulia;

1301 Atwood Ave Suite 101W

02919 Johnston, RI, United States
Vitaly Bulgakov, Professor

Parametric Technology Corp.;

57 Metropolitan Av.,

Ashland, MA, USA

Nikolai P. Osmolovskii, Professor

Systems Research Institute

Polish Academy of Sciences;

Kazimierz Pulaski University

of Technology and Humanities in Radom;

29, ul. Malczewskiego, 26-600, Radom, Poland

Gregory P. Panasenko, Professor

Equipe d'Analise Numerique

NMR CNRS 5585

University Gean Mehnet;

23 rue. P.Michelon 42023, St.Etienne, France

Leonid A. Rozin, Professor

Department of Structural Mechanics,

Peter the Great Saint-Petersburg

Polytechnic University;

29, Ul. Politechnicheskaya,

195251 Saint-Petersburg, Russia 


\section{ADVISORY EDITORIAL BOARD \& REVIEWERS}

Robert M. Aloyan, Professor Ivanovo State Polytechnical University; 20, Ulitsa 8 Marta, Ivanovo, 153037, Russia

Vladimir I. Andreev, Professor National Research Moscow State University of Civil Engineering; Yaroslavskoe shosse 26, Moscow, 129337, Russia

Mukhtaritdin M. Batdalov, Professor Makhachkala Branch of Moscow Automobile and Road State Technical University (MADI); 1. Imam Shamil Avenue, Makhachkala, Russia

Klaus-Jurgen Bathe, Professor Massachusetts Institute of Technology; Cambridge, MA 02139, USA

Alexander T. Bekker, Professor Far Eastern Federal University; 8 Suhanova Street, Vladivostok, 690950, Russia

Jan Buynak, Professor University of Žilina; 1, Univerzitná, Žilina, 010 26, Slovakia

Evgeniy M. Chernishov, Professor Voronezh State University of Architecture and Civil Engineering; 84, 20 Let Oktyabrya st., Voronezh, 394006, Russia

Vladimir T. Erofeev, Professor Ogarev Mordovia State University; 68 Bolshevistskaya Str., Saransk 430005, Republic of Mordovia, Russia

Victor S. Fedorov, Professor Moscow State University of Railway Engineering; 9, Obraztsova Street, Moscow, 127994, Russia

Sergiy Yu. Fialko, Professor, Cracow University of Technology; 24, Warszawska Street, Kraków, 31-155, Poland

Alexander S. Gorodetsky, Professor LIRA SAPR Ltd.;

Office 212, 7a Kiyanovsky side street (pereulok), Kiev, 04053, Ukraine

Vyatcheslav A. Ilyichev, Professor Russian Academy of Architecture and Construction Sciences; 24, Ulitsa Bolshaya Dmitrovka, Moscow, 107031, Russia

Marek Iwański, Professor, Kielce University of Technology; 7, al. Tysiąclecia Państwa Polskiego Kielce, 25 -314, Poland
Sergey Yu. Kalashnikov, Professor

Volgograd State University of Architecture and Civil Engineering; 1, Academicheskaya Street, Volgograd, 400074, Russia

Nikolay I. Karpenko, Professor Research Institute of Building Physics; 21, Locomotive Travel, Moscow, 127238, Russia

Vladimir V. Karpov, Professor Saint Petersburg State University of Architecture and Civil Engineering; 4, 2-nd Krasnoarmeiskaya Steet, Saint Petersburg, 190005, Russia

Galina G. Kashevarova, Professor Perm National Research Polytechnic University; 29 Komsomolsky pros., Perm, Perm Krai, 614990, Russia

Vitaly I. Kolchunov, Professor Southwest State University; 94, 50 let Oktyabrya, Kursk, 305040, Russia

Markus König, Professor Ruhr-Universität Bochum; 150, Universitätsstraße, Bochum, 44801, Germany

Sergey B. Kositsin, Professor Moscow State University of Railway Engineering; 9, Obraztsova Street, Moscow, 127994, Russia

Amirlan A. Kusainov, Professor Kazakh Leading Academy of Architecture and Civil Engineering; 29, Toraigyrov str., Almaty, Almaty, 050043, Republic of Kazakhstan

Sergey V. Kuznetsov, Professor Institute for Problems in Mechanics of the Russian Academy of Sciences; 101-1, Prosp. Vernadskogo, Moscow, 119526, Russia

Vladimir V. Lalin, Professor Peter the Great Saint-Petersburg Polytechnic University; 29, Ul. Politechnicheskaya, St. Petersburg, 195251, Russia

Leonid S. Lyakhovich, Professor Tomsk State University of Architecture and Building; 2, Solyanaya sq., Tomsk, 634003 , Russia

Rashid A. Mangushev, Professor Saint Petersburg State University of Architecture and Civil Engineering; 4, 2-nd Krasnoarmeiskaya Steet, Saint Petersburg, 190005, Russia
Ilizar T. Mirsayapov, Professor Kazan State University of Architecture and Engineering; 1, Zelenaya Street, Kazan, 420043 , Republic of Tatarstan, Russia

Vladimir L. Mondrus, Professor National Research Moscow State University of Civil Engineering; Yaroslavskoe shosse 26, Moscow, 129337, Russia

Anatoly V. Perelmuter, Professor SCAD Soft;

Office 1,2, 3a Osvity street, Kiev, 03037, Ukraine

Alexey N. Petrov, Professor Petrozavodsk State University; 33, Lenina Prospect, Petrozavodsk, 185910, Republic of Karelia, Russia

Vladilen V. Petrov, Professor

Yuri Gagarin State Technical University of Saratov;

77 Politechnicheskaya Street,

Saratov, 410054, Russia

Jerzy Z. Piotrowski, Professor

Kielce University of Technology;

al. Tysiaclecia Państwa Polskiego 7 ,

Kielce, 25 - 314, Poland

Chengzhi Qi, Professor Beijing University of Civil

Engineering and Architecture;

1, Zhanlanlu, Xicheng District,

Beijing, China

Nikolaj N. Shaposhnikov, Professor Moscow State University of Railway Engineering; 9, Obraztsova Street, Moscow, 127994, Russia

Eun Chul Shin, Professor Incheon National University; (Songdo-dong)119 Academy-ro, Yeonsu-gu, Incheon, Korea

D.V. Singh, Professor

Vice-Chairman all India Council for Technical Education;

New Delhi, India

Waclaw Szcześniak, Professor Lublin University of Technology;

Ul. Nadbystrzycka 40,

20-618 Lublin, Poland

Tadatsugu Tanaka, Professor Tokyo University; 7-3-1 Hongo, Bunkyo, Tokyo, 113-8654, Japan

Zbigniew Wojcicki, Professor Wroclaw University of Technology; 11 Grunwaldzki Sq., 50-377, Wrocław, Poland

Askar Zhussupbekov, Professor Eurasian National University; 5, Munaitpassov street, Astana, 010000, Kazakhstan 


\title{
INVITED REVIEWERS
}

\author{
Akimbek A. Abdikalikov, Professor, Dr.Sc., \\ Kyrgyz State University of Construction, Transport and Architecture n.a. N. Isanov; \\ 34 Maldybayeva Str., Bishkek, 720020, Biskek, Kyrgyzstan \\ Vadim K. Akhmetov, Professor, Dr.Sc. \\ National Research Moscow State University of Civil Engineering; \\ 26, Yaroslavskoe Shosse, Moscow, 129337, Russia \\ Ján Čelko, Professor, PhD, Ing. \\ University of Žilina; Univerzitná 1, 010 26, Žilina, Slovakia \\ Stanislaw Jemioło, Professor, Dr.Sc., \\ Warsaw University of Technology; 1, Pl. Politechniki, 00-661, Warsaw, Poland
}

Konstantin I. Khenokh, M.Ing., M.Sc.

Trinity Industries, Inc.; 2525 Stemmons Freeway, Dallas, Texas 75207, USA

Christian Koch, Dr.-Ing.

Ruhr-Universität Bochum;

Lehrstuhl für Informatik im Bauwesen, Gebäude IA, 44780, Bochum, Germany

Gaik A. Manuylov, Professor, $\mathrm{PhD}$

Moscow State University of Railway Engineering; 9, Obraztsova Street, Moscow, 127994, Russia

Alexander S. Noskov, Professor, Dr.Sc.

Ural Federal University named after the first President of Russia B.N. Yeltsin;

19 Mira Street, Ekaterinburg, 620002, Russia

Nelli N. Rogacheva, Professor, Dr.Sc.

National Research Moscow State University of Civil Engineering;

26, Yaroslavskoe Shosse, Moscow, 129337, Russia

Grzegorz Świt, Professor, Dr.hab. Inż.,

Kielce University of Technology; 7, al. Tysiąclecia Państwa Polskiego, Kielce, 25 - 314, Poland

Josef Vičan, Professor, PhD, Ing.

University of Žilina; Univerzitná 1, 010 26, Žilina, Slovakia

Artur Zbiciak, Associate Professor

Warsaw University of Technology; 1, Pl. Politechniki, 00-661, Warsaw, Poland

\section{AIMS AND SCOPE}

The aim of the Journal is to advance the research and practice in structural engineering through the application of computational methods. The Journal will publish original papers and educational articles of general value to the field that will bridge the gap between high-performance construction materials, large-scale engineering systems and advanced methods of analysis.

The scope of the Journal includes papers on computer methods in the areas of structural engineering, civil engineering materials and problems concerned with multiple physical processes interacting at multiple spatial and temporal scales. The Journal is intended to be of interest and use to researches and practitioners in academic, governmental and industrial communities. 


\section{ОБЩАЯ ИНФОРМАЦИЯ О ЖУРНАЛЕ \\ International Journal \\ for Computational Civil and Structural Engineering}

(МежАународный журнал по расчету гражАанских и строительных конструкций)

Международный научный журнал “International Journal for Computational Civil and Structural Engineering (Международный журнал по расчету гражданских и строительных конструкций)" (IJCCSE) является ведущим научным периодическим изданием по направлению «Инженерные и технические науки», издаваемым, начиная с 1999 года. В журнале на высоком научно-техническом уровне рассматриваются проблемы численного и компьютерного моделирования в строительстве, актуальные вопросы разработки, исследования, развития, верификации, апробации и приложений численных, численно-аналитических методов, программно-алгоритмического обеспечения и выполнения автоматизированного проектирования, мониторинга и комплексного наукоемкого расчетно-теоретического и экспериментального обоснования напряженно-деформированного (и иного) состояния, прочности, устойчивости, надежности и безопасности ответственных объектов гражданского и промышленного строительства, энергетики, машиностроения, транспорта, биотехнологий и других высокотехнологичных отраслей.

В редакционный совет журнала входят известные российские и зарубежные деятели науки и техники. Основной критерий отбора статей для публикации в журнале - их высокий научный уровень, соответствие которому определяется в ходе высококвалифицированного рецензирования и объективной экспертизы, поступающих в редакцию материалов.

Журнал входит в Перечень ВАК РФ ведущих речензируемых научных изданий, в которых должны быть опубликованы основные научные результаты диссертаций на соискание ученой степени кандидата наук, на соискание ученой степени доктора наук. В Российской Федерации журнал индексируется Российским индексом научного цитирования (РИНЦ). Журнал входит в базу данных Russian Science Citation Index (RSCI), полностью интегрированную с платформой Web of Science. Журнал имеет международный статус и высылается в ведущие библиотеки и научные организации мира.

Издатели журнала - Издательство Ассоциации строительных высших учебных заведений /ACВ/ (Россия, г. Москва) и Издательский дом Begell House Inc. (США, г. НьюЙорк). Партнерами издания является Российская академия архитектуры и строительных наук (РААСН) и Научно-исследовательский иентр СтаДиО (ЗАО НИЦ СтаДиО).

Цели журнала - демонстрировать в публикациях российскому и международному профессиональному сообществу новейшие достижения науки в области вычислительных методов решения фундаментальных и прикладных технических задач, прежде всего в области строительства.

Задачи журнала:

- предоставление российским и зарубежным ученым и специалистам возможности публиковать результаты своих исследований;

- привлечение внимания к наиболее актуальным, перспективным, прорывным и интересным направлениям развития и приложений численных и численно-аналитических методов решения фундаментальных и прикладных технических задач, совершенствования технологий математического, компьютерного моделирования, разработки и верификации реализующего программно-алгоритмического обеспечения;

- обеспечение обмена мнениями между исследователями из разных регионов и государств. 
Тематика журнала. К рассмотрению и публикации в журнале принимаются аналитические материалы, научные статьи, обзоры, рецензии и отзывы на научные публикации по фундаментальным и прикладным вопросам технических наук, прежде всего в области строительства. В журнале также публикуются информационные материалы, освещающие научные мероприятия и передовые достижения Российской академии архитектуры и строительных наук, научно-образовательных и проектно-конструкторских организаций.

Тематика статей, принимаемых к публикации в журнале, соответствует его названию и охватывает направления научных исследований в области разработки, исследования и приложений численных и численно-аналитических методов, программного обеспечения, технологий компьютерного моделирования в решении прикладных задач в области строительства, а также соответствующие профильные специальности, представленные в диссертационных советах профильных образовательных организациях высшего образования.

Редакционная политика. Политика редакционной коллегии журнала базируется на современных юридических требованиях в отношении авторского права, законности, плагиата и клеветы, изложенных в законодательстве Российской Федерации, и этических принципах, поддерживаемых сообществом ведущих издателей научной периодики.

За публикацию статей плата с авторов не взымается. Публикация статей в журнале бесплатная. На платной основе в журнале могут быть опубликованы материалы рекламного характера, имеющие прямое отношение к тематике журнала.

Авторам. Прежде чем направить статью в редакцию журнала, авторам следует ознакомиться со всеми материалами, размещенными в разделах сайта журнала (интернет-сайт Российской академии архитектуры и строительных наук (http://raasn.ru); подраздел «Издания PAACH» или интернет-сайт Издательства ACB (http://iasv.ru); подраздел «Журнал IJCCSE»): с основной информацией о журнале, его целями и задачами, редакционной политикой, порядком рецензирования направляемых в журнал статей и пр.

Подписка. Журнал зарегистрирован в Федеральном агентстве по средствам массовой информации и охраны культурного наследия Российской Федерации. Индекс в общероссийском каталоге РОСПЕЧАТЬ - 18076.

По вопросам подписки на международный научный журнал "International Journal for Computational Civil and Structural Engineering (Международный журнал по расчету гражданских и строительных конструкций)" обращайтесь в Агентство «Роспечать» (Официальный сайт в сети Интернет: http://www.rosp.ru/) или в издательство Ассоциации строительных вузов (АСВ) в соответствии со следующими контактными данными:

\section{$O O O$ «Издательство АСВ»}

Юридический адрес: 129337, Россия, г. Москва, Ярославское ш., д. 26, офис 705;

Фактический адрес: 129337, Россия, г. Москва, Ярославское ш., д. 19, корп. 1, 5 этаж, офис 12 (ТЦ Мебель России);

Телефоны: +7 (925) 084-74-24, +7 (926) 010-91-33

Интернет-сайт: www.iasv.ru. Адрес электронной почты: iasv@iasv.ru.

\section{Контактная информация.}

По всем вопросам работы редакции, рецензирования, согласования правки текстов и публикации статей следует обращаться к главному редактору журнала Сидорову Владимиру Николаевичу (e-mail: sidorov.vladimir@gmail.com, iasv@iasv.ru, sidorov@raasn.ru) или техническому редактору журнала Кайтукову Таймуразу Батразовичу (адреса электронной почты: kaytukov@raasn.ru; tkaytukov@gmail.com). Кроме того, по указанным вопросам, а также по вопросам размещения в журнале рекламных материалов можно обращаться к генеральному директору ООО «Издательство АСВ» Никитиной Надежде Сергеевне (адреса электронной почты: iasv@iasv.ru,nsnikitina@mail.ru,ijccse@iasv.ru). 
Журнал становится технологичнее. Издательство АСВ с сентября 2016 года является членом Международной ассоциации издателей научной литературы (PILA), осуществляющей свою деятельность на платформе CrossRef. Оригинальным статьям, публикуемым в журнале, будут присваиваться уникальные номера (индексы DOI - Digital Object Identifier), что значительно облегчит поиск метаданных и местонахождение полнотекстового произведения. DOI - это система определения научного контента в сети Интернет.

CrossRef - независимая организация, образованная в 2000 году по инициативе группы научных издателей. Инициатором разработки технологии стало издательство Wiley. Texнология использования ссылок (DOI) принадлежит агентству CrossRef.

Publishers International Linking Association (PILA) - Международная ассоциация по связям издателей - является управляющей структурой агентства CrossRef - агрегатора и регистратора DOI, а также международной базой научных статей и их метаданных (www.crossref.org). На настоящий момент в базе несколько десятков миллионов материалов.

Использование этой технологии позволит представить информацию о журнале на международной платформе издателей научной литературы CrossRef, обеспечит удобный и принятый в мировом научном сообществе доступ к полнотекстовой электронной версии. Это значительно облегчит поиск научной информации, что способствует повышению наукометрических показателей авторов статей, публикуемых в журнале. Если, например, у произведения российского автора есть DOI и если на его работу имеется ссылка в публикации зарубежного коллеги в журнале, включенном в Web of Science и Scopus, то такое произведение автоматически попадает в эти базы данных и получает в нем собственный индекс цитируемости.

Bсе журналы, индексируемые в международной базе научного цитирования Web of Science и большинство периодических изданий, индексируемых в международной базе Scopus, имеют DOI. Использование цифрового идентификатора говорит прежде всего о технологическом качестве научного издания и является неотъемлемым атрибутом системы научной коммуникации за счет эффективного обеспечения процессов обмена научной информацией.

С октября 2016 года стал возможным прием статей на рассмотрение и рецензирование через он-лайн систему приема статей Open Journal Systems на специализированном сайте журнала: http://ijccse.iasv.ru/index.php/IJCCSE. Автор здесь имеет возможность следить за продвижением статьи в редакции в личном кабинете Open Journal Systems и получать соответствующие уведомления по электронной почте.

Официальные Интернет-ресурсы журнала. Официальными интернет-сайтами журнала являются:

http://ijccse.iasv.ru/index.php/IJCCSE (реализована функиия подачи статей через спеичиализированную он-лайн систему);

http://raasn.ru/public.php (подраздел «Журнал International Journal for Computational Civil and Structural Engineering (Международный журнал по расчету гражданских и строительных конструкций)» раздела «Издания РААСН» официального Интернет-сайта Российской академии архитектуры и строительных наук). 


\section{International Journal for \\ Computational Civil and Structural Engineering}

(МежАународный журнал по расчету гражАанских и строительных конструкций)

Volume 12, Issue 4

2016

\section{TABLE OF CONTENTS (СOДEPЖAHИE)}

Mathematical Modeling of Elastically Fixed Wall Longitudinal Oscillations of Wedge-Shaped Channel Under Foundation Vibration

Lev I. Mogilevich, Victor S. Popov, Lev N. Rabinsky

Reliability Analysis of Soil Bases According to the Deformation Criterion

Vladimir S. Utkin, Anastasia A. Kaberova, Sergey A. Solovyev

The Extended Shell Theory of Vekua-Amosov Type

and the Low-Order Plate Models

Sergey I. Zhavoronok

Физически нелинейные задачи для неоднородных толстостенных оболочек

В.И. Андреев, Л.С. Полякова

К вопросу о верификации многоуровневых подходов к локальному расчету строительных конструкций

Часть 1: Дискретный (численный) подход

Моджтаба Аслами, П.А. Акимов, Ж.И. Мсхалая

К вопросу о верификации многоуровневых подходов к локальному расчету строительных конструкций

Часть 2: Дискретно-континуальный (численно-аналитический) подход

Моджтаба Аслами, П.А. Акимов, В.Н. Сидоров, Т.Б. Кайтуков

Методика численного моделирования напряженно-деформированного состояния системы «основание - плотина - водохранилище» при сейсмических воздействиях

А.М. Белостоикий, Д.С. Дмитриев, Нгуен Тай Нанг Лыонг

Алгоритмическая и программная реализация задач проектировочного расчета стержневых конструкций

Т.Л. Дмитриева, Ле Чан Минь Дат

Численные исследования элементов усиления железобетонных колонн

Л.В. Енджиевский, Ю.С. Сергиенко 
Расчет бесконечной ледовой плиты, ослабленной отверстием

О явлении потери устойчивости продольно сжатой круговой цилиндрической оболочки Часть 2: Максвеллова сила и энергетический барьер Г.А. Мануйлов, С.Б. Косицын, М.М. Бегичев

Численное моделирование нестационарных упругих контурных напряжений в оболочке реакторного отделения атомной станции с фундаментом и основанием (полуплоскость) при ударе самолета B.K. Мусаев

Апробация методики численного моделирования статического и сейсмического напряженно-деформированного состояния на трехмерной системе «основание - плотина - водохранилище» ГЭС Нам Чиен (Вьетнам)

Нгуен Тай Нанг Лыонг

Численный анализ динамических характеристик элемента трубопроводной системы многоэтажного жилого дома Л.Г. Пастухова, А.А. Секачева

Определение напряженно-деформированного состояния армированных плит из нелинейного материала с учетом воздействия агрессивных сред А.А. Трещев, А.В. Башкатов, В.Г. Теличко

Вязкое демпфирование в платформенных моделях систем «сооружение - основание»

А.Г. Тяпин

Экспериментальный стенд для исследования особенностей предкритических и критических деформационных процессов в железобетонных конструкциях (математическое моделирование, структурная схема)

И.Н. Шардаков, А.П. Шестаков, Г.Н. Гусев, М.Л. Бартоломей, Г.Г. Камеварова, Р.В. Цветков, И.О. Глот

Сообщение о проведении выборов членов Российской академии архитектуры и строительных наук (РААСН) в 2017 году

Сообщение о проведении конкурса на медали и дипломы Российской академии архитектуры и строительных наук (РААСН) за 2016 год 


\title{
MATHEMATICAL MODELING OF ELASTICALLY FIXED WALL LONGITUDINAL OSCILLATIONS OF WEDGE-SHAPED CHANNEL UNDER FOUNDATION VIBRATION
}

\author{
Lev I. Mogilevich ${ }^{1}$, Victor S. Popov ${ }^{1}$, Lev. N. Rabinsky ${ }^{2}$ \\ ${ }^{1}$ Yuri Gagarin State Technical University of Saratov, Saratov, RUSSIA \\ ${ }^{2}$ Moscow Aviation Institute (National Research University), Moscow, RUSSIA
}

\begin{abstract}
The mathematical modeling problem of elastically fixed wall longitudinal oscillations of narrow wedge-shaped channel with highly viscous incompressible liquid inside it and set on the vibrating foundation is set up and analytically solved. This problem is considered in a flat setting for stationary harmonic oscillations regime. The considered mathematical model presents the Navier-Stokes equations and the continuity equation for thin layer of highly viscous incompressible liquid, as well as elastically fixed channel wall dynamics equation. The no-slip conditions for viscous liquid and the condition of free leakage of liquid at channel edges are chosen as boundary ones. The complex of dimensionless variables for the considered problem is suggested. The analytical expressions of channel wall movements and liquid layer hydrodynamic parameters are obtained. The calculations demonstrating the channel wall inclination impact on elastically fixed wall longitudinal oscillations damping are made.
\end{abstract}

Keywords: mathematical modeling, hydroelasticity, highly viscous liquid, wedge-shaped channel, elastically fixed wall, vibration, foundation

\section{МАТЕМАТИЧЕСКОЕ МОДЕЛИРОВАНИЕ ПРОДОЛЬНЫХ КОЛЕБАНИЙ УПРУГОЙ СТЕНКИ КАНАЛА КЛИНОВИДНОЙ ФОРМЫ ПРИ ВИБРАЦИЯХ УПРУГОГО ОСНОВАНИЯ}

\begin{abstract}
Л.И. Могилевич ${ }^{I}$, В.С. Попов ${ }^{1}$, Л.Н. Рабинский
${ }^{1}$ Саратовский государственный технический университет им. Ю. А. Гагарина, г. Саратов, РОССИЯ

${ }^{2}$ Московский авиационный институт (государственный технический университет), г. Москва, РОССИЯ

Аннотация: Получена постановка и аналитическое решение задачи математического моделирования продольных колебаний упругой стенки узкого клиновидного канала, заполненного высоковязкой несжимаемой жидкостью и расположенного на вибрирующем основании. Рассматриваются стационарные плоские гармонические колебания. Математическая модель образована уравнениями Навье-Стокса и уравнением неразрывности для тонкого слоя жидкости, а также уравнениями движения упругой стенки. В качестве краевых условий для течения вязкой жидкости поставлены условия отсутствия проскальзывания и условия свободного течения на стенках канала. Введена система безразмерных переменных задачи. Получены аналитические выражения для перемещений стенки канала и гидродинамические параметры для слоя жидкости. Показано, что наклон стенки канала влияет на демпфирование колебаний стенки.
\end{abstract}

Ключевые слова: математическое моделирование, гидроупругость, высоковязкие жидкости, каналы клиновидной формы, упругие стенки, колебания, упругие основания

\section{INTRODUCTION}

Mathematical modeling problems of elastic systems and liquid interaction are of significant importance for contemporary engineering tech- nologies development. The investigations devoted to hydroelastic behavior of constructions interacting with perfect fluid deserve to be singled out. For example, the bending oscillations of channel wall, taken as a beam and interacting 
with a perfect liquid, is solved in reference [1]. On the basis of this solution, the model of internal combustion engine liner with water cooling is constructed and the approaches to defining its cavitations resource are suggested. Reference [2] deals with modeling oscillations of the plate plunged into a perfect incompressible liquid with free surface. Reference [3] suggests and studies the mathematical model for investigation of a round plate vibration on a free surface of an ideal incompressible liquid. The problem is considered for the case when the liquid volume is limited by a rigid bottom and cylinder surface. Reference [4] suggests and investigates the mathematical model of the plate chaotic oscillations, the plate interacting with perfect incompressible liquid stream. References $[5,6]$ suggest the mathematical model of transient interaction of shallow shells and an incompressible ideal liquid using the transient functions approach.

At the same time, the references, mentioned above, do not deal with liquid damping characteristics conditioned by its viscosity. References $[7,8]$ deal with investigating dynamics stationary problem when viscous incompressible liquid is situated in the channel with absolutely rigid walls, including the case of one wall inclination within the theory of hydrodynamic lubrication. The infinitely long beam harmonic bending vibration on viscous liquid layer is considered in reference [9]. This reference studies the channel, formed by parallel walls, one of which is modeled as an infinite beam. The investigation of elastically fixed wall transverse oscillations of channel, formed by two parallel walls of finite sizes, is carried out in reference [10]. Hydroelastic oscillations of cantilevered beam in unlimited volume of viscous incompressible liquid are considered in reference [11]. Reference [12] suggests the model for investigating dynamic interaction of coaxial vibrating discs with viscous incompressible liquid between them. This reference considers the case, when one disc is taken as an elastic one. Reference [13] solves the analogues problem for the two plates of finite sizes. The investigation of beam hydroelastic oscillations in viscous liquid stream with application to piezoelectric elements, which can be used for obtaining energy from the stream, is made in reference [14]. The problem of bending hydroelastic oscillation plate, forming one of the narrow channel parallel walls under viscous liquid pulsating layer impact, is solved in reference [15]. The transition to onemass model for investigating transverse oscillations of an elastic cylinder surrounded by viscous liquid layer, is suggested in [16]. The considered model was used for investigating internal combustion engine liner with water cooling. A approximated numerical model of unsteady interaction of a pressure wave on a middlethickness shell was used in [17] to suppress unphysical solution oscillations.

The mathematical model for investigating bending hydroelastic oscillations of the plate on elastic foundation interacting, with pulsating viscous layer, is considered in $[18,19]$. Reference [20] deals with the mathematical model of the plate longitudinal oscillations with the set sagging form in viscous liquid stream flowing in the channel, formed by two parallel rigid walls. The article under consideration studies the impact of rigidly fixed channel wall inclination on longitudinal oscillations of its elastically fixed wall, interacting with highly viscous incompressible liquid layer under foundation vibration, the channel being put on the above mentioned foundation.

\section{STATEMENT OF THE PROBLEM}

Let us consider the narrow channel, presented in fig.1. The channel is formed by two walls 1 and 2 , put into one casing. The casing is set up on the foundation, vibrating in longitudinal direction. The channel is completely filled with highly viscous incompressible liquid 3 . The channel wall 2 is wedge-shaped and rigidly fixed with the casing. We take into account channel wall 2 inclination by considering parameter $\theta$. Channel wall 1 is elastically fixed with the casing and possesses geometrical sizes $b \times 2 \ell$. The elastic 
Mathematical Modeling of Elastically Fixed Wall Longitudinal Oscillations of Wedge-Shaped Channel

Under Foundation Vibration

fixation of channel wall 1 is made in such a way that it can move only in longitudinal direction. Further we take $b>>2 \ell$, in other worlds, we consider only a flat problem, where liquid layer average thickness $\delta_{0}$ is considerably less than $2 \ell$. We take the leakage at channel edges into the casing butt ends cavities to be free, the cavities being filled with the same liquid with constant pressure $p_{0}$. Let us introduce Cartesian coordinate system $O x z$ center, which coincides with the impenetrable channel wall $l$ surface center, contacting with liquid (see fig.1).

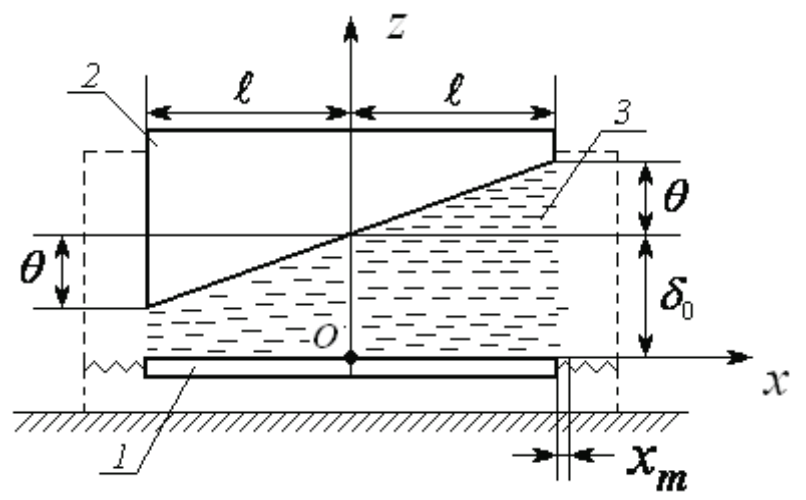

Figure 1. The scheme of wedge-shaped channel with elastically fixed wall, the channel being put on vibrating foundation.

By taking into account that transient processes will go out in the course of time we will restrict to the consideration the stationary harmonic oscillations study [21].

We present the law of vibrating foundation movement as a harmonic law

$$
x_{0}=E_{x} f_{0}(\omega t), f_{0}(\omega t)=\sin (\omega t),
$$

then vibro-acceleration takes the form of

$$
\ddot{x}_{0}=E_{x} \frac{d^{2} f_{0}(\omega t)}{d t^{2}}=-E_{x} \omega^{2} \sin (\omega t),
$$

where $E_{x}=k g / \omega^{2}$ is the amplitude foundation longitudinal oscillations, $g$ is the gravitational acceleration, $k$ is the vibro-overloading coefficient, $\omega$ is the oscillation frequency, $t$ is the time, $f_{0}(\omega t)$ is the law of motion. The equations of highly viscous incompressible liquid dynamics in a narrow channel take the form of $[8,22]$

$$
\begin{gathered}
\frac{1}{\rho} \frac{\partial p}{\partial x}=-\ddot{x}_{0}+v\left(\frac{\partial^{2} u_{x}}{\partial x^{2}}+\frac{\partial^{2} u_{x}}{\partial z^{2}}\right), \\
\frac{1}{\rho} \frac{\partial p}{\partial z}=v\left(\frac{\partial^{2} u_{z}}{\partial x^{2}}+\frac{\partial^{2} u_{z}}{\partial z^{2}}\right), \\
\frac{\partial u_{x}}{\partial x}+\frac{\partial u_{z}}{\partial z}=0
\end{gathered}
$$

where $p$ is the pressure, $\rho, v$ are the density and the kinematical coefficient of the liquid viscosity correspondingly, $u_{x}, u_{z}$ are the liquid velocity vector projections on coordinates axis.

The boundary conditions of these equations are the no-slip conditions for viscous liquid

$$
\begin{gathered}
u_{x}=\frac{d x_{1}}{d t}, \quad u_{z}=0 \quad \text { at } \quad z=0 ; \\
u_{x}=0, \quad u_{z}=0 \quad \text { at } \quad z=\delta_{0}+\frac{\theta x}{\ell},
\end{gathered}
$$

and the conditions of free liquid leakage at channel edges (conditions for pressure at channel edges)

$$
\begin{gathered}
p=p_{0}+\ddot{x}_{0} \rho \ell \text { at } \quad x=-\ell, \\
p=p_{0}-\ddot{x}_{0} \rho \ell \text { at } \quad x=\ell .
\end{gathered}
$$

Here $x_{1}=x_{m} f(\omega t)$ is the elastically fixed channel wall law of motion, $x_{m}$ is the elastically fixed channel wall oscillations amplitude.

We present the dynamic equation of elastically fixed channel wall in the form of

$$
m\left(\frac{d^{2} x_{1}}{d t^{2}}+\ddot{x}_{0}\right)+n x_{1}=N
$$

where $m$ is the wall mass, $n$ is the elastic connection rigidity, $N$ is the force, acting on the 
wall from the side of highly viscous liquid in the channel. The expression for the force $N$ takes the form of

$$
\begin{aligned}
N=b \int_{-\ell}^{\ell} q_{x y} d x, \quad q_{x y} & =\rho v\left(\frac{\partial u_{z}}{\partial x}+\frac{\partial u_{x}}{\partial z}\right) \\
\text { at } \quad z & =0,
\end{aligned}
$$

here $q_{x y}$ shear stress, influencing the elastically fixed wall from the highly viscous liquid.

\section{MATHEMATICAL MODEL}

Let us introduce dimensionless variables and the small parameter into the consideration

$$
\begin{gathered}
\psi=\frac{\delta_{0}}{\ell} \ll 1, \quad \tau=\omega t, \quad \xi=\frac{x}{\ell}, \quad \zeta=\frac{z}{\delta_{0}} ; \\
u_{z}=x_{m} \omega \psi U_{\zeta}, \quad u_{x}=x_{m} \omega U_{\xi}, \\
p=p_{0}-\ddot{x}_{0} \rho x+\frac{x_{m} \rho \nu \omega}{\delta_{0} \psi} P, \quad \varepsilon=\frac{\theta}{\delta_{0}} .
\end{gathered}
$$

Here $\psi$ is the small parameter, characterizing the problem.

Substituting (7) into equations (2), boundary conditions (3), (4) and expression for the force (6), we obtain dynamic equations of highly viscous incompressible liquid:

$$
\begin{gathered}
\frac{\partial P}{\partial \xi}=\psi^{2} \frac{\partial^{2} U_{\xi}}{\partial \xi^{2}}+\frac{\partial^{2} U_{\xi}}{\partial \zeta^{2}}, \\
\frac{\partial P}{\partial \zeta}=\psi^{2}\left[\psi^{2} \frac{\partial^{2} U_{\zeta}}{\partial \xi^{2}}+\frac{\partial^{2} U_{\zeta}}{\partial \zeta^{2}}\right], \\
\frac{\partial U_{\xi}}{\partial \xi}+\frac{\partial U_{\zeta}}{\partial \zeta}=0
\end{gathered}
$$

with boundary conditions

$$
\begin{gathered}
U_{\xi}=\frac{d f}{d \tau}, U_{\zeta}=0 \quad \text { at } \quad \zeta=0, \\
U_{\xi}=0, U_{\zeta}=0 \quad \text { at } \quad \zeta=1+\varepsilon \xi,
\end{gathered}
$$

$$
P=0 \quad \text { at } \quad \xi=-1, \quad P=0 \quad \text { at } \quad \xi=1
$$

and expression for the force, acting on the elastically fixed channel wall from the side of highly viscous liquid in the channel

$$
N=\left.\frac{b \ell \rho v x_{m} \omega}{\delta_{0}} \int_{-1}^{1}\left(\psi^{2} \frac{\partial U_{\zeta}}{\partial \xi}+\frac{\partial U_{\xi}}{\partial \zeta}\right)\right|_{\zeta=0} d \xi
$$

Further, we notice, that the members at $\psi^{2}$ in equations (8) and expression for the force (11) for a thin layer of highly viscous incompressible liquid may be omitted in the considered setting. As a result, we get dynamics equations of highly viscous incompressible in a narrow channel

$$
\frac{\partial P}{\partial \xi}=\frac{\partial^{2} U_{\xi}}{\partial \zeta^{2}}, \frac{\partial P}{\partial \zeta}=0, \frac{\partial U_{\xi}}{\partial \xi}+\frac{\partial U_{\zeta}}{\partial \zeta}=0,
$$

with boundary conditions (9), (10) and the expression for the force $N$

$$
N=\left.\frac{b \ell \rho v x_{m} \omega}{\delta_{0}} \int_{-1}^{1} \frac{\partial U_{\xi}}{\partial \zeta}\right|_{\zeta=0} d \xi
$$

The solution of equations (12) with boundary conditions (9), (10) takes the form of

$$
\begin{gathered}
U_{\xi}=\frac{d f}{d \tau}\left(1-\frac{\zeta}{1+\varepsilon \xi}\right)-\frac{1}{2} \frac{\partial P}{\partial \xi} \zeta(1+\varepsilon \xi-\zeta), \\
U_{\zeta}=-\frac{d f}{d \tau} \frac{1}{(1+\varepsilon \xi)^{2}} \frac{\varepsilon \zeta^{2}}{2}+ \\
+\frac{1}{2} \frac{\partial^{2} P}{\partial \xi^{2}}\left(\frac{\zeta^{2}}{2}(1+\varepsilon \xi)-\frac{\zeta^{3}}{3}\right)+\frac{1}{2} \frac{\partial P}{\partial \xi} \frac{\varepsilon \zeta^{2}}{2}, \\
P=\frac{3 \varepsilon\left(\xi^{2}-1\right)}{(1+\varepsilon \xi)^{2}} \frac{d f}{d \tau} .
\end{gathered}
$$

In so doing, we should notice, that

$$
\left.\frac{\partial U_{\xi}}{\partial \zeta}\right|_{\zeta=0}=-\frac{d f}{d \tau}\left(\frac{1}{(1+\varepsilon \xi)}+3 \varepsilon \frac{\xi+\varepsilon}{(1+\varepsilon \xi)^{2}}\right) .
$$


Mathematical Modeling of Elastically Fixed Wall Longitudinal Oscillations of Wedge-Shaped Channel Under Foundation Vibration

Equation (5) with consideration of (13), (15) and (7) takes the form of

$$
m \frac{d^{2} x_{1}}{d t^{2}}+2 K \frac{d x_{1}}{d t}+n x_{1}=-m \ddot{x}_{0}
$$

where

$$
2 K=\frac{4 \ell b \rho \vee}{\delta_{0}}\left(\frac{1}{\varepsilon} \ln \left(\frac{1+\varepsilon}{1-\varepsilon}\right)-\frac{3}{2}\right) .
$$

The solution of equation (16) with consideration of the set vibration foundation law (1) takes the form of

$$
\begin{gathered}
x_{1}=\frac{E_{z} \omega^{2} m}{\left(n-m \omega^{2}\right)^{2}+(2 K \omega)^{2}} \times \\
\times\left[\left(n-m \omega^{2}\right) \sin \omega t-2 K \omega \cos \omega\right]= \\
=\frac{k g m}{n} A(\omega) \sin (\omega t+\phi(\omega)), \\
A(\omega)=\frac{1}{\sqrt{\left(1-m \omega^{2} / n\right)^{2}+(2 K \omega / n)^{2}}} \\
\operatorname{tg} \phi(\omega)=\frac{2 K \omega}{m \omega^{2}-n} .
\end{gathered}
$$

Here $A(\omega)$ is the amplitude frequency characteristic of elastically fixed wall displacements of narrow wedge-shaped channel, $\phi(\omega)$ is the phase frequency characteristic of elastically fixed wall displacements of narrow wedgeshaped channel.

It may be noted, that $A(\omega) \rightarrow 1$ at $\omega \rightarrow 0$ and $A(\omega) \rightarrow 0$ at $\omega \rightarrow \infty$.

To obtain the law of pressure change along wedge-shaped channel in the form of

$$
\begin{gathered}
p=p_{0}-\ddot{x}_{0} \rho x+ \\
+\frac{k g m}{\delta_{0}^{2}} \Pi(\omega, x) \cos (\omega t+\phi(\omega)), \\
\Pi(\omega, x)=\frac{3 \varepsilon\left((x / \ell)^{2}-1\right)}{(1+\varepsilon(x / \ell))^{2}} \frac{\rho \vee \ell \omega}{n} A(\omega) .
\end{gathered}
$$

we use the obtained expression (17) for elastically fixed wall displacements. Here $\Pi(\omega, x)$ is the frequency dependent function of pressure distribution along narrow wedge-shaped channel.

It may be noted, that $\Pi(\omega, x) \rightarrow 0$ at $\omega \rightarrow 0$ and $\Pi(\omega, x) \rightarrow 0$ at $\omega \rightarrow \infty$.

By using the expression for pressure (19), we find pressure force from the side of the liquid, acting on channel wall 1

$$
\begin{aligned}
& F_{z}^{(1)}=-2 b \ell p_{0}-6 \frac{k g m}{\delta_{0}^{2}} \frac{\rho \nu \ell \omega}{n} A(\omega) \times \\
& \times\left[\frac{2}{\varepsilon}-\frac{1}{\varepsilon^{2}} \ln \left(\frac{1+\varepsilon}{1-\varepsilon}\right)\right] \cos (\omega t+\phi(\omega)) .
\end{aligned}
$$

Vertical projection of pressure force (support force), acting on channel wall 2 , takes the form of

$$
\begin{gathered}
F_{z}^{(2)}=2 b \ell p_{0}+6 \frac{k g m}{\delta_{0}^{2}} \frac{\rho v \ell \omega}{n} A(\omega) \times \\
\times\left[\frac{2}{\varepsilon}-\frac{1}{\varepsilon^{2}} \ln \left(\frac{1+\varepsilon}{1-\varepsilon}\right)\right] \cos (\omega t+\phi(\omega)),
\end{gathered}
$$

and horizontal projection of pressure force (shear force), acting on channel wall 2, is obtained as,

$$
\begin{aligned}
& F_{x}^{(2)}=-2 \varepsilon b \delta_{0} p_{0}-6 \frac{k g m}{\delta_{0}} \frac{\rho v \varepsilon \omega}{n} A(\omega) \times \\
& \times\left[\frac{2}{\varepsilon}-\frac{1}{\varepsilon^{2}} \ln \left(\frac{1+\varepsilon}{1-\varepsilon}\right)\right] \cos (\omega t+\phi(\omega)) .
\end{aligned}
$$

By considering the special case of $\varepsilon \rightarrow 0$ (the channel formed by two parallel walls), we get the following expressions for wall displacements and liquid pressure

$$
\begin{gathered}
\tilde{x}_{1}=\frac{1}{n} \frac{k g m}{\sqrt{\left(1-m \omega^{2} / n\right)^{2}+(2 \tilde{K} \omega / n)^{2}}} \times \\
\sin \left(\omega t+\operatorname{arctg}\left(2 \tilde{K} \omega /\left(m \omega^{2}-n\right)\right)\right), \\
\tilde{p}=p_{0}-\ddot{x}_{0} \rho x,
\end{gathered}
$$


where $2 \tilde{K}=2 b \ell \rho v / \delta_{0}$.

We may note for the case under consideration $(\varepsilon \rightarrow 0)$, that liquid pressure in channel (24) is defined by the pressure at butt ends cavities and the pressure due to the vibro-acceleration of foundation (an analogue of pressure due to the gravity), while the expressions for projections of pressure force, acting on channel walls (20)(22), take the form

$$
\tilde{F}_{z}^{(1)}=-2 b \ell p_{0}, \quad \tilde{F}_{z}^{(2)}=2 b \ell p_{0}, \quad \tilde{F}_{x}^{(2)}=0 .
$$

We introduce the following dimensionless parameters

$$
\Omega^{2}=n / m, 4 D^{2}=4 K^{2} /(m n)
$$

and a variable $\eta=\omega / \Omega$ and present $A(\omega)$ in form of

$$
A(\eta)=\frac{1}{\sqrt{\left(1-\eta^{2}\right)^{2}+4 D^{2} \eta^{2}}}
$$

To define maximum of function $A(\eta)$, we shall find the derivative of the given function with respect to its argument

$$
\frac{\mathrm{d} A}{\mathrm{~d} \eta}=\frac{\left(2\left(1-\eta^{2}\right)-4 D^{2}\right) \eta}{\left(\left(1-\eta^{2}\right)^{2}+4 D^{2} \eta^{2}\right)^{3 / 2}} .
$$

By solving the equation

$$
\left(2-2 \eta^{2}-4 D^{2} \eta\right)=0
$$

we find its roots

$$
\eta_{1}=-\sqrt{1-2 D^{2}}, \quad \eta_{2}=0, \quad \eta_{3}=\sqrt{1-2 D^{2}} .
$$

It can be noted, that the first and the second roots do not possess physical meaning. The third root corresponds to maximal volume of function $A(\eta)$

$$
A(\eta)=\frac{1}{2 D \sqrt{1-D^{2}}},
$$

i.e. defines resonance oscillations [23].

Thus, the expression for resonance frequency oscillation of elastically fixed wall of wedgeshaped channel takes the form of

$$
\omega_{\max }=\Omega \sqrt{1-2 D^{2}}=\sqrt{\frac{n}{m}-\frac{2 K^{2}}{m^{2}}} .
$$

To illustrate the mathematical model usage, we shall consider the channel with parameters

$$
\begin{gathered}
\ell=0.1 \mathrm{~m}, \delta_{0} / \ell=1 / 15, \ell / b=1 / 5, \\
\rho=1.84 \cdot 10^{3} \mathrm{~kg} / \mathrm{m}^{3}, v=2.5 \cdot 10^{-4} \mathrm{~m}^{2} / \mathrm{s}, \\
m=1.97 \mathrm{~kg}, n=3.6 \cdot 10^{2} \mathrm{~kg} / \mathrm{s}^{2} .
\end{gathered}
$$

We make calculations of its amplitude frequency characteristics $A(\eta)$, depending on the parameter $\varepsilon=\theta / \delta_{0}$, characterizing the channel wall inclination (channel cone characteristic). The calculation results for $A(\eta)$ are shown in fig. 2.

\section{SUMMARY}

The article presents the mathematical model of marrow wedge-shaped channel with elastically fixed wall. The channel is filled with highly viscous incompressible liquid and put on vibrating foundation. This model was used for investigation elastically fixed channel wall oscillations. The model and the calculations showed the substantial influence of wall inclination on amplitude oscillations and the appearance of additional pressure, conditioned by wall inclination. In particular, by enlarging channel wall inclination it is possible to substantially damp its longitudinal oscillations amplitude. The analytical expressions for elastically fixed channel wall motion law, as well as the law of liquid pressure distribution along the channel are found within the worked out problem. 
Mathematical Modeling of Elastically Fixed Wall Longitudinal Oscillations of Wedge-Shaped Channel Under Foundation Vibration

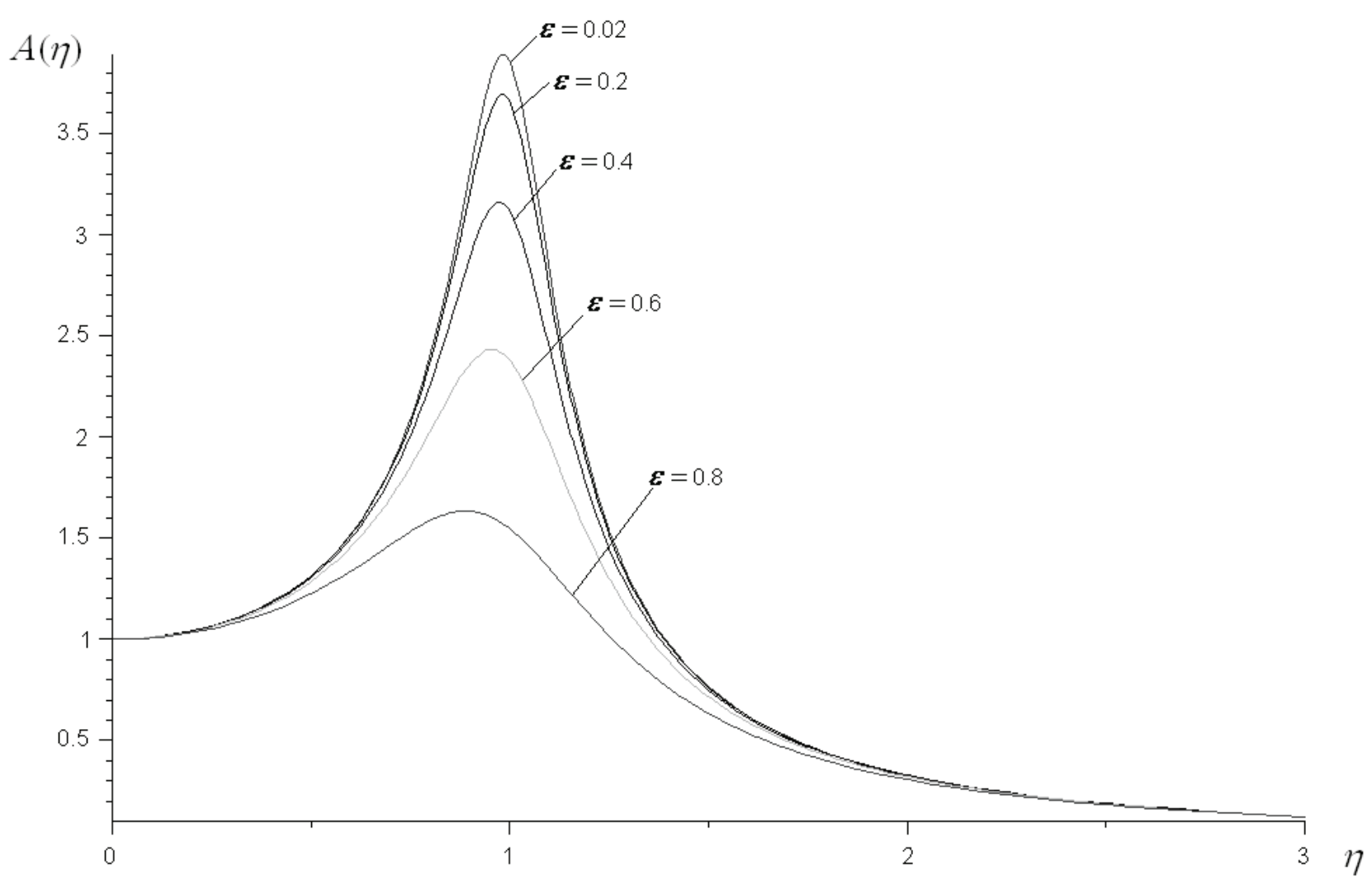

Figure 2. Dimensionless amplitude frequency characteristics $A(\eta)$.

Amplitude frequency characteristic of elastically fixed channel wall and frequency dependent function of pressure distribution along the channel were found. The analytical expression for defining channel wall oscillations resonance frequency was obtained.

The suggested model can be used for developing and investigating the effective hydrodampers for diminishing the oscillations caused by foundation vibration of various devices, machines and constructions, for example, at shock periodical influences, earthquakes and other vibration sources.

\section{ACKNOWLEDGEMENTS}

The reported study was funded by RFBR according to the research projects № 15-0101604-a and №16-01-00175-a.

\section{REFERENCES}

1. Indeitsev D.A., Polypanov I.S., Sokolov S.K. Calculation of Cavitation Life-Time of Ship Engine Liner // Problemy Mashinostraeniya i Nadezhnos'ti Mashin, Vol. 4, 1994, pp. 59-64.

2. Haddara M.R., Cao S. A Study of the Dynamic Response of Submerged Rectangular Flat Plates // Marine Structures, Vol. 9, 1996, pp. 913-933.

3. Amabili M. Vibrations of Circular Plates Resting on a Sloshing Liquid: Solution of the Fully Coupled Problem // Journal of Sound and Vibration, Vol. 245, no. 2, 2001, pp. 261-283.

4. Avramov K.V., Strel'nikova E.A. Chaotic oscillations of plates interacting on both sides with a fluid flow // International Applied Mechanics, Vol. 50, no. 3, 2014, pp. 303-309.

5. Zhavoronok S.I., Rabinskiy L.N. Axisymmetric problem of unsteady interaction 
of acoustic pressure waves with elastic shell of revolution // The Journal of Mekhanika Kompozitsionnykh Materialov i Konstruktsii, Vol. 12, no. 4, 2006, pp. 541-554.

6. Gorshkow A.G., Zhavoronok S.I., Medvedsky A.L., Rabinsky L.N. A nonsteady problem of cylindrical pressure wave's diffraction on thin elliptical shell // International Journal of Computational Civil and Structural Engineering, Vol. 3, no. 2, 2007, pp. 82-93.

7. Lamb H. Hydrodynamics, 6th edition, New York: Dover Publications, Inc., 1945.

8. Slezkin N.A. Dinamika viazkoi neszhimaemoi zhidkosti [Dynamics of a Viscous Incompressible Fluid], Gostekhizdat, Moscow, 1955 (in Russian).

9. Önsay T. Effects of layer thickness on the vibration response of a plate-fluid layer system // Journal of Sound and Vibration, Vol. 163, 1993, pp. 231-259.

10. Ageev R.V., Mogilevich L.I., Popov V.S., Popova A.A., Kondratov D.V. Mathematical Model of Pulsating Viscous Liquid Layer Movement in a Flat Channel with Elastically Fixed Wall // Applied Mathematical Sciences, Vol. 8, no. 159, 2014, pp. 78997908.

11. Faria C.T., Inman D.J. Modeling energy transport in a cantilevered Euler-Bernoulli beam actively vibrating in Newtonian fluid // Mechanical Systems and Signal Processing, Vol. 45, 2014, pp. 317-329.

12. Mogilevich L.I., Popov V.S. Investigation of the interaction between a viscous incompressible fluid layer and walls of a channel formed by coaxial vibrating discs // Fluid Dynamics, Vol. 46, no. 3, 2011, pp. 375-388.

13. Mogilevich L.I., Popov V.S., Popova A.A. Dynamics of interaction of elastic elements of a vibrating machine with the compressed liquid layer lying between them // Journal of Machinery Manufacture and Reliability, Vol. 39, no. 4, 2010, pp. 322-331.

14. Akcabay D.T., Young Y.L. Hydroelastic Response and Energy Harvesting Potential of Flexible Piezoelectric Beams in Viscous
Flow // Physics of Fluids, Vol. 24, no. 5, 2012, 054106.

15. Ageev R.V., Kuznetsova E.L., Kulikov N.I., Mogilevich L.I., Popov V.S. Mathematical model of movement of a pulsing layer of viscous liquid in the channel with an elastic wall // PNRPU Mechanics Bulletin, no. 3, 2014, pp. 17-35.

16. Mogilevich L.I., Popov V.S. Dynamics of interaction of an elastic cylinder with a layer of viscous incompressible fluid // Mechanics of Solids, Vol. 39, no. 5, 2004, pp. 146-155.

17. Egorova O.V., Zhavoronok S.I., Rabinsky L.N. Middle thickness shell's interaction with acoustical wave // Vestnik Moskovskogo Aviatsionnogo Instituta Journal, Vol. 17, no. 2, 2010, pp. 127-135.

18. Kuznetsova E.L., Mogilevich L.I., Popov V.S., Rabinsky L.N. Mathematical model of the plate on elastic foundation interacting with pulsating viscous liquid layer // Applied Mathematical Sciences, Vol. 10, no. 23, 2016, pp. 1101-1109.

19. Mogilevich L.I., Popov V.S., Popova A.A., Christoforova A.V. Mathematical Modeling of Hydroelastic Walls Oscillations of the Channel on Winkler Foundation Under Vibrations // Vibroengineering PROCEDIA, vol. 8, 2016, pp.294-299.

20. Kurzin V.B. Streamwise vibrations of a plate in a viscous fluid flow in a channel, induced by forced transverse vibrations of the plate // Journal of Applied Mechanics and Technical Physics, Vol. 52, no. 3, 2011, pp. 459-463.

21. Panovko Y.G., Gubanova I.I. Stability and Oscillations of Elastic Systems, Consultants Bureau Enterprises, Inc., New York, 1965.

22. Loitsyanskii L.G. Mechanics of Liquids and Gases, Pergamon Press, Oxford, 1966.

23. Magnus K. Schwingungen. Teubner, Stuttgart, 1976.

Mogilevich Lev Ilyich, Dr. Sci., Prof., Professor of Department of Applied Mathematics and System Analysis (School of Physics and Technology), Yuri Gagarin State Technical University of Saratov; 77 Politechnicheskaya 
Mathematical Modeling of Elastically Fixed Wall Longitudinal Oscillations of Wedge-Shaped Channel Under Foundation Vibration

street, Saratov, Russia, 410054; tel. +7(8452) 99-86-03; fax +7(8452) 99-88-10; e-mail: sstu_office@sstu.ru.

Popov Victor Sergeevich, Dr. Sci., Prof., Professor of Department of Applied Mathematics and System Analysis (School of Physics and Technology), Yuri Gagarin State Technical University of Saratov; 77 Politechnicheskaya street, Saratov, Russia, 410054; tel. +7(8452) 99-86-03; fax +7(8452) 99-88-10; e-mail: sstu_office@sstu.ru.

Rabinsky Lev Naumovich, Dr. Sci., Prof., Dean of Faculty of Applied Mechanics, Prof. of Department of a Resistance of Materials, Dynamics and Strength of Machines, Moscow Aviation Institute (National Research University); Volokolamskoe shosse, 4, Moscow, A-80, GSP-3,125993, Russia;

e-mail: mogilevich@sgu.ru,vic_p@bk.ru.

Могилевич Лев Ильич, профессор, доктор технических наук, профессор кафедры «Прикладная математика и системный анализ» Саратовского государственного технического университета имени Гагарина Ю.А.; 410054, Россия, г. Саратов, ул. Политехническая, 77; тел. +7(8452) 99-86-03; факс +7(8452) 99-8810; e-mail: sstu_office@sstu.ru.

Попов Виктор Сергеевич, профессор, доктор технических наук, профессор кафедры «Прикладная математика и системный анализ» Саратовского государственного технического университета имени Гагарина Ю.А.; 410054, Россия, г. Саратов, ул. Политехническая, 77; тел. +7(8452) 99-86-03; факс +7(8452) 99-8810; e-mail: sstu_office@sstu.ru.

Рабинский Лев Наумович, профессор, доктор физикоматематических наук, профессор кафедры сопротивления материалов, динамики и прочности машин Московский авиационный институт (государственный технический университет); Волоколамское шоссе, д. 4, г. Москва, А-80, ГСП-3, 125993; телефоны: +7 (499) 158-43-33, 158-58-70, 158-00-02; факс +7(499) 158-2977; e-mail: mogilevich@sgu.ru,vic_p@bk.ru. 


\title{
RELIABILITY ANALYSIS OF SOIL BASES ACCORDING TO THE DEFORMATION CRITERION
}

\author{
Vladimir S. Utkin ${ }^{1}$, Anastasia A. Kaberova ${ }^{2}$,Sergey A. Solovyev ${ }^{1}$ \\ ${ }^{1}$ Vologda State University, Vologda, RUSSIA \\ ${ }^{2}$ JSC "SKDM", Vologda, RUSSIA
}

\begin{abstract}
The article describes the methods of reliability analysis of structures soil bases with limited statistical information about random controlled parameters in design mathematical model of limit states. The proposed methods take into account the variability of the soil layers thicknesses. The article also illustrates the application of the evidence theory for calculation the statistical expected value of reliability with statistical data in the form of a subset of intervals. The numerical examples are given.
\end{abstract}

Keywords: soil bases, foundation, reliability, fuzzy variables, evidence theory, safety

\section{РАСЧЕТ НАДЕЖНОСТИ ГРУНТОВЫХ ОСНОВАНИЙ ПО КРИТЕРИЮ ДЕФОРМАЦИЙ}

\author{
В.С. Уткин ${ }^{1}$, А.А. Каберова ${ }^{2}$, С.А. Соловьев ${ }^{1}$ \\ ${ }^{1}$ Вологодский государственный университет, г. Вологда, РОССИЯ \\ ${ }^{2} \mathrm{AO} «$ СКДМ», г. Вологда, РОССИЯ
}

\begin{abstract}
Аннотация: В статье предлагаются методы расчет надежности грунтовых оснований зданий и сооружений при ограниченной статистической информации о контролируемых параметрах в расчетной математической модели предельных состояний. Разработанные в статье методы учитывают изменчивость толщин слоев грунта. Также проиллюстрировано применение теории свидетельств Демпстера-Шефера для определения статистического математического ожидания надежности при наличии данных в виде подмножества интервалов. Приведены численные примеры.
\end{abstract}

Ключевые слова: грунтовые основания, надежность, нечеткие переменные, теория свидетельств Демпстера-Шефера, безопасность

\section{INTRODUCTION}

Soil bases are understood as the soil mass which is deformed by efforts/loads on foundation (footing) from the above-foundation (above-footing) constructions. The reliability of soil bases is a measure of safety and a component of reliability system: which consisting of a soil base, a foundation and an above-foundation structure. Therefore, it is necessary to understand that the reliability of the soil bases, in fact, is the reliability of sequential system of elements: soil base, foundation and above-foundation structure. It's impossible to speak about the reliability of the whole system, with the absence of information of the soil base reliability value. Moreover, unreliability of the soil base causes emergence of such deformations and stresses in foundation and abovefoundation structure at which they become unreliable, even in the case when their reliability is provided itself. At the same time, unreliability of foundations and the above-foundation structure usually has small effect on soil bases reliability, if it is was provided at the design and operation stages with all set of loads and soil conditions. Thus, ensuring of the soil bases reliable work should be considered at the same time as a condition of reliable work of the whole system: soil base - foundation - above-foundation structure. The probability of non-failure is calculated as 


$$
P=P_{1} \cdot P_{2} \cdot P_{3}
$$

where $P_{i}$ is the probability of non-failure of the $i$-element of the sequential system.

\section{THE RELEVANCE OF THE ARTICLE}

The problem of soil bases reliability analysis at the operation stage gains special importance with the changing of the soil bases properties, which is caused by external and internal influence of natural and technogenic factors: underflooding, earthquakes, explosions, fires, etc. The analysis of the soil bases reliability is necessary also in the design of buildings reconstruction, when changing their functional purpose, which leading to the increase of the loads on the soil bases. According to the requirements of the Interstate Standard GOST 31937-2011 (came into force in 01.01.2014), it is necessary to give an assessment of technical condition of the structure (including a soil base), when capital repair or reconstruction is planned. Russian Building Code SP 22.13330.2011 (further designated as SP) "Soil bases of buildings and structures" requires designing the soil bases according to deformation and bearing capacity criteria. The purpose of the soil bases calculation on deformations is to restrict absolute or relative movement (settlement) of the soil bases and abovefoundation construction according to limits, which guaranteed safe operation of buildings and structures. However, the quantitative safety measure in SP is absent. The Russian Federation Law No. 384 "Technical regulations of buildings and structures safety" contains the requirements for mechanical (constructional) safety for buildings and structures, which should defined by calculations or experiments; and also a probabilistic assessment and estimation of safety risk.

Interstate Standard GOST 27751-2014 "Reliability of structures and foundations" is defined reliability as "the ability of structure to carry out the demanded functions during the lifetime". One of the reliability measures is the probability of non-failure or the probability of failure. It is offered to consider the methods of soil bases reliability analysis for buildings and structures at the design and operation stages according to the deformation criterion (the foundation settlement). Reliability analysis (assessment of probability of non-failure) of the soil bases is a difficult problem in many cases. Statistical information of random variables (controlled parameters) in mathematical models of limit states is a necessary for the reliability analysis of any structure. Information on random variables is considered full if it is possible to reveal the distribution function of a random variable, dependence (independence) of parameters and to estimate quantitative values of function parameters with sufficient accuracy. The Russian Interstate Standard GOST 27751-2014 recommends carrying out calculation of structures and soil bases reliability by mean of probabilistic methods "in the presence of sufficient data on variability of key parameters in calculations".

Probabilistic methods of reliability analysis received a certain theoretical justification and practical application in engineering calculations [1, 2, 3 etc.]. Works by N. N. Yermolaev, V.V. Mikheev, V.I. Sheinin etc. [2, 3 etc.] were devoted to the soil bases reliability analysis with a probabilistic approach. In practice, we can meet the situations when it is possible to receive only limited (incomplete) statistical information of random variables. It is difficult to justify the application of the probabilistic methods of reliability analysis having such limited information, which often based on assumptions. New directions in mathematics, such as "The evidence theory" [4, 5 etc.]; "Theory of fuzzy sets" [6]; "Theory of possibility" [7]; "Theory of interval statistical models" [8], etc. have gained development recently. New methods of reliability analysis of structure and machine parts $[9,10$ etc.] were developed on the basis of these theories. New approach to reliability calculations can be found in works $[11,12]$ for the soil bases and foundations. 


\section{THE MATHEMATICAL MODEL OF LIMIT STATE}

If the condition

$$
p<R,
$$

where $p$ is value of stress under foundation and $R$ is the normative strength of soil base, is satisfied, then reliability analysis according to deformation criterion is defined by the inequality:

$$
s \leq s_{u}
$$

where $s$ is the general settlement of soil base which is subject to determination; $s_{u}$ is the ultimate sag which is set by the State Standards (SP, items 5.6.46-5.6.50, for example).

The design scheme of soil bases is presented in the form of linearly deformable half-space in the design of the soil bases by SP (Figure 1).

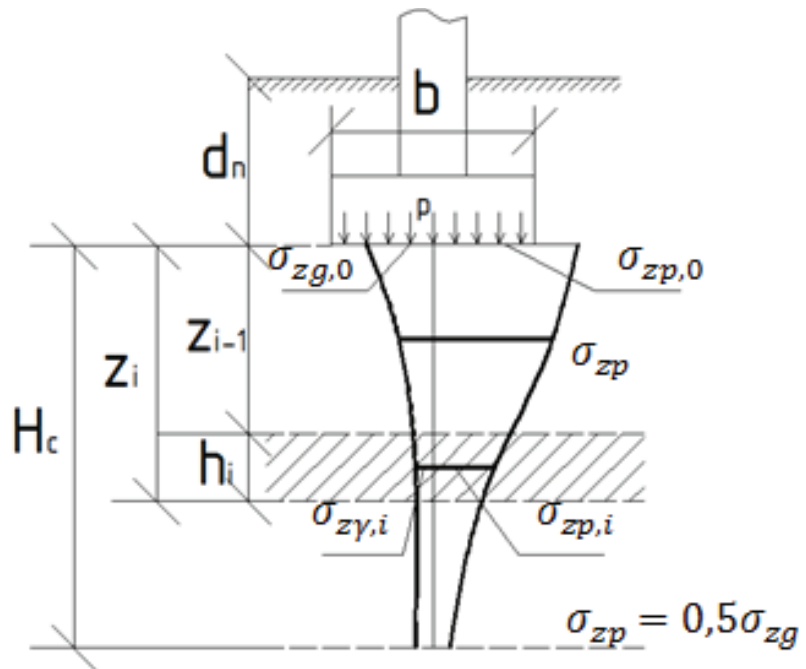

Figure 1. Design scheme of the soil bases.

Generally by SP, a settlement of a soil bases is calculated by eq. (2):

$$
s=\beta \sum_{i=1}^{n} \frac{\left(\sigma_{z p, i}-\sigma_{z \gamma, i}\right) \cdot h_{i}}{E_{i}}+\beta \sum_{i=1}^{n} \frac{\sigma_{z \gamma} \cdot h_{i}}{E_{e, i}} .
$$

Values of parameters in eq. (2) and ways of their determination (calculation) are described in SP. According to the SP item 5.6.35, it is specified that a settlement of a soil base $s$ with $p \leq \sigma_{z g, 0}$ where $p$ is an average stress under a foundation base and $\sigma_{z g, 0}$ is a vertical stress from a soil body weight on the foundation base mark, is determined by the equation:

$$
s=\beta \sum_{i=1}^{n} \frac{\sigma_{z p, i} \cdot h_{i}}{E_{i}},
$$

We consider that the formulation $p \leq \sigma_{z g, 0}$ is wrong. The condition $p \geq \sigma_{z g, 0}$ must be kept, to what we adhere further.

We consider thus a soil base, where $p>>\sigma_{z g, 0}$. According to the SP, parameter

$$
\sigma_{z \gamma, i}=\alpha_{i} \cdot \sigma_{z g, o}
$$

in (2) is small in comparison with pressure $p$ and $\sigma_{z \gamma, i}$. Then, the equation (2) will have a form:

$$
\tilde{s}=\beta \sum_{i=1}^{n} \frac{\tilde{\sigma}_{z p, i} \cdot \tilde{h}_{i}}{\tilde{E}_{i}},
$$

We meet such option in calculations of soil bases chimneys, masts, some high-rise buildings, supports for temporary bridges, etc.

According to the SP -

$$
\tilde{\sigma}_{z p, i}=\alpha_{i} \cdot \tilde{p}
$$

Value of pressure under the base foundation $\tilde{p}$ is calculated on design stages by loads sets or at an operation stage by measurement by various methods using the special measuring devices.

Deformation (elastic) module $\widetilde{E}_{i}$ is possible to determine by compression tests of soil, test of a stamp by static loading in field conditions (according to Russian State Standard GOST 20276-2012 "Soil. Methods of field definition of characteristics of durability and deformability"), by means of pressuremeter, etc. Soil layer 
thickness $\tilde{h}_{i}$ is defined by various methods: by direct linear measurements with drilling wells or by a comparative assessment of the deformation module, for example. According to the item 6.1.5.1, SP 47.13330.2012 "Engineering Researches for Construction" delimitation (determination) accuracy between layers of soil is determined by range from $0,25-0,5$ meters $(9.84$ 19.69 inch), which testify the random character of parameter $\tilde{h}_{i}$.

We'll consider the values $\tilde{p}, \tilde{E}_{i}$ and $\tilde{h}_{i}$ like random (fuzzy) values in soil base reliability analysis. We have a clear boundary of layers and thickness of soil $i$-layer $h_{i}$ in uniform soil. So, it is possible to accept it as determined value, just as it was accepted in the examples [13] in analysis of a multilayered soil base. In some cases, there is no clear boundary between layers of soils in multilayered soil base. Therefore, soil layer thickness under the base is characterized by an expected value and other statistical characteristics of random variable.

Let's consider soil bases reliability analysis by deformation (the settlement) criterion taking into account variability of $h_{i}$ under the foundation. Then, the mathematical model of the limit state (1) with (4) and

$$
\tilde{\sigma}_{z p, i}=\alpha_{i} \cdot \tilde{p}
$$

will assume a form:

$$
\sum_{i=1}^{n} \frac{\alpha_{i} \cdot \tilde{p} \cdot \tilde{h}_{i}}{\tilde{E}_{i}} \leq s_{u} / \beta,
$$

where $\alpha$ is a coefficient, which is accepted according to the table from SP, depending on a form of the foundation base and relative depth

$$
\xi=2 z / b
$$

of the soil base layer; $\tilde{p}$ is pressure under a foundation base. $\tilde{p}$ is accepted according to statistical data (a priori information) at a design stage. At a stage of operation, $\widetilde{p}$ is measured by various methods [14]. In practice [13], "it is widely used at calculation of foundation settlement " by the following formula:

$$
\tilde{\sigma}_{z p}=K \frac{\tilde{p}}{z^{2}},
$$

where $K$ is coefficient, which value is determined for simplification of calculations by the table III.1 [13] depending on value of the relation $r / z$, where $r$ is across distance from the axis $Z$ passing through the point of force application $p ; z$ is a distance from the considered foundation point to the foundation base.

At designation of

$$
\tilde{H}_{i}=\tilde{h}_{i} \cdot \alpha_{i}
$$

in (5), we receive mathematical model for reliability analysis:

$$
\sum_{i=1}^{n} \frac{\tilde{p} \cdot \tilde{H}_{i}}{\tilde{E}_{i}} \leq s_{u} / \beta,
$$

\section{THE RELIABILITY ANALYSIS OF SOIL BASES}

Let's consider an option, where $\tilde{p}, \tilde{H}_{i}, \widetilde{E}_{i}$ are defined (are measured) by the small number of values. It does not allow using a probabilistic method of reliability analysis. We will use methods of the possibilities theory [7] for their description. According to terminology of "the theory of possibilities", parameter X is called a «fuzzy variable». For the description of fuzzy variables, we'll use distribution function of possibility [7, 9, 10 etc.]:

$$
\begin{gathered}
\pi_{X}(x)=\exp \left[-\left(\frac{x-a_{x}}{b_{x}}\right)^{2}\right], \\
\text { where } \quad a_{x}=0.5\left(X_{\max }+X_{\min }\right)
\end{gathered}
$$




$$
b_{x}=0.5\left(X_{\max }-X_{\min }\right) / \sqrt{-\ln \alpha} ;
$$

risk level (cut level) $\alpha \in[0 ; 1]$; Value of $\alpha$ is set by [15]. Function (7) is represented on Figure 2.

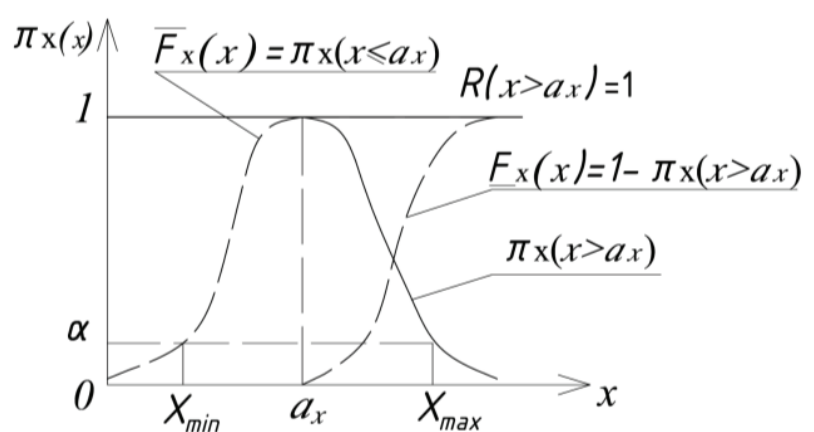

Figure 2. Distribution function of possibilities

$$
\pi_{X}(x) \text {. }
$$

From Fig. 2, we see that at $\pi_{X}(x)=1$ we have conditional "average" value of fuzzy variable $a_{x}$. In the soil base reliability analysis for model (6) with nonlinear dependence between fuzzy variables, we use the principle of generalization of Zaden L. [6] in the fuzzy sets theory. For this purpose, we create a fuzzy function from (6) as:

$$
Y(y)=\tilde{p} \sum_{i=1}^{n} \frac{\tilde{H}_{i}}{\tilde{E}_{i}} \leq s_{u} / \beta,
$$

Beforehand, we shall show finding of the inverse function $x$ from (7). The inverse function from $x$ can be found as a result of mathematical actions in the form

$$
x=a_{x} \pm b_{x} \cdot \theta
$$

where $\quad \theta=\sqrt{-\ln \pi_{X}(x)}=\sqrt{-\ln \alpha_{X}}$

$$
\pi_{X}(x)=e^{-\theta_{\min }^{2}} \text {. }
$$

By analogy for $x$, we shall find inverse functions for $\tilde{p}, \tilde{H}_{i}, \widetilde{E}_{i}$ in (6), if we accepted their description concerning the function (7) with the same risk level $\alpha$ for all fuzzy variables. Inverse functions of fuzzy variables $\tilde{p}, \tilde{H}_{i}, \tilde{E}_{i}$ assume the form:

$$
\begin{gathered}
p=a_{p} \pm b_{p} \cdot \theta, H_{i}=a_{H_{i}} \pm b_{H_{i}} \cdot \theta, \\
p=a_{p} \pm b_{p} \cdot \theta, H_{i}=a_{H_{i}} \pm b_{H_{i}} \cdot \theta,
\end{gathered}
$$

where

$$
\begin{gathered}
a_{p}=0.5 \cdot\left(p_{\max }+p_{\min }\right), \\
b_{p}=0.5 \cdot\left(p_{\max }-p_{\min }\right) / \sqrt{-\ln \alpha}, \\
a_{H_{i}}=0.5 \cdot\left(H_{i, \max }+H_{i, \min }\right), \\
b_{H_{i}}=0.5 \cdot\left(H_{i, \max }-H_{i, \min }\right) / \sqrt{-\ln \alpha}, \\
a_{E_{i}}=0.5 \cdot\left(E_{i, \max }+E_{i, \min }\right), \\
b_{E_{i}}=0.5 \cdot\left(E_{i, \max }-E_{i, \min }\right) / \sqrt{-\ln \alpha} .
\end{gathered}
$$

We shall find inverse functions for $Y(y)$ from (8) through inverse functions of fuzzy arguments.

$$
\left\{\begin{array}{l}
y_{l}=\left(a_{p}-b_{p} \cdot \theta\right) \cdot \frac{a_{H_{i}}-b_{H_{i}} \cdot \theta}{a_{E_{e, i}}+b_{E_{e, i}} \cdot \theta} \\
y_{r}=\left(a_{p}+b_{p} \cdot \theta\right) \cdot \frac{a_{H_{i}}+b_{H_{i}} \cdot \theta}{a_{E_{e, i}}-b_{E_{e, i}} \cdot \theta}
\end{array}\right.
$$

The principle of the plus and minus sings arrangement is described in [6].

Firstly, we shall find conditional "average" $a_{y}$ value of function $\pi_{Y}(y)$ at

$$
\pi_{Y}(y)=1 \text { or } \theta=\sqrt{-\ln \pi_{Y}(y)}=0 .
$$

If it turns out from (9) or (10) with a glance at (8)

$$
a_{y}=a_{p} \cdot \sum_{i=1}^{n} \frac{a_{H_{i}}}{a_{E_{i}}} \leq s_{u} / \beta \text {, }
$$

then the right branch of function $\pi_{Y}(y)$ is considered where $R=1$, i.e. possibility of nonfailure of a soil base is equal to one. The possibility of failure (by analogy with 


$$
\left.\pi_{X}(x)=e^{-\theta_{\min }^{2}}\right) Q=\pi_{Y}\left(y>a_{y}\right)=e^{-\theta_{\min }^{2}}
$$

for the right branch (see Figure 2), where $\theta_{\text {min }}$ is found by the minimum value. Necessity of non-failure of soil base is calculate as [7]

$$
N=1-Q
$$

We will find values of $\theta$ for calculation $Q$. We use the equation (10) for this purpose taking into account (8)

$$
\text { (at } \left.a_{y} \leq s_{u} / \beta\right)
$$

accepting that

$$
y_{n p}=S_{u} / \beta .
$$

Value of

$$
N=1-e^{-\theta_{\min }^{2}}
$$

corresponds to the lower value of probability of non-failure

$$
\underline{P}=N \text {. }
$$

The upper value of probability of non-failure operation is

$$
\bar{P}=R=1 \text { at } a_{y}<s_{u} / \beta \text {. }
$$

Reliability of the soil base by a criterion of the general settlement presented by interval of probabilities values

$$
[\underline{P} ; \bar{P}] \text { or }\left[1-e^{-\theta_{\min }^{2}} ; 1\right] \text {. }
$$

We have

$$
\bar{Q}=1 \text { at } a_{y} \geq s_{u} / \beta \text {, }
$$

i.e. possibility of refusal is equal to one. We shall find $\theta_{\min }$ from (10) at

$$
y_{\text {лев }}=s_{u} / \beta
$$

and possibility of non-failure

$$
R=e^{-\theta_{\min }^{2}} .
$$

Reliability of the soil base is characterized by an interval of values

$$
\left[1 ; e^{-\theta_{\min }^{2}}\right]
$$

Now we shall consider an algorithm of of the soil base reliability analysis on the general settlement by the offered method at an example. We shall consider an option with two layers, where the top layer is uniform soil, which lies on soil (rock) with the deformation module $E>100 M P$, to reduce calculations and the article. Thickness of the first soil layer $h_{1}$ is appointed on the condition of

$$
h_{1}=0,4 \cdot b
$$

and it is the determinate value because of

$$
H_{1}=h_{1} \cdot \alpha_{1} \text {. }
$$

The second layer have random (fuzzy) thickness $\tilde{h}_{2}$ and it everywhere is less than $0,4 \cdot b$. The value

$$
\tilde{H}_{2}=\tilde{h}_{2} \cdot \alpha_{2}
$$

is a fuzzy variable because of small number of measurements of $\tilde{h}_{2}$. The soils can be nonuniform in practice, and in these cases a number 
of layers thicknesses $h_{i}$ are random variables or fuzzy variables, depending on the calculation method.

It is also allowed to calculate vertical stress using average values (according to SP). However, such remarks not allowed in the reliability analysis. Therefore, it is necessary to consider variability of a layer height in reliability analysis, as it is made on the lower example.

\section{THE NUMERCAL EXAMPLE}

Let $b=2 \mathrm{~m} \quad$ (78.74 inch), then $h_{1}=i d e m=0,4 \cdot b=0,8 \mathrm{~m} \quad(31.49$ inch $)$, and value $h_{2}$ is revealed from a series of small number of measurements near a base contour. $h_{2, \text { min }}=0,2 \mathrm{~m} \quad(7.87$ inch $), \quad h_{2, \text { max }}=0,5 \mathrm{~m}$ (19.68 inch) and it is a fuzzy variable. The equation (6) in an expanded form is

$$
\tilde{p}\left(\frac{H_{1}}{\tilde{E}_{1}}+\frac{\tilde{H}_{2}}{\tilde{E}_{2}}\right) \leq s_{u} / \beta .
$$

Let $\quad p_{\max }=12,1 \quad \mathrm{MPa} \quad\left(\begin{array}{lll}1.77 & \mathrm{ksi}\end{array}\right)$, $p_{\text {min }}=7,6 \mathrm{MPa} \quad(1.11 \mathrm{ksi}), \quad E_{1, \max }=8 \mathrm{MPa}$ $(1.17 \mathrm{ksi}), \quad E_{1, \min }=5,4 \mathrm{MPa} \quad(0.79 \mathrm{ksi})$, $E_{2, \text { max }}=10 \mathrm{MPa}(1.46 \mathrm{ksi}), E_{2, \text { min }}=8 \mathrm{MPa}$ $(1.17 \mathrm{ksi}), E_{3}>100 \mathrm{MPa}(14.62 \mathrm{ksi})$. Let risk level is $\alpha=0,1$. According to SP: $s_{u}=0,1 \mathrm{~m}$ (3.94 inch), $\quad \beta=0,8$. Then $s_{u} / \beta=0,1 / 0,8=0,125 \mathrm{~m}$ (4.92 inch). Using the table values of $\alpha_{i}$ from SP, we shall calculate $H_{1}=h_{1} \cdot \alpha_{1}=0,8 \cdot 0,8=0,64 \mathrm{~m}$ (25.19 inch), $H_{2, \text { max }}=h_{2, \max } \cdot \alpha_{2}=0,5 \cdot 0.567=0,284 \mathrm{~m} \quad(11.18$ inch), $H_{2, \min }=h_{2, \min } \cdot \alpha_{2}=0,2 \cdot 0,488=0,098$ $\mathrm{m}$ (3.86 inch).

We can calculate the values: $a_{p}=9,85 \mathrm{MPa}$ $\left(\begin{array}{ll}1.43 \mathrm{ksi}\end{array}\right), \quad b_{p}=1,48 \mathrm{MP} \quad(0.21 \quad \mathrm{ksi})$, $a_{H_{2}}=0,19 \mathrm{~m}$ (7.48 inch), $b_{H_{2}}=0,061 \mathrm{~m}$ (2.4 inch), $a_{E_{1}}=6,7 \mathrm{MPa} \quad(0.97 \quad \mathrm{ksi})$, $b_{E_{1}}=0,855 \mathrm{MPa}(0.12 \mathrm{ksi}), a_{E_{2}}=9 \mathrm{MPa}(1.30$ ksi), $\quad b_{E_{2}}=0,659 \mathrm{MPa} \quad(0.09 \quad \mathrm{ksi}) . \quad$ As $a_{y}=0,020(0.79$ inch $)<0,125 \mathrm{~m}$ (4.92 inch), so $R=1$. From Eq. (10), we have: $y_{n p}=0,125$. Then we calculate $\theta_{1}=2,72 ; \theta_{2}=-1240,29$ and $Q=e^{-\theta_{\min }^{2}}=e^{-2,72^{2}}=6,1 \cdot 10^{-4} \quad$ and $N=1-Q=1-0,00061=0,99939$. Lower value of probability of non-failure operation of the soil base is $\underline{P}=N=0.99939$, and top value of probability is $\bar{P}=R=1$. The soil base reliability by criterion of deformation (the general settlement) is characterized by the interval of nonfailure probability $[0,99939 ; 1]$ or the interval of failure probability $[0 ; 0,00061]$.

\section{DEMPSTER-SHAFER THEORY (EVIDENCE THEORY)}

Is it possible to consider the soil base reliability (probability of failure), which we received in an example in accordance with the results of calculations, sufficient for safe operation of the soil base in system - soil base - the foundation above-foundation construction? The answer of this question depends on many factors. For example, it depends on the responsibility of the construction on safety (nuclear power plant and the hospital case or a house and etc.) the existing norms on reliability of objects, the region with seismic activity, etc.

According to [16], probability of failure of a soil base must not be more $10^{-5}$ without preliminary signal. If the probability of failure is more then $10^{-3}$, then the soil base exploitation is not allowed. It is necessary to carry out several tests to the basis reliability analysis during some time in various conditions in important design cases. The reliability results in the form of intervals $[N ; R]_{i}$ will be different. Therefore, the interval is one of a random variable indicators in an in- 
terval form. The statistical analysis of such values is carried out by methods of the evidence theory by Dempster-Shafer [4, 5, 17, 18 etc.]. The statistical analysis of this information is required to get a statistical expected value of reliability in the form of one interval. We show it on a simple example of reliability analysis according to information in form of a subset of reliability intervals received as the results of tests and calculations. Let values of reliability intervals $\quad[0,99939 ; 1,0] ; \quad[0,99938 ; 1,0]$; $[0,99937 ; 1,0]$. The statistical expected value of intervals (for example the subset intervals) is found by equations [18]:

$$
\left\{\begin{array}{l}
\underline{E} X=\int_{\Omega} \omega \frac{d \underline{F}(x)}{d x} d \omega=\sum_{i=1}^{n} m\left(A_{i}\right) \inf A_{i} \\
\bar{E} X=\int_{\Omega} \omega \frac{d \bar{F}(x)}{d x} d \omega=\sum_{i=1}^{n} m\left(A_{i}\right) \sup A_{i}
\end{array}\right.
$$

where $A_{i}$ is a subset of set $\Omega ; X$ is indiscrete random variable; $\mathrm{E} X$ is mathematical expectation of intervals total (upper and lower); $m\left(A_{i}\right)$ is basic probability which is found by the formula

$$
m\left(A_{i}\right)=C_{i} / N
$$

where $N$ is number of intervals, $C_{i}$ is generally, number of tests (calculations) in one of subsets $A_{i} \leq \Omega$. By the data of the example condition: $C_{i}=1, N=3$. For the data provided in the example of intervals we have

$$
m\left(A_{1}\right)=\frac{1}{3}, m\left(A_{2}\right)=\frac{1}{3}, m\left(A_{3}\right)=\frac{1}{3}
$$

and by the (11) we'll get

$$
\overline{\mathrm{E}} X=(1,0+1,0+1,0) \cdot \frac{1}{3}=1,0
$$

and

$$
\begin{aligned}
\underline{E} X=(0,99939 & +0,99938+0,99937) \cdot \frac{1}{3}= \\
& =0,99938 .
\end{aligned}
$$

The statistical expected value of reliability is $[0,99938 ; 1]$. It is possible to increase the number of intervals of assessment reliability or increase the accuracy of measurements for refinement the reliability interval but it is depends on the period related with time and an economic component.

\section{CONCLUSIONS}

1. The article described the new method of soil base reliability analysis by the deformation (the settlement) criterion with limited statistical information about controlled parameters in design mathematical model of limit state.

2. The algorithm of soil bases reliability analysis shown at the.

3. The offered method of soil bases reliability analysis can be used for reliability analysis with other data and for other types of soil bases.

\section{REFERENCES}

1. Xiao-hui Tan, Na Hu, Dan Li etc. TimeVariant Reliability Analysis of Unsaturated Soil Slopes Under Rainfall. // Geotechnical and Geological Engineering, 2013, Vol. 31, No. 1, pp. 319-327.

2. Ермолаев Н.Н., Михеев В.В. Надежность оснований сооружений. - М.: Стройиздат, 1976. - 152 с

3. Afeez Adefemi Bell. Reliability assessment of reddish brown tropical soil as a liner material. // Geotechnical and Geological Engineering, 2013, Vol. 31, No. 1, pp. 35-45.

4. Уткин В.С., Соловьев С.А. Определение несущей способности и надежности стальной балки на стадии эксплуатации с использованием теории свидетельств 
Демпстера-Шефера. // Деформация и разрушение материалов, 2015, № 7, с. 1015.

5. Уткин В.С., Соловьев С.А. Определение надежности и несущей способности элементов конструкций с использованием теории свидетельств ДемпстераШефера // Строительная механика и расчет сооружений, 2015, №5, с. 38-45.

6. Zaden L.A. Fuzzy Sets. // Information and Control, 1965, Vol. 8, No. 3, pp. 338-353.

7. Dubois D., Prade H. Possibility Theory, Probability Theory and Multiple-valued Logics: A Clarification. // Annals of Mathematics and Artificial Intelligence, 2001, No. 32, pp. 35-66.

8. Кузнецов В.П. Интервальные статистические модели: монография. - М.: АСB, 1998. - 304 c.

9. Уткин В.С. Новые методы расчетов надежности строительных конструкций. Учебное пособие. - Вологда: ВоГТУ, 2011. - 79 c.

10. Уткин В.С. Расчет надежности механических систем при ограниченной статистической информации. Вологда: ВоГТУ, 2008. 188 c.

11. Уткин В.С., Каберова А.А., Соловьев С.А. Расчет надежности грунтовых оснований зданий и сооружений по несущей способности при реконструкции // Сейсмостойкое строительство. Безопасность сооружений. 2016. № 3. С. 51-58.

12. Уткин В.С., Каберова А.А., Соловьев C.A. Расчет надежности основания фундамента, сложенного просадочными грунтами, по критерию деформаций // Геотехника. 2016. №3. С. 18-25.

13. Цытович Н.А. Механика грунтов. М.: Высшая школа, 1979. 271 с.

14. Тензо-М. Тензодатчики. Тензорезисторный извлекаемый датчик давления грунта ДДГЛ [Электронный ресурс]. Режим доступа: tenso-m.ru/pages/21. Дата обращения: 26.02.2015.
15. Уткин В.С., Соловьев С.А., Каберова А.А. Значение уровня среза (риска) при расчете надежности несущих элементов возможностным методом // Строительная механика и расчет сооружений. 2015. №6. С. 63-67.

16. Казачек В.Г., Нечаев Н.В., Нотенко С.Н. и др. Обследование и испытание зданий и сооружений. Под ред. В.И. Римшина. Учебник для вузов 3-е издание. М.: Высшая школа, 2007. 655 с.

17. Shafer G.A. Mathematical Theory of Evidence. Princeton University Press, 1976. 297 p.

18. Уткин Л.В. Анализ риска и принятие решений при неполной информации. СПб: Наука, 2007. 404 с.

Уткин Владимир Сергеевич, доктор технических наук, профессор кафедры промышленного и гражданского строительства Вологодского государственного университета; 160000, Россия, г. Вологда, ул. Ленина, д. 15; тел. +7(8172) 51-83-96,

e-mail: utkinvogtu@mail.ru

Каберова Анастасия Андреевна, инженер, АО «СКДМ»; 160000, Россия, г. Вологда, Набережная 6 Армии, д. 201, тел. +7(8172) 2732 82,

e-mail: kaberovanas@yandex.ru

Соловьев Сергей Александрович, аспирант кафедры промышленного и гражданского строительства Вологодского государственного университета; 160000, Россия, г. Вологда, Набережная 6 Армии, д. 201, тел. +7(8172) 27-32-82; e-mail: ser6sol@yandex.ru

Vladimir S. Utkin, Dr. Sc., Professor, Department of Industrial and Civil Engineering, Vologda State University; 15, Lenin Street, Vologda, 160000, Russia; Phone/fax: +7(8172) 5183 96, e-mail: utkinvogtu@ mail.ru

Anastasia A. Kaberova, engineer, JSC "SKDM", Vologda; 201, VI Army promenade, Vologda, 160000, Russia. Phone/fax: +7(8172) 27-32-82;

e-mail: kaberovanas@yandex.ru

Sergey A. Solovyev, Ph.D. Student, Department of Industrial and Civil Engineering, Vologda State University; 15, Lenin Street, Vologda, 160000, Russia; Phone/fax: +7 (8172) 51-83-96; e-mail: ser6sol@yandex.ru 


\title{
THE EXTENDED SHELL THEORY OF VEKUA-AMOSOV TYPE AND THE LOW-ORDER PLATE MODELS
}

\author{
Sergey I. Zhavoronok \\ Institute of Applied Mechanics of Russian Academy of Sciences, Moscow, RUSSIA \\ National Research Moscow State University of Civil Engineering, Moscow, RUSSIA \\ Moscow Aviation Institute (National Research University), Moscow, RUSSIA
}

\begin{abstract}
The extended shell theory of I.N. Vekua - A.A. Amosov type is formulated in terms of the Lagrangian variational formalism of analytical mechanics and accounts for the boundary conditions on shell's faces as constraint equations. The hierarchy of thick shell and plate models based on the extended theory allows one to obtain the classical plate model as a lowest-order approximation of the three-dimensional elasticity problem with no supplementary assumptions.
\end{abstract}

Keywords: extended shell theory, refined plate theory, analytical mechanics of continua, constraint equations, Lagrangian formalism

\section{РАСШИРЕННАЯ ТЕОРИЯ ОБОЛОЧЕК ТИПА И.Н. ВЕКУА - А.А. АМОСОВА И МОДЕЛИ ПЛАСТИН НИЗШИХ ПОРЯДКОВ}

\author{
С.И. Жаворонок \\ Институт прикладной механики Российской академии наук, г. Москва, РОССИЯ \\ Национальный исследовательский Московский государственный строительный университет, \\ г. Москва, РОССИЯ \\ Московский авиационный институт (Национальный исследовательский университет), г. Москва, РОССИЯ
}

Резюме: На базе лагранжева формализма аналитической механики континуальных систем построена расширенная теория оболочек типа И.Н. Векуа - А.А. Амосова, учитывающая краевые условия на лицевых поверхностях оболочки в форме уравнений связей. Иерархия моделей толстостенных оболочек и плит, полученная на основе расширенной теории оболочек, включает в себя классическую теорию пластин как низшее приближение трехмерной задачи теории упругости без введения каких-либо дополнительных допущений.

Ключевые слова: расширенная теория оболочек, уточненные теории пластин, аналитическая механика континуальных систем, уравнения связей, формализм Лагранжа

\section{INTRODUCTION}

As it was noted recently $[1,2]$, “...one should keep in mind that during the past two centuries only small progress has been achieved in better $2 \mathrm{D}$ modelling of the shell internal state in the boundary zone of width of the order of a few shell thicknesses, where all the fields are essentially three-dimensional" [1, p. 5]. To model adequately three-dimensional stress and strain state in such area as boundary layers, the improved two-dimensional theories are required; “...without a breakthrough in better 2D modelling of the boundary zone any refinement of existing 2D shell models in the interior domain may not be regarded as satisfactory" [1, p. 5]. Moreover, not only the near-boundary stress state analysis requires the qualitative refinement of classical theories. The solution for some buckling problems for functionally graded plates and shells made from metal-ceramics systems becomes impossible without higher-order approximations (see [3-6]). For instance, it is shown that for the compressed square 
$\mathrm{Al} / \mathrm{Al}_{2} \mathrm{O}_{3}$ plate [4] with power law distribution of elastic moduli through the thickness at least the third order theory is required to estimate corresponding critical stresses; the author considers the fifth order theory as "... sufficient with respect to the accuracy of the solutions" if the power law index is equal to 10 [4, p. 140]. The same results were obtained considering the vibrations of functionally graded systems [3-5]. On the other hand the classical theory as well as the $1^{\text {st }}$-order shear deformation theory cannot be applied to the high-frequency vibrations investigation even for thin-walled structures [7] where the near-face boundary layers exist [8]. The higher-order theories of shells and plates can be also implemented in the civil engineering practice where most structural elements are relatively thick. Use of the finite element method allows one to obtain quantitative results but has nothing to do with the qualitative analysis.

To construct a higher-order shell theory, one can use various approaches that can be provisionally subdivided to three main concepts [9]. The first concept so-called the "engineering" one consists in the system of assumptions; "... it depends on the intuition of engineers to construct a sound theory ... it is needless to say that this approach can not be systemized and is prone to errors" ([9], p. 755). The second and the third concepts are based on the strong mathematical formalism; they can be defined as "induction" and "reduction". The "induction", or "direct approach" consists in the deformable surface with a set of directors defined at each point [10-13]; the simplest one is the 2D Cosserat continuum $[10,11]$. The "reduction" is based on the dimensional reduction of the $3 \mathrm{D}$ problem of solid mechanics and uses various decompositions of unknowns with respect to the coordinate normal to a midurface [3-6, 8, 9, 14-24]. Monomial functions [3-6, 8, 9, 14-16], orthogonal polynomials [17-21], or trigonometric functions can be used as a basis together with Galerkin $[17,18,20]$ or variational $[19,20]$ approaches. It has to be noted that both "induction" and "reduction" give the shell model of the same type, a two-dimensional manifold with a set of fields defined on them, or (form the practical p. o. v.) a material surface with a set of mechanical properties corresponding to these fields (e. g. tangential stiffness, bending stiffness, warping stiffness, etc.).

The well-known asymptotic approach $[25,26]$ is the efficient alternative of the formal reduction; it offers asymptotically correct low-order theories but results complex higher-order models. On the other hand, the "formal reduction" allows one to obtain the "hierarchical" set of theories by truncating series at different orders; it is "... capable of approximating the solution of the full, three-dimensional problem... in various norms, a feature not shared by models obtained from, for example, asymptotic expansion ..." [21]. Instead of different reduction methods both "formal" and asymptotic theories are based on the same dimensional reduction idea; but their lowest-order models are qualitatively different. The asymptotic method gives the classical Kirchhoff theory contrarily to the "formal" approach that result the well-know $1^{\text {st }}$ order shear deformation theory if the linear approximation is used. Moreover, this model requires the "plane stress assumption" artificially introduced [9, 21]: $\bar{v}=v /(1+v)$, otherwise the bending stiffness is drastically overestimated [9]. Thus, the classical and asymptotically correct Kirchhoff's theory cannot be included in the formally defined system of shell models, and the hierarchy of theories seems to be incomplete.

On the one hand, the consistent Kirchhoff model of a plate can be obtained by using of the second-order theory of Vekua type [17] where the boundary conditions on the faces of a shell can be satisfied through the supplementary degrees of freedom. On the other hand, a lot of modifications of such a theory were proposed; this is, for instance, an extended shell theory of Vekua $[17,22]$ that uses series residuals to satisfy the boundary conditions; it results the properly defined bending stiffness for the first-order theory. Another way so-called "correction solution" was developed by A. A. Amosov [20] who improved the Vekua hierarchy by properly ac- 
counting for the through-thickness metric variation [20, 27-30].

A modified reduction approach can be based on the Lagrangian formalism of analytical mechanics; the shell model is defined within the configuration space with a set of field variables and the scalar generator function [31-35]; the field variables are determined as biorthogonal expansion factors with respect to the polynomials of the normal coordinate (classic theories) or piecewise functions (finite layer model). This formalism allows one to represent the boundary conditions on faces being "lost" after dimensional reduction as constraint equations for field variables; thus, the extended variational shell model becomes constrained [36-38].

Below the extended $\mathrm{N}^{\text {th }}$ order theory of thick anisotropic shells of Vekua - Amosov type is briefly described, and the dynamical bending of an orthotropic plate is considered as a simplest example. It is shown that the Kirchhoff plate model follows directly from the $1^{\text {st }}$ order plate theory if the boundary conditions are considered as constraints.

\section{THE EXTENDED SHELL THEORY OF N-TH ORDER}

Let the shell be a three-dimensional body with smooth faces $S_{ \pm} \neq \varnothing$ and a lateral surface $S_{B}$ [31-33], where $S$ is the smooth base surface and $2 h=h_{+}-h_{-}$is the shell thickness [31]:

$$
\begin{gathered}
V \subset \mathbb{R}^{3}, \bar{V}=V \cup \partial V, \partial V=S_{B} \oplus S_{ \pm} ; \\
\forall M_{*} \in \bar{V} \mathbf{R}\left(M_{*}\right)=\mathbf{r}(M)+\xi^{3} \mathbf{n}(M), \\
M \in S, \quad \xi^{3} \in\left[h_{-}(M), h_{+}(M)\right] .
\end{gathered}
$$

The mathematical model of a shell consists in the two-dimensional manifold $S$ where $\bar{S}=S \cup\left(\partial S=S \cap S_{B}\right)$, with the curvilinear chart $\xi^{\alpha}, \alpha=1,2[31,32]$ :

$$
\forall M \in \bar{S} \mathbf{R}(M) \equiv \mathbf{r}\left(\xi^{\alpha}\right) ; \quad \xi^{\alpha} \in D_{\xi} \subseteq \mathbb{R}^{2}
$$

The tangent bundle $T_{M} S$ with the covariant base vectors $\mathbf{r}_{\alpha}=\partial_{\alpha} \mathbf{r}, \partial_{\alpha} \equiv \partial / \partial \xi^{\alpha}$ is defined by the metrics $a_{\alpha \beta}=\mathbf{r}_{\alpha} \cdot \mathbf{r}_{\beta}$ and the curvature $b_{\alpha \beta}$; $\mathbf{n}=\left(\mathbf{r}_{1} \times \mathbf{r}_{2}\right) / \sqrt{a}$ is the normal unit vector; $a=\operatorname{det} a_{\alpha \beta}$. The holonomic basis of the spatial frame $\xi^{\alpha}, \xi^{3}$ at the point $M_{*} \in \bar{V} \backslash S$ consists in the vectors $\mathbf{R}_{\alpha}=\partial_{\alpha} \mathbf{R}\left(\xi^{\beta}, \xi^{3}\right), \mathbf{R}_{3}=\mathbf{n}$ $[29,31]$. The spatial "nabla" operator can be defined as follows [31, 32]:

$$
\begin{gathered}
\nabla=\mathbf{R}^{\alpha} \partial_{\alpha}+\mathbf{n} \partial_{3}=\mathbf{r}^{\beta} A_{\cdot \beta}^{\alpha \cdot}\left(\partial_{\alpha}+h_{\alpha} \partial_{\zeta}\right)+h^{-1} \mathbf{n} \partial_{\zeta}, \\
{[-1,1] \ni \zeta=\left(\xi^{3}-\bar{h}\right) / h} \\
h_{\alpha}=-\partial_{\alpha}(\ln h), 2 \bar{h}=h_{+}+h_{-}, A_{\cdot \beta}^{\alpha \cdot}=\mathbf{R}^{\alpha} \cdot \mathbf{r}_{\beta} .
\end{gathered}
$$

The problem statement can be based on the Hamilton principle [31-33]:

$$
\begin{gathered}
\delta \mathcal{H} \equiv \delta \int_{t_{0}}^{t_{1}}\left(\int_{V} \mathcal{L}_{V} d V+\int_{\partial V} \mathcal{L}_{\partial V} d S\right) d t=0 \\
\left.\mathbf{u}\right|_{t=t_{0}}=\mathbf{U}_{0},\left.\dot{\mathbf{u}}\right|_{t-t_{0}}=\mathbf{V}_{0}
\end{gathered}
$$

The volumetric and surface Lagrangian densities can be written as follows [33]:

$$
\begin{gathered}
\mathcal{L}_{V}=\frac{1}{2}\left[\rho \dot{\mathbf{u}} \cdot \dot{\mathbf{u}}-(\nabla \otimes \mathbf{u})^{\mathrm{T}}: \mathbf{C}:(\nabla \otimes \mathbf{u})\right]+ \\
+\rho \mathbf{F} \cdot \mathbf{u} ; \quad \mathcal{L}_{\partial V}=\left.\mathbf{q}\right|_{\partial V} \cdot \mathbf{u},
\end{gathered}
$$

$\mathbf{u}=u_{\alpha} \mathbf{r}^{\alpha}+u_{3} \mathbf{n}$ is the displacement. Let $\mathbf{s}$ be a stress tensor and $\mathbf{C}$ be an elastic constants tensor. The boundary conditions on $S_{ \pm}$can be written in the following form:

$$
\left.\left.\mathbf{S}\right|_{ \pm} \cdot \mathbf{n}\right|_{ \pm} \equiv \mathbf{C}:\left.(\nabla \otimes \mathbf{u})\right|_{ \pm}=\mathbf{q}_{ \pm}
$$

where $\mathbf{F}, \mathbf{q}_{ \pm}$are resultant forces, and $\mathbf{n}_{ \pm}$is the normal unit at a point $M_{ \pm} \in S_{ \pm}$.

The Lagrangian formalism of analytical dynamics allows one to introduce the 
configuration manifold $\Omega$ with the generalized coordinates [31, 32, 36-38]:

$$
\mathbf{u}^{(k)}: D_{\xi} \times \mathbb{R}_{+} \rightarrow T_{M} S, \quad k \in \mathbb{N} \cup\{0\},
$$

so that $\mathbf{u}=\mathbf{u}\left(\mathbf{u}^{(k)}\right)$, and the tangent fibration $T_{q} \Omega$ can be defined with the base vectors

$$
\mathrm{p}_{(k)}=\partial \mathbf{u} / \partial \mathbf{u}^{(k)}
$$

For linear systems such as (2.1) $\Omega$ is an euclidian space; the reduction consists in the projection of $\Omega$ onto its subspace $\Omega_{N}$ $(k=0 \ldots N)$ [34, 35]. To construct a shell theory, the biorthogonal system $\mathrm{p}_{(k)}(\zeta)$, $\mathrm{p}^{(k)}(\zeta)$ is used, and $\mathbf{u}^{(k)}$ are field variables of the $1^{\text {st }}$ kind: $\mathbf{u}^{(k)}=\left(\mathbf{u}, \mathrm{p}^{(k)}\right)_{1}[31,32]$. Here $(u, v)_{1}$ is the scalar product [32], the vector $\mathbf{u}$ is referenced to the basis $\mathbf{r}^{\alpha}, \mathbf{n}$, and its components $u_{i}\left(\xi^{\alpha}, \zeta\right)$ are supposed to be square integrable over $[-1,1] \ni \zeta[31,32]$. The densitiy of Lagrangian can be now defined on $\bar{S}$ as follows $[33,35]$ :

$$
\begin{gathered}
\mathcal{L}_{\partial \mathrm{S}}\left(u_{i}^{(k)}\right)=q_{B(k)}^{i} u_{i}^{(k)} \\
\mathcal{L}_{\mathrm{S}}\left(u_{i}^{(k)}, \dot{u}_{i}^{(k)}, \bar{\nabla}_{\alpha} u_{i}^{(k)}\right)=\frac{1}{2} \rho_{(k)}^{(m)} \dot{u}_{(m)}^{i} \dot{u}_{i}^{(k)}- \\
-\frac{1}{2} h^{-1}\left(\bar{C}_{(k m)}^{i 3 j \gamma} \bar{\nabla}_{\gamma} u_{j}^{(m)}+\bar{C}_{(k m)}^{i 3 j} u_{j}^{(m)}\right) D_{(n \cdot)}^{(\cdot k)} u_{i}^{(n)}- \\
-\frac{1}{2}\left(\overline{\bar{C}}_{(k m)}^{\alpha \beta j \gamma} \bar{\nabla}_{\gamma} u_{j}^{(m)}+\bar{C}_{(k m)}^{\alpha \beta j} u_{j}^{(m)}\right) \times \\
\times\left(\bar{\nabla}_{\beta} u_{\alpha}^{(k)}+H_{\beta(n)}^{(\cdot k)} u_{\alpha}^{(n)}-b_{\alpha \beta} u_{3}^{(k)}\right)- \\
-\frac{1}{2}\left(\overline{\bar{C}}_{(k m)}^{3 \beta j \gamma} \bar{\nabla}_{\gamma} u_{j}^{(m)}+\bar{C}_{(k m)}^{3 \beta j} u_{j}^{(m)}\right) \times \\
\times\left(\bar{\nabla}_{\beta} u_{3}^{(k)}+H_{\beta(n \cdot)}^{(\cdot k)} u_{3}^{(n)}+b_{\beta}^{\alpha} u_{\alpha}^{(k)}\right)+F_{(k)}^{i} u_{i}^{(k)}
\end{gathered}
$$

Finally, the Lagrange equations (2.5), (2.6), their natural boundary conditions (2.7), and the initial conditions (2.8) can be represented in the following formulation [36]:

$$
\begin{gathered}
\rho_{(k)}^{(m)} \ddot{u}_{(m)}^{\alpha}=\bar{\nabla}_{\beta} \tilde{s}_{(k)}^{\alpha \beta}-H_{\beta(k \cdot)}^{(\cdot m)} \tilde{s}_{(m)}^{\alpha \beta}+ \\
-b_{\beta}^{\alpha} \tilde{s}_{(k)}^{3 \beta}-h^{-1} D_{(k \cdot)}^{(\cdot m)} \tilde{s}_{(m)}^{\alpha 3}+\tilde{P}_{(k)}^{\alpha} ; \\
\rho_{(k)}^{(m)} \ddot{u}_{(m)}^{3}=\bar{\nabla}_{\beta} \tilde{s}_{(k)}^{3 \beta}-H_{\beta(k \cdot)}^{(\cdot m)} \tilde{s}_{(m)}^{3 \beta}+ \\
+b_{\alpha \beta} \tilde{s}_{(k)}^{\alpha \beta}-h^{-1} D_{(k \cdot)}^{(\cdot m)} \tilde{s}_{(m)}^{33}+\tilde{P}_{(k)}^{3} ;
\end{gathered}
$$




$$
\begin{gathered}
\left.\left(\tilde{s}_{(k)}^{i \beta} v_{\beta}-q_{B(k)}^{i}\right) \delta u_{i}^{(k)}\right|_{M \in \Gamma}=0 ; \\
\left.u_{i}^{(k)}\right|_{t=t_{0}}=U_{i}^{(k)} ;\left.\dot{u}_{i}^{(k)}\right|_{t=t_{0}}=V_{i}^{(k)} ; \\
i=1,2,3 ; \quad \beta=1,2 ; \quad k=0 \ldots N .
\end{gathered}
$$

The shell model consists in (2.5)-(2.8), the constraint equations (2.4), and the constitutive equations [32] accounting the kinematic relationships (see [31, 32]):

$$
\begin{gathered}
\tilde{s}_{(k)}^{i j}=\overline{\bar{C}}_{(k m)}^{i j \gamma \delta} \bar{\nabla}_{\delta} u_{\gamma}^{(m)}+\overline{\bar{C}}_{(k m)}^{i j 3 \delta} \bar{\nabla}_{\delta} u_{3}^{(m)}+ \\
+\bar{C}_{(k m)}^{i j \gamma} u_{\gamma}^{(m)}+\bar{C}_{(k m)}^{i j 3} u_{3}^{(m)}+\lambda_{\varepsilon}^{ \pm} \overline{\bar{C}}_{ \pm(k)}^{i j}+\lambda_{3}^{ \pm} \overline{\bar{C}}_{ \pm(k)}^{3 i j} .
\end{gathered}
$$

The generalized forces denoted by $\tilde{s}_{(k)}^{i j}$ account for the boundary conditions on the faces $S_{ \pm}$by means of Lagrange multipliers [37]. The members $\overline{\bar{C}}_{ \pm(k)}^{l i j} \lambda_{l}^{ \pm}$can be treated as reaction forces generated by the nonholonomic constraints (2.4).

The initial-boundary value problem (2.4)-(2.8) of the extended shell theory of $\mathrm{N}^{\text {th }}$ order coincides with the "elementary" one [31-44] if $\lambda_{i}^{ \pm} \equiv 0$ and the constraints (2.4) are neglected (i. e. the dynamic bondary conditions on $S_{ \pm}$are satisfyed approximately due to the convergence of the two-dimensional solutions sequience as $N \rightarrow \infty$, e. g. see $[27,28,30])$.

\section{FIRST-ORDER PLATE THEORY}

Let us consider the bending of an orthotropic plate as one of simplest examples. The $1^{\text {st }}$ order model is defined within two field variables, $u_{\alpha}^{(1)}$ and $u_{3}^{(0)}$. The Legendre polynomials are used as a basis, so that

$$
\mathrm{p}_{(0)}=1, \quad \mathrm{p}_{(1)}=\zeta
$$

The equilibrium equations (2.5), (2.6), the constitutive equations (2.8), and the constraints (2.4) can be written as follows:

$$
\begin{gathered}
\frac{2}{3} \rho h a^{\alpha \beta} \ddot{u}_{\beta}^{(1)}=\bar{\nabla}_{\beta} \tilde{s}_{(1)}^{\alpha \beta}-h^{-1} \tilde{s}_{(0)}^{3 \alpha} ; \\
2 \rho h \ddot{u}_{3}^{(0)}=\bar{\nabla}_{\alpha} \tilde{s}_{(0)}^{3 \alpha}+q^{3}, \\
q^{3}=q_{+}^{3}+q_{-}^{3} \equiv 2 q_{+}^{3} ; \\
\tilde{s}_{(1)}^{\alpha \beta}=\frac{2}{3} h\left(C^{\alpha \beta \gamma \delta} \bar{\nabla}_{\delta} u_{\gamma}^{(1)}+C^{\alpha \beta 33} \lambda_{3}\right), \\
\lambda_{3}=\lambda_{3}^{+}+\lambda_{3}^{-} ; \\
\tilde{s}_{(0)}^{3 \alpha}=2 h C^{\alpha 3 \beta 3}\left(\bar{\nabla}_{\beta} u_{3}^{(0)}+h^{-1} u_{\beta}^{(1)}+\lambda_{\beta}\right), \\
\lambda_{\beta}=\lambda_{\beta}^{+}-\lambda_{\beta}^{-} ; \\
C^{\alpha 3 \beta 3}\left(\bar{\nabla}_{\beta} u_{3}^{(0)}+h^{-1} u_{\beta}^{(1)}\right)=0, \quad q_{ \pm}^{\alpha} \equiv 0 ; \\
C^{33 \gamma \delta} \bar{\nabla}_{\delta} u_{\gamma}^{(1)}=\frac{1}{2} q^{3},
\end{gathered}
$$

here the symmetry conditions for the plate bending problem are taken into account. The dynamic equations (3.1), (3.2) corresponding to the $1^{s_{t}}$ order shear deformation theory as well as the constitutive ones (3.3), (3.4) can be obtained neglecting constraints (3.5), (3.6).

The constraint (3.5) is a straight normal condition; we eliminate $u_{\alpha}^{(1)}$ accounting for it:

$$
u_{\alpha}^{(1)}=-h \bar{\nabla}_{\alpha} u_{3}^{(0)} .
$$

Now we can introduce the bending moment (3.2) by the equation

$$
M^{\alpha \beta}:=\tilde{s}_{(1)}^{\alpha \beta}=-\frac{2}{3} h\left(C^{\alpha \beta \gamma \delta} \bar{\nabla}_{\gamma} \bar{\nabla}_{\delta} u_{3}^{(0)}-C^{33 \gamma \delta} \lambda_{3}\right) .
$$

As a result of (3.5) the shear strain vanishes; the shear force (3.3) can be defined as a reaction corresponding to the constraint (3.5):

$$
Q^{\alpha}:=\tilde{s}_{(0)}^{3 \alpha}=2 h C^{\alpha 3 \beta 3} \lambda_{\beta},
$$

and $Q^{\alpha}$ can be derived directly from the dynamic equation (3.1): 


$$
Q^{\alpha}=h \bar{\nabla}_{\beta} \tilde{s}^{\alpha \beta}+\frac{2}{3} \rho h^{3} a^{\alpha \beta} \bar{\nabla}_{\beta} \ddot{u}_{3}^{(0)} .
$$

The constraint (3.6) can be represented as

$$
h C^{33 \gamma \delta} \bar{\nabla}_{\gamma} \bar{\nabla}_{\delta} u_{3}^{(0)}=-\frac{1}{2} q^{3}
$$

the equation (3.8) is similar to the incompressibility condition (i.e. the dilatation is constrained). On the other hand we can interpret the multiplier $\lambda_{3}$ as a compliance of the constraint (3.8) in the direction normal to the mid-plane $S$ $[36,37]$. The corresponding reaction force is a pressure $p^{*}$ on planes $S_{\zeta}$ parallel to $S$; it is defined as

$$
p *=-C^{3333} \lambda_{3}
$$

Taking into account (3.8) we have

$$
\lambda_{3}=h \prod_{3333} \mathrm{C}^{33 \gamma \delta} \bar{\nabla}_{\gamma} \bar{\nabla}_{\delta} u_{3}^{(0)} .
$$

Finally the bending moment $M^{\alpha \beta}$ as well as the force $Q^{\alpha}$ can be represented as follows:

$$
\begin{aligned}
M^{\alpha \beta}= & -\frac{2}{3} h^{2} \hat{C}^{\alpha \beta \gamma \delta} \bar{\nabla}_{\gamma} \bar{\nabla}_{\delta} u_{3}^{(0)} ; \\
Q^{\alpha}= & -\frac{2}{3} h^{3} \bar{C}^{\alpha \beta \gamma \delta} \bar{\nabla}_{\beta} \bar{\nabla}_{\gamma} \bar{\nabla}_{\delta} u_{3}^{(0)}+ \\
& +\frac{2}{3} h^{3} \rho a^{\alpha \beta} \bar{\nabla}_{\beta} \ddot{u}_{3}^{(0)},
\end{aligned}
$$

$\widehat{C}^{\alpha \beta \gamma \delta}$ corresponds to the plane stress state:

$$
\bar{C}^{\alpha \beta \gamma \delta}=C^{\alpha \beta \gamma \delta}-C^{\alpha \beta 33} \Pi_{3333} C^{33 \gamma \delta},
$$

and $h$ is the semi-thickness of the plate. Thus the bending stiffness is properly defined with no supplementary assumption contrarily to the elementary $1^{\text {st }}$ order theory [17, 21, 39-41].

Considering (3.9) and (3.10), the scalar dynamic equation can be derived from (3.2):

$$
\begin{aligned}
& 2 \rho h \ddot{u}_{3}^{(0)}-\frac{2}{3} h^{3} \rho a^{\alpha \beta} \bar{\nabla}_{\alpha} \bar{\nabla}_{\beta} \ddot{u}_{3}^{(0)}= \\
= & -\frac{2}{3} h^{3} \bar{C}^{\alpha \beta \gamma \delta} \bar{\nabla}_{\alpha} \bar{\nabla}_{\beta} \bar{\nabla}_{\gamma} \bar{\nabla}_{\delta} u_{3}^{(0)}+q^{3} .
\end{aligned}
$$

The obtained dynamic equation (3.11) corresponds to the Kirchhoff's plate theory taking into account the rotatory inertia.

The natural boundary conditions (2.7) corresponding to the $1^{\text {st }}$ order theory of plates can be written in the following general representation:

$$
\begin{gathered}
\left.\frac{2}{3} h^{2} \bar{C}^{\alpha \beta \gamma \delta} v_{\beta} \bar{\nabla}_{\gamma} \bar{\nabla}_{\delta} u_{3}^{(0)} \delta\left(\nabla_{\alpha} u_{3}^{(0)}\right)\right|_{\partial S}=q_{B(1)}^{\alpha} ; \\
-\frac{2}{3} h^{3}\left(\hat{C}^{\alpha \beta \gamma \delta} \bar{\nabla}_{\beta} \bar{\nabla}_{\gamma} \bar{\nabla}_{\delta} u_{3}^{(0)}-\right. \\
\left.-\rho \bar{\nabla}_{v} \ddot{u}_{3}^{(0)}\right) \delta u_{3}^{(0)} \mid=q_{B(0)}^{3} .
\end{gathered}
$$

where $v_{\beta}$ is the normal unit to the contour $\partial S$ of the mid-plane and $\bar{\nabla}_{v}$ denotes the normal covariant derivative on the contour.

As a result, the initial-boundary value problem statement for the extended first-order theory of orthotropic plates consists in the dynamic equation (3.11), the boundary conditions (3.12) and (3.13) and the following initial conditions at the point $t=t_{0}$ :

$$
\left.u_{3}^{(0)}\right|_{t=t_{0}}=U_{3},\left.\quad \dot{u}_{3}^{(0)}\right|_{t=t_{0}}=V_{3} .
$$

\section{CONCLUSIONS}

An extended linear shell model of $\mathrm{N}^{\text {th }}$ order accounting for the boundary conditions on shell's faces is formulated as a constrained twodimensional continuum system on the basis of the Lagrange variational formalism of analytical dynamics. The model consists in the set of field variables corresponding to the biorthogonal expansion factors of the displacement, the constraint equations derived from the boundary conditions on the faces, and the twodimensional density of Lagrangian. It can be shown that the constraints change the model qualitatively. For instance the extended $1^{\text {st }}$ order theory of plates corresponds to the Kirchhoff model with no one supplementary assumption, $i$. e. it is asymptotically consistent contrarily to the elementary theory that corresponds to the $1^{\text {st }}$ 
order shear model. Moreover, such a theory does not overestimate the plate bending stiffness without artificially modified Poisson ratio, $\bar{v}=v /(1+v)[21]$, and the shear force is defined as a reaction of the straight normal constraint by the Lagrange multiplier. As a result, the known "quasi-contradiction" of the constitutive and dynamic equations in the Kirchhoff plate model is now eliminated. Thus, the proposed approach allows one to construct the full hierarchy of models including the lowest-order ones.

\section{ACKNOWLEDGEMENTS}

This investigation was financially supported by Russian Foundation for Basic Researches under grants Nr. 16-01-00751-a, Nr. 16-01-00641-a, and Nr. 16-01-00809-a).

\section{REFERENCES}

1. Eremeyev V., Pietraszkiewicz W. Refined theories of plates and shells // ZAMM. Vol. 94, 2014, pp. 5-6.

2. Kienzler R., Altenbach H., Otts I. Theories of Plates and Shells: Critical Review and New Applications, Springer-Verlag, Berlin, 2004.

3. Matsunaga H. Free vibration and stability of functionally graded plates according to a 2D higher-order deformation theory // Composite Structures. Vol. 82, 2008, pp. 499512.

4. Matsunaga H. Free vibration and stability of functionally graded shallow shells according to a 2D higher-order deformation theory // Composite Structures. Vol. 84, 2008, pp. 132-146.

5. Matsunaga H. Free vibration and stability of functionally graded circular cylindrical shells according to a 2D higher-order deformation theory // Composite Structures. Vol. 88, 2009, pp. 519-531.
6. Matsunaga H. Thermal buckling of functionally graded plates according to a 2D higher-order deformation theory // Composite Structures. Vol. 90, 2009, pp. 76-86.

7. Carrera E., Brischetto S. Importance of higher order modes and refined theories in free vibration analysis of composite plates // J. Applied Math. Vol. 77, 2010, 011013 (14 $\mathrm{pp).}$

8. Batra R.C., Aimmanee S. Vibrations of thick isotropic plates with higher order shear and normal deformable plate theories // Comput. and Struct. Vol. 83, 2005, pp. 934955.

9. Kienzler R.J. Consistent theories of isotropic and anisotropic plates // Theor. Appl. Mechanics. Vol. 50, No. 3, 2012, pp. 755768.

10. Zhilin P.A. Mechanics of deformable directed surfaces // Int. J. Sol. Struct. Vol. 12, 1976, pp. 635-648.

11. Altenbach H., Eremeyev V. On the linear theory of micropolar plates // ZAMM, Vol.89, No. 4, 2009, pp. 242-256.

12. Pietraszkiewicz W., Konopinska V. On unique kinematics for the branching shells // Int. J. Solids and Structures. Vol. 48, No. 14, 2011, pp. 2238-2244.

13. Eremeyev V.A., Zubov L.M. Mechanics of Elastic Shells (in Russian), Moscow: Nauka, 2008.

14. Kilchevskiy N.A. Fundamentals of the Analytical Mechanics of Shells (in Russian), AN UkrSSR, Kiev, 1963.

15. Vassiliev V.V., Lurie S.A. On the problem of construction of non-classical plate theories // Mechanics of Solids. No. 2, 1990, pp. 158-167.

16. Vassiliev V.V., Lurie S.A. On the problem of refinement of shallow shell theories // Mechanics of Solids. No. 6, 1990, pp. 139146.

17. Vekua I.N. Shell Theory: General Methods of Construction, Pitman Advanced Publ. Program, Boston, 1985.

18. Guliaev V.I., Bazhenov B.A., Lizunov P.P. Nonclassical Shell Theory and Applications 
in Engineering (in Russian), Lvov, Vishcha Shkola, 1978.

19. Khoma I.Yu. General Anisotropic Shell Theory (in Russian), Kiev, Naukova Dumka, 1986.

20. Amosov A.A. An approximate threedimensional theory of thick plates and shells // Structural Mechanics and Design of Buildings, No. 5, 1987, pp. 37-42.

21. Schwab C., Wright S. Boundary layer approximation in hierarchical beam and plate models // J. of Elasticity. Vol. 38, 1995, pp. $1-40$.

22. Gordeziani D., Avalishvili M., and Avalishvili G. Hierarchical models for elastic shells in curvilinear coordinates // Comput. Math. Appl. Vol. 51, 2006, pp. 1789-1808.

23. Meunargia T. Some general methods for constructing the theory of shells // J. Math. Sci. Vol.157, No. 1, 2009, pp. 1-15.

24. Meunargia T. On some refined theories of plates and shells // Bull. TICMI, Vol. 14, 2010, pp. 24-32.

25. Goldenweiser A.L. Theory of Thin Elastic Shells. Int. Ser. of Monograph in Aeronautics and Astronautics, Pergamon Press, N.Y., 1961.

26. Berdichevskiy V.L. Variational methods of constructing models of shells // J. of Applied Mathematics and Mechanics. Vol. 36 (1972), pp. 743-758.

27. Amosov A.A., Zhavoronok S.I. An approximate high-order theory of thick anisotropic shells. // International Journal of Computational Civil and Structural Engineering. Vol. 1, 2003, pp. 28-38.

28. Amosov A.A., Zhavoronok S.I., Leontiev K.A. About solving some problem on the stressed-strained state thick anisotropic shells of revolution in three-dimensional statement // The Journal of Mekhanika Kompozitsionnykh Materialov i Konstruktsii. Vol. 10, No. 3, 2004, pp. 301-310.

29. Zhavoronok S.I. High-order anisotropic shells models // The Journal of Mekhanika Kompozitsionnykh Materialov i Konstruktsii. Vol. 14, No. 4, 2008, pp. 561-571.
30. Zhavoronok S.I., Leontiev A.N., Leontiev K.A. Analysis of thick-walled rotation shells based on Legendre polynomials // International Journal of Computational Civil and Structural Engineering. Vol. 6, Issue 1\&2, 2010, pp. 105-111.

31. Zhavoronok S.I. Generalized Lagrange equations of the second kind of threedimensional anisotropic shell theory // The Journal of Mekhanika Kompozitsionnykh Materialov i Konstruktsii. Vol. 17, No. 1, 2011, pp. 116-132.

32. Zhavoronok S.I. A Vekua-type linear theory of thick elastic shells // ZAMM. Vol. 94, Nos. 1-2, 2014, pp. 164-184.

33. Zhavoronok S.I. Variational formulations of Vekua-type shell theories and some their applications. Shell Structures: Theory and Applications, Vol. 3, eds: W. Pietraszkiewicz, J. Gorski, CRC Press / Balkema, Taylor \& Francis Gr., Leiden, 2014, pp. 341344.

34. Zhavoronok S.I. Kinematics of normal modes in elastic layer for some wavenumbers investigation based on $\mathrm{N}$-th order three-dimensional shells' theory // The Journal of Mekhanika Kompozitsion-nykh Materialov i Konstruktsii. Vol. 18, No. 1, 2012, pp. 45-56.

35. Zhavoronok S.I. A Formulation of the three-dimensional approximated shells theory of $\mathrm{N}$-th order using generalized displacements and its application to steady dynamics // The Journal of Mekhanika Kompozitsionnykh Materialov i Konstruktsii. Vol.18, No. 3, 2012, pp. 333-344.

36. Zhavoronok S.I. On the variational formulation of the extended thick anisotropic shells theory of I. N. Vekua type // Procedia Engineering. Vol. 111, 2015, pp. 888-895.

37. Zhavoronok S. I. The generalized Lagrange equations of the second kind for the extended three-dimensional Nth order theory of anisotropic shells // The Journal of Mekhanika Kompozitsionnykh Materialov i Konstruktsii. Vol. 21, No. 3, 2015, pp. 370-381. 
38. Zhavoronok S.I. High-order shell theory based on the analytical continuum dynamics formalism // XXIV ICTAM, 21-26.08.2016, Montreal, Canada, Book of Papers, 19981999, 2016.

39. Kienzler R. On consistent plate theories // Archive of Applied Mechanics. Vol. 72, 2002, pp. 229-247.

40. Kienzler R., Schneider P. Comparison of various linear plate theories in the light of a consistent second-order approximation, Shell Structures: Theory and Applications, Vol. 3, eds: W. Pietraszkiewicz, J. Gorski, CRC Press / Balkema, Taylor \& Francis Gr., Leiden, 2014, pp. 109-112.

41. Schneider P., Kienzler R., Böhm M. Modeling of consistent second-order plate theories for anisotropic materials // ZAMM. Vol. 94, No. 1-2, 2014, pp. 21-42.

Zhavoronok Sergey Igorevich, Ph.D., Senior researcher of the Department of Mechanics of Smart and Composite Materials and Systems, Institute of Applied Mechanics of Russian Academy of Sciences; Associate Professor of the Department of Strength of Materials, Machine Dynamics and Strength, Moscow Aviation Institute (National Research University); Associate Professor of Department of Higher Mathematics and Computer Science, National Research University - Moscow State University of Civil Engineering. Address: Leningradskiy Prospekt 7, 125040, Moscow, Russia; Phone: +7(495) 941-1777; +7(916) 1342843; e-mail: Zhavoronok@iam.ras.ru,

zhavor71@mail.ru

Жаворонок Сергей Игоревич, кандидат физикоматематических наук, старший научный сотрудник Института прикладной механики Российской академии наук (ИПРИМ РАН), доцент кафедры «Сопротивление материалов, динамика и прочность машин» Московского авиационного института (Национального исследовательского университета), доцент кафедры прикладной математики Национального исследовательского Московского государственного строительного университета; 125040, Россия, г. Москва, Ленинградский проспект, д. 7; тел. +7(495) 941-1777; +7(916) 134-2843; e-mail: Zhavoronok@iam.ras.ru, zhavor71@mail.ru 


\title{
ФИЗИЧЕСКИ НЕЛИНЕЙНЫЕ ЗАДАЧИ ДЛЯ НЕОДНОРОДНЫХ ТОЛСТОСТЕННЫХ ОБОЛОЧЕК
}

\author{
В.И. Андреев, Л.С. Полякова \\ Национальный исследовательский Московский государственный строительный университет, \\ г. Москва, РОССИЯ
}

\begin{abstract}
Аннотация: В работе предлагается численный метод решения задач расчета цилиндрической и сферической толстостенных оболочек из физически нелинейных неоднородных материалов. При этом используется метод последовательных приближений. Результаты численного расчета сравниваются с аналитическими решениями тестовых задач. Полученные результаты можно считать вполне удовлетворительными. Актуальность решаемой задачи объясняется изменением механических свойств материалов под действием различных физических полей (температура, влажность, излучения и т. д.).
\end{abstract}

Ключевые слова: нелинейно упругий материал, неоднородность, численный метод, диаграмма деформирования

\section{PHYSICALLY NONLINEAR PROBLEMS FOR INHOMOGENEOUS THICK-WALLED SHELLS}

\author{
Vladimir I. Andreev, Lyudmila S. Polyakova \\ National Research Moscow State University of Civil Engineering, Moscow, RUSSIA
}

\begin{abstract}
The paper proposes the numerical method of solution the problems of calculation the stress state in thick-walled cylinders and spheres from physically nonlinear inhomogeneous material. It uses the method of successive approximations. Numerical calculation results are compared with analytical solutions of test problems. The obtained results can be considered quite satisfactory. The urgency of solved problem due to the change of mechanical properties of materials under the influence of different physical fields (temperature, humidity, radiation, etc.).
\end{abstract}

Key words: nonlinearity, inhomogeneity, numerical method, stress-strain diagrams

\section{ВВЕДЕНИЕ}

Так как характер деформирования многих материалов существенно отличается от линейного, представляет интерес разработка методов расчета напряженнодеформированного состояния тел с учетом неоднородности их механических характеристик. В статье рассматривается задача расчета толстостенного цилиндра и шара из неоднородного материала, который имеет нелинейный характер деформирования. Задача рассматривается в осесимметричной и центрально-симметричной постановках.

\section{1. ПОСТАНОВКА ЗАДАЧИ}

На рисунке 1 изображена диаграмма деформирования нелинейно упругого материала, которая описывается соотношением [1]:

$$
\sigma_{i}=f\left(\varepsilon_{i}\right)=E \varepsilon_{i}-A \varepsilon_{i}^{\alpha} .
$$

При изменении условий нагружения (наличие физических полей - температурного, радиационного, влажности и пр.) диаграмма может изменяться, что может быть учтено заменой в (1) постоянных $E, A$ и $\alpha$ на функции $E(r), A(r)$ и $\alpha(r)$. 


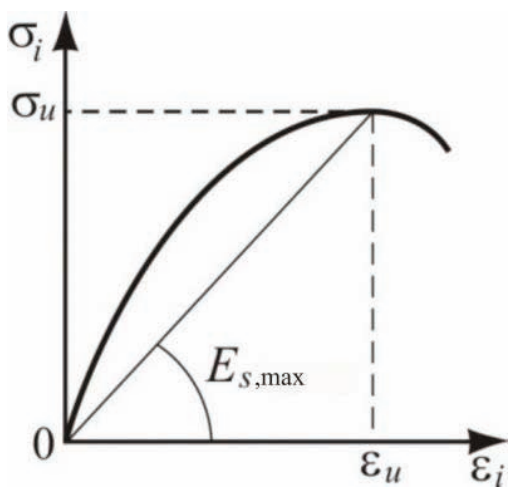

Рисунок 1. Нелинейная диаграмма деформирования; $E_{s, \max }=\sigma_{u} / \varepsilon_{u}-$ секущий модуль в момент разрушения.

В работах $[2,8,9]$ были получены аналитические решения подобных задач для несжимаемого материала, в которых неоднородность учитывалась с помощью следующих зависимостей:

$$
\begin{gathered}
E(r)=E_{0}\left[1+\left(k_{E}-1\right)(a / r)^{m_{E}}\right], \\
A(r)=E_{0}\left[1+\left(k_{A}-1\right)(a / r)^{m_{A}}\right], \\
\alpha=\text { const. }
\end{gathered}
$$

при условиях $k_{E}=k_{A}, m_{E}=m_{A}$. Выбранные функции неоднородности $E(r)$ и $A(r)$, а также $\alpha$ позволяют описать широкий спектр экспериментальных данных, полученных для различных воздействий $[3,4,6,7]$.

Полученные с указанными ограничениями аналитические решения могут служить в качестве тестовых при разработке численного метода расчета.

Ниже излагается численный метод решения задачи для неоднородных цилиндрических оболочек из нелинейно упругого материала. Используется метод последовательных приближений [5].

\section{2. МЕТОД РЕШЕНИЯ}

Приведем решения аналогичных задач методом последовательных приближений и сравним результаты. Рассмотрим случай, когда

$$
\begin{gathered}
\alpha=3, k_{E}=k_{A}=0,5, m_{E}=m_{A}=2, \\
E_{0}=3 \cdot 10^{4} \mathrm{M \Pi а}, A_{0}=2 \cdot 10^{5} \mathrm{M} \text { Па }, v=0,5
\end{gathered}
$$

при следующих граничных условиях:

$$
\begin{gathered}
r=a, \quad \sigma_{r}=p_{a}=0 ; \\
r=b \rightarrow \infty, \sigma_{r}=p_{b}=-p .
\end{gathered}
$$

Эти граничные условия описывают задачу о концентрации напряжений вблизи отверстия в бесконечном массиве. Нагрузка $p$ определяется соотношением $p / \sigma_{u, \max }=0,5$, где $\sigma_{u, \max }-$ интенсивность напряжений в момент разрушения при $r \rightarrow \infty$.

В соответствии с приведенными значениями констант диаграмма деформирования описывается соотношением

$\sigma_{i}=E_{0}\left[1-\frac{a^{2}}{2 r^{2}}\right] \varepsilon_{i}-A_{0}\left[1-\frac{a^{2}}{2 r^{2}}\right] \varepsilon_{i}^{\alpha}$

На рисунке 2 показано, как изменяется диаграмма деформирования материала вдоль радиуса.

Метод последовательных приближений заключается в следующем [3,5]. На нулевом этапе ищется решение задачи для линейноупругого материала. Вычислив интенсивности напряжений и деформаций, можно в каждой точке тела определить секущий модуль $E_{s}$ :

$$
E_{s}^{0}=\frac{\sigma_{i}^{(0)}}{\varepsilon_{i}^{(0)}}=\frac{f\left(\varepsilon_{i}^{(0)}\right)}{\varepsilon_{i}^{(0)}}
$$

где функция $f$ определяется по диаграмме $\sigma_{i}-\varepsilon_{i}$, а нулевой индекс вверху означает решение на нулевом этапе.

На следующем шаге итерационного процесса снова решается линейно-упругая задача, когда закон Гука задается в форме: 


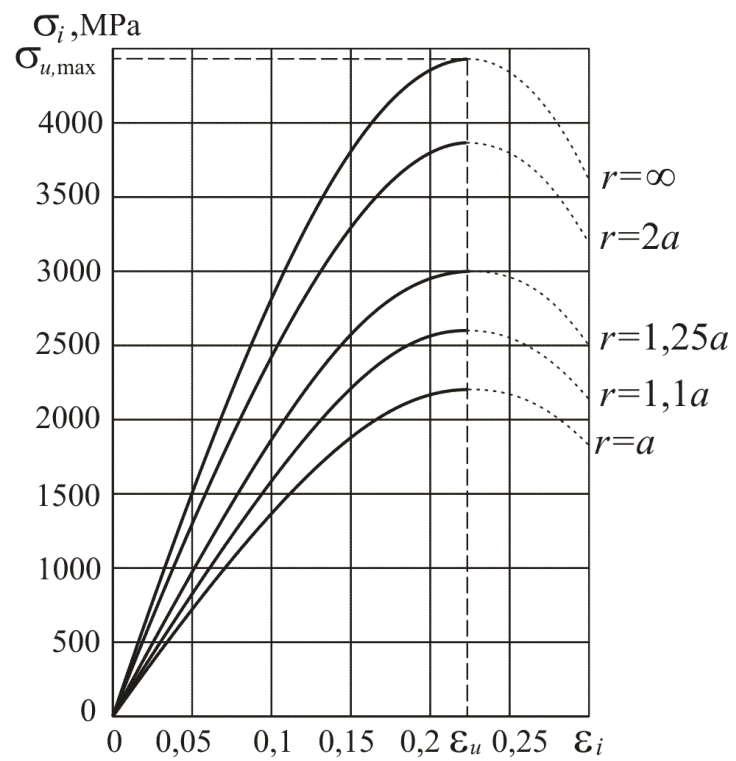

Рисунок 2. Диаграмма деформирования неоднородного материала на различных расстояниях от контура отверстия

$$
\begin{array}{r}
\varepsilon_{\xi}=\frac{1}{E_{1}}\left[\sigma_{\xi}-v_{1}\left(\sigma_{\eta}+\sigma_{\zeta}\right)\right], \\
\varepsilon_{\eta}=\frac{1}{E_{1}}\left[\sigma_{\eta}-v_{1}\left(\sigma_{\zeta}+\sigma_{\xi}\right)\right], \\
\varepsilon_{\zeta}=\frac{1}{E_{1}}\left[\sigma_{\zeta}-v_{1}\left(\sigma_{\xi}+\sigma_{\eta}\right)\right],
\end{array}
$$

где $\xi, \eta, \zeta$ соответствует $r, \theta, z$ в цилиндрических координатах и $r, \theta, \varphi$ в сферических координатах. Упругие характеристики в (6) описываются равенствами [3]:

$$
\begin{gathered}
\frac{1}{E_{1}}=\frac{1}{E_{s}}+\frac{1-2 v_{0}}{3 E_{0}}, \\
v_{1}=\frac{1-\frac{2\left(1-2 v_{0}\right) E_{s}}{3 E_{0}}}{2+\frac{2\left(1-2 v_{0}\right) E_{s}}{3 E_{0}}}=\frac{3 E_{1}}{2 E_{s}}-1 .
\end{gathered}
$$

В эти соотношения входит вычисленный секущий модуль $E_{s}$. Поскольку эта величина, а также фиктивные значения модуля упругости $E_{1}$ и коэффициента Пуассона $v_{1}$ в общем случае в каждой точке тела различ- ны, на втором и последующих этапах итерационного процесса необходимо решать задачу теории упругости с переменными характеристиками, или задачу теории упругости непрерывно неоднородных тел.

При отыскании решения на $n$-ном этапе секущий модуль вычисляется на основании решения, полученного на (n-1) этапе. Каждый последующий шаг итерационного процесса приближает решение к точному.

Для оценки точности метода приведем решение двух задач - осесимметричной (для толстостенного цилиндра) и центральносимметричной (для толстостенного полого шара), и сравним результат с аналитическими решениями, приведенными в $[2,8,9]$.

\section{3. РЕШЕНИЕ И РЕЗУЛЬТАТ}

\section{1. Осесимметричная задача.}

Разрешающее уравнение на нулевом шаге, при $v_{0}=0.5[3]$ :

$$
r \sigma_{r}^{\prime \prime}+\left(3-r \frac{E^{\prime}}{E}\right) \sigma_{r}^{\prime}=0
$$

Решение уравнения (8) имеет вид:

$$
\sigma_{r}=C_{2}+C_{1} \int \frac{E(r) d r}{r^{3}}
$$

Подставив (9) в граничные условия (3), получим выражения для напряжений:

$$
\begin{gathered}
\sigma_{r}^{(0)}=-\frac{p}{3}\left(3-\frac{4 a^{2}}{r^{2}}+\frac{a^{4}}{r^{4}}\right), \\
\sigma_{\theta}^{(0)}=-\frac{p}{3}\left(3+\frac{4 a^{2}}{r^{2}}-\frac{3 a^{4}}{r^{4}}\right) .
\end{gathered}
$$

Учитывая предположение о несжимаемости материала $\left(v_{0}=0.5\right)$, т. е. отсутствии объемных деформаций $\left(\varepsilon_{z}+\varepsilon_{\theta}+\varepsilon_{r}=0 \quad\right.$ и $\left.\varepsilon_{z}=0 \Rightarrow \varepsilon_{\theta}=-\varepsilon_{r}\right)$, получим: 
$\varepsilon_{i}^{(0)}=\frac{2 \sqrt{3}}{3} \varepsilon_{r}^{(0)}=\frac{2 \sqrt{3}}{3} \frac{p}{E(r)}\left(\frac{2 a^{2}}{r^{2}}-\frac{a^{4}}{r^{4}}\right)$.

Для секущего модуля получим выражение:

$$
E_{s}^{(0)}=\left(1-\frac{a^{2}}{2 r^{2}}\right)\left(E_{0}-\frac{16 A_{0} p^{2}}{3 E_{0}^{2}} \cdot \frac{a^{4}}{r^{4}}\right) \text {. }
$$

На первом и последующих этапах итерационного процесса уравнение (8) решалось методом прогонки с переменным шагом. На рисунке 3 показаны диаграммы напряжений, полученных в пятом приближении. Для сравнения на этом же графике показаны напряжения, полученные на нулевом шаге (соответствуют линейно-упругому материалу) и результаты аналитического расчета.

Наибольшее отличие численного решения от аналитического наблюдается в вершине кривой, где оно не превышает $3 \%$.

\section{2. Центрально-симметричная задача}

Разрешающее уравнение на нулевом шаге, при $v_{0}=0.5[3]$ :

$$
r \sigma_{r}^{\prime \prime}+\left(4-r \frac{E^{\prime}}{E}\right) \sigma_{r}^{\prime \prime}=0 .
$$

Решение уравнения (8) имеет вид:

$$
\sigma_{r}=C_{2}+C_{1} \int \frac{E(r) d r}{r^{4}}
$$

Подставив (14) в граничные условия (3), получим выражения для напряжений:

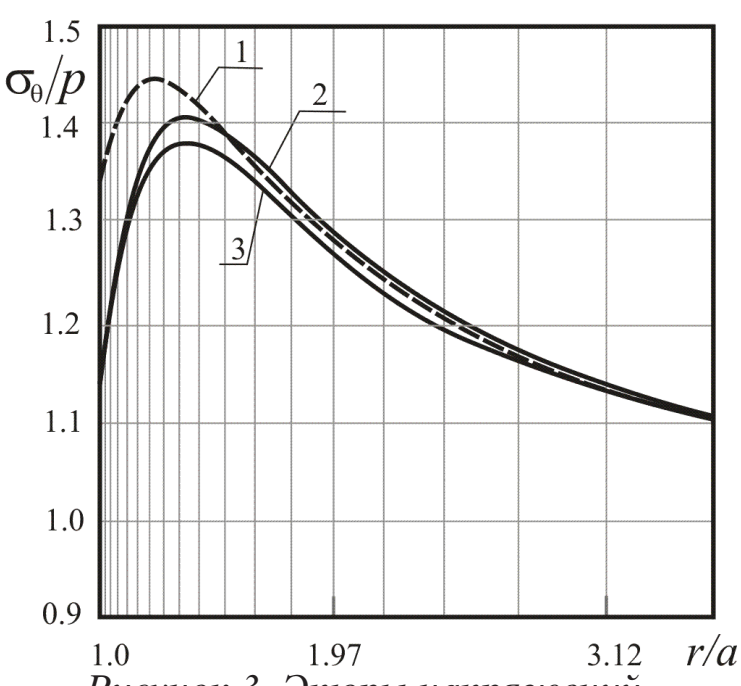

Рисунок 3. Эпюры напряжений в иилиндрической оболочке: 1 - линейный неоднородный материал; 2 - нелинейный неоднородный материал, численное решение; 3 - нелинейный неоднородный материал, аналитическое решение.

$$
\begin{aligned}
& \sigma_{r}^{(0)}=-\frac{p}{7}\left(7-\frac{10 a^{3}}{r^{3}}+\frac{3 a^{5}}{r^{5}}\right), \\
& \sigma_{\theta}^{(0)}=-\frac{p}{7}\left(7+\frac{5 a^{3}}{r^{3}}-\frac{9 a^{5}}{2 r^{5}}\right) .
\end{aligned}
$$

Учитывая предположение о несжимаемости материала $\left(v_{0}=0.5\right)$, т. е. отсутствии объемных деформаций $\left(\varepsilon_{\varphi}+\varepsilon_{\theta}+\varepsilon_{r}=0 \quad\right.$ и $\left.\varepsilon_{\varphi}=\varepsilon_{\theta} \Rightarrow \varepsilon_{r}=-2 \varepsilon_{\theta}\right)$, получим:

$$
\begin{gathered}
\varepsilon_{r}^{(0)}=\frac{p}{E(r)} \frac{15}{7}\left(\frac{a^{3}}{r^{3}}-\frac{a^{5}}{2 r^{5}}\right), \\
\varepsilon_{\theta}^{(0)}=\frac{1}{2} \varepsilon_{r}^{(0)}=\frac{p}{E(r)} \frac{15}{14}\left(\frac{a^{3}}{r^{3}}-\frac{a^{5}}{2 r^{5}}\right), \\
\varepsilon_{i}^{(0)}=\frac{p}{E(r)} \frac{15}{7}\left(\frac{a^{3}}{r^{3}}-\frac{a^{5}}{2 r^{5}}\right) .
\end{gathered}
$$

Для секущего модуля получим выражение:

$$
E_{c}^{(0)}=\left(1-\frac{a^{2}}{2 r^{2}}\right)\left(E_{0}-\frac{225 A_{0} p^{2}}{49 E_{0}^{2}} \cdot \frac{a^{6}}{r^{6}}\right) .
$$


На первом и последующих этапах итерационного процесса уравнение (13) решалось методом прогонки с переменным шагом. На рисунке 4 показаны диаграммы напряжений, полученных в пятом приближении. Для сравнения на этом же графике показаны напряжения, полученные на нулевом шаге (соответствуют линейно-упругому материалу) и результаты аналитического расчета. Сходимость численного метода при решении центрально-симметричной задачи лучше, чем в случае цилиндрической оболочки. Наибольшее отклонение численного решения от аналитического для сферической оболочки составляет $0,7 \%$. Полученные в обоих случаях результаты можно считать вполне удовлетворительными. Особенно это относится к точке $r=a$, где численное и аналитическое решения практически совпадают.

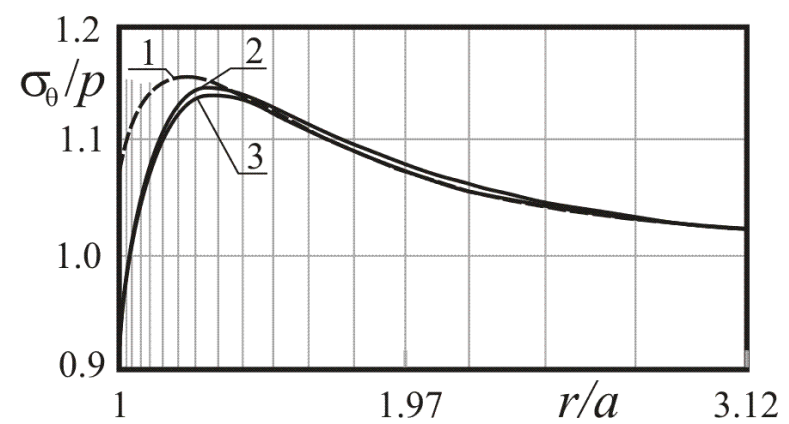

Рисунок 4. Эпюры напряжений в сферической оболочке: 1 - линейный неоднородный материал; 2 - нелинейный неоднородный материал, численное решение; 3 - нелинейный неоднородный материал, аналитическое ремение.

\section{ВЫВОДЫ}

Разработан численный метод решения плоской осесимметричной и центральносимметричной задач для толстостенных оболочек из физически нелинейного радиально неоднородного материала. Этот метод можно применять при произвольных функциях $E(r), A(r)$ и $\alpha(r)$, которые зависят от изменения механических характеристик $\sigma_{u}(r)$ и $\varepsilon_{u}(r)$, определяемых экспериментально.

\section{СПИСОК ЛИТЕРАТУРЫ}

1. Лукаш П.А. Основы нелинейной строительной механики. - М.: Стройиздат, 1978. - 208 c.

2. Андреев В.И., Полякова Л.С. Аналитическое решение физически нелинейной задачи для неоднородной толстостенной цилиндрической оболочки. // Вестник МГСУ, 2015, №11.

3. Андреев В.И. Некоторые задачи и методы механики неоднородных тел. - М.: ACB, 2002. - 288 c.

4. Баклашов И.В., Картозия Б.А. Механика горных пород. - М.: Недра, 1975. - 272 с.

5. Биргер И.А. Некоторые общие методы решения задач теории пластичности //ПММ. 1951. Т.15. Вып.6. С. 765-770.

6. Колчин Г.Б. Плоские задачи теории упругости неоднородных тел. - Кишинев: Штиинца, 1977. - 119 с.

7. Менцель В., Шрейнер В. Закономерности механического поведения каменных солей в лабораторных и натурных условиях //Механика горных пород - АлмаАта: Наука, 1975. С. 64-78.

8. Andreev V.I. Equilibrium of a Thick-walled Sphere of Inhomogeneous Nonlinear-elastic Material // Applied Mechanics and Materials Vols. 423-426, 2013, pp. 1670-1674.

9. Andreev V.I., Polyakova L.S. Calculation of a Thick-walled Inhomogeneous Cylinder of Nonlinear-elastic Material //Advanced Materials and Structural Engineering, 2015, pp. 715-718.

Андреев Владимир Игоревич, академик РААСН, профессор, доктор технических наук, заведующий кафедрой сопротивления материалов; Национальный исследовательский Московский государственный строительный университет, (НИУ МГСУ), Россия, 129337, г. Москва, Ярославское шоссе, д. 26; e-mail: asv@mgsu.ru.

Полякова Людмила Сергеевна, магистр кафедры сопротивления материалов, НИУ МГСУ, Россия, 129337, г. Москва, Ярославское шоссе, д. 26; e-mail: 1.poliakova@inbox.ru.

Andreev Vladimir I., Full Member of the Russian Academy of Architecture and Construction Sciences, Professor, Doctor of Technical Sciences, Head of the Department of Strength of Materials, National Research Moscow State University of Civil Engineering (MSUCE); Russia, 129337, Moscow, Yaroslavskoye sh., 26; e-mail: asv@mgsu.ru.

Polyakova Lyudmila, S., MA student of Department of Strength of Materials, MSUCE, Russia, 129337, Moscow, Yaroslavskoye sh., 26; e-mail: 1.poliakova@inbox.ru. 


\title{
К ВОПРОСУ О ВЕРИФИКАЦИИ МНОГОУРОВНЕВЫХ ПОДХОДОВ К ЛОКАЛЬНОМУ РАСЧЕТУ СТРОИТЕЛЬНЫХ КОНСТРУКЦИЙ ЧАСТЬ 1: ДИСКРЕТНЫЙ (ЧИСЛЕННЫЙ) ПОДХОД
}

\author{
Моджтаба Аслами ${ }^{1}$, П.А. Акимов $2,3,4,5$ Ж.И. Мсхалая ${ }^{4}$ \\ ${ }^{1}$ Университет Фесы, г. Феса, ИРАН \\ ${ }^{2}$ Российская академия архитектуры и строительных наук, г. Москва, РОССИЯ \\ ${ }_{3}^{3}$ ЗАО «Научно-исследовательский центр «СтаДиО», г. Москва, РОССИЯ \\ ${ }^{4}$ Национальный исследовательский Московский государственный строительный университет, \\ г. Москва, РОССИЯ \\ ${ }^{5}$ Научно-исследовательский институт строительной физики Российской академии архитектуры \\ и строительных наук, г. Москва, РОССИЯ
}

\begin{abstract}
Аннотация: Настоящая статья посвящена актуальным вопросам верификации многоуровневого дискретного (численного) подхода к локальному расчету строительных конструкций. Рассматриваются верификационные примеры решения одномерных и двумерных краевых задач строительной механики (расчеты балки Бернулли на упругом основании, стержня, балок-стенок и пластин). Для сопоставлений используются результаты полученные с помощью стандартного метода конечных элементов (МКЭ). Анализ полученных результатов демонстрирует преимущества предлагаемого авторами многоуровневого дискретного подхода.
\end{abstract}

Ключевые слова: многоуровневый дискретный подход, локальный расчет, строительные конструкции, численные методы, вейвлет-анализ, верификация, метод конечных элементов

\section{ABOUT VERIFICATION OF MULTILEVEL METHODS OF LOCAL STRUCTURAL ANALYSIS PART 1: DISCRETE (NUMERICAL) METHOD}

\author{
Mojtaba Aslami', Pavel A. Akimov ${ }^{2,3,4,5}$, Zhiuli I. Mskhalaya ${ }^{4}$ \\ ${ }^{1}$ Fasa University, Fasa, IRAN \\ ${ }^{2}$ Russian Academy of Architecture and Construction Sciences, Moscow, RUSSIA \\ ${ }^{3}$ Scientific Research Center "StaDyO", Moscow, RUSSIA \\ ${ }^{4}$ National Research Moscow State University of Civil Engineering, Moscow, RUSSIA \\ ${ }^{5}$ Research Institute of Building Physics of Russian Academy \\ of Architecture and Construction Sciences, Moscow, RUSSIA
}

\begin{abstract}
The distinctive paper is devoted to actual aspects of verification of multilevel discrete (numerical) method of local structural analysis. Solutions of one-dimensional and two-dimensional verification samples (analysis of column, beam, deep beams, plates) are under consideration. Conventional finite element method (FEM) is used for verification purposes. The presented samples show some of the advantages of the suggested approach to numerical local structural analysis.
\end{abstract}

Keywords: multilevel discrete method, local structural analysis, numerical methods, wavelet analysis, verification, finite element method

\section{1. ВВЕДЕНИЕ}

Современные численные и численно-анали- тические методы позволяют определять напряженно-деформированное состояние (НДС) строительных конструкций, зданий и соору- 


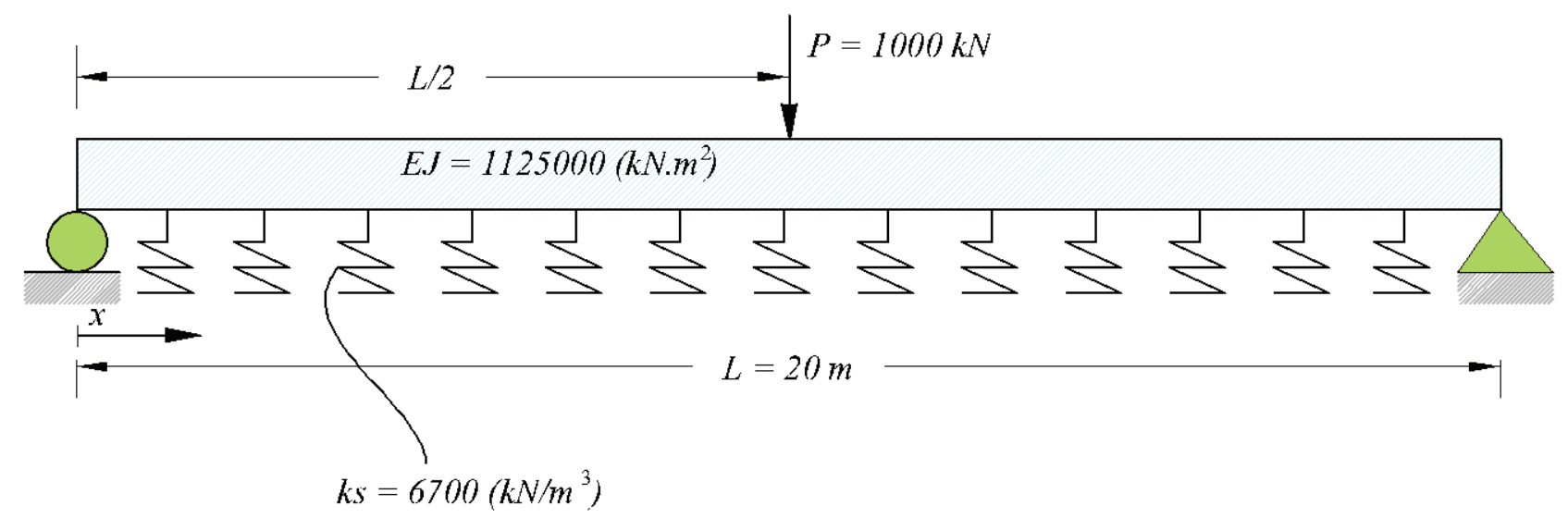

Рисунок 2.1. Задача о статическом расчете балки Бернулли на упругом основании.

жений, в том числе повышенной ответственности (уникальных, большепролетных, высотных), что может привести на практике к вычислительным схемам исключительно большой размерности [4,5,15-17]. Вместе с тем, квалифицированному расчетчику известно [1,6-8,10-14], что наиболее опасным с точки зрения прочности является напряженно-деформированное состояние (НДС) в относительно небольшом числе локальных зон конструкций, причем расположение этих зон, как правило, известно заранее. В частности, к последним следует отнести зоны краевого эффекта, т.е. разного рода углы, трещины, щели, места контактов и связей различных конструктивных элементов, места локальных изменений, обусловленных реконструкцией объекта (например, пробивка новых проходов, снос опор, усиления и т.д.) и др. Таким образом, особую актуальность приобретает локальный расчет строительных объектов, тем более актуальная, позволяющий, кроме того, при корректной реализации существенно сократить количество неизвестных. В работах П.А. Акимова, Моджтаба Аслами, А.Б. Золотова и М.Л. Мозгалевой [1,2,4-8,10-14] были предложены дискретный (численных) и дискретно-континуальный (численно-аналитический) подходы к локальному расчету строительных конструкций, основанные на использовании аппарата кратномасштабного вейвлет-анализа. Последний позволяет всесторонне оценить влияние различных с точки зрения локализации факторов, является весьма эффективным математическим инструментарием [3,9]. В настоящей статье излагаются некоторые вопросы верификации многоуровневого дискретного подхода к локальному расчету строительных конструкций.

\section{2. ЛОКАЛЬНЫЙ РАСЧЕТ БАЛКИ БЕРНУЛЛИ НА УПРУГОМ ОСНОВАНИИ}

Рассмотрим задачу локального статического расчета балки Бернулли [2,7,11,12] (рис. 2.1) длиной $l=20$ м, имеющей изгибную жесткость $E J=1125000$ кН·м ${ }^{2}$ и ширину $0.50 \mathrm{M}$, которая лежит на упругом основании (параметр $k_{s}=6700 \kappa \mathrm{H} / \mathrm{M}^{3}$ ). Пусть требуется получить высокоточное решение в интервале $8 \leq k_{s} \leq 12$.

На рис. 2.2 и 2.3 представлены выборочные результаты расчета в зоне гарантированной точности, полученные при использовании модуля FDMDWTB комплекса программ MWBDASA2D [6,7].

Для более детальной проверки и сопоставления полученных результатов в таблице 2.1 для данного примера приведено сравнение значений изгибающего момента и поперечных сил, полученных при помощи многоуровневого дискретного подхода (FDMDWT) и метода конечных разностей (FDM) $[1,2,6,7]$. 
К вопросу о верификации многоуровневых подходов к локальному расчету строительных конструкций Часть 1: Дискретный (численный) подход

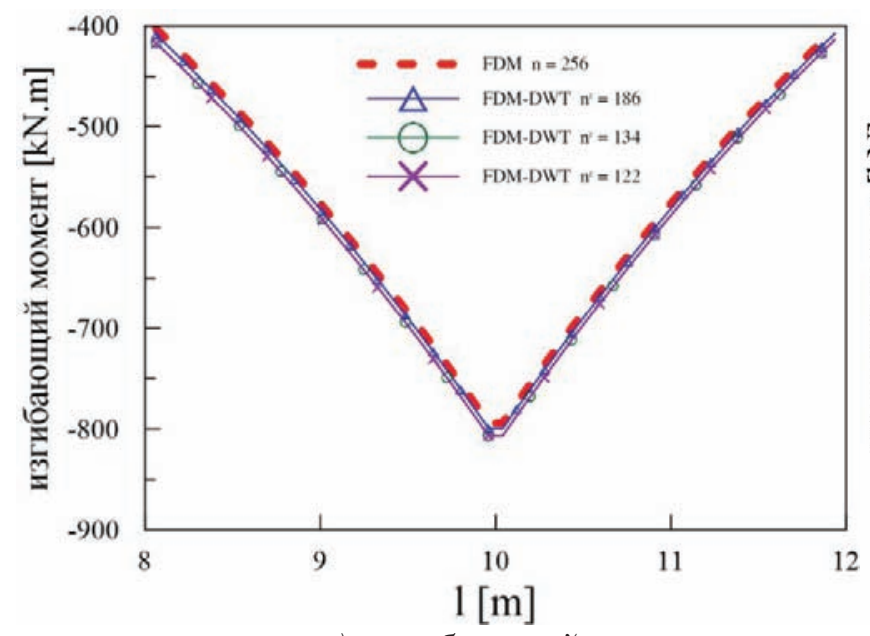

а) изгибающий момент

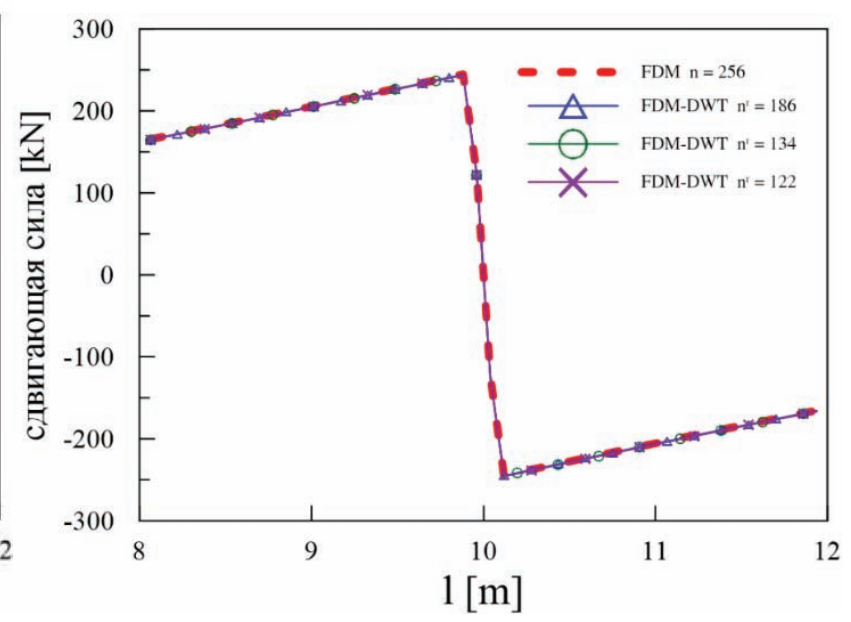

б) поперечная сила

Рисунок 2.2. Сопоставление результатов расчета (изгибающие моменты и поперечныле силь) в зоне гарантированной точности.

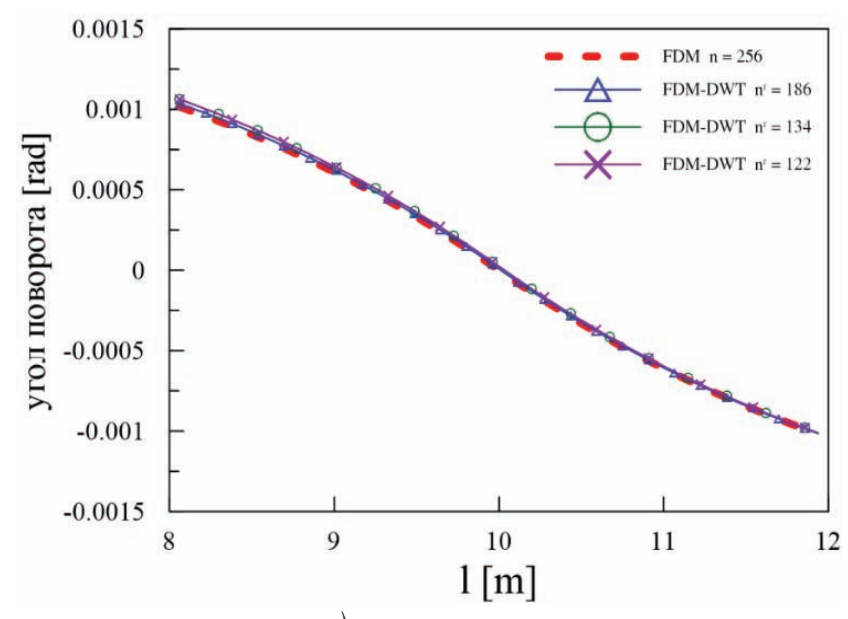

a) угол поворота

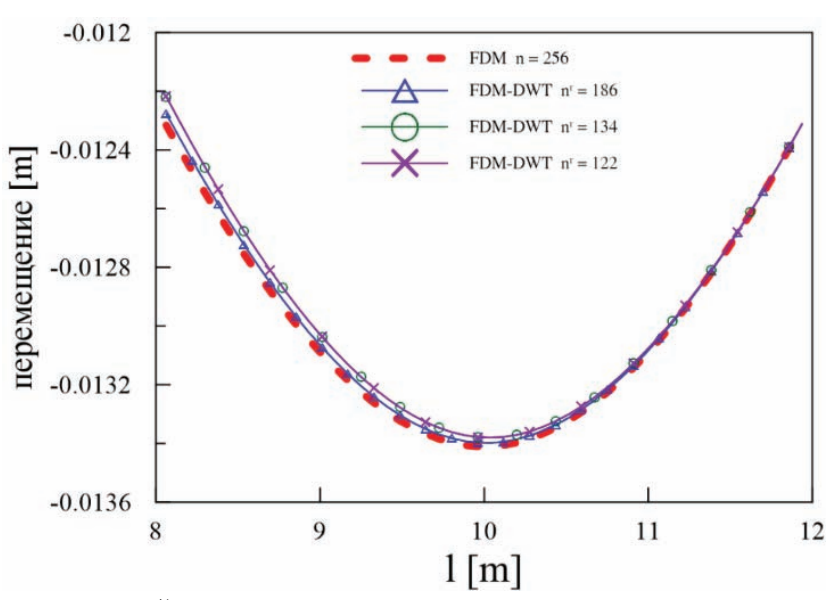

б) вертикальное перемещуение

Рисунок 2.3. Сопоставление результатов расчета (угль поворота и перемещения) в зоне гарантированной точности.

\section{3. ЛОКАЛЬНЫЙ РАСЧЕТ ОДНОМЕРНОГО СТЕРЖНЯ}

Рассмотрим задачу локального статического расчета стального стержня переменной высоты, нагруженного сосредоточенной силой как это показано на рис. 3.1 [1,2,6,7].

Пусть модуль упругости стержня $E=200$ ГПа. Положим, что требуется получить высокоточное локальное решение в зонах, примыкающих к местам опирания стержня. Будем использовать многоуровневый дискретный подход к локальному расче- ту строительных конструкций, основанный на использовании техники метода конечных элементов (FEM-DWT) [6,7].

Выполнены сопоставления исходного (эталонного) решения (без редукции), полученного по методу конечных элементов при использовании 256 узлов, с соответствующими редуцированными (FEM-DWT) при различных параметрах редукции. Результаты сопоставлений (в части нормальных напряжений) представлены на рис. 3.2. Следует отметить, что в зоне гарантированной точности результаты, полученные с использованием много- 
уровневого дискретного подхода к локаль- ному расчету строительных конструкций Таблича 2.1. Сопоставления результатов расчета.

\begin{tabular}{|c|c|c|c|c|c|c|}
\hline & Метод & $\begin{array}{c}\text { Количе- } \\
\text { ство эле- } \\
\text { ментов }\end{array}$ & $\begin{array}{l}\text { Изгибающий } \\
\text { момент (кНм) }\end{array}$ & $\begin{array}{c}\text { Расхождение } \\
\text { результатов, } \\
\%\end{array}$ & $\begin{array}{c}\text { Попереч- } \\
\text { ная сила } \\
(\kappa \mathrm{H}) \\
\end{array}$ & $\begin{array}{c}\text { Расхождение } \\
\text { результатов, } \\
\%\end{array}$ \\
\hline \multirow{5}{*}{1} & FDM & 64 & -763 & - & 227 & - \\
\hline & \multirow{4}{*}{$\begin{array}{l}\text { FDM- } \\
\text { DWT }\end{array}$} & 64 & -763 & 0.0 & 227 & 0.0 \\
\hline & & 47 & -768 & +0.7 & 227 & 0.0 \\
\hline & & 39 & -768 & +0.7 & 227 & 0.0 \\
\hline & & 35 & -768 & +0.7 & 227 & 0.0 \\
\hline \multirow{5}{*}{2} & FDM & 128 & -784 & - & 238 & - \\
\hline & \multirow{4}{*}{$\begin{array}{l}\text { FDM- } \\
\text { DWT }\end{array}$} & 128 & -784 & 0.0 & 238 & 0.0 \\
\hline & & 93 & -790 & +0.8 & 238 & 0.0 \\
\hline & & 76 & -794 & +1.3 & 238 & 0.0 \\
\hline & & 64 & -794 & +1.3 & 238 & 0.0 \\
\hline \multirow{8}{*}{3} & FDM & 256 & -794 & - & 245 & - \\
\hline & \multirow{7}{*}{$\begin{array}{l}\text { FDM- } \\
\text { DWT }\end{array}$} & 256 & -794 & 0.0 & 245 & 0.0 \\
\hline & & 186 & -799 & +0.6 & 244 & -0.4 \\
\hline & & 151 & -804 & +1.3 & 244 & -0.4 \\
\hline & & 134 & -806 & +1.5 & 244 & -0.4 \\
\hline & & 126 & -807 & +1.6 & 244 & -0.4 \\
\hline & & 122 & -807 & +1.6 & 243 & -0.8 \\
\hline & & 110 & -831 & +4.7 & 241 & -1.7 \\
\hline
\end{tabular}

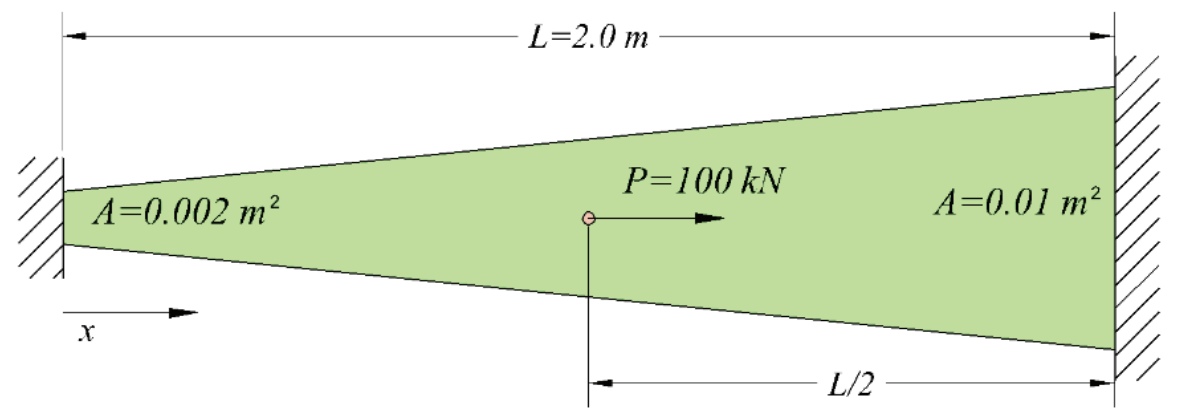

Рисунок 3.1. Задача о статическом расчете одномерного стержня.

(модуль $1 D F E M D W T$ ), хорошо согласуются с соответствующими результатами расчета по методу конечных элементов даже при значительной редукции и соответственно существенном сокращением размерности решаемой задачи.

На рис. 3.3 и в таблице 3.1 приведены сопоставления значений максимального напряжения в балке (координата $x=0.0$ ), полученного с использованием многоуровневого дискретного подхода к локальному расчету строительных конструкций (при значительной редукции и, как следствие, существенно меньшем объеме вычислительной работы) и метода конечных элементов.

В целом, как и в предыдущем примере, следует отметить хорошую согласованность полученных результатов. 
К вопросу о верификации многоуровневых подходов к локальному расчету строительных конструкций Часть 1: Дискретный (численный) подход

Таблица 3.1. Сопоставление максимальных напряжений в конструкичи ( $x=0.0)$.

$\begin{array}{cc}\text { Метод } & \text { Многоуровневый } \\ \text { конечных } & \text { подход к локальному } \\ \text { элементов } & \text { расчету } \\ \text { (FEM) } & \text { (FEM-DWT) }\end{array}$

\section{Максимальное напряжение $\left(k N / m^{2}\right)$}

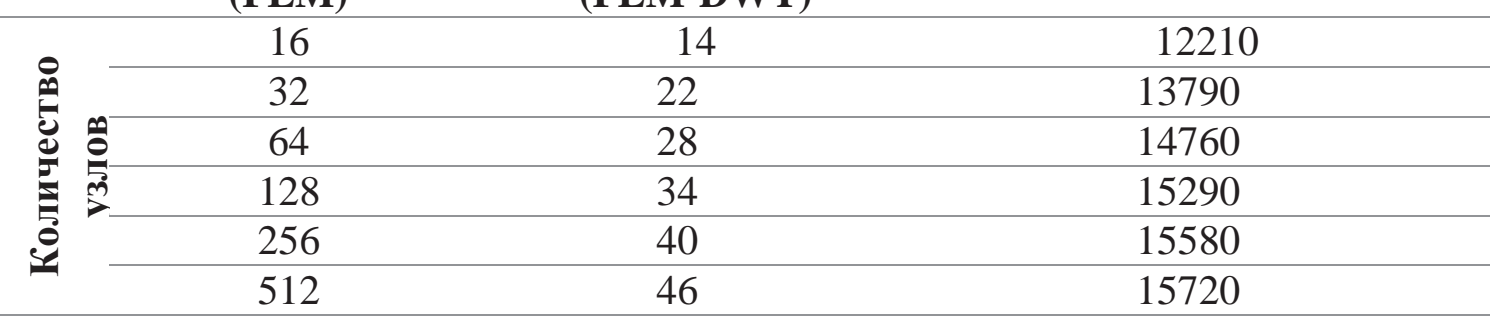

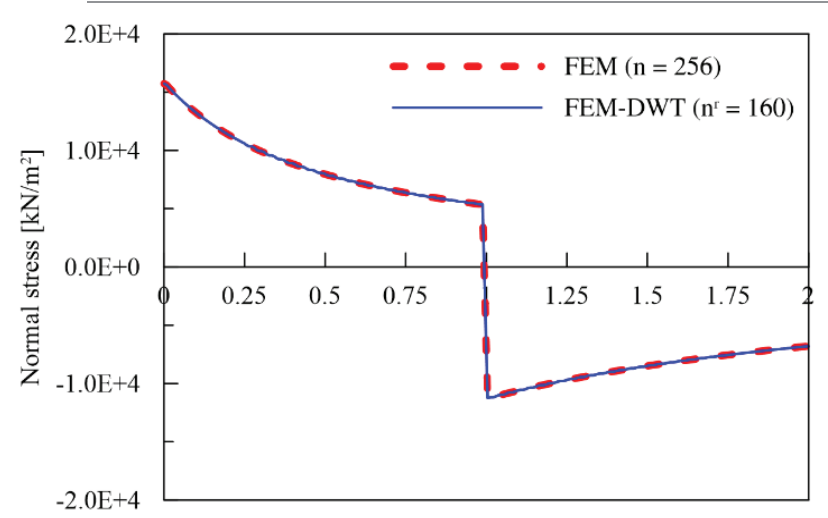

а) первый уровень редукции

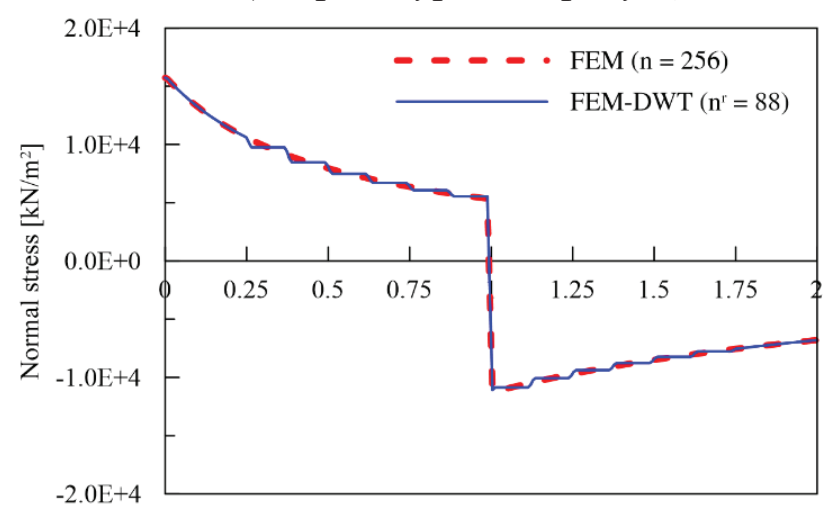

в) третий уровень редукичии

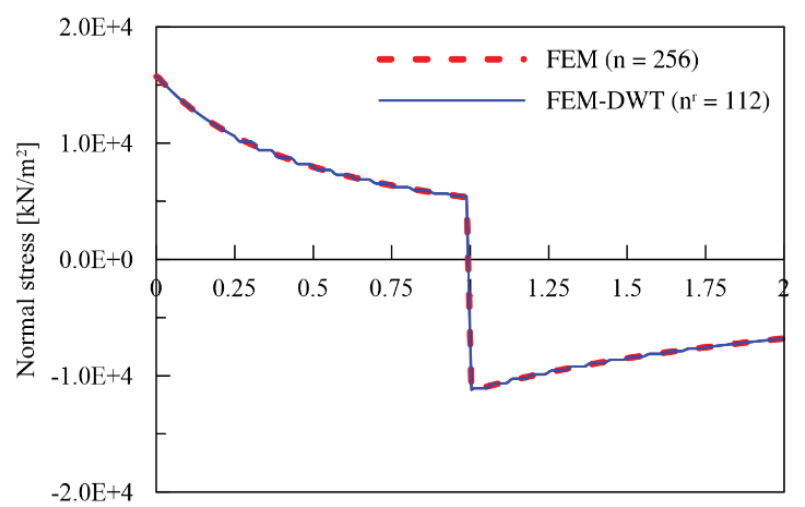

б) второй уровень редукции

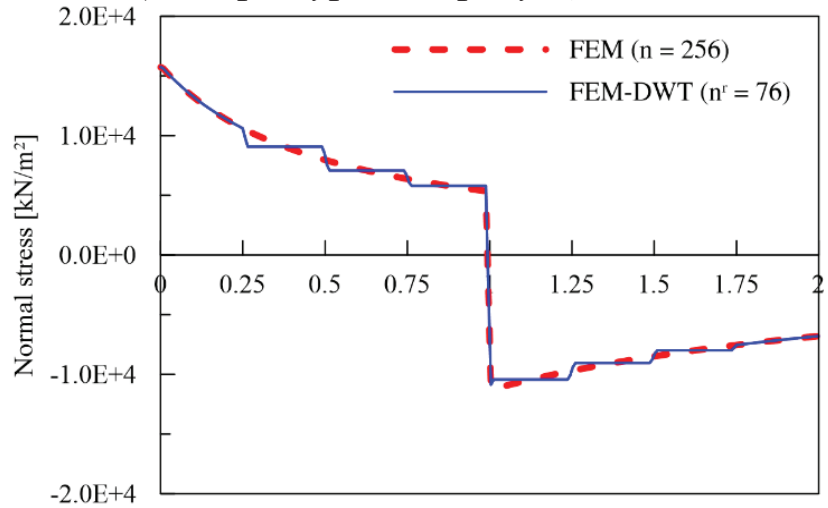

г) четвертый уровень редукции

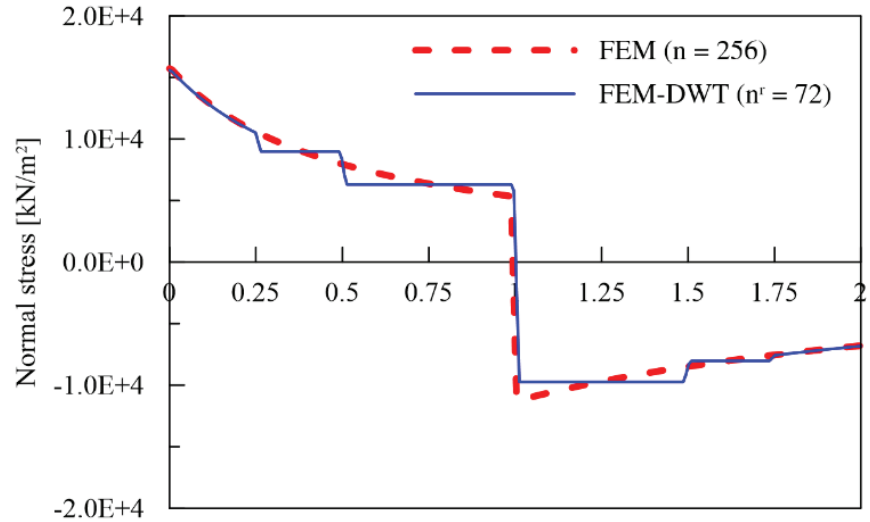

д) пятый уровень редукиии

Рисунок 3.2. Сопоставление результатов расчета в зоне гарантированной точности. 


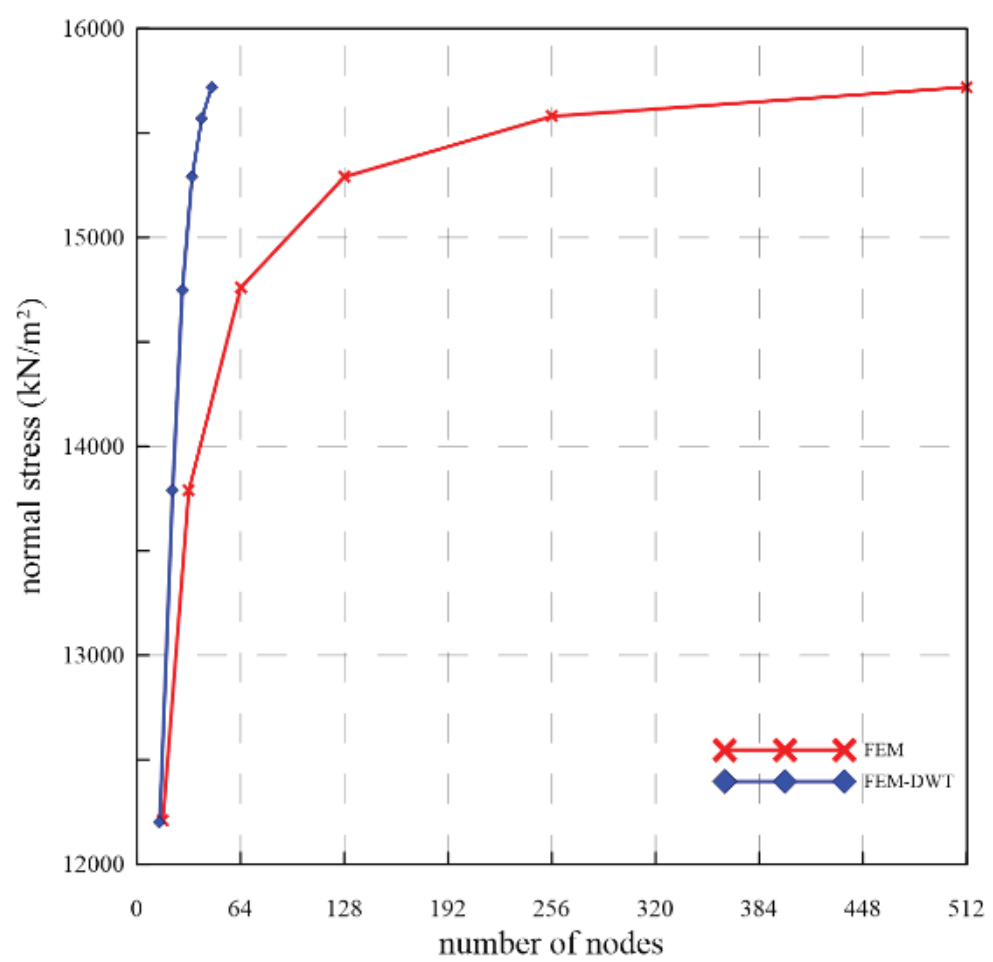

Рисунок 3.3. Сопоставление максимальных напряжений в конструкции $(x=0.0)$.

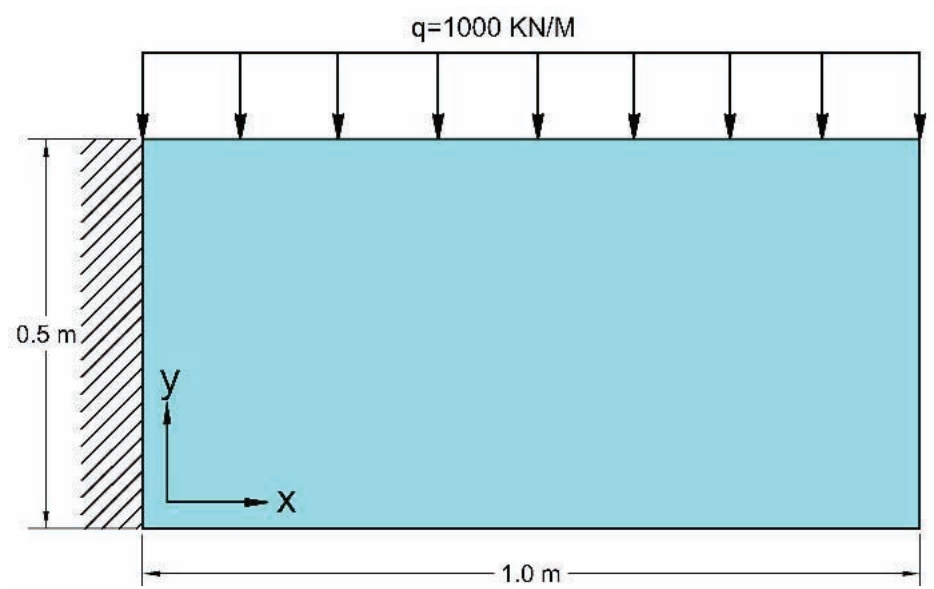

Рисунок 4.1. Задача о статическом расчете балки-стенки.

\section{4. ЛОКАЛЬНЫЙ РАСЧЕТ}

БАЛКИ-СТЕНКИ В УСЛОВИЯХ ДЕЙСТВИЯ РАВНОМЕРНО РАСПРЕДЕЛЕННОЙ НАГРУЗКИ

Рассмотрим задачу локального статического расчета балки стенки (плоское напряженное состояние) в условиях действия распределенной нагрузки $q=1000 \kappa \mathrm{H} / \mathrm{m}$, толщина пластины условно полагается единичной, модуль упругости и коэффициент Пуассона равны $E=200$ ГПа и $v=0.3$ (рис. 4.1).

Для решения данной задачи используется многоуровневый дискретный подход к локальному расчету строительных конструкций (модуль 2DFEMDWT комплекса программ MWBDASA2D [6,7]), основанный на использовании техники метода конечных элементов (FEM-DWT) (применяются изопараметрические четырехузловые конечные элементы). 
К вопросу о верификации многоуровневых подходов к локальному расчету строительных конструкций Часть 1: Дискретный (численный) подход

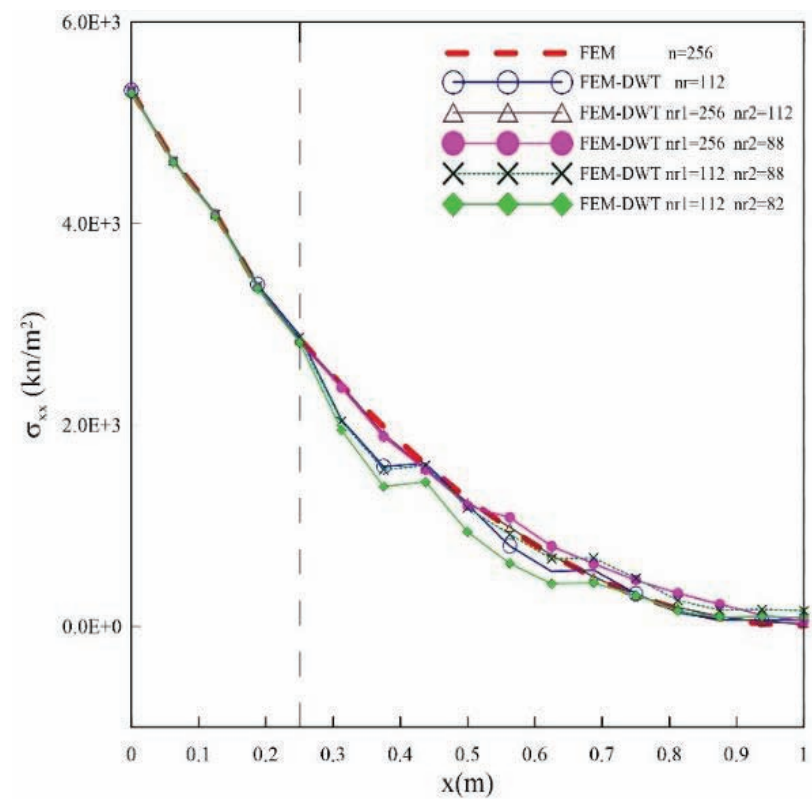

a) $\sigma_{x x}$

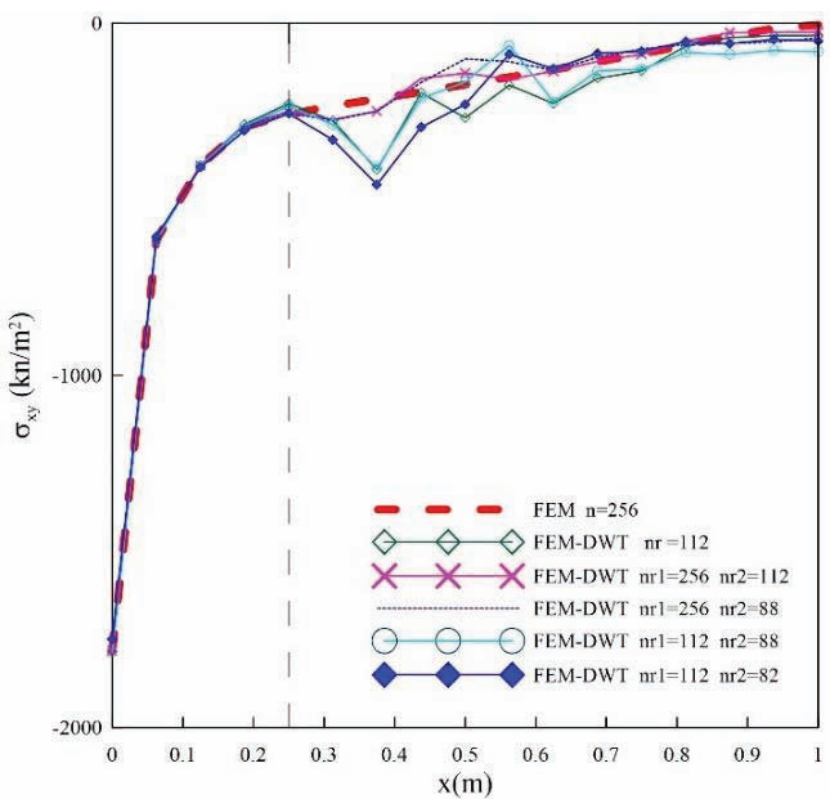

б) $\sigma_{x y}$

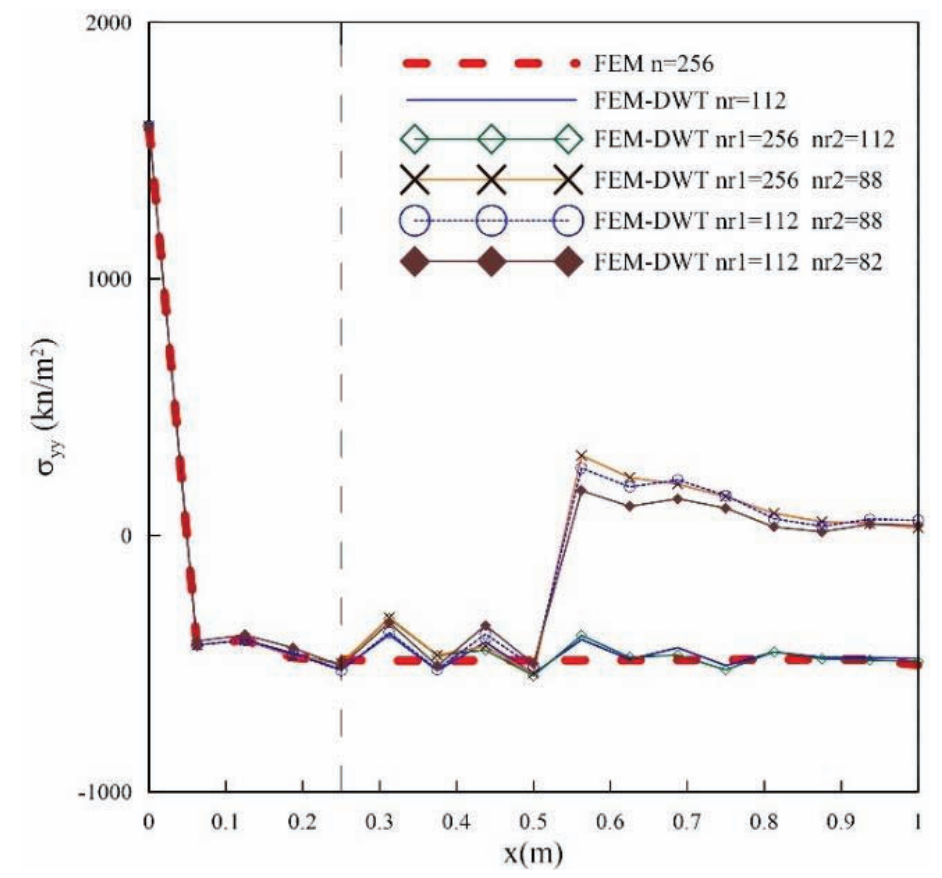

в) $\sigma_{y y}$

Рисунок 4.2. Сопоставление напряжений по сечению $y=0.47$ м.

Положим, что зона гарантированной точности, в которой требуется построить локальное решение задается неравенствами $0 \leq x \leq 0.3$ и $0 \leq y \leq 0.5$.

Выполнены сопоставления исходного (эталонного) решения (без редукции), полученного по МКЭ при использовании 256 узлов, с соответствующими редуцированными (FEM-
DWT) при следующих различных параметрах редукции [6,7]:

- осреднение по узлам, количество узлов после осреднения $-n_{r}=112$;

- осреднение по степеням свободы, количество узлов после осреднения в направлении осей $x$ и $y-n_{r 1}$ и $n_{r 2}$ соответственно. 

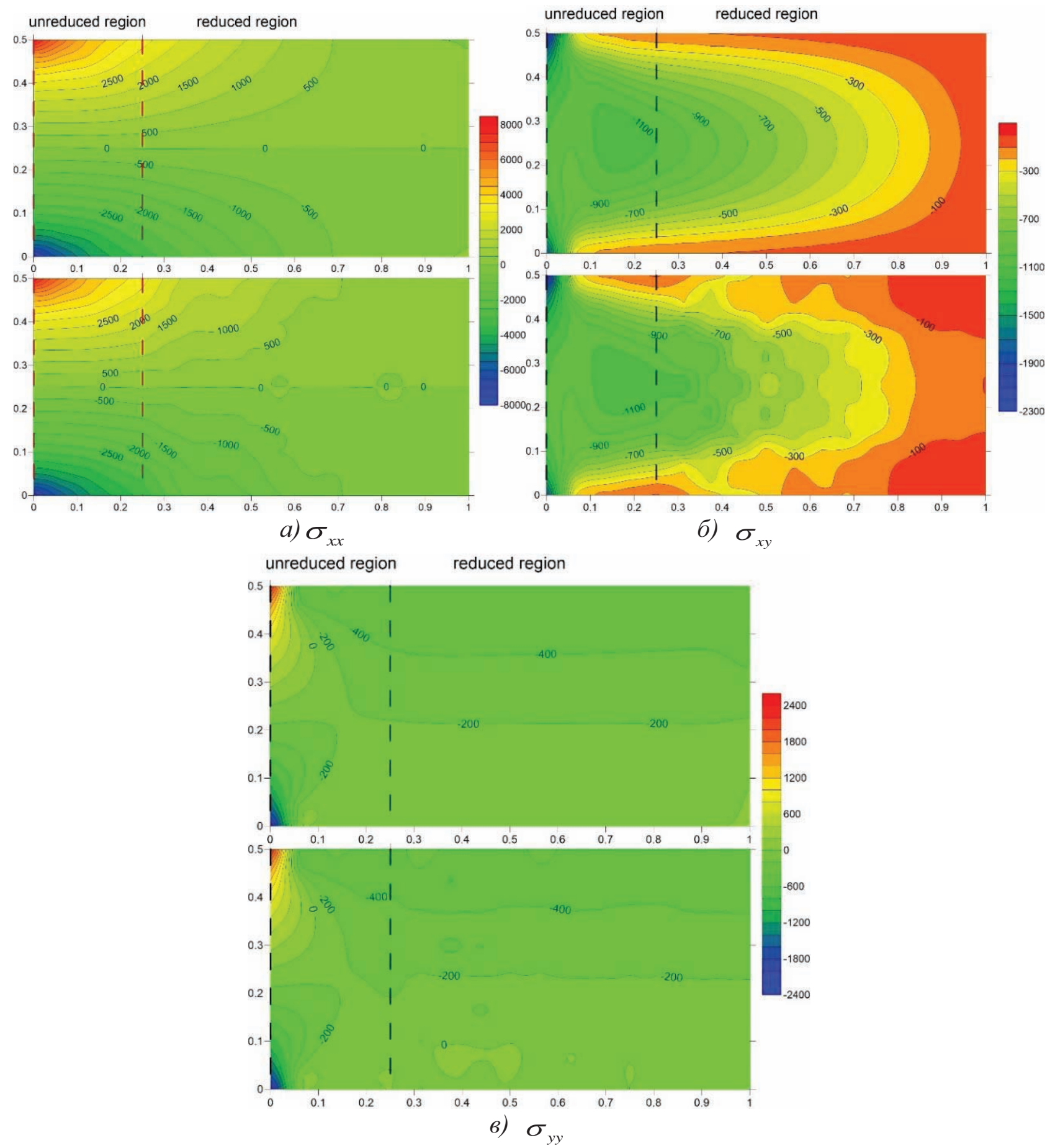

Рисунок 4.3. Сопоставление напряжений по сечению $y=0.47$ м (при $\left.n_{r}=112\right)$.

На рис. 4.2 и 4.3 представлены выборочные сопоставления некоторых результатов (в части напряжений), в частности, по сечению $y=0.47 \mathrm{M}$.

Следует отметить, что в целом, имеет место хорошая согласованность результатов в зоне гарантированной точности даже при значительной редукции. 
К вопросу о верификации многоуровневых подходов к локальному расчету строительных конструкций Часть 1: Дискретный (численный) подход

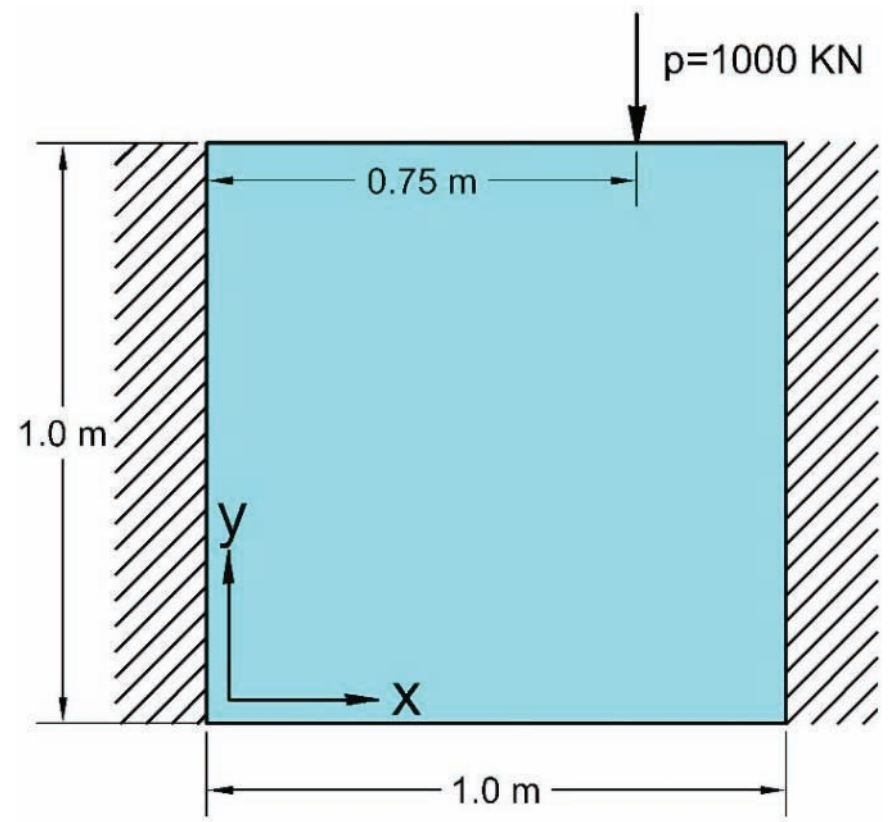

Рисунок 5.1. Задача о статическом расчете балки-стенки.

\section{5. ЛОКАЛЬНЫЙ РАСЧЕТ \\ БАЛКИ-СТЕНКИ В УСЛОВИЯХ ДЕЙСТВИЯ СОСРЕДОТОЧЕННОЙ НАГРУЗКИ}

Рассмотрим задачу локального статического расчета балки стенки (плоское напряженное состояние) в условиях действия сосредоточенной силы $p=1000$ кН, толщина пластины условно полагается единичной, модуль упругости и коэффициент Пуассона соответственно равны $E=200$ ГПа и $v=0.3$ (рис. 5.1).

Для решения данной задачи используется многоуровневый дискретный подход к локальному расчету строительных конструкций (модуль 2DFEMDWT комплекса программ MWBDASA2D [6,7]), основанный на использовании техники метода конечных элементов (FEM-DWT).

Положим, что зона гарантированной точности, в которой требуется построить локальное решение задается неравенствами $0.5 \leq x \leq 1.0$ и $0.5 \leq y \leq 1.0$.

На этапе построения дискретной модели используются изопараметрические четырехузловые конечные элементы.

Выполнены сопоставления исходного (эталонного) решения (без редукции), полученно- го по методу конечных элементов при использовании 256 узлов, с соответствующими редуцированными (FEM-DWT) при различных параметрах редукции $[1,2,6,7]$ :

- осреднение по узлам, количество узлов после осреднения $-n_{r}=112$;

- осреднение по узлам, количество узлов после осреднения $-n_{r}=91$.

На рис. 5.2-5.5 представлены выборочные сопоставления результатов (в части напряжений).

\section{6. ЛОКАЛЬНЫЙ РАСЧЕТ \\ ТОНКИХ ПЛАСТИН В УСЛОВИЯХ ДЕЙСТВИЯ РАСПРЕДЕЛЕННОЙ НАГРУЗКИ}

Рассмотрим задачу локального статического расчета тонкой пластины в условиях действия распределенной нагрузки $q=100$ кН/м, толщина пластины равна $h=0.01$ м, модуль упругости и коэффициент Пуассона соответственно равны $E=30$ ГПа и $v=0.3$ (рис. 6.1). Зона гарантированной точности, где требуется построить локальное решение задается неравенствами $0 \leq x \leq 0.5,0 \leq y \leq 0.33$. 

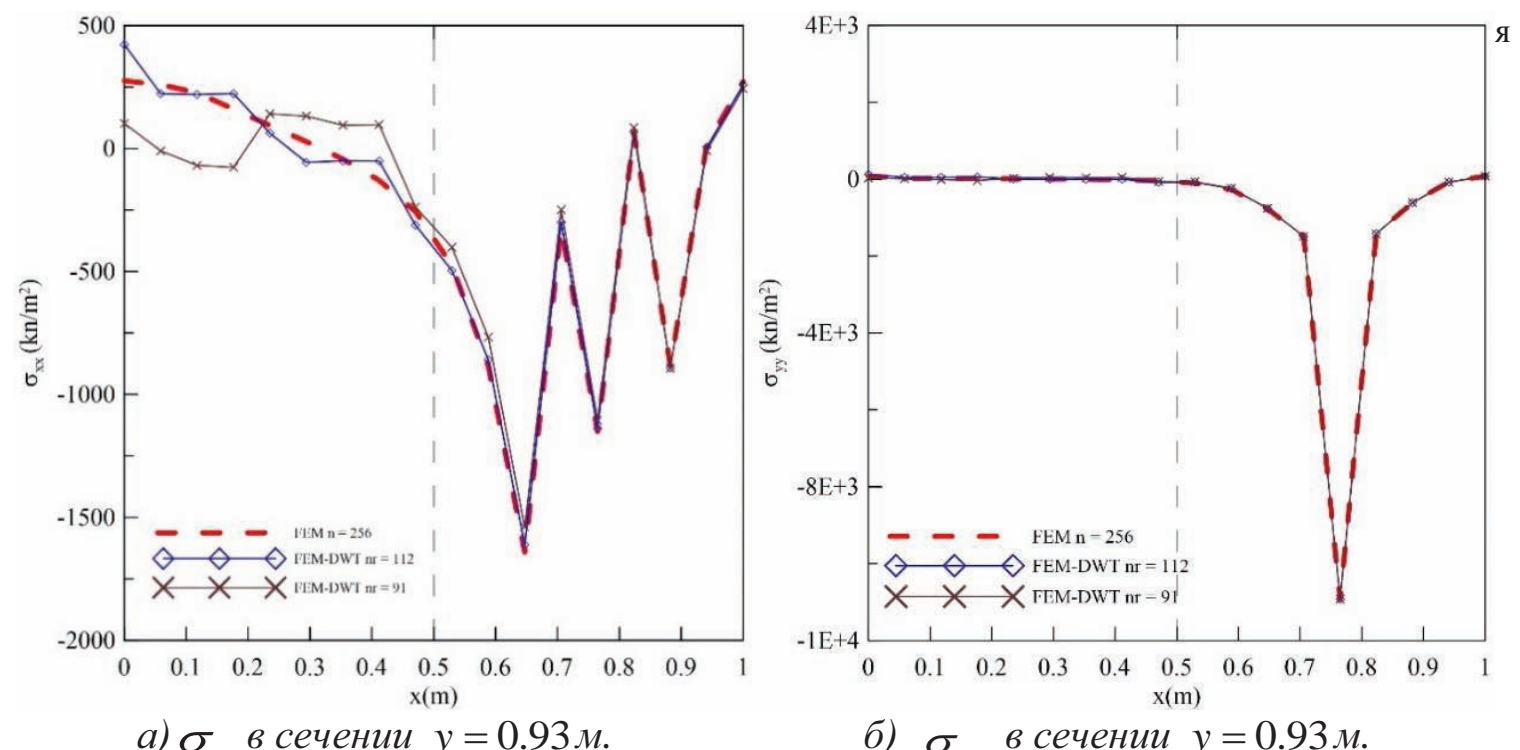

а) $\sigma_{x x}$ в сечении $y=0.93$ м.
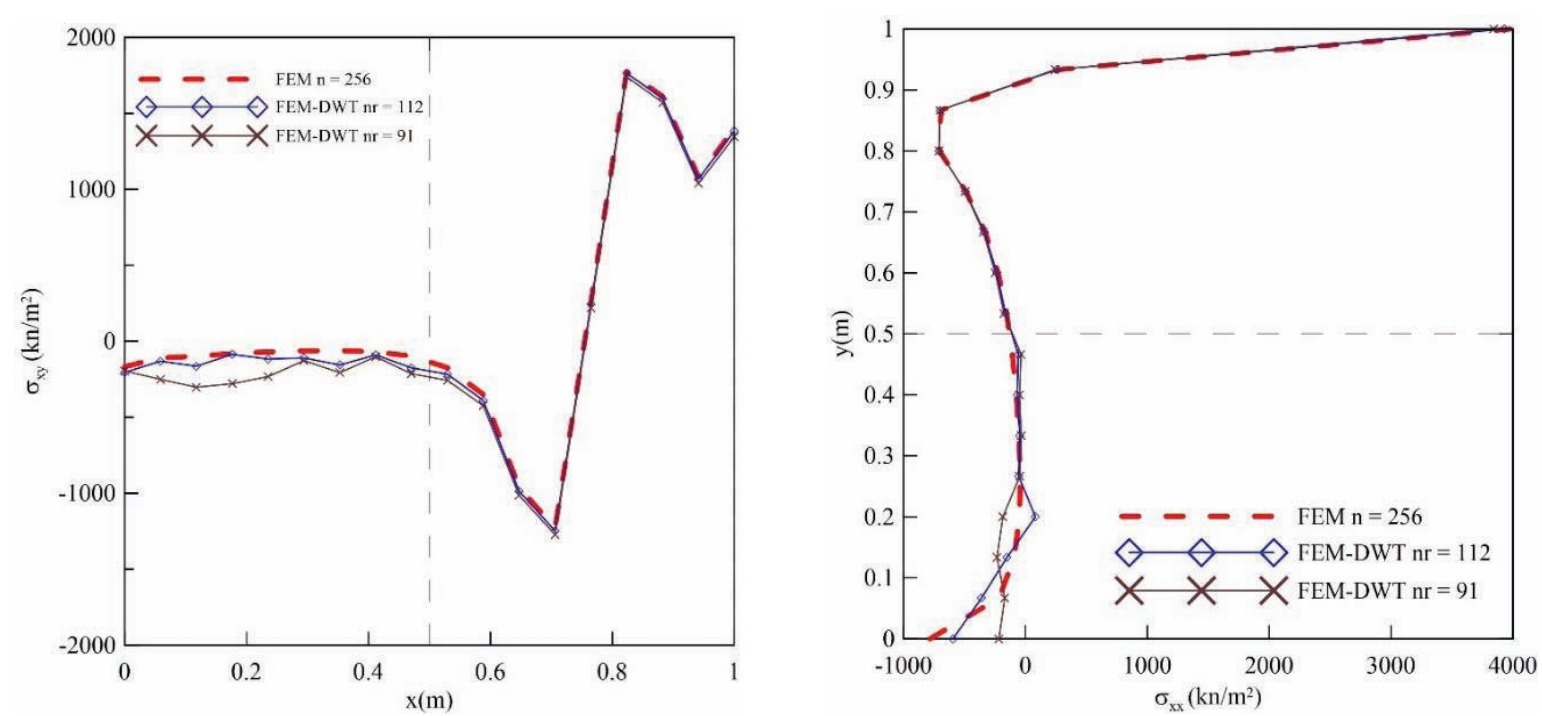

в) $\sigma_{x y}$ в сечении $y=0.93 \mathrm{M}$.

г) $\sigma_{x х}$ в сечении $x=1.0$ м.
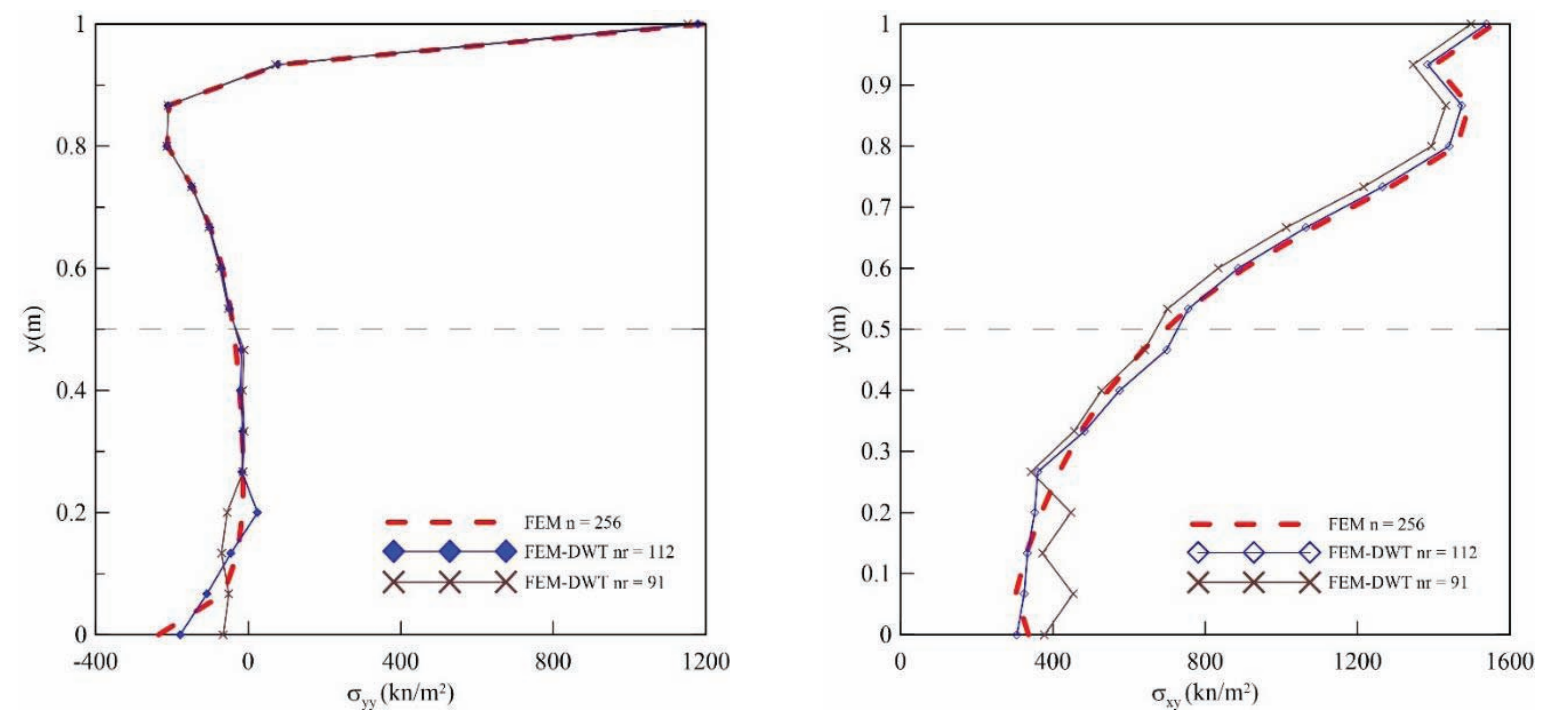

д) $\sigma_{y y}$ в сечении $x=1.0 м$.

е) $\sigma_{x y}$ в сечении $x=1.0 \mathrm{M}$.

Рисунок 5.2. Сопоставление напряжений. 


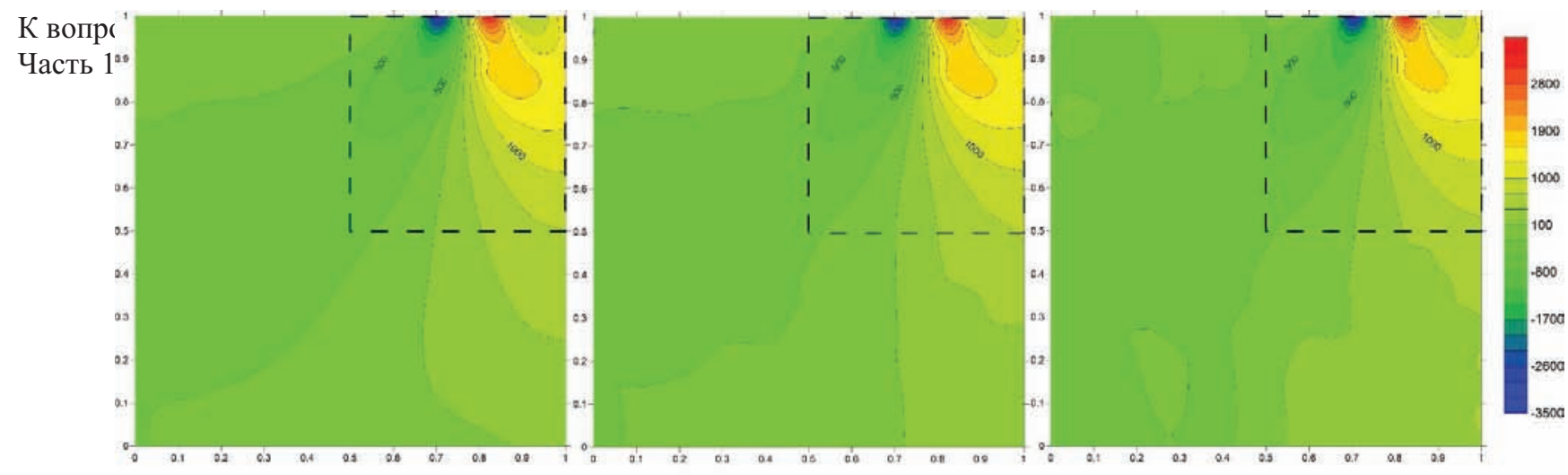

a)

b)

c)

Рисунок 5.3. Сопоставление узловых напряэсений $\sigma_{x y}$ :

a) FEM;

b) FEM-DWT $n_{r}=112$;

c) FEM-DWT $n_{r}=91$.

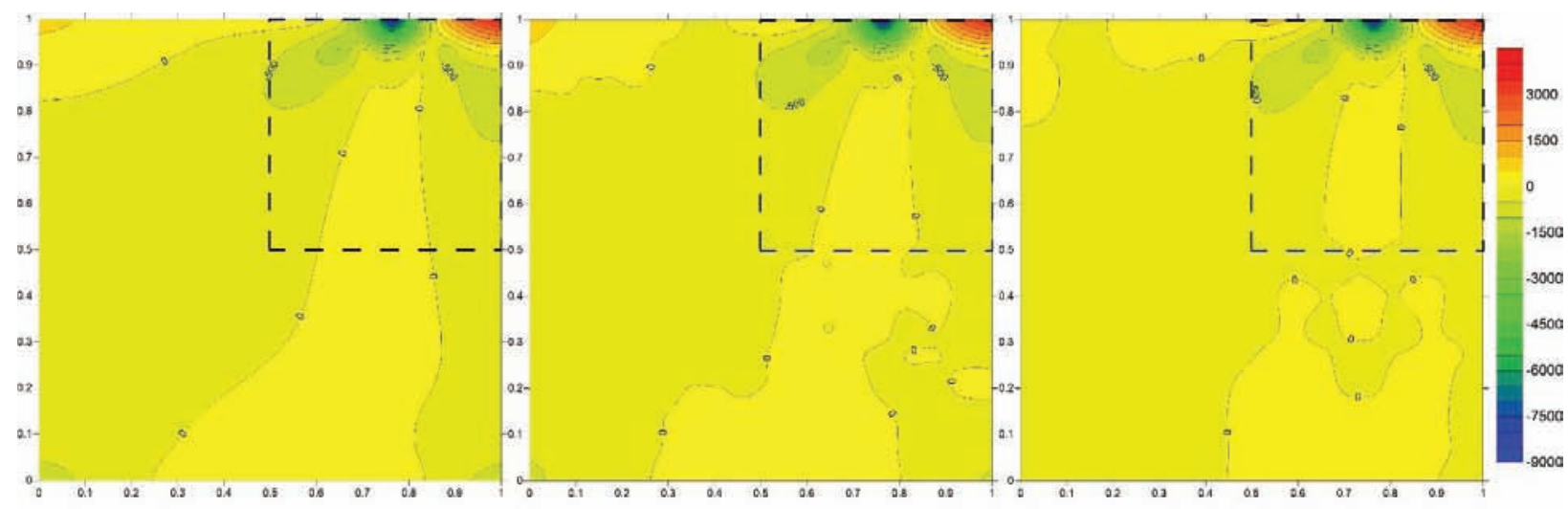

a)

b)

c)

Рисунок 5.4. Сопоставление узловых напряжений $\sigma_{x x}$ :

a) FEM;

b) FEM-DWT $n_{r}=112$;

c) FEM-DWT $n_{r}=91$.

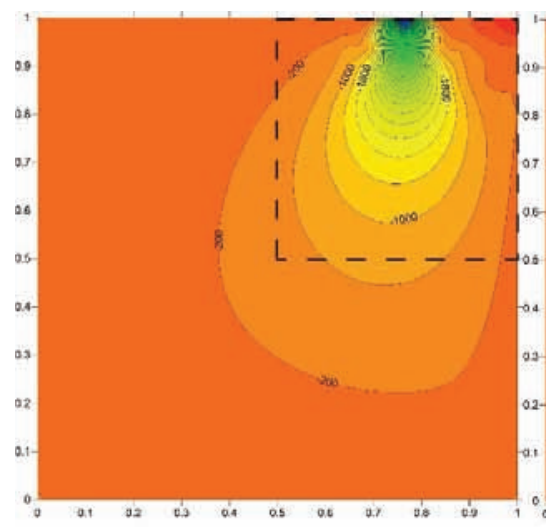

a)

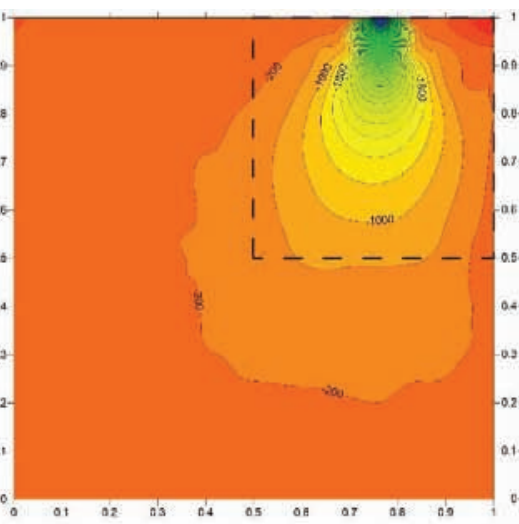

b)

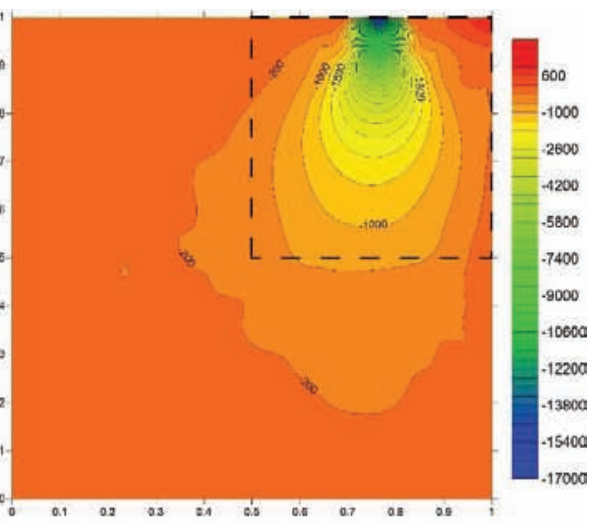

c)

Рисунок 5.5. Сопоставление узловых напряжений $\sigma_{y y}$ :

a) FEM;

b) FEM-DWT $n_{r}=112$;

Volume 12, Issue 4, 2016

c) FEM-DWT $n_{r}=91$. 


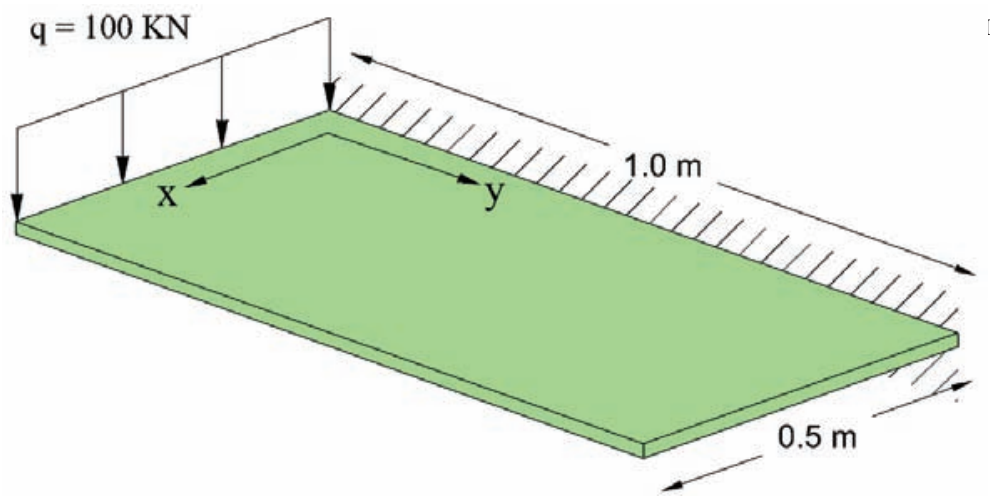

гов, Ж.И. Мсхалая

Рисунок 6.1. Задача о статическом расчете тонкой пластинь.
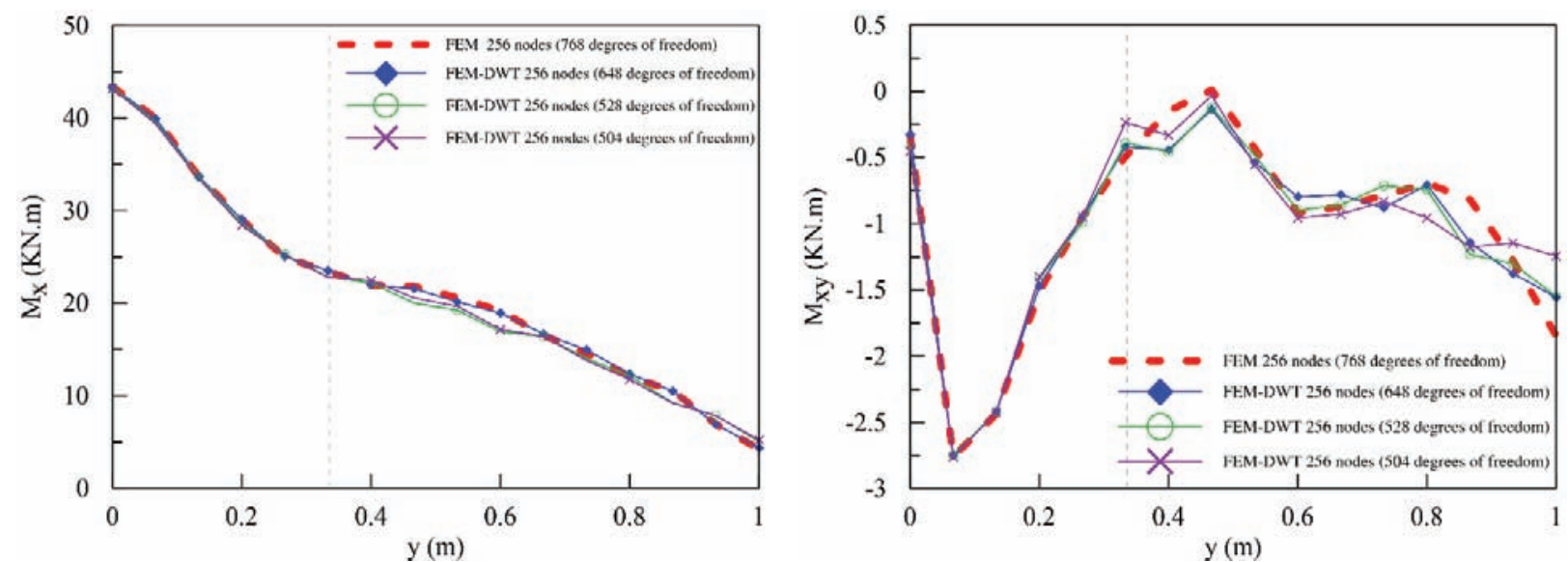

a)
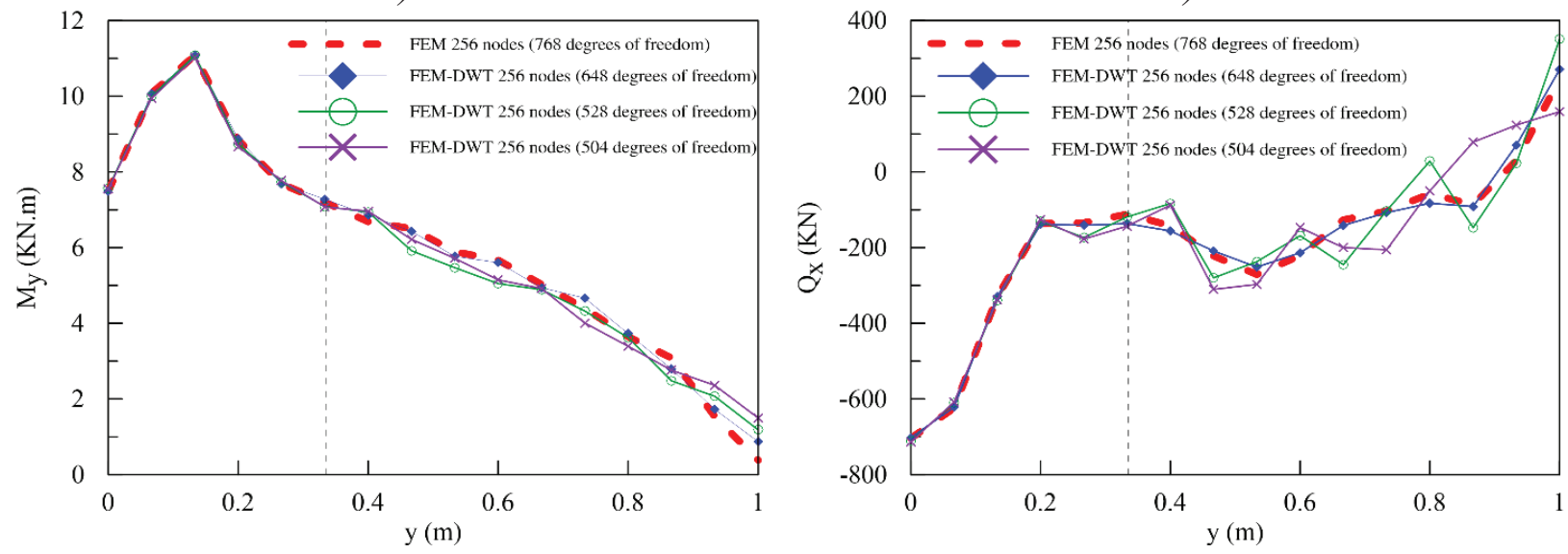

b)

2)

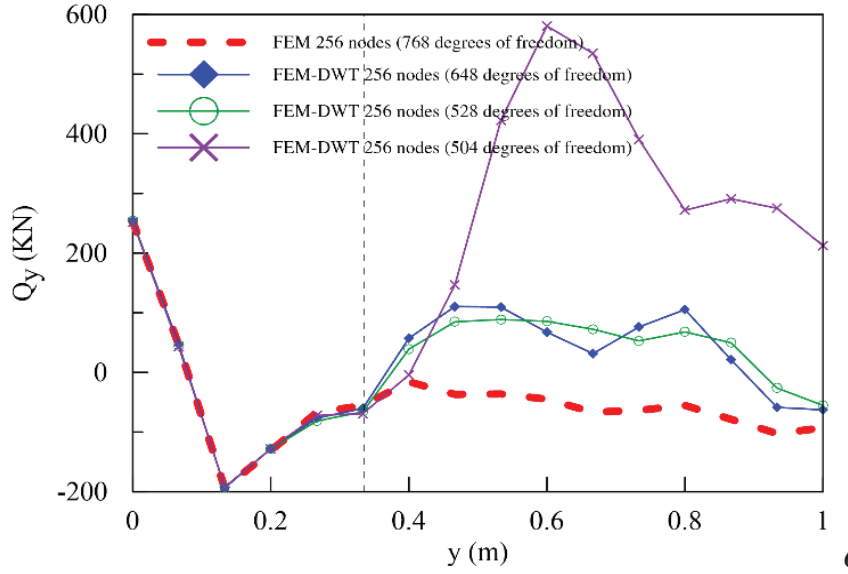

d)

Рисунок 6.2. Сопоставление изгибающих и крутящего моментов, поперечных сил. 
К вопросу о верификации многоуровневых подходов к локальному расчету строительных конструкций

Часть 1: Дискретный (численный) подход

Для решения данной задачи используется многоуровневый дискретный подход к локальному расчету строительных конструкций (модуль FEMDWP комплекса программ $M W B D A S A 2 D[6,7])$, основанный на использовании техники метода конечных элементов (FEM-DWT). Выполнены сопоставления исходного (эталонного) решения (без редукции), полученного по методу конечных элементов при использовании 256 узлов, с соответствующими редуцированными (FEMDWT) при различных параметрах редукции (c учетом схемы нагружения конструкции максимальные значения изгибающих и крутящего моментов и поперечных сил наблюдаются вблизи опорного закрепления, что предопределило выбор сечения для сопоставления ( $x=0.03$; в направлении $y$; рис. 6.2-6.3).

Следует отметить, что в рассматриваемой зоне имеет место хорошая согласованность результатов, полученных по многоуровневому дискретному подходу к локальному расчету строительных конструкций (FEM-DWT), в том числе и при значительной редукции, и методу конечных элементов [15-17].

\section{7. ЛОКАЛЬНЫЙ РАСЧЕТ ТОНКИХ ПЛАСТИН В УСЛОВИЯХ ДЕЙСТВИЯ СОСРЕДОТОЧЕННОЙ НАГРУЗКИ}

Рассмотрим задачу локального статического расчета тонкой пластины в условиях действия сосредоточенной нагрузки силы $p=100$ кН, толщина пластины равна $h=0.05$ м, модуль упругости и коэффициент Пуассона соответственно равны $E=30$ ГПа и $v=0.3$ (рис. 7.1).

Пусть зона гарантированной точности, в которой требуется построить локальное решение задается неравенствами $2.3 \leq x \leq 3.0$ и $0 \leq y \leq 1.4$.

Для решения данной задачи используется многоуровневый дискретный подход к локальному расчету строительных конструкций (модуль FEMDWP комплекса программ
$M W B D A S A 2 D$ [7]), основанный на использовании техники метода конечных элементов (FEM-DWT).

Выполнены сопоставления исходного (эталонного) решения (без редукции), полученного по методу конечных элементов при использовании 256 узлов, с соответствующими редуцированными (FEM-DWT) при различных параметрах редукции (рис. 7.2-7.4) $[1,2,6,7]$.

На рис. 7.2-7.4 представлены выборочные сопоставления результатов (в части изгибающего и крутящих моментов и поперечных сил), в частности, по сечениям $y=0.0$ м и $x=2.3$ м.

Следует отметить, что в рассматриваемой зоне имеет место хорошая согласованность результатов, полученных по многоуровневому дискретному подходу к локальному расчету строительных конструкций (FEM-DWT), в том числе и при значительной редукции, и методу конечных элементов [4,5,15-17].

\section{ЗАМЕЧАНИЕ}

Исследование выполнено за счет средств Государственной программы Российской Федерации «Развитие науки и технологий» на 2013-2020 годы в рамках Плана фундаментальных научных исследований Министерства строительства и жилищнокоммунального строительства Российской Федерации на 2016 год, тема 7.1.1 «Разработка многоуровневого подхода к исследованию напряженно-деформированного состояния конструкций в рамках единой иерархически выстроенной расчетной модели на основе совместного применения дискретно-континуального метода конечных элементов и метода конечных элементов». 
Моджтаба Аслами, П.А. Акимов, Ж.И. Мсхалая
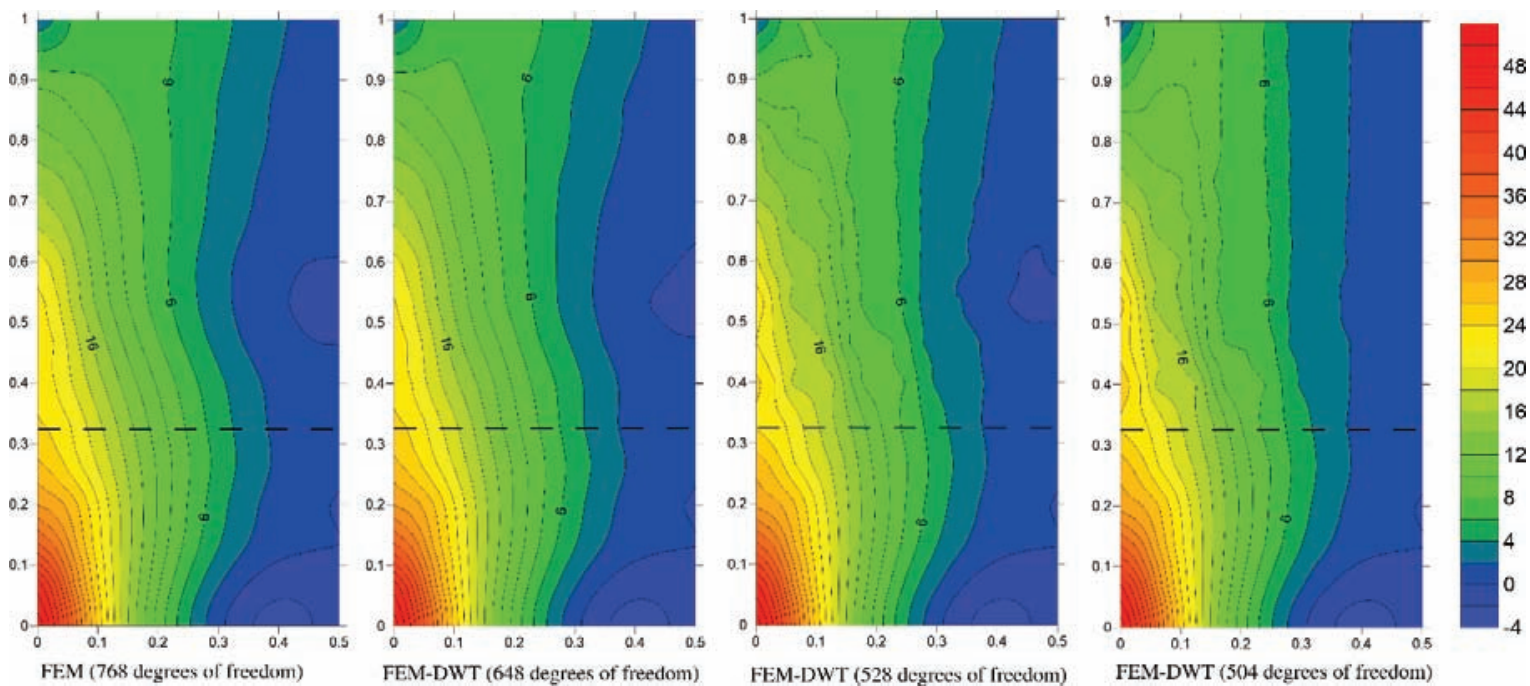

a) $M_{x}$
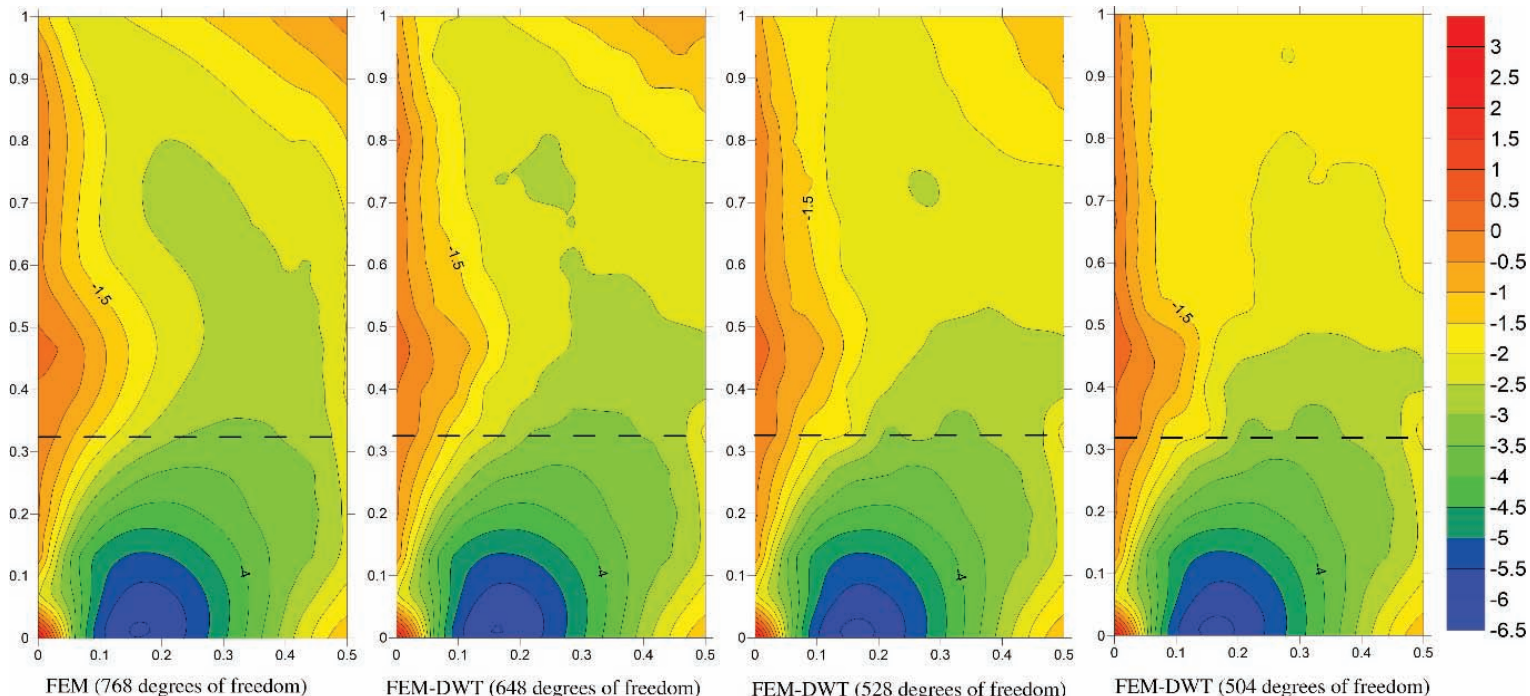

б) $M_{x y}$

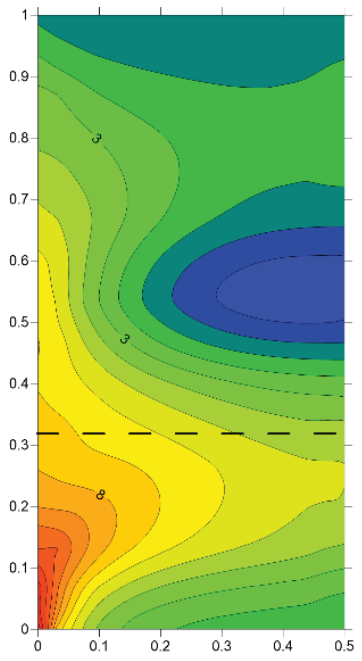

FEM (768 degrees of freedom)

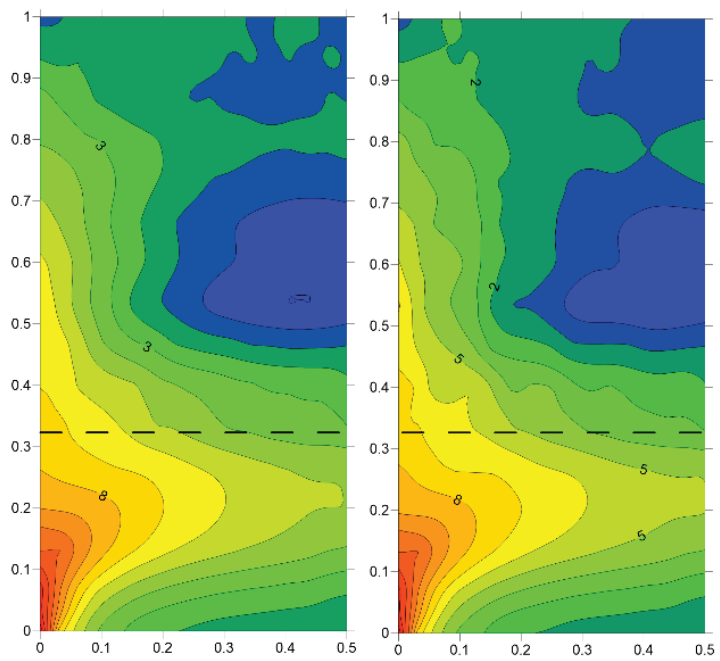

FEM-DWT (648 degrees of freedom)
FEM-DWT (528 degrees of freedom)

в) $M_{y}$

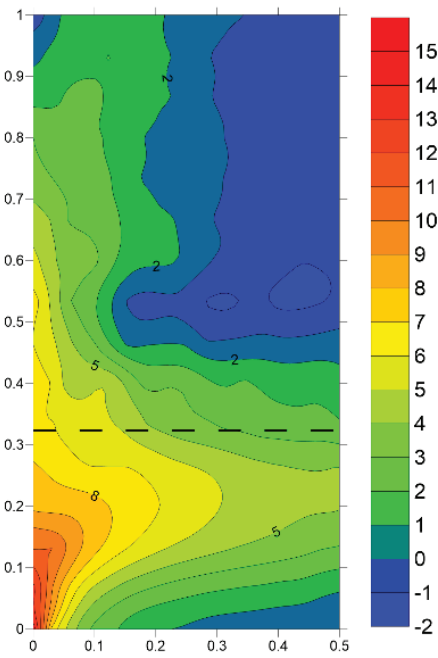

FEM-DWT (504 degrees of freedom) 
К вопросу о верификации многоуровневых подходов к локальному расчету строительных конструкций Часть 1: Дискретный (численный) подход

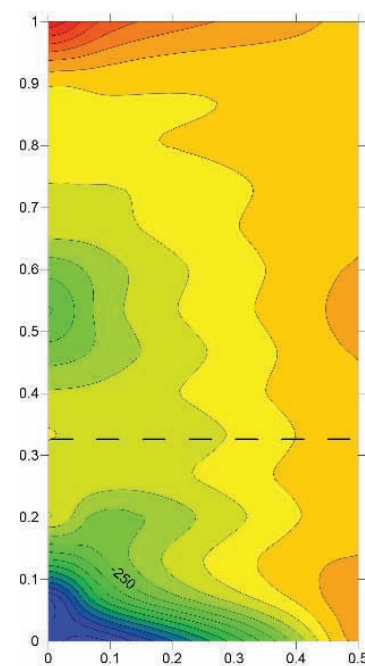

FEM (768 degrees of freedom)

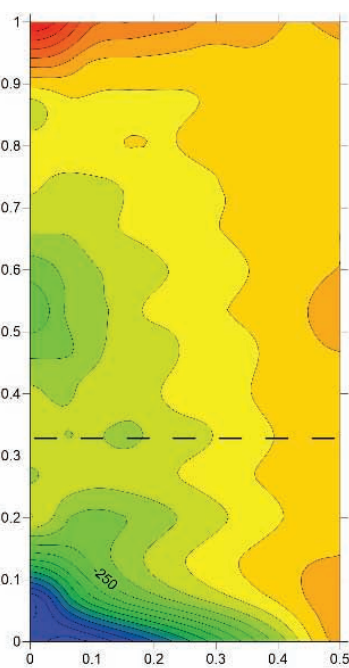

FEM-DWT (648 degrees of freedom)

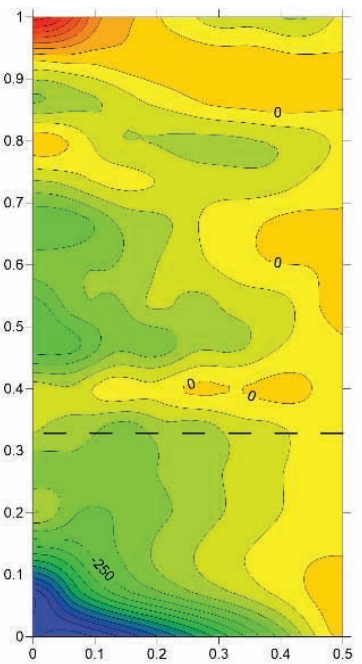

FEM-DWT (528 degrees of freedom)

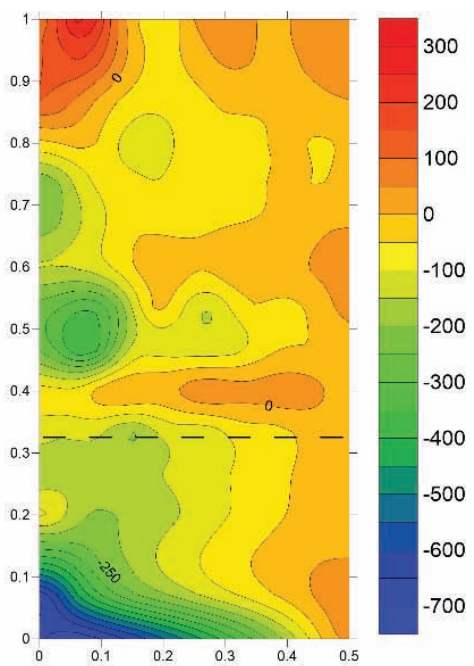

FEM-DWT (504 degrees of freedom)

2) $Q_{x}$

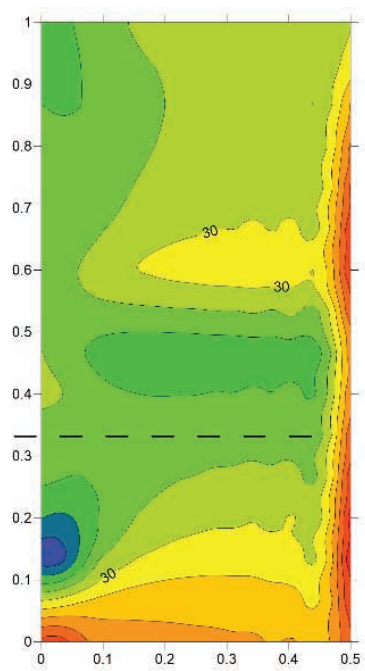

FEM (768 degrees of freedom)

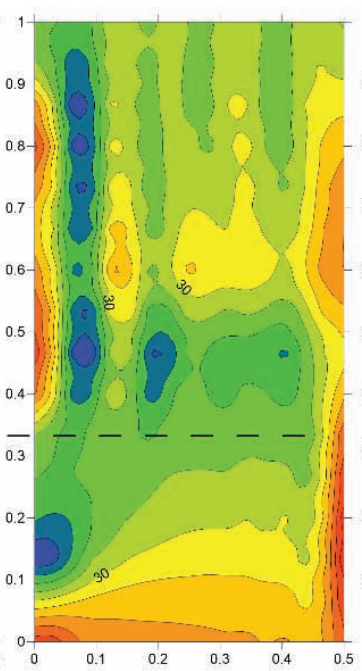

FEM-DWT (648 degrees of freedom)

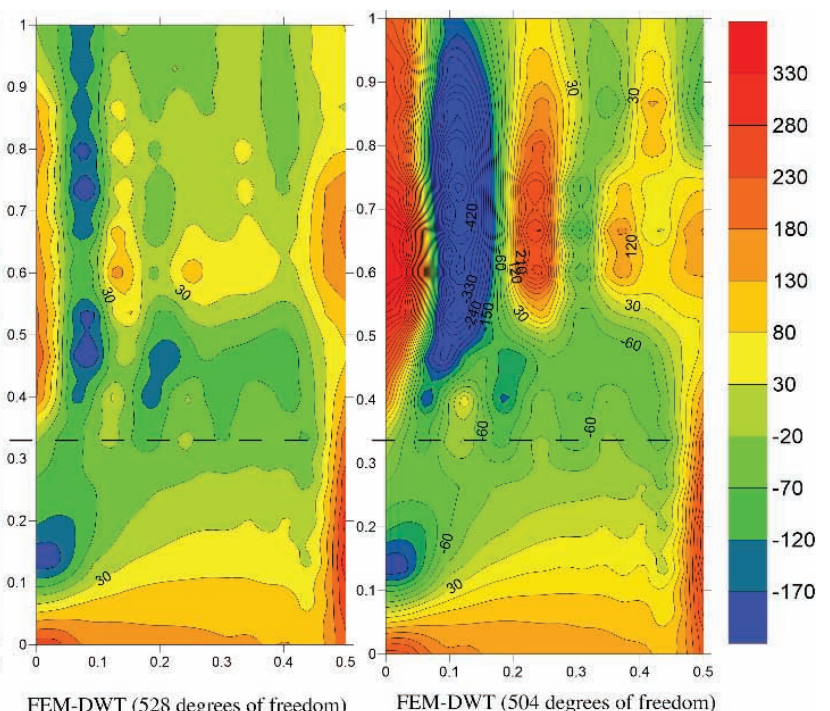

d) $Q_{y}$

Рисунок 6.3. Сопоставление изгибающих и крутящего моментов и поперечных сил.

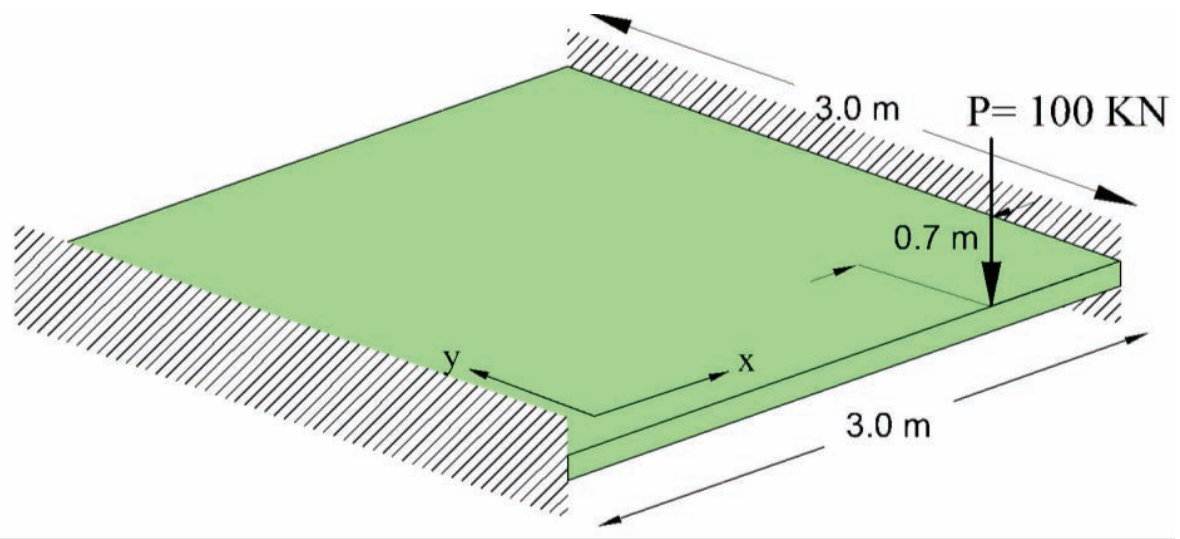

Рисунок 7.1. Задача о статическом расчете тонкой пластины. 

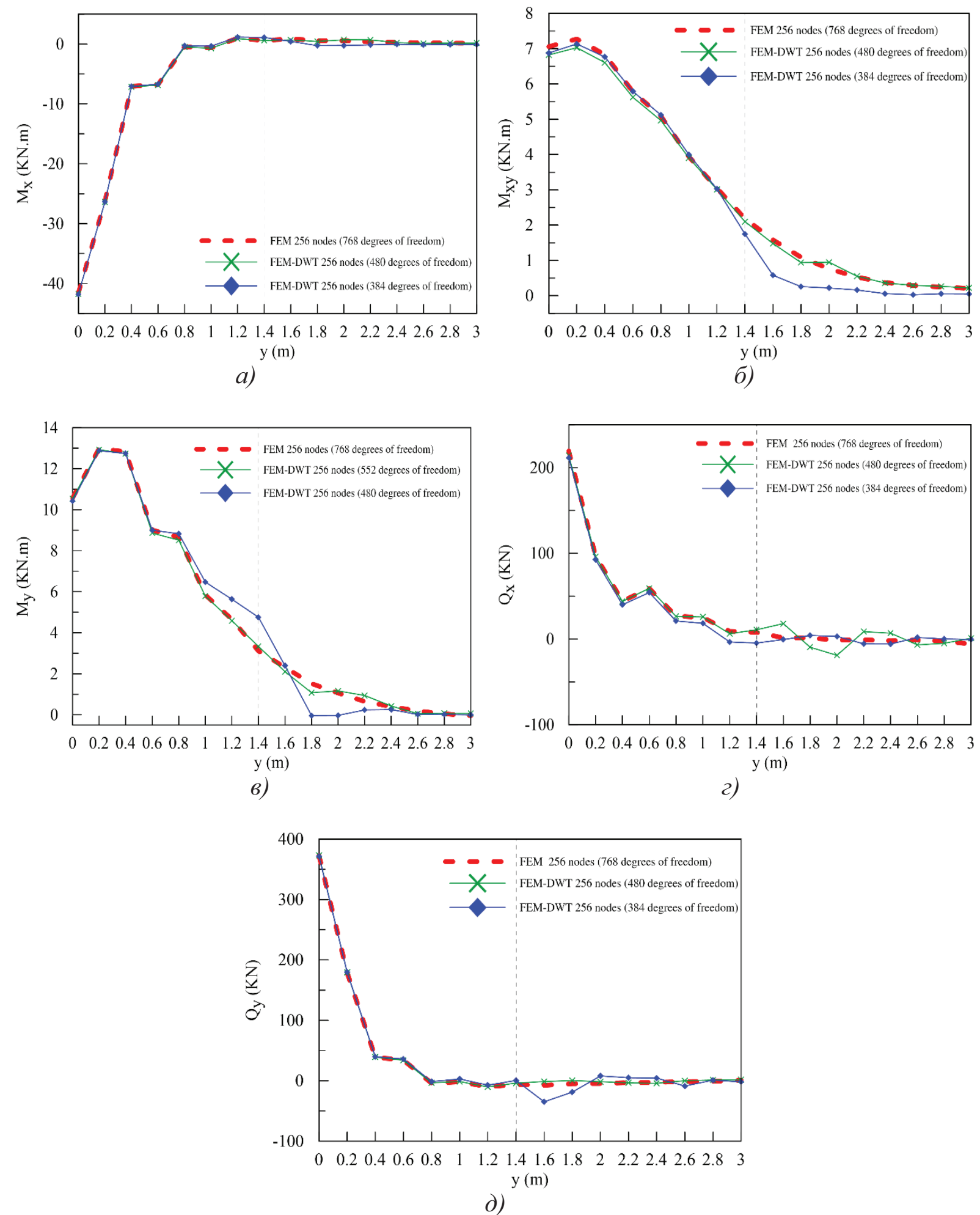

Рисунок 7.2. Сопоставление изгибающих и крутящего моментов и поперечных сил по сечению $x=2.3$ м вдоль $y$. 
К вопросу о верификации многоуровневых подходов к локальному расчету строительных конструкций Часть 1: Дискретный (численный) подход

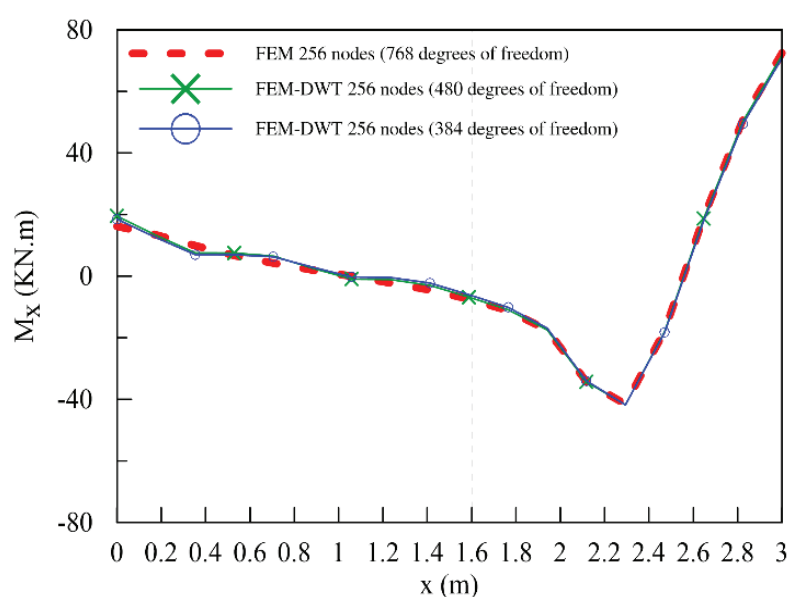

a)

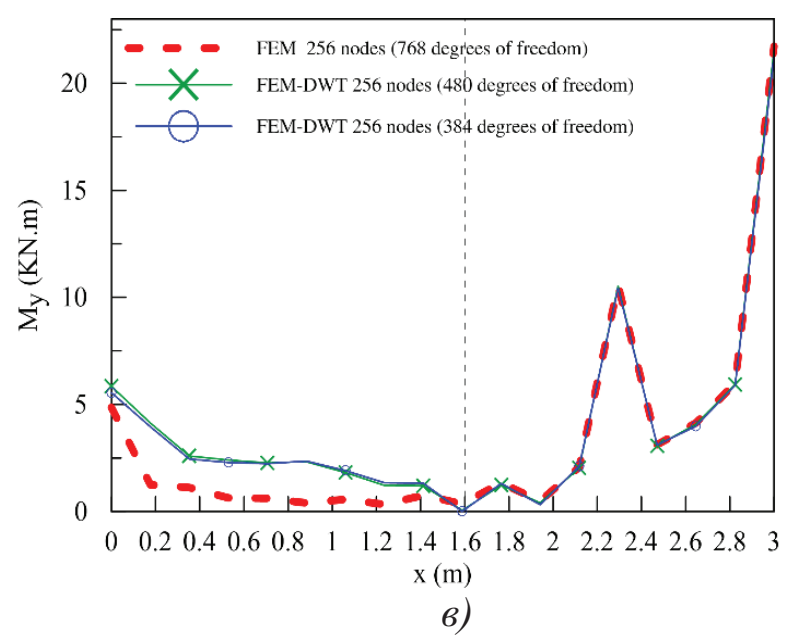

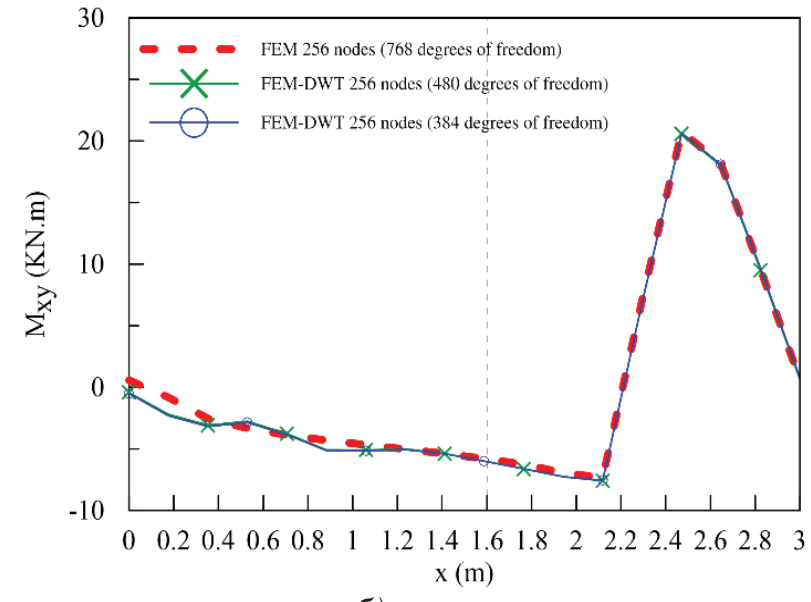

б)

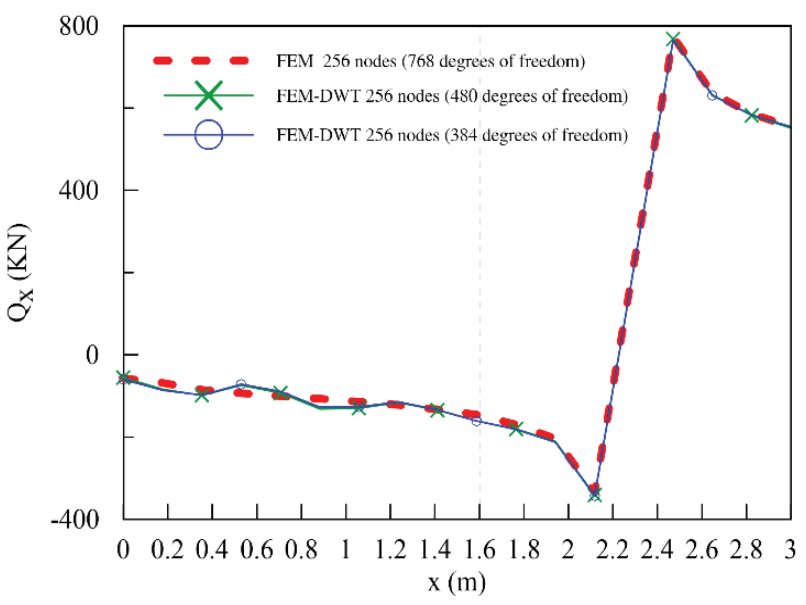

2)

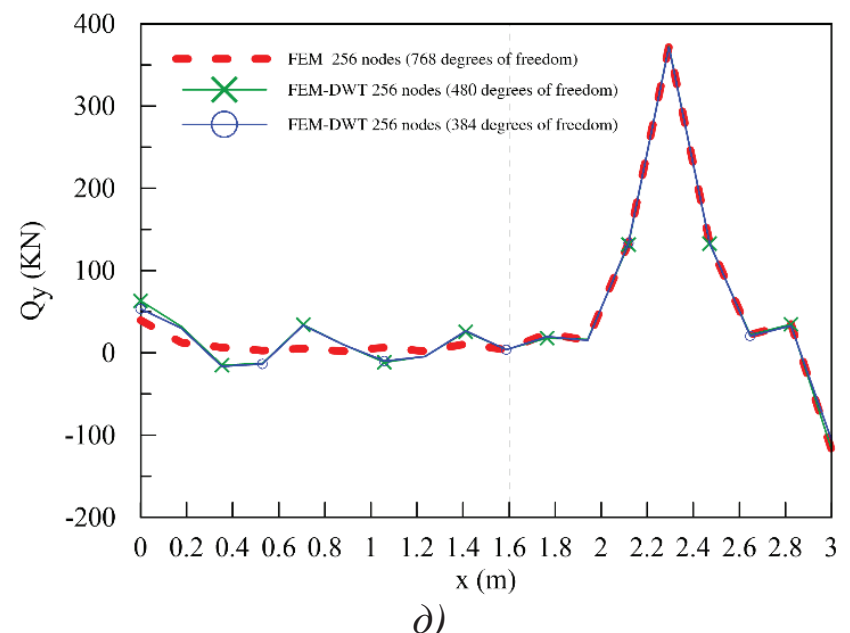

Рисунок 7.3. Сопоставление изгибающих и крутящего моментов и поперечных сил по сечению $y=0.0$ м вдоль $x$. 


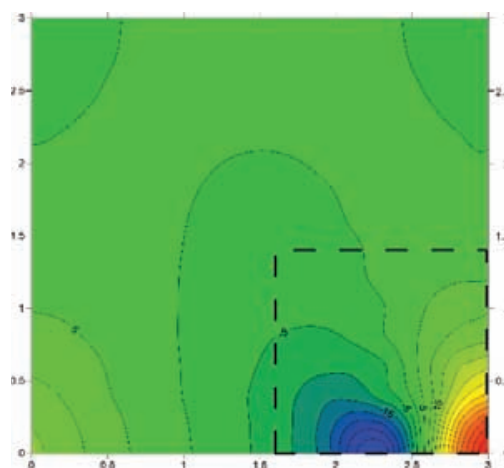

FEM (768 degrees of freedom)

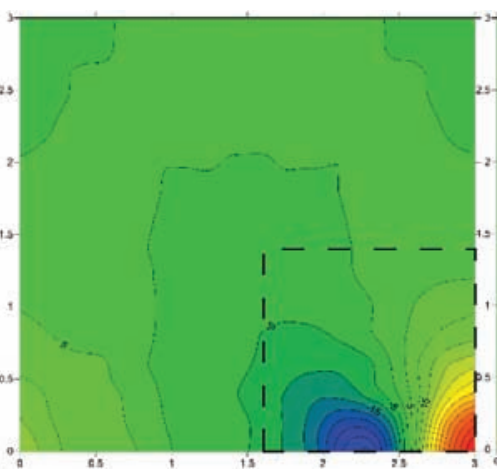

FEM-DWT (480 degrees of freedom)

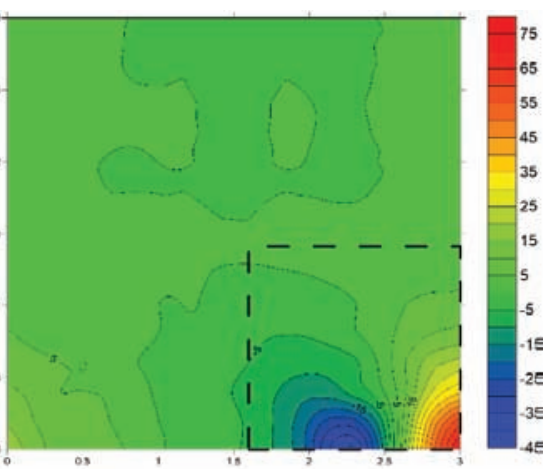

FEM (384 degrees of freedom)

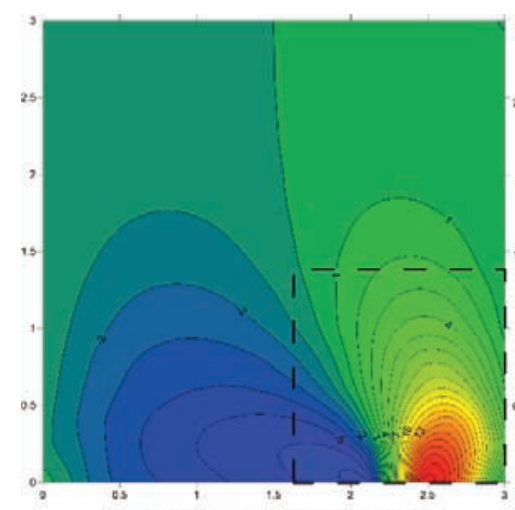

FEM (768 degrees of freedom)

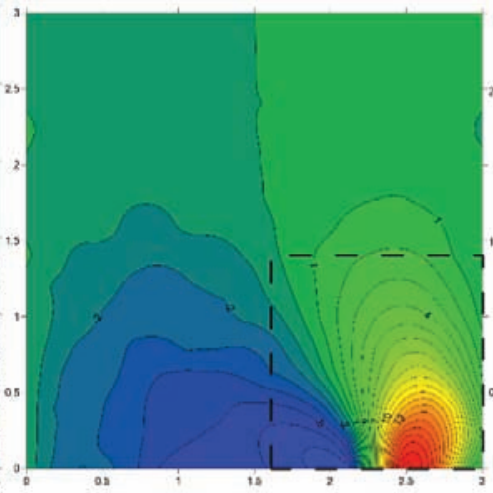

FEM-DWT (480 degrees of freedom)

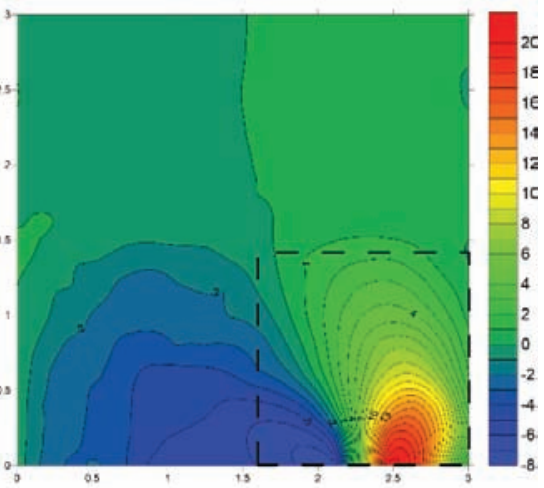

FEM (384 degrees of freedom)

б) $M_{x y}$

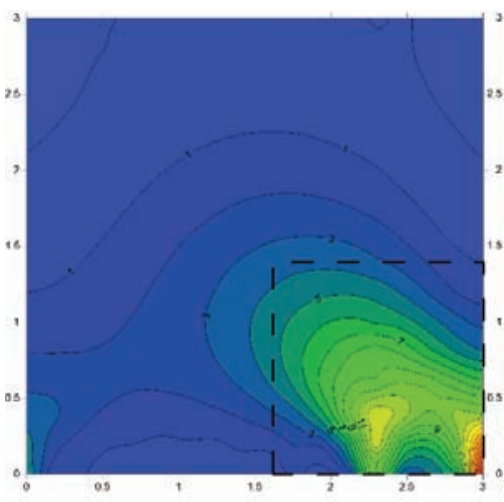

FEM (768 degrees of freedom)

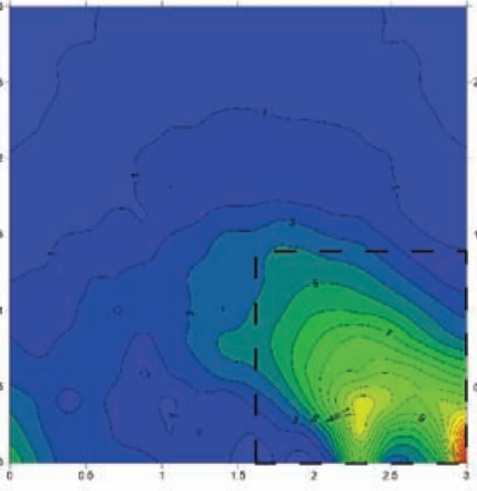

FEM-DWT (480 degrees of freedom)

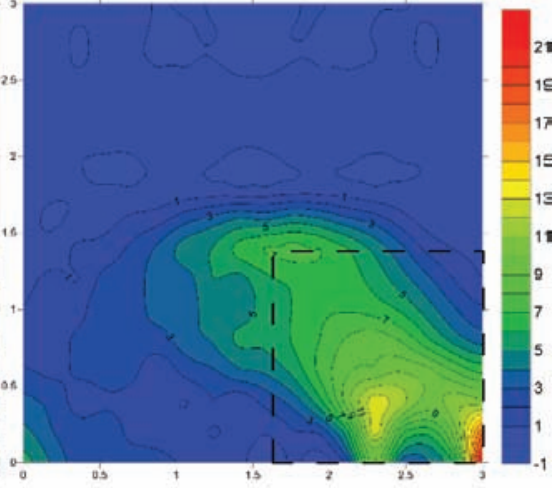

FEM (384 degrees of freedom)

в) $M$

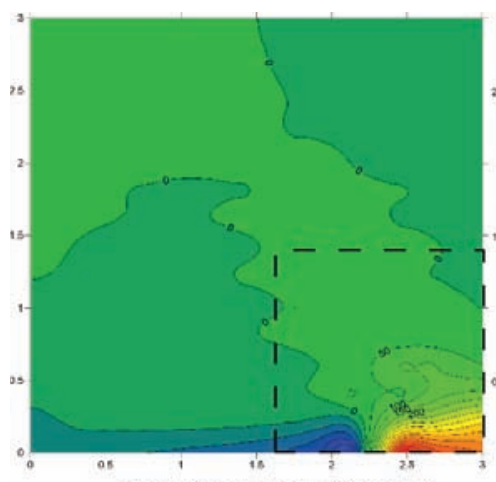

2) $Q_{x}$

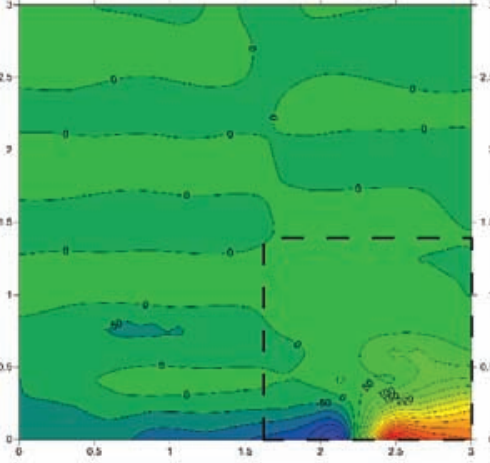

FEM-DWT (480 degrees of freedom)

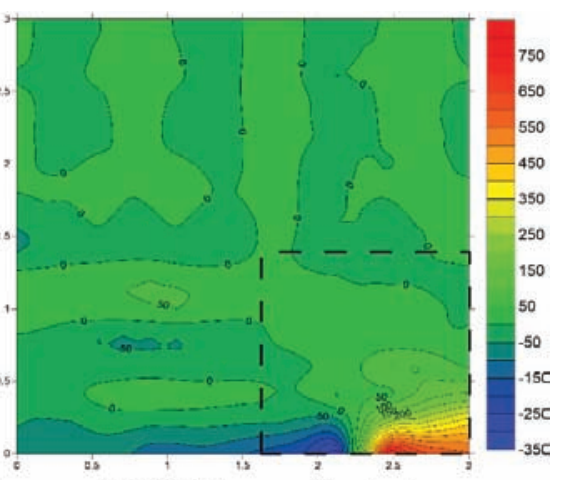

FEM (384 degrees of freedom) 
К вопросу о верификации многоуровневых подходов к локальному расчету строительных конструкций Часть 1: Дискретный (численный) подход

d) $Q_{y}$

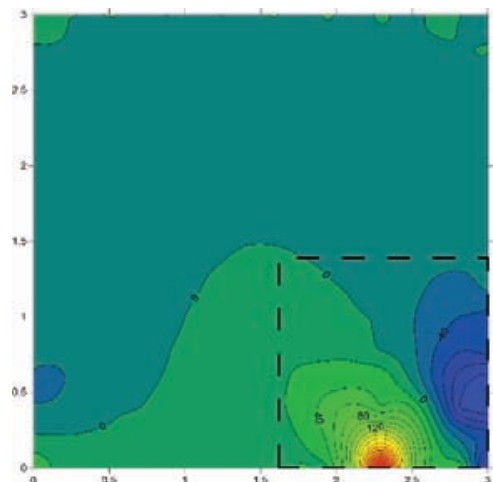

FEM (768 degrees of freedom)

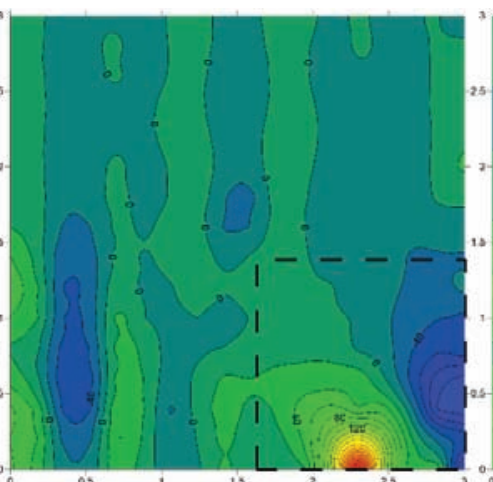

FEM-DWT (480 degrees of freedom)

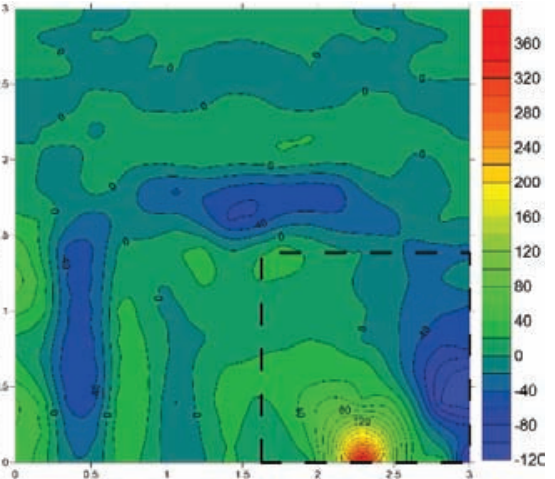

FEM (384 degrees of freedom)

Рисунок 7.4. Сопоставление изгибающих и крутящего моментов и поперечных сил.

\section{СПИСОК ЛИТЕРАТУРЫ}

1. Акимов П.А., Мозгалева М.Л. Многоуровневые дискретные и дискретноконтинуальные методы локального расчета строительных конструкций. - М.: Издательство МИСИ-МГСУ, 2014.-632с.

2. Акимов П.А., Мозгалева М.Л., Кайтуков Т.Б., Негрозов О.А., Моджтаба Аслами. $\mathrm{O}$ верификации вейвлетреализаций многоуровневых дискретных и дискретно-континуальных методов локального расчета строительных конструкций. // Строительная механика и расчет сооружений, №5, 2014, с. 14-20.

3. Добеши И. Десять лекций по вейвлетам. - Ижевск: НИЦ «Регулярная и хаотическая динамика», 2001. - 464 с.

4. Золотов А.Б., Акимов П.А. Практические методы расчета строительных конструкций. Численно-аналитические методы: Монография - М.: Издательство ACB, 2006. - 208 c.

5. Золотов А.Б., Акимов П.А., Сидоров В.Н., Мозгалева М.Л. Численные и аналитические методы расчета строительных конструкций. - М.: Издательство ACB, 2009. - 336 c.

6. Моджтаба Аслами. Многоуровневые дискретные и дискретно-континуальные подходы к локальному расчету строительных конструкций. Диссертация на соискание ученой степени кандидата технических наук по специальности
05.13.18 - «Математическое моделирование, численные методы и комплексы программ». - М.: НИУ МГСУ, 2015. $321 \mathrm{c}$.

7. Моджтаба Аслами. Многоуровневые дискретные и дискретно-континуальные подходы к локальному расчету строительных конструкций. // International Journal for Computational Civil and Structural Engineering / Международный журнал по расчету гражданских и строительных конструкций, Volume 11, Issue 1, 2015, pp. 64-79.

8. Мозгалева М.Л., Золотов А.Б. Использование дискретного базиса Хаара для получения локального решения краевых задач. // International Journal for Computational Civil and Structural Engineering. Volume 5, Issue 1\&2, 2009, pp. 127-132.

9. Чуи К. Введение в вейвлеты. - М.: Мир, 2001. - 412 c.

10. Akimov P.A., Aslami M. Theoretical Foundations and Applications of Multilevel Discrete and Discrete-Continual Methods of Local Structural Analysis. // Materials Physics and Mechanics, 26 (2016) pp. 1-4.

11. Akimov P.A., Mojtaba Aslami. Theoretical Foundations of Correct Wavelet-Based Approach to Local Static Analysis of Bernoulli Beam. // Applied Mechanics and Materials, Vols. 580-583 (2014) pp. 29242927.

12. Akimov P.A., Mojtaba Aslami. About Verification of Correct Wavelet-Based Ap- 
proach to Local Static Analysis of Bernoulli Beam. // Applied Mechanics and Materials, Vols. 580-583 (2014) pp. 3013-3016.

13. Mojtaba Aslami, Akimov P.A. Waveletbased finite element method for multilevel local plate analysis. // Thin-Walled Structures, Volume 98, Part B (2015-2016) pp. 392-402.

14. Mojtaba Aslami, Akimov P.A., Kaytukov T.B. About verification of multilevel wavelet-based numerical method of local structural analysis for two-dimensional problems. // Procedia Engineering, Vol. 111 (2015), pp. 57-64.

15. Zienkiewicz O.C., Morgan K. Finite Elements and Approximation. Dover Publications, 2006, 352 pages.

16. Zienkiewicz O.C., Taylor R.L., Zhu J.Z. The Finite Element Method: Its Basis and Fundamentals. Butterworth-Heinemann, Sixth edition, 2005, 752 pages.

17. Zienkiewicz O.C., Taylor R.L. The Finite Element Method for Solid and Structural Mechanics. Volume 2. ButterworthHeinemann, Sixth Edition, 2005, 736 pages.

Моджтаба Аслами, кандидат технических наук; доцент Университета Фесы; Феса, Иран;

e-mail: aslami.mojtaba@gmail.com

Акимов Павел Алексеевич, член-корреспондент РА$\mathrm{ACH}$, профессор, доктор технических наук; главный ученый секретарь РАACH; заместитель генерального директора по науке ЗАО «Научно-исследовательский центр СтаДиО», профессор кафедры прикладной математики Национального исследовательского Московского государственного строительного университета (НИУ МГСУ); 107031, г. Москва, ул. Большая Дмитровка, д. 24, стр. 1; тел. +7(495) 625-71-63; факс +7 (495) 650-27-31; e-mail: akimov@raasn.ru, pavel.akimov@gmail.com.

Мсхалая Жиули Ивлианович, доцент, кандидат технических наук; доцент кафедры прикладной математики Национального исследовательского Московского государственного строительного университета (НИУ МГСУ); 129337, г. Москва, Ярославское шоссе, дом 26; тел. +7(499) 183-59-94; факс +7 (499) 183-5994; e-mail: KafedraIPM@mgsu.ru.
Mojtaba Aslami, Ph.D., Associate Professor, Fasa University, Fasa, Iran;

e-mail: aslami.mojtaba@gmail.com

Pavel A. Akimov, Corresponding Member of Russian Academy of Architecture and Construction Sciences, $\mathrm{PhD}$, Professor, Chief Scientific Secretary of Russian Academy of Architecture and Construction Sciences; StaDyO Research \& Engineering Center; Department of Applied Mathematics, Moscow State University of Civil Engineering; 24, Ul. Bolshaya Dmitrovka, 107031, Moscow, Russia; phone +7(495) 625-71-63; fax: +7 (495) 650-27-31; e-mail: akimov@ raasn.ru,

pavel.akimov@gmail.com.

Zhiuli I. Mskhalaya, Ph.D., Associate Professor of Department of Applied Mathematics; National Research Moscow State University of Civil Engineering; 26, Yaroslavskoe Shosse, Moscow, 129337, Russia; phone +7(499) 183-59-94; fax: +7 (499) 183-59-94;

e-mail: KafedraIPM@mgsu.ru. 


\title{
К ВОПРОСУ О ВЕРИФИКАЦИИ МНОГОУРОВНЕВЫХ ПОДХОДОВ К ЛОКАЛЬНОМУ РАСЧЕТУ СТРОИТЕЛЬНЫХ КОНСТРУКЦИЙ ЧАСТЬ 2: ДИСКРЕТНО-КОНТИНУАЛЬНЫЙ (ЧИСЛЕННО-АНАЛИТИЧЕСКИЙ) ПОДХОД
}

\author{
Моджтаба Аслами ${ }^{1}$, П.А. Акимов ${ }^{2,3,4,5}$, В.Н. Сидоров \\ ${ }^{1}$ Университет Фесы, г. Феса, ИРАН \\ ${ }^{2}$ Российская академия архитектуры и строительных наук, г. Москва, РОССИЯ \\ 3 ЗАО «Научно-исследовательский центр «СтаДиО», г. Москва, РОССИЯ \\ ${ }^{4}$ Национальный исследовательский Московский государственный строительный университет, \\ г. Москва, РОССИЯ \\ ${ }^{5}$ Научно-исследовательский институт строительной физики Российской академии архитектуры \\ и строительных наук, г. Москва, РОССИЯ \\ ${ }^{6}$ Московский архитектурный институт (государственная академия), г. Москва, РОССИЯ
}

\begin{abstract}
Аннотация: Настоящая статья посвящена актуальным вопросам верификации многоуровневого дискретно-континуального (численно-аналитического) подхода к локальному расчету строительных конструкций. Рассматриваются верификационные примеры решения двумерных краевых задач строительной механики (расчеты балок-стенок и пластин). Для сопоставлений используются результаты полученные с помощью дискретно-континуального метода конечных элементов (ДКМКЭ) и метода конечных элементов (МКЭ). Анализ полученных результатов демонстрирует преимущества предлагаемого авторами многоуровневого дискретно-континуального подхода.
\end{abstract}

Ключевые слова: многоуровневый дискретно-континуальный подход, локальный расчет, строительные конструкции, численно-аналитические методы, вейвлет-анализ, верификация, дискретно-континуальный метод конечных элементов, метод конечных элементов

\section{ABOUT VERIFICATION OF MULTILEVEL METHODS OF LOCAL STRUCTURAL ANALYSIS PART 2: DISCRETE-CONTINUAL (SEMIANALYTICAL) METHOD}

\author{
Mojtaba Aslami ${ }^{1}$, Pavel A. Akimov ${ }^{2,3,4,5}$, Vladimir N. Sidorov ${ }^{6}$, \\ Taymuraz B. Kaytukov ${ }^{2,4}$ \\ ${ }^{1}$ Fasa University, Fasa, IRAN \\ ${ }^{2}$ Russian Academy of Architecture and Construction Sciences, Moscow, RUSSIA \\ ${ }^{3}$ Scientific Research Center "StaDyO", Moscow, RUSSIA \\ ${ }^{4}$ National Research Moscow State University of Civil Engineering, Moscow, RUSSIA \\ ${ }^{5}$ Research Institute of Building Physics of Russian Academy \\ of Architecture and Construction Sciences, Moscow, RUSSIA \\ ${ }^{6}$ Moscow Institute of Architecture (state academy), Moscow, RUSSIA
}

\begin{abstract}
The distinctive paper is devoted to actual aspects of verification of multilevel discrete-continual (semianalytical) method of local structural analysis. Solutions of two-dimensional verification samples (analysis of deep beams and plates) are under consideration. Discrete-continual finite element method (DCFEM) and finite element method (FEM) is used for verification purposes. The presented samples show some of the advantages of the suggested approach to semianalytical local structural analysis.
\end{abstract}

Keywords: multilevel discrete-continual method, local structural analysis, semianalytical methods, wavelet analysis, verification, discrete-continual finite element method, finite element method 


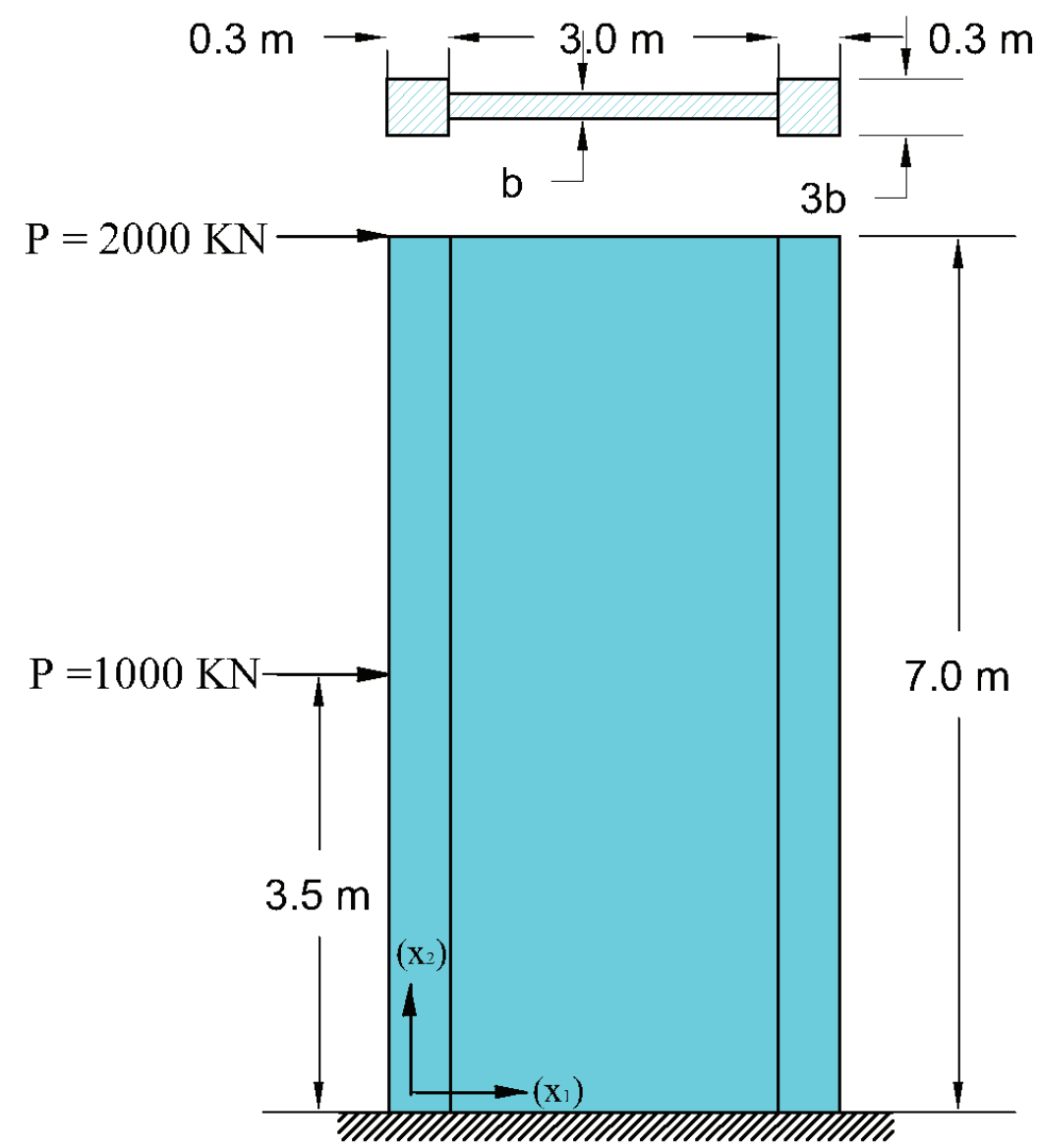

Рисунок 1.1. Задача о статическом расчете диафрагмы жесткости.

\section{1. РАСЧЕТ ДИАФРАГМЫ ЖЕСТКОСТИ В УСЛОВИЯХ ДЕЙСТВИЯ ДВУХ СОСРЕДОТОЧЕННЫХ СИЛ}

Рассмотрим задачу локального статического расчета бетонной диафрагмы жесткости, нагруженной парой сосредоточенных сил (рис. 1.1). Модуль упругости и коэффициент Пуассона материала конструкции (стенка) равны соответственно $E_{\text {wall }}=26500 \mathrm{MПа} \mathrm{и}$ $v=0.3$. Модуль упругости колонн при проведении расчетного обоснования задавали равным $E_{\text {column }}=3 E_{\text {wall }}[1,2,8,9,11,12]$.

Дискретная и дискретно-континуальная модели конструкции в рамках метода конечных элементов (МКЭ) и дискретноконтинуального метода конечных элементов (ДКМКЭ) [1,8,9,21-23] показаны на рис. 1.2.
Для решения рассматриваемой двумерной (плоской) задачи используется дискретноконтинуальный метод конечных элементов (модуль 2DDCFEM комплекса программ MWBDASA2D [8,9]).

Выполнены сопоставления решений, полученных по указанному ДКМКЭ и МКЭ. На рис. 1.4 указанные сопоставления представлены для сечений $x_{1}=0.1$ м и $x_{2}=0.3$ м. Следует отметить хорошую согласованность результатов. На рис. 1.3 приведены изополя соответствующих напряжений.

В целом, следует отметить хорошую согласованность получаемых результатов, некоторые различия в зонах краевого эффекта обусловлены аналитическим (более точным по сравнению с МКЭ [21-23]) характером решений, найденным с помощью дискретноконтинуального метода конечных элементов. 
К вопросу о верификации многоуровневых подходов к локальному расчету строительных конструкций Часть 2: Дискретно-континуальный (численно-аналитический) подход

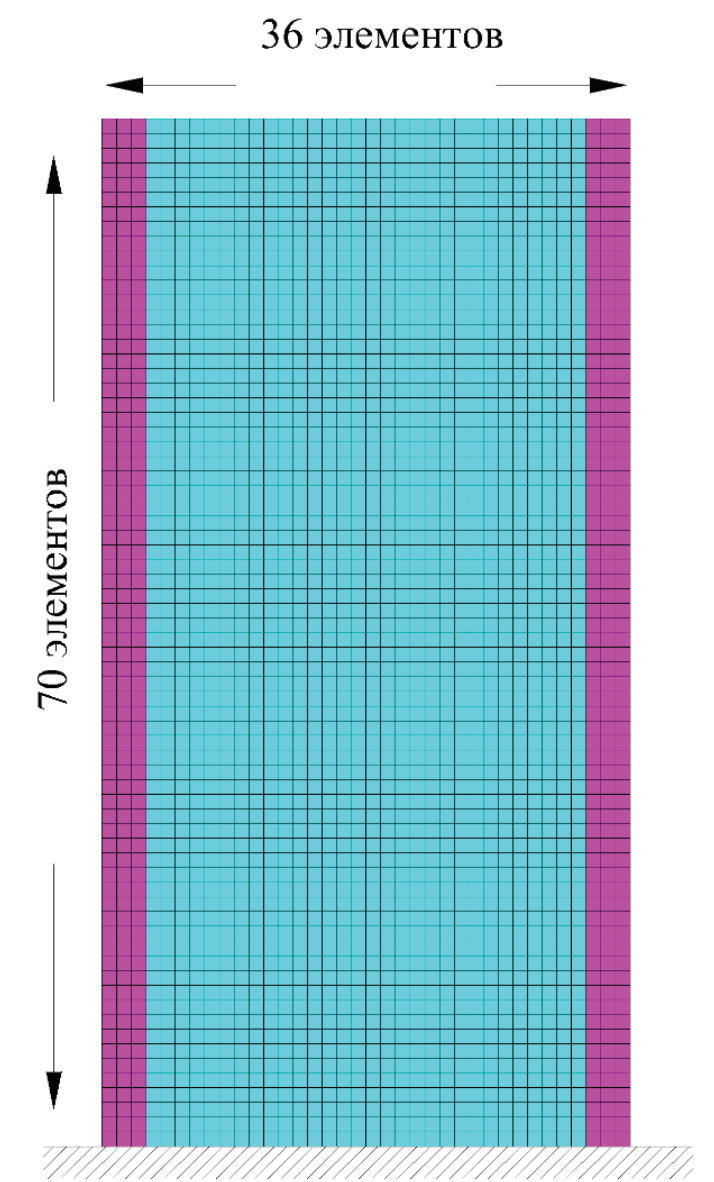

МКЭ дискретизация 2520 элементов
МКЭ дискретизация 36 элементов

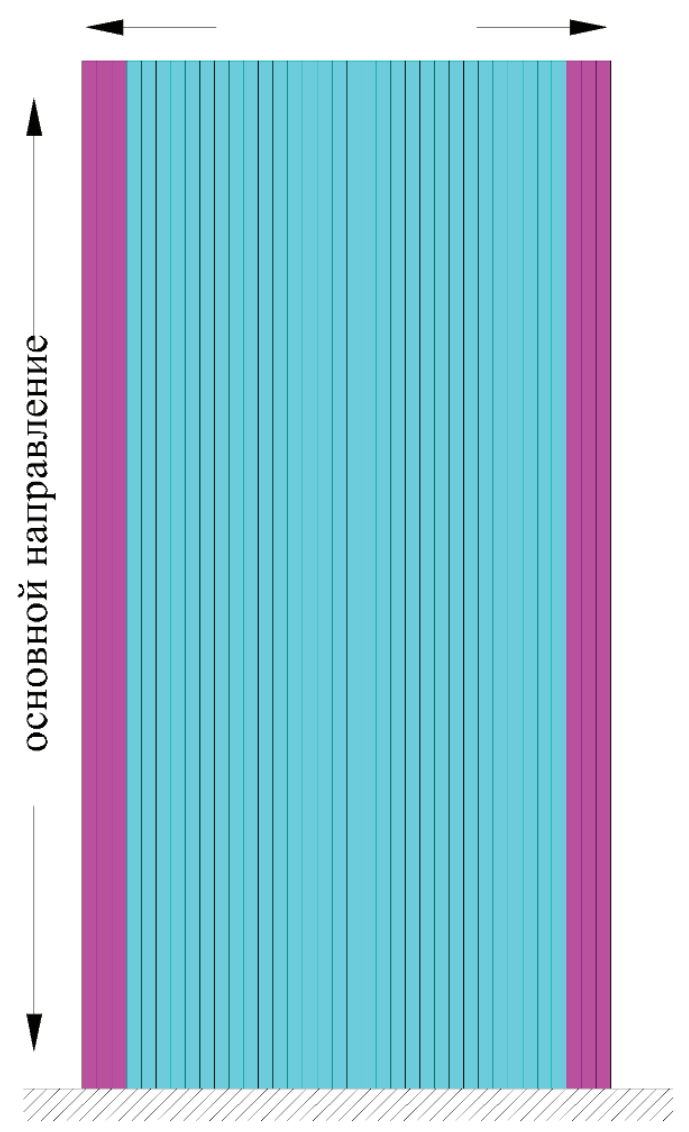

сетка ДКМКЭ

Рисунок 1.2. Дискретная и дискретно-континуальная модели конструкции.

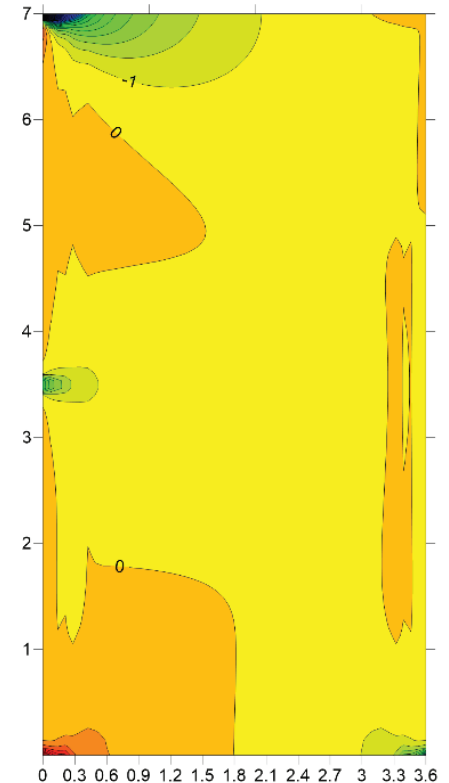

$\sigma_{11}[\mathrm{MPa}]$
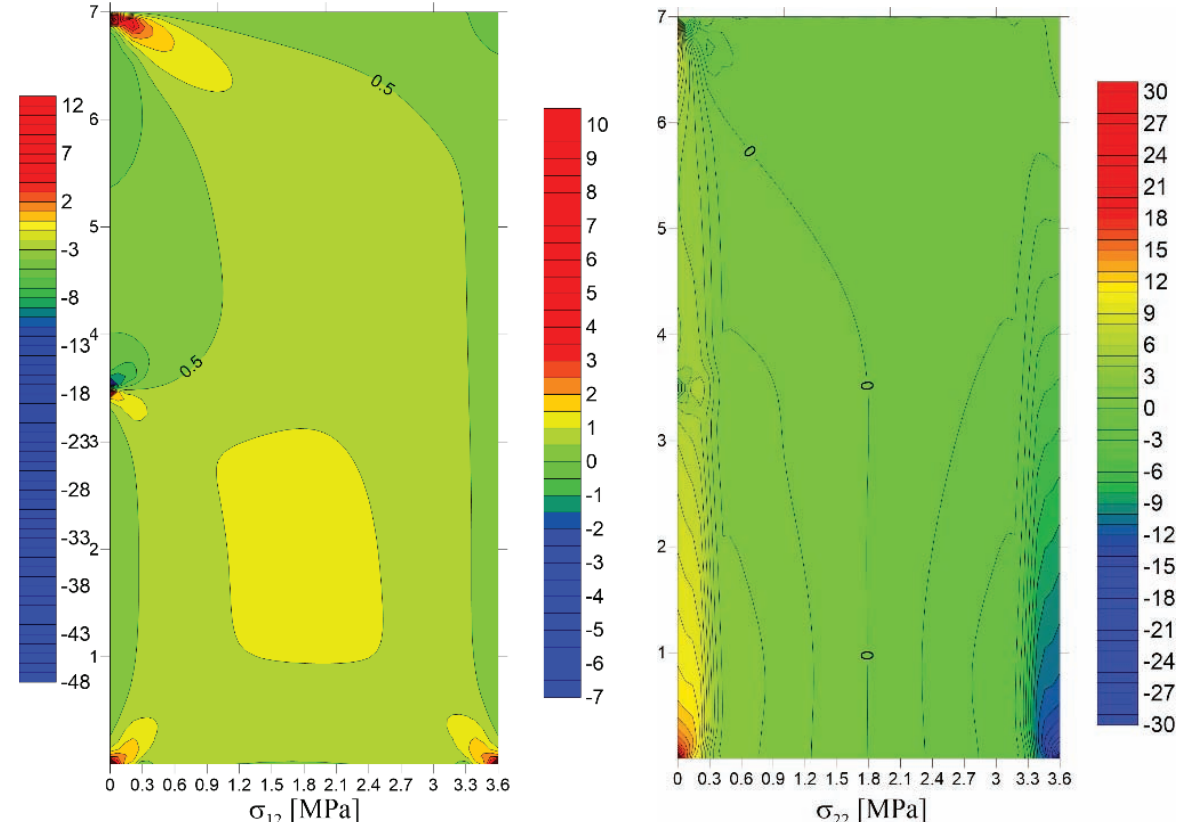

$0 \quad 0.30 .60 .91 .21 .51 .82 .12 .42 .7 \quad 3 \quad 3.33 .6$ $\sigma_{22}[\mathrm{MPa}]$

Рисунок 1.3. Изополя напряжений в конструкичии. 

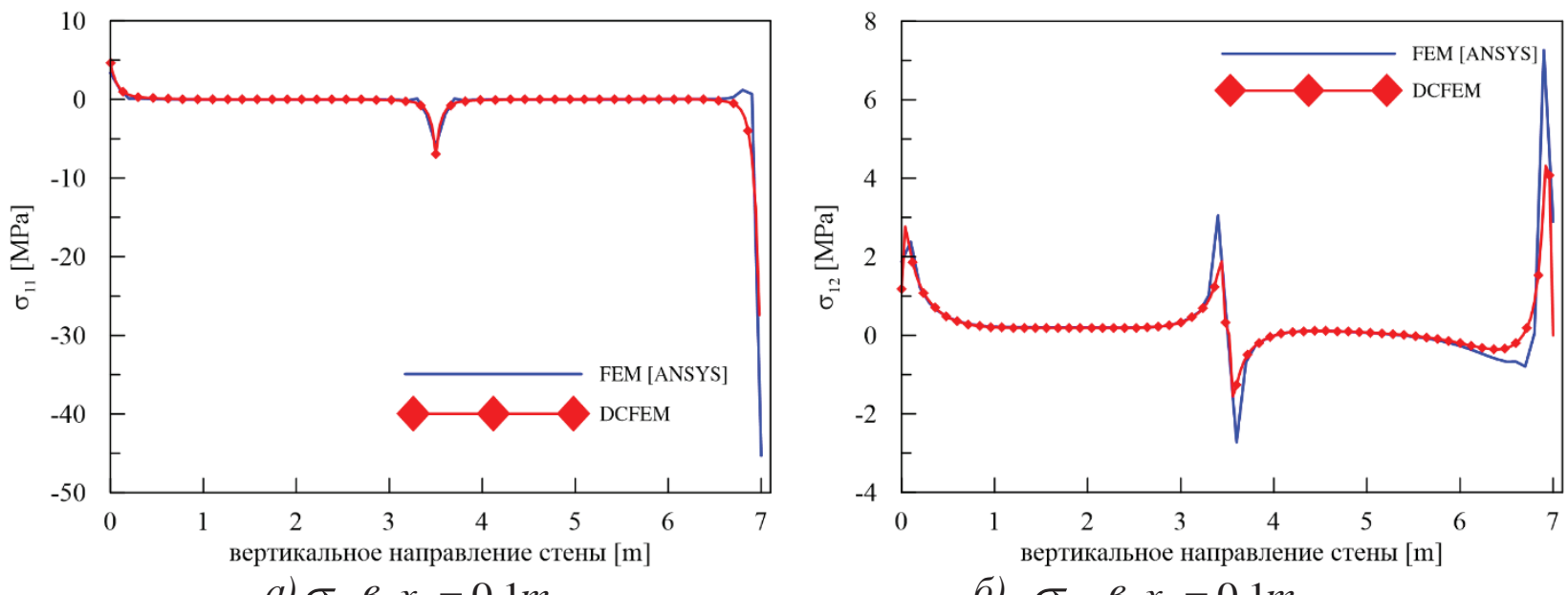

a) $\sigma_{11} B \quad x_{1}=0.1 m$

б) $\sigma_{12}$ в $\quad x_{1}=0.1 m$
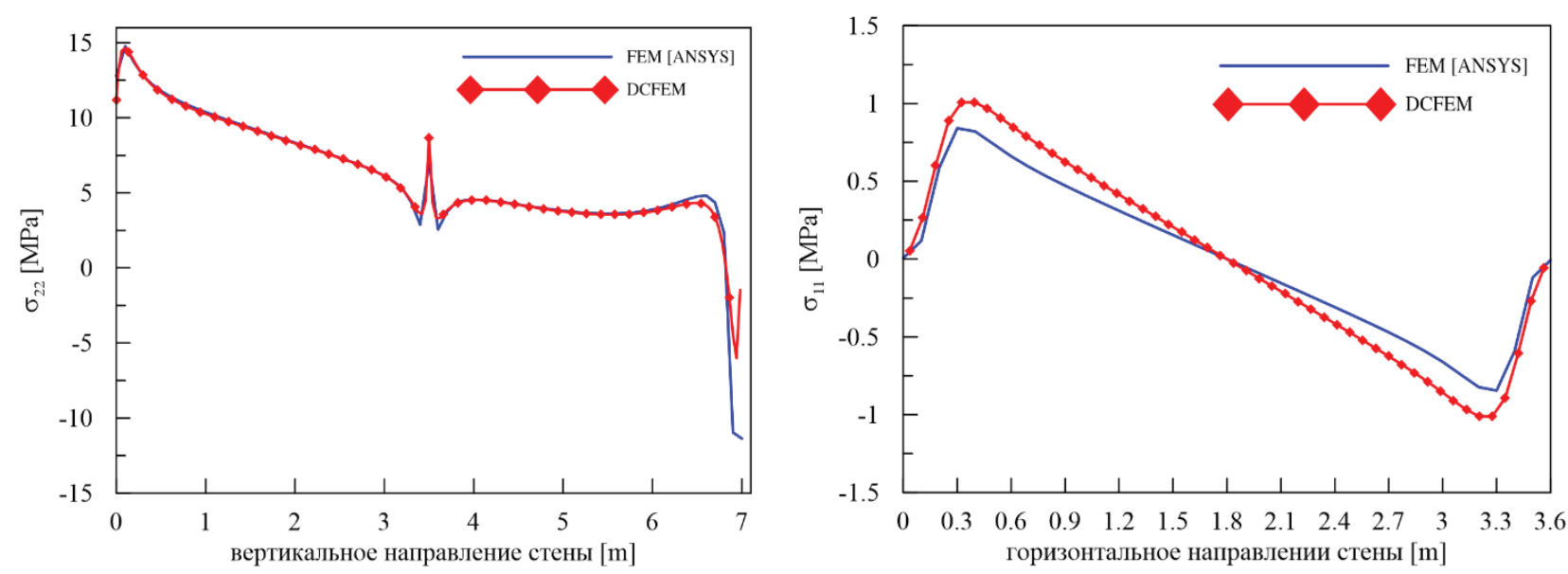

в) $\sigma_{22}$ B $x_{1}=0.1 \mathrm{~m}$

2) $\sigma_{11}$ \& $\quad x_{2}=0.3 m$

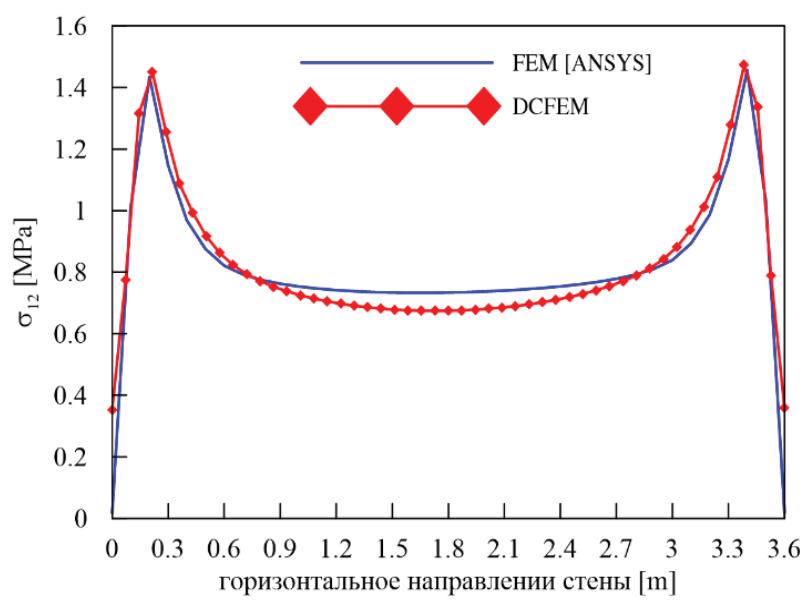

d) $\sigma_{12}$ \& $x_{2}=0.3 m$

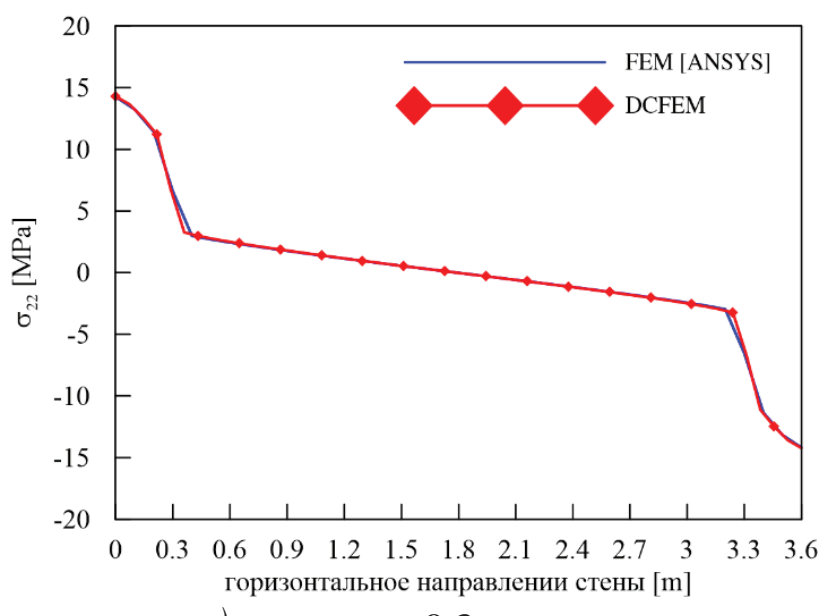

Рисунок 1.4. Сопоставление решений (в части напряжений), полученных по МКЭ и ДКМКЭ по сечениям конструкции $x_{2}=0.3$ м и $x_{1}=0.1$ м. 
К вопросу о верификации многоуровневых подходов к локальному расчету строительных конструкций Часть 2: Дискретно-континуальный (численно-аналитический) подход

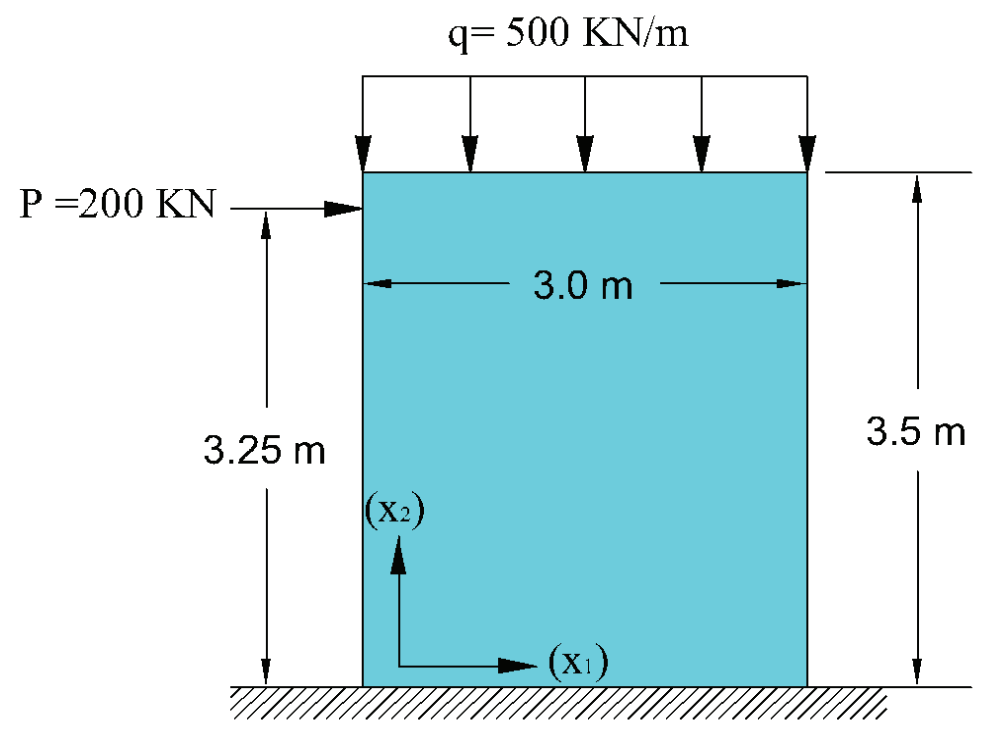

Рисунок 2.1. Задача о статическом расчете диафрагмы жесткости.

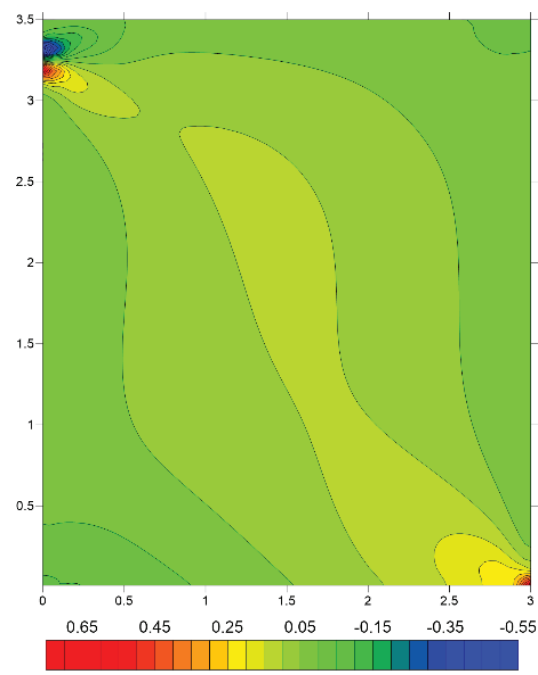

$\sigma_{12}[\mathrm{KPa}]$

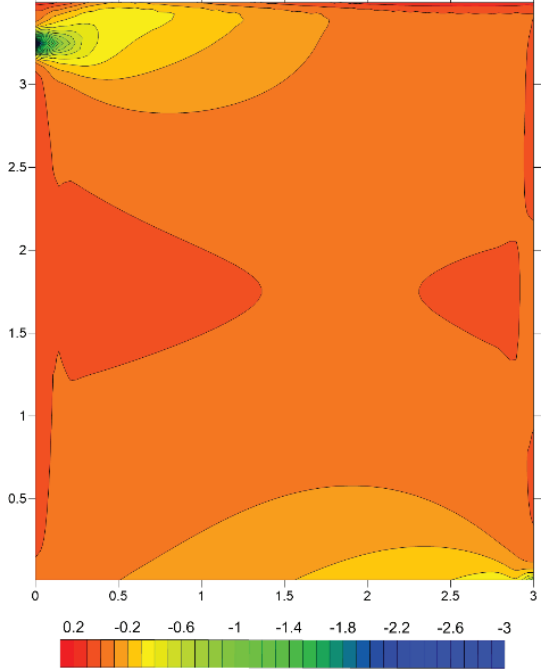

$\sigma_{11}[\mathrm{KPa}]$

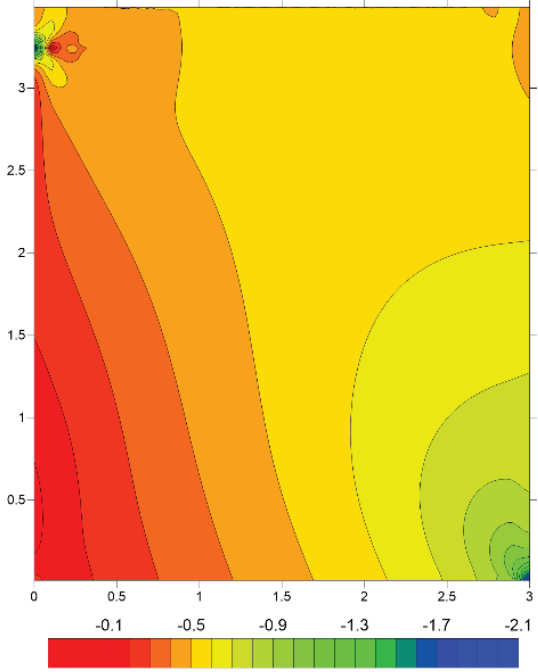

$\sigma_{22}[\mathrm{KPa}]$

Рисунок 2.2. Изополя напряжений в конструкции.

2. РАСЧЕТ ДИАФРАГМЫ ЖЕСТКОСТИ В УСЛОВИЯХ ДЕЙСТВИЯ СОСРЕДОТОЧЕННОЙ И РАСПРЕДЕЛЕННОЙ СИЛ

Рассмотрим задачу локального статического расчета бетонной диафрагмы жесткости, нагруженной сосредоточенной и распределенной сил (рис. 2.1).

Положим, что диафрагма имеет постоянную толщину $t=0.15$ м, модуль упругости и коэффициент Пуассона материала конструкции (стенка) равны соответственно $E_{\text {wall }}=26500$ МПа и $v=0.3$.

Для решения рассматриваемой двумерной (плоской) задачи используется дискретноконтинуальный метод конечных элементов (модуль 2DDCFEM комплекса программ MWBDASA2D [8,9]).

В верификационных целях также проводился контрольный расчет конструкции с использованием метода конечных элементов, причем при построении соответствующей дискретной (конечноэлементной) модели диа- 


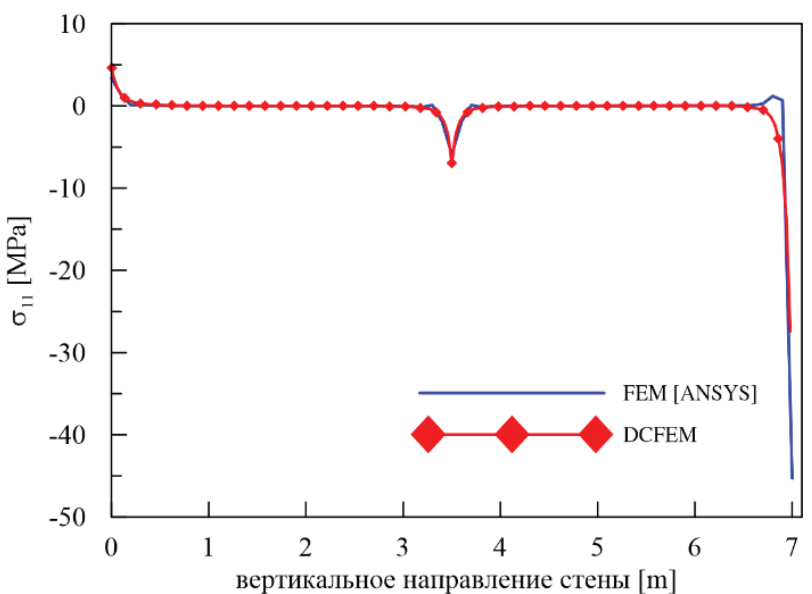

a) $\sigma_{11}$ B $x_{1}=0.1 \mathrm{~m}$

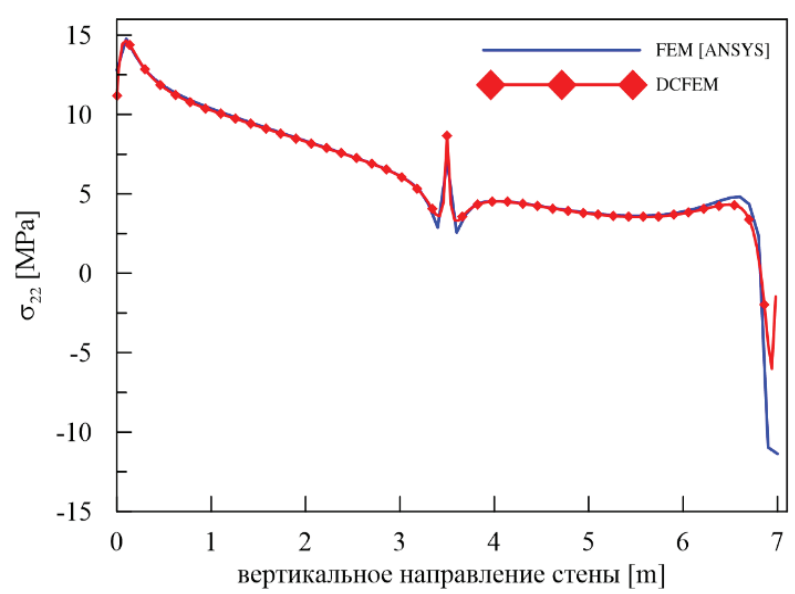

в) $\sigma_{22}$ в $x_{1}=0.1 \mathrm{~m}$

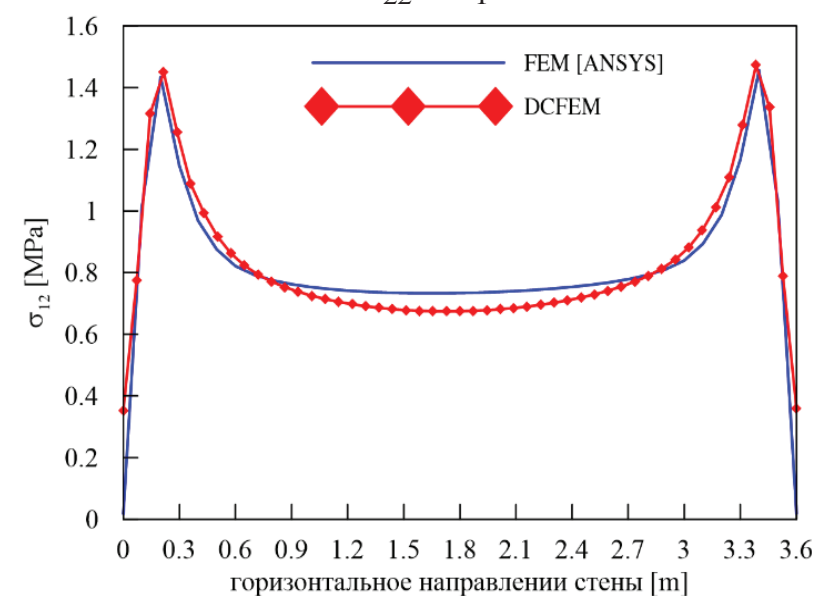

д) $\sigma_{12}$ в $x_{2}=0.3 m$

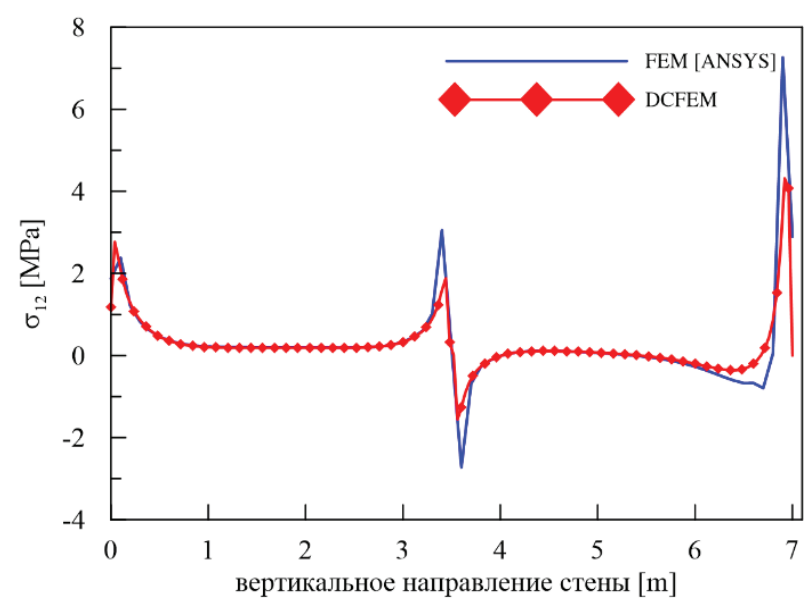

б) $\sigma_{12}$ в $x_{1}=0.1 m$

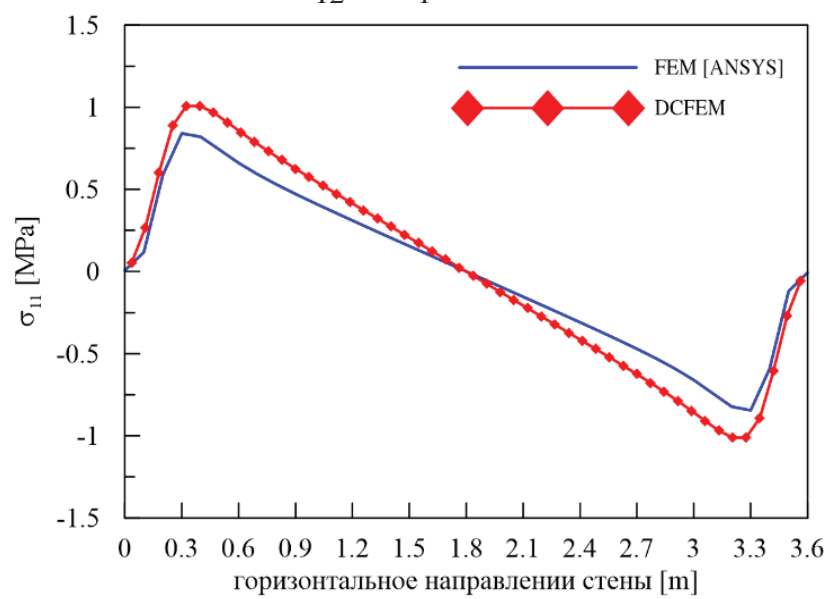

г) $\sigma_{11}$ в $x_{2}=0.3 m$

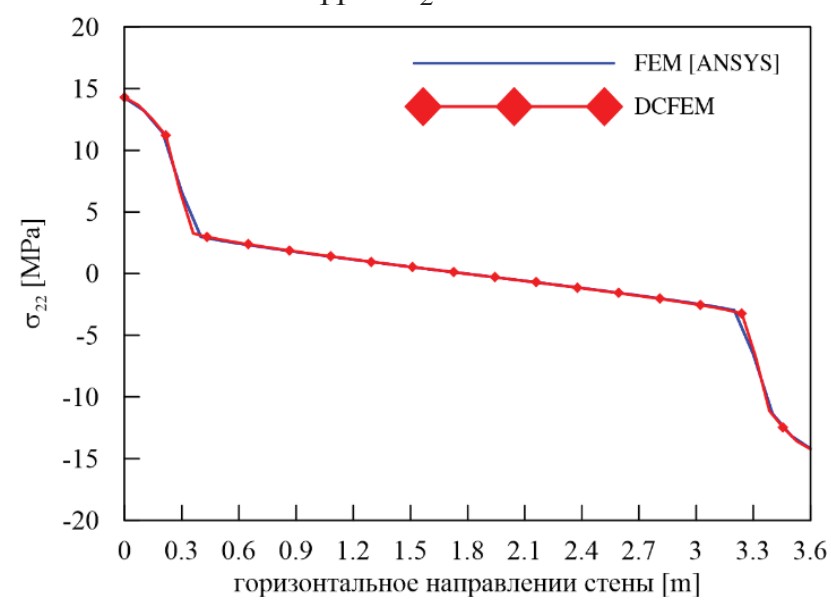

e) $\sigma_{22} x_{2}=0.3 \mathrm{~m}$

Рисунок 2.3. Сопоставление решений (в части напряжений), полученных по МКЭ и ДКМКЭ по сечениям конструкиии $x_{2}=0.3$ м и $x_{1}=0.1$ м.

фрагмы жесткости были использованы 4200 изопараметрических четырехузловых конечных элементов, тогда как для формирования дискретно-континуальной модели потребо- валось лишь 60 дискретно-континуальных конечных элементов $[8,9,11,12]$.

Выполнены сопоставления решений, полученных по обоим указанным методам. На рис. 
К вопросу о верификации многоуровневых подходов к локальному расчету строительных конструкций Часть 2: Дискретно-континуальный (численно-аналитический) подход

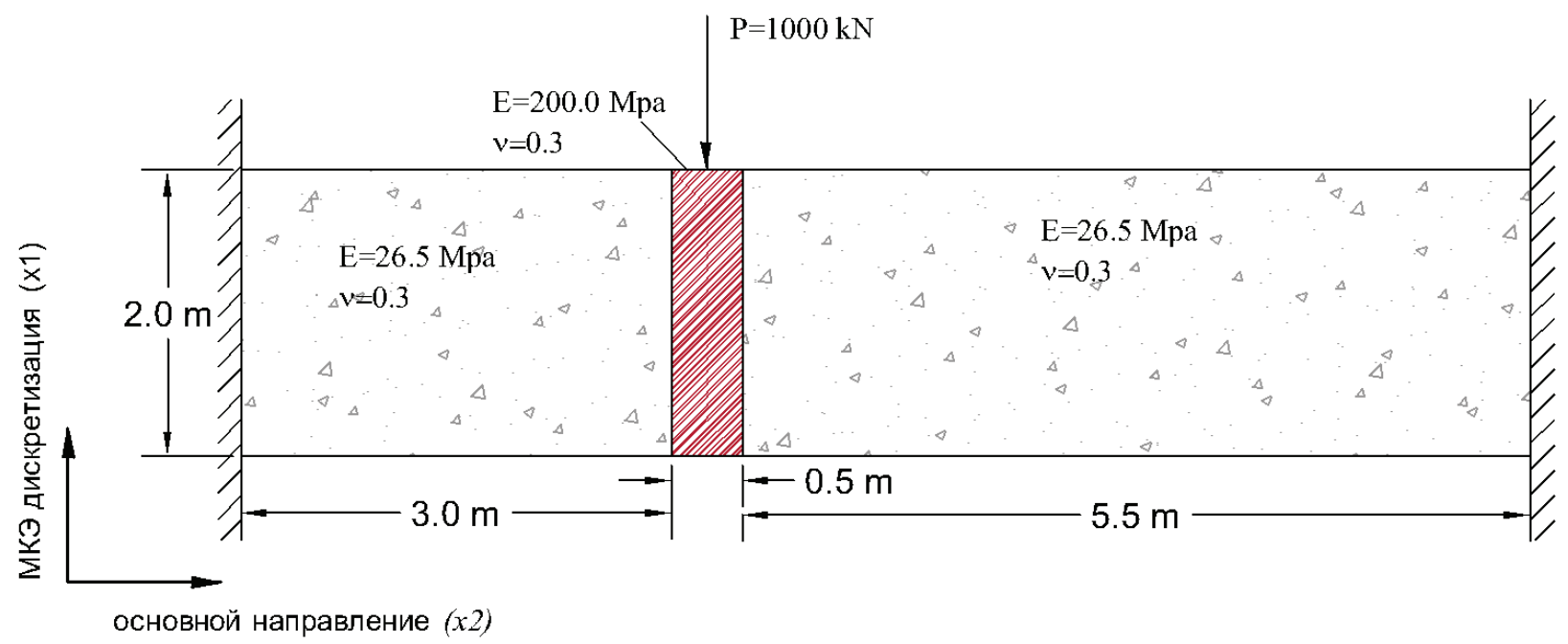

Рисунок 3.1. Задача о статическом расчете балки-стенки.

2.3 указанные сопоставления представлены для сечений $x_{1}=0.05$ м и $x_{2}=0.2$ м. Следует отметить хорошую согласованность результатов. На рис. 2.2 приведены изополя соответствующих напряжений.

\section{3. ЛОКАЛЬНЫЙ РАСЧЕТ БАЛКИ-СТЕНКИ}

Рассмотрим задачу локального статического расчета балки-стенки, показанной на рис. 3.1 (зона гарантированной точности выделена цветом).

Для решения данной задачи используется многоуровневый дискретно-континуальный подход к локальному расчету строительных конструкций, основанный на использовании техники метода конечных элементов (DCFEM-DWT; реализующий комплекс программ MWBDCASA2D [8,9]).

Выполнены сопоставления решений, полученных по МКЭ (при построении дискретной модели использовались изопараметрические четырехузловые конечные элементы), с соответствующими редуцированными решениями в рамках указанного подхода (использованы 31 дискретно-континуальный конечный элементов, имеем соответственно 32 узловых линии) $[1-5,8,9,13,15,16,19]$.
На рис. 3.2 представлены выборочные сопоставления результатов (в части напряжений), в частности, по сечениях $x_{2}=0.15$ м и $x_{1}=1.94$ м. В зоне гарантированной точности результаты, по существу, идентичны даже при значительной редукции неизвестных.

На рис. 3.3 показаны изополя напряжений в балке-стенке.

\section{4. РАСЧЕТ ТОНКОЙ ПЛАСТИНЫ}

Рассмотрим задачу статического расчета тонкой прямоугольной пластины, лежащей на двухпараметрическом упругом основании при двух вариантах нагружения [8,9].

Первый вариант нагружения - приложена равномерно распределенная нагрузка по всей поверхности пластины ( $q=23.94$ кН/ $\left.\mathrm{M}^{2}\right)$.

Второй вариант нагружения - приложена сосредоточенная нагрузка в центре пластины $(P=133.34$ кН $)$.

Модуль упругости и коэффициент Пуассона грунта составляют соответственно $E_{s}=68950 \kappa \mathrm{H} / \mathrm{M}^{2}$ и $v_{s}=0.25$, а модуль упругости и коэффициент Пуассона материала пластины равны $E_{p}=20685000 \kappa \mathrm{H} / \mathrm{M}^{2} \quad$ и $v_{p}=0.2$, толщина пластины $\Delta_{k}=0.1524 \mathrm{M}$. Мощность (глубина) учитываемого массива 
Моджтаба Аслами, П.А. Акимов, В.Н. Сидоров, Т.Б. Кайтуков
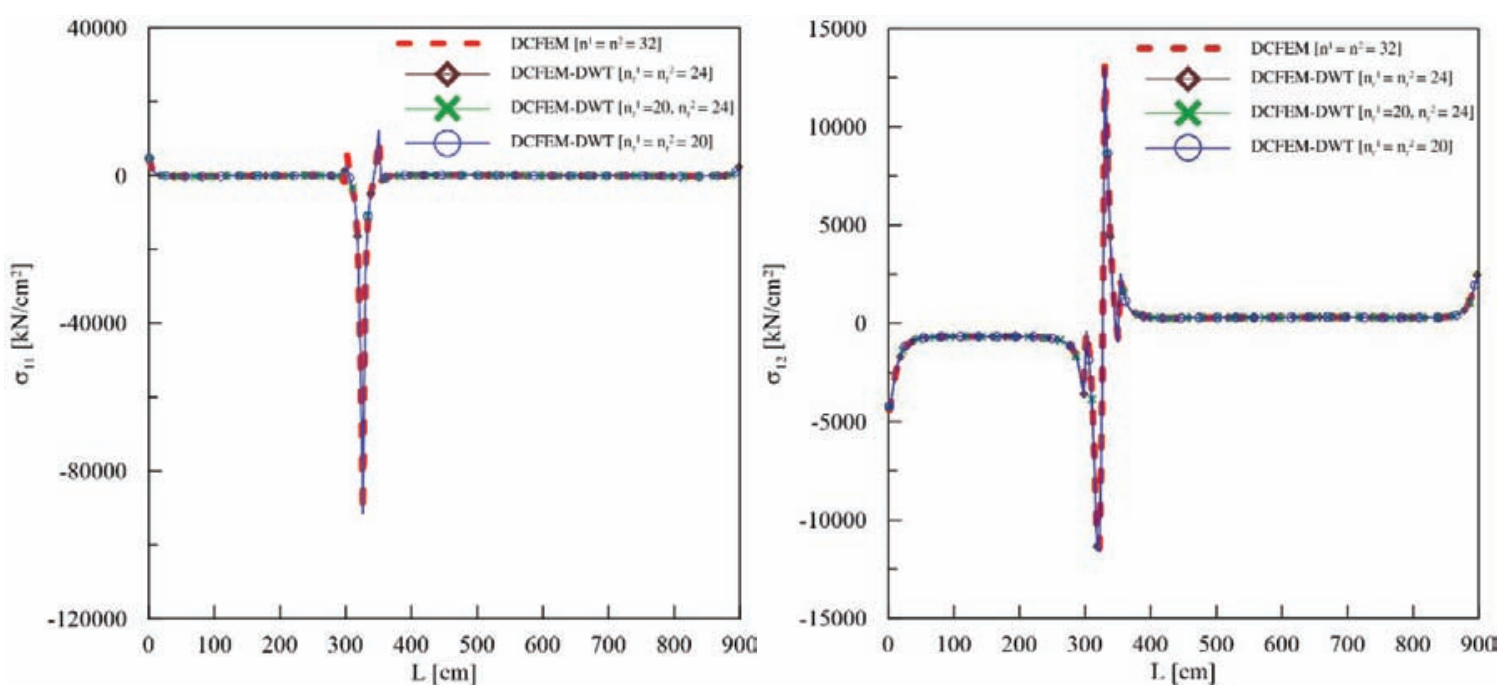

a) $\sigma_{11} B \quad n=31$

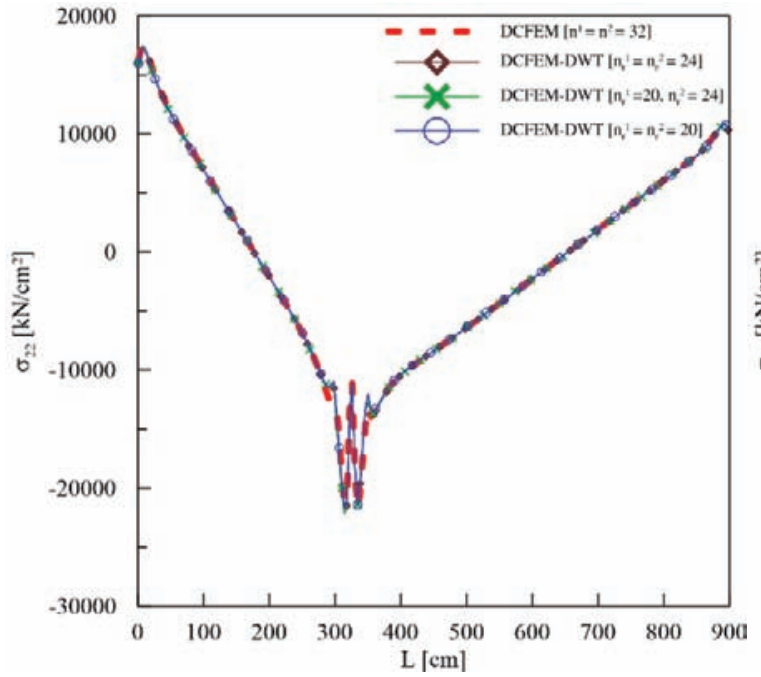

б) $\sigma_{12}$ в $n=31$

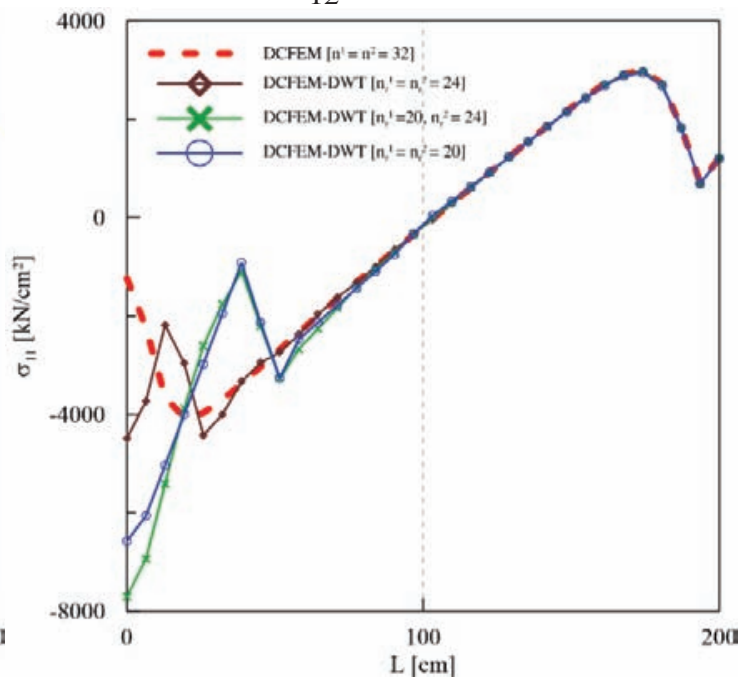

в) $\sigma_{22}$ b $n=31$

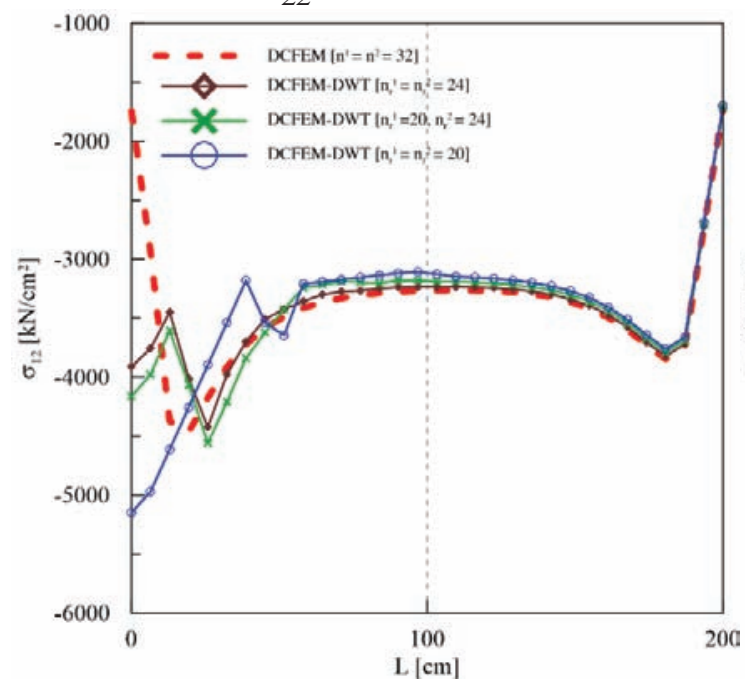

2) $\sigma_{11}$ в $\quad x_{2}=0.15 \mathrm{~m}$

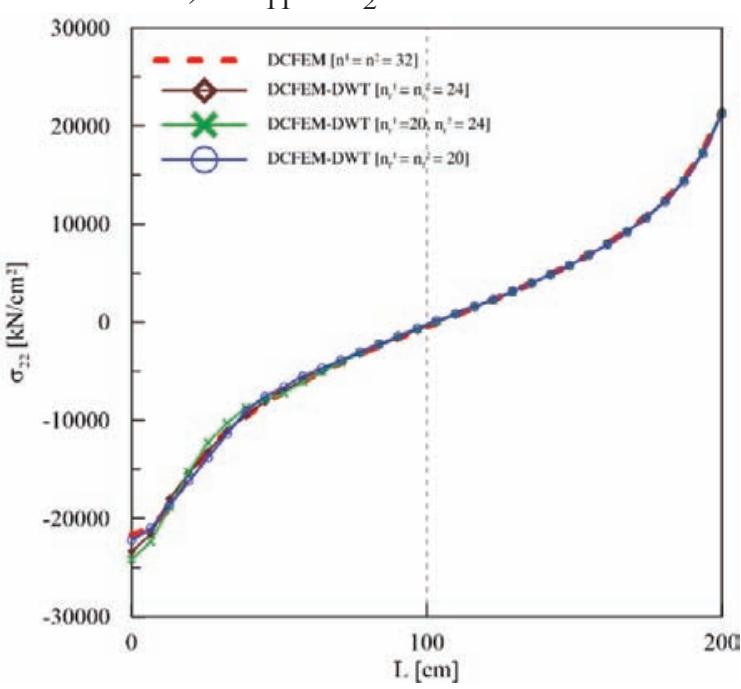

d) $\sigma_{12}$ B $x_{2}=0.15 \mathrm{~m}$

e) $\sigma_{22}$ в $x_{2}=0.15 \mathrm{~m}$

Рисунок 3.2. Сопоставление решений (в части напряжений), полученных по МКЭ и ДКМКЭ по сечениям конструкции $x_{2}=0.15$ м и $x_{1}=1.94 \mathrm{M}$. 
К вопросу о верификации многоуровневых подходов к локальному расчету строительных конструкций Часть 2: Дискретно-континуальный (численно-аналитический) подход
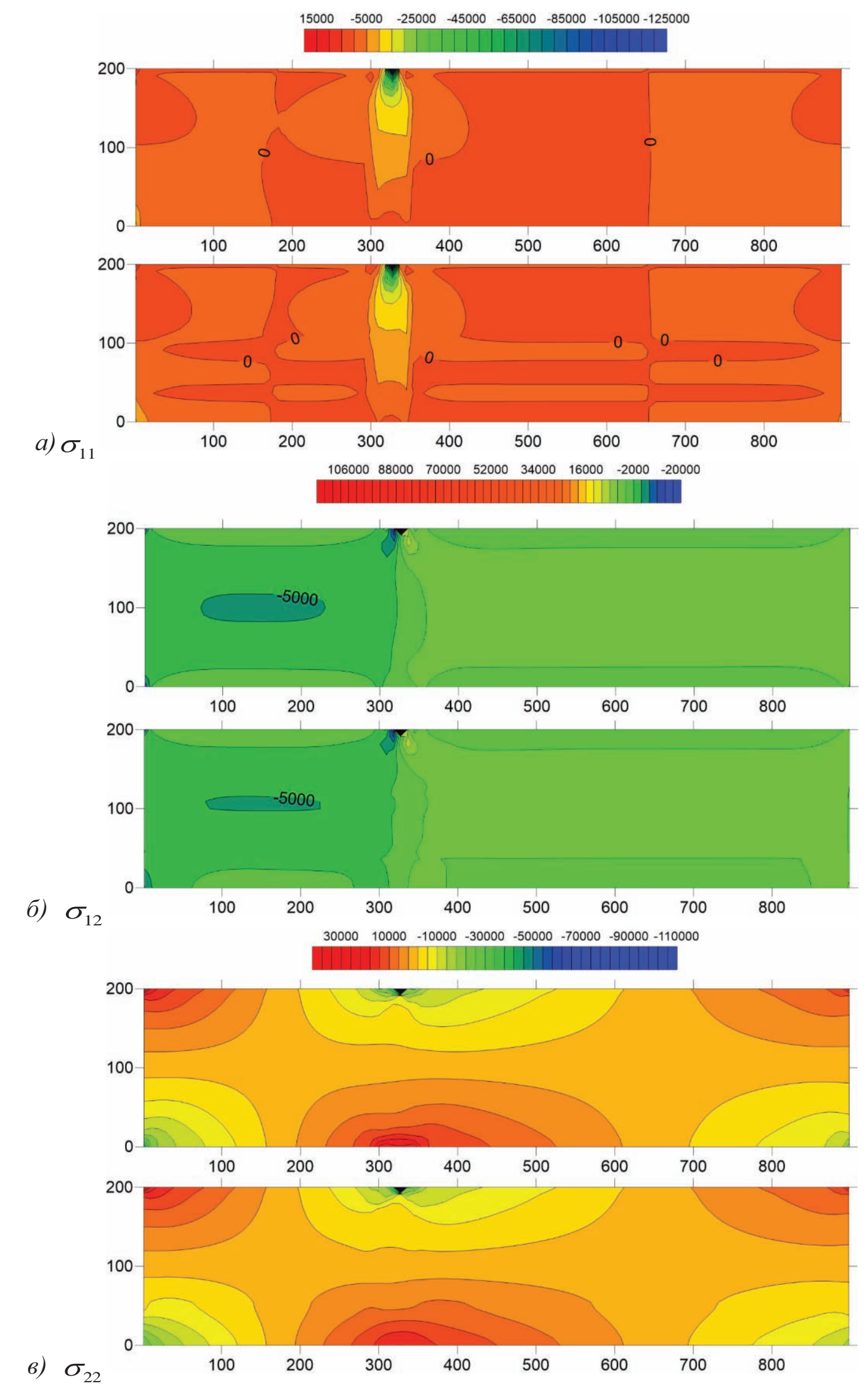

Рисунок 3.3. Изополя напряжений в конструкиии. 
Таблица 3.1. Параметры основания, максимальные перемещения и изгибающие моменты в пластине под действием распределенной нагрузки.

\begin{tabular}{|c|c|c|c|c|c|}
\hline$H, \mathrm{M}$ & Источник & $k_{s}, \mathrm{\kappa H} / \mathrm{m}^{3}$ & $2 t_{s}, \mathrm{\kappa Н} / \mathrm{м}$ & $w, \mathrm{~cm}$ & $M_{2}, \mathrm{\kappa H} / \mathrm{M} / \mathrm{M}$ \\
\hline 3.048 & $\begin{array}{c}\text { Ozgan and Daloglu [20] } \\
\text { DCFEM }\end{array}$ & 28207 & 26839 & $\begin{array}{l}0.0874 \\
0.0854\end{array}$ & $\begin{array}{c}0.049 \\
0.0875\end{array}$ \\
\hline 6.096 & $\begin{array}{c}\text { Ozgan and Daloglu [20] } \\
\text { DCFEM }\end{array}$ & 13744 & 50620 & $\begin{array}{l}0.1533 \\
0.1456\end{array}$ & $\begin{array}{l}0.2794 \\
0.3236\end{array}$ \\
\hline 9.144 & $\begin{array}{c}\text { Ozgan and Daloglu [20] } \\
\text { DCFEM }\end{array}$ & 9337 & 71390 & $\begin{array}{l}0.1918 \\
0.1834\end{array}$ & $\begin{array}{l}0.3917 \\
0.4021\end{array}$ \\
\hline 15.24 & $\begin{array}{c}\text { Ozgan and Daloglu [20] } \\
\text { DCFEM }\end{array}$ & 5924 & 105889 & $\begin{array}{l}0.2238 \\
0.2269\end{array}$ & $\begin{array}{l}0.4311 \\
0.4134\end{array}$ \\
\hline
\end{tabular}

Таблица 3.2. Параметры основания, максимальные перемещения и изгибающие моменты в пластине под действием сосредоточенной нагрузки.

\begin{tabular}{|c|c|c|c|c|c|}
\hline$H, \mathrm{~m}$ & Источник & $k_{s}, \mathrm{\kappa H} / \mathrm{M}^{3}$ & $2 t_{s}, \mathrm{\kappa H} / \mathrm{M}$ & $w, \mathrm{~cm}$ & $M_{2}, \mathrm{\kappa H} / \mathrm{M} / \mathrm{M}$ \\
\hline \multirow{2}{*}{3.048} & $\begin{array}{c}\text { Ozgan and Daloglu [20] } \\
\text { DCFEM }\end{array}$ & 31804 & 18990 & $\begin{array}{l}0.0857 \\
0.0889\end{array}$ & $\begin{array}{l}17.633 \\
23.011\end{array}$ \\
\hline \multirow{2}{*}{6.096} & $\begin{array}{c}\text { Ozgan and Daloglu [20] } \\
\text { DCFEM }\end{array}$ & 24092 & 23763 & $\begin{array}{l}0.0884 \\
0.0981\end{array}$ & $\begin{array}{l}17.128 \\
22.165\end{array}$ \\
\hline \multirow{2}{*}{9.144} & $\begin{array}{c}\text { Ozgan and Daloglu [20] } \\
\text { DCFEM }\end{array}$ & 23557 & 24221 & $\begin{array}{l}0.0885 \\
0.0988\end{array}$ & $\begin{array}{l}17.073 \\
22.086\end{array}$ \\
\hline \multirow{2}{*}{15.24} & $\begin{array}{c}\text { Ozgan and Daloglu [20] } \\
\text { DCFEM }\end{array}$ & 23530 & 24245 & $\begin{array}{l}0.0885 \\
0.0989\end{array}$ & $\begin{array}{l}17.070 \\
22.082\end{array}$ \\
\hline
\end{tabular}

полагалась равной $H=3.048,6.096,9.144$, 15.240 м. Параметры отпора грунта $\left(k_{s}\right.$ и $\left.2 t_{s}\right)$ принимались в соответствии с [11].

Параметры отпора грунта $\left(k_{s}\right.$ и $\left.2 t_{s}\right)$ принимались в соответствии с $[8,9]$.

Для решения данной задачи используется многоуровневый дискретно-континуальный подход к локальному расчету строительных конструкций, основанный на использовании техники метода конечных элементов (модуль DCFEMP комплекса программ MWBDCASA2D [8,9]).

В приводимых ниже таблицах 3.1 и 3.2 представлено сопоставление полученных результатов (в части вертикального перемещения и изгибающего момента) с известными решениями других авторов.

При построении дискретно-континуальной модели было использовано 16 дискретноконтинуальных конечных элементов, далее рассматривались различные варианты редук- ции $[7,14]$. На рис. 4.1 представлены выборочные сопоставления результатов (в части изгибающих и крутящего моментов и поперечных сил) для двух указанных вариантов (вариант 1 (в.1) и вариант 2 (в.2)), в частности, для первого дискретно-континуального конечного элемента. Соответствующие изополя изгибающих моментов приведены на рис. $4.2[8,9]$.

\section{5. ЛОКАЛЬНЫЙ РАСЧЕТ ТОНКОЙ ПЛАСТИНЫ}

Рассмотрим задачу локального статического расчета тонкой прямоугольной пластины, показанной на рис. 5.1.

Для решения данной задачи используется многоуровневый дискретно-континуальный подход к локальному расчету строительных конструкций, основанный на использовании техники метода конечных элементов (DCFEM 
К вопросу о верификации многоуровневых подходов к локальному расчету строительных конструкций Часть 2: Дискретно-континуальный (численно-аналитический) подход
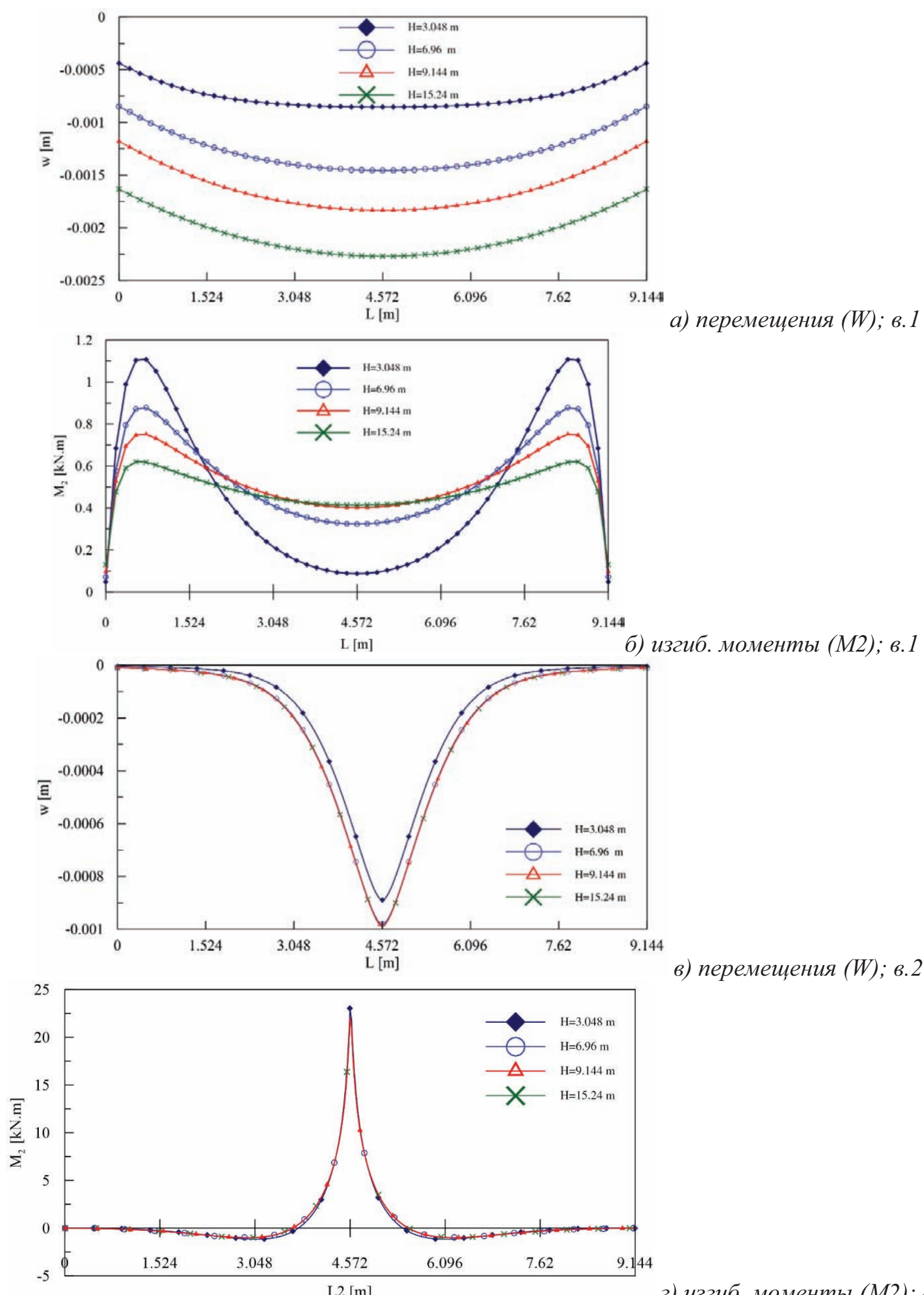

в) перемещения $(W) ; в .2$

г) изгиб. моменты (М2); в.2

Рисунок 4.1. Сопоставление решений, полученных по МКЭ и ДКМКЭ. 

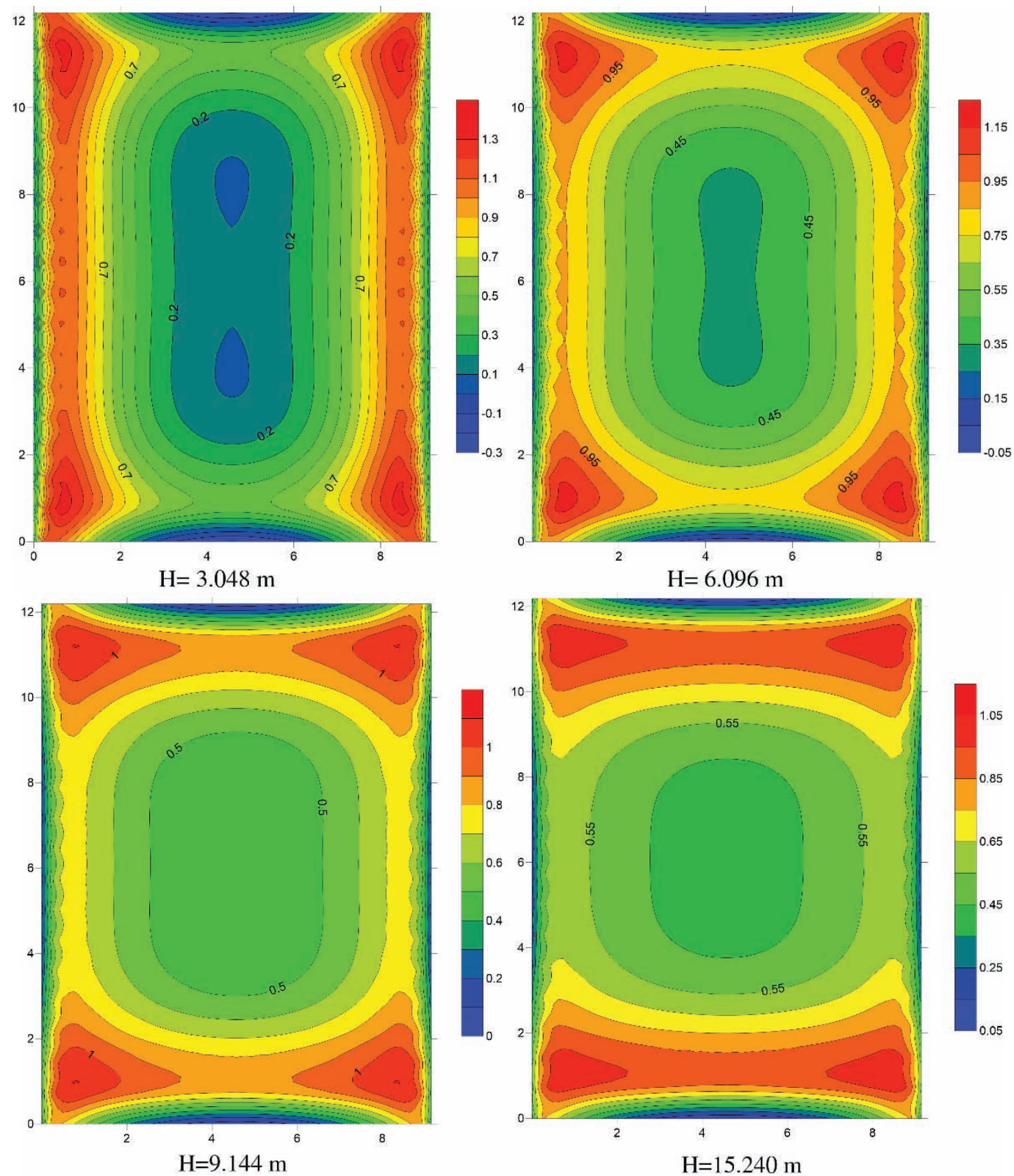

Рисунок 4.2. Изополя изгибающих моментов $\left(M_{2}\right)$ в конструкции.

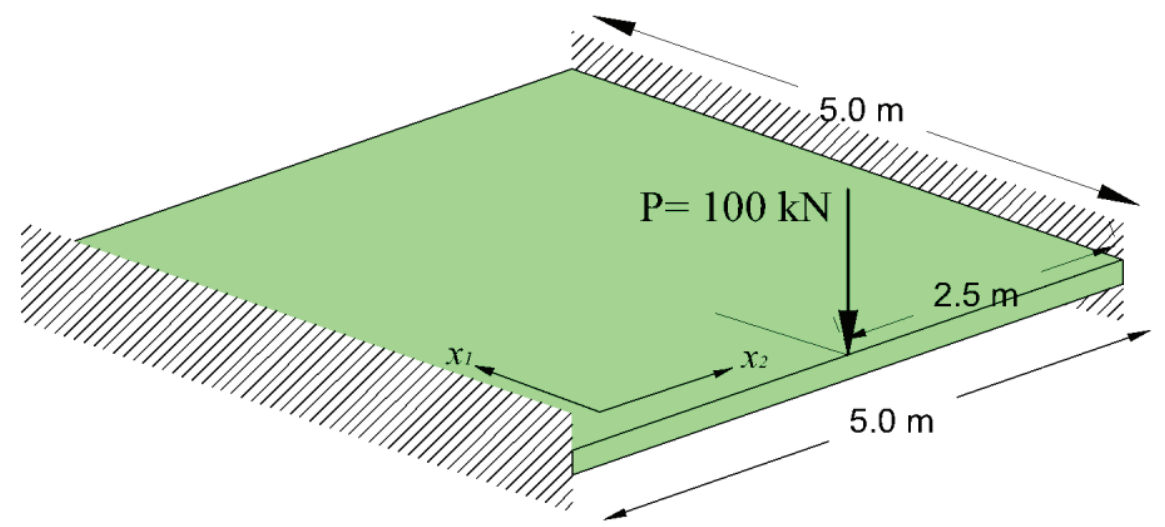

Рисунок 5.1. Задача о статическом расчете тонкой пластины. 
К вопросу о верификации многоуровневых подходов к локальному расчету строительных конструкций Часть 2: Дискретно-континуальный (численно-аналитический) подход

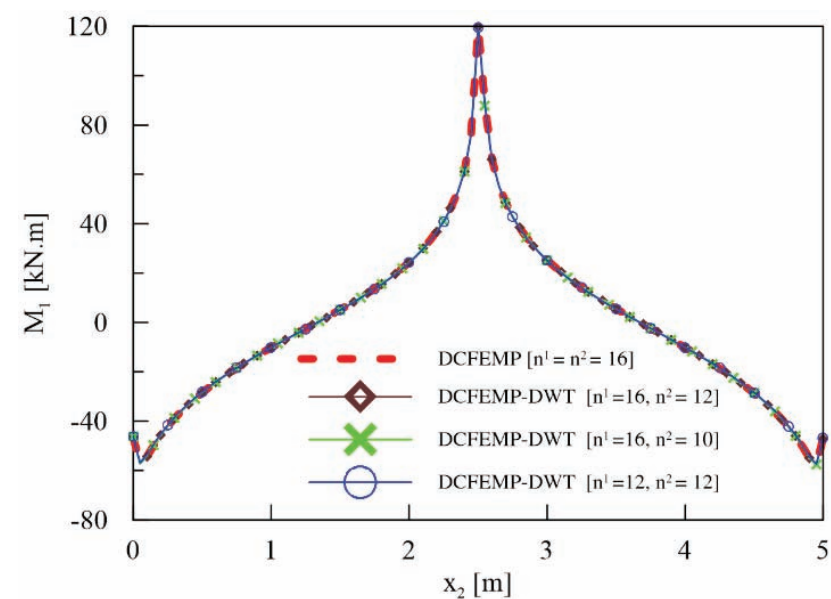

a)

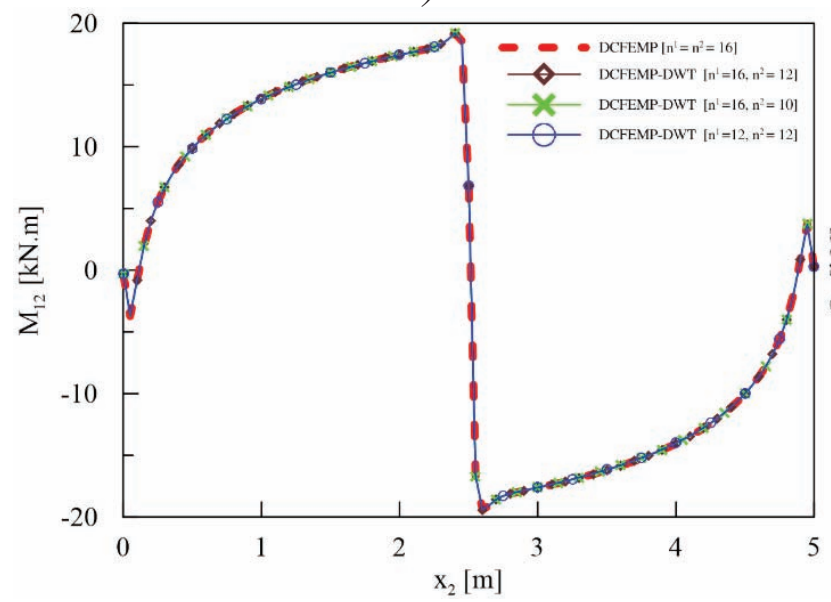

b)

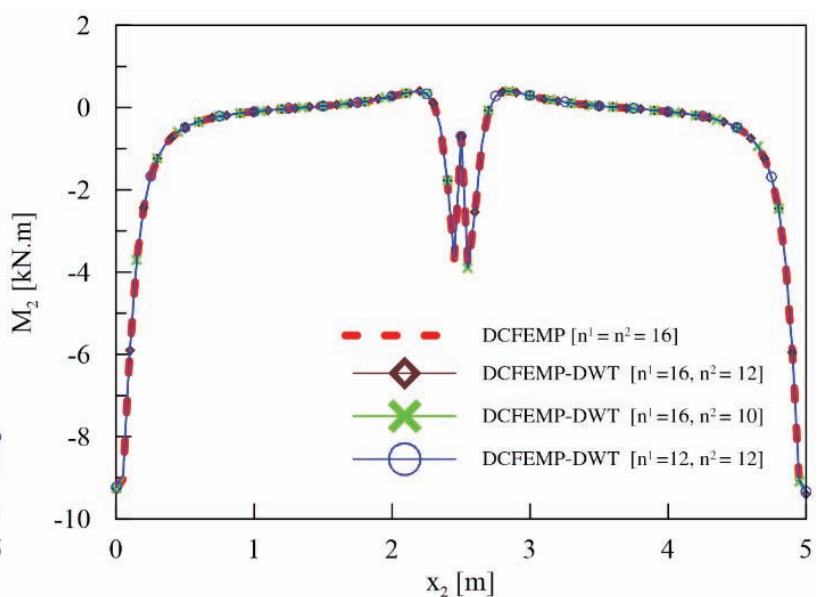

б)

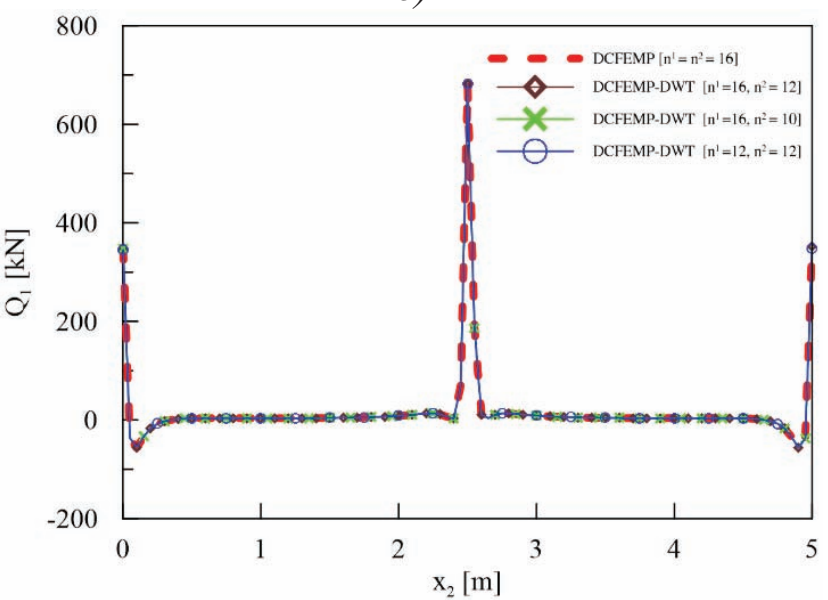

2)

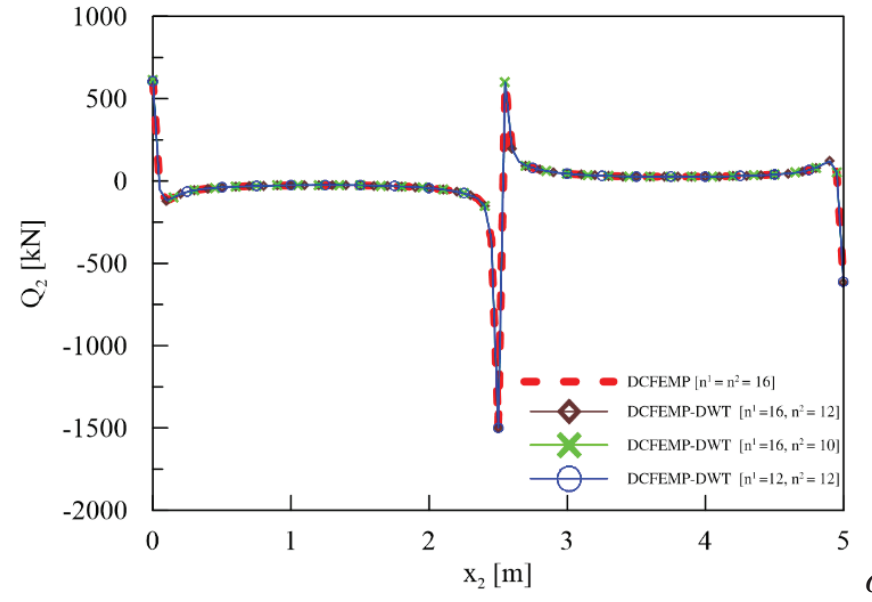

d)

Рисунок 5.2. Сопоставление решений (в части изгибающих и крутящего моментов и поперечных сил), полученных по ДКМКЭ и многоуровневому дискретно-континуальному подходу к локальному расчету строительных конструкций для сечения $x_{1}=0.0$.

DWT). Выполнены сопоставления решений, полученных по МКЭ (при построении дискретной модели использовались изопараметрические четырехузловые конечные элемен- ты) [1,8,9]. При построении дискретноконтинуальной модели было использовано 16 дискретно-континуальных конечных элементов, далее рассматривались различные 

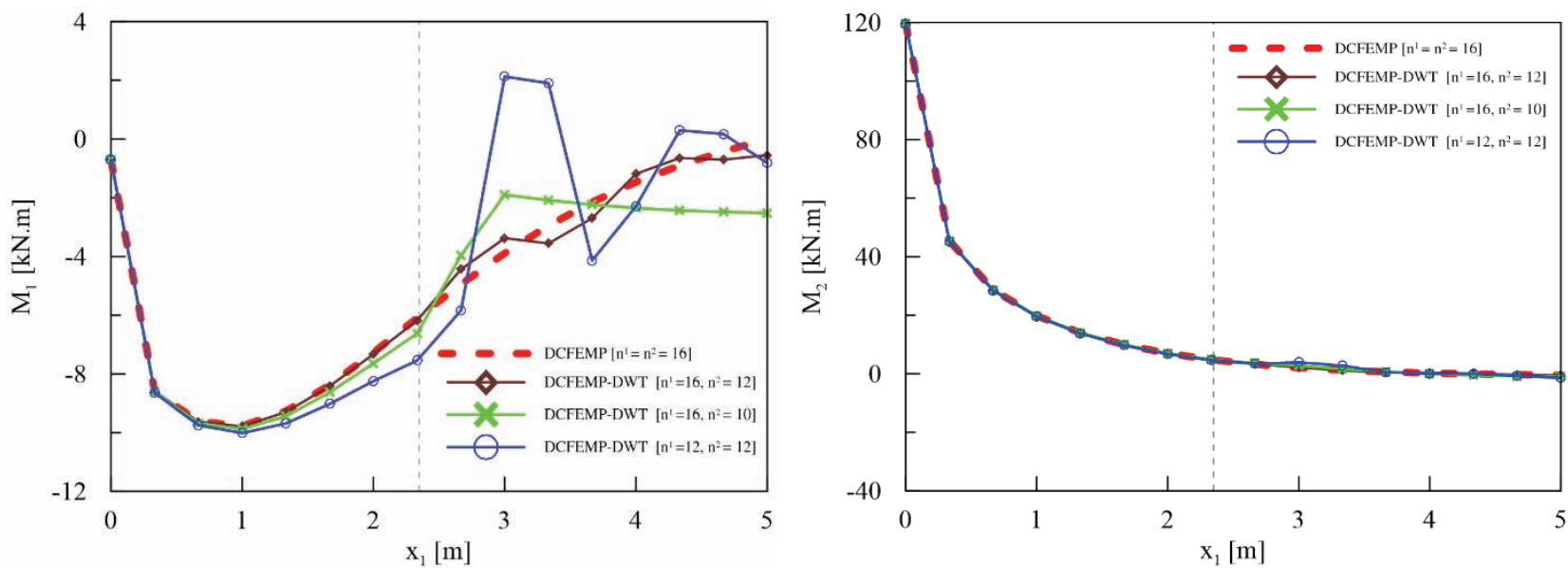

a)

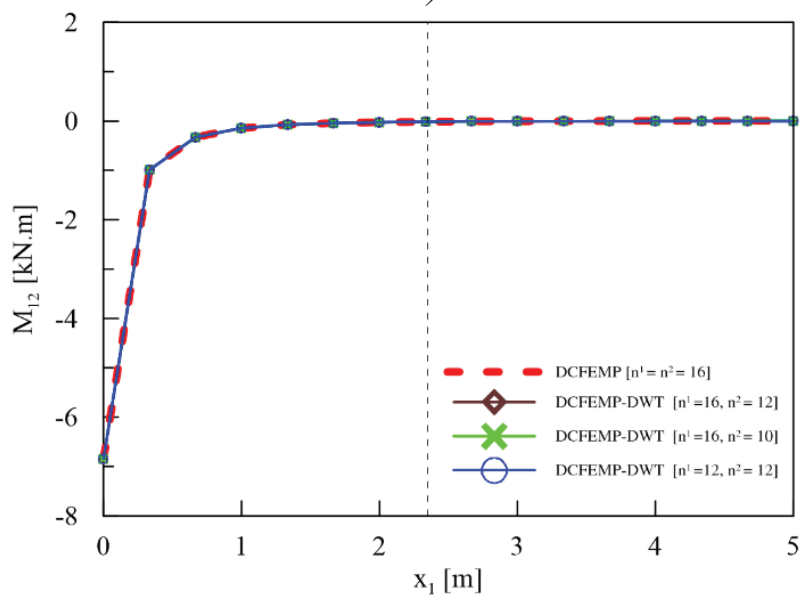

б)

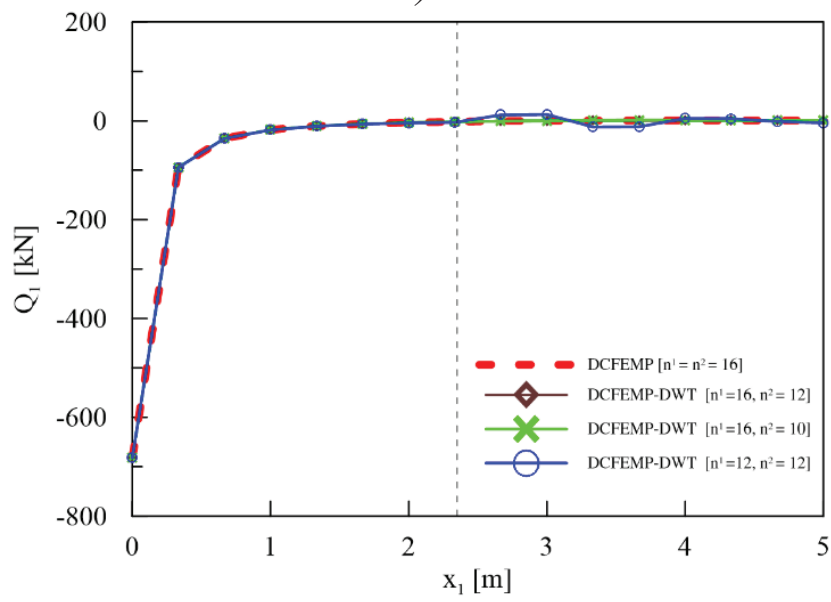

в)

2)

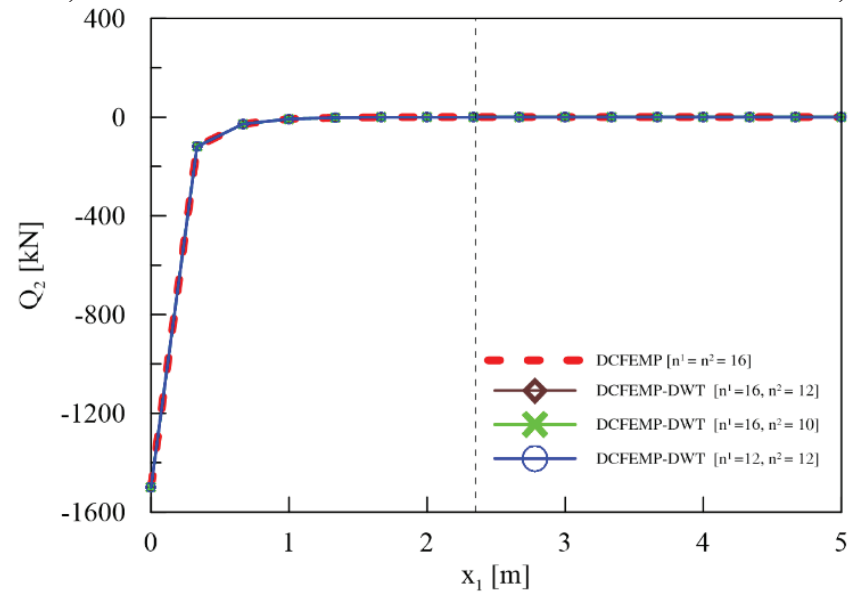

d)

Рисунок 5.3. Сопоставление решений (в части изгибающих и крутящего моментов и поперечных сил), полученных по ДКМКЭ и многоуровневому дискретно-континуальному подходу к локальному расчету строчтельных конструкиий для сечения $x_{2}=2.5$.

варианты редукции (локализация по степеням свободы). На рис. 14 и 15 представлены сопоставления результатов (в части изгибающих и крутящего моментов и поперечных сил), в частности, для сечений $x_{1}=0.0$ м и $x_{2}=2.5$ м. На рис. 16 приведено сопоставление соответствующих изополей. 
К вопросу о верификации многоуровневых подходов к локальному расчету строительных конструкций Часть 2: Дискретно-континуальный (численно-аналитический) подход
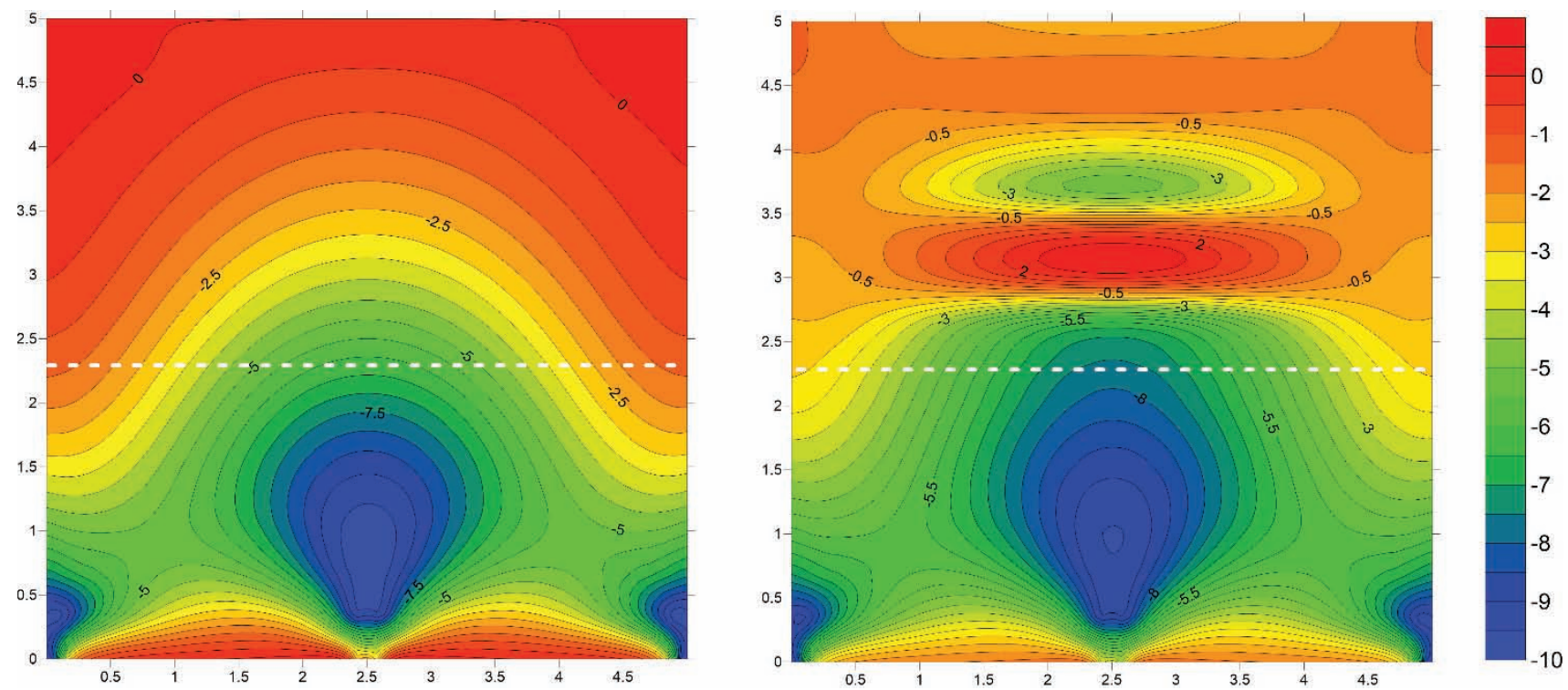

a) $M_{1}[\mathrm{kN} . \mathrm{m}]$
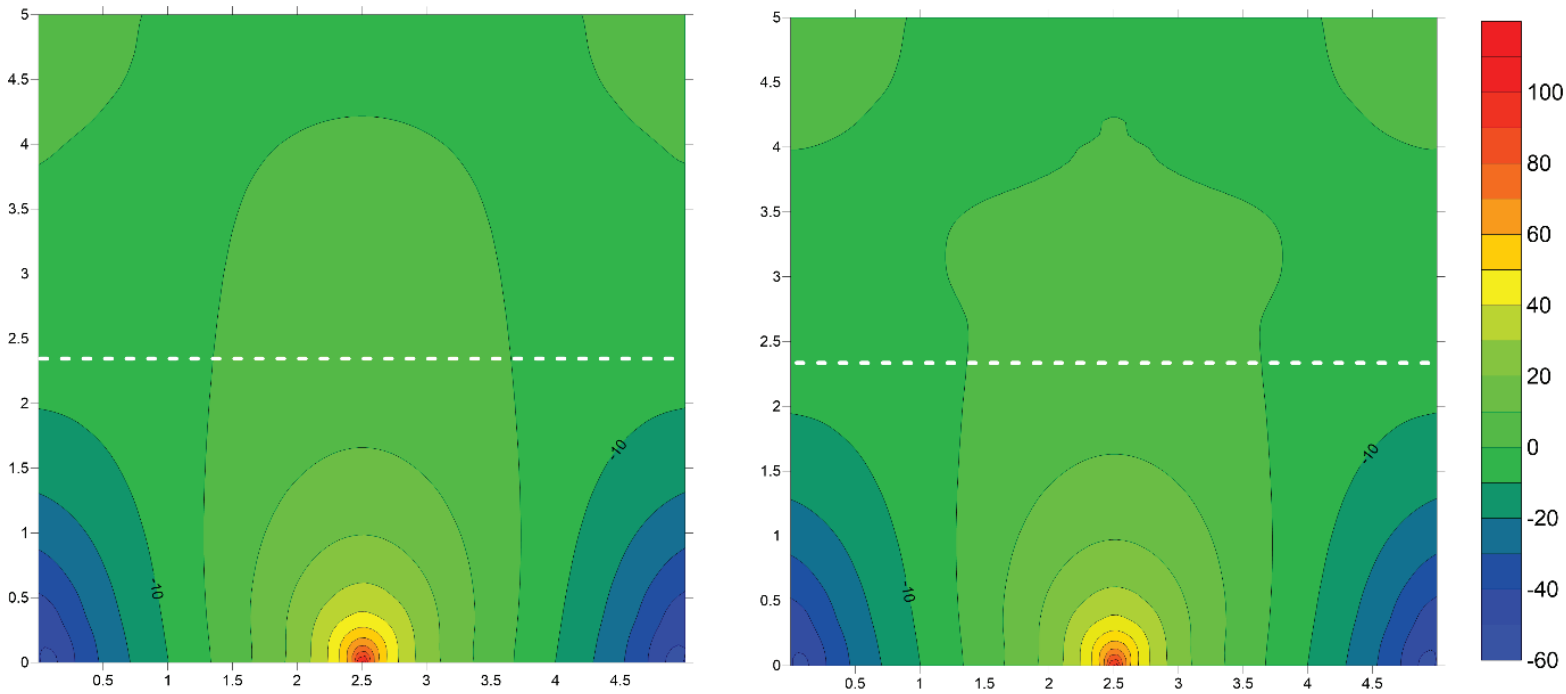

б) $M_{2}[\mathrm{kN} . \mathrm{m}]$
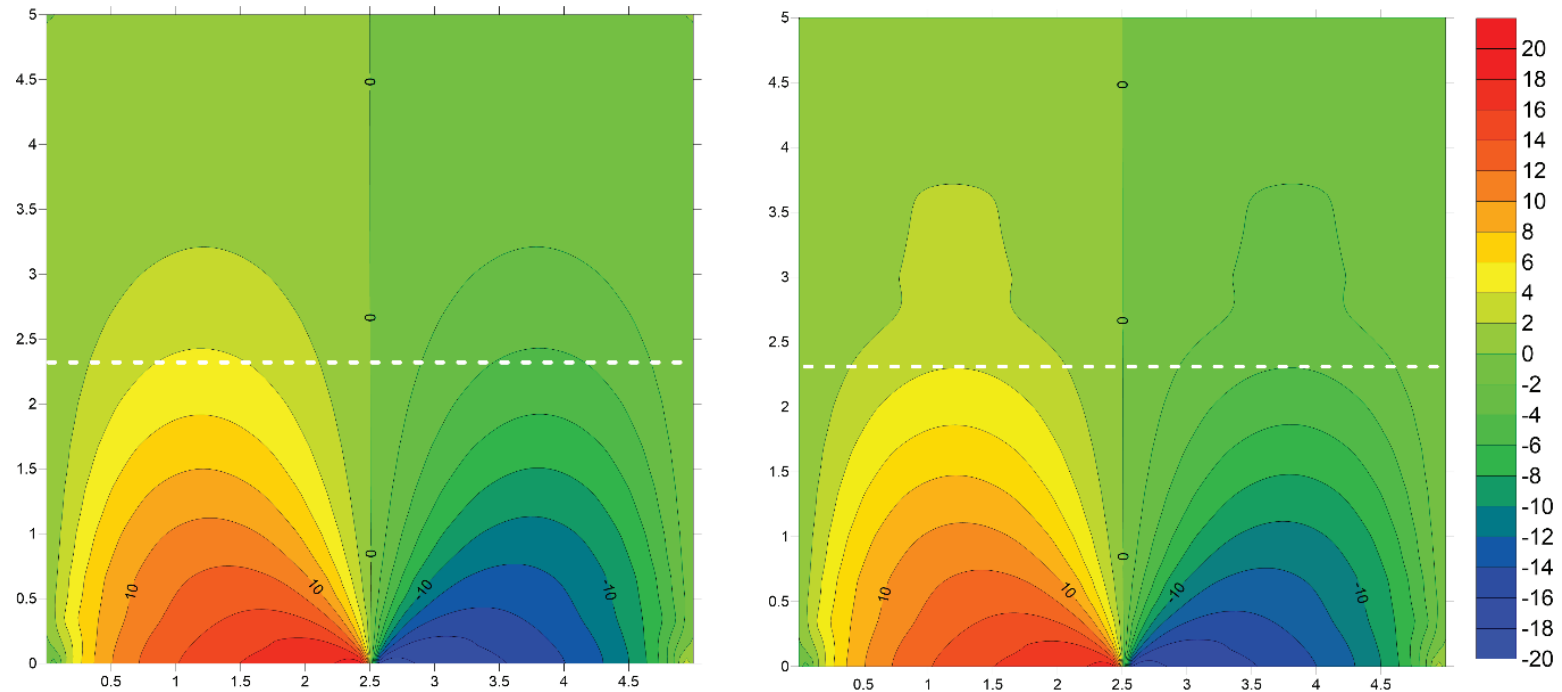

Volume 12, Issue 4, 2016 
в) $M_{12}[\mathrm{kN} . \mathrm{m}]$
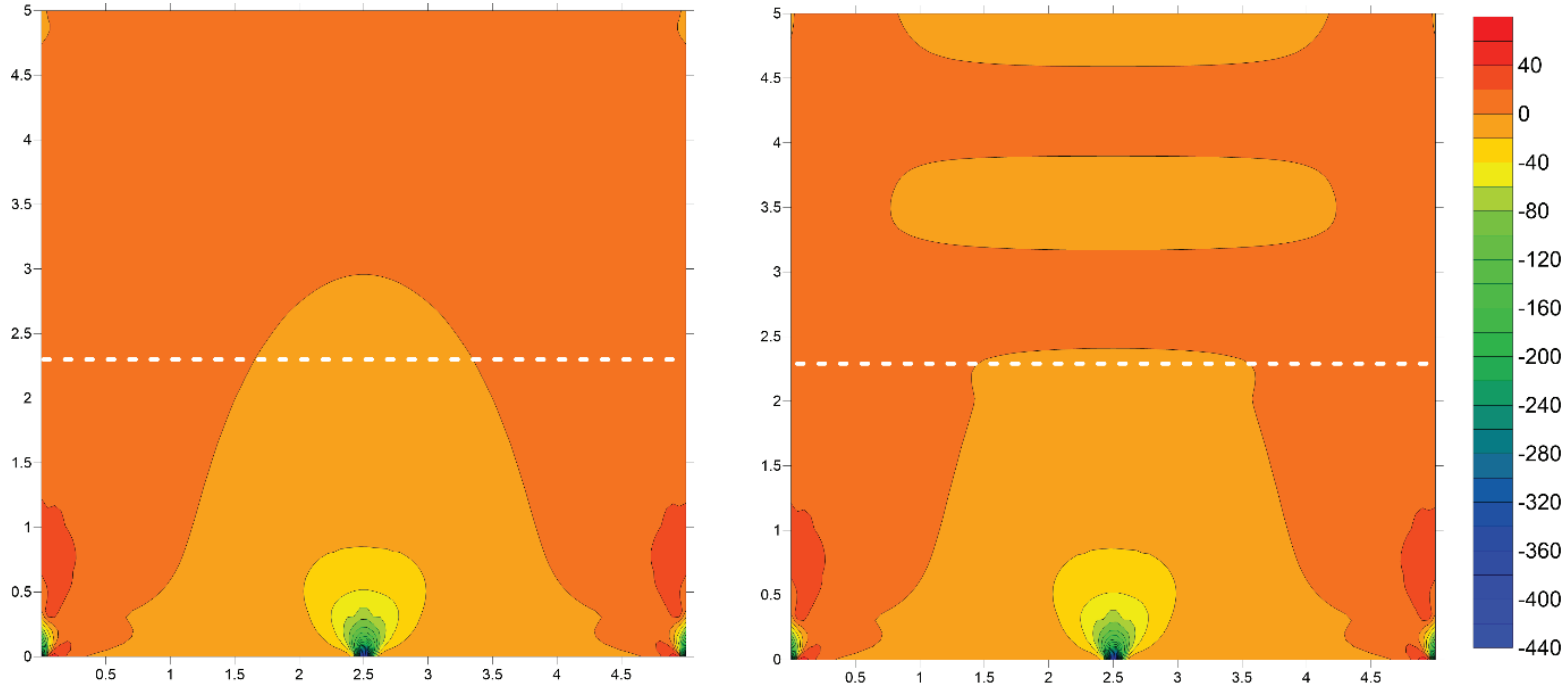

d) $Q_{1}[\mathrm{kN}]$
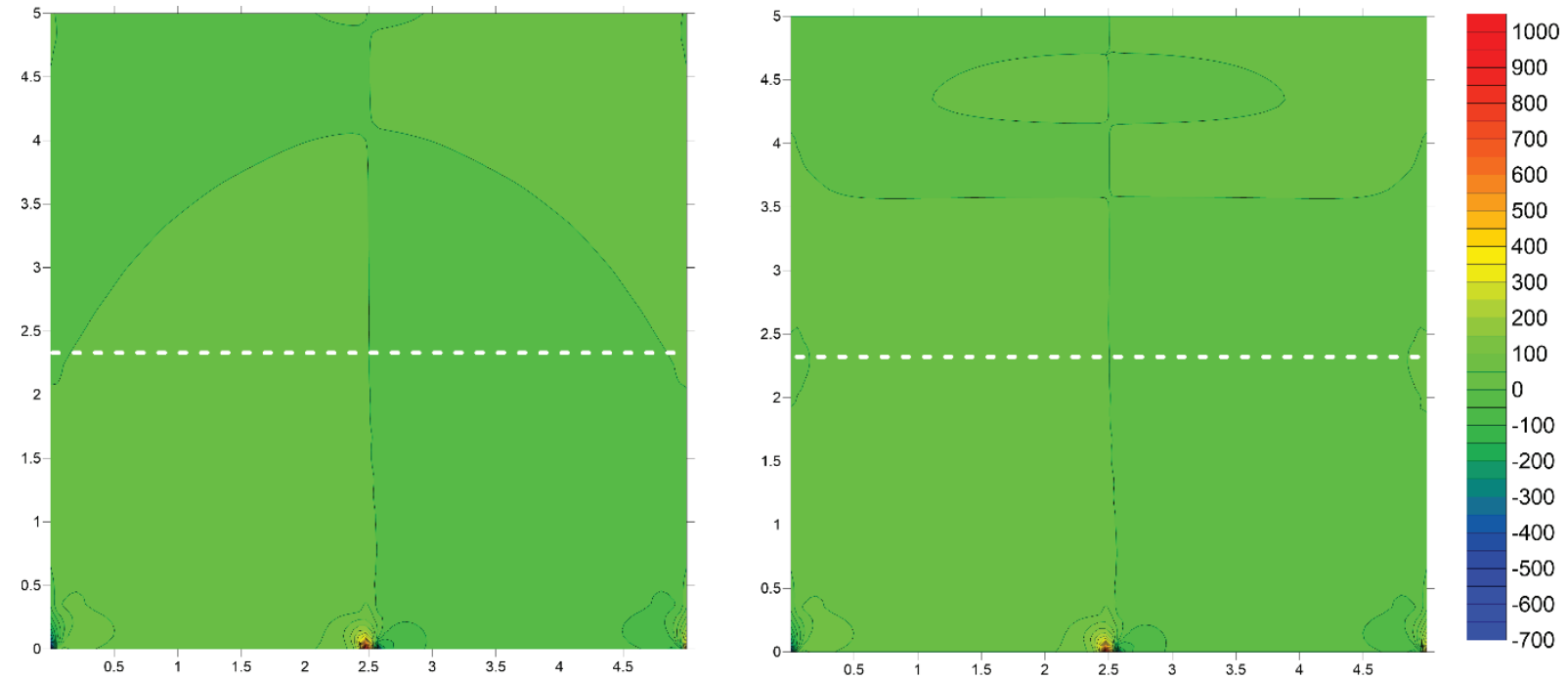

e) $Q_{2}[\mathrm{kN}]$

Рисунок 5.5. Сопоставление изополей решений (в части изгибающих и крутящего моментов u поперечных сил), полученных по ДКМКЭ и многоуровневому дискретно-континуальному подходу к локальному расчету строительных конструкиий.

Следует отметить, что в зоне гарантированной точности результаты, по существу, идентичны даже при значительной редукции неизвестных $[1,6,17,18]$.

\section{ЗАМЕЧАНИЕ}

Исследование выполнено за счет средств Государственной программы Российской
Федерации «Развитие науки и технологий» на 2013-2020 годы в рамках Плана фундаментальных научных исследований Министерства строительства и жилищнокоммунального строительства Российской Федерации на 2016 год, тема 7.1.1 «Разработка многоуровневого подхода к исследованию напряженно-деформированного состояния конструкций в рамках единой иерархически выстроенной расчетной моде- 
К вопросу о верификации многоуровневых подходов к локальному расчету строительных конструкций

Часть 2: Дискретно-континуальный (численно-аналитический) подход

ли на основе совместного применения дискретно-континуального метода конечных элементов и метода конечных элементов».

\section{СПИСОК ЛИТЕРАТУРЫ}

1. Акимов П.А., Мозгалева М.Л. Многоуровневые дискретные и дискретноконтинуальные методы локального расчета строительных конструкций. - М.: Издательство МИСИ-МГСУ, 2014.-632с.

2. Акимов П.А., Мозгалева М.Л., Кайтуков Т.Б., Негрозов О.А., Моджтаба Аслами. $\mathrm{O}$ верификации вейвлетреализаций многоуровневых дискретных и дискретно-континуальных методов локального расчета строительных конструкций. // Строительная механика и расчет сооружений, №5, 2014, с. 14-20.

3. Акимов П.А., Мозгалева М.Л., Моджтаба Аслами, Негрозов О.А., Щербина С.В. О верификации дискретно-континуального метода конечных элементов для задач статического расчета балок-стенок с кусочно-постоянными физико-геометрическими параметрами вдоль основного направления. Сопоставления с программным комплексом ANSYS Mechanical. // International Journal for Computational Civil and Structural Engineering, Volume 10, Issue 1, 2014, pp. 33-45.

4. Акимов П.А., Мозгалева М.Л., Моджтаба Аслами, Негрозов О.А., Щербина С.В. О верификации дискретно-континуального метода конечных элементов для задач статического расчета балок-стенок с постоянными физикогеометрическими параметрами вдоль основного направления. Сопоставления с программным комплексом ANSYS Mechanical. // International Journal for Computational Civil and Structural Engineering, Volume 10, Issue 1, 2014, pp. 18-32.

5. Акимов П.А., Мозгалева М.Л., Сидоров В.Н., Моджтаба Аслами, Негрозов
O.А. Усовершенствованная вейвлетреализация дискретно-континуального метода конечных элементов для локального решения двумерных задач расчета конструкций. // International Journal for Computational Civil and Structural Engineering, Volume 10, Issue 2, 2014, pp. 2937.

6. Акимов П.А., Мозгалева М.Л., Сидоров В.Н., Моджтаба Аслами, Негрозов O.А. Усовершенствованная вейвлетреализация дискретно-континуального метода конечных элементов для локального решения трехмерных задач расчета конструкций. // International Journal for Computational Civil and Structural Engineering, Volume 10, Issue 2, 2014, pp. 3846.

7. Акимов П.А., Мозгалева М.Л., Сидоров В.Н., Моджтаба Аслами, Негрозов О.А. Усовершенствованная вейвлетреализация дискретно-континуального метода конечных элементов для локального решения задач расчета тонких пластин. // International Journal for Computational Civil and Structural Engineering, Volume 10, Issue 2, 2014, pp. 47-55.

8. Моджтаба Аслами. Многоуровневые дискретные и дискретно-континуальные подходы к локальному расчету строительных конструкций. Диссертация на соискание ученой степени кандидата технических наук по специальности 05.13.18 - «Математическое моделирование, численные методы и комплексы программ». - М.: НИУ МГСУ, 2015. $321 \mathrm{c}$.

9. Моджтаба Аслами. Многоуровневые дискретные и дискретно-континуальные подходы к локальному расчету строительных конструкций. // International Journal for Computational Civil and Structural Engineering / Международный журнал по расчету гражданских и строительных конструкций, Volume 11, Issue 1, 2015, pp. 64-79. 
10. Akimov P.A., Aslami M. Theoretical Foundations and Applications of Multilevel Discrete and Discrete-Continual Methods of Local Structural Analysis. // Materials Physics and Mechanics, 26 (2016) pp. 1-4.

11. Akimov P.A., Aslami M., Mozgaleva M.L., Mskhalaya Z.I. Semianalytical Analysis of Shear Walls with the Use of Discrete-Continual Finite Element Method. Part 1: Mathematical Foundations. // MATEC Web Conferences, Vol. 86 (2016) 8 pages.

12. Akimov P.A., Aslami M., Mozgaleva M.L. Semianalytical Analysis of Shear Walls with the Use of Discrete-Continual Finite Element Method. Part 2: Numerical Examples, Future Development. // MATEC Web Conferences, Vol. 86 (2016) 8 pages.

13. Akimov P.A., Belostosky A.M., Mozgaleva M.L., Mojtaba Aslami, Negrozov O.A. Correct Multilevel Discrete-Continual Finite Element Method of Structural Analysis. // Advanced Materials Research, Vol. 1040 (2014) pp. 664-669.

14. Akimov P.A., Mozgaleva M.L., Aslami M., Negrozov O.A. Local High-Accuracy Plate Analysis Using Wavelet-Based Multilevel Discrete-Continual Finite Element Method. // Key Engineering Materials, Vol. 685 (2016) pp. 962-966.

15. Akimov P.A., Mozgaleva M.L., Mojtaba Aslami, Negrozov O.A. About Verification of Discrete-Continual Finite Element Method for Two-Dimensional Problems of Structural Analysis. Part 2: Deep Beam with Piecewise Constant Physical and Geometrical Parameters Along Basic Direction. // Advanced Materials Research, Vols. 10251026 (2014) pp. 95-103.

16. Akimov P.A., Mozgaleva M.L., Mojtaba Aslami, Negrozov O.A. About Verification of Discrete-Continual Finite Element Method of Structural Analysis. Part 1: TwoDimensional Problems. // Procedia Engineering, Vol. 91 (2014) pp. 2-7.

17. Akimov P.A., Mozgaleva M.L., Mojtaba Aslami, Negrozov O.A. Modified Wavelet- based Multilevel Discrete-Continual Finite Element. Part 1: Continual and DiscreteContinual Formulations of the Problems Method for Local Structural Analysis. // Applied Mechanics and Materials, Vols. 670-671 (2014) pp. 720-723.

18. Akimov P.A., Mozgaleva M.L., Mojtaba Aslami, Negrozov O.A. Modified Waveletbased Multilevel Discrete-Continual Finite Element. Part 2: Reduced Formulations of the Problems in Haar Basis Method for Local Structural Analysis. // Applied Mechanics and Materials, Vols. 670-671 (2014) pp. 724-727.

19. Akimov P.A., Mozgaleva M.L., Mojtaba Aslami, Negrozov O.A. Wavelet-Based Discrete-Continual Finite Element Method of Local Structural Analysis for TwoDimensional Problems. // Procedia Engineering, Vol. 91 (2014) pp. 8-13.

20. Ozgan Korhan, Ayse T. Daloglu. Effect of transverse shear strains on plates resting on elastic foundation using modified Vlasov model. // Thin -Walled Structures 46.11 (2008): $1236-1250$.

21. Zienkiewicz O.C., Morgan K. Finite Elements and Approximation. Dover Publications, 2006, 352 pages.

22. Zienkiewicz O.C., Taylor R.L., Zhu J.Z. The Finite Element Method: Its Basis and Fundamentals. Butterworth-Heinemann, Sixth edition, 2005, 752 pages.

23. Zienkiewicz O.C., Taylor R.L. The Finite Element Method for Solid and Structural Mechanics. Volume 2. ButterworthHeinemann, Sixth Edition, 2005, 736 pages.

Моджтаба Аслами, кандидат технических наук; доцент Университета Фесы; Феса, Иран;

e-mail: aslami.mojtaba@gmail.com

Акимов Павел Алексеевич, член-корреспондент РА$\mathrm{ACH}$, профессор, доктор технических наук; главный ученый секретарь РАAСН; заместитель генерального директора по науке ЗАО «Научно-исследовательский центр СтаДиО», профессор кафедры прикладной математики Национального исследовательского Московского государственного строительного университета (НИУ МГСУ); 107031, г. Москва, ул. Большая 
К вопросу о верификации многоуровневых подходов к локальному расчету строительных конструкций Часть 2: Дискретно-континуальный (численно-аналитический) подход

Дмитровка, д. 24, стр. 1; тел. +7(495) 625-71-63; факс +7 (495) 650-27-31; e-mail: akimov@raasn.ru, pavel.akimov@gmail.com.

Сидоров Владимир Николаевич, советник РААСН, профессор, доктор технических наук, Московский архитектурный институт (государственная академия), кафедра высшей математики и строительной механики, 107031, Россия, г. Москва, ул. Рождественка, дом 11/4, корп. 1, стр. 4; e-mail: sidorov.vladimir@gmail.com

Кайтуков Таймураз Батразович, советник РААСН, кандидат технических наук, доцент, доцент кафедры прикладной математики Национального исследовательского Московского государственного строительного университета, заместитель главного ученого секретаря Российской академии архитектуры и строительных наук; Россия, 129337, г. Москва, Ярославское шоссе, д. 26; тел/факс: +7 (499) 183-59-94;

e-mail: tkaytukov@gmail.com.

Mojtaba Aslami, Ph.D., Associate Professor, Fasa University, Fasa, Iran;

e-mail: aslami.mojtaba@gmail.com

Pavel A. Akimov, Corresponding Member of the Russian Academy of Architecture and Construction Sciences, $\mathrm{PhD}$, Professor, Chief Scientific Secretary of Russian Academy of Architecture and Construction Sciences; StaDyO Research \& Engineering Center; Department of Applied Mathematics, Moscow State University of Civil Engineering; 24, Ul. Bolshaya Dmitrovka, 107031, Moscow, Russia; phone +7(495) 625-71-63; fax: +7 (495) 650-27-31; e-mail: akimov@ raasn.ru,

pavel.akimov@gmail.com.

Vladimir N. Sidorov, Advisor of the Russian Academy of Architecture and Construction Sciences, Professor, Dr.Sc., Department of Advanced Mathematics and Structural Mechanics; Moscow Institute of Architecture (State Academy); 11/4, Building 4, Ulitsa Rozhdestvenka, Moscow, 107031, Russia;

e-mail: sidorov.vladimir@gmail.com

Taymuraz B. Kaytukov, Advisor of Russian Academy of Architecture and Construction Sciences, PhD, Associate Professor, Department of Applied Mathematics, National Research Moscow State University of Civil Engineering; Vice Executive Scientific Secretary of Russian Academy of Architecture and Construction Sciences; 26, Yaroslavskoe Shosse, Moscow, 129337, Russia, phone/fax: +7(499) 183-59-94, e-mail: tkaytukov@gmail.com. 


\title{
МЕТОДИКА ЧИСЛЕННОГО МОДЕЛИРОВАНИЯ НАПРЯЖЕННО-ДЕФОРМИРОВАНОГО СОСТОЯНИЯ СИСТЕМЫ «ОСНОВАНИЕ - ПЛОТИНА - ВОДОХРАНИЛИЩЕ» ПРИ СЕЙСМИЧЕКИХ ВОЗДЕЙСТВИЯХ
}

\author{
А.М. Белостоцкий ${ }^{1,2,3,4}$, Д.С. Дмитриев ${ }^{2}$, \\ Нгуен Тай Нанг Льонг ${ }^{1}$ \\ ${ }^{1}$ Национальный исследовательский Московский государственный строительный университет, \\ г. Москва, РОССИЯ \\ $23 \mathrm{AO}$ «Научно-исследовательский центр СтаДиО», г. Москва, РОССИЯ \\ ${ }^{3}$ Научно-исследовательский институт строительной физики Российской академии архитектуры \\ и строительных наук, г. Москва, РОССИЯ \\ ${ }^{4}$ Пермский национальный исследовательский политехнический университет, г. Пермь, РОССИЯ
}

Аннотация: в статье рассматриваются вопросы численного моделирования взаимодействия гидротехнического сооружения (плотины) с водохранилищем при сейсмических воздействиях. Приведены основополагающие уравнения, описывающие поведение жидкости, сооружения (конструкций) и конечноэлементные аналоги соответствующих уравнений, а также граничные условия на контактных поверхностях. Кратко описаны прямые и итерационные алгоритмы решения связанных задач гидроупругости. Изложены вопросы объединения несвязанных конечноэлементных сеток.

Ключевые слова: математическое моделирование, численные методы, метод конечных элементов, связанные системы «сооружение-жидкость», уравнение Навье-Стокса, эйлерова постановка,

лагранжева постановка, акустическая жидкость, уравнения связи

\section{NUMERICAL METHOD MODELING OF STRAIN-STRESS STATE SYSTEM "FOUNDATION - DAM - RESERVOIR" AT SEISMIC IMPACTS}

\author{
Alexander M. Belostotsky ${ }^{1,2,3,4}$, Dmitry S. Dmitriev², \\ Nguyen Tai Nang Luong ${ }^{1}$ \\ ${ }^{1}$ National Research Moscow State University of Civil Engineering, Moscow, RUSSIA \\ ${ }^{2}$ StaDyO Research \& Engineering Center, Moscow, RUSSIA \\ ${ }^{3}$ Research Institute of Building Physics of Russian Academy \\ of Architecture and Construction Sciences, Moscow, RUSSIA \\ ${ }^{4}$ Perm National Research Polytechnic University, Perm, RUSSIA
}

\begin{abstract}
This article discusses the finite element modeling of the interaction of structures with the fluid. Presents the fundamental equations describing the fluid behavior, structures (structures) and finite-element analogues of the corresponding equations and boundary conditions on the contact surfaces. Briefly describe direct and iterative algorithms for solving problems related hydroelasticity. Touched upon the unification of unrelated finite element meshes.
\end{abstract}

Key words: math modeling, numerical methods, related production, finite element method, system «construction-fluid», Navier-Stokes equations, Euler formulation, Lagrangian formulation, acoustic fluid, communication equation 
Методика численного моделирования напряженно-деформированного состояния системы «основание - плотина - водохранилище» при сейсмических воздействиях

\section{1. ВВЕДЕНИЕ}

Проектирование и расчетное обоснование сооружений, непосредственно взаимодействующих с жидкостью, будь то водоподпорные гидросооружения или различного типа резервуары, представляет собой достаточно нетривиальную задачу ввиду известных волновых эффектов в жидкости. Задачи такого типа обладают значительной актуальностью и несомненной практической значимостью, связанной, в частности, с потребностями в дальнейшем совершенствовании и развитии методов решения и проектирования сооружений такого типа.

На сегодняшний день сформировались и развиваются три основных направления численного решения связанных задач гидроупругости:

1. Постановка Эйлера [1], описывающая состояние жидкости в теоретически естественных узловых переменных (давление или потенциал скоростей), а сооружения и основания - через узловые перемещения.

2. Постановка Лагранжа [2], с представлением и жидкости, и сооружений (с основанием) уравнениями в перемещениях.

3. Смешанная лагранжева-эйлеровая постановка, в зарубежной литературе называемая Arbitrary Lagrangian-Eulerian Formulation (постановка ALE), сочетает в себе оба подхода. Постановки типа ALE ориентированы на изучение сложных течений, где чисто лагранжевы и чисто эйлеровы постановки не могут быть применены. Однако для задач, где основной интерес представляют прочность и надёжность достаточно жестких сооружений, контактирующих с жидкостью (в том числе гидротехнические сооружения), вполне применима эйлерова постановка.

Для решения связанных задач «сооружение - жидкость» (Fluid-Structure Interaction (FSI)) могут использоваться различные алгоритмы, причем выбор наиболее эффективного из них определяется характером каждой конкретной задачи. Если поведение жидкости описывается уравнениями НавьеСтокса или Эйлера, можно выделить два основных случая. Первый случай имеет место при слабом взаимодействии между сооружением и жидкостью (области, которые занимают сооружение и жидкость деформируются слабо) - здесь принято использовать итерационные алгоритмы, когда уравнения, описывающие поведение жидкости и сооружения, решаются отдельно, после чего параметры решения последовательно согласуются, пока соответствующий процесс не сойдется. Второй случай, напротив, возникает при сильном взаимодействии жидкости с сооружением, в результате чего могут возникать значительные деформации - здесь принято использовать прямые алгоритмы, когда уравнения, описывающие поведение жидкости и сооружения, решаются совместно.

$\mathrm{B}$ настоящей статье описана методика численного (конечноэлементного) моделирования взаимодействия сооружения с жидкостью в эйлеровой постановке. Поведение жидкости описывается уравнениями Навье-Стокса, определяющие уравнения для сооружения могут быть как линейными, так и нелинейными, а используемые алгоритмы решения прямые.

\section{2. ОПРЕДЕЛЯЮЩИЕ УРАВНЕНИЯ}

В акустической среде, уравнения динамики необходимо рассматривать вместе с уравнениями импульса и неразрывности. Для вывода волнового уравнения акустической жидкости используются следующие допущения:

- акустическая жидкость сжимаемая (плотность изменятся за счёт изменения давления);

- средняя плотность является единой на всей акустической жидкости.

Уравнение неразрывности имеет вид:

$$
\frac{\partial \rho}{\partial t}+\frac{\partial\left(\rho v_{x}\right)}{\partial x}+\frac{\partial\left(\rho v_{y}\right)}{\partial y}+\frac{\partial\left(\rho v_{z}\right)}{\partial z}=0
$$

где $v_{x}, v_{y}, v_{z}$ - компоненты вектора скорости в направлениях глобальных осей $\mathrm{x}$, у и $\mathrm{z}$ соответственно; $\rho$ - плотность; $t$ - время.

Уравнение движения Навье-Стокса имеет вид:

$$
\begin{gathered}
\frac{\partial \rho v_{x}}{\partial t}+\frac{\partial\left(\rho v_{x} v_{x}\right)}{\partial x}+\frac{\partial\left(\rho v_{y} v_{x}\right)}{\partial y}+\frac{\partial\left(\rho v_{z} v_{x}\right)}{\partial z}= \\
=\rho g_{x}-\frac{\partial P}{\partial x}+R_{x}+\frac{\partial}{\partial x}\left(\mu_{e} \frac{\partial v_{x}}{\partial x}\right)+\frac{\partial}{\partial y}\left(\mu_{e} \frac{\partial v_{y}}{\partial y}\right) \\
+\frac{\partial}{\partial z}\left(\mu_{e} \frac{\partial v_{z}}{\partial z}\right)+T_{x} \\
\frac{\partial \rho v_{y}}{\partial t}+\frac{\partial\left(\rho v_{x} v_{y}\right)}{\partial x}+\frac{\partial\left(\rho v_{y} v_{y}\right)}{\partial y}+\frac{\partial\left(\rho v_{z} v_{y}\right)}{\partial z}= \\
=\rho g_{y}-\frac{\partial P}{\partial y}+R_{y}+\frac{\partial}{\partial x}\left(\mu_{e} \frac{\partial v_{y}}{\partial x}\right)+\frac{\partial}{\partial y}\left(\mu_{e} \frac{\partial v_{y}}{\partial y}\right) \\
+\frac{\partial}{\partial z}\left(\mu_{e} \frac{\partial v_{y}}{\partial z}\right)+T_{y}
\end{gathered}
$$




$$
\begin{gathered}
\frac{\partial \rho \mathrm{v}_{\mathrm{z}}}{\partial \mathrm{t}}+\frac{\partial\left(\rho \mathrm{v}_{\mathrm{x}} \mathrm{v}_{\mathrm{z}}\right)}{\partial \mathrm{x}}+\frac{\partial\left(\rho \mathrm{v}_{\mathrm{y}} \mathrm{v}_{\mathrm{z}}\right)}{\partial \mathrm{y}}+\frac{\partial\left(\rho \mathrm{v}_{\mathrm{z}} \mathrm{v}_{\mathrm{z}}\right)}{\partial \mathrm{z}}= \\
=\rho \mathrm{g}_{\mathrm{z}}-\frac{\partial \mathrm{P}}{\partial \mathrm{z}}+\mathrm{R}_{\mathrm{z}}+\frac{\partial}{\partial \mathrm{x}}\left(\mu_{\mathrm{e}} \frac{\partial \mathrm{v}_{\mathrm{y}}}{\partial \mathrm{x}}\right)+\frac{\partial}{\partial \mathrm{y}}\left(\mu_{\mathrm{e}} \frac{\partial \mathrm{v}_{\mathrm{y}}}{\partial \mathrm{y}}\right) \\
+\frac{\partial}{\partial \mathrm{z}}\left(\mu_{\mathrm{e}} \frac{\partial \mathrm{v}_{\mathrm{z}}}{\partial \mathrm{z}}\right)+\mathrm{T}_{\mathrm{z}}
\end{gathered}
$$

где $\mathrm{g}_{\mathrm{x}}, \mathrm{g}_{\mathrm{y}}, \mathrm{g}_{\mathrm{z}}$ - компоненты ускорения свободного падения; $\rho$ - плотность; $\mu_{\mathrm{e}}$ - вязкость жидкости; Исходя из выше изложенного волновое уравнение акустической среды имеет вид:

$$
\begin{gathered}
\nabla\left(\frac{1}{\rho_{0}} \nabla \mathrm{P}\right)-\frac{1}{\rho_{0} \mathrm{c}^{2}} \frac{\partial^{2} \mathrm{P}}{\partial \mathrm{t}^{2}}+\nabla\left[\frac{4 \mu}{3 \rho_{0}} \nabla\left(\frac{1}{\rho_{0} \mathrm{c}^{2}} \frac{\partial \mathrm{P}}{\partial \mathrm{t}}\right)\right]= \\
=-\frac{\partial}{\partial \mathrm{t}}\left(\frac{\mathrm{Q}}{\rho_{0}}\right)+\nabla\left[\frac{4 \mu}{3 \rho_{0}} \nabla\left(\frac{\mathrm{Q}}{\rho_{0}}\right)\right]
\end{gathered}
$$

где с - скорость звука в акустической жидкости; $\rho_{0}-$ средняя плотность акустической жидкости; $\mu$ - динамическая вязкость акустической жидкости; P - акустическое давление; Q - масса источника в уравнении непрерывности; $\mathrm{t}$ - время.

\section{3. ДИСКРЕТИЗАЦИЯ ВОЛНОВОГО УРАВНЕНИЯ}

Для дискретизации уравнения (2.1) необходимо использовать матричные операторы градиент и дивергенция.

$$
\begin{gathered}
\nabla \cdot()=\{L\}^{T}=\left|\frac{\partial}{\partial x} \frac{\partial}{\partial y} \frac{\partial}{\partial z}\right| \\
\nabla()=\{L\}
\end{gathered}
$$

Интегрируя уравнение (2.1) по объёму V и применяя теорему Гаусса-Остроградского получаем:

$$
\begin{gathered}
\int_{V} \frac{1}{\mathrm{c}^{2}} \delta \mathrm{P} \frac{\partial^{2} \mathrm{P}}{\partial \mathrm{t}^{2}} \mathrm{dV}+\int_{\mathrm{V}}\{\mathrm{L}\}^{\mathrm{T}} \delta \mathrm{P}\{\mathrm{L}\} \mathrm{PdV}= \\
=\int_{\Gamma}\{\mathrm{n}\}^{\mathrm{T}} \delta \mathrm{P}\{\mathrm{L}\} \mathrm{Pd} \Gamma
\end{gathered}
$$

где $\delta \mathrm{P}$ - виртуальное изменение давления; Г - поверхности, для которых существует производная давления по нормали к ним; $\{\mathrm{n}\}$ - нормаль к поверхности $Г$.
В задачах взаимодействия «сооружение - жидкость», поверхность Г рассматривается, как граничная между разными средами. Для упрощения дальнейших выкладок, применим соотношение связи нормального градиента давлений, с нормальными ускорениями твёрдого тела на границе:

$$
\{\mathrm{n}\}^{\mathrm{T}} \nabla P=-\rho\{\mathrm{n}\} \frac{d^{2}\{u\}}{d t^{2}}
$$

где $\{u\}$ - вектор перемещения твердого тела. В матричном выражении уравнение (3.4) перепишется:

$$
\{\mathrm{n}\}^{\mathrm{T}}\{L\} P=-\rho\{n\}^{T} \frac{d^{2}\{u\}}{d t^{2}}
$$

Подставляя выражение (3.5) в выражение (3.3) получим:

$$
\begin{gathered}
\int_{V} \frac{1}{c^{2}} \delta P \frac{\partial^{2} P}{\partial t^{2}} d V+\int_{V}\{L\}^{T} \delta P\{L\} P d V= \\
=\int_{\Gamma} \rho \delta P\{n\}^{T} \frac{\partial^{2} u}{\partial t^{2}} d \Gamma
\end{gathered}
$$

\section{4. ВЫВОД МАТРИЦ АКУСТИЧЕСКОЙ СРЕДЫ}

Уравнение (3.6) содержит давление Р и смещения компонентов перемещений, в качестве зависимых переменных. Для реализации метода конечных элементов необходимо ввести функции формы, при помощи которых значения давления и перемещений внутри конечного элемента выражаются через его узловые значения:

$$
\begin{gathered}
P=\{N\}^{T}\left\{P_{e}\right\} \\
u=\left\{N^{\prime}\right\}^{T}\left\{u_{e}\right\}
\end{gathered}
$$

где $\{N\}$ - вектор функций форм для определения давления; $\left\{N^{\prime}\right\}$ - матрица функций форм для определения перемещений; $\left\{P_{e}\right\}$ - вектор узловых значений давления; $\left\{u_{e}\right\}=\left\{u_{e x}\right\},\left\{u_{e y}\right\},\left\{u_{e z}\right\}$ - вектор узловых значений перемещений.

Учитывая уравнения (4.1) и (4.2), производные переменных и виртуальное изменение давления определятся как: 
Методика численного моделирования напряженно-деформированного состояния системы «основание - плотина - водохранилище» при сейсмических воздействиях

$$
\begin{aligned}
& \frac{\partial^{2} P}{\partial t^{2}}=\{N\}^{T}\left\{\ddot{P}_{e}\right\} \\
& \frac{\partial^{2} u}{\partial t^{2}}=\left\{N^{\prime}\right\}^{T}\left\{\ddot{u}_{e}\right\} \\
& \delta P=\{N\}^{T}\left\{\delta P_{e}\right\}
\end{aligned}
$$

Применим оператор $\{L\}$ к матрицам функций форм:

$$
\{B\}=\{L\}\{N\}^{T}
$$

Подставляя (1) и (2) в уравнение (2) и учитывая (3)-(6) получаем:

$$
\begin{aligned}
& \int_{V} \frac{1}{c^{2}}\left\{\delta P_{e}\right\}^{T}\{N\}\{N\}^{T} d V\left\{\ddot{P}_{e}\right\} \\
& +\int_{V}\left\{\delta P_{e}\right\}^{T}\{B\}^{T}\{B\} d V\left\{P_{e}\right\} \\
& +\int_{\Gamma} \rho\left\{\delta P_{e}\right\}^{T}\{N\}\{n\}^{T}\left\{N^{\prime}\right\}^{T} d \Gamma\left\{\ddot{u}_{e}\right\}
\end{aligned}
$$

С учетом диссипации энергии за счет демпфирования, в матричном виде уравнение (4.7) может быть переписано:

$$
\begin{array}{r}
\left\{M_{e}\right\}\left\{\ddot{P}_{e}\right\}+\left\{C_{e}\right\}\left\{\dot{P}_{e}\right\}+\left\{K_{e}\right\}\left\{P_{e}\right\} \\
+\rho\left\{R_{e}\right\}^{T}\left\{\ddot{u}_{e}\right\}=0
\end{array}
$$

где

$$
\left\{M_{e}\right\}=\int_{V} \frac{1}{c^{2}}\{N\}\{N\}^{T} d V
$$

- матрица масс акустической жидкости;

$$
\left\{C_{e}\right\}=\frac{\beta}{c} \int_{\Gamma}\{N\}\{N\}^{T} d \Gamma
$$

- матрица демпфирования акустической жидкости;

$$
\left\{K_{e}\right\}=\int_{V}\{B\}^{T}\{B\} d V
$$

- матрица жесткости акустической жидкости;

Сравнивая интеграл (5.4) с уравнением (4.8), становится ясно, что:

$$
\left\{F_{e p}\right\}=\left\{R_{e}\right\}\left\{P_{e}\right\}
$$

где

где $\sigma\left\{u_{e}\right\}$ - тензор напряжений сооружения; $\left\{u_{e}\right\}$ вектор узловых значений перемещений сооружения; $\left\{u_{f}\right\}$ - вектор узловых значений перемещений акустической жидкости; $\{n\}$ - нормаль к поверхности контакта. Из уравнения (5.2) следует, что на границе меду двух сред нет трения.

Для того, чтобы полностью описать взаимодейбавляется давление жидкости, действующее на границе. Тогда уравнение движения твёрдого тела аписывается следующим образом:

$$
\begin{gathered}
\left\{M_{e u}\right\}\left\{\ddot{u}_{e}\right\}+\left\{C_{e u}\right\}\left\{u_{e}\right\}+\left\{K_{e u}\right\}\left\{u_{e}\right\} \\
=\left\{F_{e u}\right\}+\left\{F_{e p}\right\}
\end{gathered}
$$

где

$$
\left\{F_{e p}\right\}=\int_{\Gamma}\left\{N^{\prime}\right\} P\{n\} d \Gamma \text {, }
$$

$\left\{N^{\prime}\right\}$ - матрица функуций форм перемещений; $\{n\}$ - нормаль к поверхности Г.

Учитывая аппроксимацию давления через функции формы, получим:

$$
\left\{F_{e p}\right\}=\int_{\Gamma}\left\{N^{\prime}\right\}\{N\}^{T}\{n\} d \Gamma\left\{P_{e}\right\}
$$

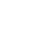




$$
\left\{R_{e}\right\}=\int_{\Gamma}\{N\}\{n\}^{T}\left\{N^{\prime}\right\}^{T} d \Gamma
$$

- связующая матрица акустической жидкости. С учетом этого уравнение (5.3) перепишется следующим образом:

$$
\begin{array}{r}
\left\{M_{e u}\right\}\left\{\ddot{u}_{e}\right\}+\left\{C_{e u}\right\}\left\{u_{e}\right\}+\left\{K_{e u}\right\}\left\{u_{e}\right\} \\
-\left\{R_{e}\right\}\left\{P_{e}\right\}=\left\{F_{e u}\right\}
\end{array}
$$

Таким образом конечно-элементная формулировка взаимодействия акустической жидкости с твердым телом, с учетом уравнений (4.8) и (5.6), принимает вид:

$$
\begin{aligned}
& \left|\begin{array}{cc}
\left\{M_{e u}\right\} & 0 \\
\rho\left\{R_{e}\right\}^{T} & \left\{M_{e}\right\}
\end{array}\right|\left\{\frac{\left\{\ddot{u}_{e}\right\}}{\left\{\ddot{P}_{e}\right\}}\right\} \\
& +\left|\begin{array}{cc}
\left\{C_{e u}\right\} & 0 \\
0 & \left\{C_{e}\right\}
\end{array}\right|\left\{\frac{\left\{\dot{u}_{e}\right\}}{\left\{\dot{P}_{e}\right\}}\right\} \\
& +\left|\begin{array}{cc}
\left\{K_{e u}\right\} & -\left\{R_{e}\right\} \\
0 & \left\{K_{e}\right\}
\end{array}\right|\left\{\frac{\left\{u_{e}\right\}}{\left\{P_{e}\right\}}\right\}=\left\{\frac{F_{e u}}{0}\right\}
\end{aligned}
$$

где $\left\{M_{e u}\right\},\left\{C_{e u}\right\},\left\{K_{e u}\right\}$ - матрицы масс, сопротивлений и жесткости конструкции соответственно; $\left\{M_{e}\right\},\left\{C_{e}\right\},\left\{K_{e}\right\}$ - матрицы масс, сопротивлений и демпфирования акустической жидкости соответственно; $\left\{R_{e}\right\}$ - связующая матрица взаимосвязи “давление-перемещение”; $\rho$ - средняя плотность акустической жидкости; $\left\{u_{e}\right\},\left\{\dot{u}_{e}\right\},\left\{\ddot{u}_{e}\right\}$ - векторы перемещений, скоростей и ускорений конструкции; $\left\{P_{e}\right\},\left\{\dot{P}_{e}\right\},\left\{\ddot{P}_{e}\right\}$ - давление и его производные по времени; $\left\{F_{e u}\right\}$ - вектор приложенных к конструкции сил.

В результате решения (5.7) определяются значения перемещений в конструкции и давления в акустической жидкости. Для гармонического и модального анализа разработаны процедуры симметризации уравнения (5.7) [8].

Для построения матрицы $\left\{R_{e}\right\}$, “перевязывающей” уравнения движения твердого тела (сооружения, основания) и акустических колебаний жидкости на поверхностях их контакта, используются специальные изопараметрические контактные элементы сирендипова семейства. Узлы контактного элемента геометрически попарно совпадают и образуют два слоя. Узлы первого слоя имеют по 3 степени свободы, соответствующие поступательным перемещениям по направлениям осей глобальной декартовой системы координат, а узлы второго - по одной степени свободы, соответствующей давлению в жидкости. Для построения конечноэлементной модели связанной задачи контактный элемент стыкуется узлами первого слоя с конечными элементами сооружения (основания), а узлами второго слоя - с конечными элементами, моделирующими жидкость.

\section{6. ОБЪЕДИНЕНИЕ НЕСВЯЗАННЫХ СЕТОК НА КОНТАКТАХ}

На практике, как правило, удобно дискретизировать сооружение и жидкость, используя совершенно разные, не согласованные аппроксимирующие сетки конечных элементов. Вместе с тем, очевидно, что вне зависимости от характера конечноэлементных сеток, применяемых на этапе дискретизации жидкости и сооружения, должны выполняться уравнения (5.1) и (5.2) на поверхности их контакта.

При решении связанных задач «сооружение-жидкость» часто возникает необходимость объединения фрагментов несвязанных конечноэлементных сеток, имеющих общую границу [6,7]. Это объединение проводится за счет создания связей между степенями свободы одного узла с одной или несколькими степенями свободы других узлов.

Пусть, например, перемещение UX узла $n$ составляет $30 \%$ от перемещения UX узла $k$. Математическое уравнение для этого имеет вид:

$$
U X_{k}=0.3 \cdot U X_{n}
$$

Уравнение (3.16) может быть переписано в стандартную форму уравнения ограничения:

$$
0=1 \cdot U X_{k}-0.3 \cdot U X_{n}
$$

Число, лежащее слева от знака равенства, называется постоянным числом уравнения, числа, используемые для умножения на значения степени свободы, именуются коэффициентами. Замена значения коэффициента -0.3 в уравнении (6.1) на -1, позволяет непосредственно связать перемещения UX узла $k$ с перемещениями узла $n$. Подобный тип простого уравнения ограничений имеет название «связь» и является специальным слу- 
Методика численного моделирования напряженно-деформированного состояния системы «основание - плотина - водохранилище» при сейсмических воздействиях

чаем уравнений ограничения, в котором коэффициенты всегда имеют значения 1 и -1 . Так создается решение, в котором поведение одного узла непосредственно связано с поведением других узлов.

\section{7. ЗАКЛЮЧЕНИЕ}

Описанная методика численного (конечноэлементного) моделирования связанных систем «основание - плотина - водохранилище», основанная на постановке Эйлера для жидкости, позволяет описать состояние жидкости через узловые давления (акустическая жидкость), а сооружения и основания - через узловые перемещения. Данный подход значительно упрощает уравнения гидроупругости, но для задач, где основной интерес представляет напряженно-деформированное состояние достаточно жесткого сооружения, контактирующего с жидкостью (в том числе гидросооружения), данный численный метод вполне эффективен.

Вместе с тем, очевидно, что решение связанных задач системы «сооружение-жидкость», как научное направление не является исчерпанным и попрежнему обладает актуальностью и практической значимостью, а также является предметом интенсивных теоретических и экспериментальных исследований [9-17].

\section{СПИСОК ЛИТЕРАТУРЫ}

1. Белостоцкий А.М. Численное моделирование статического динамического напряженно деформированного состояния пространственных систем «сооружение - основание водохранилище» с учетом нелинейных эффектов открытия закрытия швов и макротрещин. Дис. на соиск. учен. степ. д-ра техн. наук: 05.23.07. МГУП, М., 1998. - 367с.

2. Wilson E.L., Khalvati M. Finite elements for dynamics of fluid system. // Int. J. Num. Meth. Eng., 1983, v. 19, pp. 1657-1668.

3. Atluri S.N. Alternate stress and conjugate strain measures, and mixed variational formulations involving rigid rotations, for computational analysis of finitely deformed solids, with application to plates and shells. // I. Theory. Computers \& Structures, 1984, Vol. 18, pp. 93-116.
4. Bathe K.J. Finite Element Procedures, PrenticeHall, New York, 1996.

5. Bathe K.J., Zhang H., Zhang X. Some advances in the analysis of fluid flows. // Computers \& Structures, 1997, Vol. 64, pp. 909-930.

6. ICOLD, International Benchmark Workshop on Numerical Analysis of Dams. Theme A-Fluid Structure Interaction Arch Dam - Reservoir at Seismic loading. Graz University of Technology, 2013, pp. 15-188.

7. Белостоцкий А.М., Нгуен Тай Нанг Лыонг, Дмитриев Д.С., Щербина С.В. Сравнительный анализ методик численного моделирования динамики трехмерных систем «основание - арочная плотина - водохранилище» при сейсмических воздействиях. // International Journal for Computational Civil and Structural Engineering / Международный журнал по расчету гражданских и строительных конструкций, Volume 12, Issue 2, 2016 , pp. 65-74.

8. ANSYS Mechanical APDL. PDF Documentation for Release 15.0.

9. Белостоцкий А.М., Акимов П.А., Кайтуков Т.Б., Афанасьева И.Н., Вершинин B.B., Усманов А.P., Щербина С.B. К вопросу о численном моделировании поведении резервуара с понтоном (плавающей крышей) при сейсмическом воздействии. // International Journal for Computational Civil and Structural Engineering, Volume 10, Issue 2, 2014, pp. 6478.

10. Белостоцкий А.М., Акимов П.А., Кайтуков Т.Б., Афанасьева И.Н., Вершинин В.В., Дмитриев Д.С., Усманов А.P., Чужинов А.С., Щербина С.В. Постановка, конечноэлементная аппроксимация и алгоритмы решения задач расчетного обоснования связанных систем «сооружение - жидкость». // Строительная механика и расчет сооружений, №5, 2014, c. 21-27.

11. Белостоцкий А.М., Акимов П.А., Кайтуков Т.Б., Афанасьева И.Н., Вершинин B.B., Усманов А.P., Щербина С.В. О численном моделировании поведения связанных систем «сооружение - жид- 
кость». Часть 1: Математические постановки задач. // International Journal for Computational Civil and Structural Engineering, Volume 11, Issue 3, 2015, pp. 3746.

12. Белостоцкий А.М., Акимов П.А., Кайтуков Т.Б., Афанасьева И.Н., Вершинин В.В., Усманов А.P., Щербина С.В. О численном моделировании поведения связанных систем «сооружение - жидкость». Часть 2: Аппроксимация и численное решение. // International Journal for Computational Civil and Structural Engineering, Volume 11, Issue 3, 2015, pp. 4758.

13. Белостоцкий А.М., Акимов П.А., Кайтуков Т.Б., Афанасьева И.Н., Вершинин В.В., Усманов А.P., Щербина С.В. О численном моделировании поведения связанных систем «сооружение - жидкость». Часть 3: Особенности моделирования жидкости. // International Journal for Computational Civil and Structural Engineering, Volume 11, Issue 3, 2015, pp. 5968.

14. Белостоцкий А.М., Акимов П.А. Научно-исследовательский центр СтаДиО. 25 лет на фронте численного моделирования. // International Journal for Computational Civil and Structural Engineering / Международный журнал по расчету гражданских и строительных конструкций, Volume 12, Issue 1, 2016, pp. $8-45$.

15. Belostosky A.M., Akimov P.A., Kaytukov T.B., Afanasyeva I.N., Usmanova A.R., Scherbina S.V., Vershinin V.V. About Finite Element Analysis of Fluid - Structure Interaction Problems. // Procedia Engineering, Vol. 91 (2014) pp. 37-42.

16. Belostotskiy A.M., Akimov P.A., Afanasyeva I.N., Usmanov A.R., Scherbina S.V., Vershinin V.V. Numerical simulation of oil tank behavior under seismic excitation fluid - structure interaction problem solution. // Procedia Engineering, Vol. 111 (2015) pp. 115-120.
17. Belostosky A.M., Akimov P.A., Afanasyeva I.N. Multilevel Methodology of Numerical Seismic Analysis of Coupled Systems "Foundation - Shell - Pontoon (Floating Roof) -Column(s) - Fluid". // Procedia Engineering, Vol. 153 (2016) pp. 8994.

Белостоцкий Александр Михайлович, член-корреспондент РААСН, доктор технических наук, професcop, генеральный директор $3 \mathrm{AO}$ «Научно-исследовательский центр СтаДиО», профессор кафедры прикладной математики, научный руководитель Научнообразовательного центра компьютерного моделирования, Национальный исследовательский Московский государственный строительный университет; г. Москва ул. 3-я Ямского Поля, д.18, 8 этаж, офис 810, тел. +7 (495) 706-88-10;

e-mail: stadyo@stadyo.ru,niccm@mgsu.ru

Дмитриев Дмитрий Сергеевич, ведущий инженер-расчетчик ЗАО «Научно-исследовательский центр СтаДиО», г. Москва ул. 3-я Ямского Поля, д.18, 8 этаж, офис 810, тел. +7 (495) 706-88-10;

e-mail: stadyo@stadyo.ru

Нгуен Тай Нанг Лыонг, аспирант кафедры прикладной математики Национального исследовательского Московского государственного строительного университета; 129337, Россия, г. Москва, Ярославское шоссе, дом 26; e-mail: KafedraIPM@mgsu.ru

Alexander M. Belostotsky, Corresponding Member of Russian Academy of Architecture and Construction Sciences, Doctor of Science, Professor, Director of StaDyO Research \& Engineering Center; Science Director of Research \& Education Center of Computation Simulation, National Research Moscow State University of Civil Engineering, 26, Yaroslavskoe Shosse, Moscow, 129337, Russia; phone/fax: +7 (499) 929-50-17;

e-mail: stadyo@stadyo.ru,niccm@mgsu.ru

Dmitry S. Dmitriev, StaDyO Research \& Engineering Center; office 810, 8th floor, 18, 3-ya Ulitsa Tverskogo Polya, Moscow, 125040, Russia; tel. +7 (495) 706-88-10, e-mail: stadyo@stadyo.ru

Nguyen Tai Nang Luong, Ph.D Student, Department of Applied Mathematics and Computer Sciences, National Research Moscow State University of Civil Engineering; 26, Yaroslavskoe Shosse, Moscow, 129337, Russia; 
DOI:10.22337/1524-5845-2016-12-4-87-94

\title{
АЛГОРИТМИЧЕСКАЯ И ПРОГРАММНАЯ РЕАЛИЗАЦИЯ ЗАДАЧ ПРОЕКТИРОВОЧНОГО РАСЧЕТА СТЕРЖНЕВЫХ КОНСТРУКЦИЙ
}

\author{
Т.Л. Дмитриева, Ле Чан Минь Дат \\ Иркутский национальный исследовательский технический университет, г. Иркутск, РОССИЯ
}

\begin{abstract}
Аннотация: Разработан алгоритм оптимального проектирования пространственных стержневых систем. Алгоритм основан на совместном использовании метода конечных элементов и методов нелинейного программирования. Решены прикладные задачи.
\end{abstract}

Ключевые слова: оптимальное проектирование конструкций, нелинейное программирование, метод конечных элементов, пространственные стержневые системы

\section{ALGORITHMIC AND PROGRAMMATIC REALIZATION FOR TASK PROJECTING CALCULATION DESIGNS OF ROD STRUCTURES}

\author{
Tatyana L. Dmitrieva, Le Tran Minh Dat \\ Irkutsk National Research Technical University, Irkutsk, RUSSIA
}

\begin{abstract}
A design optimization algorithm of the spatial structure has been developed. The algorithm is based on the finite element method and the nonlinear programming method. Applied tasks were solved.
\end{abstract}

Key words: optimum design of structures, nonlinear programming, the finite element method, spatial structure

\section{1. СВЯЗЬ ЗАДАЧИ ПРОЕКТИРОВОЧНОГО РАСЧЕТА С ЗАДАЧАМИ ОПТИМИЗАЦИИ}

Вопросы оптимального проектирования, очень популярные и активно исследуемые начиная со второй половины XX века, в настоящее время часто вызывают некоторый скепсис. Это связано, с одной стороны, со сложностью их алгоритмической и программной реализации, а с другой, с проблемами их восприятия со стороны инженеров-проектировщиков.

Между тем, разработка любого проекта подразумевает его оптимальное решение. Проектировщик решает эту задачу, используя свой либо чужой опыт, а также вариантный подход. Поэтому задачу оптимального проектирования можно сформулировать как задачу формализации проектировочного расчета конструкций согласно некоторому заданному критерию оптимальности (или рациональности). Причем в нашем случае предполагается, что оптимизация выполняется не с нуля, а в рамках некоторого заданного проекта и служит инструментом для его улучшения.

Алгоритм решения такой задачи выдвигает определенные требования к подготовке исходных положений, которые можно сформулировать следующим образом:

- геометрические и физические параметры исходного проекта должны быть разделены на фиксированные и варьируемые на заданном диапазоне;

- требуется выбрать и формализовать критерий оптимальности в виде функции варьируемых параметров;

- необходимо определить перечень требований к поведению конструкции; 
- задаются погрешности в соблюдении этих требований.

Итак, прежде всего, проектировщику предстоит разделить все параметры конструкций сооружения на варьируемые, которые необходимо подобрать на заданном интервале, и уже принятые, т.е. фиксированные. Выбор варьируемых параметров зависит от материала и вида конструкции. Это могут быть не только параметры сечений, но и геометрические параметры сооружения (высота этажа, размер пролета, угол наклона стропильных конструкций и т.д.). Они могут изменяться как на непрерывном, так и на дискретном интервале, например, согласно сортаментам. При этом надо понимать, что с учетом типоразмеров число варьируемых параметров даже для сложных сооружений должно быть достаточно ограничено, измеряясь десятками, но не сотнями.

Проектировочный расчёт предполагает подбор варьируемых параметров согласно принятому критерию (назовем его критерий оптимальности). Большинство известных алгоритмов оптимизации используют в качестве такого критерия расход материала конструкций, хотя это может быть любой другой критерий, который можно формализовать как функцию варьируемых параметров. Самым полноценным критерием, безусловно, является стоимостной, но формализовать его в полной мере с учетом расходов на технологию возведения сооружения, транспортных расходов и т. п. достаточно сложно.

Далее, из предлагаемого списка необходимо сформировать требования, предъявляемые к поведению проектируемой конструкции. Это, как правило, стандартные нормативные требования по прочности, жесткости и устойчивости, формализованные в виде ограничений неравенств.

И наконец, необходимо задать допустимую погрешность в реализации этих требований.

Таким образом, задачу оптимального проектирования можно представить как задачу автоматизации проектировочного расчета, где должен быть выполнен заданный критерий оптимальности при условии выполнения заданных требований к поведению конструкции с заданной точностью.

\section{2. ФОРМАЛИЗАЦИЯ ЗАДАЧИ ПРОЕКТИРОВОЧНОГО РАСЧЕТА ПРИ СТАТИЧЕСКИХ ВОЗДЕЙСТВИЯХ}

Приведём формализацию задачи проектировочного расчета в форме задачи нелинейного программирования:

$$
\begin{aligned}
& \text { Найти min } f(x, D(x)), \quad x \in E^{n x}, \\
& \text { при ограничениях } \\
& g_{j}(x, D(x)) \leq 0, \quad j=1,2 \ldots m, \\
& x_{i}^{L} \leq x_{i} \leq x_{i}^{U}, \quad i=1,2 \ldots n x .
\end{aligned}
$$

Критерий оптимальности здесь реализован в виде целевой функции $f(x)$, которую для получения оптимального решения необходимо минимизировать.

Вектор варьируемых параметров обозначен как вектор основных неизвестных $X$. Интервал изменения этих параметров определяется векторами $X^{L}-X^{U}$.

Функции ограничений $g_{j}(x)$ формализуют нормативные требования по прочности и устойчивости. Предусмотрены также ограничения на перемещения узлов.

Важной особенностью постановки задачи (1-3) является тот факт, что функции ограничений имеют неявную связь с варьируемыми параметрами через параметры состояния $D(x)$, которые могут представлять собой перемещения узлов $(\delta)$, внутренние силовые факторы $(s)$, напряжения $(\sigma)$, деформация $(\varepsilon)$ и т.д.:

$$
\{D(x)\}=\varphi(\delta, s, \sigma, \varepsilon)
$$

определяемые решением задачи конечноэлементного анализа в линейной постановке:

$$
[K(x)]\{\delta\}=\{F(x)\}
$$




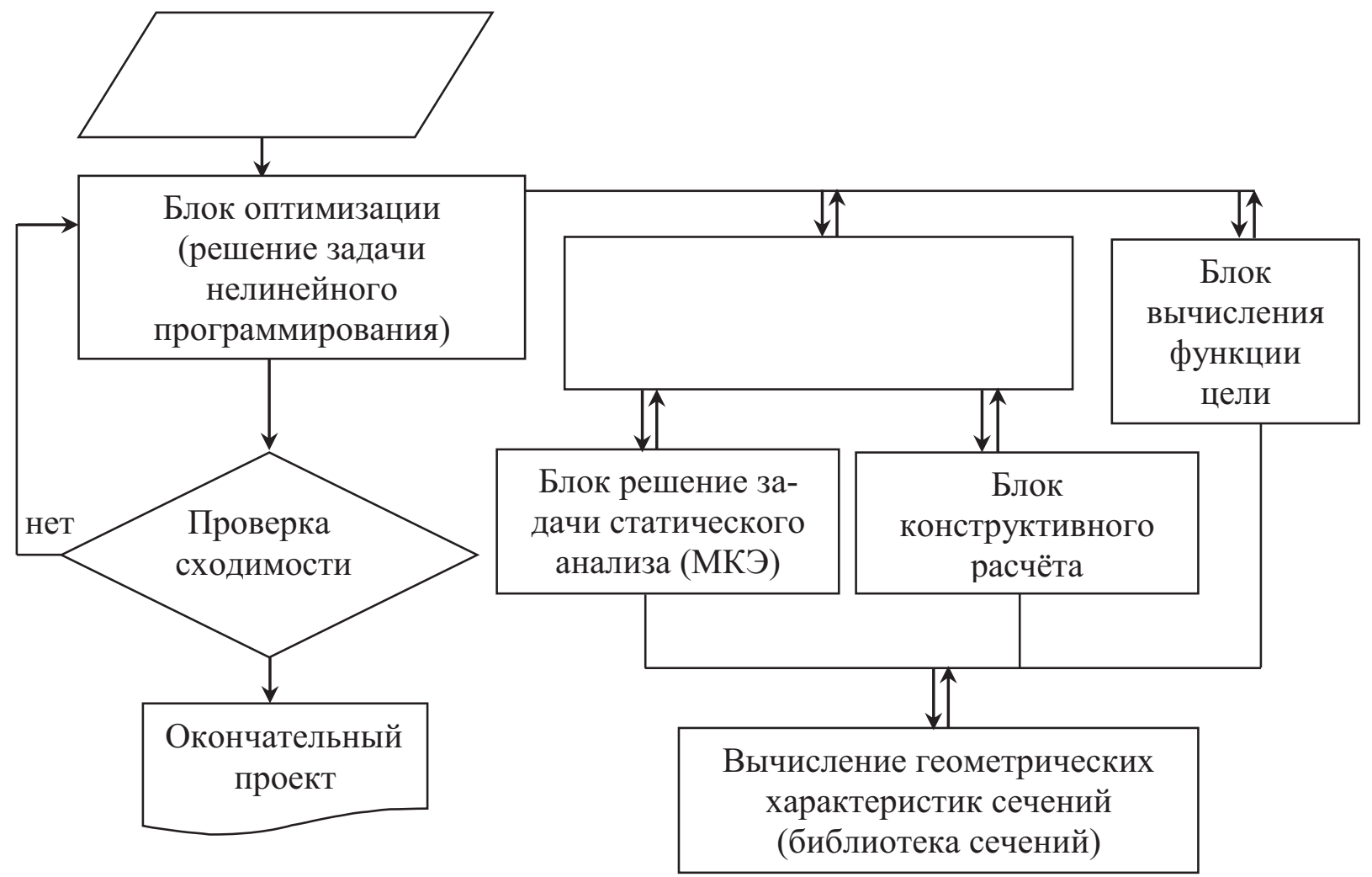

Рисунок 1. Алгоритм проектировочного расчета согласно заданному критерию оптимальности.

где $[K(x)]$ - матрица жесткости системы; $\{F(x)\}$ - вектор внешней нагрузки

\section{3. АЛГОРИТМ ЗАДАЧИ ПРОЕКТИРОВОЧНОГО РАСЧЕТА}

На рис. 1 показана блок-схема алгоритма решения этой задачи, каждый блок которой может функционировать автономно, что повволяет легко расширять возможности этого алгоритма. Особенность данного подхода состоит в том, что каждое обращение к функциям ограничений $g(x)$ требует решение задачи конечно-элементного анализа. Алгоритм дает хорошую сходимость на внешних итерациях поискового процесса, но требует больших вычислительных затрат для задач, где конечно-элементная модель имеет большую размерность.

Основным блоком алгоритма является блок оптимизации, где реализовано решение стандартной задачи нелинейного программирования [1]

найти $\min f(x), x \in E^{n x}$,

при ограничениях

$$
\begin{gathered}
g_{j}(x) \leq 0, \quad j=1,2, \ldots, m ; \\
x_{i}^{L} \leq x_{i} \leq x_{i}^{U}, \quad i=1,2, \ldots, n x .
\end{gathered}
$$

Поисковые алгоритмы оптимизации, заложенные в этом блоке, основаны на сведении условно-экстремальной задачи (4-6) к задаче на безусловный экстремум. Такой переход возможен с помощью функции Лагранжа:

$$
F_{L}(x)=f(x)+\{Y\}^{T}[\delta]\left\{g^{*}\right\},
$$


где $\{Y\}$ - множители Лагранжа (двойственные переменные); $[\delta]$ - диагональная матрица, элементы которой $\delta_{j j}=1$, если соответствующее ограничение является активным, иначе, $\delta_{j j}=0$.

Однако область применения функции ограничивается задачами выпуклого программирования, что не всегда имеет место в задачах оптимизации конструкций. Для построения алгоритмов, обладающих более широкой областью сходимости, функцию Лагранжа модифицируют путем добавления к ней штрафа за невыполнение условий (5). Модифицированная функция Лагранжа (МФЛ) $F p$ имеет вид:

$$
F_{P}(x)=k_{f} \cdot F_{L}(x)+P(x),
$$

где $P(x)$ - штрафная добавка:

$$
P(x)=0,5\left\{g^{*}\right\}^{T}[\delta][k]\left\{g^{*}\right\} .
$$

Выражения (8) и (9) содержат регулирующие коэффициенты: $k_{f}$ - коэффициент нормировки функции Лагранжа; $[k]$ - диагональная матрица штрафных коэффициентов.

На основе алгоритма, приведенного на рис. 1, разработаны программные комплексы оптимального проектирования стальных и железобетонных конструкций $[2,3,5,5]$, которые позволяют решать задачи проектировочного расчета реальных строительных конструкций. Приведем некоторые примеры. Пример 1. Оптимизация 25-ти стержневой пространственной металлической конструкции (рис. 2 а, б).

Рассмотрена тестовая задача оптимизации пространственной конструкции при двух случаях загружений силовой статической нагрузкой (рис. 2 a, б).

- первый случай загружения: $F_{1}=22,25$ кН, $F_{2}=89$ кН;

- второй случай загружения: $F_{1}=22,25 \mathrm{\kappa H}, F_{3}$ $=4,45 \mathrm{\kappa H}, F_{4}=44,5 \mathrm{\kappa H}, F_{5}=2,225 \mathrm{\kappa H}$.

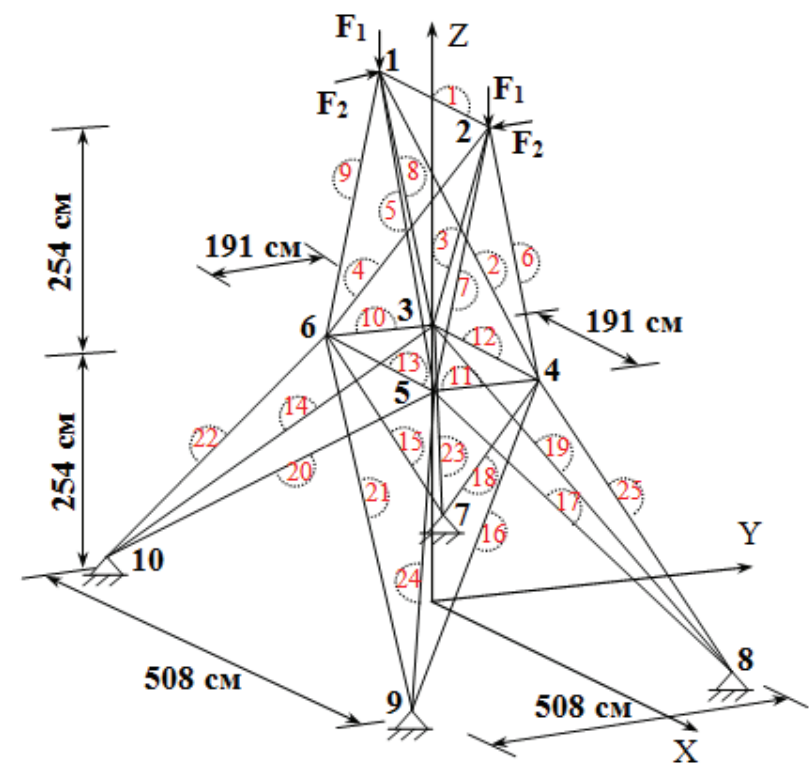

Рисунок 2,a. Первый случай загружения.

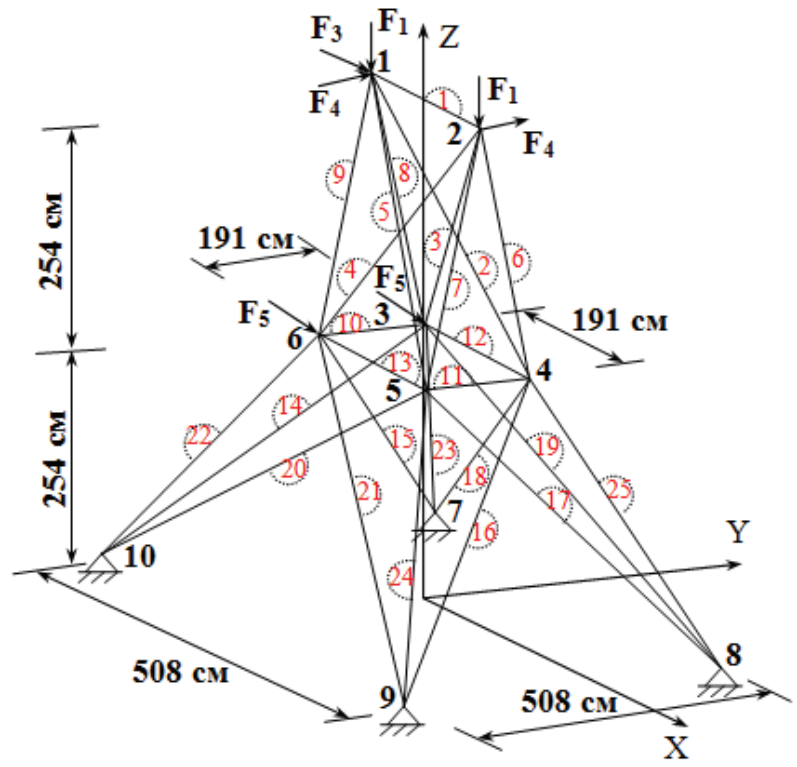

Рисунок 2,б. Второй случай загружения.

Элементы конструкции сгруппированы по типу сечений (8 групп).

Целевая функция представляет собой объём конструкции:

$$
f(x)=\sum_{i=1}^{25} A_{i}(x) \cdot l_{i}(x)
$$

где $A_{i}(x), l_{i}(x)$ - площадь поперечного сечения и длина $i$-ого элемента. 
Таблица 1.Сравнение полученных результатов оптимизации

\begin{tabular}{|c|c|c|c|c|c|c|c|}
\hline \multirow{2}{*}{ Показатели } & \multicolumn{2}{|c|}{ Вариант 1} & \multicolumn{2}{|c|}{ Вариант 2} & \multirow{2}{*}{$\begin{array}{l}\text { Решение } \\
\text { в ANSYS }\end{array}$} & \multirow{2}{*}{$\begin{array}{c}\text { Адели и } \\
\text { Камаль }\end{array}$} & \multirow{2}{*}{$\begin{array}{l}\text { Адели, } \\
\text { Х. Парк }\end{array}$} \\
\hline & Случай 1 & Случай 2 & Случай 1 & Случай 2 & & & \\
\hline Сечение & 0 & $\square$ & 0 & $\square$ & O & - & - \\
\hline $\begin{array}{l}\text { Координаты } \\
\text { узлов } 3,4,5 \text {, } \\
6 \quad \text { по оси } Z\end{array}$ & $254 \mathrm{~cm}$ & $254 \mathrm{~cm}$ & $280 \mathrm{~cm}$ & $270 \mathrm{~cm}$ & $254 \mathrm{~cm}$ & $254 \mathrm{~cm}$ & $254 \mathrm{~cm}$ \\
\hline $\begin{array}{l}\text { Число } \\
\text { итераций }\end{array}$ & 6 & 4 & 3 & 3 & 8 & - & 77 \\
\hline $\begin{array}{l}\text { Число } \\
\text { обращений к } \\
\text { функциям } \\
\text { ограничений }\end{array}$ & 16743 & 13285 & 7027 & 13178 & - & - & - \\
\hline $\begin{array}{l}\text { Оптимальный } \\
\text { объём, } \text { см }^{3}\end{array}$ & 88134,02 & 87418,36 & 79024,99 & 82784,12 & 87784,12 & 89425,20 & 90383,19 \\
\hline Объём \% & $97,51 \%$ & $96,8 \%$ & $87,43 \%$ & $91,59 \%$ & $97,12 \%$ & $98,94 \%$ & $100 \%$ \\
\hline
\end{tabular}

Функиии ограничений:

а) проверка на прочность $i$-й группы конструкции:

$g_{j}(x)=N_{i}(x) /\left(\left[\sigma_{i}\right] \cdot A_{i}(x)\right)-1 \leq 0, \quad i=1,2, \ldots, 16$

Здесь $N_{i}(x)$ - значение продольной силы базового элемента в $i$-ой группе.

$\left[\sigma_{i}\right]$ - предельные напряжения для сжатых и растянутых элементов в каждой группе.

б) ограничение в виде допусков на перемещение узлов 1, 2 по направлениям $X, Y$ :

$g_{k}(x)=\delta_{x i}(x) /[\delta]-1 \leq 0, i=1,2 ; k=17,19,21,23$.

$g_{h}(x)=\delta_{y i}(x) /[\delta]-1 \leq 0, i=1,2 ; \quad h=18,20,22,24$.

где $[\delta]=0,89 \mathrm{~cm}$.

Для обеспечения устойчивости работы алгоритмов в качестве метода безусловной минимизации использован метод деформируемого многогранника. Максимальная невязка в ограничениях задана $\varepsilon_{g}=10^{-4}$. Задача решена в двух вариантах.

Вариант 1. Варьирование параметрами сечений элементов.

Число варьируемых параметров - 8; общее число ограничений -24 .

Вариант 2. Варьирование параметрами сечений элементов и координатами узлов Кроме варьирования параметрами сечений, здесь варьируется - координата группы узлов 3, 4, 5, 6 по направлению $Z$ с дискретным шагом 10 см. Число варьируемых параметров увеличивается до 9-ти; общее число ограничений - 24.

В каждом варианте рассмотрены два случая, в которых прияты кольцевое и коробчатое сечение.

Выполнено сравнение результатов оптимальных решений в 2-х вариантах с результатами, полученными в других источниках. Данные приведены в табл. 1. Отметим, что варьирование координатой узлов конструкции привело к уменьшению её объёма.

На основе полученных исследований сделан вывод, что использование алгоритмов НЛП на основе модифицированной функции Лагранжа дают лучшую сходимость и результативность чем алгоритмы, заложенные в других источниках.

Пример 2. Оптимизация пространственного каркаса двухэтажного здания

Здесь варьируются параметры сечений, а также угол наклона ригеля каркаса $\alpha$ (рис. 3 ). 
Таблица 2. Результаты оптимизации пространственного каркаса.

\begin{tabular}{|l|c|c|}
\hline \multicolumn{1}{|c|}{ Показатели } & Вариант 1 & Baриант 2 \\
\hline Угол наклона $\alpha$ (град.) & $27^{0}$ & $18^{0}$ \\
\hline Число итераций & 5 & 10 \\
\hline Число обращения к функциям ограничений & 170163 & 4896 \\
\hline Макс. невязка в ограничениях & $-0,46 \cdot 10^{-3}$ & $-0,87 \cdot 10^{-2}$ \\
\hline Объем $\left(\mathrm{cm}^{3}\right)$ & 986987,702 & 1065521,59 \\
\hline Объем $(\%)$ & $79,69 \%$ & $100 \%$ \\
\hline
\end{tabular}

Приняты следующие пределы изменения угла этого угла: $\alpha_{\min }=5^{0}, \alpha_{\max }=45^{0}$.

Координаты остальных узлов при этом фиксированы.

Минимизируется объём каркаса:

$$
f(x)=\sum_{i=1}^{34} A_{i}(x) \cdot l_{i}(x),
$$

где $A_{i}(x), l_{i}(x)$ - площадь поперечного сечения и длина $i$-го элемента.

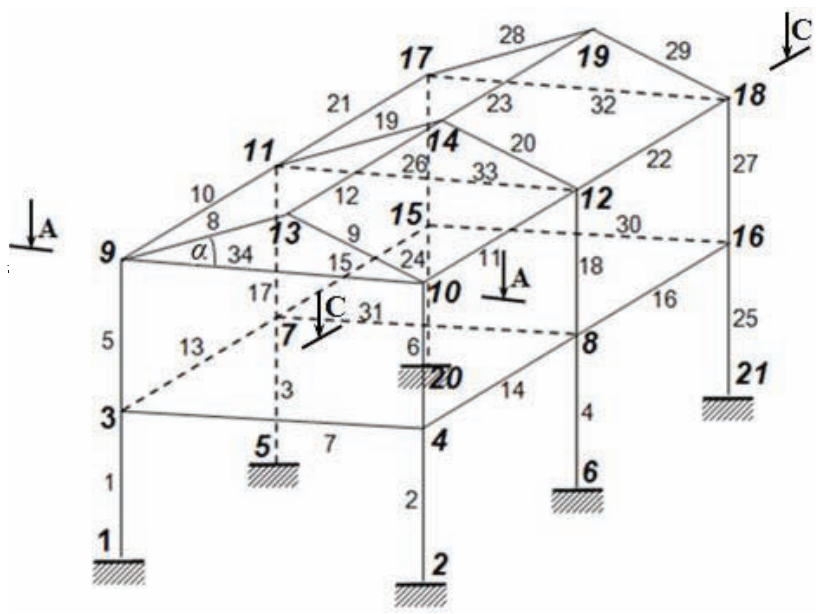

Рисунок. 3. Расчетная схема пространственного каркаса.

Ограничения представлены в виде:

a) проверок по прочности и местной устойчивости согласно нормам СП 16.13330.2011 «Стальные конструкции»;

б) допусков на перемещение узлов 9, 11, 17 по направлениям $x$ и $y$.

Задача решена в двух вариантах.

Вариант 1. Непрерывное изменение варьируемых параметров сечений.
Вариант 2. Параметры сечений принимались согласно сортаменту.

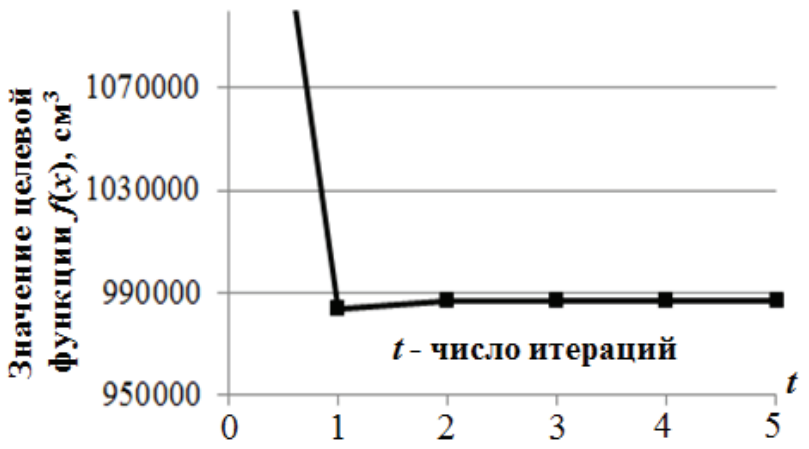

Рисунок 4. Изменение целевой функиии на итерациях (вариант 1).

На рис. 4 показано изменение целевой функции на итерациях при непрерывном изменении параметров (вариант 1).

Видно, что уже первая итерация поискового процесса даёт решение близкое к оптимальному. Выполнено исследование оптимального решения на единственность для варианта 1. Результаты показали, что получено практическое совпадение оптимальных значений целевой функции (разброс составил $0,0016 \%)$.

В дискретном варианте получено 3 локальных решения, дающих значения целевой функции с разбросом в 5,7\%. В этом случае оптимальный проект может быть выбран с точки зрения таких показателей, как наименьшее значение целевой функции, высокая точность в невязках ограничений и т.д. Оптимальный объём конструкции в первом варианте имеет отличие по сравнению со вторым на 7,3\%. 
Таблица 3. Сравнение полученных результатов оптимизации.

\begin{tabular}{|l|c|c|c|}
\hline \multicolumn{1}{|c|}{ Показатели } & $\begin{array}{c}\text { Непрерывное } \\
\text { варьирование }\end{array}$ & $\begin{array}{c}\text { Дискретное } \\
\text { варьирование }\end{array}$ & $\begin{array}{c}\text { Значение } \\
\text { в типовом проекте }\end{array}$ \\
\hline Число итераций & 2 & 6 & - \\
\hline $\begin{array}{l}\text { Число обращения к функциям } \\
\text { ограничений }\end{array}$ & 151486 & 20925 & - \\
\hline Невязка в ограничениях $\mathrm{g}_{\max }$ & $0,33 \cdot 10^{-4}$ & $-0,49 \cdot 10^{-3}$ & 304528,302 \\
\hline Объем опоры $\left(\mathrm{cm}^{3}\right)$ & 232837,32 & 273525,29 & 2421 \\
\hline Масса опоры (кг) & 1851,1 & 2174,5 & $100 \%$ \\
\hline Масса \% & $76,46 \%$ & $89,82 \%$ & - \\
\hline
\end{tabular}

Пример 3. Оптимизачии опоры линии электропередачи напряжением $35 \kappa B$.

Задача имеет существенно большую размерность по сравнению с предыдущими примерами (255 элементов и 97 узлов).

Целевая функиия:

$$
f(x)=\sum_{i=1}^{255} A_{i}(x) \cdot l_{i}
$$

где $A_{i}, l_{i}$ - площадь поперечного сечения и длина $i$-го элемента опоры.

Функции ограничений представлены в виде проверки по прочности и жесткости (допуска на перемещение узлов $41,79,51,97)$. Задача решена в непрерывном и дискретном изменении варьируемых параметров.

а) Непрерывное изменение параметров сечений. В этом варианте для каждого случая загружения варьировалась ширина (высота) равнополочного уголка, толщина принималась как часть ширины $t=H / 12$. Число варьируемых - 8; число ограничений - 36; максимальная невязка в ограничениях $-10^{-3}$.

б) Дискретное изменение параметров сечений. В реальном проектировании опор ЛЭП параметры сечения меняются дискретно, поэтому в данном случае сечение варьировалось согласно сортаментам. Число варьируемых 8 ; число ограничений - 36 ; максимальная невязка в ограничениях $-10^{-2}$.

Рассмотрено сравнение полученных оптимальных решений со значениями, соответствующими типовому проекту (100\%) (табл. 3). Авторский результат продемонстрировал снижение объема, вычисленного при дискретном варьировании параметров сечений, по сравнению с типовым проектом на $10 \%$.

Таким образом, данный пример иллюстрирует, что разработанный алгоритм и программный комплекс оптимизации позволяет решать практические задачи проектировочного расчета реальных конструкций, получая при этом оптимальные проекты с заданной точностью в невязках ограничений согласно заданному критерию оптимальности.

\section{4. ОСНОВНЫЕ ВЫВОДЫ}

Предложен алгоритм решения задач проектировочного расчета конструкций с заданным критерием оптимальности, соединяющий в себе блок конструктивного расчета, блок конечно-элементного анализа и блок оптимизации. Каждый из этих блоков может функционировать автономно, что дает широкую возможность к его совершенствованию, как в области добавления нормативных требований к конструкциям различного вида, так и в направлении развития конечноэлементной модели. Рассмотренные примеры проектировочного расчета пространственных металлических конструкций с большим количеством узлов позволили выявить особенности предложенного алгоритма. 


\section{СПИСОК ЛИТЕРАТУРЫ}

1. Дмитриева Т.Л., Ле Чан Минь Дат, Нгуен Ван Ты. Реализация алгоритмов численной оптимизации в современных программных комплексах. - Иркутск: издво ИРНИТУ, 2015. - 160 с.

2. Дмитриева Т.Л. Алгоритм автоматизированного проектирования ферм минимального веса. // Известия вузов. Строительство. № 3, 2010, с. 98-105.

3. Дмитриева Т.Л. Программный комплекс расчета и оптимизации строительных конструкций «РОСК». // Известия вузов. Инвестиции. Строительство. Недвижимость.№1(1), 2011, с. 31-38.

4. Дмитриева Т.Л., Ле Чан Минь Дат. Решение тестовых задач оптимизации пространственных металлических конструкций. // «Современные технологии. Системный анализ. Моделирование»: науч. журнал. ИрГУПС, № 4 (48), 2015, с. 14-20.

5. Дмитриева Т.Л., Нгуен Ван Ты. Математическое моделирование процессов автоматизированного проектирования железобетонных конструкций каркасов многоэтажных зданий с оптимальными параметрами. // Известия вузов. Строительство. № 1 (673), 2015 , с. 5-14.

6. Дмитриева Т.Л., Ле Чан Минь Дат. Алгоритмы решения задачи оптимизации пространственной металлической конструкции и их программная реализация. // Вестник ИрГТУ, №4(111), 2016, c. $75-82$.

Дмитриева Татьяна Львовна, доктор технических наук, профессор кафедры сопротивления материалов ФГБОУ ВО «Иркутский национальный исследовательский технический университет», Россия, 664074, г. Иркутск, ул. Лермонтова, 83, e-mail: dmitrievat@list.ru.

Ле Чан Минь Дат, аспирант кафедры сопротивления материалов и строительной механики ФГБОУ ВО «Иркутский национальный исследовательский технический университет», Россия, 664074, г. Иркутск, ул.
Лермонтова 83, e-mail: letranminhdat@gmail.com.

Dmitrieva Tatiana Lvovna, Doctor of Technical Sciences, Professor, Department of the strength of materials and structural mechanics, Irkutsk National Research Technical University, Russia, 664074, Irkutsk City, street Lermontova, 83, e-mail: dmitrievat@list.ru.

Le Tran Minh Dat, PhD student at Department of the strength of materials and structural mechanics, Irkutsk National Research Technical University, Russia, 664074, Irkutsk City, street Lermontova, 83, e-mail: letranminhdat@gmail.com 


\title{
ЧИСЛЕННЫЕ ИССЛЕДОВАНИЯ ЭЛЕМЕНТОВ УСИЛЕНИЯ ЖЕЛЕЗОБЕТОННЫХ КОЛОНН
}

\author{
Л.В. Енджиевский, Ю.С. Сергиенко \\ Сибирский Федеральный университет, г. Красноярск, РОССИЯ
}

\begin{abstract}
Аннотация: Выполнены численные исследования элементов стального упора на основе которых можно подобрать оптимальную конструкцию детали при предполагаемом воздействии на нее домкрата.
\end{abstract}

Ключевые слова: усиление, железобетонная колонна, конечный элемент, численные исследования

\section{NUMERICAL STADY OF ELEMENTS STRENGTHENING REINFORCED CONCRETE COLUMNS}

\author{
L.V. Endzhievsky, Y.S. Sergienko \\ Siberian Federal University, Krasnoyarsk, RUSSIA
}

\begin{abstract}
The numerical study of the steel elements will go on the basis of which it is possible to choose the optimal design details in the alleged action of a jack.
\end{abstract}

Keywords: strengthening, reinforced concrete column, finite element, numerical study

Из большого многообразия вариантов усиления железобетонных колонн обращено внимание на стальные преднапряженные обоймы. Для создания предварительного напряжения мы разработали способ [1], в котором одним из ключевых элементов является инвентарный стальной упор, передающий нагрузку от домкрата на стальную обойму в момент создания преднапряжения. Предлагаемый способ предполагает использование двух стальных съемных упоров, устанавливаемых с двух торцов усиливаемой колонны, которые после завершения исследований демонтируются с целью повторного использования при усилении других колонн, включая и использование на других объектах. Конструкция упора должна быть минимальная по весу с обеспечением несущей способности и долговечности даже в условиях монтажа с максимально допустимыми отклонениями.

В способе создания предварительного напряжения [1] элементы упора запроектированы в виде горизонтальной пластины с ребром жесткости, на которую будет осу- ществляться воздействие перемещаемого штока домкрата и двух вертикальных пластин, между которыми устроена поперечная упорная планка обоймы колонны (рис. 1).

Численные исследования элементов упора выполнены в программных комплексах SCAD Office и ЛИРА-САПР. Моделирование расчетной схемы, включающей поперечную планку стальной обоймы, упорный элемент и сварные швы, выполнялось из конечных элементов 36 и 34 типа (8-ми и 6-ти узловые изопараметрические конечные элементы) (рис. 2).

Определение предельной допустимой нагрузки на элементы стального упорного устройства и анализ напряженнодеформированного состояния, оптимальных параметров детали и сварных швов выполнялись в указанных программных комплекcax. Размеры и исследуемые параметры стального упора приведены на рис. 3.

Результаты исследований - максимальные напряжения в элементах стального упора и места их концентрации (рис. 4), перемеще- 

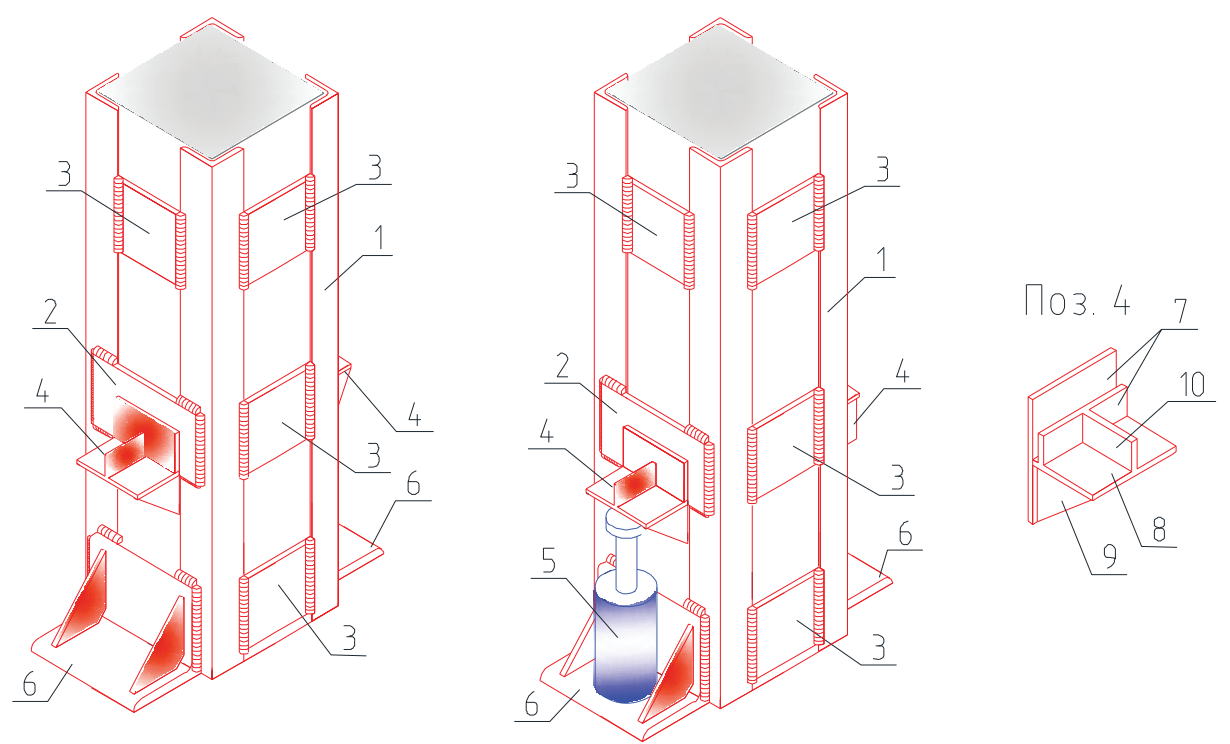

Рисунок 1. Схема создания предварительного напряжения при усилении средней колонны с помощью упорных элементов: 1 - вертикальные уголки стальной обоймы; 2 -поперечные планки, для установки стального упора; 3 - поперечные планки стальной обоймы;;

4 - упорный элемент; 5 - домкрат; 6 - нижний опорный уголок;

7 - вертикальные пластины; 8-горизонтальные пластины; 9 - нижние ребра жесткости; 10 - верхнее ребро жесткости.

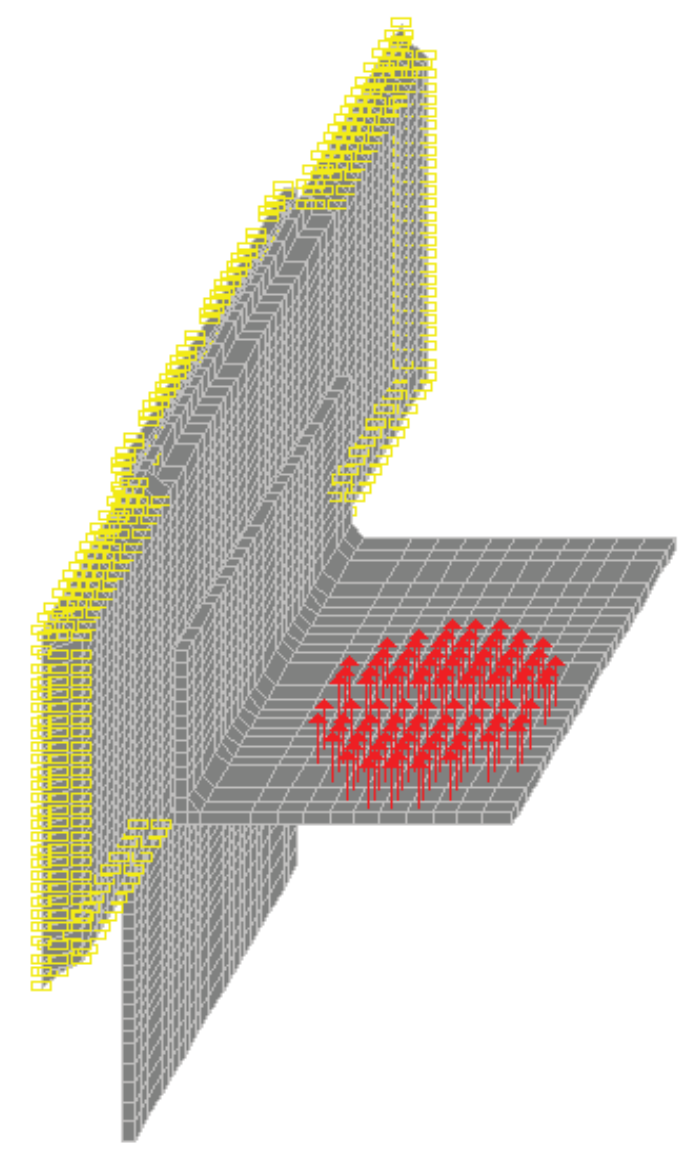

Рисунок 2. Расчетная схема стального упора без ребер жесткости. 

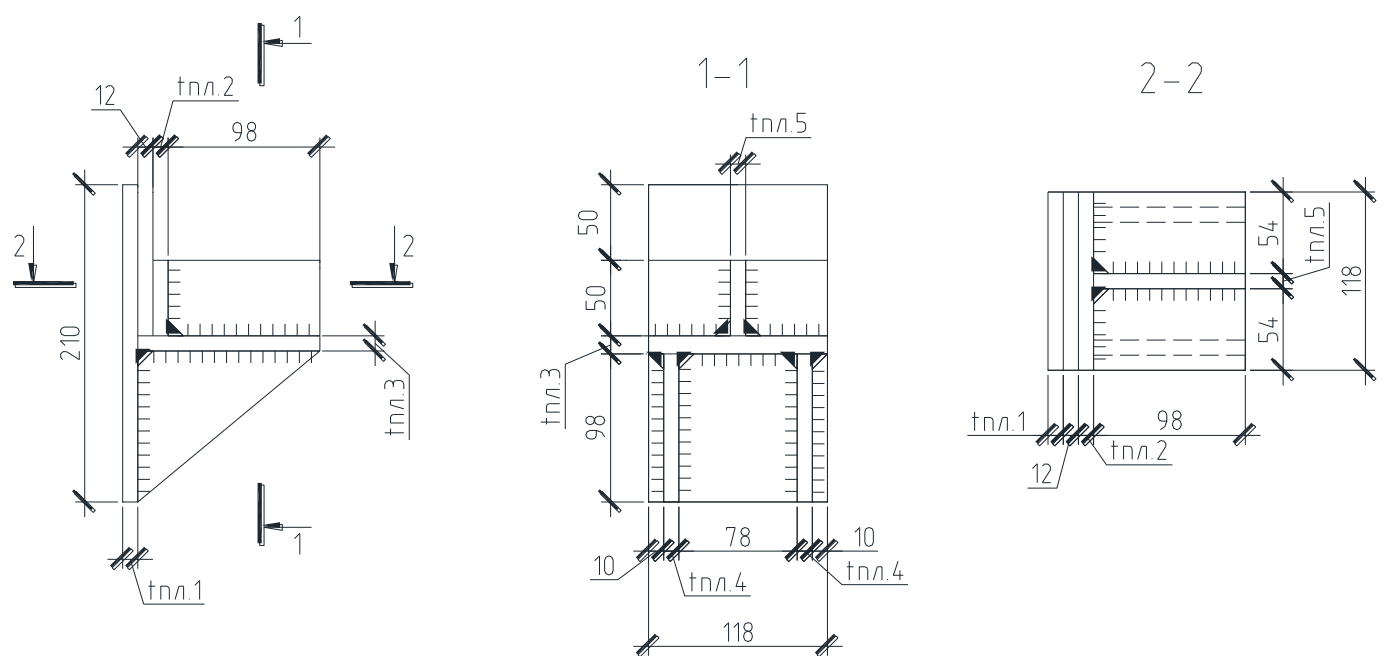

Рисунок 3. Размеры и исследуемые параметры стального упора.

tn1, tn2 - толщины вертикальных пластин стального упора,

tn3 - толщина горизонтальной пластины, tn4 - толщины нижних вебер жесткости, tn5 - верхнего ребра жесткости
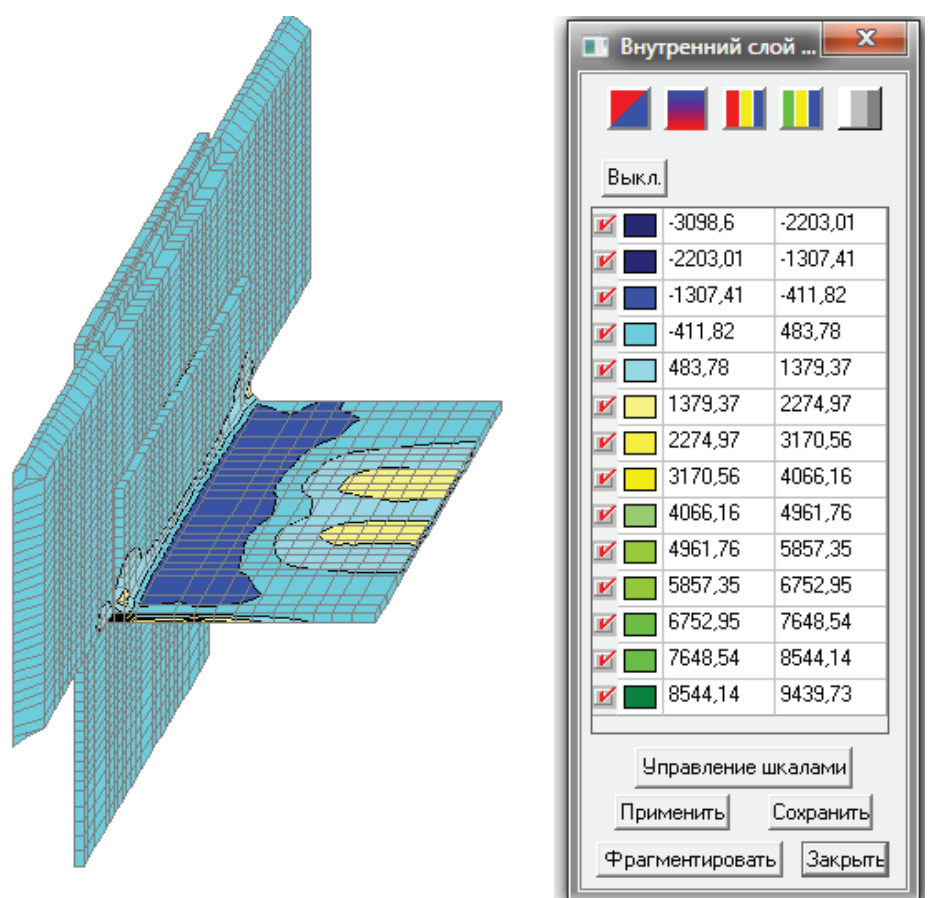

Рисунок 4. Изополя и изолинии главных напряжений $S 1$ при нагрузке 7,5 m, H/мм² Максимальное напряжение 373,4 Mnа.

ния (рис. 5) для определения предельно допустимой нагрузки на упорный элемент, варьируя характеристики упорного элемента. Результаты численных исследований приведены в табл. 1. При максимальном напряжении разрушающая нагрузка составила 13,41 тс. Сталь принималась марки С390 по ГОСТ 27772.

На основе полученных результатов можно подобрать оптимальную конструкцию детали при предполагаемом воздействии на нее домкрата. 

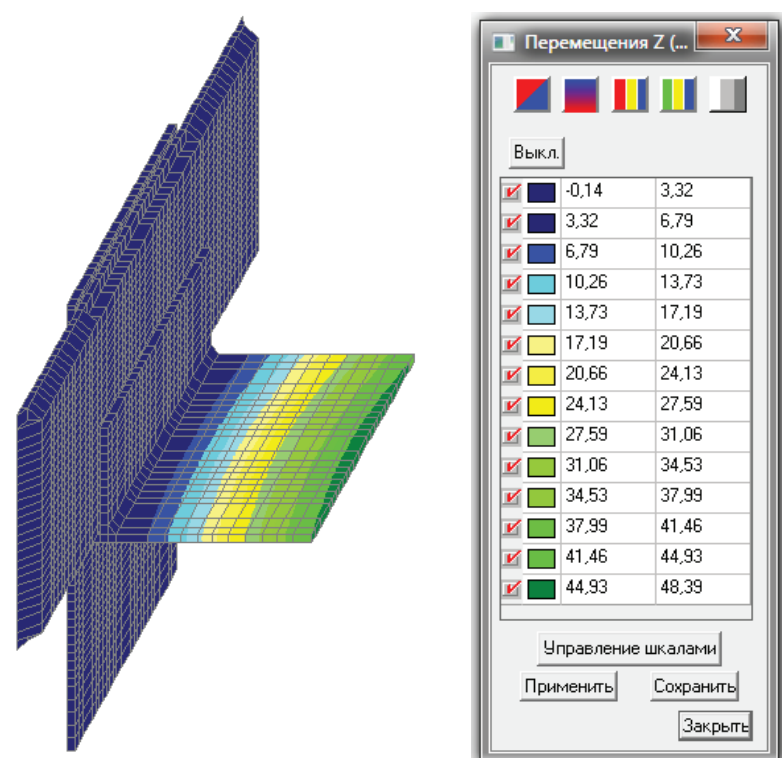

Рисунок 5. Изополя перемещений элементов стального упора при нагрузке 7,5 m, мм.

Таблииа 1. Результаты численных исследований.

\begin{tabular}{|c|c|c|c|c|}
\hline $\begin{array}{c}\text { Толщины } \\
\text { пластин упора, } \\
\text { мм }\end{array}$ & $\begin{array}{c}\text { Максимальная } \\
\text { нагрузка } \\
\text { от домкрата, т }\end{array}$ & $\begin{array}{c}\text { Ребро жесткости } \\
\text { верхнее }\end{array}$ & $\begin{array}{c}\text { Ребра } \\
\text { жесткости } \\
\text { нижние }\end{array}$ & $\begin{array}{c}\text { Катет } \\
\text { сварного } \\
\text { шва, мм }\end{array}$ \\
\hline 4 & 7,5 & - & - & 4 \\
\hline 4 & 8,63 & - & + & 4 \\
\hline 4 & 9,58 & + & + & 4 \\
\hline 5 & 9,75 & - & - & 5 \\
\hline 5 & 10,81 & - & + & 5 \\
\hline 5 & 11,69 & + & + & 5 \\
\hline 6 & 11,25 & - & + & 6 \\
\hline 6 & 12,32 & - & + & 6 \\
\hline 6 & 13,41 & + & + \\
\hline
\end{tabular}

\section{СПИСОК ЛИТЕРАТУРЫ}

1. Устройство для усиления несущих конструкций [Текст] : пат. 2555179 Рос. Федерация : МПК Е 04 G23/02 / Юрченко А.А., Енджиевский Л.В., Сергиенко Ю.С.; заявитель и патентообладатель Федеральное государственное автономное образовательное учреждение высшего профессионального образования «Сибирский федеральный университет». № 2014119735/03; заявл. 16.05.2014 ; опубл. 10.07.2015, Бюл. № 9.
Сергиенко Юлия Сергеевна, аспирант кафедры «Строительных конструкций и управляемых систем, Сибирский федеральный университет; 660041, г. Красноярск, пр. Свободный 82, кор. «А», ауд.4-04; тел.:8 (391) 206-26-92; e-mail: SDeordiev@sfu-kras.ru

Енджиевский Лев Васильевич, член-корреспондент PAACH, профессор, доктор технических наук.

Y.S. Sergienko, Ph.D. student, Department of Construction Structures and Control Systems, Siberian Federal University; apt 4-04, Building “A”, Krasnoyarsk, 660041, Russia; tel. +7(391)206-26-92;

e-mail: SDeordiev@sfu-kras.ru

Lev V. Endzhievsky, Corresponding Member of the Russian Academy of Architecture and Construction Sciences, Professor, Doctor of Technical Sciences. 
DOI:10.22337/1524-5845-2016-12-4-99-102

\title{
РАСЧЕТ БЕСКОНЕЧНОЙ ЛЕДОВОЙ ПЛИТЫ, ОСЛАБЛЕННОЙ ОТВЕРСТИЕМ
}

\author{
Е.Б. Коренева \\ Национальный исследовательский Московский государственный строительный университет, \\ г. Москва, РОССИЯ
}

\begin{abstract}
Аннотация: Изучается ледовая плита с отверстием, имеющим форму круга. Эта пластина рассматривается как бесконечная; ее толщина плавно уменьшается по направлению к краю отверстия. Предлагается аналитическая методика; получены решения в замкнутом виде.
\end{abstract}

Ключевые слова: бесконечная ледовая плита, отверстие, переменная толщина.

\section{ANALYSIS OF AN INFINITE ICE PLATE WITH AN OPENING}

\author{
Elena B. Koreneva \\ National Research Moscow State University of Civil Engineering, Moscow, RUSSIA
}

\begin{abstract}
Ice plate with an opening in a form of a circle is under study. The mentioned plate is considered as an infinite one. Its thickness decrease by the direction of the boundary of the opening. Analytical method is proposed. The solutions are given in the closed form.
\end{abstract}

Key words: infinite ice plate, opening, variable thickness.

\section{1. ВВЕДЕНИЕ}

В литературе имеются публикации, связанные с изучением прочности ледового покрова. В частности, известна задача Герца, где подобная конструкция рассчитывается как пластина постоянного сечения, лежащая на упругом основании, свойства которого описываются моделью Винклера с коэффициентом постели, равном объемному весу воды. Ниже рассмотрим расчет на прочность бесконечной ледовой плиты, содержащей отверстие в форме круга.

Толщина пластины в части, примыкающей к отверстию, имеет переменную толщину, которая уменьшается по направлению к внутреннему контуру (рис. 1). Пусть изучаемая конструкция загружена моментами и силами, равномерно распределенными вдоль внутреннего контура отверстия.

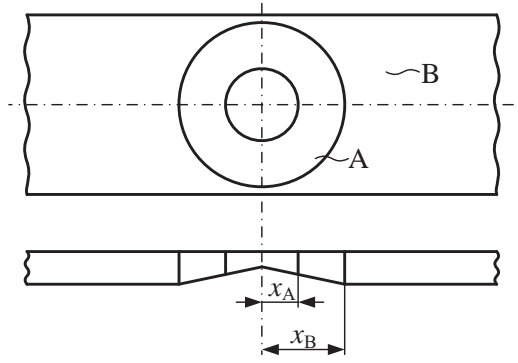

Рисунок 1. Бесконечная ледовая плита с отверстием.

\section{2. РАСЧЕТ ВНУТРЕННЕГО УЧАСТКА ПЛИТЫ}

Будем рассчитывать внутренний участок А, примыкающий к отверстию, как кольцевую плиту радиально-переменной толщины, лежащую на упругом винклеровском основании. Пусть толщина изменяется от внутрен- 
него контура к месту сочетания с пластиной постоянного сечения по закону

$$
h=h_{0} x^{4 / 3},
$$

где $x=r / r_{0} ; h_{0}, r_{0}$ - постоянные.

В месте примыкания к «наружной» зоне В, которая будет рассчитываться как изотропная плита постоянного сечения, лежащая на упругом винклеровском основании, будет учтено, что прогибы, углы поворота и усилия остаются непрерывными.

Приведем дифференциальное уравнение, описывающее изгиб внутренней части плиты, примыкающей к отверстию:

$$
\begin{gathered}
D\left[\frac{d^{4} w}{d r^{4}}+\frac{2}{r} \frac{d^{3} w}{d r^{3}}-\frac{1}{r^{2}} \frac{d^{2} w}{d r^{2}}+\frac{1}{r^{3}} \frac{d w}{d r}\right]+ \\
+\frac{d D}{d r}\left[2 \frac{d^{3} w}{d r^{3}}+\frac{(2+\sigma)}{r} \frac{d^{2} w}{d r^{2}}-\frac{1}{r^{2}} \frac{d w}{d r}\right]+ \\
+\frac{d^{2} D}{d r^{2}}\left[\frac{d^{2} w}{d r^{2}}+\frac{\sigma}{r} \frac{d w}{d r}\right]=q_{\text {акт }}-c w .
\end{gathered}
$$

Здесь $D$ - цилиндрическая жесткость, $\sigma$ - коэффициент Пуассона, $c$ - коэффициент постели. Толщине (1) соответствует жесткость, изменяющаяся по степенному закону

$$
D=D_{0} x^{4},
$$

где $D_{0}-$ постоянная.

Учитывая (3), уравнение (2) принимает вид:

где

$$
\begin{aligned}
& \frac{d^{4} w}{d x^{4}}+\frac{10}{x} \frac{d^{3} w}{d x^{3}}+\frac{(19+4 \sigma)}{x^{2}} \frac{d^{2} w}{d x^{2}}+ \\
& +\frac{3(4 \sigma-1)}{x^{3}} \frac{d w}{d x}+\frac{\beta^{4}}{x^{4}} w=\frac{q r_{0}^{4}}{D_{0}},
\end{aligned}
$$

Проинтегрируем уравнение (4); ему соответствует однородное дифференциальное уравнение с переменными коэффициентами, которое с помощью подстановок $z=e^{x}$ и $w=u e^{-z}$ можно свести к дифференциальному уравнению с постоянными коэффициентами.

Согласно теории уравнений, принадлежащих к классу Эйлера, его интеграл можно записать в форме:

$$
w=x^{-1}\left[A_{1} x^{\alpha_{1}}+A_{2} x^{\alpha_{2}}+A_{3} x^{\alpha_{3}}+A_{4} x^{\alpha_{4}}\right],
$$

где $\alpha_{1,2,3,4}= \pm \sqrt{(3-2 \sigma) \pm 2 \sqrt{4\left(1-2 \sigma-\sigma^{2}\right)-\beta^{4}}}$.

Отметим, что

$$
\alpha_{1}=-\alpha_{2}, \alpha_{3}=-\alpha_{4} \text {. }
$$

Уравнение изогнутой срединной поверхности внутренней части А изучаемой плиты с отверстием, при ее загружении при $x=x_{\mathrm{A}}$ равномерно распределенными моментами $M_{1}$ и силами $Q_{1}$, имеет вид:

$$
\begin{aligned}
& w=w_{0} w_{1}\left(x_{\mathrm{A}} ; x\right)+\vartheta_{0} r_{0} w_{2}\left(x_{\mathrm{A}} ; x\right)- \\
& -M_{1} r_{0}^{2} w_{3}\left(x_{\mathrm{A}} ; x\right)-Q_{1} r_{0}^{3} w_{4}\left(x_{\mathrm{A}} ; x\right),
\end{aligned}
$$

где $w_{0}, \vartheta_{0}$ - соответственно прогиб и угол поворота плиты при $x=x_{1} ; w_{1}, w_{2}, w_{3}, w_{4}-$ фундаментальные функции, свойства которых описаны в [1]. Указанные функции определяются выражениями:

$$
\begin{gathered}
w_{1}\left(x_{1} ; x\right)=Z_{1}\left(x_{1} ; x\right) ; \\
w_{2}\left(x_{1} ; x\right)=Z_{2}\left(x_{1} ; x\right)-\frac{\sigma}{x_{1}} Z_{3}\left(x_{1} ; x\right)+ \\
+\frac{(1+\sigma)}{x_{1}^{2}} Z_{4}\left(x_{1} ; x\right) ; \\
w_{3}\left(x_{1} ; x\right)=-\frac{1}{D\left(x_{1}\right)}\left\{Z_{3}\left(x_{1} ; x\right)-\right. \\
\left.-\left[\frac{1}{x_{1}}+\frac{1}{D\left(x_{1}\right)} \frac{d D\left(x_{1}\right)}{d x}\right] Z_{4}\left(x_{1} ; x\right)\right\} ; \\
w_{4}\left(x_{1} ; x\right)=-\frac{1}{D\left(x_{1}\right)} Z_{4}\left(x_{1} ; x\right),
\end{gathered}
$$

где $Z_{1,2,3,4}\left(x_{1} ; x\right)$ - функции Коши, которые для плиты с цилиндрической жесткостью (3), 
лежащей на упругом винклеровском основании, получены в следующем виде:

$$
\begin{aligned}
& Z_{1}\left(x_{1} ; x\right)=\frac{x_{1}}{2\left(\alpha_{3}^{2}-\alpha_{1}^{2}\right)}\left\{\frac { \alpha _ { 3 } ^ { 2 } } { \alpha _ { 1 } } \left[\left(\alpha_{1}+1\right) x_{1}^{-\alpha_{1}} x^{\alpha_{1}-1}+\right.\right. \\
& \left.+\left(\alpha_{1}-1\right) x_{1}^{\alpha_{1}} x^{-\alpha_{1}-1}\right]- \\
& \left.-\frac{\alpha_{1}^{2}}{\alpha_{3}}\left[\left(\alpha_{3}+1\right) x_{1}^{-\alpha_{3}} x^{\alpha_{3}-1}+\left(\alpha_{3}-1\right) x_{1}^{\alpha_{3}} x^{-\alpha_{3}-1}\right]\right\} \text {; } \\
& Z_{2}\left(x_{1} ; x\right)=\frac{x_{1}^{2}}{2\left(\alpha_{3}^{2}-\alpha_{1}^{2}\right)}\left\{\frac{\left(\alpha_{3}^{2}-3 \alpha_{1}\right)}{\alpha_{1}} x_{1}^{-\alpha_{1}} x^{\alpha_{1}-1}-\right. \\
& -\frac{\left(\alpha_{3}^{2}-3 \alpha_{1}\right)}{\alpha_{1}} x_{1}^{-\alpha_{1}} x^{-\alpha_{1}-1}-\frac{\left(\alpha_{1}^{2}-3 \alpha_{1}\right)}{\alpha_{3}} x_{1}^{-\alpha_{3}} x^{\alpha_{1}-1}+ \\
& \left.+\frac{\left(\alpha_{1}^{2}-3 \alpha_{1}\right)}{\alpha_{3}} x_{1}^{-\alpha_{3}} x^{-\alpha_{3}-1}\right\} \text {; } \\
& Z_{3}\left(x_{1} ; x\right)=\frac{x_{1}^{3}}{2\left(\alpha_{3}^{2}-\alpha_{1}^{2}\right)}\left\{-\frac{x_{1}^{-\alpha_{1}}\left(6+\alpha_{1}\right)}{\alpha_{1}} x_{1}^{-\alpha_{1}-1}+\right. \\
& +\frac{x_{1}^{-\alpha_{1}}\left(6-\alpha_{1}\right)}{\alpha_{1}} x^{-\alpha_{1}-1}+\frac{x_{1}^{-\alpha_{3}}\left(6+\alpha_{3}\right)}{\alpha_{3}} x^{\alpha_{3}-1}- \\
& \left.-\frac{x_{1}^{-\alpha_{3}}\left(6-\alpha_{3}\right)}{\alpha_{3}} x^{-\alpha_{3}-1}\right\} \\
& Z_{4}\left(x_{1} ; x\right)=\frac{x_{1}^{4}}{2\left(\alpha_{3}^{2}-\alpha_{1}^{2}\right)}\left\{\frac{x_{1}^{-\alpha_{1}} x^{\alpha_{1}-1}}{\alpha_{1}}+\frac{x_{1}^{\alpha_{1}} x^{-\alpha_{1}-1}}{\alpha_{1}}+\right. \\
& \left.+\frac{x_{1}^{-\alpha_{1}} x^{\alpha_{3}-1}}{\alpha_{3}}-\frac{x_{1}^{\alpha_{3}} x^{-\alpha_{3}-1}}{\alpha_{3}}\right\} \text {. }
\end{aligned}
$$

Величины $w_{0}$ и $\vartheta_{0}$ в формуле (6) определяются из условий при $x \rightarrow \infty$. При расчете в формулах (7)-(8) положим $x_{1}=x_{\mathrm{A}}$.

\section{3. РАСЧЕТ ВНЕШНЕГО УЧАСТКА ПЛИТЫ}

Внешняя часть ледовой плиты рассматривается как бесконечная пластина, лежащая на упругом винклеровском основании, с отверстием, радиус которого равен $x_{\mathrm{B}}$. Как известно, дифференциальное уравнение, описывающее изгиб подобной пластины, интегрируется в функциях Бесселя [2]. Для учета сопряжения с внутренней кольцевой частью переменной толщины воспользуемся фундаментальными функциями, которые имеют другой вид, чем приведенные выше для пластины переменной толщины, но обладают подобными свойствами.

Приведем дифференциальное уравнение, описывающее осесимметричную деформацию подобной пластины:

$$
\begin{gathered}
D \Delta_{r} \Delta_{r} w+c w=q, \\
\Delta_{r}=\frac{d^{2}}{d r^{2}}+\frac{1}{r} \frac{d}{d r} .
\end{gathered}
$$

где

Введем соответственно новое обозначение и безразмерную координату

$$
l=\sqrt[4]{\frac{D}{c}} ; \quad x=\frac{r}{l}
$$

Уравнение (9) запишем в следующем виде:

$$
\Delta^{2} w+w=\frac{q l^{4}}{D} .
$$

Соответствующее (10) однородное уравнение можно свести к решению системы двух дифференциальных уравнений второго порядка:

$$
\frac{d^{2} w}{d x^{2}}+\frac{1}{x} \frac{d w}{d x} \pm i w=0
$$

Решение этой системы дифференциальных уравнений выражается в функциях Бесселя и имеет следующий вид:

$$
\begin{aligned}
& w=A_{1} J_{0}(x \sqrt{i})+A_{2} J_{0}(x \sqrt{-i})+ \\
& +A_{3} H_{0}^{(1)}(x \sqrt{i})+A_{4} H_{0}^{(1)}(x \sqrt{-i}) .
\end{aligned}
$$

Поскольку решение (12) должно быть действительно, а функции $J_{0}(x \sqrt{ \pm i}), H_{0}^{(1)}(x \sqrt{ \pm i})$ комплексные, то константы $A_{1}, A_{2}, A_{3}, A_{4}$ должны быть комплексными числами. Запишем это решение через действительные функции. Используем обозначения, принятые в [2]: 


$$
\begin{gathered}
u_{0}(x)=\operatorname{Re} J_{0}(x \sqrt{i}), v_{0}(x)=\operatorname{Im} J_{0}(x \sqrt{i}), \\
f_{0}(x)=\operatorname{Re} H_{0}^{(1)}(x \sqrt{i}), g_{0}(x)=\operatorname{Im} H_{0}^{(1)}(x \sqrt{i}) .
\end{gathered}
$$

Тогда (12) определяется следующим выражением:

$$
\begin{gathered}
w=B_{1} u_{0}(x)+B_{2} v_{0}(x)+ \\
+B_{3} f_{0}(x)+B_{4} g_{0}(x) .
\end{gathered}
$$

Здесь функции $u_{0}, v_{0}, f_{0}, g_{0}$ и постоянные $B_{1}, B_{2}, B_{3}, B_{4}$ - действительны.

Для рассмотрения внешней части бесконечной плиты с круговым отверстием также используем фундаментальные функции, обладающие известными свойствами, так как последние относятся к решению (11) и выражаются через функции Бесселя. В месте сопряжения двух участков при $x=x_{\mathrm{B}}$ прогиб, угол поворота, изгибающий момент, поперечная сила $\left(w_{\mathrm{B}}, \vartheta_{\mathrm{B}}, M_{\mathrm{B}}, Q_{\mathrm{B}}\right.$ соответственно) определяются с помощью зависимостей (6) для кольцевой пластины переменной толщины при $x=x_{\mathrm{B}}$. Фундаментальные функции $Y_{\mathrm{i}}\left(x_{\mathrm{B}} ; x\right)$ для внешнего участка определяются выражениями:

$$
\begin{aligned}
Y_{1}\left(x_{\mathrm{B}} ; x\right) & =\frac{\pi x_{\mathrm{B}}}{2}\left[-u_{0}{ }^{\prime}\left(x_{\mathrm{B}}\right) g_{0}(x)-v_{0}{ }^{\prime}\left(x_{\mathrm{B}}\right) f_{0}(x)+\right. \\
+ & \left.f_{0}{ }^{\prime}\left(x_{\mathrm{B}}\right) v_{0}(x)+g_{0}{ }^{\prime}\left(x_{\mathrm{B}}\right) u_{0}(x)\right], \\
Y_{2}\left(x_{\mathrm{B}} ; x\right) & =\frac{\pi x_{\mathrm{B}}}{2}\left[-u_{0}\left(x_{\mathrm{B}}\right) g_{0}(x)-v_{0}\left(x_{\mathrm{B}}\right) f_{0}(x)+\right. \\
& \left.+f_{0}\left(x_{\mathrm{B}}\right) v_{0}(x)+g_{0}\left(x_{\mathrm{B}}\right) u_{0}(x)\right], \\
Y_{3}\left(x_{\mathrm{B}} ; x\right) & =\frac{\pi x_{\mathrm{B}}}{2}\left[u_{0}{ }^{\prime}\left(x_{\mathrm{B}}\right) f_{0}(x)-v_{0}{ }^{\prime}\left(x_{\mathrm{B}}\right) g_{0}(x)-\right. \\
& \left.-f_{0}{ }^{\prime}\left(x_{\mathrm{B}}\right) u_{0}(x)+g_{0}{ }^{\prime}\left(x_{\mathrm{B}}\right) v_{0}(x)\right], \\
Y_{4}\left(x_{\mathrm{B}} ; x\right) & =\frac{\pi x_{\mathrm{B}}}{2}\left[u_{0}\left(x_{\mathrm{B}}\right) f_{0}(x)-v_{0}\left(x_{\mathrm{B}}\right) g_{0}(x)-\right. \\
& \left.-f_{0}\left(x_{\mathrm{B}}\right) u_{0}(x)+g_{0}\left(x_{\mathrm{B}}\right) v_{0}(x)\right] .
\end{aligned}
$$

Между функциями $Y_{\mathrm{i}}\left(x_{\mathrm{B}} ; x\right)$ существуют следующие дифференциальные зависимости:

$$
\begin{gathered}
\Delta Y_{1}=Y_{3}, \Delta Y_{2}=Y_{4}, \\
\Delta Y_{3}=-Y_{1}, \Delta Y_{4}=-Y_{2} .
\end{gathered}
$$

Приведем выражение для прогиба при $x=x_{\mathrm{B}}$ :

$$
\begin{aligned}
& w=w_{\mathrm{B}} Y_{1}\left(x_{\mathrm{B}} ; x\right)+\vartheta_{\mathrm{B}} l Y_{2}\left(x_{\mathrm{B}} ; x\right)+ \\
& +M_{\mathrm{B}} l^{2} Y_{3}\left(x_{\mathrm{B}} ; x\right)+Q_{\mathrm{B}} l^{3} Y_{4}\left(x_{\mathrm{B}} ; x\right) .
\end{aligned}
$$

Изгибающие моменты и поперечные силы определяются по следующим формулам:

$$
\begin{gathered}
M_{1}=-D\left(\frac{d^{2} w}{d r^{2}}+\frac{\sigma}{r} \frac{d w}{d r}\right), \\
M_{2}=-D\left(\sigma \frac{d^{2} w}{d r^{2}}+\frac{1}{r} \frac{d w}{d r}\right) \\
Q_{1}=-D \frac{d}{d r}\left(\frac{d^{2} w}{d r^{2}}+\frac{1}{r} \frac{d w}{d r}\right)
\end{gathered}
$$

\section{4. ЗАКЛЮЧЕНИЕ}

Итак, в замкнутом виде получено решение задачи о ледовой пластине с круговым отверстием; в области отверстия толщина пластины переменна и плавно уменьшается по направлению к внутреннему контуру.

\section{СПИСОК ЛИТЕРАТУРЫ}

1. Коренева Е.Б. Аналитические методы расчета пластин переменной толщины и их практические приложения. - М.: АСВ, 2009. - 240 с.

2. Коренев Б.Г. Некоторые задачи теории упругости и теплопроводности, решаемые в бесселевых функциях. - М.: ФИЗМАТГИЗ, 1960. - 458 с.

Коренева Елена Борисовна, доктор технических наук, профессор кафедры прикладной математики, Национальный исследовательский Московский государственный строительный университет, 129337, Россия, г. Москва, Ярославское шоссе, дом 26, e-mail: elena.koreneva2010@yandex.ru.

Elena B. Koreneva, Department of Applied Mathematics, National Research Moscow State University of Civil Engineering, 26, Yaroslavskoe Shosse, 129337, Moscow, RUSSIA, e-mail: elena.koreneva2010@yandex.ru. 
DOI:10.22337/1524-5845-2016-12-4-103-115

\title{
О ЯВЛЕНИИ ПОТЕРИ УСТОЙЧИВОСТИ ПРОДОЛЬНО СЖАТОЙ КРУГОВОЙ ЦИЛИНДРИЧЕСКОЙ ОБОЛОЧКИ. ЧАСТЬ 2: МАКСВЕЛЛОВА СИЛА И ЭНЕРГЕТИЧЕСКИЙ БАРЬЕР
}

\author{
Г.А. Мануйлов, С.Б. Косицын, М.М. Бегичев \\ Московский государственный университет путей сообщения Императора Николая II (МГУПС (МИИТ)), \\ г. Москва, РОССИЯ
}

\begin{abstract}
Аннотация: Для круговой упругой кинематически продольно сжатой цилиндрической оболочки на основе анализа данных экспериментов рассматриваются особенности формирования начального послекритического равновесия, образования и развития ромбо-треугольных вмятин в процессе хлопка по нагрузке, а также характерные свойства кусочно-гладких кривых послехлопковых равновесий. Дано определение нагрузки резкого уменьшения энергетического барьера - максвелловой силы и описаны способы ее вычисления. Численные результаты сравниваются с экспериментальными.
\end{abstract}

Ключевые слова: потеря устойчивости, цилиндрическая оболочка, послекритическое равновесие

\section{ON INSTABILITY OF CIRCULAR AXIALLY COMPRESSED CYLINDRICAL SHELL. PART 2: MAXWELL LOAD AND ENERGY BARRIER}

\author{
Gaik A. Manuylov, Sergey B. Kosytsyn, Maxim M. Begichev \\ Moscow State University of Railway Engineering (MIIT), Moscow, RUSSIA
}

\begin{abstract}
On the formation features of the initialpostbuckling equilibrium, development of diamond- triangular dents, characteristic properties ofpostbuckling equilibrium curves for circular elastic axially compressed cylindrical shell under kinematic loading. The definition of sharp decrease of energy barrier - Maxwell load is given with the methods of its calculation. The numerical results are compared with experimental.
\end{abstract}

Key words: buckling, cylindrical shell, postbuckling equilibrium.

\section{1. ЭНЕРГЕТИЧЕСКИЙ БАРЬЕР И МАКСВЕЛЛОВА СИЛА}

Недоверие исследователей и проектировщиков к достаточно высоким значениям верхних критических нагрузок объясняется очень малым энергетическим барьером, отделяющим исходное устойчивое осесимметричное докритическое равновесие от серии далеких и сильно деформированных устойчивых и несимметричных равновесий. Последние отличаются друг от друга количеством окружных волн и числом продольных полуволн. Для металлических оболочек любое сильно деформированное равновесие фактически означает разрушение (в силу развития больших пластических деформаций).

Чтобы попасть в область притяжения потенциальной ямы упомянутых сильно деформированных равновесий при действии внешних статических возмущений, оболочка должна предварительно оказаться в некотором седловом (неустойчивом) равновесии, которое расположено на энергетическом барьере (водоразделе). Наименьший уровень энергетического барьера определяется уровнем критериального седла на водоразделе. 
Новые идеи энергетического характера в решении этой задачи появились в 1941 - 42гг благодаря работам К. Фридрихса и ТзяньСюэ-Сеня $[1,2]$. В юбилейном сборнике, посвященном 60-тилетию Т. Кармана, К. Фридрихс опубликовал статью, в которой на основании анализа изменений полной потенциальной энергии упругих систем, имеющих «характеристику» с участком рестабилизации была высказана мысль о существовании такой промежуточной нагрузки $P_{M}$, при которой полная потенциальная энергия рестабилизированного равновесия (《далекого» от докритического) впервые будет равна аналогичной величине для исходного устойчивого докритического равновесия. При увеличении нагрузки $\left(\mathrm{P} *>\mathrm{P}_{\mathrm{M}}\right)$ полная потенциальная энергия далекого устойчивого равновесия станет меньше энергии исходного равновесия. Иначе говоря, потенциальная яма далекого и сильно деформированного устойчивого равновесия окажется более глубокой по сравнению с потенциальной ямой исходного докритического равновесия. На основании термодинамических представлений Дж.К. Максвелла, К. Фридрихс заключил, что упругая система с такой характеристикой при нагрузках, превышающих «максвеллову силу» $P_{M}$ (английский термин), всегда будет стремиться попасть в положение равновесия с наименьшим уровнем полной энергии. Используя это заключение Фридрихса, Тзянь в статье 1942г [2] пришел к выводу о том, что упругая система при нагрузках выше «максвелловой силы» $P_{M}$, может быть «выбита» со дна потенциальной ямы неким динамическим возмущением, достаточным для преодоления энергетического барьера и перехода в область притяжения далекого устойчивого и сильно деформированного равновесия. Потому Тзянь назвал «максвеллову силу» критериальной энергетической силой (что на наш взгляд более правильно). Отметим, что для выхода на энергетический барьер необязательно воздействие в виде динамического возмущения. Упругая система может преодолеть этот барьер и под действием статиче- ских возмущений, путем перехода через неустойчивое седловое равновесие, о чем говорилось выше.

Для более четкого понимания «знергетических» идей К. Фридрихса и Тзяня рассмотрим простейший вариант характеристики равновесных состояний, соответствующей кинематическому нагружению продольным сжатием круговой цилиндрической оболочки (рис. 1).

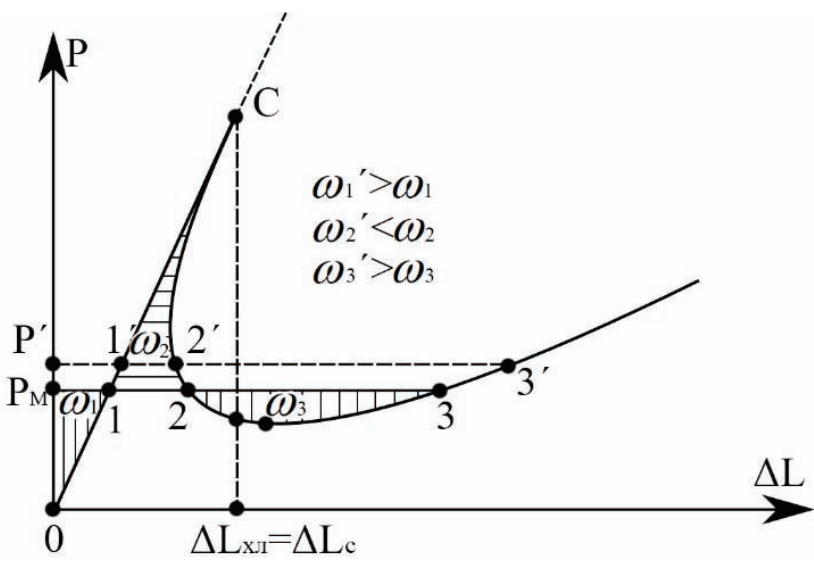

Рисунок 1. Полная потенциильная энергия оболочки в различных состояниях равновесия и определение максвеловой силь.

Здесь точки 1 и 3 есть точки устойчивых равновесий (докритического1 и далекого «сильно деформированного», соответственно) при нагрузке, равной «максвелловой силе» $P_{M}$.

Точка 2 есть точка неустойчивого равновесия, точки $\mathrm{C}$ и $\mathrm{L}$ есть точка бифуркации и точка минимума на кривой равновесий оболочки. Обозначим площади заштрихованных областей $01 \mathrm{P}, 1 \mathrm{C} 2$, и $2 \mathrm{~L} 3$ чере $3 \omega_{1}, \omega_{2}, \omega_{3}$, coответственно. Полная потенциальная энергия докритического равновесия в точке 1 равна $V_{1}=-\omega_{1}$, для точки 2 эта же энергия есть $V_{2}=-\omega_{1}+\omega_{2}$, для точки 3 полная энергия равна $V_{3}=-\omega_{1}+\omega_{2}-\omega_{3}$. По определению «максвелловасила» $P_{M}$ есть величина нагрузки, при которой площади $\omega_{2}$ и $\omega_{3}$ равны. 
О явлении потери устойчивости продольно сжатой круговой цилиндрической оболочки Часть 2: Максвеллова сила и энергетический барьер
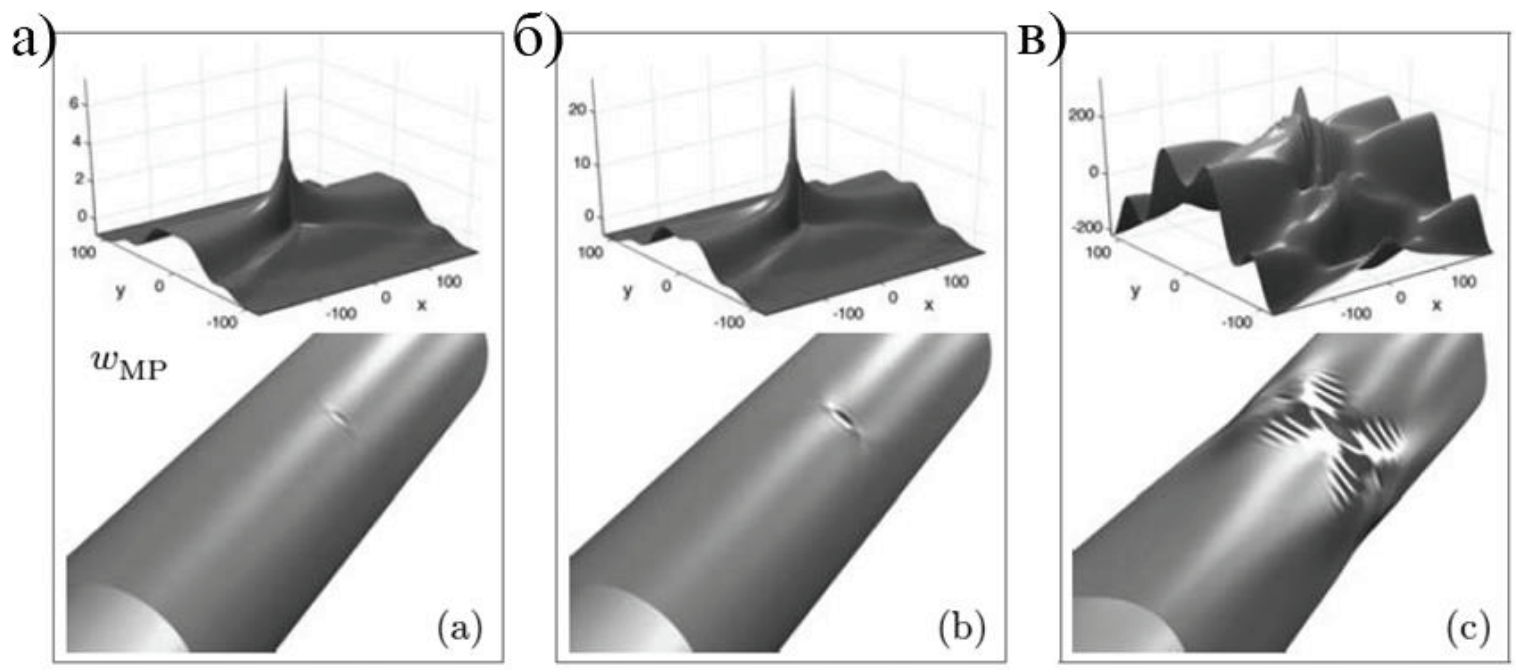

Рисунок 2. Развитие вмятин при переходе оболочки через энергетический барьер:

а) седловое равновесие; б) развитие седлового равновесия;

в) сильнодеформированное равновесие в результате хлопка.

В силу этого при нагрузке, равной «максвелловой силе», потенциальная энергия далёкого устойчивого равновесия в точке 3 оказывается равной потенциальной энергии устойчивого докритического равновесия в точке 1:

$$
V_{3}=V_{1}=-\omega_{1}
$$

Если же нагрузка $P^{\prime}$ больше «максвелловой силы» $\left(P^{\prime}>P_{M}\right.$, пунктирная линия и точки $1^{\prime}$, $2^{\prime}$ и $3^{\prime}$ на рис. 1), то в этом случае площадь $\omega_{3}{ }^{\prime}$ будет больше площади $\omega_{2}{ }^{\prime}$. Вследствие этого потенциальная энергия в точке 3' окажется меньше потенциальной энергии докритического равновесия в точке 1 ' $\left(V_{3}{ }^{\prime}<V_{1}{ }^{\prime}\right)$.

Приведенные рассуждения привели Тзяня к формулировке его «энергетического принципа», который часто критикуют за неконструктивный характер (неизвестна величина минимального энергетического барьера, как найти форму неустойчивого равновесия в критериальном седле, как выйти на энергетический барьер, и т.д.). ТзяньСюэ-Сень прожил 98 лет (1911-2009г), возможно, так и не узнав, что его «критериальная энергетическая сила» (или «максвеллова сила») $P_{M}$ за последние 30 лет стала объектом многих исследований как применительно к задаче устойчивости круговой продольно сжатой цилиндрической оболочки, так и в задачах устойчивости равновесия оболочек с другими формами срединной поверхности, имеющих участки рестабилизации на кривых равновесий.

\section{2. О МИНИМАЛЬНОМ ЭНЕРГЕТИЧЕСКОМ БАРЬЕРЕ И СООТВЕТСТВУЮЩЕМ СЕДЛОВОМ РАВНОВЕСИИ ОБОЛОЧКИ}

В последние 20-25 лет было установлено, что при переходе через максвеллову силу резко уменьшается энергетический барьер докритического равновесия. Объясняется это тем, что при нагрузках больше максвелловой силы существуют неустойчивые локализованные равновесия в виде одиночных вмятин на поверхности оболочки. Вмятины имеют сравнительно малые размеры и правильную форму. Именно эти седловые равновесия определяют величину минимального энергетического барьера.

Это важное открытие, по-видимому, впервые было опубликовано в работах Дж. Ханта с соавторами [3-7], а также в работах Ван Дер Хейдена и Дж.М.Т. Томпсона [8, 9], выполненных в начале 2000-х годов. 


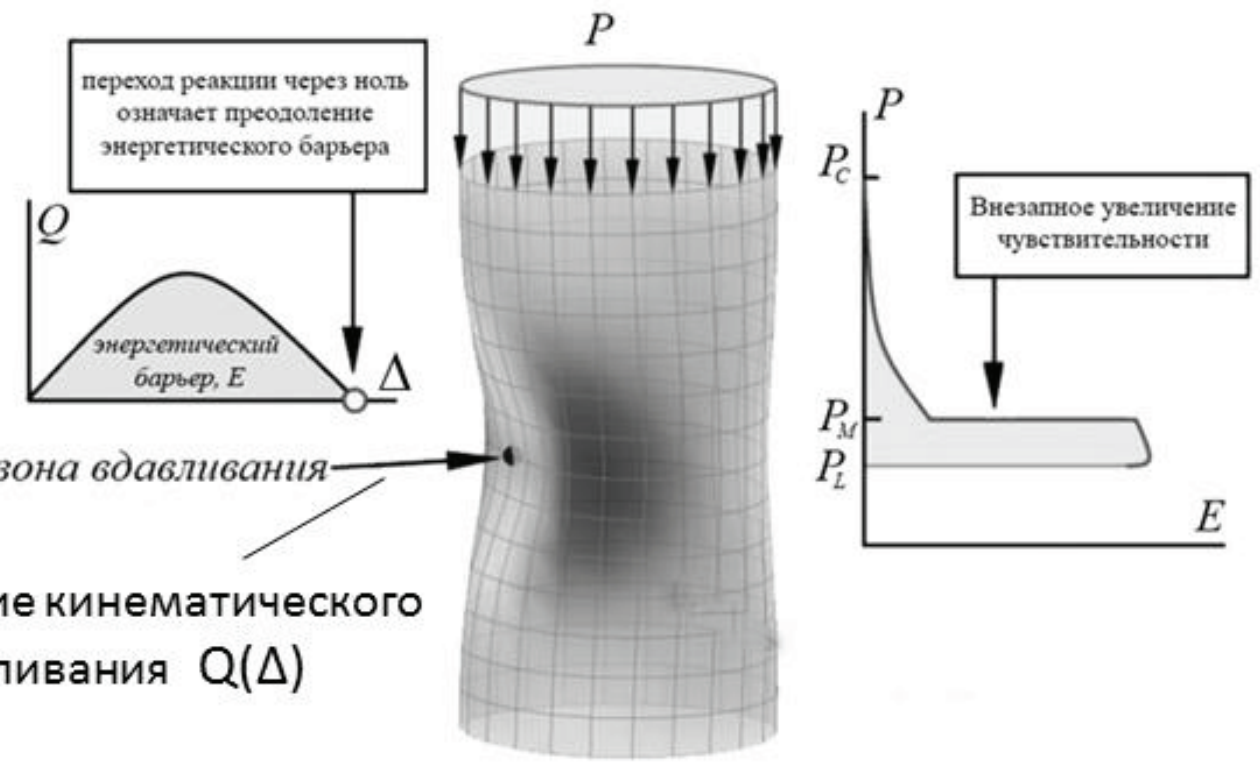

Рисунок 3. Определение максвелловой силь при помощи кинематического вдавливания (согласно Дж.М.Т. Томпсону)
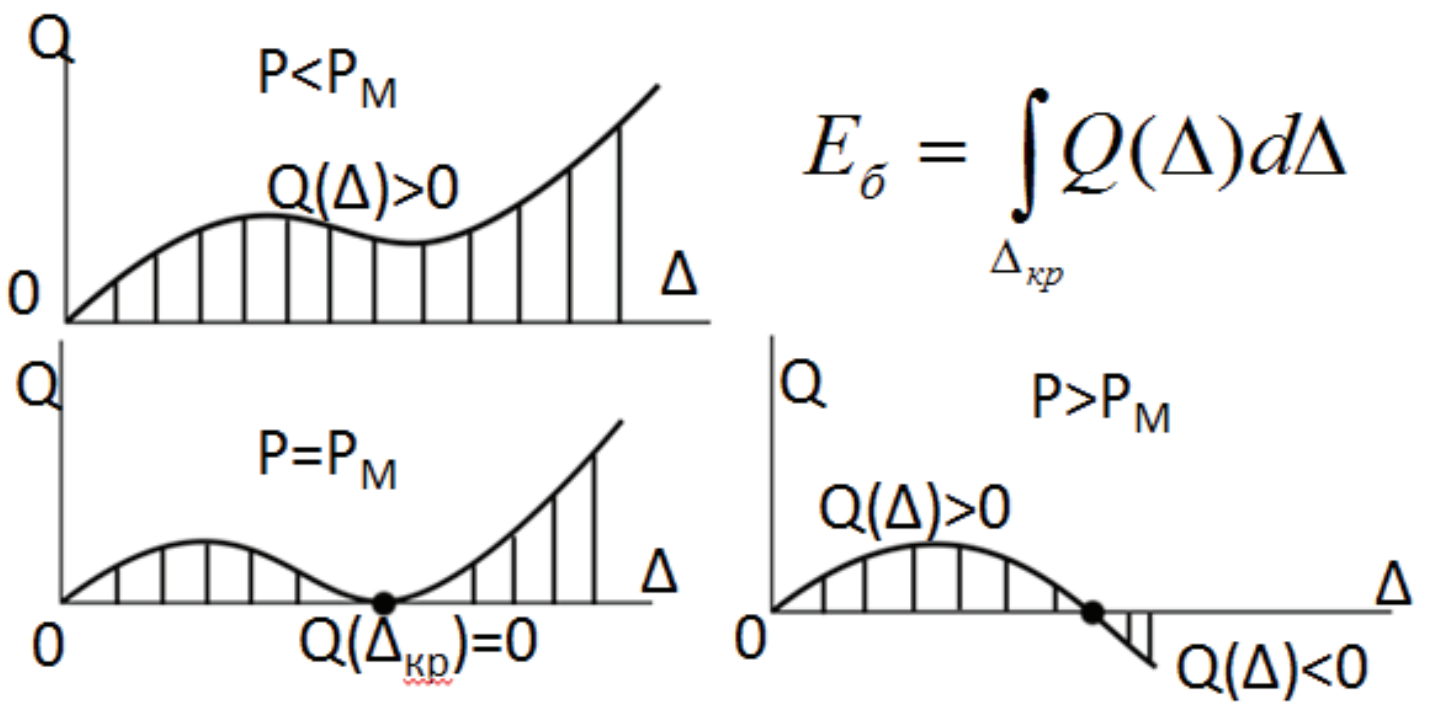

Рисунок 4. Возможные графики изменения реакции $Q(\Delta)$ в зависимости от глубиньл сосредоточенного вдавливания $\triangle$ при различных продольных сжимающих нагрузках.

В 2006 году Ю. Хорак и соавторы [10] доказали существование неустойчивого седлового решения на энергетическом «водоразделе» в виде малой одиночной, но весьма глубокой вмятины на поверхности оболочки. Эти же авторы разработали специальный вариант mountainpass-алгоритма (ранее предложенного в работе [11]).

Дискретный алгоритм типа «спуска» позволяет численно определить координаты точки максимума функционала полной энергии оболочки, а также получать представление о форме (профиле) упомянутой седловой вмятины. На рис. 2 показаны этапы развития такой вмятины при переходе через энергетический барьер и переход оболочки в результате хлопка в далекое сильнодеформированное равновесие.

Однако при помощи какого поперечного воздействия можно создать такую вмятину, 
О явлении потери устойчивости продольно сжатой круговой цилиндрической оболочки

Часть 2: Максвеллова сила и энергетический барьер

определяющую критериальное седловое равновесие оболочки? В.Г. Паламарчук ([12], 1970 г.) утверждал, что среди поперечных возмущающих воздействий есть такое, которое приводит к развитию деформаций оболочки по пути преодоления минимального энергетического барьера. Поскольку профиль седловой вмятины очень близок к профилю вмятины, создаваемой давлением сосредоточенной силой, приложенной перпендикулярно к поверхности оболочки, то Дж.М.Т. Томпсон [8, 9] предложил исследовать минимальный энергетический барьер оболочки при помощи «сосредоточенного кинематического вдавливания» в наиболее слабой точке поверхности цилиндрической оболочки (рис. 3). Тогда форма графика изменения усилия вдавливания $\mathrm{Q}(\Delta)$ как функция от глубины вмятины $\Delta$ будет меняться в зависимости от того, больше или меньше нагрузка сжатия оболочки Р по сравнению с максвелловой силой. На рис. 4 представлены, полученные в данной работе грфики $\mathrm{Q}(\Delta)$ при нагрузке сжатия $\mathrm{P}<\mathrm{P}_{\mathrm{M}}, \mathrm{P}=\mathrm{P}_{\mathrm{M}}, \mathrm{P}>\mathrm{P}_{\mathrm{M}}$. Сравнение этих графиков показало, что при нагрузках $\mathrm{P} \geq \mathrm{P}$ мможно достигнуть критического вдавливания $\Delta_{\text {кр}}$, соответствующего обращению в ноль усилия вдавливания $\left(\mathrm{Q}\left(\Delta_{\mathrm{\kappa p}}\right)=0\right)$. Этот момент соответствует выходу оболочки на неустойчивое седловое равновесие. Далее последует хлопок и оболочка переходит в далекое, устойчивое, но сильно деформированное равновесие.

Величина энергетического барьера, отделяющего исходное осесимметричное равновесие сжатия от седлового есть площадь графика функции $\mathrm{Q}(\Delta)$ на участке $0-\Delta_{\text {кр. Как }}$ следует из сравнения графиков на рис. 4 при малом сжатии $\left(\mathrm{P}<\mathrm{P}_{\mathrm{M}}\right)$ эта площадь (т.е. величина энергетического барьера $\mathrm{E}_{\delta}$ ) во много раз больше соответствующих площадей графиков функции $\mathrm{Q}(\Delta)$ при сжатии $\mathrm{P}$, равном или большем максвелловой силы $(\mathrm{P} \geq \mathrm{P} M)$. Поэтому при нагрузках меньших максвелловой силы $\left(\mathrm{P}<\mathrm{P}_{\mathrm{M}}\right)$ возможность нарушения безопасной эксплуатации «совершенной» (условно) оболочки действием внешних по- перечных возмущений гораздо меньше по сравнению с аналогичными возможностями при более высоких нагрузках $\left(\mathrm{P}>\mathrm{P}_{\mathrm{M}}\right)$.

По-видимому, впервые перепад энергетического барьера (по величине глубины критической вмятины $\Delta_{\text {кр}}$ ) был экспериментально исследован в 1976 г М. Эсслингер и Б. Гейером [13]. На рис. 5, взятом из этой работы, показано резкое уменьшение глубины критического вдавливания $\Delta_{\text {кр }}=5,2$ мм при $\mathrm{P}=51$ кг до $\Delta_{\text {кр }}=0,9$ мм при $\mathrm{P}=55$ кг. Аналогично уменьшился и энергетический барьер исходного равновесия. По современным представлениям эти изменения связаны с переходом нагрузки через значение, равное максвелловой силе $\mathrm{P}_{\mathrm{M}}$. Экспериментально эта сила для испытанной оболочки из майлара должна быть в пределах 51 кг $<\mathrm{P}_{\mathrm{M}}<55$ кг.

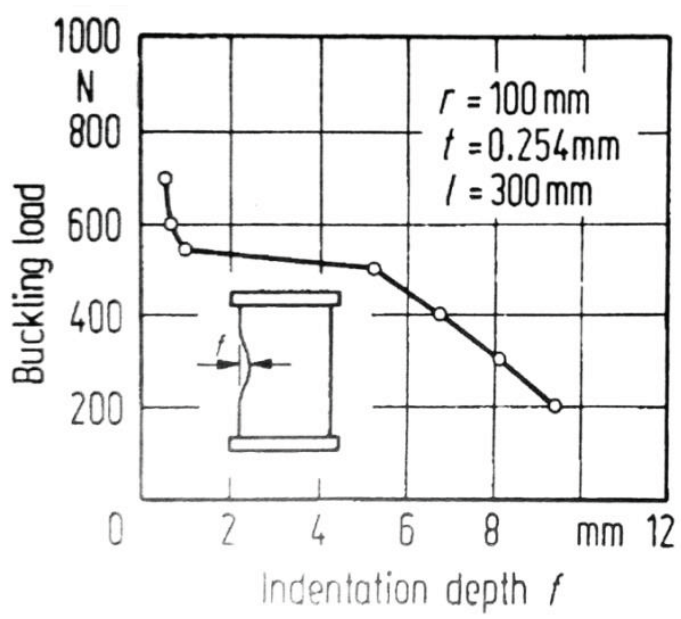

Рисунок 5. График резкого уменьшения глубины критического вдавливани на основе экспериментов М. Эсслингер и Б. Гейера.

Классическая критическая нагрузка для этой оболочки составила $\mathrm{P}_{\text {кр.кл }}=134,8$ кг, а отношение $\mathrm{P}_{\mathrm{M}} / \mathrm{P}_{\text {кр.кл }}=0,378$.

Авторы данной работы [14] выполнили ряд расчетов, моделирующих кинематическое вдавливание в наиболее «слабой» точке поверхности оболочки М. Эсслингер и Б. Гейера (одна из точек средней линии по высоте оболочки). Нагрузки сжатия Р были равны 30, 50, 55, 56 и 60 кг. Соответствующие кривые зависимости реактивного усилия $\mathrm{Q}(\Delta)$ 
при разных Р представлены на рис. 6. Из этих графиков следует, что при $\mathrm{P}=30,50,55$ кг усилие $\mathrm{Q}(\Delta)$ сохраняет свой знак при всех значениях глубины вдавливания $\Delta$. Более того, при $\mathrm{P}=50$ и 55 кг после некоторого уменьшения (при глубинах вмятины $\approx 1,5-2$ мм) это усилие увеличивается. При Р 56 кг усилие вдавливания $\mathrm{Q}(\Delta)$ обращается в ноль в точке касания $\left(\Delta_{\text {кр }} \approx 2\right.$ мм или $\Delta_{\text {кр }} \approx 8 \delta$, $\delta=0,254$ мм - толщина оболочки). Это значение нагрузки есть численное значение максвелловой силы для данной оболочки. Расхождение с экспериментальным результатом

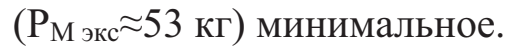

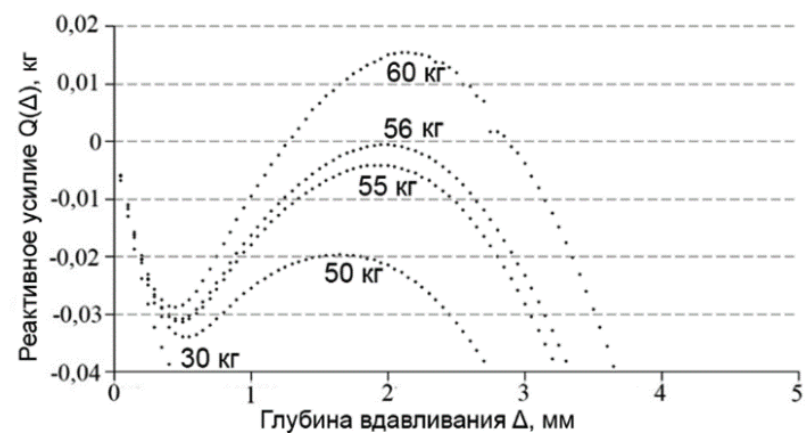

Рисунок 6. Изменение реактивной сильл в зависимости от глубины вдавливания для оболочки М. Эсслингер и Б. Гейером.

Заметим, что авторы [13] ясно представляли, что превышение «силы резкого перепада величины критического вдавливания» позволяет оболочке терять устойчивость исходного равновесия даже при действии очень малых внешних возмущающих воздействий. М. Эсслингер и Б. Гейер исследовали серию оболочек из майлара одинакового радиуса $\mathrm{R}=100$ мм и одинаковой толщины $(\delta=0,254$ Mм), но разной длины $50 \leq \mathrm{L} \leq 400$ мм. Для всех этих оболочек были вычислены упомянутые критические нагрузки резкого перепа-

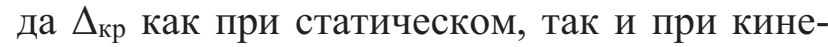
матическом нагружениях (рис. 7). Верхняя кривая на этом рисунке дает численные результаты определения максвелловой силы, полученные авторами настоящей работы. Как следует из рисунка, эта кривая достаточно хорошо согласуется с эксперимен- тальными данными для удлиненных оболочек $(150 \leq \mathrm{L} \leq 400 \mathrm{Mm})$. Для более коротких оболочек «численная» кривая проходит несколько выше значений, полученных экспериментально.

Здесь интересно отметить, что нижняя кривая есть график так называемой «характеристической силы», соответствующей самой крайней левой точке условной характеристики оболочки (рис. 7, правый верхний угол).

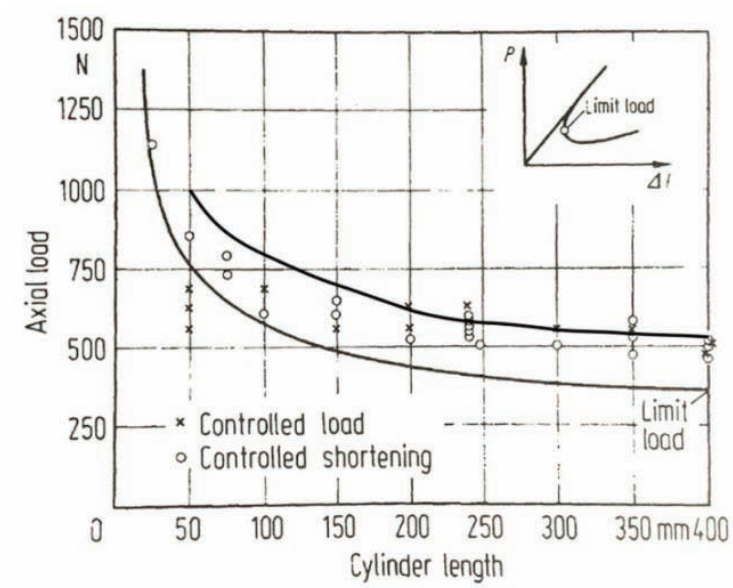

Рисунок 7. Сравнение величины максвелловой и характеристической сил для серии изилиндрических оболочек.

Эта кривая принималась авторами [13] как совокупность безопасных нагрузок на оболочку (поскольку она проходит ниже кривой максвелловых сил и соответствует сжимающим нагрузкам из области весьма больших энергетических барьеров.

\section{3. ЧИСЛЕННОЕ ОПРЕДЕЛЕНИЕ МАКСВЕЛЛОВОЙ СИЛЫ И ЭНЕРГЕТИЧЕСКОГО БАРЬЕРА}

Для построения графика изменения энергетического барьера по мере роста сжимающей нагрузки была выполнена серия численных расчетов для модельной шарнирно закрепленной оболочкой (E=100000 Мпа, $v=0,3$, $\mathrm{L}=4 \mathrm{R}, \mathrm{R}=250$ мм, $\delta=1$ мм) при различных уровнях сжимающей нагрузки (рис. 8). Для этой оболочки $\mathrm{P}_{\text {кр.кл }}=380,08$ кН, геометриче- 
О явлении потери устойчивости продольно сжатой круговой цилиндрической оболочки

Часть 2: Максвеллова сила и энергетический барьер

ски нелинейный расчет (NASTRAN) дал $\mathrm{P}_{\text {кр}}=360$ кН. Моделировалось кинематическое вдавливание в средней точке на поверхности оболочки и фиксировалось изменение соответствующего реактивного усилия $\mathrm{Q}(\Delta)$. Момент обращения этой реакции в ноль соответствовал моменту перехода оболочки в состояние критического седлового равновесия.

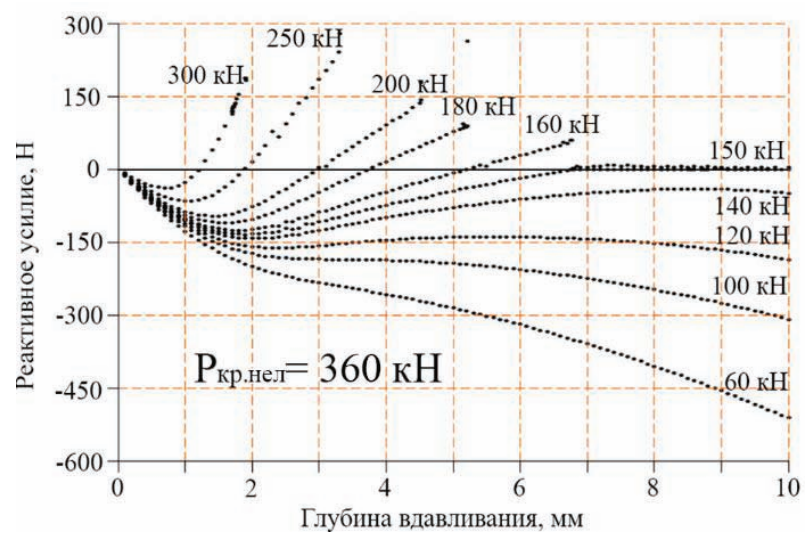

Рисунок 8. Кривые изменения усилия вдавливания $Q$ в зависимости от его глубины вмятины $\triangle$ при различных нагрузках продольного сжатия.

Из сравнения графиков изменения усилий $\mathrm{Q}(\Delta)$ следует, что энергетический барьер (5,29 кН*мм) при сжатии в 150 кН значительно меньше аналогичного барьера для нагрузки сжатия 140 кН. При нагрузке в 146,5 кН реактивное усилие $\mathrm{Q}(\Delta)$ впервые обратилось в ноль при достижении глубины вдавливания 6,8 мм. Эта сила есть сила резкого перепада энергетического барьера (т.е. максвеллова сила). Из рис 9 видно, что по мере приближения сжимающей нагрузки к критической величина энергетического барьера катастрофически падает. Так при нагрузке 250 кН ( 0,658Р кр.кл) величина энергетического барьера в 6 раз меньше энергитического барьера при нагрузке сжатия 150

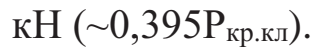

Заметим, что приведенные результаты определения энергетического барьера были получены при двусторонней связи с оболочкой поперечного кинематического воздействия.
Двусторонняя связь при потере устойчивости «удерживала» оболочку от хлопка в далекое сильно деформированное равновесие. Чтобы «увидеть» результат такого хлопка были выполнены расчеты с GAP-элементом, который обеспечивал только сосредоточенное кинематическое вдавливание перпендикулярно поверхности оболочки. Такое воздействие могло быть только до момента потери устойчивости оболочки, пока усилие вдавливания (т.е. сжатие) не обратилось в ноль, а оболочка еще оказывает сопротивление. Как только усилие вдавливанияQ $\left(\Delta_{\text {кр }}\right)=0$ оболочка становилась «свободной» и могла «прощелкнуть» в далекое сильно деформированное равновесие.

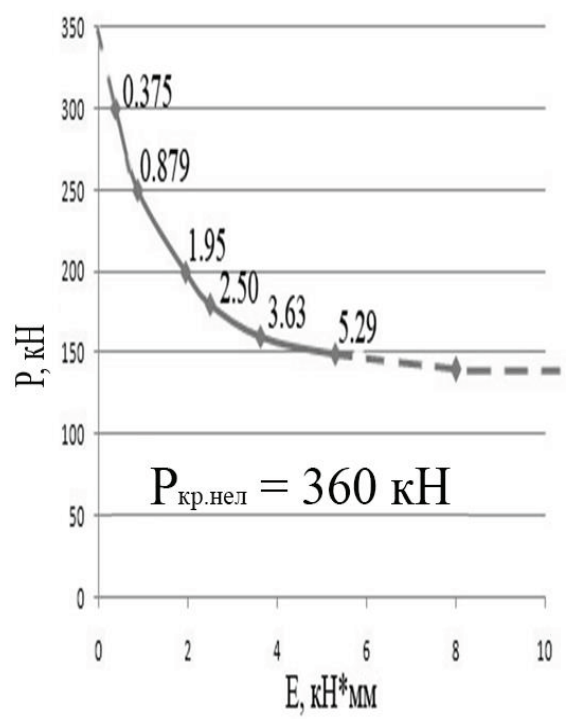

Рисунок 9. График изменения величинь энергетического барьера Е исходного равновесия модельной оболочки в зависимости от величины сжимающей нагрузки $P$.

Этот процесс и наблюдался при реализации вдавливания с GAP-элементом на модельной оболочке $(\mathrm{L} / \mathrm{R}=4)$ при нагрузках близких к максвелловой силе (рис. 10). Величина максвелловой силы $\mathrm{P}_{\mathrm{M}}$, как и при вдавливании без GAP-элемента, составила $\sim 146,5$ кН. Уже при сжатии в 150 кН из графиков на рис. 10 видно, что когда величина вдавливания оказалась равной критической $\left(\Delta_{\text {кр }}(\mathrm{P}=146,5\right.$ кН) $\approx 6,8$ мм), реактивное усилие вдавливания 
обратилось в ноль и в дальнейшем не изменялось.

При несколько большем усилии $(\mathrm{P}=160$ кН) величина критического вдавливания была, естественно, меньше, чем при нагрузке, равной максвелловой силе $\left(\Delta_{\text {кр }} \approx 5,2\right.$ мм, т.2 на графике). Этому состоянию оболочки соответствует седловое равновесие 2 с едва за- метной, но глубокой вмятиной. Оболочка считалась абсолютно упругой.Поэтому произошло прощелкивание в далекое сильно деформированное равновесие 3 с большим количеством разнокалиберных вмятин и выпучин на поверхности оболочки, фактически означающих ее разрушение (рис. 10).

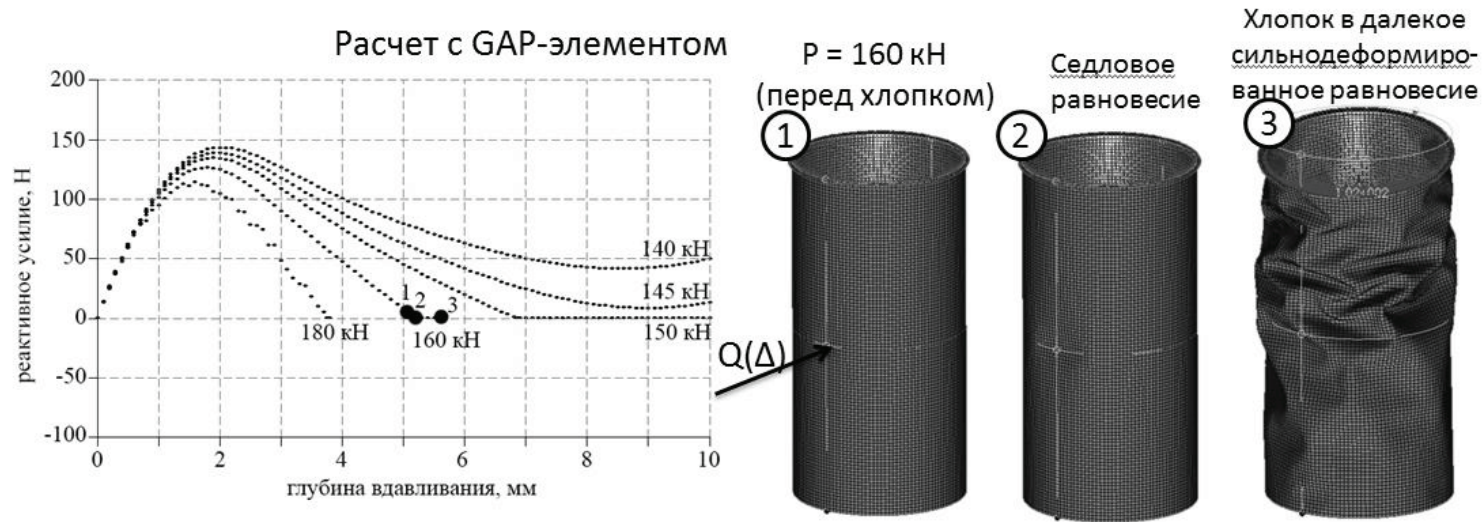

Рисунок 10. Графики зависимости реактивного усилия от глубины вдавливания при различных сжимающих нагрузках, деформированный вид оболочки в характерных точках графика.

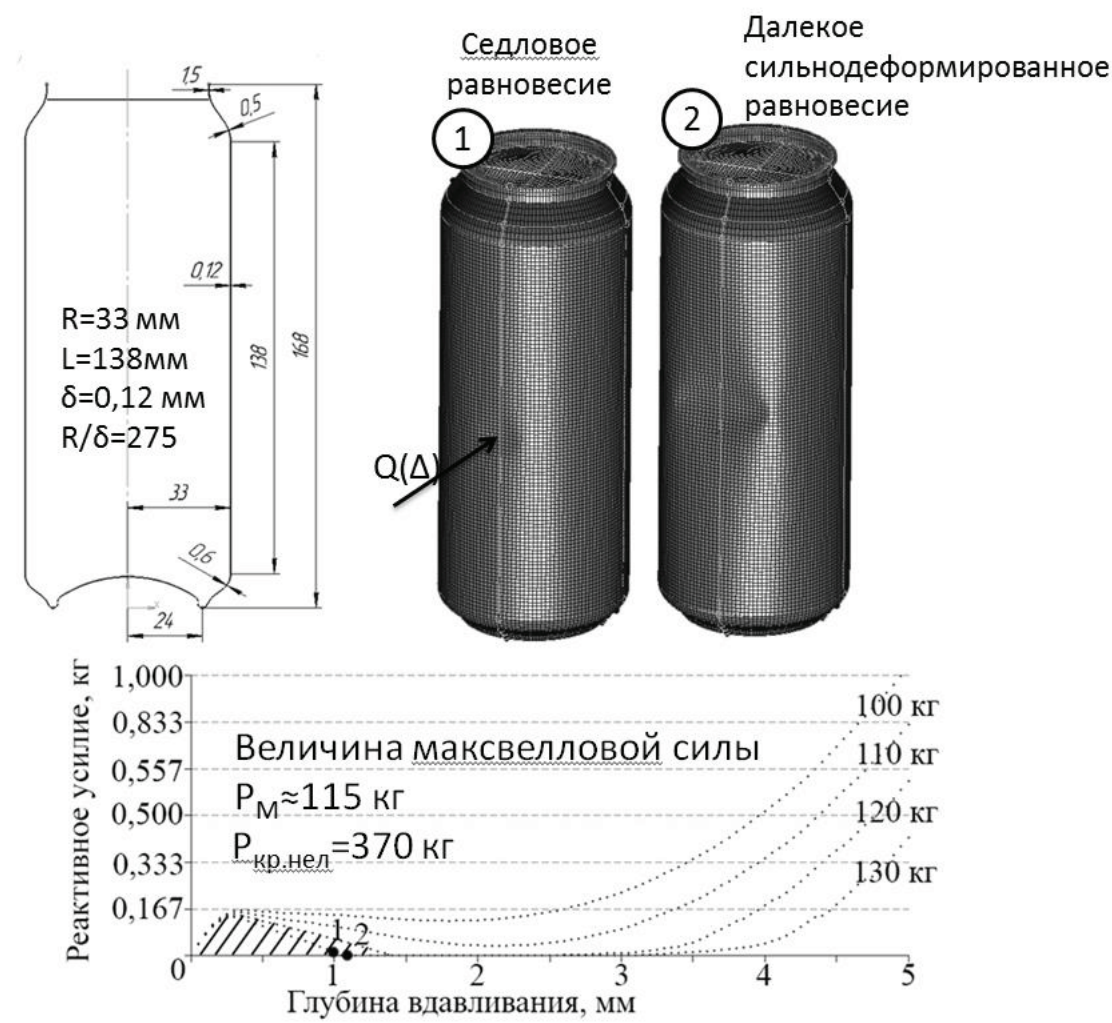

Рисунок 11. Результаты определения величины нагрузки перепада энергетического барьера для оболочки из дюралюмния. 
О явлении потери устойчивости продольно сжатой круговой цилиндрической оболочки

Часть 2: Максвеллова сила и энергетический барьер

На рис 11 показаны результаты поперечного вдавливания и последующего «хлопка» дюралюминиевой оболочки $(\mathrm{E}=, \mathrm{v}=)$, геометрические параметры которой указаны на рис. Графики изменения реактивной поперечной силы при значениях усилия сжатия, близких к максвелловой силе, показаны на рис. Величина максвелловой силы ( $\mathrm{P}_{\mathrm{M}} \approx 115$ кг) составила $\approx 0,31$ от критической бифуркационной нагрузки, полученной из геометрически нелинейного расчета (Р бифнел $=370$ кг).

Для сжатия, превышающего максвеллову силу $(\mathrm{P}=130$ кг >Рм) было проведено численное исследование процесса вдавливания с GAP-элементом и последующего прощелкивания оболочки. Здесь седловое равновесие на энергетическом водоразделе было достигнуто при критическом вдавливании $\Delta_{\text {кр}}=1$ мм (точка 1 на диаграмме). Далее произошел хлопок оболочки в далекое сильно деформированное равновесие 2 (точка 2 на диаграмме). На рис. 11 (точка 2) видно, что в послехлопковом равновесии появилась одна обширная вмятина значительной глубины.

\section{4. О РАЗРЫВАХ КРИВЫХ ПОСЛЕХЛОПКОВЫХ РАВНОВЕСИЙ И «СНЕЙК-РЕШЕНИЯХ»}

В конце I части данной работы при анализе экспериментальных кривых послехлопковых равновесий В. Тилеманна, а также Н. Ямаки, обсуждались разрывы устойчивых участков равновесий с пвмятинами по окружности и скачкообразные перестройки в равновесия с n-1 вмятинами при одновременном уменьшением нагрузки сжатия. Эти разрывы есть точки пересечения двух равновесных кривых, после которых на каждой кривой равновесие становилось неустойчивым. При этом неустойчивая часть ветви «равновесийп-1» проходит несколько ниже устойчивой ветви «равновесийп». Однако в эксперименте неустойчивые ветви равновесий не наблюдаемы. Все устойчивые «n-1 ветви» заканчиваются в левых предельных точках, после прохождения которых равновесия на этих ветвях становятся неустойчивыми.

Описанные особенности кривых экспериментальных послехлопковых равновесий были численно смоделированы Дж. Хантом и его сотрудниками (Л. Нетто, А. Чампнуэй, В. Бадд, Г. Лорд, М. Пельтьер[3-7]).Цели работы этой группы - определение величины «максвелловой» силы, установление факта существования локализованных решений, исследование так называемых «снейкрешений». Наличие неустойчивых участков равновесных кривых «снейковых» решений объясняет «разрывы» в экспериментальных кривых (рис. 12).

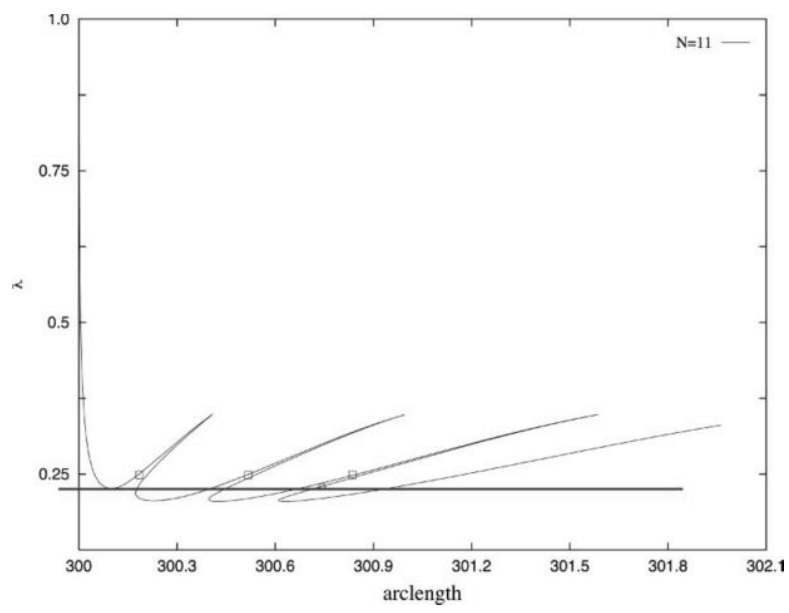

Рисунок 12. Полученные Дж. Хантом с соавторами «снейк-решения», описывающие перестройки форм

закритического равновесия для оболочки Н. Ямаки с параметром $Z=1000$.

Что же касается положения средней линии, делящей площади под снейк-решениями пополам и тем самым (по Дж. Ханту) представляющей уровень нагрузки, равной максвелловой силе для оболочки Н. Ямаки $(\mathrm{Z}=1000)$, то этот вывод представляется спорным. Дело в том, что проведение аналогичной средней линии через экспериметнальные кривые если и дает прямую (?!), то она окажется наклонной (см. часть Інастоящей статьи, рис. 16) к оси укорочения, а не параллельной ей. 


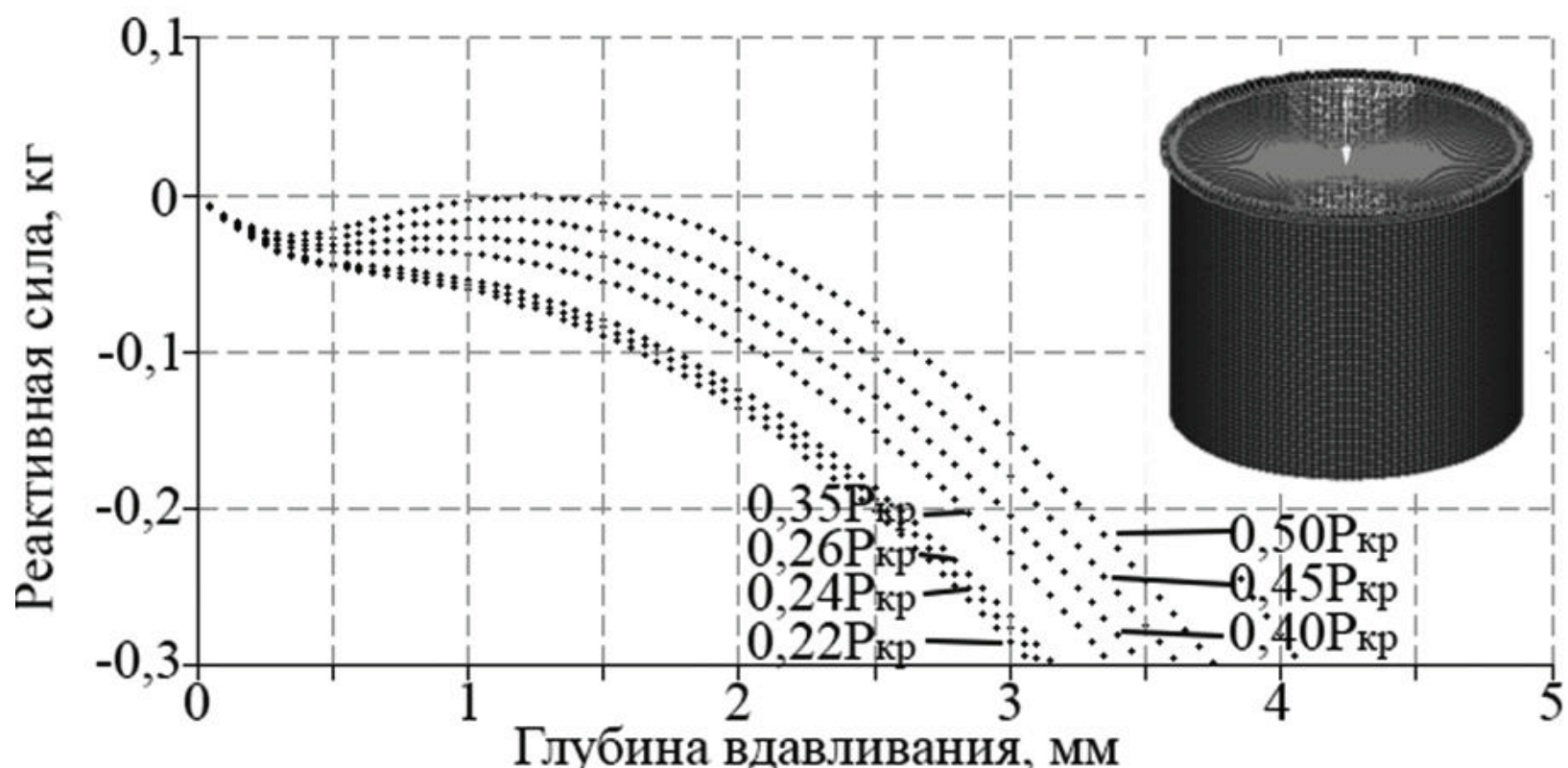

Рисунок 13. Изменение реактивной силь $Q в$ зависимости от глубины вдавливания $\triangle$ для оболочки, рассмотренной Н Ямаки $(Z=1000)$.

Такая прямая не может соответствовать одной нагрузке (в частности максвелловой силе). Кроме тогоснейк-решения если и существуют, то только при кинематическом нагружении, тогда как максвеллова сила существует как при силовом, так и кинематическом нагружениях. Именно в условиях силового нагружения был впервые экспериментально обнаружен резкий перепад энергетического барьера [13].

На рис. 13 представлены графики изменения реактивного усилия $\mathrm{Q}(\Delta)$ при кинематическом вдавливании в средней точке оболочки Н. Ямаки $(\mathrm{Z}=1000)$. Сосредоточенное вдавливание выполнялось при сжимающих нагрузках, значения которых составляли $0,22,0,24,0,26,0,35,0,4,0,45,0,48$ от классической критической нагрузки $\mathrm{P}_{\text {кр.кл }} \approx 131,5$ кг. Как видно из графиков, впервые реактивное усилие вдавливания обращается в ноль при глубине вдавливания $\Delta_{\text {кр }}=1,1$ мм (учетверенная толщина оболочки), и сжимающей нагрузке $\mathrm{P} \approx 63$ кг. Следовательно для данной оболочки Н. Ямакимаксвеллова сила $\mathrm{P}_{\mathrm{M}}$ $\approx 63$ кг, что сосотавляет 0,48Р кр.кл, а не
0,245Р кр.кл, как это следует из результатов Дж. Ханта с соавторами [3-6].

\section{5. МАКСВЕЛЛОВА СИЛА И ОЦЕНКИ ДОПУСТИМЫХ НАЧАЛЬНЫХ НЕСОВЕРШЕНСТВ}

В заключение рассмотрим возможность оценки допустимой величины начальных геометрических несовершенств в виде вмятин при помощи максвелловой силы, вычисленной для идеальной оболочки. В процессе нагружения продольным сжатием оболочки с несовершенствами, размеры и глубина вмятин увеличивается вплоть до достижения максимальной нагрузки $\mathrm{P}^{*}$ в предельной точке (верхняя критическая нагрузка). Если эта нагрузка больше максвелловой силы $\left(\mathrm{P}^{*}>\mathrm{P}_{\mathrm{M}}\right)$, то начальное несовершенство в виде рассмотренных вмятин допустимо и не угрожает безопасной эксплуатации оболочки при условии, что эксплуатационная нагрузка сжатия меньше величины $\mathrm{P}_{\mathrm{M}}$. 
О явлении потери устойчивости продольно сжатой круговой цилиндрической оболочки

Часть 2: Максвеллова сила и энергетический барьер
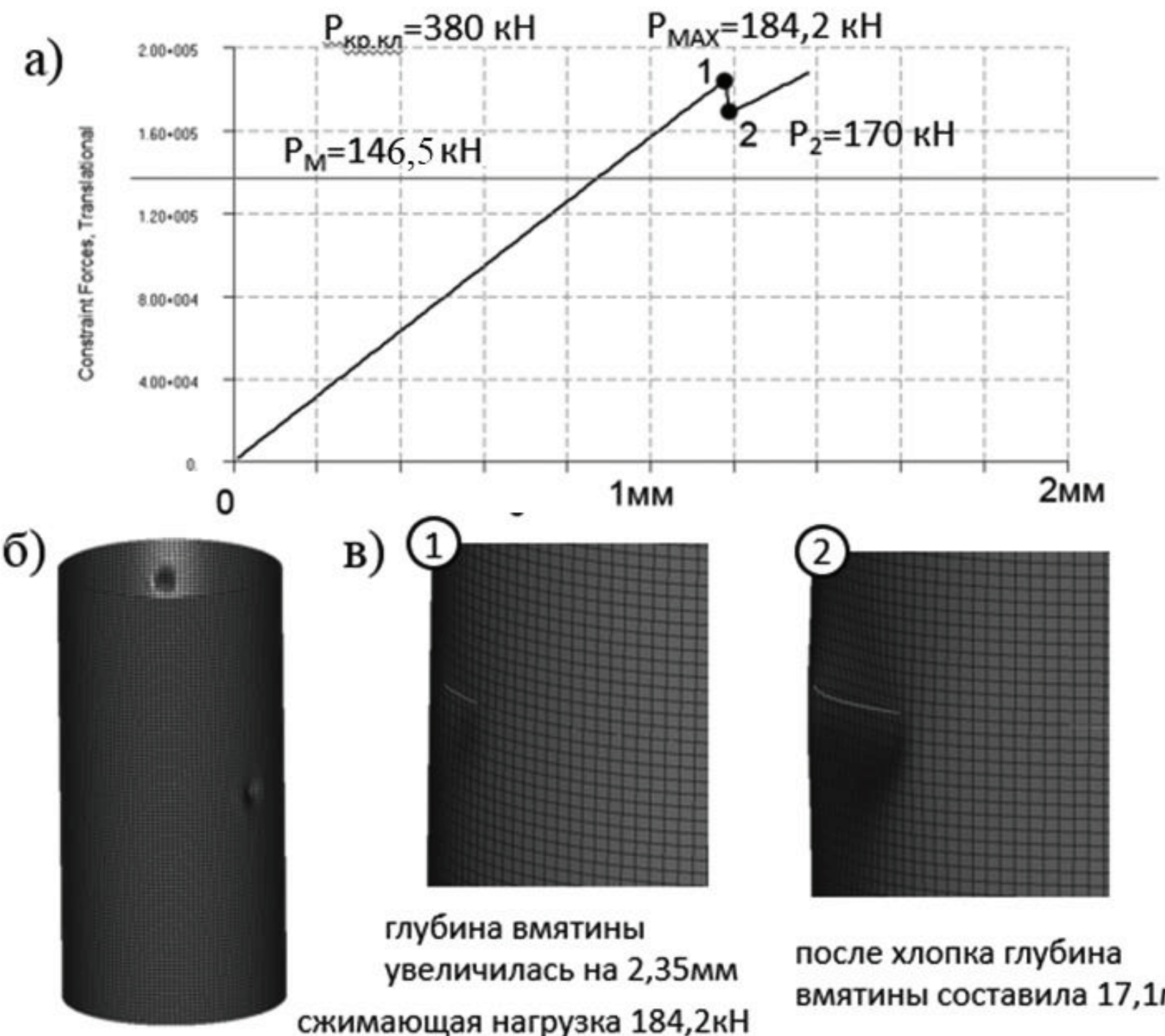

B)

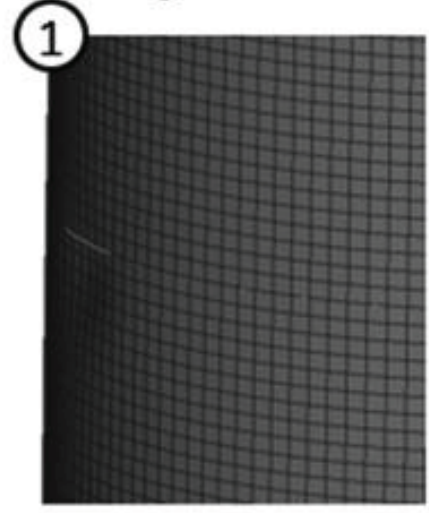

глубина вмятины

увеличилась на 2,35мм

сжимающая нагрузка 184,2кH

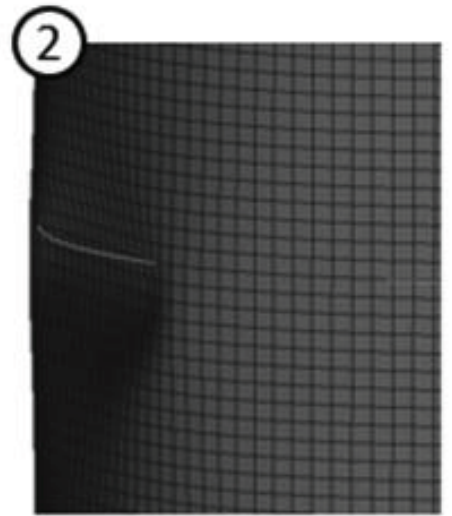

после хлопка глубина вмятины составила 17,1мм

Рисунок 14. Исследование влияния вмятин глубиной 1мм на поверхности модельной оболочки на величину критической нагрузки: а) график изменения нагрузки при кинематическом нагружении оболочки; б) вид вмятины в характерных точках графика;

в) исходный вид вмятин

В противном случае, когда верхняя критическая нагрузка меньше максвелловой силы $\left(\mathrm{P}^{*}<\mathrm{P}_{\mathrm{M}}\right)$, рассмотренное несовершенство недопустимо, и нагрузки на оболочку с вмятинами должны быть существенно меньше величины $\mathrm{P}_{\mathrm{M}}$.

На рис. 14 показана ранее рассмотренная модельная оболочка $(\mathrm{L} / \mathrm{R}=4)$ с тремя достаточно произвольно расположенными вмятинами, имеющими глубину, равную толщине оболочки ( $\delta=1$ мм). При кинематическом нагружении оболочки продольным сжатием была достигнута верхняя критическая нагрузка в предельной точке, равная $\mathrm{P}^{*}=184,02$ кН. При этом глубина средней вмятины увеличилась, далее произошел сравнительно малый скачек по нагрузке до значения $\mathrm{P}_{2}=170$ кН.

Глубина средней вмятины в результате скачка увеличилась на 17 мм (рис. 14). Поскольку критическая нагрузка в предельной точке оказалась выше максвелловой силы, то рассмотренные начальные вмятины глубиной 1 мм можно считать безопасными для данной оболочки при условии, что эксплуатационная нагрузка Р не будет превышать величину 
максвелловой силы (т.е. $\mathrm{P}<\mathrm{P}_{\mathrm{M}}=146,5$ кН). Аналогичные численные исследования для более короткой оболочки ( $\mathrm{L} / \mathrm{R}=2)$ с такими же тремя вмятинами показали, что если глубина всех вмятин $\sim 0,5$ мм, то критическая нагрузка в предельной точке составила 212 кН. Это значение оказалось больше величины максвелловой силы для этой оболочки без вмятин ( $\mathrm{P}_{\mathrm{M}} \approx 187$ кН<Р*). Следовательно, такие начальные вмятины не создают опасность потери устойчивости оболочки при нагрузках, меньших максвелловой силы. Однако увеличение начальной глубины вмятин до 1мм приводит к уменьшению критической нагрузки в предельной точке $\left(\mathrm{P}^{*}=197\right.$ кН). Этавеличина находится уже в опасной близости к максвелловой силе и, по мнению авторов, оболочку, имеющую вмятины с такой начальной глубиной, лучше не допускать в эксплуатацию при «рабочих» нагрузках сжатия близких к величине $\mathrm{P}_{\mathrm{M}} \sim 160-170$ кН.

Следует ожидать, что те же 3 вмятины, но с большей начальной глубиной (1,5-2 мм) снизят критическую нагрузку сжатия в предельной точке еще больше, и она окажется меньше максвелловой силы $\left(\mathrm{P}^{*}<\mathrm{P}_{\mathrm{M}}\right)$. В соответствии с изложенным оболочку с такими «большими» несовершенствами нельзя допускать к эксплуатации даже при нагрузках меньших максвелловой силы, но достаточно близких к ней. Естественно, что эти предварительные рекомендации необходимо обосновать результатами дальнейших более широких численных и экспериментальных исследований.

\section{СПИСОК ЛИТЕРАТУРЫ}

1. Friedrichs K.O. On the minimum buckling load for spherical shells // Theodore von Kármán Anniversary Volume, California Institute of Technology, Pasadena, Calif., 1941. - pp. 258-272.

2. Tsien H.S. Theory for the buckling of thin shells // J. Aero. Sci., 9, 1942. P 373-384.
3. Hunt G.W., Lord G.J., Champneys A.R. Homoclinic and heteroclinic orbits underlying the post-buckling of axially-compressed cylindrical shells // Comput. Methods Appl. Mech. Eng., 170. -1999. - pp. 239-251.

4. Hunt G.W., Lucena Neto E. Maxwell critical loads for axially loaded cylindrical shells // ASME J. Appl. Mech., 60(3). 1993. - 702-706.

5. Hunt G.W. Reflections and symmetries in space and time // IMA Journal of Applied Mathematics 76, 2-26. -2011

6. Hunt G.W., Lord G.J., Peletier M.A. Cylindrical shell buckling: a characterization of localization and periodicity // Discrete \& Continuous Dynamical Systems, Series B, 3. - 2003. - pp. 505-518.

7. Budd C.J., Hunt G.W., Kuske R. Asymptotics of cellular buckling close to the Maxwell load // Proceedings of the Royal Society A, 457. - 2001. - pp. 2935-2964.

8. Thompson J.M.T., Van der Heijden G.H.M Quantified "shock-sensitivity" above the Maxwell load // Int. J. Bifurcation and Chaos, 24 (3). - 2014.

9. Thompson J.M.T. Advances in shell buckling: theory and experiments // Int. J. Bifurcation and Chaos, 2015

10. Horak J., Lord G.J., Peletier M.A. Cylinder buckling: the mountain pass as an organizing centre. // SIAM Journal Applied Mathematics 66. - 2006. - pp. 1793-1824.

11. Choi Y.S., McKenna P.J. A mountain pass method for the numerical solution of semilinear elliptic problems // Nonlinear Analysis, Theory, Methods \& Applications, Vol. 20, No. 4, pp. 417-437, 1993.

12. Паламарчук В.Г. Процесс выпучивания круговой цилиндрической оболочки при осевом сжатии // Труды седьмой Всесоюзной конференции по теории оболочек и пластин. М.: Наука 1970, с 460-464.

13. Esslinger M., Geier B. Calculated postbuckling loads as lower limits for the buckling loads of thin-walled circular cylinders // Buckling of structures - Proceedings of 
О явлении потери устойчивости продольно сжатой круговой цилиндрической оболочки Часть 2: Максвеллова сила и энергетический барьер

the Symposium, Cambridge, Mass, 1974. pp. 274-290.

14. Мануйлов Г.А., Косицын С.Б., Бегичев М.М. О явлении потери устойчивости продольно сжатой круговой цилиндрической оболочки. Часть 1: О послекритическом равновесии оболочки. // International Journal for Computetional Civil and Structural Engineering / Международный журнал по расчету гражданских и промышленных конструкций, 2016, Volume 12, Issue 3, pp. 58-72.

Мануйлов Гайк Александрович, кандидат технических наук, доцент, доцент кафедры «Строительная механика» Московского государственного университета путей сообщения Императора Николая II (МГУПС (МИИТ)); 127994, г. Москва, ул. Образцова, 9, стр. 9; тел./факс +7(499) 972-49-81

Косицын Сергей Борисович, доктор технических наук, профессор, заведующий кафедрой «Теоретическая механика» Московского государственного университета путей сообщения Императора Николая II (МГУПС (МИИТ)); 127994, г. Москва, ул. Образцова, 9, стр. 9; тел./факс +7(499) 978-16-73;

E-mail: kositsyn-s@yandex.ru, kositsyn-s@mail.ru

Бегичев Максим Михайлович, кандидат технических наук, доцент кафедры «Теоретическая механика» Московского государственного университета путей сообщения Императора Николая II (МГУПС (МИИТ)); 127994, г. Москва, ул. Образцова, 9, стр. 9; тел./факс +7(499) 978-16-73; E-mail: noxonius@ mail.ru

Gaik A. Manuylov, Ph.D., Associate Professor, Department of Structural Mechanics, Moscow State University of Railway Engineering (MIIT); 127994, Russia, Moscow, 9b9 Obrazcova Street; phone/fax +7(499)972-49-81.

Sergey B. Kosytsyn, Dr.Sc., Professor, Head of Department of Theoretical Mechanics, Moscow State University of Railway Engineering (MIIT); 127994, Russia, Moscow, 9b9 Obrazcova Street; phone/fax: +7(499) 978-1673; E-mail: kositsyn-s@yandex.ru, kositsyn-s@mail.ru

Maxim M. Begichev, Ph.D., Associate Professor, Department of Theoretical Mechanics, Moscow State University of Railway Engineering (MIIT); 127994, Russia, Moscow, 9b9 Obrazcova Street; phone/fax: +7(499) 97816-73; E-mail: noxonius@mail.ru. 


\title{
ЧИСЛЕННОЕ МОДЕЛИРОВАНИЕ НЕСТАЦИОНАРНЫХ УПРУГИХ КОНТУРНЫХ НАПРЯЖЕНИЙ В ОБОЛОЧКЕ РЕАКТОРНОГО ОТДЕЛЕНИЯ АТОМНОЙ СТАНЦИИ С ФУНДАМЕНТОМ И ОСНОВАНИЕМ (ПОЛУПЛОСКОСТЬ) ПРИ УДАРЕ САМОЛЕТА
}

\author{
B.K. Mycaes \\ Российский университет дружбы народов, г. Москва, РОССИЯ
}

\begin{abstract}
Аннотация: Приводится информация о применении численного моделирования для определения волн напряжений при ударных нагрузках. Рассматриваются некоторые вопросы в области определения контурных напряжений в защитной оболочке реакторного отделения атомной станции с помощью волновой теории ударной безопасности. Для решения волновой задачи теории упругости применяется метод конечных элементов в перемещениях. Задача решается методом сквозного счета, без выделения разрывов. Основные соотношения метода конечных элементов получены с помощью принципа возможных перемещений. Исследуемая расчетная область имеет 1096 узловых точек. Получены контурные напряжения в защитной оболочке реакторного отделения атомной станции.
\end{abstract}

Ключевые слова: математическое моделирование, контурные напряжения, оболочка реакторного отделения атомной станции, ударные воздействия, волновая теория, динамическая теория упругости, перемещение, скорость перемещений, ускорение, метод конечных элементов, комплекс программ, узловые точки, явная двухслойная схема, несущая способность

\section{NUMERICAL SIMULATION OF NON-STATIONARY ELASTIC CONTOUR STRESSES IN THE SHELL OF THE REACTOR COMPARTMENT OF THE NUCLEAR POWER STATION WITH THE FOUNDATION AND BASIS (HALF-PLANE) AT IMPACT OF AIRCRAFT}

\author{
Vyacheslav K. Musayev \\ Russian University of friendship of peoples, Moscow, RUSSIA
}

\begin{abstract}
Provides information on the application of numerical simulation for determination of stress waves at shock loadings. Discusses some of the issues in the field of determination of the contour of stresses in the containment of the reactor compartment of the nuclear power station with the wave theory of shock safety. For the solution of wave problems in the theory of elasticity applied finite element method in displacements. The task is solved by the method of end-to-end account, without the allocation of gaps. The main ratios of the finite element method is obtained using the principle of virtual work. The study estimated the region has 1096 anchor points. Received the grid voltages in the containment of the reactor Department of the nuclear station.
\end{abstract}

Key words: mathematical modeling, grid voltages,

the shell of the reactor Department of the nuclear station-impact, wave theory, dynamical theory of elasticity, displacement, velocity, displacement, acceleration, finite element method, complex programs, anchor points,

an explicit two-layer scheme, bearing capacity 
Численное моделирование нестационарных упругих контурных напряжений в оболочке реакторного отделения атомной станции с фундаментом и основанием (полуплоскость) при ударе самолета

\section{ПОСТАНОВКА ЗАДАЧИ ПРИ УДАРНЫХ УПРУГИХ ВОЛНОВЫХ ВОЗДЕЙСТВИЯХ}

Моделирование широко применяется при решении научных и прикладных задач. В данном случае выделим физические и математические модели. Математические модели являются наиболее характерными в естественнонаучных исследованиях. Физические модели имитируют часть свойств исследуемого объекта. В настоящее время обеспечение безопасности уникальных объектов является приоритетной задачей фундаментальной и прикладной науки.

Приводится постановка, метод решения и результаты в виде контурных напряжений для задачи об ударном воздействии на защитную оболочку реакторного отделения атомной станции с фундаментом и грунтовым основанием.

Постановки, численные методы, технология программных комплексов и анализ результатов решения нестационарных волновых задач для областей различной формы рассмотрены в следующих работах [1-57].

Для решения задачи о моделировании упругих волн в деформируемых областях сложной формы рассмотрим некоторое тело $\Gamma$ в прямоугольной декартовой системе координат XOY (рис. 1), которому в начальный момент времени $t=0$ сообщается механическое воздействие.

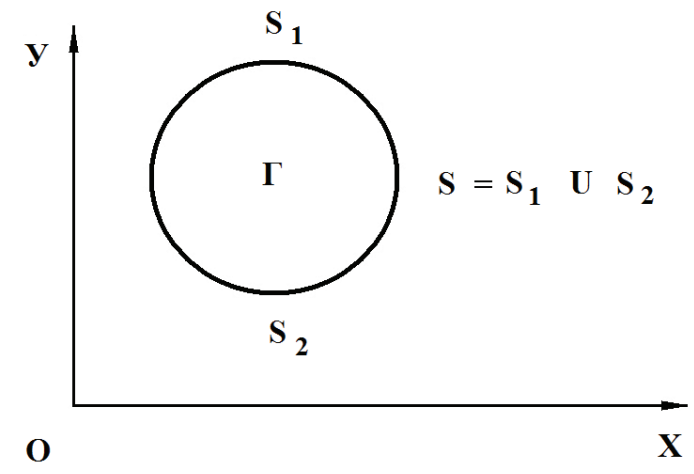

Рисунок 1. Некоторое тело Г в прямоугольной декартовой системе координат ХOY .
Предположим, что тело $\Gamma$ изготовлено из однородного изотропного материала, подчиняющегося упругому закону Гука при малых упругих деформациях.

Точные уравнения двумерной (плоское напряженное состояние) динамической теории упругости имеют вид

$$
\begin{gathered}
\frac{\partial \sigma_{x}}{\partial X}+\frac{\partial \tau_{x y}}{\partial Y}=\rho \frac{\partial^{2} u}{\partial t^{2}}, \\
\frac{\partial \tau_{y x}}{\partial X}+\frac{\partial \sigma_{y}}{\partial Y}=\rho \frac{\partial^{2} v}{\partial t^{2}},(x, y) \in \Gamma, \\
\sigma_{x}=\rho C_{p}^{2} \varepsilon_{x}+\rho\left(C_{p}^{2}-2 C_{s}^{2}\right) \varepsilon_{y}, \\
\sigma_{y}=\rho C_{p}^{2} \varepsilon_{y}+\rho\left(C_{p}^{2}-2 C_{s}^{2}\right) \varepsilon_{x}, \\
\tau_{x y}=\rho C_{s}^{2} \gamma_{x y}, \\
\varepsilon_{x}=\frac{\partial u}{\partial X}, \quad \varepsilon_{y}=\frac{\partial v}{\partial Y}, \\
\frac{\partial u}{\partial Y}+\frac{\partial v}{\partial X}, \quad(x, y) \in(\Gamma \cup S),
\end{gathered}
$$

где: $\sigma_{x}, \sigma_{y}$ и $\tau_{x y}-$ компоненты тензора упругих напряжений; $\varepsilon_{x}, \varepsilon_{y}$ и $\gamma_{x y}-$ компоненты тензора упругих деформаций; $u$ и $v$ - составляющие вектора упругих перемещений вдоль осей $O X$ и $O Y$ соответственно; $\rho$ - плотность материала;

$$
C_{p}=\sqrt{\frac{E}{\rho\left(1-v^{2}\right)}}
$$

- скорость продольной упругой волны;

$$
C_{S}=\sqrt{\frac{E}{2 \rho(1+v)}}
$$

- скорость поперечной упругой волны; v коэффициент Пуассона; $E$ - модуль упругости; $S\left(S_{1} \cup S_{2}\right)$ - граничный контур тела $\Gamma$. 
Систему (1) в области, занимаемой телом $\Gamma$, следует интегрировать при начальных и граничных условиях.

Начальные условия в области $\Gamma$ зададим в виде

$$
\begin{gathered}
\left.u\right|_{t=0}=u_{0},\left.v\right|_{t=0}=v_{0}, \\
\left.\dot{u}\right|_{t=0}=\dot{u}_{0},\left.\dot{v}\right|_{t=0}=\dot{v}_{0},(x, y) \in \Gamma,
\end{gathered}
$$

где: $u_{0}, v_{0}, \dot{u}_{0}$ и $\dot{v}_{0}-$ заданные в области $\Gamma$ функции.

Граничные условия зададим в виде:

составляющих компонентов тензора упругих напряжений на границе $S_{1}$

$$
\begin{gathered}
\sigma_{x} l+\tau_{x y} m=A_{x}, \\
\tau_{x y} l+\sigma_{y} m=A_{y},(x, y) \in S_{1}
\end{gathered}
$$

составляющих компонентов вектора упругих перемещений на границе $S_{2}$

$$
u=B_{x}, \quad \mathrm{v}=\mathrm{B}_{\mathrm{y}}, \quad(x, y) \in S_{2},
$$

где: $l$ и $m$ - направляющие косинусы; $A_{x}$, $A_{y}, B_{x}$ и $B_{y}-$ заданные на границе $\mathrm{S}$ функции.

\section{РАЗРАБОТКА МЕТОДИКИ И АЛГОРИТМА}

Для решения двумерной нестационарной динамической задачи математической теории упругости с начальными и граничными условиями (1-4) используем метод конечных элементов в перемещениях.

Возросшая популярность метода конечных элементов, как наиболее эффективного математического аппарата для инженерного проектирования, сделала актуальным создание специализированных вычислительных систем автоматической подготовки входных данных, решение дифференциальных уравнений и обработку выходных результатов.
Задача решается методом сквозного счета, без выделения разрывов.

Основные соотношения метода конечных элементов получены с помощью принципа возможных перемещений.

Принимая во внимание определение матрицы жесткости, вектора инерции и вектора внешних сил для тела $\Gamma$, записываем приближенное значение уравнения движения в теории упругости

$$
\begin{gathered}
\bar{H} \overrightarrow{\dot{\Phi}}+\bar{K} \vec{\Phi}=\vec{R}, \\
\left.\vec{\Phi}\right|_{t=0}=\vec{\Phi}_{0},\left.\quad \overrightarrow{\dot{\Phi}}\right|_{t=0}=\overrightarrow{\dot{\Phi}}_{0},
\end{gathered}
$$

где: $\bar{H}$ - диагональная матрица инерции; $\bar{K}$ - матрица жесткости; $\vec{\Phi}$ - вектор узловых упругих перемещений; $\overrightarrow{\dot{\Phi}}$ - вектор узловых упругих скоростей перемещений; $\overrightarrow{\ddot{\Phi}}-$ вектор узловых упругих ускорений; $\vec{R}-$ вектор внешних узловых упругих сил.

Соотношение (5) система линейных обыкновенных дифференциальных уравнений второго порядка в перемещениях с начальными условиями.

Таким образом, с помощью метода конечных элементов в перемещениях, линейную задачу с начальными и граничными условиями (14) привели к линейной задаче Коши (5). Для интегрирования уравнения (5) конечноэлементным вариантом метода Галеркина приведем его к следующему виду

$$
\bar{H} \frac{d}{d t} \overrightarrow{\dot{\Phi}}+\bar{K} \vec{\Phi}=\vec{R}, \frac{d}{d t} \vec{\Phi}=\overrightarrow{\dot{\Phi}}
$$

Интегрируя по временной координате соотношение (6) с помощью конечноэлементного варианта метода Галеркина, получим двумерную явную двухслойную конечноэлементную линейную схему в перемещениях для внутренних и граничных узловых точек

$$
\begin{gathered}
\overrightarrow{\dot{\Phi}}_{i+1}=\overrightarrow{\dot{\Phi}}_{i}+\Delta t \bar{H}^{-1}\left(-\bar{K} \vec{\Phi}_{i}+\vec{R}_{i}\right) \\
\vec{\Phi}_{i+1}=\vec{\Phi}_{i}+\Delta t \overrightarrow{\dot{\Phi}}_{i+1}
\end{gathered}
$$


Численное моделирование нестационарных упругих контурных напряжений в оболочке реакторного отделения атомной станции с фундаментом и основанием (полуплоскость) при ударе самолета

Основные соотношения метода конечных элементов в перемещениях получены с помощью принципа возможных перемещений и конечноэлементного варианта метода Галеркина.

Система уравнений (7) должна давать решение, сходящееся к решению исходной системы (1-4).

Шаг по временной переменной координате $\Delta t$ определяем из следующего соотношения

$$
\Delta t=k \frac{\min \Delta l_{i}}{C_{p}}(i=1,2,3, \ldots),
$$

где $\Delta l$ - длина стороны конечного элемента. Результаты численного эксперимента показали, что при $k=0,5$ обеспечивается устойчивость двумерной явной двухслойной конечноэлементной линейной схемы в перемещениях для внутренних и граничных узловых точек на квазирегулярных сетках.

Предложен квазирегулярный подход к решению системы линейных обыкновенных дифференциальных уравнений второго порядка в перемещениях с начальными условиями и к аппроксимации исследуемой области.

Для аппроксимации по пространственным координатам применяются треугольные конечные элементы с тремя узловыми точками с линейной аппроксимацией упругих перемещений и прямоугольные конечные элементы с четырьмя узловыми точками с билинейной аппроксимацией упругих перемещений.

По временной переменной исследуемая область разбивается на линейные конечные элементы.

На основе метода конечных элементов в перемещениях разработана методика, разработан алгоритм и составлен комплекс программ для решения двумерных линейных и нелинейных задач при различных начальных и граничных условиях, для областей сложной формы.

Комплексы программ написаны на алгоритмическом языке Фортран-90.

\section{ОПРЕДЕЛЕНИЕ КОНТУРНЫХ НАПРЯЖЕНИЙ В ЗАЩИТНОЙ ОБОЛОЧКЕ РЕАКТОРНОГО ОТДЕЛЕНИЯ АТОМНОЙ СТАНЦИИ}

Рассматривается задача об ударе самолета (рис. 3) на систему сооружение-фундаментоснование (Архангельская атомная станция) при воздействии $\sigma_{01}(\mathrm{H}=69,9$ м) (рис. 2).

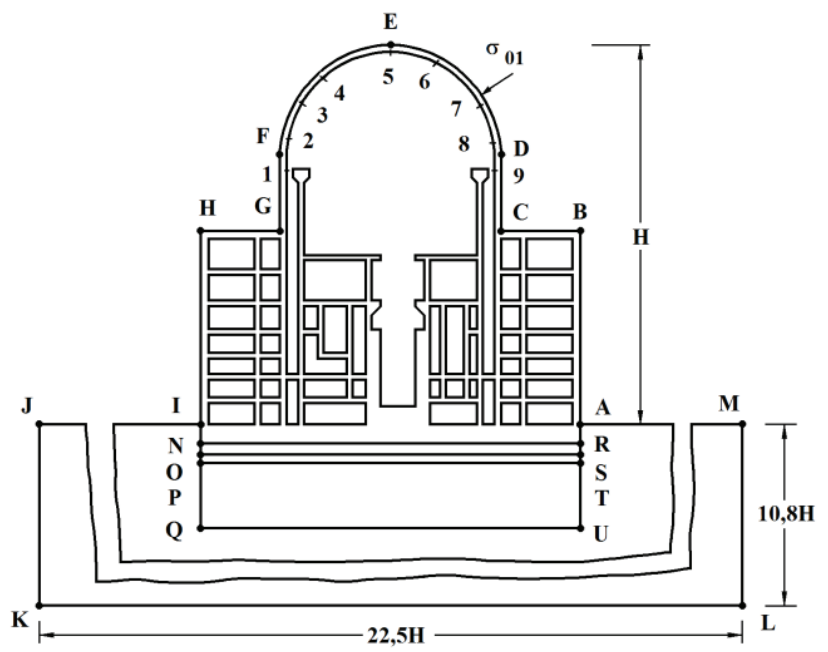

Рисунок 2. Постановка задачи для Архангельской атомной станщии.

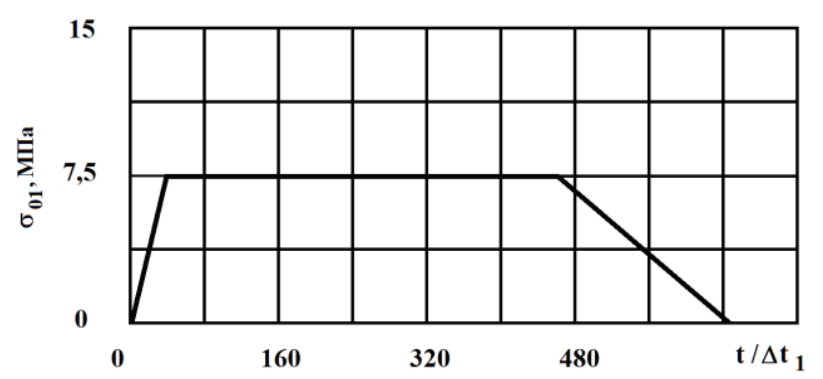

Рисунок 3. Воздействие $\sigma_{01}$.

Начальные условия приняты нулевым. Граничные условия для контура $J K L M$ (рис. 2) при $t>0 \quad u_{i}=v_{i}=\dot{u}_{i}=\dot{v}_{i}=0$. Отраженные волны от контура $J K L M$ не доходят до исследуемых точек при $0 \leq n_{1} \leq 2000$. На границах материалов с разными физическими свойствами приняты условия непрерывности перемещений (1-ABCDEFGHIN $R$ и SOPT; 2-RNOS ; 3-MAUQIJKL;4-TPQU ). 


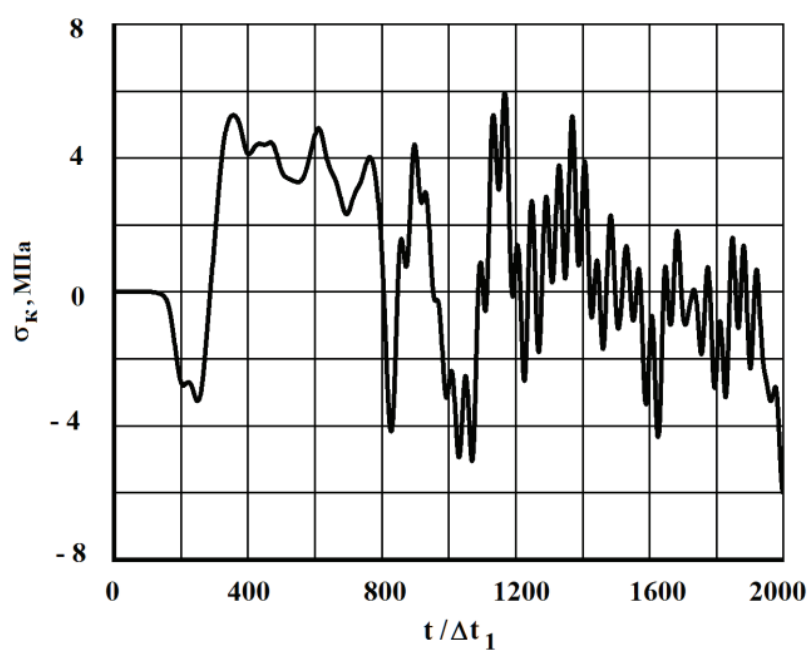

Рисунок 4. Изменение упругого контурного напряжения $\sigma_{k}$ в точке 1 во времени $t / \Delta t_{1}$.

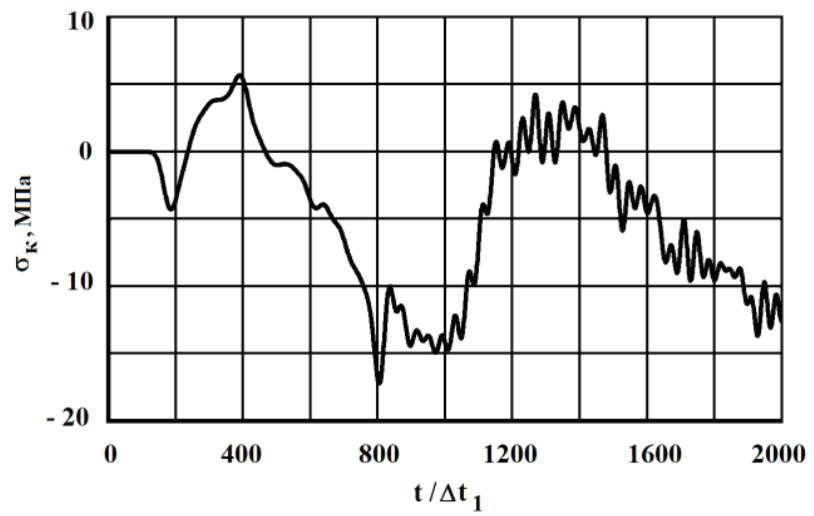

Рисунок 5. Изменение упругого контурного напряжения $\sigma_{k}$ в точке 2 во времени $t / \Delta t_{1}$.

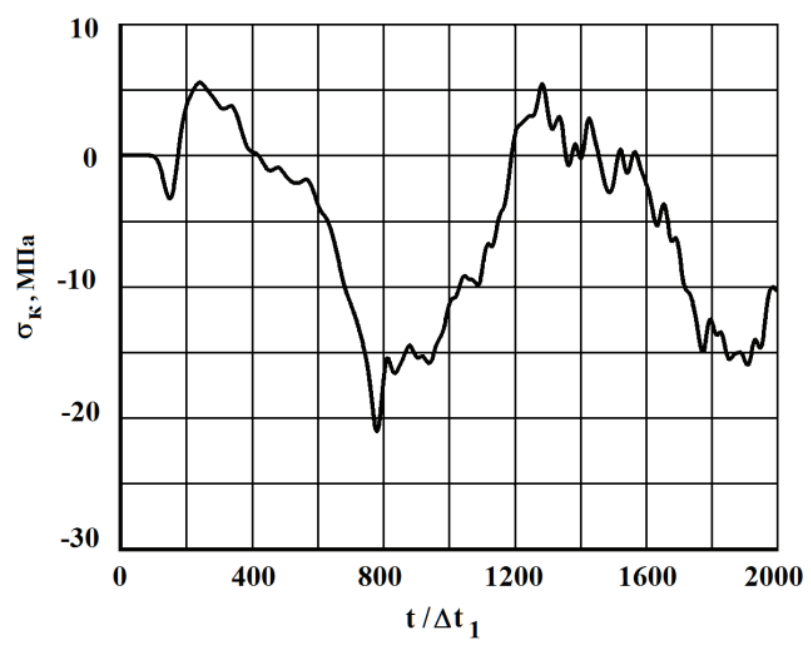

Рисунок 6. Изменение упругого контурного напряжения $\sigma_{k}$ в точке 3 во времени $t / \Delta t_{1}$.

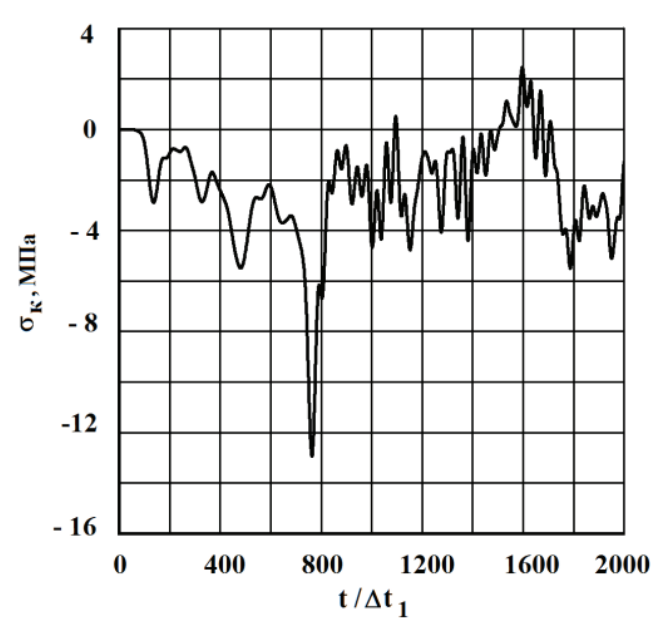

Рисунок 7. Изменение упругого контурного напряжения $\sigma_{k}$ в точке 4 во времени $t / \Delta t_{1}$.

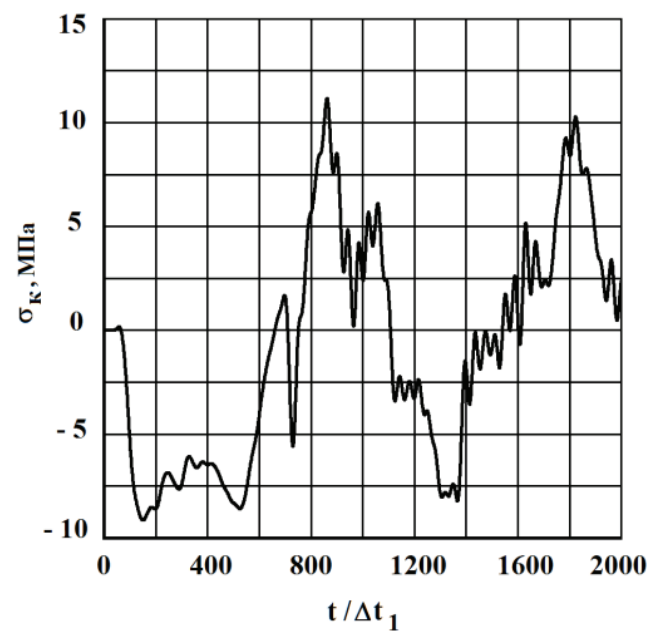

Рисунок 8. Изменение упругого контурного напряжения $\sigma_{k}$ в точке 5 во времени $t / \Delta t_{1}$.

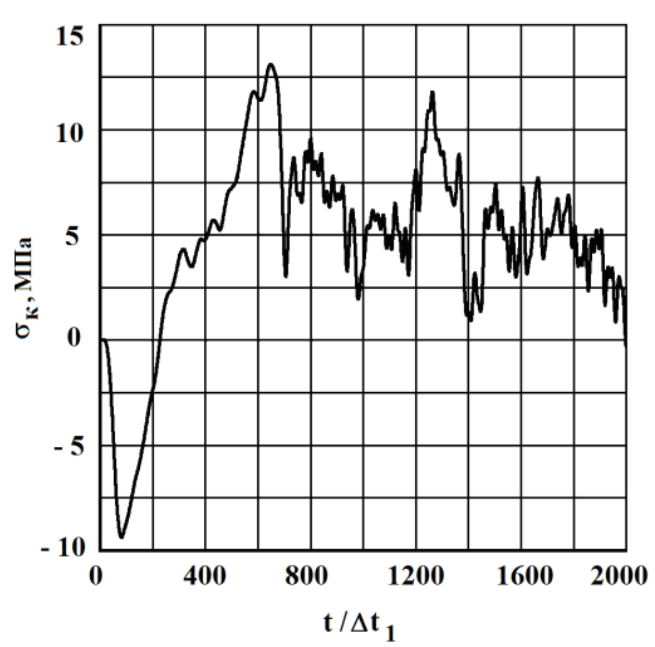

Рисунок 9. Изменение упругого контурного напряжения $\sigma_{k}$ в точке 6 во времени $t / \Delta t_{1}$. 
Численное моделирование нестационарных упругих контурных напряжений в оболочке реакторного отделения атомной станции с фундаментом и основанием (полуплоскость) при ударе самолета

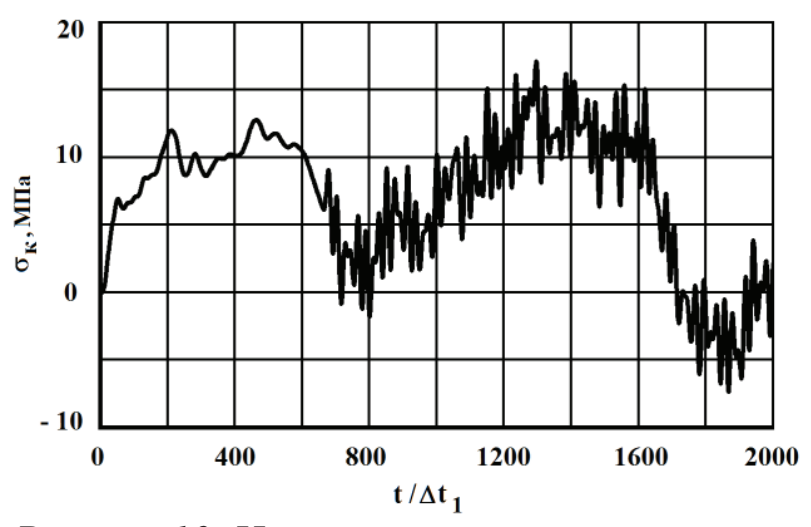

Рисунок 10. Изменение упругого контурного напряжения $\sigma_{k}$ в точке 7 во времени $t / \Delta t_{1}$.

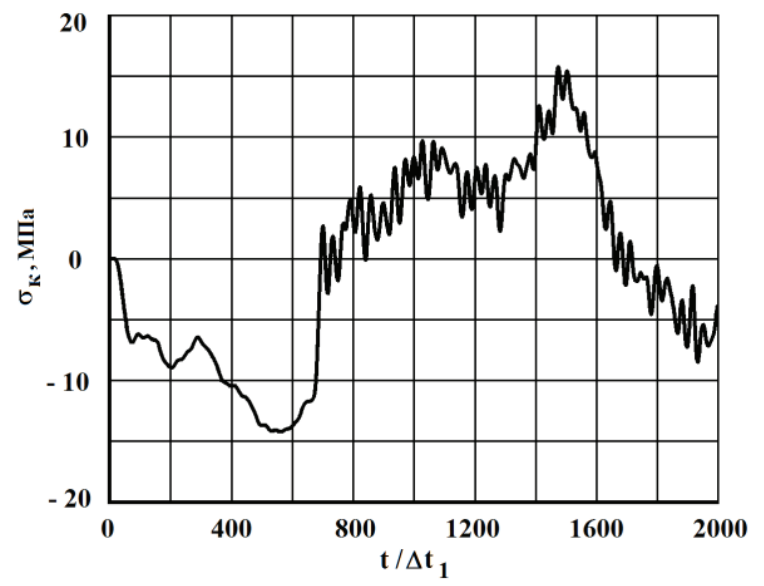

Рисунок 11. Изменение упругого контурного напряжения $\sigma_{k}$ в точке 8 во времени $t / \Delta t_{1}$.

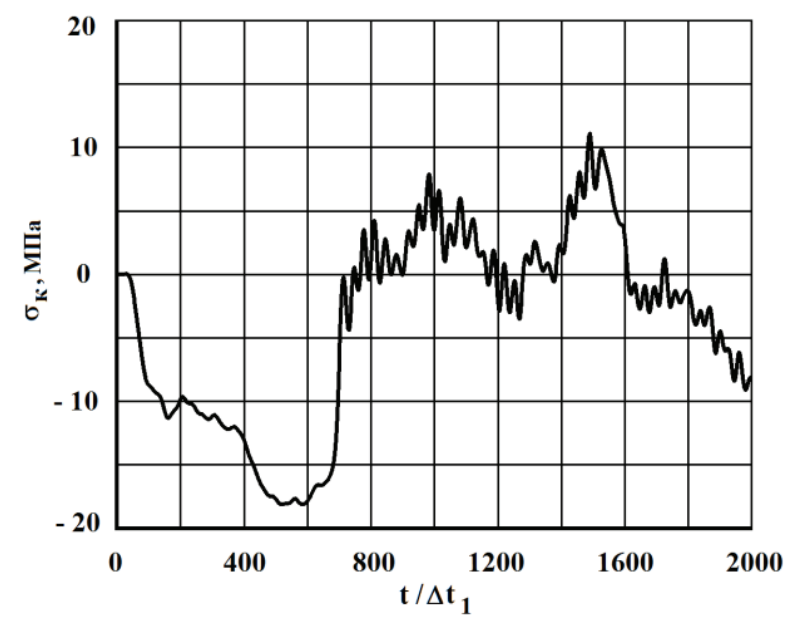

Рисунок 12. Изменение упругого контурного напряжения $\sigma_{k}$ в точке 9 во времени $t / \Delta t_{1}$.
Исследуемая расчетная область имеет 1096 узловых точек. Получены контурные напряжения в защитной оболочке реакторного отделения атомной станции.

На рис. 4-12 показано изменение контурных напряжений $\sigma_{k}$ в защитной оболочке во времени $t / \Delta t_{1}$.

\section{ВЫВОД}

На основании проведенных исследований можно сделать следующий вывод:

Полученные результаты позволяют оценить несущую способность защитной оболочки реакторной отделения атомной станции.

\section{СПИСОК ЛИТЕРАТУРЫ}

1. Филоненко-Бородин М. М. Теория упругости. - М.: Гостехиздат, 1947. $300 \mathrm{c}$.

2. Кольский Г. Волны напряжений в твердых телах. - М.: Иностранная литератуpa, 1955. - 192 c.

3. Тимошенко С.П., Гудьер Д. Теория упругости. - М.: Наука, 1975. - 576 с.

4. Зенкевич О. Метод конечных элементов в технике. - М: Мир, 1975. - 543 с.

5. Ионов В.И., Огибалов П.М. Напряжения в телах при импульсивном нагружении. - М.: Высшая школа, 1975. - 464 с.

6. Тихонов А.Н., Самарский А.А. Уравнения математической физики. - М.: Наука, 1977. - 736 с.

7. Бате К., Вилсон Е. Численные методы анализа и метод конечных элементов. М.: Стройиздат, 1982. - 448 с.

8. Филиппов И.Г., Немчинов В.В. Однородный разностный алгоритм решения плоских динамических задач теории упругости в областях сложной формы // Тезисы докладов конференции по распространению упругих и упругопластических волн. - Фрунзе: Фрунзенский по- 
литехнический институт, 1983, ч. 1, с. 75-76.

9. Хан Х. Теория упругости. - М.: Мир, 1988. - 344 c.

10. Мусаев В.К. Решение задачи дифракции и распространения упругих волн методом конечных элементов // Строительная механика и расчет сооружений, 1990, №4, с. 74-78.

11. Musayev V.K. Structure design with seismic resistance foundations // Proceedings of the ninth European conference on earthquake engineering. - Moscow: TsNIISK, 1990, V. 4-A, pp. 191-200.

12. Musayev V.K. Testing of stressed state in the structure-base system under nonstationary dynamic effects // Proceedings of the second International conference on recent advances in geotechnical earthquake engineering and soil dynamics. - Sent Louis: University of Missouri-Rolla, 1991. - V. 3, pp. 87-97.

13. Мусаев В.К. Математическое моделирование ударных воздействий на реакторное отделение атомной станции // Тезисы докладов XXXII научной конференции факультета физико-математических и естественных наук. Часть 2. Математические секции. - М.: РУДН, 1996, с. 78-79.

14. Мусаев В.К. Численное решение волновых задач теории упругости и пластичности // Вестник Российского университета дружбы народов. Серия прикладная математика и информатика, 1997, №1, с. 87-110.

15. Мусаев В.К. Конечноэлементное моделирование задачи об ударе самолета на защитную оболочку реакторного отделения атомной станции // Пленарные доклады и тезисы выступлений Международного симпозиума «Партнерство во имя жизни - снижение риска чрезвычайных ситуаций, смягчение последствий аварий и катастроф». - М.: ВНИИ ГОЧС, 1998, c. 207.
16. Мусаев В.К., Хомяков Н.Н., Хомяков Д.Н. Моделирование безопасности системы «Реакторное отделениефундамент-основание» атомной станции при падении самолета // Материалы всероссийской научно-технической конференции «Проблемы прогнозирования, предотвращения и ликвидации последствий чрезвычайных ситуаций». - Уфа: НИИБЖД РБ, 2000, с. 89-90.

17. Филиппов В.М., Мусаев В.К., Кузькина Т.П., Киревнина Е.И., Золотова С.И. Метод конечных элементов в задачах об ударе самолета о систему «Защитная оболочка реакторного отделения-фундамент-основание» атомной станции // Материалы XI Международной конференции «Применение новых технологий в образовании». - Троицк: Байтик, 2000, с. 217-218.

18. Musayev V.K. Problem of the building and the base interaction under seismic loads // Proceedings of the $12^{\text {th }}$ World Conference on Earthquake Engineering. 2741. - Auckland: University of Canterbury, 2000, pp. 1-6.

19. Мусаев В.К. Математическое моделирование безопасности системы «Защитная оболочка реакторного отделенияфундамент-основание» на ударные воздействия в детерминированной постановке // Проблемы управления безопасностью сложных систем. Материалы VIII Международной конференции. - М.: РГГУ, 2000, с. 326-327.

20. Мусаев В.К. О математическом мониторинге защиты населения и территорий от чрезвычайных ситуаций природного и техногенного характера в детерминированной постановка // Оценка и управление природными рисками. Материалы Общероссийской конференции «РИСК2000». - М.: Анкил, 2000, с. 420-424.

21. Мусаев В.К. О безопасности реакторного отделения атомной станции при воздействии импульса МАГАТЭ // Техносферная безопасность - II часть: матери- 
Численное моделирование нестационарных упругих контурных напряжений в оболочке реакторного отделения атомной станции с фундаментом и основанием (полуплоскость) при ударе самолета

алы седьмой Всероссийской научнопрактической конференции. - Ростов-наДону: Ростовский государственный строительный университет, 2002, с. 205212.

22. Горшков А.Г., Медведский А.Л., Рабинский Л.Н., Тарлаковский Д.В. Волны в сплошных средах. - М.: Физматлит, 2004. -472 c.

23. Мусаев В.К., Жидков Е.П., Севастьянов Л.А. Вычислительные методы теоретической физики в задачах моделирования катастроф // Вестник Российского университета дружбы народов. Серия проблемы комплексной безопасности. 2005, №1, c. 9-12.

24. Мусаев В.К. Численное решение некоторых задач безопасности жизнедеятельности с помощью метода конечных элементов // Вестник Российского университета дружбы народов. Серия проблемы комплексной безопасности, 2005, №1, c. 17-23.

25. Мусаев В.К. Определение контурных напряжений в защитной оболочке реакторного отделения атомной станции при ударном воздействии // Вестник Российского университета дружбы народов. Серия проблемы комплексной безопасности, 2005, №2, с. 91-101.

26. Мусаев В.К. Математическое моделирование в задачах расчета несущей способности уникальных сооружений при нестационарных динамических воздействиях // Об опыте декларирования промышленной безопасности. Оценка риска аварий на опасных производственных объектах. Тематический семинар. Москва, 26-27 октября 2005 года: Тезисы докладов. - М.: Федеральное государственное унитарное предприятие «Научно-технический центр по безопасности в промышленности», 2005, с. 6164.

27. Мусаев В.К. Нормативная база расчета на безопасность уникальных сооружений. - М.: РУДН, 2005. - 28 с.
28. Мусаев В.К. О некоторых возможностях математического моделирования и численного компьютерного эксперимента // Вестник Российского университета дружбы народов. Серия проблемы комплексной безопасности, 2006, №1, с. 8186.

29. Мусаев В.К. Математическое моделирование упругих волн напряжений в сложных деформируемых телах // Вестник Российского университета дружбы народов. Серия проблемы комплексной безопасности, 2007, №1, с. 62-76.

30. Мусаев В.К. Об оценке достоверности и точности численного решения нестационарных динамических задач // Вестник Российского университета дружбы народов. Серия проблемы комплексной безопасности, 2007, №3, с. 48-60.

31. Мусаев В.К. О сходимости одномерной явной двухслойной конечноэлементной линейной схемы в перемещениях для внутренних узловых точек на равномерной линейной сетке // Вестник Российского университета дружбы народов. Серия проблемы комплексной безопасности, 2008, №1, с. 52-56.

32. Мусаев В.К. Об устойчивости одномерной явной двухслойной конечноэлементной линейной схемы в перемещениях для внутренних узловых точек на равномерной линейной сетке // Вестник Российского университета дружбы народов. Серия проблемы комплексной безопасности, 2008, №1, с. 57-60.

33. Мусаев В.К. О сходимости двумерной явной двухслойной конечноэлементной линейной схемы в перемещениях для внутренних узловых точек на равномерной прямоугольной сетке // Вестник Российского университета дружбы народов. Серия проблемы комплексной безопасности, 2008, №3, с. 60-68.

34. Мусаев В.К. Об устойчивости двумерной явной двухслойной конечноэлементной линейной схемы в перемещениях для внутренних узловых точек на 
равномерной прямоугольной сетке // Вестник Российского университета дружбы народов. Серия проблемы комплексной безопасности, 2008, №3, с. 6975.

35. Мусаев В.К. Численное, аналитическое и экспериментальное решение задачи о концентрации нестационарных динамических напряжений в свободном круглом отверстии // Вестник Российского университета дружбы народов. Серия проблемы комплексной безопасности, 2008, №4, c. 67-71.

36. Мусаев В.К. О достоверности результатов численного моделирования при решении нестационарных волновых задач // Труды Международной научнопрактической конференции «Инженерные системы-2009». - М.: РУДН, 2009, c. 373-377.

37. Мусаев В.К. О некоторых положениях в области обеспечения безопасности атомных электрических станций // Безопасность и экология технологических процессов и производств. Материалы Всероссийской научно-практической конференции. - Поселок Персиановский Ростовской области: Донской государственный аграрный университет, 2009, с. 69-73.

38. Мусаев В.К. О безопасности защитной оболочки реакторного отделения атомной станции по несущей способности при ударных воздействиях от самолета // Безопасность и экология технологических процессов и производств. Материалы Всероссийской научно-практической конференции. - Поселок Персиановский Ростовской области: Донской государственный аграрный университет, 2009, с. 170-177.

39. Мусаев В.К. Оценка достоверности и точности результатов вычислительного эксперимента при решении задач нестационарной волновой теории упругости // Научный журнал проблем комплексной безопасности, 2009, №1, с. 55-80.
40. Мусаев В.К. О достоверности результатов численного метода решения сложных задач волновой теории упругости при ударных, взрывных и сейсмических воздействиях // Ученые записки Российского государственного социального университета, 2009, №5, с. 21-33.

41. Мусаев В.К. Моделирование волн напряжений в сложных областях с помощью метода вычислительной механики // Исследования по теории сооружений, 2010, №2, с. 44-52.

42. Мусаев В.К. Вычислительный эксперимент в задачах моделирования нестационарных волн напряжений в областях сложной формы // Исследования по теории сооружений, 2010, №2, с. 138-149.

43. Ситник С.В., Сазонов К.Б., Шиянов С.М., Куранцов В.В., Кормилицин А.И. Моделирование волнового напряженного состояния в объектах сложной формы с помощью численного метода Мусаева В.К. в перемещениях // Техносферная безопасность, надежность, качество, энерго- и ресурсосбережение: Т38. Материалы Международной научнопрактической конференции. Выпуск XIII. T. 2. - Ростов-на-Дону: Ростовский государственный строительный университет, 2011, с. 262-268.

44. Мусаев В.К. Моделирование контурных напряжений в оболочке реакторного отделения атомной станции при ударе самолета с помощью волновой теории // Ревальвация публичной безопасности. Материалы Международной научной конференции. - Гожув Влкп.-Познань, Польша: Институт научных исследований и экспертиз, 2011, с. 285-294.

45. Мусаев В.К. О моделировании волновых контурных напряжений в оболочке реакторного отделения атомной станции при ударных воздействиях // Вестник НИЦ строительство. Исследования по теории сооружений, 2011, №3-4, с. 3542. 
Численное моделирование нестационарных упругих контурных напряжений в оболочке реакторного отделения атомной станции с фундаментом и основанием (полуплоскость) при ударе самолета

46. Мусаев В.К. Моделирование контурных напряжений в оболочке реакторного отделения атомной станции при ударе самолета с помощью волновой теории ударной безопасности // Инновационные технологии в развитии строительства, машин и механизмов для строительства и коммунального хозяйства, текущего содержания и ремонта железнодорожного пути. Сборник трудов международной научно-технической конференции. Смоленск: Смоленский филиал МИИТ, 2012, c. 247-253.

47. Тахо-Годи А.3. О методе решения нестационарных волновых задач с помощью численного метода Мусаева В.К. в перемещениях // Безопасность и экология технологических процессов и производств. Материалы Всероссийской научно-практической конференции. - Поселок Персиановский Ростовской области: Донской государственный аграрный университет, 2012, с. 73-77.

48. Куранцов В.А., Денисюк Д.А., Денисенков А.Н., Савичев В.А., Акатьев C.В. Моделирование нестационарных волн напряжений в деформируемых объектах сложной формы с помощью численного метода Мусаева В.К. в перемещениях // Безопасность и экология технологических процессов и производств. Материалы Всероссийской научнопрактической конференции. - Поселок Персиановский Ростовской области: Донской государственный аграрный университет, 2012, с. 213-216.

49. Ситник С.В., Сущев Т.С., Денисенков А.Н., Савичев В.А., Акатьев Д.В. Достоверность результатов численного метода Мусаева В.К. в перемещениях при математическом моделировании отражения упругих волн напряжений в виде функции Хевисайда от свободной поверхности пластинки // Безопасность и экология технологических процессов и производств. Материалы Всероссийской научно-практической конференции. -
Поселок Персиановский Ростовской области: Донской государственный аграрный университет, 2012, с. 263-267.

50. Ситник С.В., Куранцов В.А., Денисюк Д.А., Денисенков А.Н., Савичев В.А. Достоверность результатов численного метода Мусаева В.К. в перемещениях при математическом моделировании отражения упругих волн напряжений в виде функции Хевисайда от жесткой поверхности пластинки // Безопасность и экология технологических процессов и производств. Материалы Всероссийской научно-практической конференции. Поселок Персиановский Ростовской области: Донской государственный аграрный университет, 2012, с. 267-271.

51. Ситник С.В., Сущев Т.С., Кормилицин А.И., Шиянов С.М., Акатьев Д.В. Достоверность результатов численного метода Мусаева В.К. в перемещениях при математическом моделировании отражения упругих волн напряжений в виде дельта функции от свободной поверхности пластинки // Безопасность и экология технологических процессов и производств. Материалы Всероссийской научно-практической конференции. Поселок Персиановский Ростовской области: Донской государственный аграрный университет, 2012, с. 287-292.

52. Ситник С.В., Денисюк Д.А., Денисенков А.Н., Савичев В.А., Акатьев Д.В. Достоверность результатов численного метода Мусаева В.К. в перемещениях при математическом моделировании отражения упругих волн напряжений в виде дельта функции от жесткой поверхности пластинки // Безопасность и экология технологических процессов и производств. Материалы Всероссийской научно-практической конференции. - Поселок Персиановский Ростовской области: Донской государственный аграрный университет, 2012, с. 297-302.

53. Шиянов С.М., Шепелина П.В., Куранцов В.В., Кормилицин А.И. О роли 
волн напряжений в разрушении несущих конструкций сложных технических систем // Двойные технологии, №4, 2012, с. $58-60$.

54. Мусаев В.К. О моделировании отражения упругих волн напряжений от свободной поверхности деформируемой области // Двойные технологии, 2012, №4, c. $61-64$.

55. Мусаев В.К. Математическое моделирование системы «сооружениеокружающая среда» на ударные, взрывные и сейсмические воздействия // Информационно-телекоммуникационные технологии и математическое моделирование высокотехнологичных систем. Материалы Всероссийской конференции с международным участием. - М.: РУДН, 2013, с. 197-200.

56. Тахо-Годи А.3. Математическое моделирование нестационарных волн напряжений в деформируемых областях с помощью численного метода Мусаева В.К. в перемещениях // Фундаментальные исследования, 2013, №1 (часть 1), с. 159162.

57. Nemchinov V.V. Numerical methods for solving flat dynamic elasticity problems // International Journal for Computational Civil and Structural Engineering, 2013, Volume 9, Issue 1, pp. 90-97.

Мусаев Вячеслав Кадыр оглы, Почетный работник высшего профессионального образования Российской Федерации, доктор технических наук, профессор, профессор кафедры экологического мониторинга и прогнозирования Российского университета дружбы народов; 117198, Россия, г. Москва, ул. МиклухоМаклая, 6; тел. +7(926) 5670558;

e-mail: musayev-vk@yandex.ru

Musayev Vyacheslav Kadyr ogly. Honorary worker of higher professional education of the Russian Federation, Doctor of Technical Sciences, Professor, Professor of the chair of ecological monitoring and prognostication of the Russian University of friendship of peoples; 6, st. Miklukho-Maklay, 117198, Moscow, Russia;

tel.+7(926)5670558; e-mail: musayev-vk@yandex.ru 


\title{
АПРОБАЦИЯ МЕТОДИКИ ЧИСЛЕННОГО МОДЕЛИРОВАНИЯ СТАТИЧЕКОГО И СЕЙСМИЧЕСКОГО НАПРЯЖЕННО-ДЕФОРМИРОВАННОГО СОСТОЯНИЯ НА ТРЕХМЕРНОЙ СИСТЕМЕ «ОСНОВАНИЕ - ПЛОТИНА - ВОДОХРАНИЛИЩЕ» ГЭС НАМ ЧИЕН (ВЬЕТНАМ)
}

\author{
Нгуен Тай Нанг Льонг \\ Национальный исследовательский Московский государственный строительный университет, \\ г. Москва, РОССИЯ
}

\begin{abstract}
Аннотация: в статье представлена апробация разработанной методики уточненного численного моделирования трехмерного статического и динамического напряженно-деформированного состояния (НДС) системы «основание -арочная плотина - водохранилище» с учетом сейсмического воздействия, заданного трехкомпонентной акселерограммой, на примере ГЭС Нам Чиен во Вьетнаме.
\end{abstract}

Ключевые слова: математическое моделирование, численные методы, метод конечных элементов, блочный метод Ланцоша, неявная схема Ньюмарка, сейсмостойкость, собственные частоты и формы колебаний, акселерограммы, статическое и динамическое напряженно-деформированное состояние

\section{APPROBATION METHOD OF NUMERICAL SIMULATION OF STRAIN-STRESS STATIC AND SEISMIC ANALYSIS OF THREE-DIMENSIONAL SYSTEM «FOUNDATION - DAM - RESERVOIR» ON THE EXAMPLE OF NAM CHIEN HYDRO POWER PLANT (VIETNAM)}

\author{
Nguyen Tai Nang Luong \\ National Research Moscow State University of Civil Engineering, Moscow, RUSSIA
}

\begin{abstract}
The article presents a comparative analysis of solutions of problems on the verification methodology developed by the refined numerical simulation of the static and dynamic stress-strain state (SSS) of threedimensional system "foundation - dam - reservoir" Nam Chien (Vietnam) at seismic impacts given threecomponent accelerograms
\end{abstract}

Key words: math modeling, numerical methods, finite element method, natural frequencies and modes, static and dynamic strain-stress state

\section{1. ВВЕДЕНИЕ}

Разработанная и верифицированная автором комплексная методика численного моделирования трехмерного статического и динамического напряженно-деформированного состояния (НДС) систем «основание - плотина - водохранилище» [4] апробирована на ГЭС Нам Чиен, построенной в Социалисти- ческой Республике Вьетнам.

Значительная часть Вьетнама располагается в сейсмически активных районах. В настоящее время на этих территориях в больших масштабах ведется строительство. Вследствие этого остро встает и проблема обеспечения сейсмостойкости этих сооружений. ГЭС Нам Чиен, деривационного типа, мощностью 200 МВт расположена на реке Нам 

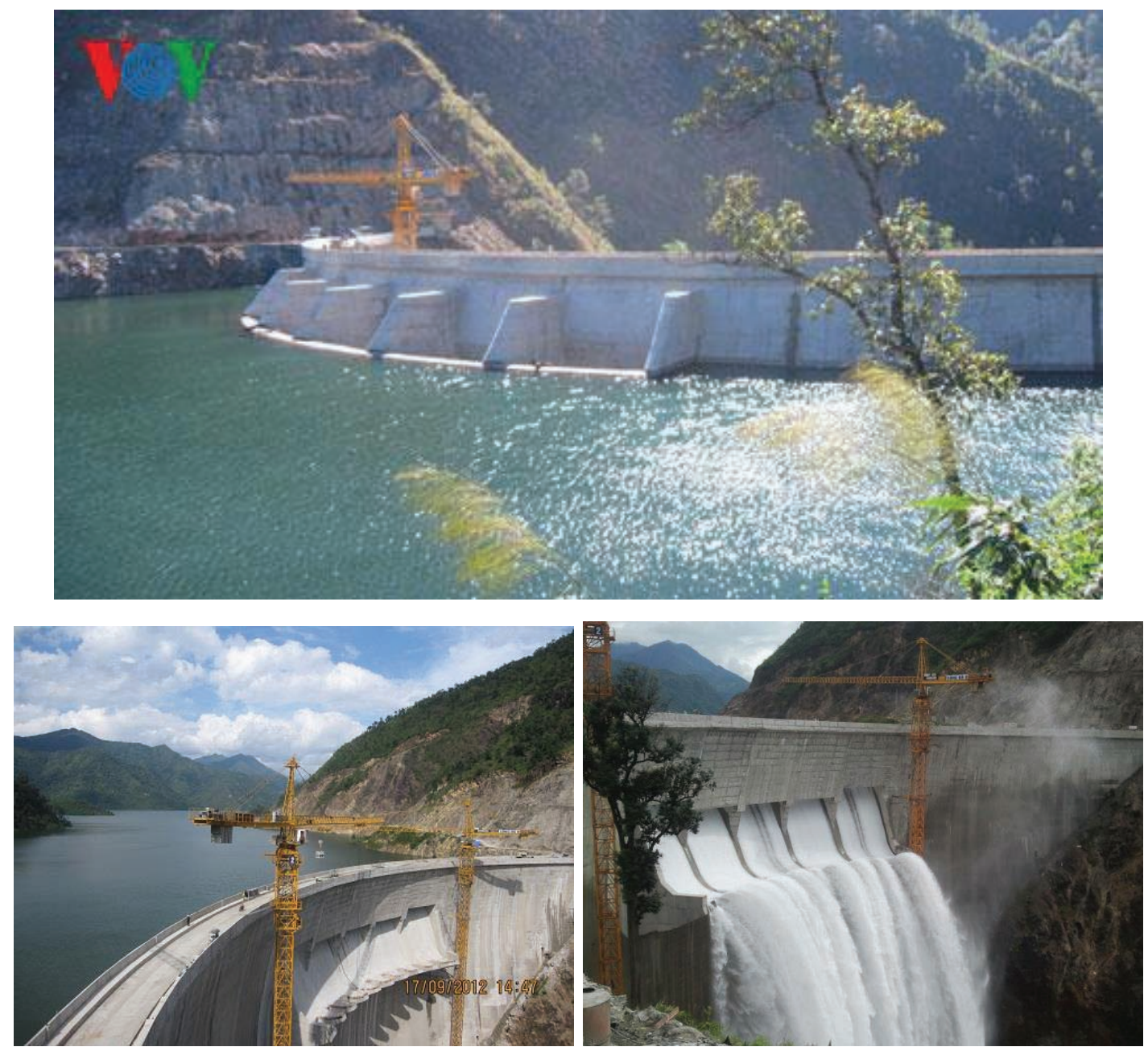

Рисунок 1. Фотография ГЭС Нам Чиен на 2012 г. Общие виды с верховой и низовой граней.

Чиен в районе города Шон Ла (рис 1). В состав гидроузла входят: здание ГЭС с двумя турбинами Пелтон мощностью 100 МВТ каждая при напоре 666,42 м, железобетонная арочная плотина высотой 132,5 м с водосбросом расхода 2619 м3/с и подводящего туннеля длиной около 10 км и диаметром 3,8 м. Среднегодовая выработка ГЭС 789,28 ГВт.ч. при расходе — 35,61 м3/с. Установленная сейсмичность района ГЭС составляет 7 баллов.

\section{2. ПОСТАНОВКА ЗАДАЧИ}

Ставятся следующие задачи:

1) разработать подробную объемную динамическая конечноэлементная модель си- стемы «основание - арочная плотина - водохранилище» эксплуатируемой ГЭС Нам Чиен, обоснованно превосходящая по точности КЭ-модели, ранее разработанные для обоснования проекта.

2) выполнить вариантные расчеты системы по разработанной и верифицированной методике численного моделирования,

3) проанализировать трехмерное НДС плотины и основания при статических нагрузках и сейсмических воздействиях.

\section{3. ОПИСАНИЕ РАСЧЕТНЫХ МОДЕЛЕЙ}

На основе актуальных чертежей разработаны детальные геометрическая (рис 2) и конечноэлементная модель (рис 3-4) системы 
Апробация методики численного моделирования статического и сейсмического напряженно-деформированного состояния на трехмерной системе «основание - плотина - водохранилище» ГЭС Нам Чиен (Вьетнам)

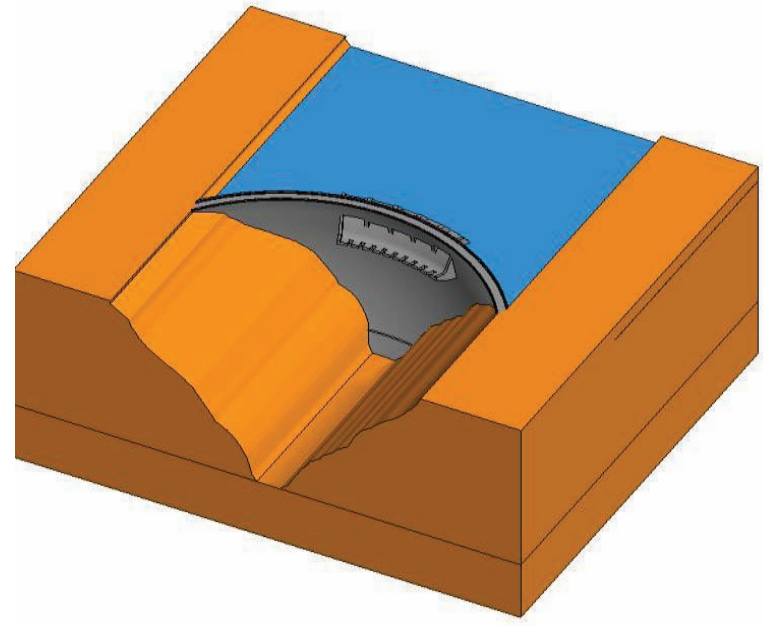

a)

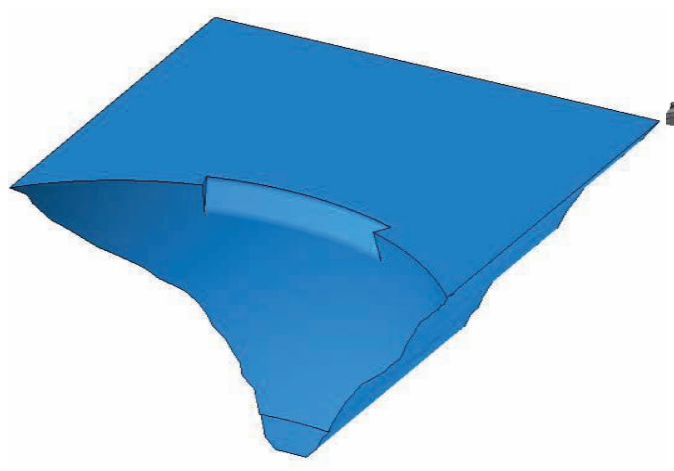

8)

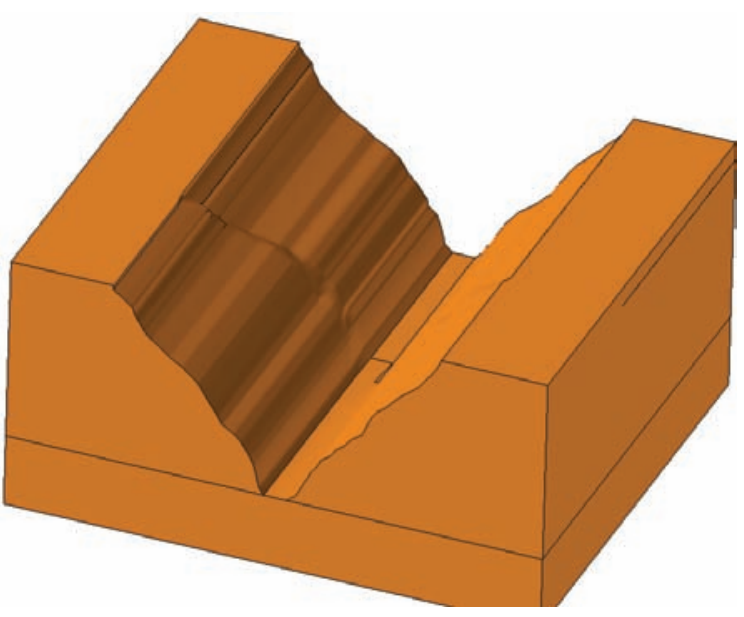

б)

Рисунок 2. Основные геометрические характеристики «основание - водохранилище - плотина» а) общая геометрия системы ОВП; б) геометрия основания; в) геометрия водохранилища; г) геометрия плотины.

«основание - плотина - водохранилище» (ОПВ). Модели создавались в препроцессоpe программного комплекса ANSYS Mechanical с использованием разработанного параметризированного макроса, написанного на внутреннем языке программирования APDL.

Моделирование массива воды выполнялось объемными 8-узловыми шестигранными изопараметрическими КЭ «акустического» типа FLUID30. Всем торцевым элементам водохранилища и элементам, контактирующим с плотиной и основанием, назначалась опция акустического анализа с FSIинтерфейсом (4 степени свободы - давление и 3 перемещения). Остальным, «внут- ренним» элементам водохранилища устанавливалась опция без FSI интерфейса (одна степень свободы - давление). Основание и плотина моделировались объемными восьмиузловыми элементами SOLID185. Контакт КЭ-моделей водной среды и плотины/основания выполнен согласованно, узел в узел.

Используются В - метод (селективный метод редуцированного интегрирования), метод равномерного редуцированного интегрирования, или метод расширенной формулировки деформаций с тремя степенями свободы перемещения. Размерность разработанной полной КЭ-модели и ее составляющих представлена в таблице 1. 


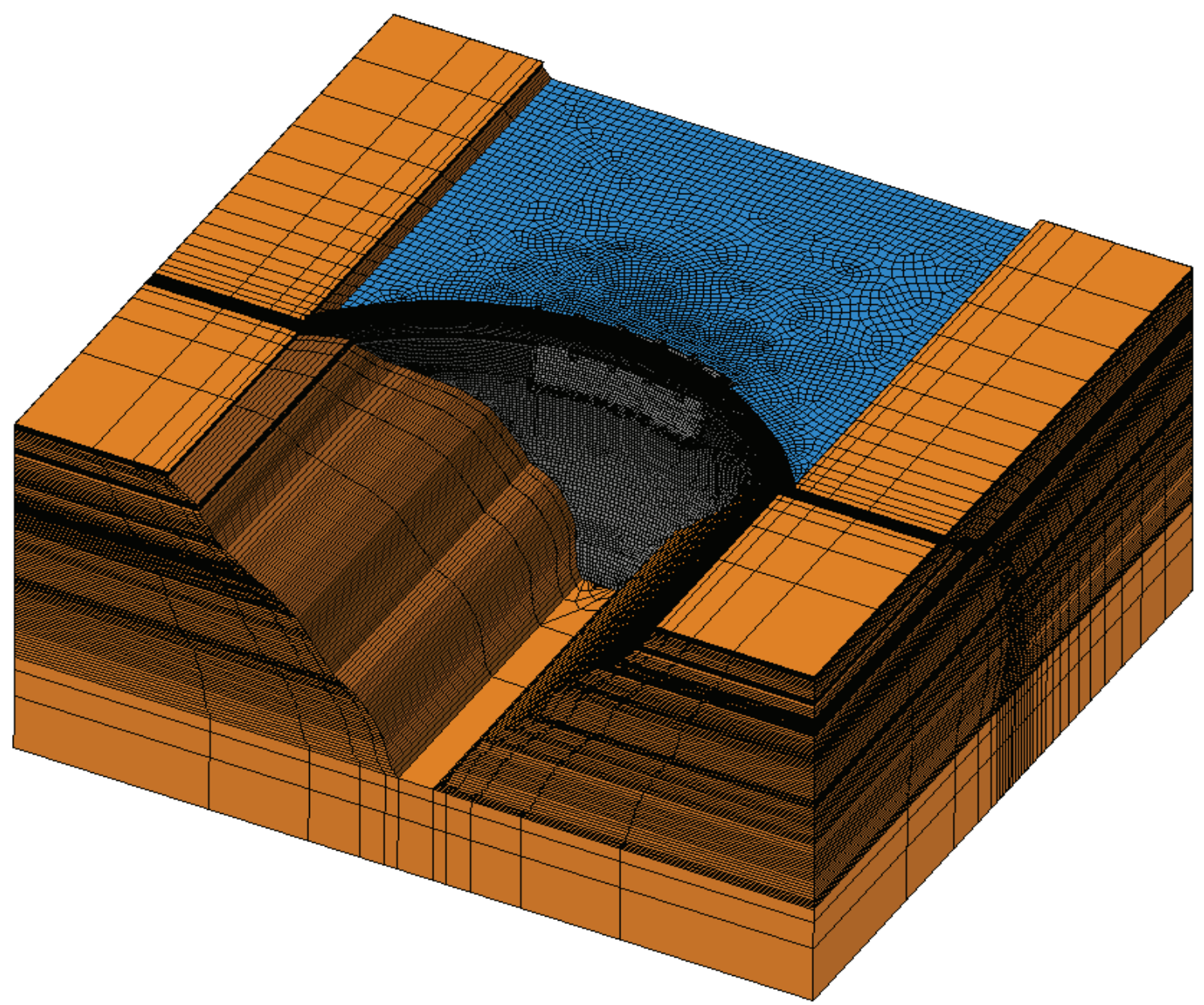

Рисунок 3. Общая КЭ-модель системы «основание - арочная плотинаводохранилище».
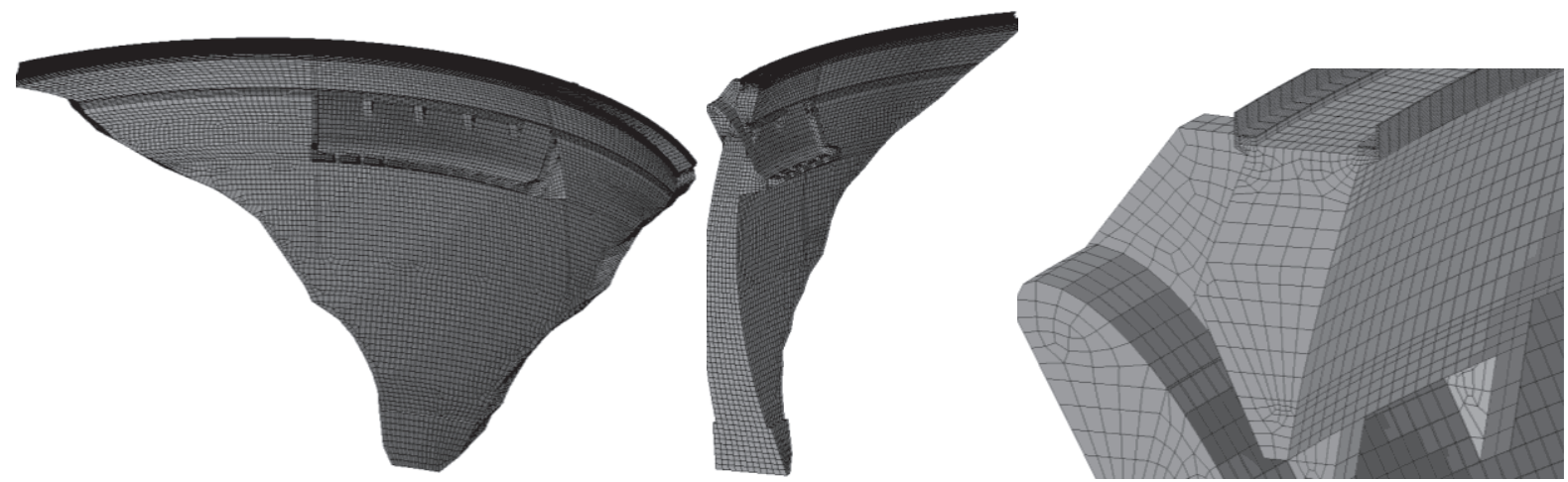

Рисунок 4. Трехмерная КЭ-модель плотины, общий вид и фрагмента.

Таблииа 1. Размерность конечноэлементной модели.

\begin{tabular}{|c|c|c|c|c|}
\hline Параметр КЭ-модели & Плотина & Основание & Водохранилище & Всего \\
\hline Количество узлов & 118916 & 47776 & 87768 & 244821 \\
\hline Количество элементов & 99460 & 37941 & 464833 & 602234 \\
\hline Тип элементов & SOLID185 & SOLID185 & FLUID30 & \\
\hline
\end{tabular}


Апробация методики численного моделирования статического и сейсмического напряженно-деформированного состояния на трехмерной системе «основание - плотина - водохранилище» ГЭС Нам Чиен (Вьетнам)

Таблича 2. Сопоставление собственных частот и форм колебаний системам «основание плотина - водохранилище» и «жесткое основание - плотина - водохранилище».

\begin{tabular}{|c|c|c|c|c|c|}
\hline $\begin{array}{c}\text { № } \\
\text { П/П } \\
\end{array}$ & 1 & 2 & 3 & 4 & 5 \\
\hline 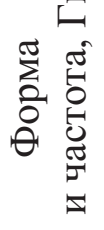 & $2.487(2.545)$ & $3.142(3.223)$ & $3.931(4.003)$ & 4.556(4.632) & $5.204(5.303)$ \\
\hline $\begin{array}{c}\text { № } \\
\text { П/П } \\
\end{array}$ & 6 & 7 & 8 & 9 & 10 \\
\hline 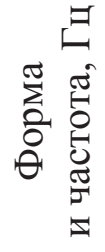 & $5.849(5.992)$ & $6.841(6.928)$ & $6.880(7.025)$ & $7.568(7.741)$ & $8.602(8.753)$ \\
\hline
\end{tabular}

4. РЕЗУЛЬТАТЫ РАСЧЕТА

СОБСТВЕННЫХ ЧАСТОТ И ФОРМ

КОЛЕБАНИЙ СИСТЕМЫ

«ОСНОВАНИЕ - ПЛОТИНА ВОДОХРАНИЛИЩЕ»

В верифицированном базовом программном комплексе ANSYS Mechanical блочным методом Ланцоша вычислены первые 40 собственных частот и форм колебаний трехмерной комбинированной системы «основание плотина - водохранилище» при полностью заполненном водохранилище. Результаты для первых 10-и из них представлены в таблице 2. Сопоставление системы ОПВ и редуцированной системы «жесткое основание - плотина - водохранилище» показывает качественное соответствие собственных форм колебаний. Различие менее $2.51 \%$ собственных частот - показатель незначительного влияния податливости основания.

\section{5. РАСЧЕТ СТАТИЧЕСКОГО И ДИНАМИЧЕСКОГО НДС СИСТЕМЫ «ОСНОВАНИЕ - АРОЧНАЯ ПЛОТИНА - ВОДОХРАНИЛИЩЕ»}

Статическая нагрузка включала собственный вес элементов системы (за исключением ос- нования) и гидростатическое давление воды. Из результатов статического расчета сопоставлены перемещения плотины для систем “основание - плотина - водохранилище" и “жесткое основание - плотина - водохранилище” в центральном сечении (таблица 3). Установлено различие в горизонтальных, вертикальных и суммарных перемещениях не более 4\%. Максимальные горизонтальные и вертикальные перемещения для системы ОПВ равны 26мм и 6мм соответственно.

Интегрирование по времени уравнений динамики КЭ-модели системы проводилось по неявной схеме Ньюмарка. Шаг принимался равным 0.01с для учета значимых собственных форм колебаний. Динамическое воздействие в виде трехкомпонентной акселерограммы (рис. 5) прикладывалось как инерционные силы. Первые 3 секунды задавалась медленно линейно возрастающая по времени статическая нагрузка (гидростатическая и собственный вес).

Динамический расчет НДС системы при статических и сейсмических воздействиях выполнен в трех вариантах:

- «основание - плотина - водохранилище»;

- «жесткое основание - плотина - водохранилище»;

- «основание-плотина» (без водохранилища). 

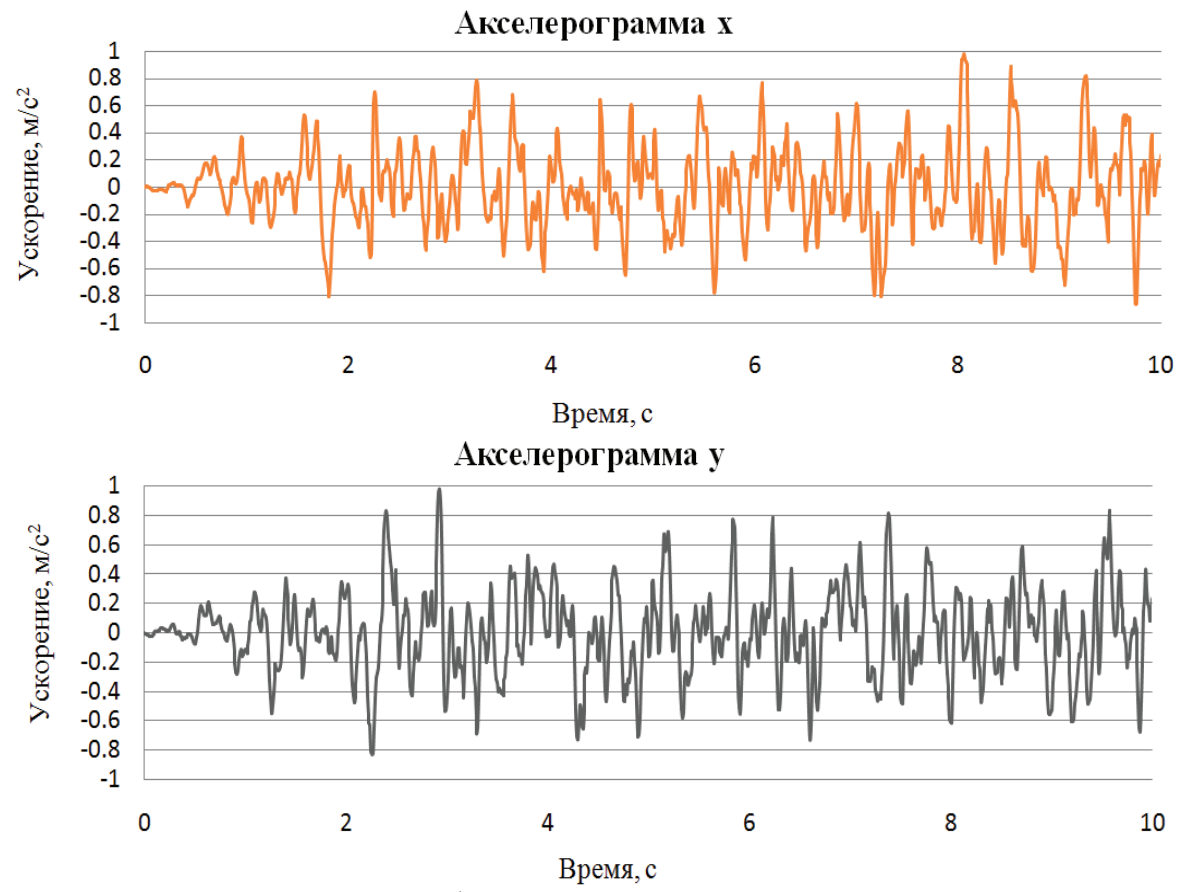

Акселерограмма z

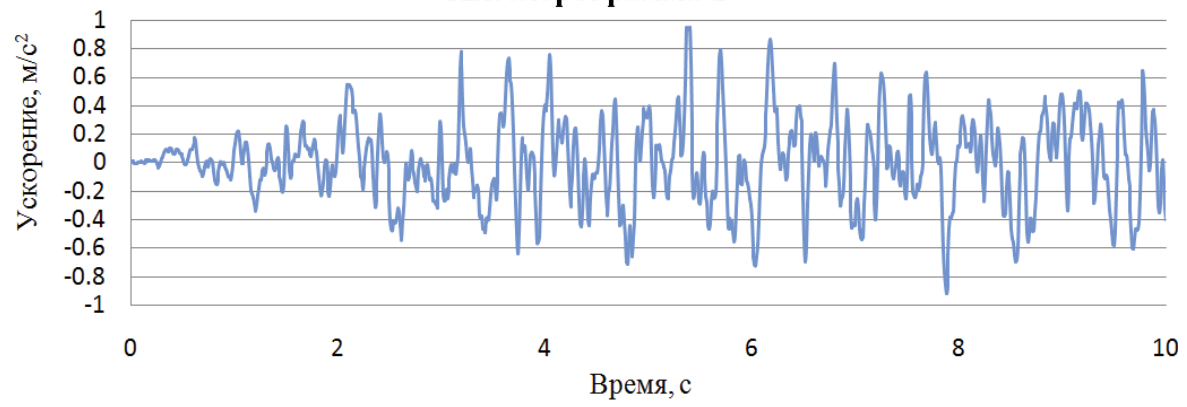

Рисунок 5. Трехкомпонентная акселерограмма.

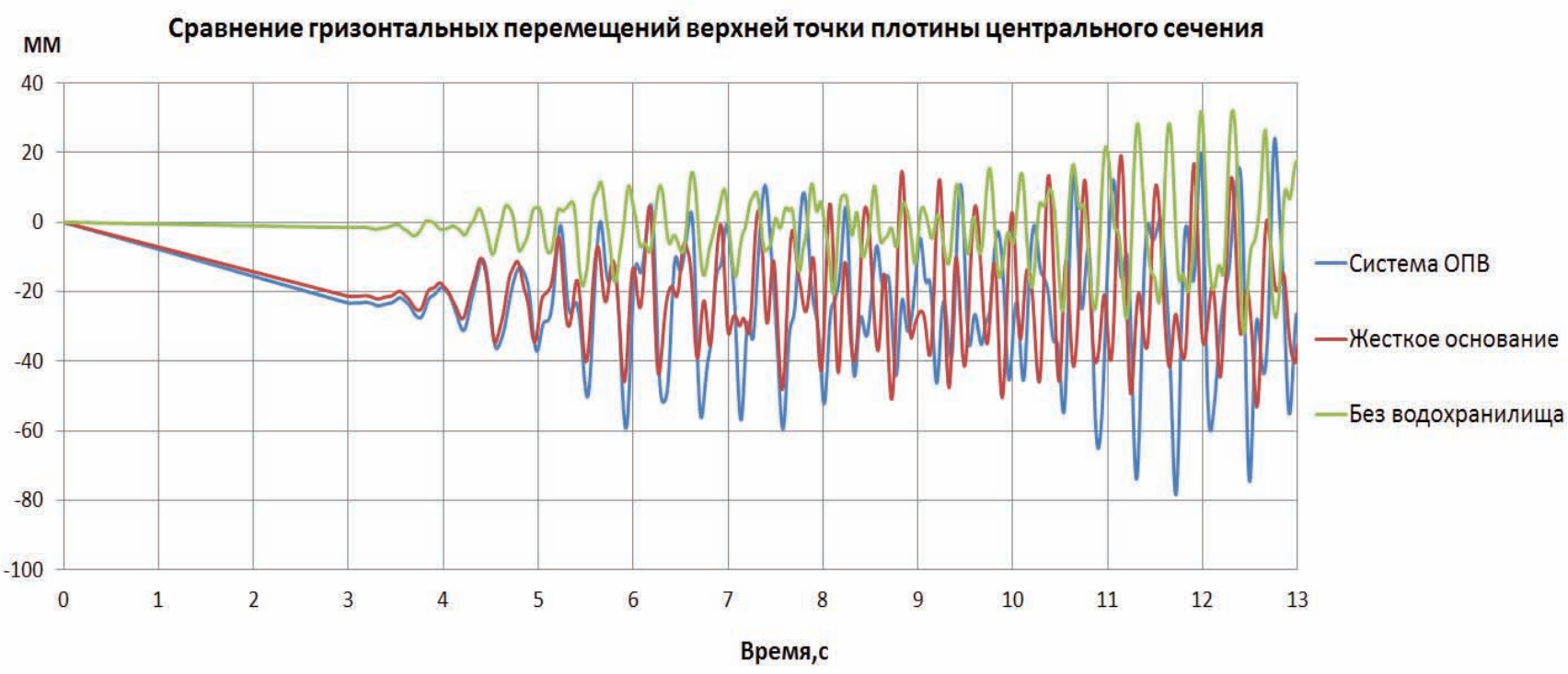

Рисунок 6. Сравнение горизонтальных динамических перемещений верхней точки плотины џентрального сечения, мм. 
Апробация методики численного моделирования статического и сейсмического напряженно-деформированного состояния на трехмерной системе «основание - плотина - водохранилище» ГЭС Нам Чиен (Вьетнам)

Таблица 3. Перемещения арочной плотины от гидростатической нагрузки и собственного веса, мм

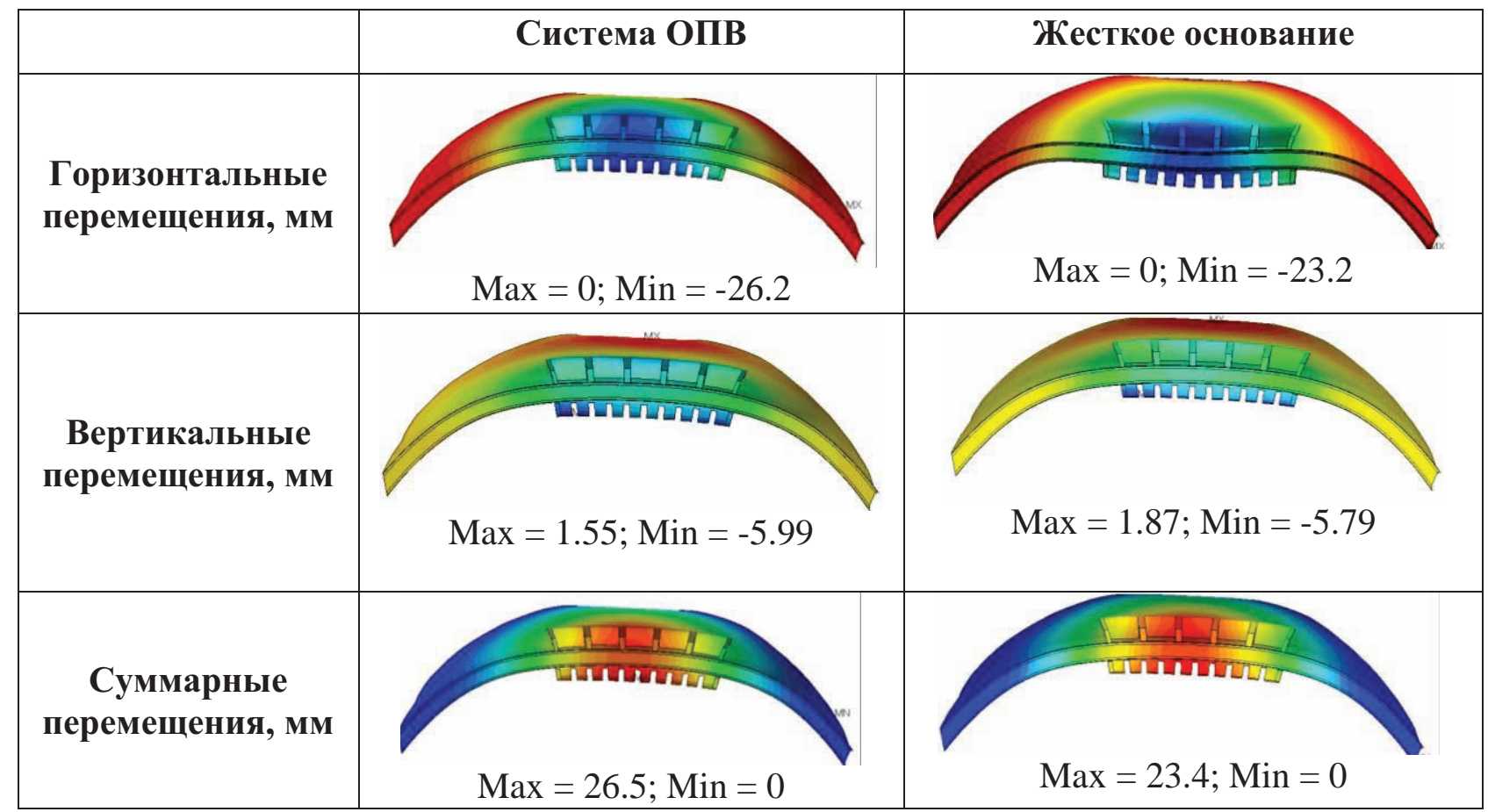

Расчетные динамические горизонтальные перемещения верхней точки центрального сечение арочной плотины для этих трех моделей представлены на рис 6.

Приведены также максимальные значения главных напряжений $\boldsymbol{\sigma}_{1}$ (таблица 4 ) и $\boldsymbol{\sigma}_{3}$ (таблица 5) и момент их реализации.

Сравнительный анализ полученных результатов привел как к ожидаемым выводам (например, реализацию наибольших значения перемещений и напряжений в системе “основание-плотина-водохранилище”), так и к выявлению значимых контактных зон арочной плотины и скального основания у верховой грани с уровнем главных напряжений, существенно превышающих предельные значения по критериям трещинообразования (растяжение) и крошения (сжатия).

\section{6. ВЫВОДЫ}

1. Разработана подробная объемная динамическая конечноэлементная модель системы "основание - арочная плотина - водо- хранилище" эксплуатируемой ГЭС Нам Чиен (Социалистическая Республика Вьетнам), эксплуатируемой с 2013 года, существенно превосходящая по точности КЭ-модели, ранее разработанные для обоснования проекта. Расчеты системы, выполненные по разработанной и верифицированной методике численного моделирования, позволили получить и проанализировать трехмерное НДС плотины при статических нагрузках и сейсмических воздействиях.

2. На вариантных моделях установлено влияние основных факторов системы (основания, водохранилище) на результирующее статическое и динамическое НДС. Выявлены локальные зоны бетонной плотины и скального основания с опасным уровнем статических и сейсмических напряжений растяжения и сжатия, чреватые разрушением материала (трещинообразованием и крошением). На этой базе разработаны рекомендации по мониторингу состояния сооружения. 


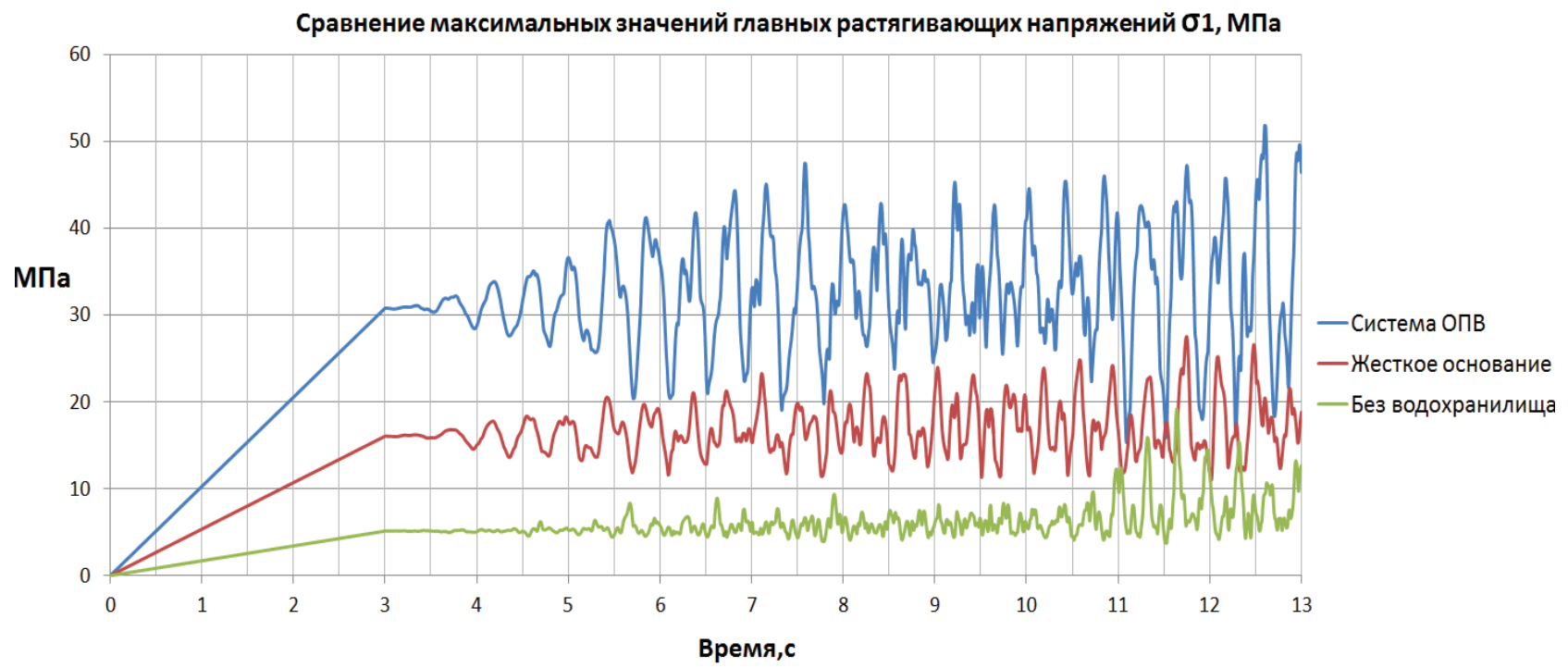

Рисунок 7. Сравнение максимальных значения главных динамических напряжений $\sigma_{1}$, МПа.

Таблица 4. Сравнение максимальных значения главных напряжения $\boldsymbol{\sigma}_{1}$, МПа.

\begin{tabular}{|c|c|c|c|}
\hline & Система ОПВ & Жесткое основание & Без водохранилища \\
\hline $\begin{array}{c}\text { Изополя } \\
\text { напряжений }\end{array}$ & & & \\
\hline Мax $\sigma_{1}$, МПа & 51.8 & 27.5 & 19.2 \\
\hline Время, с & 12.60 & 11.75 & 11.64 \\
\hline
\end{tabular}

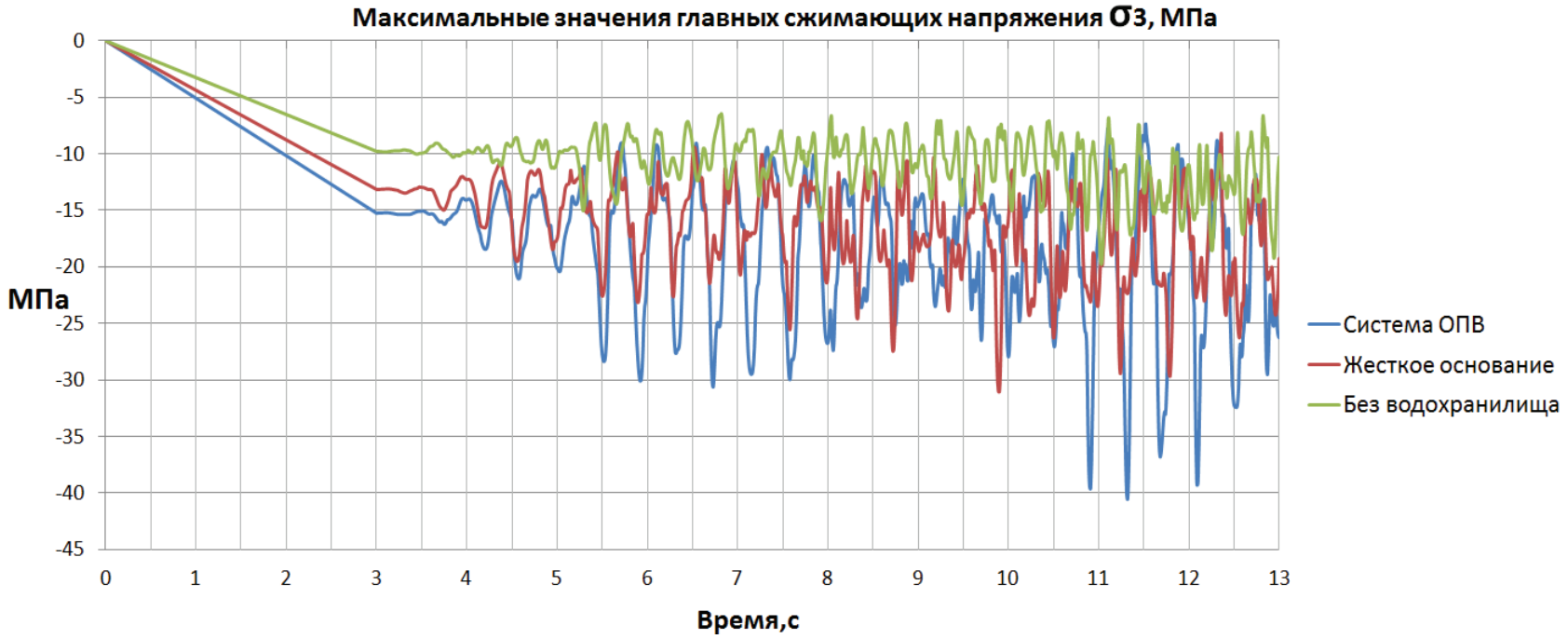

Рисунок 8. Сравнение максимальных значения главных динамических напряжения $\sigma_{3}$, МПа. 
Апробация методики численного моделирования статического и сейсмического напряженно-деформированного состояния на трехмерной системе «основание - плотина - водохранилище» ГЭС Нам Чиен (Вьетнам)

Таблича 5. Сравнение максимальных значения главных напряжений $\sigma_{3}$, МПа.

\begin{tabular}{|c|c|c|c|}
\hline & Система ОПВ & Жесткое основание & Без водохранилища \\
\hline $\begin{array}{c}\text { Изополя } \\
\text { напряжений }\end{array}$ & & & \\
\hline Min $\sigma_{3}, \mathrm{M} а \mathrm{a}$ & -40.6 & -31.1 & -19.8 \\
\hline Время,с & 11.32 & 9.90 & 11.03 \\
\hline
\end{tabular}

3. Полученные результаты позволяют усовершенствовать и конкретизировать универсальные подходы к расчету связанных трехмерных гидроупругих систем, расширить область применения современных численных методов расчета на сооружения более широкого класса.

4. Перспективой дальнейшей разработки данной темы представляется наукоемкая интеграция предложенной методики численного моделирования и параметризованных объемных расчетных КЭ-моделей системы «основание - плотина - водохранилище» в разрабатываемые и действующие системы обязательного, нормативно закрепленного мониторинга состояния уникальных гидротехнических сооружений [12-15].

\section{СПИСОК ЛИТЕРАТУРЫ}

1. Белостоцкий А.М. Численное моделирование статического динамического напряженно деформированного состояния пространственных систем «сооружение-основание-водохранилище» с учетом нелинейных эффектов открытия закрытия швов и макротрещин. Дис. на соиск. учен.степ. д-ра техн. наук: 05.23.07. МГУП, М.,1998. - 367c.

2. Белостоцкий А.М., Акимов П.А., Кай- туков Т.Б., Афанасьева И.Н., Вершинин В.В., Дмитриев Д.С., Усманов А.P., Чужинов А.С., Щербина С.В. Постановка, конечноэлементная аппроксимация и алгоритмы решения задач расчетного обоснования связанных систем «сооружение - жидкость». // Строительная механика и расчет сооружений, 2014, №5, c.21-27.

3. ICOLD, International Benchmark Workshop on Numerical Analysis of Dams. Theme A-Fluid Structure Interaction Arch Dam - Reservoir at Seismic loading. Graz University of Technology, 2013, pp. 15188.

4. Белостоцкий А.М., Дмитриев Д.С., Нгуен Тай Нанг Лыонг. Верификация методики численного моделирования динамического напряженнодеформированного состояния трехмерных систем «сооружение - жидкость». // International Journal for Computational Civiland Structural Engineering / Международный журнал по расчету гражданских и строительных конструкций, Volume 11, Issue 4, 2015, pp. 124-132.

5. Белостоцкий А.М., Акимов П.А., Кайтуков Т.Б., Афанасьева И.Н., Вершинин В.B., Усманов А.P., Щербина С.В., Мсхалая Ж.И. О методах численного моделирования связанных систем «сооружение - жидкость» International Jour- 
nal for Computational Civil and Structural Engineering / Международный журнал по расчету гражданских и строительных конструкций, Volume 10, Issue 4, 2014, pp. 54-75.

6. Parrinello F., Borino G. Lagrangian finite element modelling of dam - fluid interaction: Accurate absorbing boundary conditions. // Computers and Structures, 85, 2007, pp. 932-943.

7. СНиП 11-7-81*. Строительные нормы и правила, Глава 7. Строительство в сейсмических районах, Госстрой СССР. М.: Стройиздат, 1982, - 49 с.

8. Учет сейсмических воздействий при проектировании гидротехнических сооружений. (пособие к разделу 5: Гидротехнические сооружения СНиП II-7-81). П 17-85, - Л.:ВНИИГ, 1986. - 310с

9. Шульман С.Г. Сейсмическое давление воды на гидротехнические сооружения. - Л.: Энергия, 1970, 166 с.

10. Шульман С.Г. Расчеты сейсмостойкости гидросооружений с учетом влияния водной среды. - М.: Энергия, 1980. $336 \mathrm{c.}$

11. Westergaard H.M. Water pressure on dams during earthquakes. // Proc. Am. Soc. Civ. Engrs., 1931, v. 57, N9, pp. 1303-1318.

12. Белостоцкий А.М., Акимов П.А. Научно-исследовательский центр СтаДиО. 25 лет на фронте численного моделирования. // International Journal for Computational Civil and Structural Engineering / Международный журнал по расчету гражданских и строительных конструкций, Volume 12, Issue 1, 2016, pp. 8-45.

13. Белостоцкий А.М., Акимов П.А. Обзорно-аналитическое исследование нормативно-методической литературы в области мониторинга зданий и сооружений. // International Journal for Computational Civil and Structural Engineering / Международный журнал по расчету гражданских и строительных конструкций, Volume 12, Issue 2, 2016, pp. 42-64.

14. Белостоцкий А.М., Акимов П.А. О вопросах автоматизации систем мониторинга для оценки текущего состояния строительных конструкций зданий и сооружений. // International Journal for Computational Civil and Structural Engineering / Международный журнал по расчету гражданских и строительных конструкций, Volume 12, Issue 3, 2016 , pp. 26-34.

15. Belostosky A.M., Akimov P.A. Adaptive Finite Element Models Coupled with Structural Health Monitoring Systems for Unique Buildings. // Procedia Engineering, Vol. 153, 2016, pp. 83-88.

Нгуен Тай Нанг Лыонг, аспирант кафедры прикладной математики Национального исследовательского Московского государственного строительного университета; 129337, Россия, г. Москва, Ярославское шоссе, дом 26; тел. +7(499)183-59-94;

e-mail: tailuong2003@gmail.com

Nguyen Tai Nang Luong, Ph.D Student, Department of Applied Mathematics, National Research Moscow State University of Civil Engineering; 26, Yaroslavskoe Shosse, Moscow, 129337, Russia; tel. +7(499)183-59-94; e-mail: tailuong2003@gmail.com. 


\title{
ЧИСЛЕННЫЙ АНАЛИЗ ДИНАМИЧЕСКИХ ХАРАКТЕРИСТИК ЭЛЕМЕНТА ТРУБОПРОВОДНОЙ СИСТЕМЫ МНОГОЭТАЖНОГО ЖИЛОГО ДОМА
}

\author{
Л.Г. Пастухова, А.А. Секачева \\ Уральский федеральный университет имени первого Президента России Б.Н. Ельцина, \\ г. Екатеринбург, РОССИЯ
}

\begin{abstract}
Аннотация: Статья посвящена проблеме возникновения шума и вибрации от трубопроводных систем многоэтажных зданий. Предложен способ определения вероятности возникновения повышенных вибраций с помощью модального анализа в программном комплексе ANSYS. Представлены результаты численного анализа влияния длины и формы элемента трубопроводной системы на изменение его динамических характеристик с целью прогноза риска возможных резонансных режимов и возникновения повышенной вибрации и шума. Проведен модальный анализ участка трубы различных размеров и форм в программном комплексе ANSYS.
\end{abstract}

Ключевые слова: численный анализ, параметры собственных колебаний, трубопроводные системы, многоэтажное строительство, ПК ANSYS

\section{NUMERIC ANALYSIS OF THE DYNAMIC CHARACTERISTICS OF THE PIPELINE SYSTEM ELEMENT OF THE MULTI-STOREY BUILDING}

\author{
Lilia G. Pastukhova, Antonina A. Sekacheva \\ Ural Federal University named after the first President of Russia B.N. Yeltsin, Yekaterinburg, RUSSIA
}

\begin{abstract}
The article discusses the occurrence of noise and vibration from the piping systems of multi-storey buildings. A method for determining the probability of excessive vibrations using modal analysis software ANSYS complex. The article is dedicated to the research on the influence of length and the form of an element of a pipeline system on change of dynamic characteristics for the forecast of risk of the possible resonant modes and the emergence of excessive vibration and noise. The modal analysis of a section of the pipe of different sizes and forms using ANSYS software is carried out.
\end{abstract}

Key words: digital analysis, parameters of natural oscillations, pipeline systems, multi-storey building, PC ANSYS

\section{СОСТОЯНИЕ ВОПРОСА}

Одной из тенденций современной застройки крупных городов является строительство многоэтажных многоквартирных жилых домов.

Следует пояснить, что в 1976 году на симпозиуме СIB была принята классификация по высоте. Сооружения высотой до 30 м отнесены к зданиям повышенной этажности, до 50, 75 и 100 м соответственно, к I, II и III категориям многоэтажных зданий, свыше 100 м - к высотным. Внутри группы высотных зданий обычно прибегают к дополнительной подгруппе с градацией высоты в 100 м.

В России практика многоэтажного массового жилищного строительства и нормы проектирования ранее были ориентированы на высоту зданий до 75 м. Поэтому 
сложилась тенденция отнесения к высотным зданий выше 75 м.

По этажности существующие классификации достаточно условны и не однозначны. Например, по этажности здания классифицируются: малоэтажные (до 5 этажей), средней этажности (5-12 этажей), высотные (более 12 этажей); высотным принято называть здание высотой более 75 м (более 25 этажей) [1,2].

Как следствие, на инженерные сети и системы многоэтажек ложатся повышенные нагрузки, возрастает мощность оборудования. Поэтому очень важно эффективное проектирование и эксплуатация таких систем, обеспечивающих требуемые параметры микроклимата помещений.

Нередко возникают ситуации, при которых системы обеспечения микроклимата и комфорта становятся источником дискомфорта. Такая ситуация может быть обусловлена рядом проблем.

Одной из таких проблем является шум и вибрация от насосных установок большой мощности (свыше 400 кВт при подаче выше $\left.0,5 \mathrm{~m}^{3} / \mathrm{c}\right)$, расположенных в индивидуальных тепловых пунктах (ИТП) многоэтажных жилых домов, например, циркуляционных насосов систем теплоснабжения от крышных газовых котельных или систем водоснабжения.

Следует пояснить, что по мощности (кВТ) насосы делятся на:

- микронасосы (до 0,4 кВт);

- мелкие насосы (до 4 кВт);

- малые насосы (до 100 кВт при подаче $\left.0,5 \mathrm{~m}^{3} / \mathrm{c}\right)$;

- с средние насосы (до 400 кВт);

- крупные насосы или насосы повышенной мощности (свыше 400 кВт при подаче выше $\left.0,5 \mathrm{~m}^{3} / \mathrm{c}\right)$;

- уникальные насосы (свыше 8000 кВт при подаче свыше $20 \mathrm{~m}^{3} / \mathrm{c}$ ).

В адрес Управления Роспотребнадзора по городу Москве в Южном административном округе регулярно поступают обращения граждан, касающиеся ухудшения условий проживания, связанные с шумом, проникающим в квартиры. Так, в 2012 году в территориальный отдел поступило 252 обращения жителей округа с жалобами на ухудшение условий проживания в связи с воздействием различных физических факторов, из них 89,3 \% (223 обращения) связаны с воздействием шума и вибрации. Жалобы, связанные с воздействием шума от инженерного оборудования жилых зданий (системы отопления, бойлерных, лифтового оборудования, а также систем вентиляции и оборудования, размещенного в смежных нежилых помещениях), составили 8,3\% (21 обращение) от общего числа [4].

Например, размещение ИТП в подвале многоэтажного жилого здания приводит к тому, что показатели шума и вибрации на первом этаже превышают предельнодопустимые уровни, установленные санитарными нормами [5-7].

Следует пояснить, что имеем следующие допустимые уровни звукового давления и вибрации в помещениях жилых домов от внутренних и внешних источников постоянного шума: предельно допустимый эквивалентный уровень звука - 25 дБА, предельно допустимый эквивалентный уровень корректированного виброускорения -62 дБ [5-7].

Устранение данных нарушений связано с дополнительными финансовыми вложениями проектно-строительных и управляющих компаний.

Важно отметить, что жалобы на шум и вибрацию поступают не только от жителей первых этажей многоэтажных домов, но и от собственников квартир, расположенных на различных этажах. К примеру, основной причиной жалоб в одну из управляющих организаций г. Екатеринбурга от жителей 18этажного дома с крышной котельной, проживающих на десятом, двенадцатом и семнадцатом этажах, стали вибрация и шум от монолитных стен, смежных с коммуникационными шахтами. Экспериментальными исследованиями было 
Численный анализ динамических характеристик элемента трубопроводной системы многоэтажного жилого дома

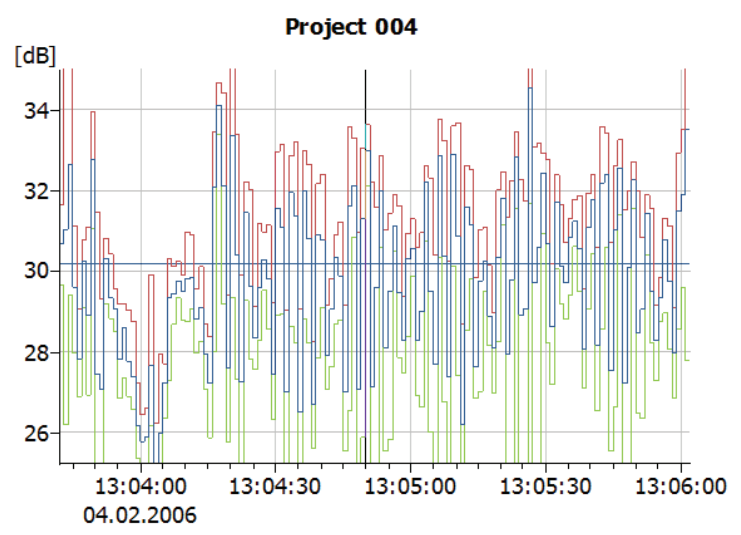

a)

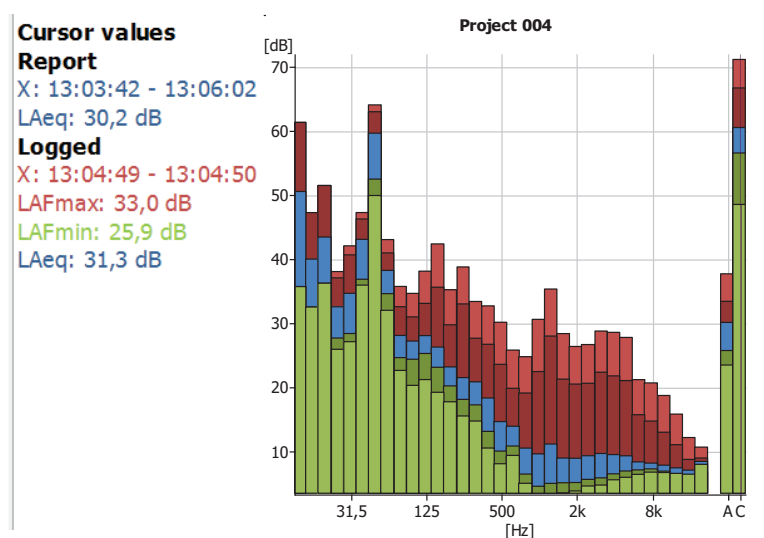

б)

Рисунок 1. Результаты экспериментальных исследований шума в 0,5 м от стены смежной с коммуникационной шахтой: а) временная характеристик; б) частотная характеристика

установлено, что шум в 0,5 м от этих стен носит низкочастотный характер (от 10 Гц) с пиками на частотах 12,5 и 50 Гц, которые соответствуют работе насосных установок (рис. 1).

Гидродинамическими источниками вибраций центробежных насосов могут быть неоднородность потока на выходе из колеса, вихреобразование в проточной части, кавитация. Анализируя течение реальной жидкости в центробежном насосе, можно назвать два основных источника возмущений, вызывающих вибрацию насоса. Первым источником являются нестационарные гидродинамические силы на лопатках направляющего аппарата и колеса насоса, возникающие вследствие потенциального взаимодействия решеток. Анализ этих сил показывает, что на направляющем аппарате они на порядок выше, чем на рабочем колесе, и их амплитуды достигают $30 \%$ от среднего значения [3].

Вторым источником вибрации являются пульсации давления жидкости в насосе, имеющие характер звуковых колебаний. Пульсации давления возникают, во-первых, при пересечении лопатками направляющего аппарата вязких слоев за лопатками колеса, при отрыве вихрей, при обтекании элементов проточной части, а во-вторых, при кавитации [3]. В первом случае амплитуды и спектр частот пульсаций давления зависит от числа оборотов насоса и числа лопаток колеса и направляющего аппарата. При кавитации наблюдаются более высокие частоты пульсаций, однако хаотическое захлопывание кавитационных пузырей, сопровождаемое излучением волн давления, создает широкий спектр возмущающих сил [3].

Распространение колебаний жидкой среды происходит в первую очередь по потоку воды, транспортируемому по трубам. Вовторых, вибрационное и шумовое воздействие воспринимают стенки трубопровода и передают на ограждающие конструкции здания - стены и перекрытия. В-третьих, все эти колебания, передаваясь воздушной среде, вызывают повышенный шум как в помещении, где установлены насосы - источники шума, так и в помещениях, смежных коммуникационными шахтами.

Передача структурного шума от стенок трубопроводов к конструкциям здания происходит по разным причинам:

- неверный или некачественный подбор насосного оборудования (при КПД менее 70 \%) и гидравлической арматуры;

- изменение проектных параметров действующих инженерных систем;

- замена старых насосных установок на новые с техническими характеристиками, 
несоответствующими работающей в доме гидросистеме;

- недостаточная виброизоляция основания установки насосов и мест соединения насосов с трубопроводом (вибровставки);

- наличие акустических мостиков - прямой контакт труб со стенами здания: стойки, металлические элементы, отсутствие гильз, которые необходимо устанавливать в местах прохождения труб через конструкции и т. Д. (устранение металлических мостиков способствует снижению уровня звука на 5-6 дБ);

- кавитация.

Кроме того, режим работы насосов различных типов может быть как постоянным, так и периодическим, вызывая постоянную и непостоянную вибрацию и шум. Таким образом, мы имеем дело с нестационарными процессами, что также осложняет решение вышеописанной проблемы.

Методом предотвращения возникновения повышенного шума и вибрации в трубопроводе является снижение пульсаций транспортируемой жидкости. Основной метод снижения пульсации жидкости или газа состоит в выполнении трубопроводной системы таким образом, чтобы исключить в ней явление резонанса, т.е. соответствующим образом выбрать ее длину и форму [3].

Таким образом, снижение вибрации и шума за счет исключения резонанса на этапе проектирования трубопроводной системы является актуальным.

Первый этап вибрационного исследования конструкции - это расчет параметров ее собственных колебаний. Его основной целью является определение степени опасности возможных резонансных режимов. Если опасные гармоники попадают в рабочий диапазон действующих внешних воздействий, то трубопровод будет подвергаться повышенной вибрации или создавать шум. В таком случае предпринимаются попытки изменить конфигурацию трубопровода таким образом, чтобы вывести его собственные частоты за рабочий диапазон, также может производиться оценка опасности резонансных колебаний по величине возникающих деформаций и напряжений в трубе [8].

Метод модального анализа получил широкое применение для изучения вибраций строительных конструкций [13-17]. Однако, как правило, для решения вибрационных задач трубопроводных систем он практически не применялся.

\section{2. МОДАЛЬНЫЙ АНАЛИЗ}

В работе предпринята попытка проведения модального анализа на численной модели элемента трубопроводной системы с помощью программного комплекса (ПК) ANSYS $[9,10,12]$ с целью определения частот и форм собственных колебаний элемента.

В процессе исследований были рассмотрены различные виды участков трубопровода:

- поворот трубы на $90^{\circ}$ с примыкающими к нему прямолинейными участками длиной $0,1 \mathrm{M}$, заполненный водой «колено» $(\mathrm{dH}=40 \mathrm{MM}, \mathrm{dy}=34 \mathrm{mM})$;

- прямолинейный участок трубы длиной 54 м, заполненный водой $(\mathrm{dH}=100 \mathrm{Mм}$, dy $=114$ мм);

- прямолинейный участок трубы длиной 54 м, незаполненный водой $(\mathrm{d} H=100$ мм, $\mathrm{dy}=114 \mathrm{Mм})$;

- прямолинейный участок трубы длиной 6 м, заполненный водой $(\mathrm{dH}=100 \mathrm{~mm}, \mathrm{dy}$ $=114 \mathrm{MM}$ );

- прямолинейный участок трубы длиной $6 \mathrm{~m}$, незаполненный водой $(\mathrm{dH}=100 \mathrm{Mм}$, $\mathrm{dy}=114 \mathrm{Mm})$;

Полагаем, что участки с двух сторон закреплены жесткой заделкой.

Задаем начальные условия: указываем материал, из которого изготовлен участок трубы (сталь), его плотность $\left(\rho=7850\right.$ кг $\left./ \mathrm{M}^{3}\right)$, a также материал содержимого участка 
Численный анализ динамических характеристик элемента трубопроводной системы многоэтажного жилого дома

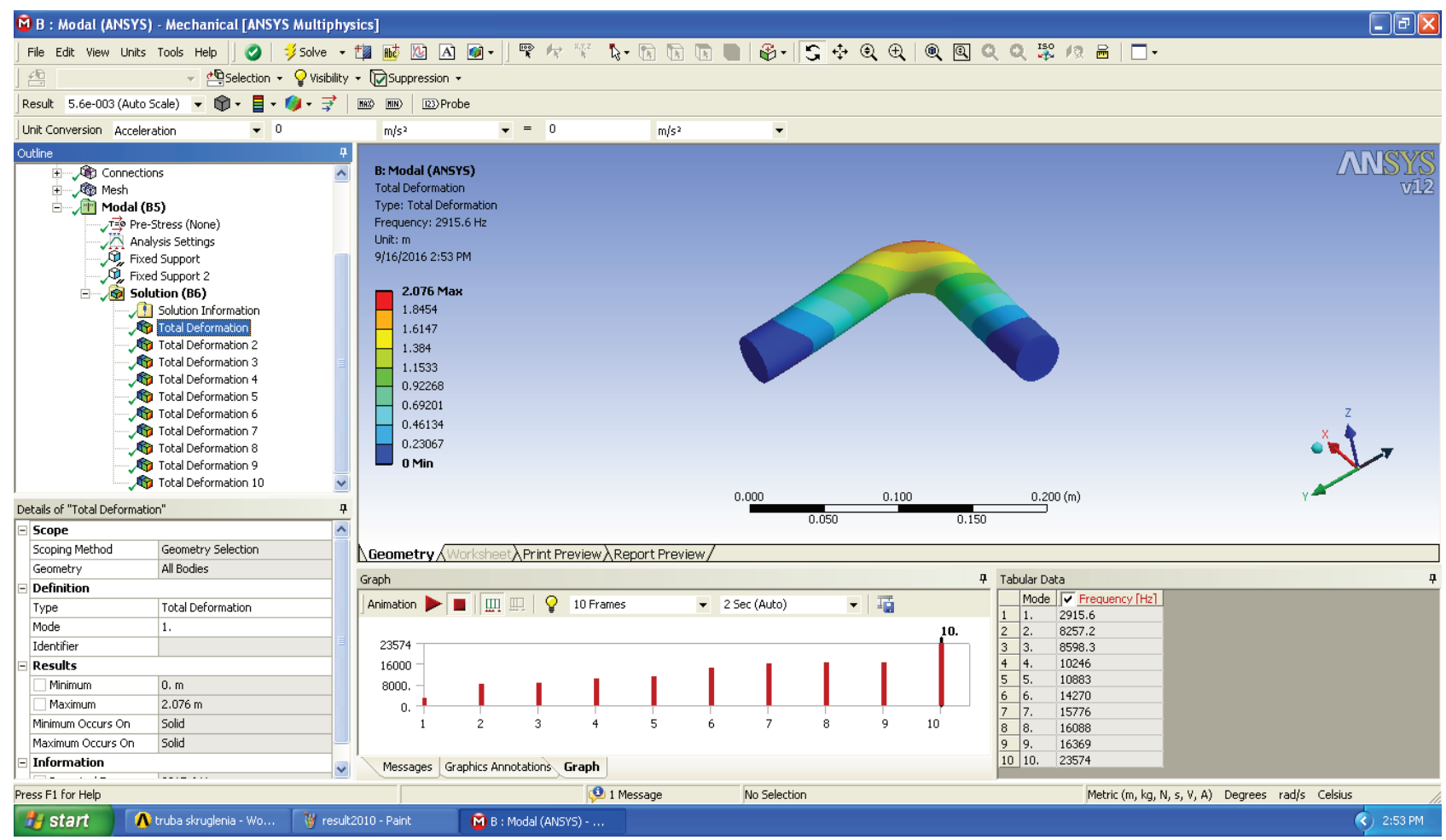

Рисунок 2. Первая форма собственных колебаний участка трубы «колено», заполненного водой.

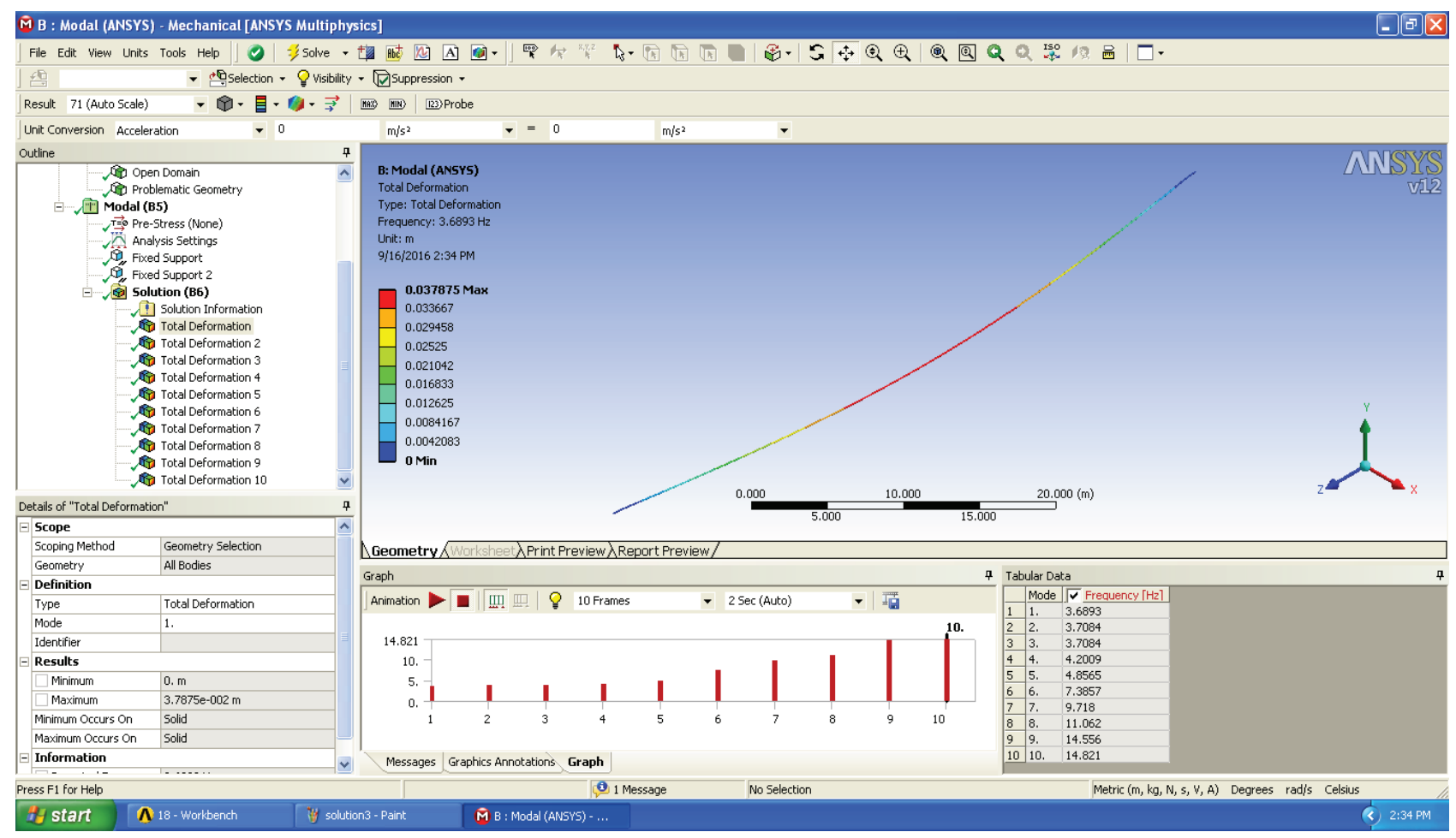

Рисунок 3. Первая форма собственных колебаний прямолинейного 54-метрового участка трубы, заполненного водой. 


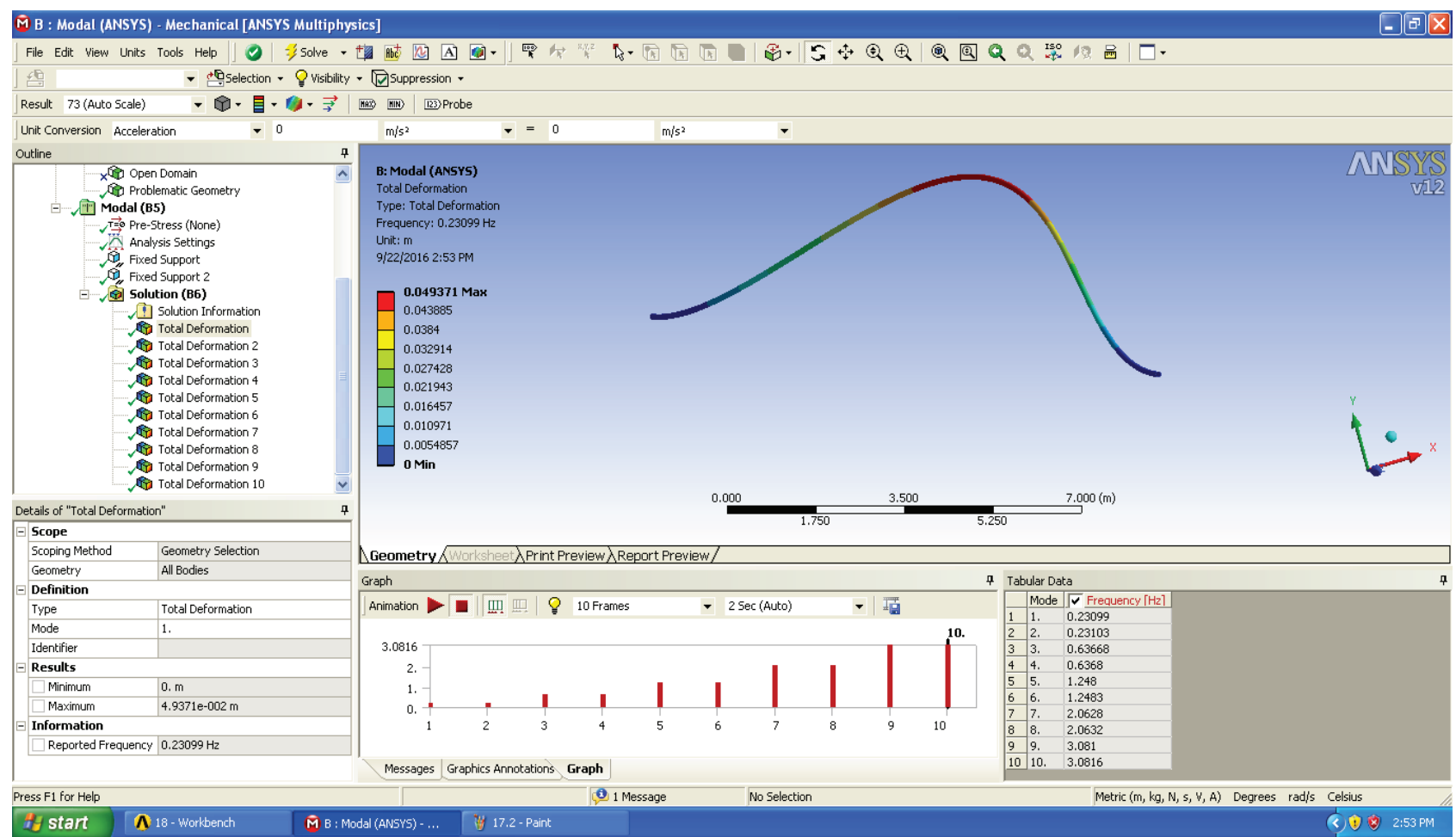

Рисунок 4. Первая форма собственных колебаний прямолинейного 54-метрового участка трубы, незаполненного водой.

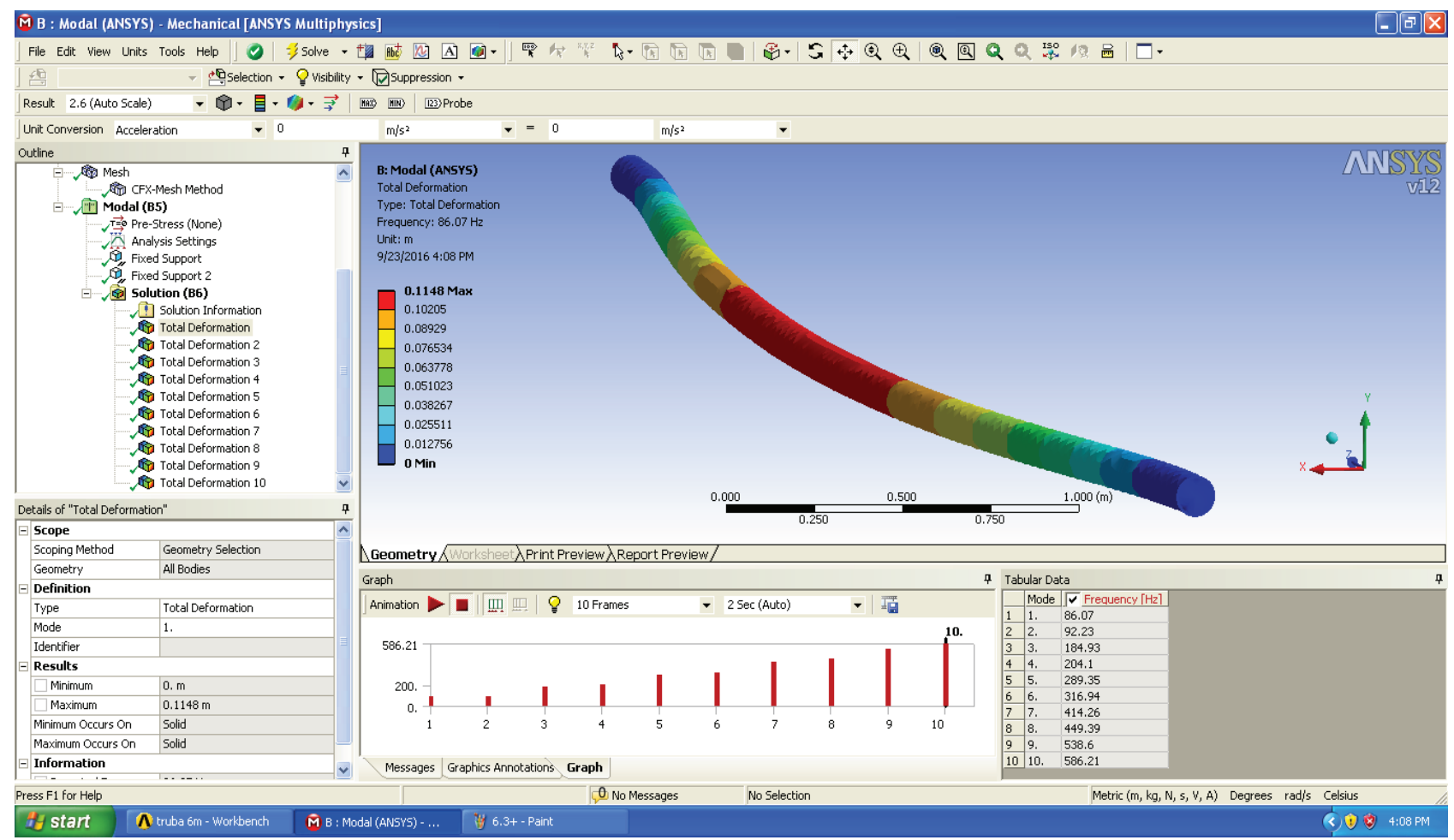

Рисунок 5. Первая форма собственных колебаний прямолинейного 6-метрового участка трубы, заполненного водой. 
Численный анализ динамических характеристик элемента трубопроводной системы многоэтажного жилого дома

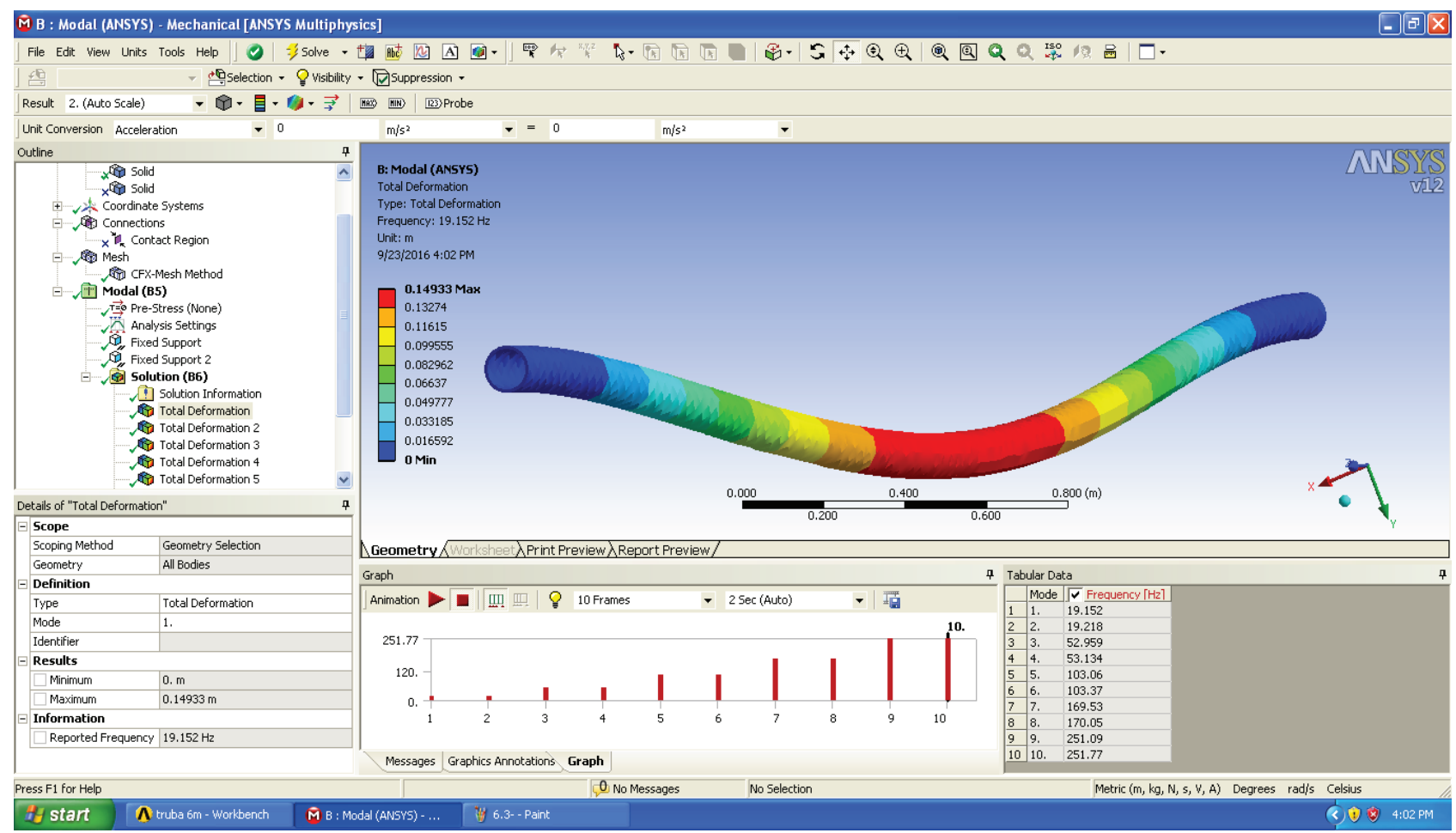

Рисунок 6. Первая форма собственных колебаний прямолинейного 6-метрового участка трубы, незаполненного водой.

Таблииа 1. Частота собственных колебаний.

\begin{tabular}{|c|c|c|c|c|c|c|c|c|c|c|c|}
\hline \multirow{2}{*}{$\begin{array}{c}\text { Вид участка } \\
\text { трубы }\end{array}$} & \multirow{2}{*}{$\begin{array}{l}\text { Запол- } \\
\text { нение } \\
\text { водой }\end{array}$} & \multicolumn{10}{|c|}{ Частота собственных колебаний (Гц) для формы собственных колебаний } \\
\hline & & 1 & 2 & 3 & 4 & 5 & 6 & 7 & 8 & 9 & 10 \\
\hline «Колено» & + & $\begin{array}{l}0,292 \\
\cdot 10^{4}\end{array}$ & $\begin{array}{l}0,826 \\
\cdot 10^{4}\end{array}$ & $\begin{array}{l}0,860 \\
\cdot 10^{4}\end{array}$ & $\begin{array}{l}0,102 \\
\cdot 10^{5}\end{array}$ & $\begin{array}{c}0,109 \\
\cdot 10^{5}\end{array}$ & $\begin{array}{l}0,143 \\
\cdot 10^{5}\end{array}$ & $\begin{array}{l}0,158 \\
\cdot 10^{5}\end{array}$ & $\begin{array}{l}0,161 \\
\cdot 10^{5}\end{array}$ & $\begin{array}{c}0,164 \\
\cdot 10^{5}\end{array}$ & $\begin{array}{l}0,236 \\
\cdot 10^{5}\end{array}$ \\
\hline $\begin{array}{c}\text { Прямолинейный } \\
54 \text {-метровый } \\
\text { участок }\end{array}$ & + & $\begin{array}{c}0,369 \\
\cdot 10^{1}\end{array}$ & $\begin{array}{c}0,371 \\
\cdot 10^{1}\end{array}$ & $\begin{array}{c}0,371 \\
\cdot 10^{1}\end{array}$ & $\begin{array}{c}0,420 \\
\cdot 10^{1}\end{array}$ & $\begin{array}{c}0,486 \\
\cdot 10^{1}\end{array}$ & $\begin{array}{c}0,739 \\
\cdot 10^{1}\end{array}$ & $\begin{array}{c}0,972 \\
\cdot 10^{1}\end{array}$ & $\begin{array}{l}0,111 \\
\cdot 10^{2}\end{array}$ & $\begin{array}{l}0,146 \\
\cdot 10^{2}\end{array}$ & $\begin{array}{c}0,148 \\
\cdot 10^{2}\end{array}$ \\
\hline 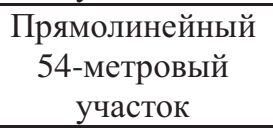 & - & 0,231 & 0,231 & 0,637 & 0,637 & $\begin{array}{l}0,125 \\
\cdot 10^{1}\end{array}$ & $\begin{array}{l}0,125 \\
\cdot 10^{1}\end{array}$ & $\begin{array}{l}0,206 \\
\cdot 10^{1}\end{array}$ & $\begin{array}{l}0,206 \\
\cdot 10^{1}\end{array}$ & $\begin{array}{c}0,308 \\
\cdot 10^{1}\end{array}$ & $\begin{array}{l}0,308 \\
\cdot 10^{1}\end{array}$ \\
\hline $\begin{array}{c}\text { Прямолинейный } \\
\text { 6-метровый } \\
\text { участок трубы }\end{array}$ & + & $\begin{array}{c}0,861 \\
\cdot 10^{2}\end{array}$ & $\begin{array}{c}0,922 \\
\cdot 10^{2}\end{array}$ & $\begin{array}{c}0,185 \\
\cdot 10^{3}\end{array}$ & $\begin{array}{c}0,204 \\
\cdot 10^{3}\end{array}$ & $\begin{array}{c}0,289 \\
\cdot 10^{3}\end{array}$ & $\begin{array}{l}0,317 \\
\cdot 10^{3}\end{array}$ & $\begin{array}{c}0,414 \\
\cdot 10^{3}\end{array}$ & $\begin{array}{c}0,449 \\
\cdot 10^{3}\end{array}$ & $\begin{array}{c}0,539 \\
\cdot 10^{3}\end{array}$ & $\begin{array}{l}0,586 \\
\cdot 10^{3}\end{array}$ \\
\hline $\begin{array}{c}\text { Прямолинейный } \\
\text { 6-метровый } \\
\text { участок трубы }\end{array}$ & - & $\begin{array}{l}0,192 \\
\cdot 10^{2}\end{array}$ & $\begin{array}{c}0,192 \\
\cdot 10^{2}\end{array}$ & $\begin{array}{c}0,530 \\
\cdot 10^{2}\end{array}$ & $\begin{array}{c}0,531 \\
\cdot 10^{2}\end{array}$ & $\begin{array}{l}0,103 \\
\cdot 10^{3}\end{array}$ & $\begin{array}{l}0,103 \\
\cdot 10^{3}\end{array}$ & $\begin{array}{c}0,170 \\
\cdot 10^{3}\end{array}$ & $\begin{array}{c}0,170 \\
\cdot 10^{3}\end{array}$ & $\begin{array}{c}0,251 \\
\cdot 10^{3}\end{array}$ & $\begin{array}{l}0,252 \\
\cdot 10^{3}\end{array}$ \\
\hline
\end{tabular}

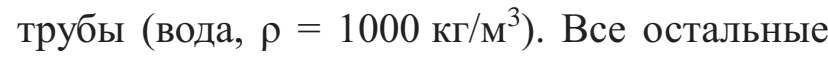
свойства материала, включенного в библиотеку материалов ПК ANSYS, заданы по умолчанию.

Полученные результаты модального анализа сведены в итоговые таблицы 1 и 2.

\section{ВЫВОДЫ}

1. При сокращении длины незакрепленной части трубопровода («прогона») в несколько раз (в данном случае в 9 раз) спектр частот ее собственных колебаний 
Таблича 2. Суммарные деформачии.

\begin{tabular}{|c|c|c|c|c|c|c|c|c|c|c|c|}
\hline \multirow{2}{*}{$\begin{array}{c}\text { Вид участка } \\
\text { трубы }\end{array}$} & \multirow{2}{*}{$\begin{array}{l}\text { Запол- } \\
\text { нение } \\
\text { водой }\end{array}$} & \multicolumn{10}{|c|}{ Суммарные деформации (м) для формы собственных колебаний } \\
\hline & & 1 & 2 & 3 & 4 & 5 & 6 & 7 & 8 & 9 & 10 \\
\hline «Колено» & + & $\begin{array}{c}0,208 \\
\cdot 10^{1}\end{array}$ & $\begin{array}{c}0,144 \\
\cdot 10^{1}\end{array}$ & $\begin{array}{c}0,195 \\
\cdot 10^{1}\end{array}$ & $\begin{array}{c}0,132 \\
\cdot 10^{1}\end{array}$ & $\begin{array}{c}0,197 \\
\cdot 10^{1}\end{array}$ & $\begin{array}{c}0,186 \\
\cdot 10^{1}\end{array}$ & $\begin{array}{c}0,226 \\
\cdot 10^{1}\end{array}$ & $\begin{array}{c}0,148 \\
\cdot 10^{1}\end{array}$ & $\begin{array}{l}0,225 \\
\cdot 10^{1}\end{array}$ & $\begin{array}{c}0,227 \\
\cdot 10^{1}\end{array}$ \\
\hline $\begin{array}{c}\text { Прямолинейный } \\
\text { 54-метровый } \\
\text { участок }\end{array}$ & + & $\begin{array}{c}0,379 \\
\cdot 10^{-1}\end{array}$ & 0,102 & 0,102 & 0,102 & $\begin{array}{c}0,378 \\
\cdot 10^{-1}\end{array}$ & $\begin{array}{c}0,379 \\
\cdot 10^{-1}\end{array}$ & $\begin{array}{c}0,378 \\
\cdot 10^{-1}\end{array}$ & $\begin{array}{c}0,378 \\
\cdot 10^{-1}\end{array}$ & $\begin{array}{c}0,377 \\
\cdot 10^{-1}\end{array}$ & $\begin{array}{c}0,380 \\
\cdot 10^{-1}\end{array}$ \\
\hline $\begin{array}{c}\text { Прямолинейный } \\
\text { 54-метровый } \\
\text { участок }\end{array}$ & - & $\begin{array}{c}0,494 \\
\cdot 10^{-1}\end{array}$ & $\begin{array}{c}0,494 \\
\cdot 10^{-1}\end{array}$ & $\begin{array}{c}0,469 \\
\cdot 10^{-1}\end{array}$ & $\begin{array}{c}0,469 \\
\cdot 10^{-1}\end{array}$ & $\begin{array}{c}0,470 \\
\cdot 10^{-1}\end{array}$ & $\begin{array}{c}0,470 \\
\cdot 10^{-1}\end{array}$ & $\begin{array}{c}0,470 \\
\cdot 10^{-1}\end{array}$ & $\begin{array}{l}0,470 \\
\cdot 10^{-1}\end{array}$ & $\begin{array}{c}0,470 \\
\cdot 10^{-1}\end{array}$ & $\begin{array}{c}0,470 \\
\cdot 10^{-1}\end{array}$ \\
\hline $\begin{array}{c}\text { Прямолинейный } \\
\text { 6-метровый } \\
\text { участок трубы }\end{array}$ & + & 0,115 & 0,112 & 0,118 & 0,125 & 0,119 & 0,121 & 0,123 & 0,120 & 0,118 & 0,122 \\
\hline $\begin{array}{c}\text { Прямолинейный } \\
\text { 6-метровый } \\
\text { участок трубы }\end{array}$ & - & 0,149 & 0,149 & 0,144 & 0,144 & 0,147 & 0,148 & 0,147 & 0,148 & 0,148 & 0,148 \\
\hline
\end{tabular}

смещается в область высоких частот. Как правило, увеличение частоты собственных колебаний соответствует уменьшению амплитуды этих колебаний. К примеру, для 54-метрового участка, заполненного водой, первая частота свободных колебаний равна 3,689 Гц, а для 6метрового участка, заполненного водой, первая частота свободных колебаний равна 86,07 Гц. Имея ввиду, что насосная установка имеет частоту вынужденных колебаний, расположенную в диапазоне 50-60 Гц (2900 об/мин), можно полагать, что возникновение резонанса маловероятно в случае использования 6метрового «прогона». Полученные результаты, позволяют судить о существовании вероятности возникновения резонанса в случае использования 54-метрового «прогона», так как частоты его собственных колебаний располагаются в том же диапазоне, что и частоты вынужденных колебаний насосной установки.

2. При уменьшении длины «прогона» трубопровода наблюдается сокращение плотности распределения собственных частот колебаний. Из таблицы видно, что первые 10 собственных частот 6метрового участка трубы, заполненного водой, лежат в диапазоне $86 . .586$ Гц (2,75 октавы), 54-метрового участка трубы, заполненного водой - в диапазоне $3,7 \ldots 14,8$ Гц (2,0 октавы).

3. При заполнении участка трубопровода определенной длины водой частота его собственных колебаний увеличивается в $1,1 \ldots 1,3$ раза, а амплитуда собственных колебаний уменьшается в среднем в $1,1 \ldots 1,3$ раза.

4. Уменьшение длины «прогона» сокращает вероятность появления резонанса в трубопроводной системе от насосной установки. На основе такого анализа можно сформулировать рекомендации по длине «прогона» для труб различных диаметров. Эти рекомендации будут полезны инженерам-проектировщикам трубопроводных систем.

Таким образом, предварительный модальный анализ может быть полезен при проектировании и моделировании вибраций и переходных процессов в элементах трубопроводных систем многоэтажных зданий, являясь первым этапом численного вибрационного исследования. 
Численный анализ динамических характеристик элемента трубопроводной системы

многоэтажного жилого дома

При наличии данных о внешних динамических воздействиях может быть проведен и следующий этап исследования с целью определения степени опасности возможных резонансных режимов.

\section{СПИСОК ЛИТЕРАТУРЫ}

1. Маклакова Т.Г., Сенин Н.И. Научнообразовательный материал «Архитектурно-конструктивные и градостроительные проблемы проектирования высотных зданий». - М.: МГСУ, 2009.

2. СП 54.13330.2011 «Здания жилые многоквартирные»//Актуализированная редакция СНиП 31-01-2003.

3. Дуров В.С., Рахмилевич 3.3., Черняк Я.С., «Эксплуатация и ремонт компрессоров и насосов: Справочное пособие»- М.: Химия, 1980. - 272 с.

4. Электронный ресурс: (http://77.rospotrebnadzor.ru/index.php/nap ravlenie/zpp/1067-fiz//) «Обзор обращений граждан с жалобами на воздействие физических факторов поступивших в территориальный отдел Управления Роспотребнадзора по городу Москве в Южном административном округе»//Управление Федеральной службы по надзору в сфере защиты прав потребителей и благополучия человека по городу Москве. Дата обращения: 27.09.2016 г.

5. СН 2.2.4/2.1.8.566-96 «Производственная вибрация, вибрация в помещениях жилых и общественных зданий».

6. СН 2.2.4/2.1.8.562-96 «Шум на рабочих местах, в помещениях жилых, общественных зданий и на территории жилой застройки».

7. СанПиН 2.1.2.2645-10 «Санитарноэпидемиологические требования к условиям проживания в жилых зданиях и помещениях» (с изменениями на 27 декабря 2010 года), утв. 10.06.2010 г.
Главным государственным санитарным врачом Российской Федерации.

8. Мягков Ю.В., Шипелов Ю.С. Определение собственных частот и форм колебаний двигателя внутреннего сгорания. // Известия Тульского государственного университета. Выпуск №3, 2014, c. 217-220.

9. Басов К.A. ANSYS справочник пользователя. - М.: ДМК Пресс, 2005. $640 \mathrm{c}$.

10. Электронный журнал для пользователей CAE-системой ANSYS [Электронный pecypc] (http://www.ansyssolutions.ru) (дата обращения: 23.05.13).

11. Бате К., Вилсон Е. Численные методы анализа и метод конечных элементов. М.:Стройиздат, 1982. - 446 с.

12. ANSYS 12.1. User's Guide. Canonsburg. 2010.

13. Шаблинский Г.Э., Зубков Д.А. Натурные и модельные исследования динамических явлений в строительных конструкциях энергетических и гражданских объектов. - М: МГСУ, 2012.

14. Андреева П.И., Ковальчук О. А. Сравнительный анализ результатов экспериментальных натурных динамических исследований и расчета динамических характеристик высотного жилого здания.// International Journal for Computational Civil and Structural Engineering, Volume 8, Issue 4, 2012, pp. 13-18.

15. Андреева П.И., Андреев М.И., Мкртычев О.В., Ковальчук О.А., Шаблинский Г.Э. Влияние геометрического дефекта купольной части защитной оболочки атомного реактора ВВЭР-1000 на динамические характеристики с у учетом статистического анализа.// International Journal for Computational Civil and Structural Engineering, Volume 11, Issue 4, 2015, pp. 29-35.

16. Чернов Ю.Т. Вибрации строительных конструкций. - М.: АCB, 2011. 
17. Болотив В.В. (ред.) Вибрации в технике:

Справочник. T. 5. Измерения и испытания /Под ред. М.Д. Генкина. Ред. совет: В.Н. Челомей (пред.). - М.: Машиностроение, 1981. - 496 с.

Пастухова Лилия Германовна, кандидат технических наук, доцент кафедры «Гидравлика»; Уральский федеральный университет имени первого Президента России Б.Н. Ельцина; 620002, Екатеринбург, ул. Мира, 19; e-mail: lilian63@bk.ru

Секачева Антонина Андреевна, магистрант кафедры «Гидравлика»; Уральский федеральный университет имени первого Президента России Б.Н. Ельцина; 620002, Екатеринбург, ул. Мира, 19; е-mail:

tonechka_marakulina@mail.ru

Lilia G. Pastukhova, Ph.D, Associate Professor of Department of Hydraulics, Ural Federal University named after the first President of Russia B.N. Yeltsin; 620002, Yekaterinburg, 19 Mira street; e-mail: lilian63@bk.ru

Antonina A. Sekacheva, master-student, Department of Hydraulics, Ural Federal University named after the first President of Russia B.N. Yeltsin; 620002, Yekaterinburg, 19 Mira street; e-mail: tonechka_marakulina@mail.ru 


\title{
ОПРЕДЕЛЕНИЕ НАПРЯЖЕННО-ДЕФОРМИРОВАННОГО СОСТОЯНИЯ АРМИРОВАННЫХ ПЛИТ ИЗ НЕЛИНЕЙНОГО МАТЕРИАЛА С УЧЕТОМ ВОЗДЕЙСТВИЯ АГРЕССИВНЫХ СРЕД
}

\author{
А.А. Трещев, А.В. Башкатов, В.Г. Теличко \\ Тульский государственный университет, г. Тула, РОССИЯ
}

\begin{abstract}
Аннотация: В настоящей статье рассматривается построение конечно-элементной модели определения напряженно-деформированного состояния армированных плит в условиях действия активной деформации и простого нагружения в сочетании с длительным воздействием хлоридсодержащей эксплуатационной среды. При решении данной задачи учитывается нелинейная чувствительность основного материала (бетона) к виду напряженного состояния, пластические деформации в арматуре, деградация защитного полимербетона при воздействии внешней агрессивной среды.
\end{abstract}

Ключевые слова: армированные плиты, нелинейные материалы, полимербетон, кинетика агрессивных сред

\section{DEFINITION OF STRESS-STRAIN STATE OF REINFORCED SLAB MADE OF NON-LINEAR MATERIAL TAKING INTO ACCOUNT EXPOSURE OF AGGRESSIVE ENVIRONMENT}

\author{
Alexander A. Treschev, Alexander V. Bashkatov, Victor G. Telichko \\ Tula state university», Tula, RUSSIA
}

\begin{abstract}
Absract: The paper aims to develop the finite element model of stress-strain state of reinforced slab under active deformation and simple loading added to long-term exposure of chloride-containing environment. The model takes into consideration non-linear sensitivity of the main material (concrete) to the type of stress state, plastic strain in reinforcement steel and degeneration of protective resin concrete on exposure to aggressive environment.
\end{abstract}

Key words: reinforced slab, non-linear materials, resin concrete, kinetics of aggressive environments

\section{1. ПОСТАНОВКА ЗАДАЧИ}

Рассматриваемые в данной работе материалы - железобетон и полимербетон, как конструкционные получили широкое распространение в строительстве сооружений всех видов и назначения. Однако присущая бетонам ярко выраженная разносопротивляемость [1,2] не позволяет использовать при прочностных расчетах конструкций классические методы механики твердого тела. Так же следует отметить, что у некоторых видов бетонов при изменении вида напряженного состояния наблюдается существенная нелинейность диаграмм деформирования [1,2]. Кроме того конструкции часто подвержены воздействию агрессивных эксплуатационных сред, вследствие чего происходит изменение физико-механических свойств материала. Одной из наиболее распространенных агрессивных эксплуатационных сред является хлорид натрия $(\mathrm{NaCl})$, который имеет как природный характер (соленая морская вода и морской туман), так и техногенный (повсеместно встречается в составе средств антиобледенителей и различных технологических сред). 
Анализ известных определяющих соотношений для материалов с усложненными свойствами, эксплуатируемых в агрессивных средах, показал, что существующие теории и методики имеют ряд недостатков, не учитывающих важных особенностей деформирования материалов, что во многих случаях приводит к значительным погрешностям получаемых аппроксимаций экспериментальных данных.

Наиболее универсальной и непротиворечивой теорией деформирования разносопротивляющихся материалов является теория Н.М. Матченко и А.А. Трещева, предложенная авторами в работе [1,2]. В своей теории авторы для описания свойств разносопротивляемости структурно изопропных и анизотропных материалов предлагают использовать нормированные пространства напряжений. Численный анализ рассматриваемой теории проводился в различных работах, среди которых исследование, проведенное в [3] при расчете НДС железобетонных плит, прошедших глубокое экспериментальное исследование в опытах Г. Баха и О. Графа, В. Гелера и Х. Амоса $[4,5]$, признанных в работе Н.И. Карпенко [6].

В свою очередь методика учета кинетики агрессивной среды предложенная в работе [7], позволяет наиболее корректно и полно описать кинетику агрессивной среды.

Таким образом, авторы представленной работы, обобщив два указанных выше направления исследования, предлагают математическую модель расчета НДС железобетонных конструкций с учетом воздействия внешних эксплуатационных сред и разносопротивляемости материала.

\section{2. КОНЕЧНО-ЭЛЕМЕНТНАЯ МОДЕЛЬ ДЕФОРМИРОВАНИЯ АРМИРОВАННЫХ ПЛИТ ИЗ НЕЛИНЕЙНОГО МАТЕРИАЛА}

Решать данную задачу предложено используя модификацию гибридных КЭ с пятью степенями свободы в узле и матрицей жесткости, полученной непосредственно для произвольного плоского треугольного элемента [8]. Данный конечный элемент разработан на основе двух модификаций гибридных КЭ, предложенных Р. Куком [9].

Построение конечно-элементной модели определения НДС слоистых армированных плит из нелинейного материала подробно описано авторами в работе [10].

Задачу изгиба железобетонных плит, независимо от геометрической конфигурации, предлагается рассматривать в условиях активной деформации и простого нагружения, при этом авторами используется потенциал деформаций, представленный в работе А.А. Трещева [1], в «рамки» которого укладываются упругопластические свойства бетона как нелинейного материала:

$$
\begin{aligned}
& W_{1}=\left(A_{e}+B_{e} \xi\right) \sigma^{2}+\left(C_{e}+D_{e} \xi+\right. \\
& \left.+E_{e} \eta \operatorname{Cos} 3 \varphi\right) \tau^{2}+\left[\left(A_{p}+B_{p} \xi\right) \sigma^{2}+\right. \\
& \left.+\left(C_{p}+D_{p} \xi+E_{p} \eta \operatorname{Cos} 3 \varphi\right) \tau^{2}\right]^{n}
\end{aligned}
$$

где $A_{e}, B_{e}, C_{e}, D_{e}, E_{e}$ и $A_{p}, B_{p}, C_{p}, D_{p}, E_{p}-$ константы потенциала, $\xi, \eta \quad-$ нормированные нормальные и касательные напряжения на октаэдрической площадке, $\sigma$ и $\tau$ - нормальные и касательные напряжения, $\varphi$ - фаза напряжений;

$$
\begin{gathered}
S_{0}=\sqrt{\sigma^{2}+\tau^{2}} ; \xi=\frac{\sigma}{S_{0}} ; \eta=\frac{\tau}{S_{0}} ; \\
\operatorname{Cos} 3 \varphi=\frac{\sqrt{2} \operatorname{det}\left(S_{i j}\right)}{\tau^{3}} ; S_{i j}=\sigma_{i j}-\delta_{i j} \sigma ; \\
\sigma=\delta_{i j} \sigma_{i j} / 3 ; \tau=\sqrt{S_{i j} S_{i j} / 3} .
\end{gathered}
$$

Дополнительно вводимые модели и допущения, а так же построение фиктивных слоев при расчете подобного рода конструкций достаточно полно приведены в работе А.А. Трещева, В.Г. Теличко, А.Н. Артемова $[3,10]$. 
Определение напряженно-деформированного состояния армированных плит из нелинейного материала с учетом воздействия агрессивных сред

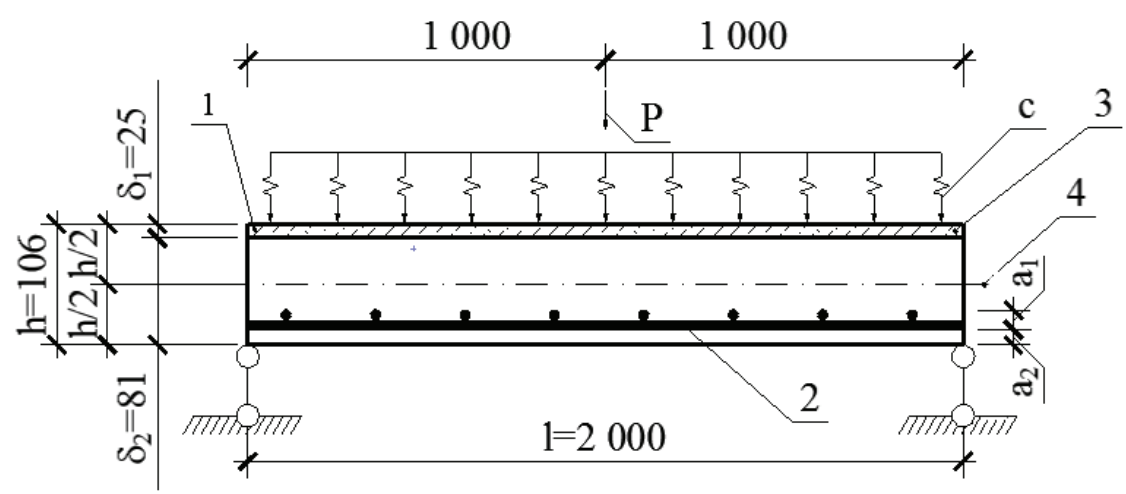

Рисунок 1. Схема рассматриваемой плиты №844.

1 - железобетонный слой плиты; 2 - армирование плиты; 3 - полимербетонный слой;

4 - срединная поверхность; P-сосредоточенная нагрузка; с-агрессивная среда;

$\delta_{1}-$ толщина полимербетонного слоя; $\delta_{2}-$ толщина железобетонного слоя;

$h$ - полная толщина плить; $a_{1}$ - толщина армированного слоя;

$a_{2}$ - толщина защитного слоя бетона для арматурной сетки.

\section{3. МОДЕЛИРОВАНИЕ}

\section{ПОЛИМЕРБЕТОННОГО СЛОЯ \\ И УЧЕТ ВОЗДЕЙСТВИЯ АГРЕССИВНОЙ ЭКСПЛУАТАЦИОННОЙ СРЕДЫ}

В рамках рассматриваемой задачи рассматривается случай, когда действие агрессивной эксплуатационной среды приходится только на полимербетонный слой, расположенный в сжатой зоне плиты. В качестве железобетонной плиты принята плита №844, подробно изученная в опытах Г. Баха и О. Графа [3], расчетная схема рассматриваемой задачи приведена на рисунке 1 .

Экспериментальные исследования показывают, что в процессе эксплуатации в материале конструкций под влиянием рабочей среды возникает неоднородность физикомеханических свойств. Неоднородное распределение свойств по сечению образца и кинетика развития этого процесса определяются сложными физико-механическими процессами, и зависят от уровня и характера напряженного состояния материала, такой вид неоднородности называют наведенной неоднородностью [12].

Наведенная неоднородность характеризуется зависимостью от координат и концентрации агрессивной среды в произвольной точке се- чения материала. Полагаем, что развитие наведенной неоднородности можно учесть введением наряду с критерием объективной прочности понятия объективных диаграмм деформирования для фиксированных моментов времени, связанных с показателем объективной прочности и кривой длительной прочности, получаемых при испытании образцов в агрессивной среде.

Примем в качестве выражения для определения глубины слоя, пораженного средой, нелинейный закон, предложенный в работе [7]:

$$
\delta(t)=\alpha \sqrt{t},
$$

где $t$ - время воздействия агрессивной среды, $\alpha$ - экспериментальный коэффициент, зависящий от конкретной пары «материал среда». Коэффициент $\alpha$ описывает химическую активность рабочей среды и силовое сопротивление конструкционного материала. С учетом деградации свойств материала, выражения переменных секущего $E_{c}$ и касательного модулей $E_{k}$ принимаем в виде, предложенным В.В. Петровым и О.В. Пениной в исследовании [7]: 


$$
\begin{aligned}
& E_{c}=E_{c}^{0} F\left(B\left(x_{3}\right)\right) ; \\
& E_{k}=E_{k}^{0} F\left(B\left(x_{3}\right)\right),
\end{aligned}
$$

где $E_{c}^{0}$ - секущий модуль материала без влияния агрессивной эксплуатационной среды, $E_{k}^{0}$ - касательный модуль без влияния агрессивной среды, $F(B)$ - функция деградации секущего и касательного модулей, $x_{3}$ - координата в направлении толщины пластины.

Результаты экспериментальных исследований композитных бетонов [13] позволили записать функцию деградации в виде:

$$
\omega_{p b}=F\left(B\left(x_{3}\right)\right)=\exp \left(-\lambda B\left(x_{3}\right)\right),
$$

где $\lambda$ - относительная скорость изменения секущего и касательного модулей.

$$
-\lambda=\frac{F^{\prime}(B)}{F(B)},
$$

где $F^{\prime}(B)$ - скорость деградации.

Заметим, что при воздействии агрессивной среды на полимербетонные слои не происходит нарушения приемлемости потенциальных определяющих соотношений, ориентированных на нелинейный дилатирующий разносопротивляющийся изотропный материал. Соответственно рассуждения, приведенные в работах $[7,13]$, справедливы и для рассматриваемого случая. Физически нелинейные свойства бетона будем аппроксимировать секущим модулем упругости $E_{c}$ и секущим коэффициентом поперечных деформаций $v_{c}$, определяемых из следующего уравнения:

$$
\begin{array}{ll} 
& e_{22}=A_{12} \sigma_{11}+A_{22} \sigma_{22}= \\
& =\left(\sigma_{22}-v_{c} \sigma_{11}\right) / E_{c}, \\
\text { т.e. } & E_{c}=1 / A_{22} ; v_{c}=-A_{12} / A_{22},
\end{array}
$$

где $A_{12}, A_{22}$ - компоненты матрицы податливостей, рассчитываемые по формулам для бетонного слоя без трещин.

С учетом изложенного, зависимости между деформациями и напряжениями для полимербетонного слоя по аналогии с моделированием слоев железобетонной плиты [3,11], представим в виде:

$$
\left\{e^{*}\right\}=\left[A^{*}\right]\left\{\sigma^{*}\right\}
$$

где

$$
\begin{aligned}
& \left\{e^{*}\right\}=\left\{\begin{array}{l}
e_{11}^{*} \\
e_{22}^{*} \\
\gamma_{12}^{*} \\
\gamma_{13}^{*} \\
\gamma_{23}^{*}
\end{array}\right\} ;\left\{\sigma^{*}\right\}=\left\{\begin{array}{l}
\sigma_{11}^{*} \\
\sigma_{22}^{*} \\
\tau_{12}^{*} \\
\tau_{13}^{*} \\
\tau_{23}^{*}
\end{array}\right\} ; \\
& {\left[A^{*}\right]=\left[\begin{array}{lllll}
A_{11}^{*} & A_{12}^{*} & 0 & 0 & 0 \\
& A_{22}^{*} & 0 & 0 & 0 \\
& & A_{66}^{*} & 0 & 0 \\
& \operatorname{Sim} & & A_{44}^{*} & 0 \\
& & & & A_{55}^{*}
\end{array}\right] ;}
\end{aligned}
$$

$$
\begin{aligned}
& A_{11}^{*}=1 /\left(E_{c} \omega_{p b}\right) ; A_{12}^{*}=-v_{c} /\left(E_{c} \omega_{p b}\right) ; \\
& A_{22}^{*}=1 /\left(E_{c} \omega_{p b}\right) ; \\
& A_{44}^{*}=A_{66}^{*}=2\left(1+v_{c}\right) /\left(E_{c} \omega_{p b}\right) ; \\
& A_{55}^{*}=2(1+v) /\left(E_{c} \omega_{p b}\right) .
\end{aligned}
$$

При этом модуль деформации бетона определим величиной $E_{c} \omega_{p b}\left(\omega_{p b}-\right.$ функция деградации [13] $\left.0<\omega_{p b} \leq 1\right)$.

В итоге для полимербетонного слоя имеем:

$$
[B]=\left[A^{*}\right]^{-1} \text {. }
$$

\section{4. РЕЗУЛЬТАТЫ РАСЧЕТА И АНАЛИЗ ПОЛУЧЕННЫХ ДАННЫХ}

При расчете были приняты следующие характеристики плиты: а) модуль упругости арматурной стали был принят равным 
Определение напряженно-деформированного состояния армированных плит из нелинейного материала с учетом воздействия агрессивных сред

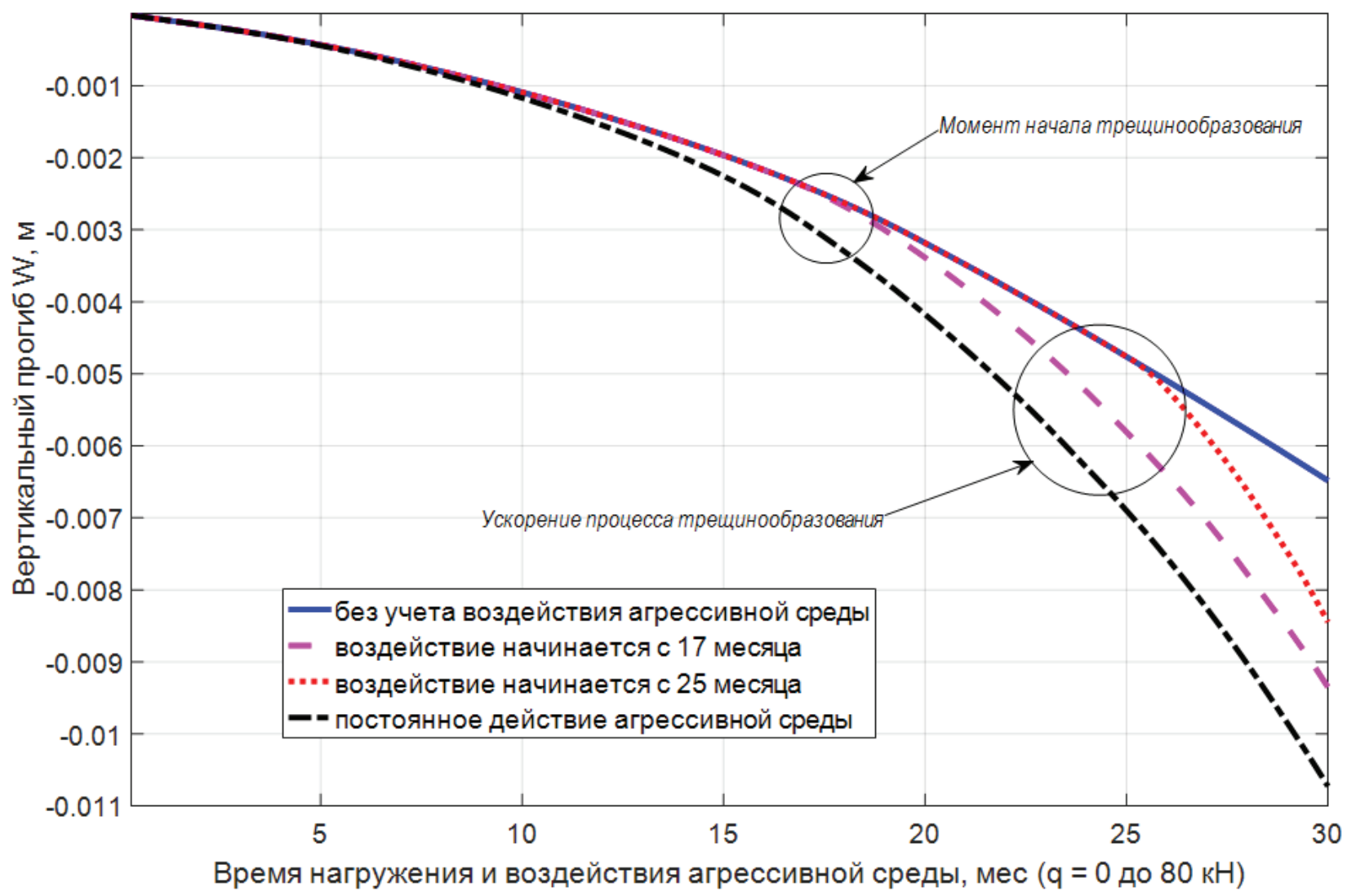

Рисунок 2. Зависимости вертикальных прогибов от момента начала и периода действия агрессивной эксплуатационной среды.

$E_{s}=2 \times 10^{5} M П а ;$ б) полимербетонный слой принят из эпоксидного бетона, модуль упругости которого составляет $E_{b}=25500 M П a$; в) толщина полимербетонного слоя - 0,025 м; г) агрессивная среда $-20 \%$ раствор $\mathrm{NaCl}, \mathrm{c}$ плотностью $\rho=1,2192 / \mathrm{cm}^{3}$. Время действия среды рассматривалось на интервале от 0 до 30 месяцев, при этом нагрузка менялась от 0 до 80 кН. Характеристики железобетонной части плиты подробно описаны в работе [6]. Полученные результаты расчета приведены на рисунке 2. При расчете задавалась сетка конечных элементов размерами $24 \times 24$ элемента, плита разбивалась на 28 фиктивных слоев.

Результаты расчета подтверждают тот факт, что учёт нелинейности диаграмм деформирования и кинетики агрессивных эксплуатационных сред вносит значительные коррек- тивы в напряжённо-деформированное состояние конструкций.

\section{СПИСОК ЛИТЕРАТУРЫ}

1. Трещев А.А. Теория деформирования и прочности материалов с изначальной и наведенной чувствительностью к виду напряженного состояния. Определяющие соотношения. - М.; Тула: РАACH; ТулГУ, 2016. $-326 \mathrm{c}$.

2. Матченко Н.М., Толоконников Л.А., Трещев А.А. Определяющие соотношения изотропных разносопротивляющихся сред. Ч. 2. Нелинейные соотношения. // Изв. РАН. МТТ, 1999, №4, с. 87-95.

3. Артемов А.Н., Трещев А.А. Поперечный изгиб железобетонных плит с учетом трещин. // Изв. вузов. Строительство, 1994, №9-10, c. 7-12. 
4. Bach G., Graf O. Versuche mit allseiting aufliegenden, quadratischen und rechteckigen Eisenbetonplatten. Berlin, 1915.

5. Gehler W., Amos H. Versuche mit kreuzweise bewerhten Platten Heft 70. Berlin, 1932.

6. Карпенко Н.И. Теория деформирования железобетона с трещинами. - М.: Стройиздат, 1976. - 208c.

7. Петров В.В., Пенина О.В. Определение долговечности и резерва несущей способности нелинейно-упругих пластинок при изгибе в агрессивных средах. // Вестник Саратовского государственного технического университета, 2008, №4, с. 16-22.

8. Теличко В.Г., Трещев А.А. Гибридный конечный элемент для расчета плит и оболочек с усложненными свойствами. // Известия вузов. Строительство, №5, 2003, c. 17-23.

9. Cook R.D. Two hybrid elements for analysis of thick thin and sandwich plates // Int. J. num. Meth. Engng., 1972, Vol. 5, pp. 277288.

10. Трещев А.А., Теличко В.Г., Башкатов A.B. Построение математической модели деформирования комплексной железобетонной плиты с полимербетонным слоем под действием агрессивной среды. // Вестник МГСУ, 2014, №3, с. 126-132.

11. Теличко В.Г., Трещев А.А. Моделирование напряженно-деформированного состояния оболочечных конструкций из железобетона. // Известия ТулГУ. Сер. Строительные материалы, конструкции и сооружения. - Тула: Изд-во ТулГУ, 2005. Вып. 8, с. 147-161.

12. Петров В.В., Иноземцев В.К., Синева Н.Ф. Теория наведенной неоднородности и ее приложения к проблеме устойчивости пластин и оболочек. Сарат. госуд. технич. ун-т, Саратов, 1996, $311 \mathrm{c.}$

13. Селяев П.В. Диаграммы деформирования композиционных материалов при воздействии жидких агрессивных сред. // Проблемы прочности элементов кон- струкций под действием нагрузок и рабочих сред: Саратов, Сарат. гос. техн. ун-т, 2006, с. 46-52.

Трещев Александр Анатольевич, советник РААСН, доктор технических наук, профессор, заведующий кафедрой строительства, строительных материалов и конструкций Федеральное государственное образовательное учреждение высшего образования «Тульский государственный университет» (ФГБОУ ВО «ТулГУ»); 300012, г. Тула, пр. Ленина, 92; тел. +7(4872) 25-71-08; e-mail: taa58@yandex.ru.

Башкатов Александр Валерьевич, ассистент кафедры строительства, строительных материалов и конструкций Федеральное государственное образовательное учреждение высшего образования «Тульский государственный университет» (ФГБОУ ВО «ТулГУ»); 300012, г. Тула, пр. Ленина, 92; тел. +7(4872) 25-7108; e-mail: a.bashkatov90@mail.ru.

Теличко Виктор Григорьевич, кандидат технических наук, доцент кафедры строительства, строительных материалов и конструкций Федеральное государственное образовательное учреждение высшего образования «Тульский государственный университет» (ФГБОУ ВО «ТулГУ»); 300012, г. Тула, пр. Ленина, 92; тел. +7(4872) 25-71-08; e-mail: katranv@yandex.ru.

Treschev, Alexander Anatolievich, Advisor of the Russian Academy of Architecture and Construction Sciences, Doctor of Technical Sciences, professor, the head of Department of Construction, Building Materials and Structures, Tula State University(TulGU); 300012, Tula, 92 Lenina prospect, 92 . Tel: +7(4872) 25-71-08;

e-mail: taa58@yandex.ru.

Bashkatov, Aleksander Valerievich, assistant, Department of Construction, Building Materials and Structures, Tula State University(TulGU); 300012, Tula, 92 Lenina prospect, 92. Tel: +7(4872) 25-71-08;

e-mail: a.bashkatov90@mail.ru.

Telichko, Victor Grigorievich, Candidate of Technical Sciences, Associate professor, Department of Construction, Building Materials and Structures, Tula State University(TulGU); 300012, Tula, 92 Lenina prospect, 92. Tel: +7(4872) 25-71-08; e-mail: katranv@ yandex.ru. 


\title{
ВЯЗКОЕ ДЕМПФИРОВАНИЕ В ПЛАТФОРМЕННЫХ МОДЕЛЯХ СИСТЕМ «СООРУЖЕНИЕ-ОСНОВАНИЕ»
}

\author{
А.Г. Тяпин \\ АО «Атомэнергопроект», г. Москва, РОССИЯ
}

\begin{abstract}
Аннотация: Системы «сооружение-основание» для динамических расчетов во многих случаях моделируются платформенными моделями, в которых основание представлено т.н. «грунтовыми» пружинами и вязкими демпферами, установленными на жесткой платформе под фундаментом. Демпферы призваны учесть как материальное демпфирование в грунте, так и волновое демпфирование в основании, возникающее даже в идеально упругой среде. Это демпфирование значительно снижает сейсмические нагрузки на сооружение, поэтому регуляторы вводили и вводят определенные ограничения на его значения. В статье обсуждается трактовка этих ограничений и рекомендации по их применению.
\end{abstract}

Ключевые слова: динамическое взаимодействие сооружений с грунтовым основанием, сейсмическое воздействие, вязкое и материальное демпфирование, модальный метод

\section{VISCOUS DAMPING IN PLATFORM MODELS OF SOIL-STRUCTURE SYSTEMS}

\author{
Alexander G. Tyapin \\ JSC “Atomenergoproject”, Moscow, RUSSIA
}

\begin{abstract}
Soil-structure systems are often analyzed using platform models, where the soil foundation is represented by the so-called "soil" springs and dashpots placed on the rigid platform under the base. Dashpots should account not only for the material damping in the soil, but also for the wave damping present even in the ideally elastic soil medium. This damping substantially decreases seismic loads impacting the structure. That is why regulatory bodies in different have tried to set up certain limitations on its value. The author in the paper comments on these limitations and gives some recommendations on their implementation.
\end{abstract}

Key words: dynamic soil-structure interaction, seismic excitation, viscous and material damping, modal approach

\section{1. ПЛАТФОРМЕННЫЕ МОДЕЛИ СИСТЕМ «СООРУЖЕНИЕ- ОСНОВАНИЕ»}

В современных моделях основания учитывается как его податливость, так и его инерционность - сочетание этих двух свойств означает возможность распространения в основании волн. В дополнение к этому, одной из главных особенностей системы «сооружение-основание» является бесконечность основания. Благодаря этой особенности в основании среди прочих волн возникают волны, уносящие энергию от фундамента со- оружения - это т.н. «волновое демпфирование». Оно значительно снижает сейсмические нагрузки на сооружение и обычно играет намного более важную роль при определении сейсмической реакции, чем традиционное материальное демпфирование.

Одним из традиционных способов учета взаимодействия сооружения с основанием является т.н. «платформенная модель» [1]. Модель сооружения ставится на жесткую платформу с помощью т.н. «грунтового подвеса» - системы специальных пружин и вязких демпферов. Вязкость «грунтовых демпферов» призвана моделировать как эффекты 
волнового демпфирования, так и влияние материального демпфирования в грунте.

Проследим, откуда взялось понятие платформенной модели. Изначально платформенная модель появилась в контактных задачах (сначала статических, потом и в динамических). Простейшая модель такого рода показана на рис.1: сила $\mathrm{P}$, действующая на штамп, вызывает перемещение V.

Принципиально важно понимать, что неподвижная платформа в данном случае нефизична: в исходной задаче нет неподвижных точек. Это всего лишь математическая аналогия.

Если основание линейное и безынерционное, его жесткость описывается оператором жесткости, не зависящим от частоты, - в простейшем случае жесткого штампа это симметричная действительная матрица размером 6 х 6 . При учете инерции линейного основания оператор жесткости зависит от частоты. Линейный оператор в частотном диапазоне превращается в оператор жесткости, подобный по формату предыдущему случаю, но отличающийся двумя особенностями: а) он зависит от частоты; б) жесткость описывается комплексными величинами. Для жесткого штампа симметричная матрица 6 х 6 называется матрицей импедансов - она комплексная и частотно-зависимая.

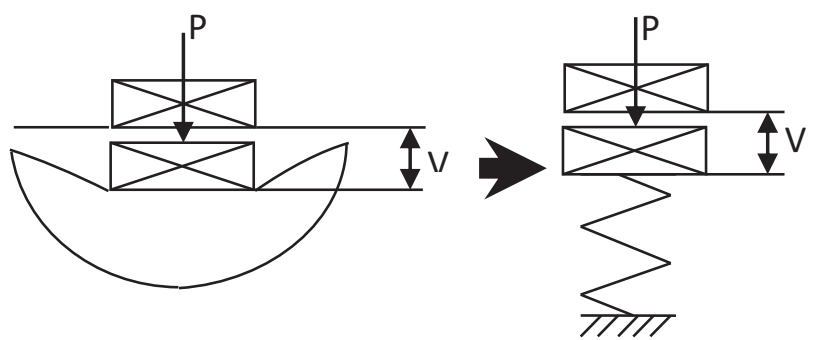

Рисунок 1. Простейшая платформенная модель в контактной задаче.

Если ввести дополнительные упрощающие предположения: однородность полупространства, поверхностное заложение и простую форму штампа в плане, то матрица импедансов упрощается [2]. В первом приближении можно считать ее диагональной. Что касается каждого диагонального элемента этой матрицы, то в первом приближении его считают постоянным в действительной части и линейным по частоте в мнимой части. Таким образом, его можно смоделировать параллельно включенными пружиной и вязким демпфером. Параметры пружин и демпферов приводятся в таблицах, приводимых в нормах (см., например, [3]). Надо только помнить, что это приближенные выражения. Они достаточно точные для горизонтальных жесткостей, менее точные для вертикальных жесткостей и еще менее точные для угловых жесткостей - двух качательных и одной крутильной.

Таким образом, линейные вязкие демпферы - это, как и платформа, всего лишь математические аналогии. Следует помнить также, что физический механизм уноса энергии от фундамента с традиционной вязкостью ничего общего не имеет - механическая энергия не переходит в тепловую, а остается механической, просто уносится на бесконечность. Волновое демпфирование существует даже в основаниях из идеально упругого материала.

Сделаем небольшое отступление. Как платформенные модели появились в задачах динамического взаимодействия сооружений с основанием при сейсмических воздействиях? Здесь проще всего воспользоваться принципом суперпозиции, разложив исходную задачу в сумму задач А и В, как показано на рис. 2.

Нагрузка $[-p(x, t)]$ на поверхности $\mathrm{Q}$ в задаче А подбирается таким специальным образом, чтобы перемещения этой поверхности были равны нулю. Это, во-первых, означает, что все перемещения внутренней части системы в исходной задаче будут в точности равны перемещениям в задаче В. Во-вторых, такое обстоятельство позволяет ослабить требования к линейности системы. Внутренняя (внутри Q) часть системы в задаче В может быть и нелинейной: принцип суперпозиции в этом частном случае все равно будет работать. 


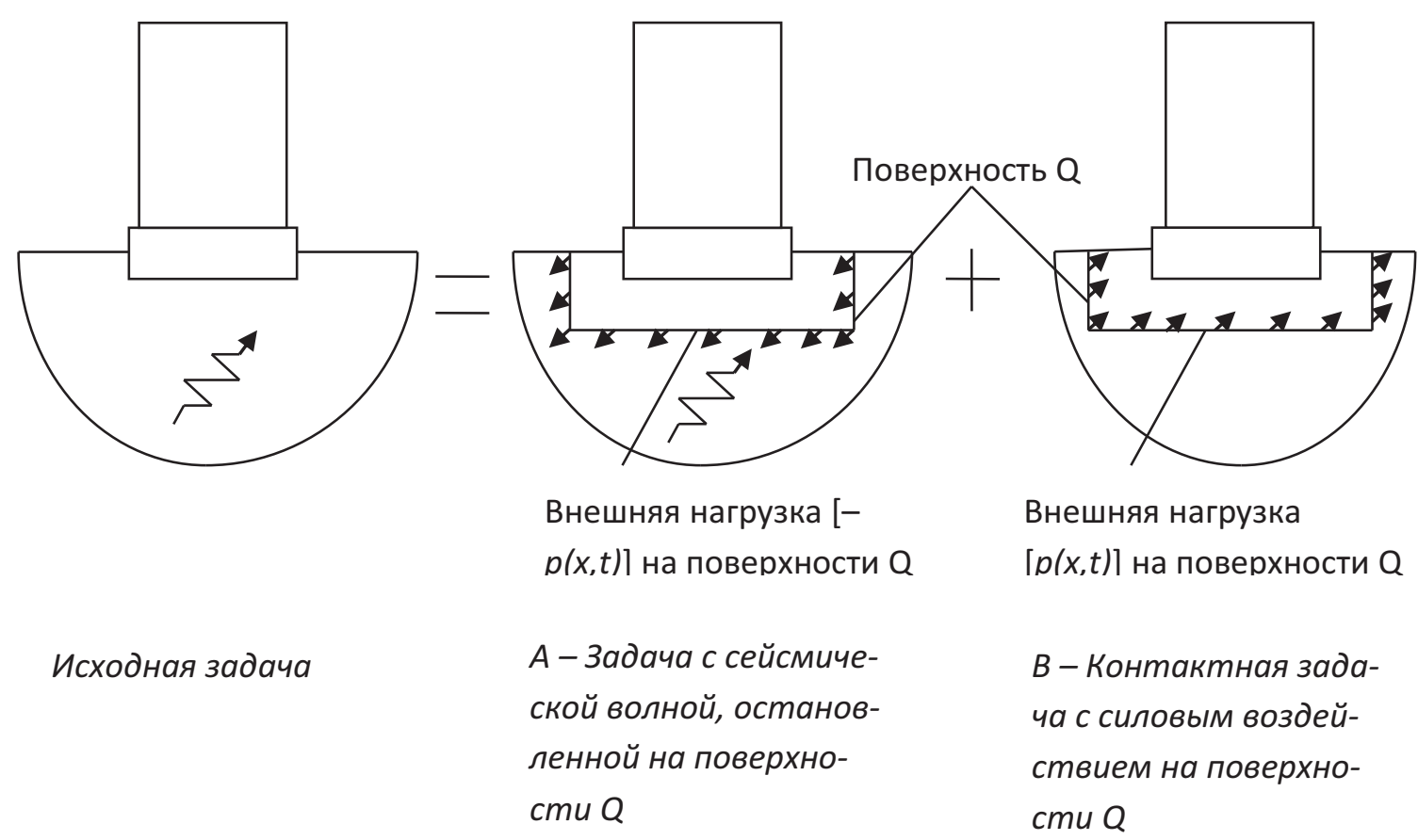

Рисунок 2. Суперпозичия двух волновых задач.

На практике расчет проводится по модели В, которая переводится в платформенную модель по типу рис.1. Но если контактная поверхность фундамента с основанием жесткая, поверхность $\mathrm{Q}$ совмещается с ней, и в итоге получается платформенная модель с шестикомпонентным силовым возбуждением и с матрицей импедансов размером 6 х 6 . Такая модель называется моделью с сосредоточенным грунтовым подвесом.

Далее при желании (это необязательная опция) можно проделать операцию замены силового возбуждения на кинематическое возбуждение платформы. При этом движение платформы в точке крепления к ней подвеса должно соответствовать движению невесомого жесткого фундамента в задаче В на рис.2. Это в общем случае шестикомпонентное движение, которое определяется в результате решения отдельной задачи о т.н. «кинематическом взаимодействии». Но в частном случае, когда жесткий фундамент расположен на поверхности основания, а в отсутствии сооружения пятно под фундаментом движется поступательно как жесткое целое, невесомый фундамент будет двигаться так же, как любая точка на поверхности основания в отсутствии сооружения. Только в этом случае и возникает платформенная модель с движением платформы, взятым с поверхности основания в отсутствии сооружения, - самая популярная платформенная модель. Эта модель проиллюстрирована на рис. 3 .

Итак, мы получили платформенную модель, где под фундаментом установлен вязкий демпфер. Расчеты платформенной модели на сейсмическое воздействие могут проводиться различными методами, самым распространенным из которых по многим причинам является модальный метод, использующий разложение по собственным формам недемпфированной системы. При этом вычисляются модальные коэффициенты демпфирования по каждой форме.

\section{2. ВЯЗКОЕ И МАТЕРИАЛЬНОЕ ДЕМПФИРОВАНИЕ}

Отметим принципиальную особенность вязкого демпфирования, которая отличает его от демпфирования материального. 


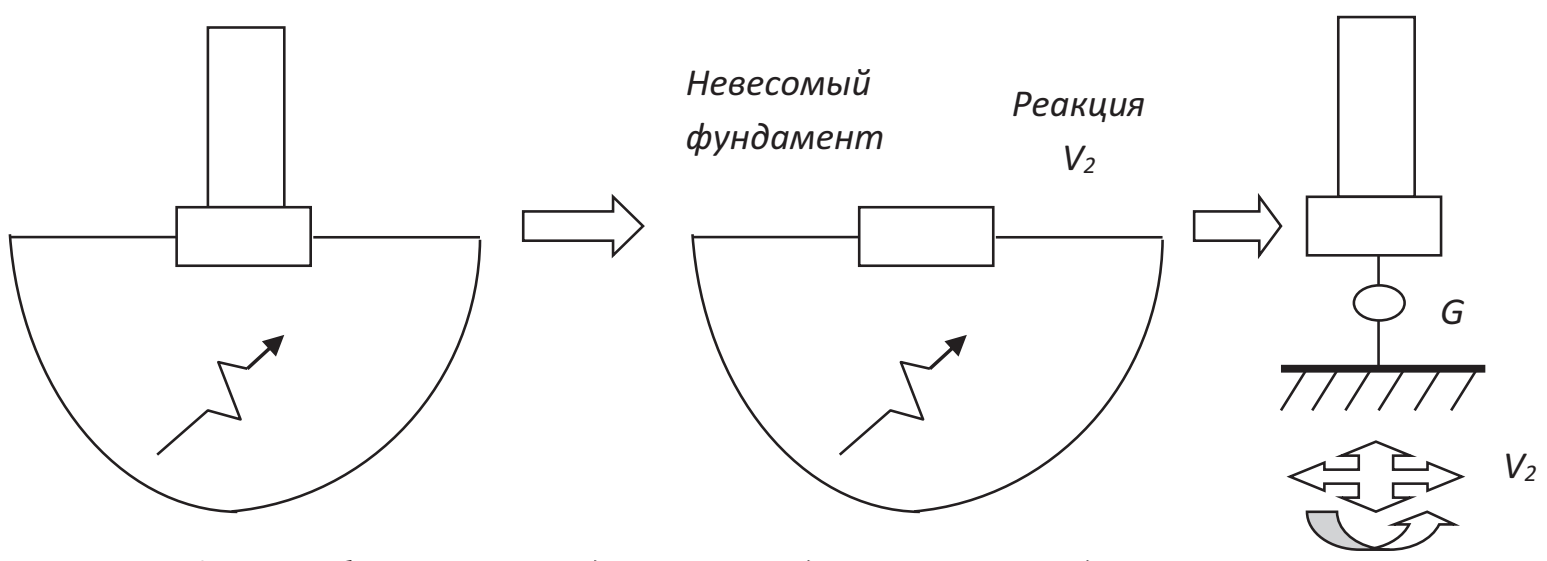

Рисунок 3. Платформенная модель с сосредоточенным подвесом и кинематическим возбуждением.

Ее непонимание часто приводит к путанице. Если в случае материального демпфирования безразмерный относительный коэффициент характеризует каждый элемент системы, то в случае вязкого демпфирования подобной характеристикой является размерная вязкость в элементе, а безразмерный коэффициент характеризует интегральные особенности системы. Проиллюстрируем это на примере одномерных колебаний простейшей платформенной модели с параллельно включенными в работу пружиной и вязким демпфером. Всем известное уравнение движения

$$
m \ddot{x}+b \dot{x}+k x=-m a_{0}
$$

в частотном диапазоне принимает вид

$$
-\omega^{2} m x+i \omega b x+k x=-m a_{0}(\omega)
$$

Здесь $x$ - относительные перемещения массы $m$ относительно платформы, которая движется с ускорением $a_{0} ; k$ - жесткость пружины; $b$ - вязкость демпфера. После традиционного приведения уравнения к безразмерному относительному демпфированию получаем

$$
-\omega^{2} x+2 i \omega \lambda \omega_{0} x+\omega_{0}^{2} x=-a_{0}(\omega)
$$

Здесь собственная частота недемпфированной системы $\omega_{0}$ и безразмерный коэффици- ент $\lambda$ демпфирования «в долях критического» равны

$$
\begin{gathered}
\omega_{0}=\sqrt{k / m} \\
\lambda=\frac{b}{2 m \omega_{0}}=\frac{b}{2 \sqrt{k m}}
\end{gathered}
$$

Отметим присутствие массы $m$ в формуле (5) для безразмерного коэффициента демпфирования $\lambda$. Оно означает, что при одной и той же физически определенной динамической жесткости подвеса

$$
D(\omega)=k+i \omega b
$$

безразмерный коэффициент демпфирования будет разным при разных массах $m$.

Теперь заменим вязкий демпфер на пластичный демпфер с безразмерным коэффициентом материального демпфирования $\gamma$. Уравнение движения в частотном диапазоне будет очень похоже на (3), отличаясь только вторым слагаемым в левой части:

$$
-\omega^{2} x+2 i \omega_{0}^{2} \gamma \frac{\sqrt{1-\gamma^{2}}}{1-2 \gamma^{2}} x+\omega_{0}^{2} x=-a_{0}(\omega)
$$

При небольших значениях $\gamma$ дробь во втором слагаемом близка к единице, поэтому часто ее просто пропускают. Но мы этого делать не будем, тем более что ранее автор в [4] написал эту дробь с ошибкой. 
В данном случае динамическая жесткость подвеса равна

$$
D(\omega)=k+i\left[2 \gamma \frac{\sqrt{1-\gamma^{2}}}{1-2 \gamma^{2}}\right] k
$$

Мы видим, что безразмерный коэффициент $\gamma$ определяется отношением мнимой и действительной частей динамической жесткости; от массы он не зависит.

Часто встречающееся отождествление безразмерных коэффициентов материального и вязкого демпфирования основано на том, что на собственной частоте $\omega=\omega_{0}$ при небольших значениях $\gamma$ передаточная функция от воздействия к реакции («резонансный пик») в обоих случаях почти совпадает. На самом же деле даже понятие «в долях критического демпфирования» для этих коэффициентов совершенно разное. Для вязкого демпфирование «критическое» значение $(\lambda=1)$ вполне достижимо физически и может даже превышаться; для материального демпфирования «критическое» значение $(\gamma=1)$ превышено быть не может ( $\gamma$ по сути есть отношение мнимой части комплексного числа к его модулю).

Вернемся к модели системы «сооружениеоснование». Из сказанного выше следует, что безразмерный коэффициент демпфирования не является характеристикой основания, а характеризует конкретное сооружение на этом основании. Само же отдельно взятое основание характеризуется размерной вязкостью. Попытки приписать основанию безразмерный коэффициент демпфирования, чтобы усреднять его потом с коэффициентами демпфирования строительных конструкций с целью получения модальных коэффициентов демпфирования, принципиально ошибочны.

Корректная оценка вклада грунтовых демпферов в коэффициенты модального демпфирования не очень сложна; этот вклад для формы номер $j$ описывается формулой

$$
\lambda_{j}^{\text {soil }}=\frac{\sum_{k=1}^{6} b_{k}^{\text {soil }}\left[\Phi_{j k}^{\text {base }}\right]^{2}}{2 \omega_{j}}
$$

Здесь $b_{k}^{\text {soil }}$ - вязкость грунтового демпфера по направлению $k$; $\Phi_{\mathrm{jk}}^{\text {base }}$ - перемещение центра фундамента по направлению $k$ в собственной форме номер $j$ (формы нормируются по массе); $\omega_{j}-$ собственная частота формы $j$ в недемпфированной системе.

Вклад грунтовых демпферов в модальное демпфирование суммируется с вкладом строительных конструкций, но обычно на практике оказывается значительно выше.

Вязкое демпфирование применяется не только в «грунтовом подвесе», но и в части платформенной модели, моделирующей сооружение. Здесь наиболее распространенной является модель Рэлея [3]. Однако надо помнить, что модель Рэлея является принципиально нефизичной в той части, где демпфирование зависит от матрицы масс. Как следствие, при переходе к другой движущейся системе координат меняются силы трения (отсюда много недоразумений). На самом деле, нефизичность модели Рэлея - плата за попытку заменить физичное материальное демпфирование в конструкционных материалах, наблюдаемое в экспериментах, вязким демпфированием. Единственное преимущество вязкого демпфирования перед материальным демпфированием, заставляющее проводить такую подмену, - удобство вязкого демпфирования при численном интегрировании уравнений движения. Впрочем, для систем с однородным демпфированием (скажем, для бетонных зданий) модель Рэлея дает результаты, мало отличающиеся от результатов с частотно-независимым материальным демпфированием.

\section{3. НОРМАТИВНЫЕ ОГРАНИЧЕНИЯ}

В связи с важной ролью демпфирования в основании для сейсмической реакции сооружения это демпфирование было предме- 
том пристального внимания регуляторов в атомной области - одной из тех областей, где взаимодействие сооружений с основанием учитывалось в сейсмических расчетах с самого начала, т.е. с 1960-х годов.

На первом этапе ограничения накладывались на физическое демпфирование в системе. Формально это выражалось в ограничениях на коэффициенты относительного модального демпфирования. Так, в немецких нормах KTA для горизонтально-качательных форм был установлен лимит относительного демпфирования в 15\% критического, а для вертикальных форм этот лимит был установлен на уровне 30\%. Формулы же для однородного упругого полупространства, прописанные в нормах, приводили к намного большим значениям коэффициентов. Эти формулы давали физические вязкости грунтовых демпферов, а коэффициенты относительного модального демпфирования затем вычислялись с учетом свойств верхнего строения. На практике полученные таким образом коэффициенты демпфирования приходилось существенно урезать, чтобы выполнить ограничения, поставленные нормами. Особенно большими оказывались модальные коэффициенты демпфирования для вертикальных колебаний.

Такая ситуация стимулировала развитие альтернативных методов расчета, не использующих модальное разложение (а следовательно, не использующих и коэффициенты модального демпфирования, попадающие под нормативные ограничения). Эти исследования привели к появлению программы SASSI в разных ее вариантах. Международная программа натурных исследований на полигоне в Лотунге (Тайвань) доказала, что волновое демпфирование действительно существует в природе, а программа SASSI дает результаты, весьма близкие к реально зафиксированным при землетрясениях.

В современных нормах осталось два ограничения на демпфирование. Первое ограничение касается материального демпфирования - точнее, «кривых деградации», применяе- мых для эквивалентного линейного расчета слоистых грунтовых площадок. Запрещено применять значения материального демпфирования более $15 \%$. Однако на вязкостях стандартных грунтовых демпферов в платформенных моделях, даваемых формулами в нормах, это никак не сказывается - там материальное демпфирование вообще не учитывается. В первом приближении можно очень приближенно считать, что материальное демпфирование складывается с вязким в терминах безразмерных коэффициентов. Если, к примеру, материальное демпфирование в сооружении и в грунте составляет 4\%, а модальное демпфирование, рассчитанное с использованием вязкого грунтового демпфера из норм, составляет 14\%, то итоговое модальное демпфирование составит 18\%.

Второе ограничение по форме напоминает ограничения 1960-х годов - вводится лимит на модальные коэффициенты относительного демпфирования (обычно это ограничение составляет 20\%). Но смысл этого ограничения по сравнению с КТА принципиально другой. Превышение этого лимита означает не требование снижения физического демпфирования, как это было с КТА (путем урезания сразу модального коэффициента или путем урезания вязкости грунтовых демпферов таким образом, чтобы все модальные коэффициенты демпфирования попали в разрешенный диапазон). Нет, теперь такое превышение означает просто необходимость отказа от использования традиционного модального метода, основанного на собственных формах недемпфированной системы с полным разделением модальных реакций. Вместо такого подхода следует применять другие подходы - например, методологию SASSI, прямое интегрирование без разложения по собственным формам, а также другие варианты модального подхода - с использованием комплексных собственных значений или же с сохранением связи между модальными реакциями. Иными словами, признано, что классический модальный метод не способен дать достоверные результаты в зада- 
чах, где взаимодействие сооружений с основанием требует учета волнового демпфирования. Индикатором же такой ситуации выступает превышение диагональными элементами матрицы демпфирования в главных координатах неких пороговых уровнях, соответствующих относительному демпфированию в $20 \%$.

\section{4. РАСЧЕТ МОДЕЛЬНОЙ СИСТЕМЫ}

Автор провел исследования на простом примере одномерных колебаний трехмассовой системы (все три массы по 1 т), установленной на платформе с помощью простейшего грунтового подвеса из пружины и вязкого демпфера. Такая система допускает аналитическое вычисление передаточных функций от колебаний платформы к колебаниям масс. Она показана на рис.4 (без демпфирования).

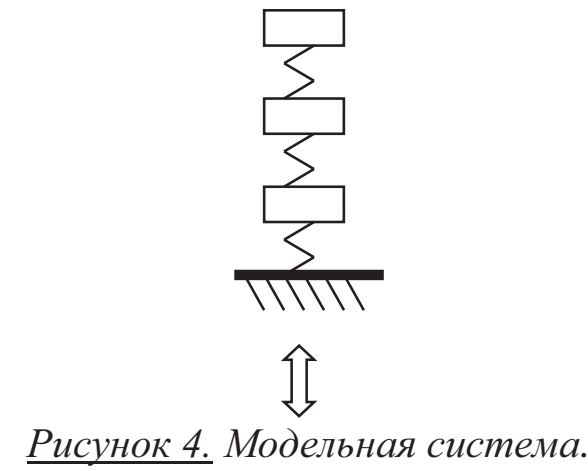

Параметры модельной системы без демпфирования приведены в Таблице 1 .

Демпфирование в «сооружении» задавалось по модели Рэлея. Вклад этого демпфирования в общую матрицу модального демпфирования в главных координатах был «почти диагональным»: квадраты внедиагональных элементов матрицы были много меньше произведения модулей соответствующих диагональных элементов.

Но вклад грунтового демпфера в общую матрицу демпфирования в главных координатах был, наоборот, «совсем недиагональным»: квадраты внедиагональных элементов в точности равнялись произведениям модулей диагональных элементов.

Сложение двух составляющих проиллюстрировано Таблицей 2.

При этом, как мы видим из таблицы 2, оказалось, что для двух первых форм вклад грунтового демпфера значительно превысил вклад «сооружения». В результате итоговая матрица демпфирования в главных координатах никак не может считаться «почти диагональной». Но как это повлияет на результаты?

Автор сравнил четыре варианта расчета. Первый вариант, который можно считать эталонным, вообще не использовал разложения по собственным формам. Демпфирование в грунтовой пружине задавалось чисто вязким, демпфирование в сооружении - чисто материальным. Вычислялись комплексные передаточные функции от колебаний платформы к колебаниям трех масс, а затем выводились их модули в зависимости от частоты.

Второй вариант расчета - «модальный с усеченным демпфированием». Матрица демпфирования в главных координатах была изменена в соответствии с традиционным модальным подходом, т.е. все ее недиагональные элементы были положены равными нулю. Диагональные элементы матрицы демпфирования тоже подверглись изменениям они были уменьшены настолько, чтобы относительное демпфирование составило 20\% (при этом уменьшать пришлось два первых элемента; третий элемент уменьшать не пришлось - он и так соответствовал критерию непревышения 20\%).

Третий вариант расчета - «полный модальный». Он повторяет второй вариант с той разницей, что диагональные элементы матрицы демпфирования были оставлены без изменений.

Наконец, четвертый вариант расчета соответствовал уменьшению физической вязкости грунтового демпфера в четыре раза. При этом если вычислить модальные коэффициенты демпфирования, то относительное 
Таблица 1. Параметры модельной системы.

\begin{tabular}{|c|c|c|c|c|c|}
\hline $\begin{array}{c}\text { Номер } \\
\text { снизу } \\
\text { вверх }\end{array}$ & $\begin{array}{c}\text { Жесткости } \\
\text { пружин, } \\
\text { кН/м }\end{array}$ & $\begin{array}{c}\text { Собственные } \\
\text { частоты, Гц }\end{array}$ & $\begin{array}{c}\text { Перемещение } \\
\text { нижней массы } \\
\text { в форме }\end{array}$ & $\begin{array}{c}\text { Перемещение } \\
\text { средней массы } \\
\text { в форме }\end{array}$ & $\begin{array}{c}\text { Перемещение } \\
\text { верхней массы } \\
\text { в форме }\end{array}$ \\
\hline 1 & $1,3 \mathrm{E} 3$ & 3,0025 & 0,4703 & 0,6069 & 0,64407 \\
\hline 2 & $3,25 \mathrm{E} 3$ & 11,297 & 0,8535 & $-0,1281$ & $-0,5051$ \\
\hline 3 & $6,75 \mathrm{E} 3$ & 20,073 & $-0,2245$ & 0,7844 & $-0,5782$ \\
\hline
\end{tabular}

Таблища 2. Сложение двух составляющих модального демпфирования.

\begin{tabular}{|c|c|c|c|}
\hline $\begin{array}{c}\text { Номер } \\
\text { формы }\end{array}$ & $\begin{array}{c}\lambda_{\mathrm{j} 0}, \% \\
(\text { бетон } 5 \%)\end{array}$ & $\mathrm{b}\left(\Phi_{\mathrm{jb}}\right)^{2} /\left(2 \omega_{\mathrm{j}}\right)$ & $\lambda_{\mathrm{j}}$ \\
\hline 1 & 0,9603 & 0,6478 & 0,65737 \\
\hline 2 & 4,0602 & 0,5670 & 0,60762 \\
\hline 3 & 4,9794 & 0,02208 & 0,071873 \\
\hline
\end{tabular}

\section{Модули передаточных функций к движению нижней массы}

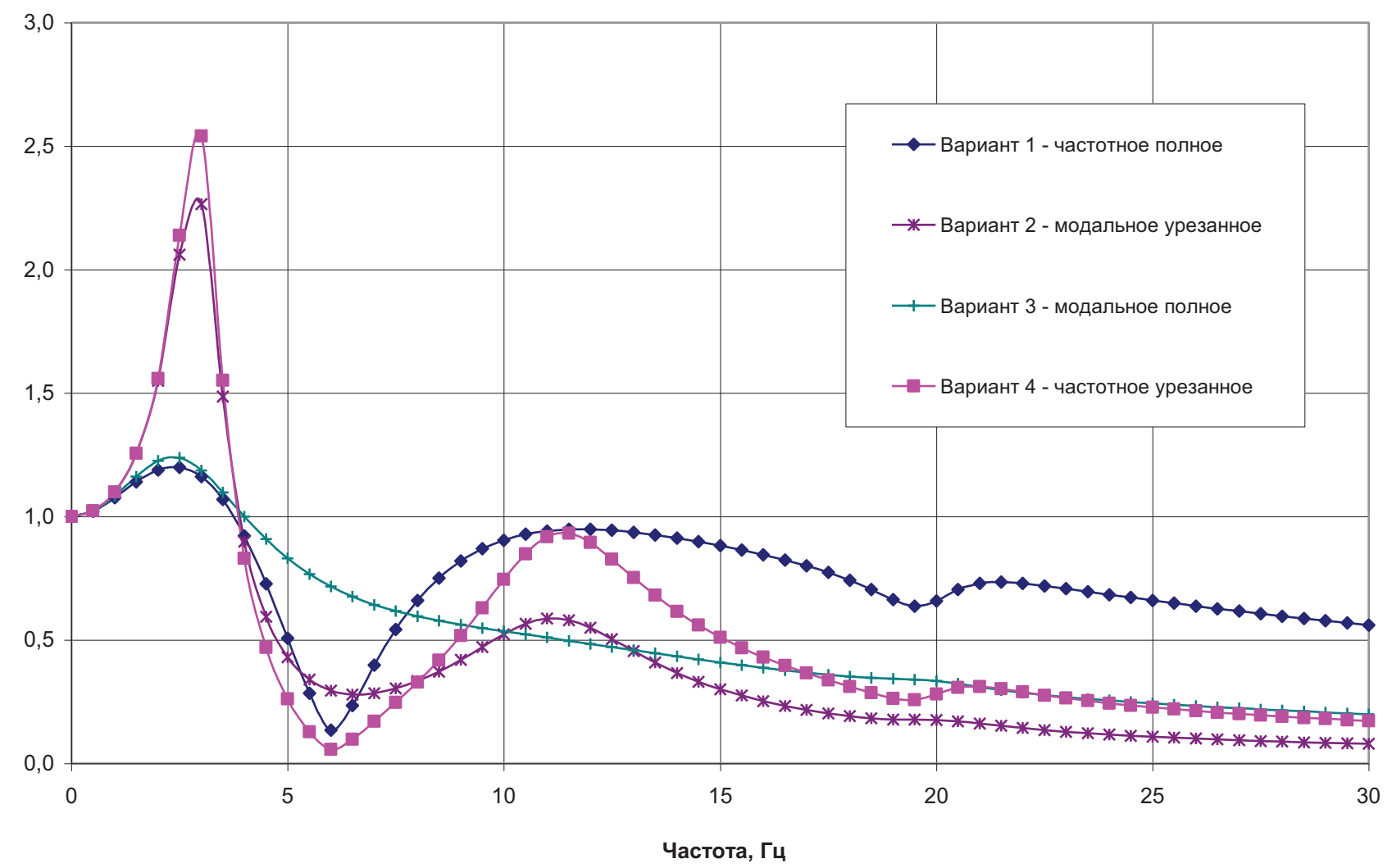

Рисунок 5. Сравнение модулей передаточных функиий для нижней массы.

демпфирование окажется в пределах 20\% даже для двух первых форм. Но разложение по формам при вычислении передаточных функций в этом варианте не применялось - в этом смысле четвертый вариант походил на первый.

Сравнение модулей передаточных функций для нижней массы показано на рис. 5. 
Анализ приведенных результатов показывает, что на низких частотах, включая область первого резонанса, все кривые ведут себя предсказуемо и «традиционно». Полный модальный подход в варианте 3 дает результаты, близкие к точным (вариант 1). Усечение демпфирования и в варианте усечения модальных коэффициентов (вариант 2), и в варианте усечения физической вязкости грунтового демпфера (вариант 4) приводит к возрастанию первого пика.

Однако при более высоких частотах проявляются интересные и неожиданные эффекты. Прежде всего, усечение демпфирования даже при одном и том же подходе к расчету (без разложения по собственным формам в вариантах 1 и 4 или с разложением по собственным формам в вариантах 2 и 3 ) уже не дает повышения модулей передаточных функций.

Кроме того, мы видим, что модальный метод (в варианте 3) дает неконсервативные результаты по сравнению с точными (вариант 1). Если сравнить между собой варианты 2 и 4 (похоже на сравнение вариантов 1 и 3, но при меньшем грунтовом демпфировании), там тоже присутствует неконсерватизм, но он меньше. Возможно, этим и объясняется выбор 20\% в качестве порога относительного демпфирования.

\section{5. КОМБИНИРОВАННЫЙ АСИМПТОТИЧЕСКИЙ МЕТОД (КАМ) КАК СПОСОБ СОХРАНЕНИЯ МОДАЛЬНОГО ПОДХОДА}

Чтобы сохранить возможность использования модального и спектрального подхода в расчетах платформенной модели системы «сооружение-основание» автор рекомендует применять комбинированный асимптотический метод (КАМ) $[1,2,4]$. Он имеет две опции.

В первой опции проводится модальный анализ модели сооружения на защемленном фундаменте. При этом определяются, в частности, модальные коэффициенты демпфиро- вания. Результаты модального анализа используются для получения матрицы «динамической инерции» - фактически для конденсации податливого верхнего строения к жесткому фундаменту. Импедансы и силовые нагрузки используются без искажений, и в результате определяется движение жесткого фундамента под сооружением. Затем проводится традиционный расчет (модальный метод вполне пригоден) сооружения на жестком фундаменте без грунтового основания с заданным движением фундамента. Новизна только в том, что это движение отличается от исходного движения свободной поверхности основания - в общем случае оно шестикомпонентное.

Вторая опция КАМ рассматривает платформенную модель с участием грунтового подвеса. Главный прием состоит в том, что создается специальный «платформенный» грунтовый подвес, отличающийся от исходного «грунтового» подвеса. В частности, он может быть представлен винклеровскими пружинами и распределенными демпферами. Демпфирование искусственно задается таким же, как в сооружении. В частности, при использовании для сооружения модели Рэлея для вязкости платформенных демпферов используется тот же коэффициент $\beta$, что и для сооружения. В итоге система оказывается однородной по демпфированию и может рассчитываться модальным методом. Интегральные динамические жесткости «платформенного» подвеса, конечно, отличаются от интегральных жесткостей исходного «грунтового» подвеса. Эта разница рассчитывается и учитывается при модификации движения платформы. Движение платформы определяется таким образом, чтобы движение фундамента соответствовало результатам расчета из первой опции КАМ.

\section{6. ВЫВОДЫ}

Перейдем к выводам. В платформенной модели системы «сооружение-основание» вяз- 
кое демпфирование может использоваться как для моделирования демпфирования в основании, так и для моделирования демпфирования в сооружении. Для моделирования волнового демпфирования в основании в простейшем случае однородного полупространства вязкое демпфирование физически подходит неплохо. Для моделирования демпфирования в сооружении вязкое демпфирование подходит меньше (модель Рэлея имеет существенные недостатки). Ограничения в настоящее время накладываются на относительное модальное демпфирование, но смысл этих ограничений - не призыв урезать демпфирование (как это было сорок лет назад), а призыв осторожно относиться к использованию модального метода в системах с неоднородным демпфированием. В то же время существует способ сохранить модальный метод и для таких систем - это комбинированный асимптотический метод $[1,4]$.

\section{СПИСОК ЛИТЕРАТУРЫ}

1. Тяпин А.Г. Платформенные модели в задачах учета взаимодействия сооружений с основанием при расчетах на сейсмические воздействия: Научное издание. - М.: АСB, 2015. - 208 c.

2. Тяпин А.Г. Расчет сооружений на сейсмические воздействия с учетом взаимодействия с грунтовым основанием. Научное издание. - М.: АСB, 2013. - 392 с.

3. Seismic Analysis of Safety-Related Nuclear Structures and Commentary. ASCE4-98. Reston, Virginia, USA. 1999.

4. Тяпин А.Г. Учет взаимодействия сооружений с основанием при расчетах на сейсмические воздействия. Руководство по расчетам. - М.: АСВ, 2014. - 136 с.

Тяпин Александр Георгиевич, доктор технических наук; главный специалист АО «Атомэнергопроект», Россия, 121352, Москва, Славянский бульвар, 1-119, тел.+7(985)998-33-21, e-mail: atyapin @bvcp.ru.
Alexander G. Tyapin, Doctor of Technical Sciences, Chief Specialist; JSC "Atomenergoproject"; 1-119, Slavyanskyi Bulvar, Moscow, 121352, Russia; tel. +7(985)998-33-21, e-mail: atyapin@bvcp.ru. 
DOI:10.22337/1524-5845-2016-12-4-163-175

\title{
ЭКСПЕРИМЕНТАЛЬНЫЙ СТЕНД \\ ДЛЯ ИССЛЕДОВАНИЯ ОСОБЕННОСТЕЙ ПРЕДКРИТИЧЕСКИХ И КРИТИЧЕСКИХ ДЕФОРМАЦИОННЫХ ПРОЦЕССОВ В ЖЕЛЕЗОБЕТОННЫХ КОНСТРУКЦИЯХ (МАТЕМАТИЧЕСКОЕ МОДЕЛИРОВАНИЕ, СТРУКТУРНАЯ СХЕМА)
}

\author{
И.Н. Шардаков, А.П. Шестаков ${ }^{1}$ Г.Н. Гусев ${ }^{1}$ М.Л. Бартоломей ${ }^{2}$, \\ Г.Г. Кашеварова ${ }^{2}$ Р.В. Цветков ${ }^{1}$ И.О. Глот 1 \\ ${ }^{1}$ Институт механики сплошных сред Уральского отделения Российской академии наук \\ (ИМСС УрО РАН), г. Пермь, РОССИЯ, \\ ${ }^{2}$ Пермский государственный национальный исследовательский университет, г. Пермь, РОССИЯ
}

\begin{abstract}
Аннотация: В работе представлены результаты математического моделирования экспериментального стенда для исследования деформационных процессов в крупномасштабных моделях строительных сооружений. Особое внимание уделено изучению предвестников разрушения модельной конструкции и ее поведению в состояниях, близких к критическим. В серии численных экспериментов изучены сценарии возникновения и развития зон разрушения в основных структурных элементах модели. Оценены масштабы и особенности конструкции испытательного стенда, варианты задания нагрузок и сценарии их приложения, точность дозирования нагрузок и способы регистрации деформационных процессов.
\end{abstract}

Ключевые слова: железобетонные конструкции, деформационные процессы, разрушение, испытательный стенд, крупномасштабная модель, математическое моделирование

\section{EXPERIMENTAL STAND FOR THE STUDY OF FEATURES OF SUBCRITICAL AND CRITICAL DEFORMATION PROCESSES IN REINFORCED CONCRETE STRUCTURES (MATHEMATICAL MODELING, STRUCTURAL SCHEME)}

\author{
Igor N. Shardakov', Alexey P. Shesnakov ${ }^{I}$, Georgiy N. Gusev ${ }^{I}$, \\ Maria L. Bartolomey², Galina G. Kashevarova', Roman V. Tsvetkov', \\ Irina O. Glot ${ }^{1}$ \\ ${ }^{1}$ Institute of Continuous Media Mechanics of the Ural Branch of Russian Academy of Science \\ (ICMM UrB RAS), Perm, RUSSIA \\ ${ }^{2}$ Perm State Research Polytechnic University, Perm, RUSSIA
}

\begin{abstract}
The paper presents the results of mathematical modeling of an experimental setup for studying deformation processes in the large-scale models of construction structures. Particular attention is given to investigation of fracture precursors in a model structure and its behavior in a near-critical state. Scenario for generation and evolution of fracture zones in the main structural elements of the model are considered in a number of numerical experiments. The analysis of the results allowed us to evaluate the sizes and peculiarities of a testing setup design, types of loads and load application scenario, accuracy of loading frequency and techniques for registering deformation processes.
\end{abstract}

Keywords: reinforced concrete structure, deformation processes, destruction, experimental setup, large-scale model, mathematical modeling 


\section{1. ВВЕДЕНИЕ}

Обеспечение безопасной эксплуатация зданий и сооружений из железобетона имеет большое значение. Для таких конструкций вопрос адекватного моделирования деформационных процессов в предкритических и критических состояниях является проблемным. Критические состояния в сооружениях реализуются по сложному пространственновременному сценарию. Это обусловлено большой совокупностью неоднородных элементов в сооружении, сложностью их взаимосвязи, а также структурными особенностями самого железобетона. Исследованию деформационных процессов в отдельных элементах конструкций, например, в балках, посвящено огромное количество работ. Однако информации по испытаниям отдельных элементов оказывается не достаточно для создания адекватной модели сложной конструкции в целом. Для достижения этой цели необходимы результаты натурного физического эксперимента на полномасштабной модели, которая представляет собой железобетонную конструкцию, состоящую из характерных структурных единиц (несколько этажей по высоте и несколько характерных блоков в горизонтальной плоскости). Для реализации таких экспериментов необходим стенд соответствующего масштаба, позволяющий осуществлять, наблюдать и регистрировать предкритические и критические деформационные состояния.

В мире существует немного лабораторий, где возможно проведение экспериментов на полномасштабных конструкциях. Например, в Италии расположена European Laboratory for Structural Assessment (ELSA) [1]. Наибольший интерес представляют исследования [2-4] сейсмических характеристик крупных фрагментов конструкций. Это связано с проблемами эксплуатации конструкций в районах сейсмической активности. Описание статических испытаний полномасштабных конструкций в литературе встречаются значительно реже. Например, в работе [5] описаны эксперименты по нагружению модели одноэтажного здания в масштабе $1: 2$.

В настоящее время в рамках проекта РНФ № 14-29-00172 в Институте механики сплошных сред УрО РАН создается экспериментальный стенд для испытания полномасштабных строительных конструкций, который позволит проводить испытания объектов из железобетона или металла в режимах квазистатического и динамического нагружения. В структуре стенда предусмотрена возможность моделирования силового и кинематического способов нагружения исследуемого объекта, что позволяет передать особенности приложения распределенных нагрузок в различных зонах объекта или неравномерность осадок его основания. Стенд позволяет подробно исследовать деформационные процессы, реализующиеся в модельной конструкции в состояниях, предшествующих критическим, фиксировать предвестники разрушения и наблюдать зарождение и развитие процессов разрушения в пространстве и времени. Регистрация этих процессов позволит получить информацию для уточнения математических моделей деформационного поведения сборно-монолитных и монолитных железобетонных конструкций. Эксперимент на крупномасштабной модели, передающей характерные особенности взаимодействии всех ее элементов, позволяет получить адекватную картину разрушения и его предвестников. Установлено, что именно такой масштаб эксперимента может обеспечить достоверность и адекватность полученных закономерностей для реальных сооружений. Это особенно справедливо в отношении исследования бетонных конструкций, поскольку в этом случае важно в модельном эксперименте сохранить реальные соотношения линейных масштабов характерных размеров: габаритов конструкции, поперечных размеров арматуры и фракций бетона. Реализация такого эксперимента позволит получить уникальные данные о прочности, надежности и долговечности железобетон- 
Экспериментальный стенд для исследования особенностей предкритических и критических деформационных процессов в железобетонных конструкциях (математическое моделирование, структурная схема)

ных конструкций в условиях статических и динамических нагрузок, а также обеспечить достоверность прогноза безопасной эксплуатации сложных инженерных и строительных сооружений.

Масштаб, габариты и функциональные особенности испытательного стенда были определены в результате математического моделирования деформационного поведения исследуемой конструкции и ее элементов от воздействия внешних силовых и кинематических факторов. В математической модели отображен процесс деформирования железобетонной конструкции, развивающийся от ее упругого состояния до полного разрушения. Анализ полученных численных результатов послужил основой для разработки структурной схемы испытательного стенда и позволил оценить его основные параметры и рабочие характеристики.

\section{2. МАТЕМАТИЧЕСКАЯ МОДЕЛЬ МОДЕЛЬНОЙ ЖЕЛЕЗОБЕТОННОЙ КОНСТРУКЦИИ}

В качестве исследуемой модельной конструкции выбран фрагмент четырехэтажного здания в масштабе 1:2. Модельный объект включает в себя 24 типовых ячейки сооружения, объединенные в 4 этажа. Общая высота модели составляет 6м, длина 9м, и ширина 6м. По всем трем направлениям присутствует фактор многоэлементности, что позволяет адекватно передать характер пространственного распределения процесса деформирования, особенно на этапах предкритического и критического состояния конструкции. Следует также отметить, что выбранный масштаб модельной конструкции позволяет при описании физикомеханических свойств ее элементов использовать сведения о свойствах типового бетона с известным соотношением размеров его фракций. Уменьшение масштаба модели при сохранении в качестве конструкционного материала типового бетона может привести к существенному искажению характера разрушения.

На рисунке 1 представлены модельная конструкция в целом и ее основные структурные элементы. Для каждого из этих фрагментов и конструкции в целом осуществлено моделирование деформационного процесса от упругого состояния до разрушения и потери несущей способности. Расчетные схемы для каждой задачи также показаны на рисунке 1. Квазистатическое нагружение осуществлялось кинематическим способом (за счет заданного перемещения отдельных частей конструкций).

Математическое моделирование деформационного процесса, включающего этап трещинообразования, осуществлено в рамках феноменологических подходов механики деформированного твердого тела. Процесс трещинообразования в бетоне моделировался как резкое снижение жесткости материала в локализованной области. Направление распространения локализованной области с пониженной жесткостью, место расположения этой области, а также закономерности снижения жесткостных характеристик материала определялись на основе критериальных тензорных соотношений.

Деформационный процесс в металлической арматуре моделировался в рамках теории упругопластичности [6].

Для математической постановки задачи используется принцип возможных перемещений [7]. Вариация работы внешних и внутренних сил для структурных элементов из железобетона представляется уравнением

$$
\delta A_{\sigma}^{c}+\delta A_{\sigma}^{r}=\delta A_{f}^{c}+\delta A_{f}^{r},
$$

где: $\delta A_{\sigma}-$ вариации работ внутренних сил; $\delta A_{f}$ - вариации работ внешних сил; верхние индексы $c$ и $r$ обозначают принадлежность к бетону и арматуре соответственно.

Для структурных элементов из бетона вариации работ внутренних и внешних сил имеют вид: 

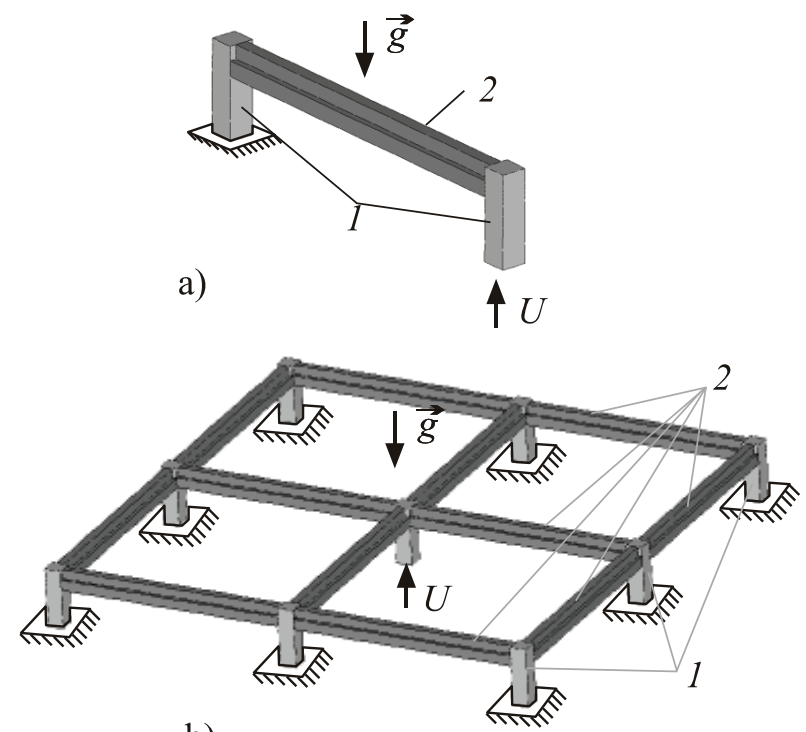

b)

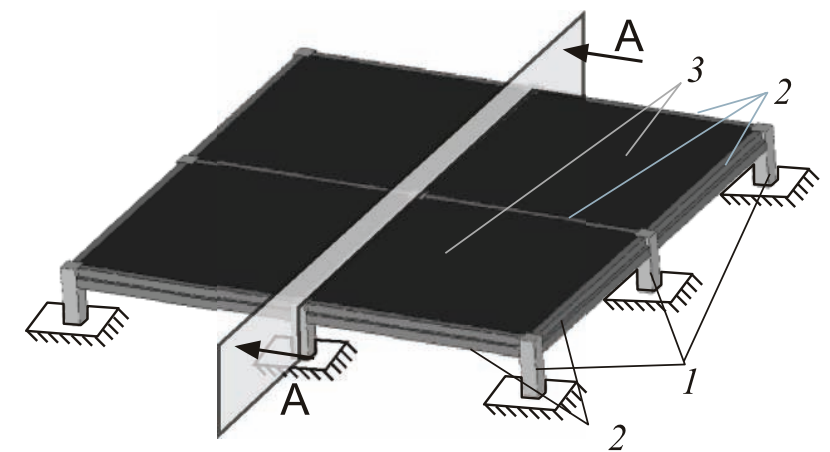

A-A

c)

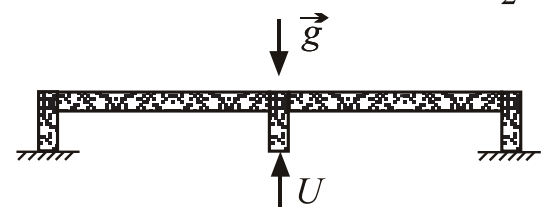

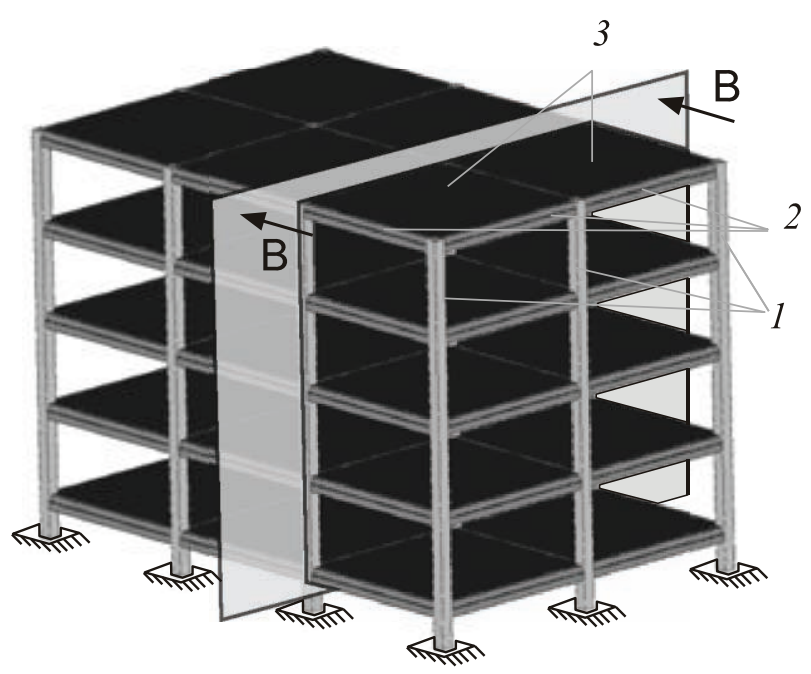

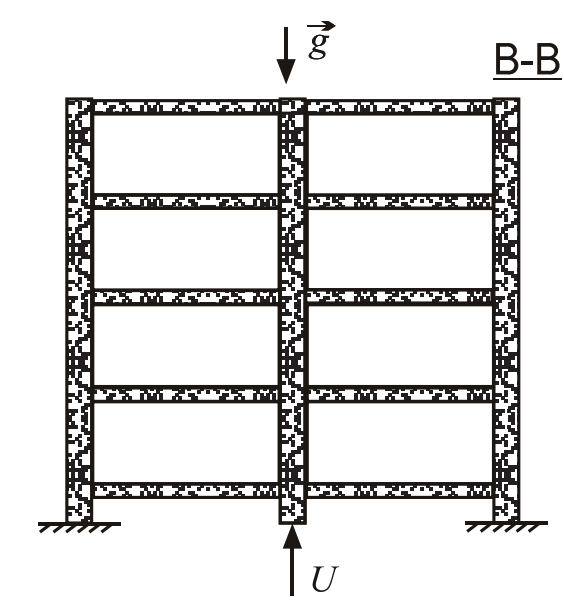

Рисунок 1. Расчетные схемы задач для основных структурных элементов модельной конструкиии: «2 колоннь - ригель» (a), «9 колонн - 12 ригелей» (b), «9 колонн -12 ригелей4 плитьл (c), модельная конструкция в сборе $(d) ; 1$ - колонныl, 2 - ригели, 3 - плиты перекрытий, $U$-заданное вертикальное смещение колонны, $g$ - направление действия собственного веса конструкиии.

$\delta A_{\sigma}^{c}=\int_{V^{c}} \sigma_{i j}^{c} \cdot \delta \varepsilon_{i j}^{c} d V, \quad \delta A_{f}^{c}=-\int_{V^{c}} \rho^{c} g_{i} \delta u_{i}^{c} d V$

Здесь $i, j$ - целочисленные индексы, принимающие значения $1,2,3$, в соответствии с осями декартовой системы координат $\left(x_{1}, x_{2}, x_{3}\right) ; u_{i}$ - компоненты вектора перемещений; $\varepsilon_{i j}, \sigma_{i j}$ - компоненты тензоров деформаций и напряжений; $g_{i}-$ компоненты вектора ускорения свободного падения, $\rho-$ плотность материала.
Компоненты тензора деформаций определяются соотношениями

$$
\varepsilon_{i j}^{c}=\frac{1}{2}\left(\frac{\partial u_{i}^{c}}{\partial x_{j}}+\frac{\partial u_{j}^{c}}{\partial x_{i}}\right)
$$

Напряженно-деформированное состояние бетона моделируется следующим образом: 
Экспериментальный стенд для исследования особенностей предкритических и критических деформационных процессов в железобетонных конструкциях (математическое моделирование, структурная схема)

1) Исходное начальное состояние описывается законом Гука для упругого изотропного материала [8]:

$$
\sigma_{i j}^{c}=\frac{E^{c}}{1+v^{c}} \varepsilon_{i j}^{c}+\frac{E^{c} v^{c}}{\left(1+v^{c}\right)\left(1-2 v^{c}\right)} \varepsilon_{k k}^{c} \delta_{i j}
$$

где модуль $E^{c}$ задается отношением $\sigma_{e} / \varepsilon_{e}$ $\left(\sigma_{e}, \varepsilon_{e}-\right.$ эквивалентные по Мизесу напряжение и деформация).

Для бетона зависимость $\sigma_{e}=f^{c}\left(\varepsilon_{e}\right)$ достаточно хорошо описывается непрерывной аппроксимацией, задаваемой тремя линейными участками. В таблице 1 приведены значения $\sigma_{e}, \varepsilon_{e}$ для точек сопряжения этих участков $\sigma_{e}=f^{c}\left(\varepsilon_{e}\right)$. Зависимость (4) будет справедлива, пока главная максимальная деформация остается меньше предельного значения: $\varepsilon_{1}<\varepsilon_{m p}$.

Таблица 1. Параметры определяющих соотношений для бетона

\begin{tabular}{|c|c|c|c|c|}
\hline$\varepsilon_{e} \times 10^{3}$ & 0 & 0,52 & 2,00 & 3,50 \\
\hline$\sigma_{e} \mathrm{MПа}$ & 0 & 16,7 & 27,0 & 28,5 \\
\hline
\end{tabular}

2) Если в какой-либо точке, принадлежащей бетону, выполняется условие $\varepsilon_{1}>\varepsilon_{m p}$, то в окрестности этой точки по направлению вектора $e_{1}$, определяющего направление первой главной деформации $\varepsilon_{1}$, модуль упругости «скачкообразно» снизит свое значение, таким образом моделируется появление трещины. Вследствие такого изменения модуля материал в указанной окрестности становится анизотропным. Величина этого модуля упругости $E_{m p}$ по направлению вектора $e_{1}$ определяется как тангенс угла наклона между секущей и осью $\varepsilon_{e 1}$ на интервале $\varepsilon_{1} \geq \varepsilon_{m p}$ : $E_{m p}=\operatorname{tg}\left(\alpha\left(\varepsilon_{1}\right)\right)$ (Рис. 2). Модуль сдвига на площадке с нормалью $e_{1}$ умножаются на ко- эффициент $\beta_{t}=0,2$, или $\beta_{c}=0,9$, соответственно, для закрытой или открытой трещины. Величины, характеризующие зависимость, представленную на рисунке 2 , для бетона будут иметь значения $\sigma_{m p}=3,74 \mathrm{MПа} \mathrm{и}$ $\varphi=0,6$.

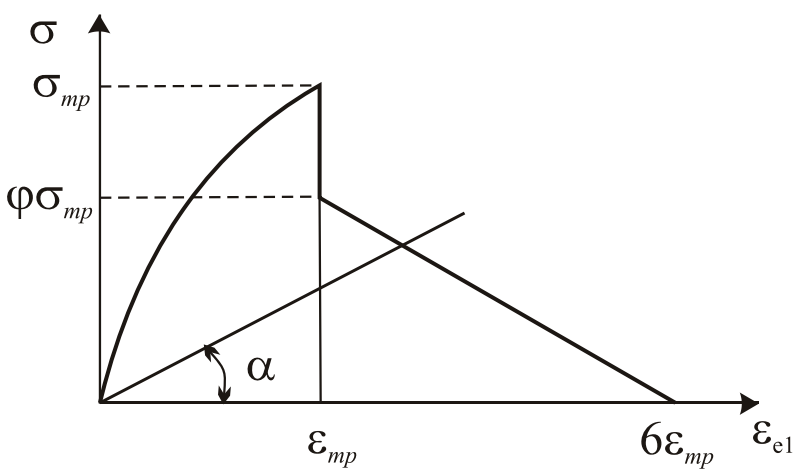

Рисунок 2. К заданию определяющих соотношений для бетона

При численной реализации данного подхода с использованием метода конечных элементов окрестностью, в пределах которой происходит изменение модуля упругости, будет являться соответствующий объем конечного элемента.

3) После образования первой трещины выполняется проверка на возможность образования второй трещины. Для этого определяется максимальное растягивающее напряжение в направлении $e_{2}$, ортогональном $e_{1}$ :

$$
\varepsilon \cdot e_{2} \cdot e_{2}=\varepsilon_{m p 2} \rightarrow \max
$$

Если величина

$$
\varepsilon_{m p 2}>\varepsilon_{m p}
$$

то в направлении $e_{2}$ образуется вторая трещина. Модули упругости в этом направлении изменятся аналогично тому, как это происходит при появлении первой трещины.

4) Так как, согласно [6], образующиеся в окрестности одной точки трещины должны быть ортогональны друг другу, то направле- 
ние третей трещины определяется условием $e_{3}=e_{1} \times e_{2}$. Если величина растягивающей деформации вдоль этого направления больше предельного значения

$$
\varepsilon_{m p 3}=\varepsilon \cdot e_{3} \cdot e_{3}>\varepsilon_{m p},
$$

то образуется трещина в третьем направлении. Детально с данным подходом к описанию возникновения и развития трещин в бетоне можно ознакомиться в работе [8].

Арматура моделируется в рамках балочной теории. Положение осевых линий арматуры определяется локальными координатами $\xi_{1}, \xi_{2}, \xi_{3}$. Для элементов стальной арматуры слагаемые вариационного уравнения записываются в виде:

$$
\begin{gathered}
\delta A_{\sigma}^{r}=\int_{L^{r}}\left(M_{i}^{r} \cdot \delta \chi_{i}^{r}+S^{r} \cdot \sigma^{r} \cdot \delta \varepsilon^{r}\right) \mathrm{d} l, \\
\delta A_{f}^{r}=-\int_{L^{r}} S^{r} \cdot \rho^{r} \cdot g_{\xi_{i}} \cdot \delta u_{\xi_{i}}^{r} \mathrm{~d} l \\
\chi^{a}=\left\{\begin{array}{c}
\frac{\partial^{2} u_{\xi_{2}}^{a}}{\partial\left(\xi_{1}\right)^{2}} \\
\frac{\partial^{2} u_{\xi_{3}}^{a}}{\partial\left(\xi_{1}\right)^{2}}
\end{array}\right\}, \quad \mathbf{M}^{a}=E^{a} \cdot\left\{\begin{array}{c}
J_{\xi_{2}}^{a} \\
J_{\xi_{3}}^{a}
\end{array}\right\} \cdot \chi^{a}, \\
\varepsilon^{a}=\frac{\partial u_{\xi_{1}}^{a}}{\partial \xi_{1}} .
\end{gathered}
$$

Здесь: $S^{r}$ - площадь поперечного сечения элемента арматуры; $J_{\xi_{2}}^{r}, J_{\xi_{3}}^{r}-$ моменты инерции поперечного сечения арматуры относительно локальных осей $\xi_{2}, \xi_{3}$ Связь напряжений и деформаций определяется в рамках теории течения [10]. Для одноосного деформирования вдоль осевых линий арматуры, в соответствии с балочной теорией, справедливо соотношение:

$$
d \sigma^{r}=\left\{\begin{array}{cc}
E(0) \cdot d \varepsilon^{r}, & |\sigma|<\sigma^{*}, \\
E(|\sigma|) \cdot d \varepsilon^{r}, & |\sigma| \geq \sigma^{*}
\end{array}\right.
$$

где: $d \sigma^{r}-$ приращение напряжений; $d \varepsilon^{r}-$ приращение деформаций; $\sigma^{*}$ - максимальное по модулю напряжение, которое испытывает материал в зоне упругого деформирования; $E(0)$ - модуль в зоне упругого деформирования;

$$
E^{r}(\sigma)=1 / \frac{\partial f(\sigma)}{\partial \sigma} \text { для }|\sigma| \geq \sigma^{*} ;
$$

$\varepsilon=f^{r}(\sigma)$ - зависимость, полученная из эксперимента на одноосное растяжение. Для арматуры эта функция задается в таблице 2.

Таблица 2. Параметры определяющих соотношений для арматурь.

\begin{tabular}{|c|c|c|c|c|c|}
\hline$\varepsilon \times 10^{3}$ & 0 & 2,1 & 4,3 & 30,0 & 153,0 \\
\hline$\sigma$ МПа & 0 & 350 & 389 & 506 & 590 \\
\hline
\end{tabular}

В соответствии с принципом возможных перемещений, на варьируемые поля перемещений накладываются ограничения, определяющие их непрерывность во всех областях элементов системы (бетон, арматура), а также на границах их контакта. Варьируемые поля перемещений должны также удовлетворять кинематическим граничным условиям, определяющим кинематическое нагружение. На всех остальных внешних поверхностях элементов системы будут выполняться естественные краевые условия, соответствующие принципу возможных перемещений.

Численное решение вариационного уравнения осуществлялось методом конечных элементов с использованием программного комплекса ANSYS. Полученная в результате применения метода конечных элементов алгебраическая система уравнений является существенно нелинейной, для ее решения используется итерационный метод НьютонаРафсона [11]. 
Экспериментальный стенд для исследования особенностей предкритических и критических деформационных процессов в железобетонных конструкциях (математическое моделирование, структурная схема)

\section{3. РЕЗУЛЬТАТЫ ЧИСЛЕННОГО АНАЛИЗА}

\section{Система "2 колонны - ригель»}

Ригели соединяют колонны, образуя каркас здания. При неравномерной осадке колонн ригель испытывает изгиб, и в нем появляются зоны растяжения и сжатия. В зонах растяжения реализуется разрушение бетона в виде трещин, а растягивающее усилие воспринимает только арматура. Дальнейшее увеличение неравномерной осадки вызывает либо разрыв растянутой арматуры, либо разрушение бетона в зоне сжатия, что приводит к существенному снижению жесткости, а иногда и к полному разрушению конструкции. Самая простая система, отражающая этот механизм, состоит из двух колонн и одного ригеля (Рис. 1a).

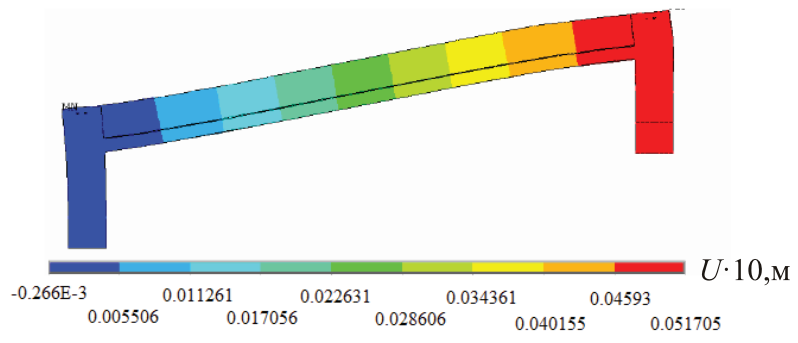

Рисунок 3. Деформированная конфигурачия системы «2 колонны - ригель» при относительном вертикальном смещении основания одной из колонн.

Нагружение системы происходит за счет вертикального перемещения $(U)$ основания правой колонны при закрепленном основании левой (Рис. 3). Моделирование деформационного процесса в такой конструкции позволило получить зависимость равнодействующей реакции на торце правой колонны вдоль её оси от перемещения основания колонны (Рис. 4a). Цифрами на графике отображены ключевые точки деформирования. Исходное состояние (конструкция под собственным весом) $1(U=0$ мм $, F=1,9 \kappa H)$. Линейное увеличение перемещений до точки $2(U=1,2 м м, F=3,7 \kappa H) \quad$ происходит без разрушений. В последующих точках $3(U=1,6 м м, F=4,0 \kappa H)$

$4(U=2,0 м м, F=4,1 \kappa H)$ происходит образование и развитие трещин в растянутых зонах бетона. В точке $5(U=3,6$ мм, $F=4,5 \kappa H)$ эти зоны существенно увеличиваются. Дальнейшее смещение колонны приводит к зарождению (точка $6(U=38,9$ мм $, F=8,2 \kappa H))$ и разрастанию (точка $7(U=38,9 \mathrm{MM}, F=8,2 \kappa H)$ ) области разрушения в сжатой зоне бетона в правой нижней части ригеля. Разрушения во второй зоне сжатия, находящейся в левой верхней части ригеля, начинается в точке $8(U=44,4 м \mathcal{M}, F=8,6 \kappa H) . \quad$ Дальнейшее увеличение перемещения приводит к снижению усилия в колонне до точки $9(U=49,7$ мм, $F=7,8 \kappa H)$ и последующей утрате несущей способности конструкции.

На рисунке $4 \mathrm{~b}$ контрастным цветом показаны области разрушения, соответствующие ключевым точкам нагружения. Фрагменты 3 , 4, 5 демонстрируют зоны разрушения, вызванного растяжением, а фрагменты 5, 7, 8, 9 - зоны разрушения, вызванного сжатием.

\section{Система «9 колонн-12 ригелей»}

Следующим по сложности конструктивным элементом является система из 9 колонн и 12 ригелей (Рис. 1b). Она отражает совместную работу ригелей и колонн первого этажа здания при вертикальном перемещении основания центральной колонны. На рисунке 5 показана конфигурация элементов системы, сформированная при таком деформировании.

Рисунок 6 представляет полученную расчетным путем зависимость усилия в центральной колонне от заданного перемещения её основания. Процесс разрушения ригелей развивается аналогично тому, как было показано в предыдущем случае. Отмеченным на рисунке 6 точкам соответствуют следующие особенности деформирования системы: точка $1(U=0, F=6,0 \kappa H)$ - система под собственным весом; 
И.Н. Шардаков, А.П. Шестаков, Г.Н. Гусев, М.Л. Бартоломей, Г.Г. Кашеварова, Р.В. Цветков, И.О. Глот

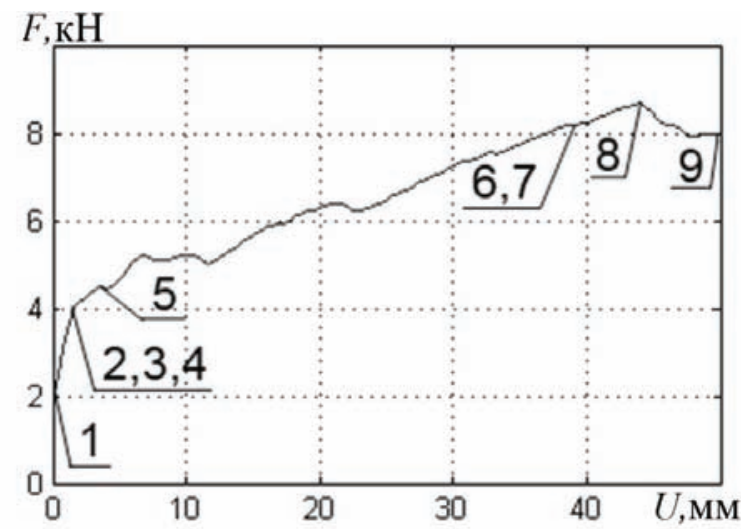

a)

$\underline{2}$

$\underline{3}$

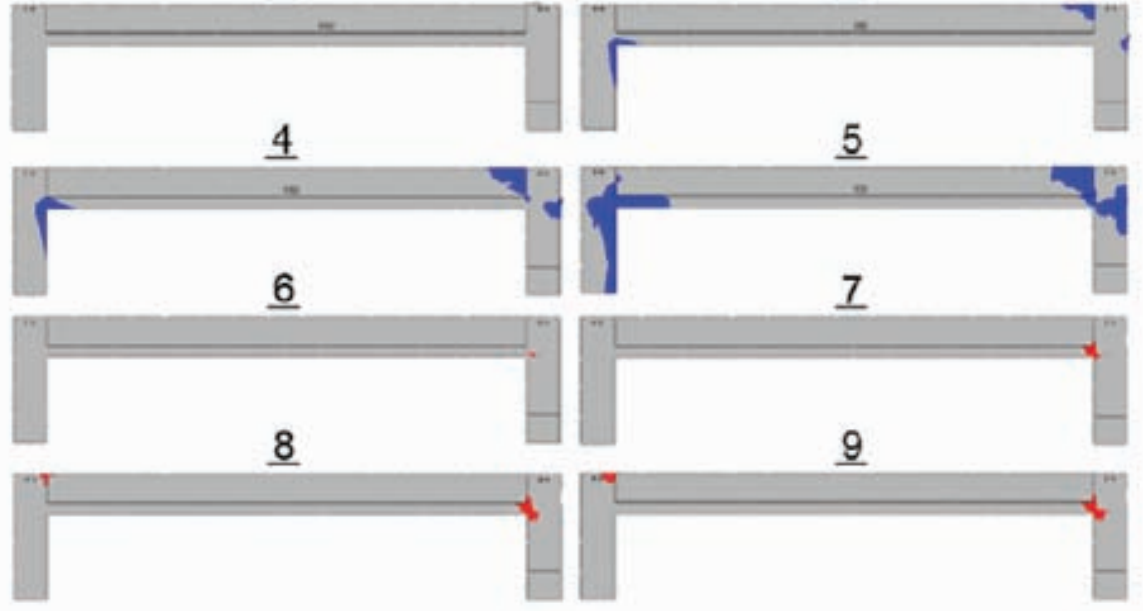

b)

Рисунок 4. Результаты моделирования деформационного прочесса в системе «2 колонны ригель» при неравномерной осадке: а-изменение усилия в колонне от заданного перемещения (цифрами отмечень ключевые точки деформирования); $b$ - области разрушения бетона в зонах сжатия (3-5) и растяжения (6-9).

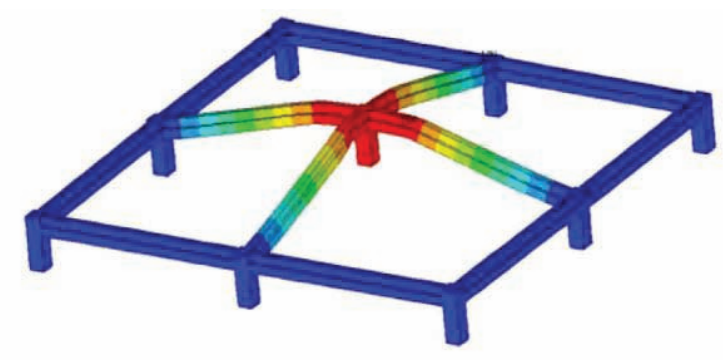

$\begin{array}{rrrrrrrrrr}-0.001131 & 0.006292 & 0.013715 & 0.021139 & 0.028562 & 0.035098 & 0.043409 & 0.050832 & 0.058236 & 0.065679 \\ U\end{array}$

Рисунок 5. Деформированная конфигурачия системы «9 колонн - 12 ригелей» при относительном вертикальном смещении центральной колонньл.

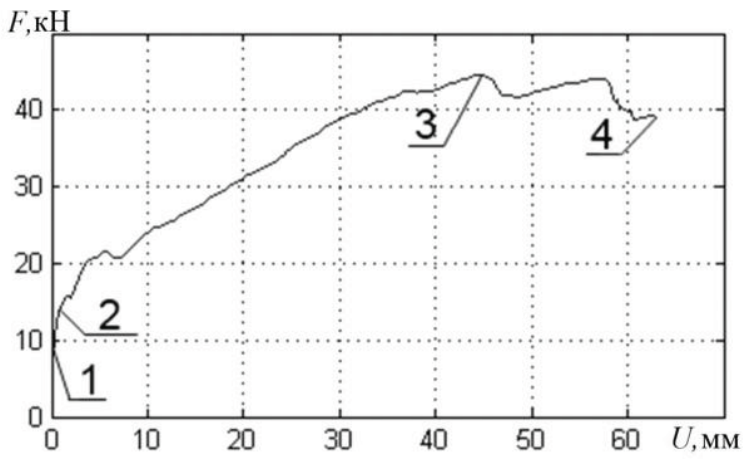

Рисунок 6. Изменение усилия в колонне от заданного перемещения ее основания (цифрами отмечены ключевые точки деформирования. 
Экспериментальный стенд для исследования особенностей предкритических и критических деформационных процессов в железобетонных конструкциях (математическое моделирование, структурная схема)

$2(U=0,8 м м, F=10,7 \kappa H)$ - начало разрушения бетона в растянутой зоне ригелей; 3 $(U=45,0$ мм, $F=44,6 \kappa H)$ - максимальное усилие, соответствующее началу разрушения в сжатой зоне ригелей; $4(U=62,9$ мм, $F=39,2 \kappa H)-$ потеря несущей способности.

\section{Система «9 колонн - 12 ригелей - 4 плиты»}

Вклад плит в работу конструкции демонстрирует система «9 колонн - 12 ригелей -4 плиты» (Рис. 1с). Эта система отличается от предыдущей наличием четырех плит перекрытия. Деформированная конфигурация системы при вертикальном смещении основания центральной колонны представлена на рисунке 7.

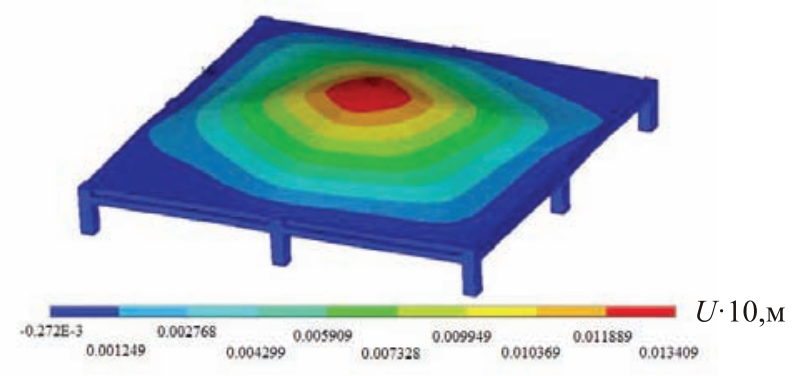

Рисунок 7. Деформированная конфигураичи системы «9 колонн - 12 ригелей -4 плиты»» при относительном вертикальном смещении ицентральной колонны.

Зависимость усилия в центральной колонне от заданного перемещения ее основания приведена на рисунке 8а. Ключевым точкам, отмеченным на графике, соответствуют следующие особенности деформирования системы: точка 1 ( $U=0, F=34,1 \kappa H)$ - система под собственным весом; $2(U=0,5 м \mu, F=69,5 \kappa H)-$ растрескивание стыков плит и ригелей и одновременное разрушение бетона в растянутой зоне; $3(U=3,1$ мм, $F=126,8 \kappa H)$ - достижение максимального усилия, с этой точки начинается процесс растрескивания плит; $4(U=4,7$ мм, $F=110,5 \kappa H) \quad-\quad$ основной процесс разрушения плит закончен;
$5(U=13,3 м м, F=107,8 \kappa H)$ - разрушение сжатой зоны ригелей и потеря несущей способности. На рисунке $8 \mathrm{~b}$, представляющем вид на конструкцию сверху, области разрушения плит перекрытия показаны черным цветом. По сравнению с аналогичной системой без плит, максимальное усилие на колонну увеличилось в 2,8 раза, при этом перемещение колонн в момент достижения максимального усилия уменьшилось в 14 раз. Это говорит о существенном влиянии плит на процесс деформирования и разрушения здания.

\section{Четырехэтажнная конструкция}

Деформирование конструкции осуществляется за счет вертикального смещения основания одной из внутренних колонн относительно остальных неподвижных (Рис. 1d).

По результатам численного моделирования получена деформированная конфигурация конструкции (Рис. 9а) и зависимость осевого усилия в основании этой колонны от заданного смещения (Рис. 9b). Особые точки деформирования

системы: $1(U=0, F=147 \kappa H)$ - конструкция нагружена собственным весом, перемещения колонны

отсутствуют; $2(U=0,5 м м, F=260 \kappa H)-$ появление первых трещин в растянутой зоне ригелей; $3(U=5,2 м \mu, F=621 \kappa H)-$ максимальное усилие в колонне, сопровождающееся появлением трещин в плитах перекрытий; $4(U=11,6 м м, F=590 \kappa H) \quad$ - разрушение ригелей в сжатой зоне.

\section{4. ЗАКЛЮЧЕНИЕ}

Анализ результатов численных экспериментов по исследованию квазистатических деформационных процессов в модельной железобетонной конструкции и в её составных частях позволяет оценить, каким образом происходит развитие процесса разрушения в элементах конструкции в пространстве и времени. 


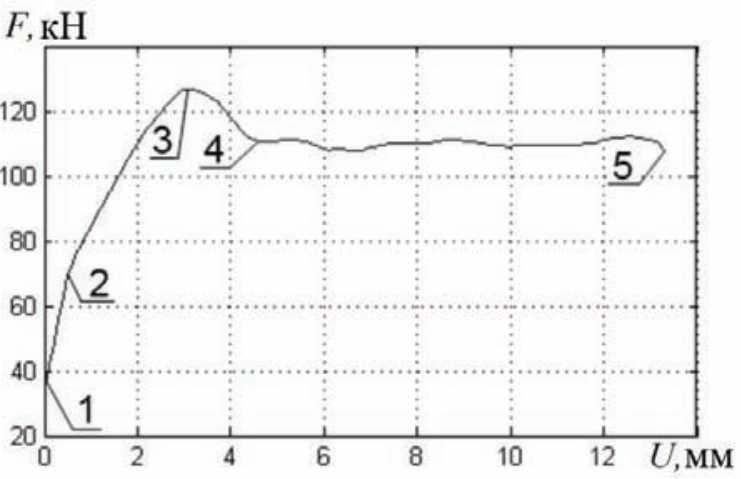

a)

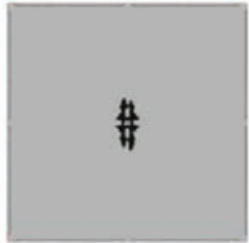

2

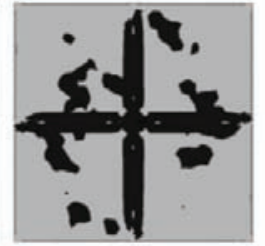

3

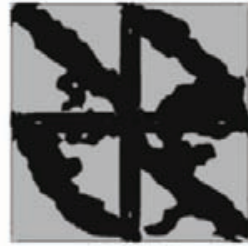

4

b)

Рисунок 8. Результаты моделирования деформационного процесса в системе "9 колонн-

12 ригелей 4 плиты»: a-изменение усилия в центральной колонне от заданного перемещчения ее основания (цифрами отмечены ключевые точки деформирования); $b$ - области разрушения на плитах перекрытия, соответствующие ключевым точкам деформирования на рисунке (a)

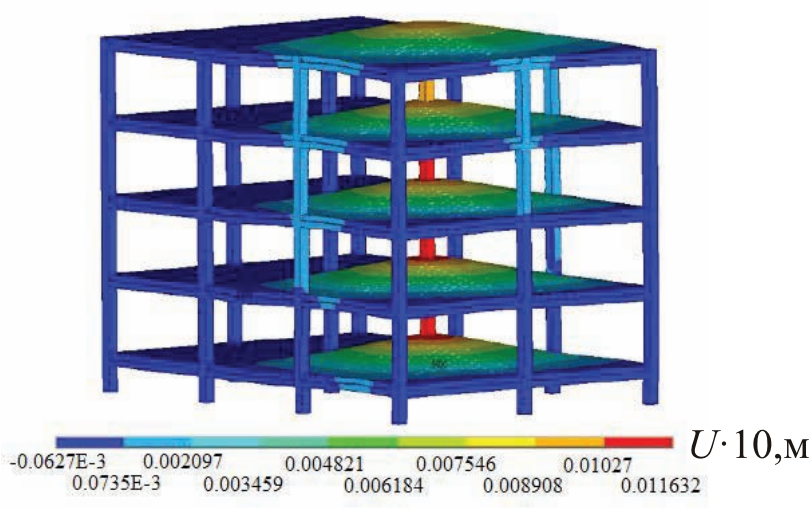

a)

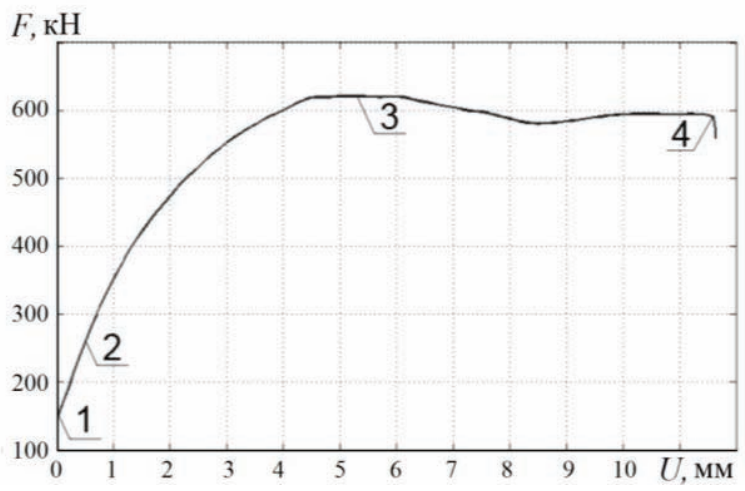

b)

Рисунок 9. Результаты моделирования деформационного процесса в четырехэтажной конструкции: $a$ - деформированная конфигурация конструкиии $b$ - изменение усилия в колонне от заданного перемещения ее основания (цифрами отмечены ключевые точки деформирования).

Сопоставление усилий, деформаций и перемещений, соответствующих ключевым точкам деформирования конструкции в целом и ее частей позволяет получить оценку внешних воздействий, при которых могут быть реализованы предкритические и критические деформационные процессы. Эти величины приведены в Таблице 3. Полученная в численных экспериментах информация дает возможность разработать сценарии приложения внешних воздействий (силовых и кинематических) в пространстве и времени, которые обеспечат развитие деформацион- ного процесса в элементах конструкции от упругого состояния до полного разрушения.

Из полученных результатов следует, что для того, чтобы гарантированно реализовать процесс разрушения в модельной железобетонной конструкции, необходимо нагружающее устройство, способное обеспечить диапазон усилий 0-1000 кН.

Для достижения контролируемого перехода конструкции из упругого состояния в стадию разрушения необходимо задавать перемещения элементов конструкции с точностью не хуже 0,1 мм. 
Экспериментальный стенд для исследования особенностей предкритических и критических деформационных процессов в железобетонных конструкциях (математическое моделирование, структурная схема)

Таблища 3. Значения сил и перемещений на различных этапах деформирования в собранной конструкичи и в её фрагментах.

\begin{tabular}{|c|c|c|c|c|}
\hline \multirow{2}{*}{$\begin{array}{c}\text { Деформируемый } \\
\text { элемент }\end{array}$} & \multicolumn{2}{|c|}{$\begin{array}{c}\text { Предельные значения } \\
\text { параметров для упругого } \\
\text { деформирования }\end{array}$} & \multirow{2}{*}{$\begin{array}{c}\text { Максимальное } \\
\text { усилие на всем } \\
\text { этапе } \\
\text { деформирования } \\
F^{*}, \text { кН } \\
\end{array}$} & \multirow{2}{*}{$\begin{array}{c}\text { Максимальное } \\
\text { перемещение на } \\
\text { всем этапе } \\
\text { деформирования } \\
U^{*}, \text { мм }\end{array}$} \\
\hline & $\begin{array}{c}\text { сила } F, \\
\text { кН }\end{array}$ & $\begin{array}{c}\text { перемещение } \\
U, \text { мм }\end{array}$ & & \\
\hline «2 колонны - ригель» & 3,7 & 1,2 & 8,6 & 50 \\
\hline «9 колонн - 12 ригелей» & 10,7 & 0,8 & 44,6 & 62 \\
\hline $\begin{array}{c}\text { «9 колонн -12 ригелей - } \\
4 \text { плиты» }\end{array}$ & 69,5 & 0,5 & 126,8 & 13 \\
\hline 4-этажная конструкция & 260,0 & 0,5 & 621,0 & 12 \\
\hline
\end{tabular}

Полученные результаты легли в основу проекта испытательного стенда для исследования деформационного поведения крупномасштабных конструкций. Эскиз стенда с размещенной внутри него модельной конструкцией показан на рисунке 10.

Для данного стенда разработана система силового и кинематического нагружения. Силовое нагружение должно осуществляться гидродомкратами и распределенными усилиями. Кинематическое воздействие будет реализовываться за счет заданного смещения отдельных колонн при фиксированном положении всех остальных. Для осуществления этого воздействия разработано специальное устройство, совмещенное с основанием стенда.

Регистрацию деформационных параметров (перемещения, деформации, углы наклона) предполагается выполнять с помощью различных датчиков и систем измерений, таких как тензометрия, фотометрия, гидронивилирование, инфракрасная термография, оптико-волоконные датчики на основе брэгговских решеток, пьезодатчики, лазерные измерители расстояния. Полученные в экспериментах закономерности будут использованы в автоматизированных системах мониторинга деформационного состояния сложных инженерных сооружений.

Работа выполнена за счет средств гранта Российского научного фонда (проект № 1429-00172).

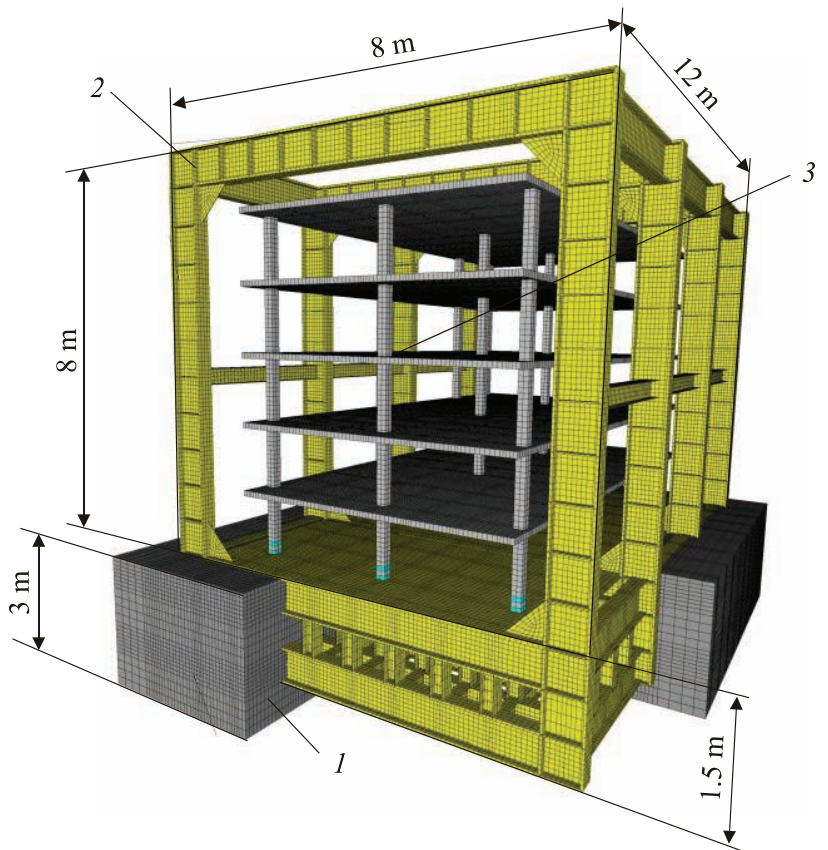

Рисунок 10. Экспериментальный стенд с модельной конструкцией:

1 - железобетонное основание стенда, 2 - силовой контур, 3 -модель 4-этажного железобетонного здания в масштабе 1:2.

\section{СПИСОК ЛИТЕРАТУРЫ}

1. Negro P., Bournas D. A, Molina F.J. Pseudodynamic test on a full-scale 3-storey precast concrete building: global response. // Eng. Str., 2013, V. 57, pp. 594-608.

2. McCrum D.P., Broderick B.M. An experimental and numerical investigation into the seismic performance of a multi-storey concentrically braced plan irregular struc- 
ture // Bull. Earthq. Eng., 2013, V. 11, pp. 2363-2385.

3. Tsai K.C., Hsiao P.C. et al. Pseudodynamic tests of full-scale CFT/BRB frame - Part 1: Specimen design, experiment and analysis // Earthq. Eng. Struct. D, 2008, V. 37, pp. 1081-1098.

4. Tsai K.C., Hsiao P.C. et al Pseudodynamic tests of full-scale CFT/BRB frame - Part 2: Seismic performance of buckling-restrained braces and connections // Earthq. Eng. Struct. D, 2008, V. 37, pp. 1099-1115.

5. Illampas R., Charmpis D.C., Ioannou I. Finite element simulation of the structural response of adobe masonry buildings subjected to lateral loading // Proceedings SAHC, Mexico, 2014, 12p.

6. Качанов Л.М. Основы теории пластичности. - М.: Наука, 1969. - 420 с.

7. Лурье А.И. Теория упругости. - М.: Наука, 1970. - 940 с.

8. Новацкий В. Теория упругости. - М.: Мир, 1975. - 872 с.

9. William K.J., Warnke E.P. Constitutive Model for the Triaxial Behavior of Concrete // Proceedings of International Association of Bridge Structural Engineering, 1974, V. 19, pp. 1-30.

10. Малинин Н.Н. Прикладная теория пластичности и ползучести. - М.: «Машиностроение», 1975. - 400 с.

11. Bathe K.J. Finite Element Procedures. Prentice-Hall. Englewood Cliffs. New Jersey, 1996.

Шардаков Игорь Николаевич, доктор физикоматематических наук; заведующий лабораторией интеллектуального мониторинга, Институт механики сплошных сред Уральского отделения Российской академии наук; 614013, Россия, г. Пермь, ул. Академика Королева, дом 1; тел. +7(342) 23783181;

e-mail: shardakov@icmm.ru

Шестаков Алексей Петрович, инженер-исследователь лаборатории интеллектуального мониторинга, Институт механики сплошных сред Уральского отделения Российской академии наук, 614013, Россия, г. Пермь, ул. Академика Королева, дом 1; тел. +7(342) 2378308; e-mail: shap@icmm.ru

Гусев Георгий Николаевич, кандидат технических наук; заведующий лабораторией строительной механики, Институт механики сплошных сред Уральского отделения Российской академии наук; 614013, Россия, г. Пермь, ул. Академика Королева, дом 1, тел. +7(342) 2378384, e-mail: gusev.g@icmm.ru

Бартоломей Мария Леонидовна, кандидат технических наук; доцент кафедры Вычислительной математики и механики, Пермский национальный исследовательский политехнический университет; 614013, Россия, г. Пермь, Комсомольский проспект, дом 29; тел. 7 (342) 2391564 e-mail: mbartolomey@ mail.ru

Кашеварова Галина Геннадьевна, советник РААСН, доктор технических наук, профессор, заведующая кафедрой «Строительные конструкции и строительная механика», Пермский национальный исследовательский политехнический университет; 614010, Россия, г. Пермь, ул. Куйбышева, 109;

тел. +7(342) 2198361, e-mail: ggkash@ mail.ru

Цветков Роман Валерьевич, кандидат технических наук; научный сотрудник лаборатории Интеллектуального мониторинга, Институт механики сплошных сред Уральского отделения Российской академии наук; 614013, Россия, г. Пермь, ул. Академика Королева, дом 1; тел. +7(342) 2378308, flower@icmm.ru

Глот Ирина Олеговна, кандидат физикоматематических наук; старший научный сотрудник лаборатории механики термопластов, Институт механики сплошных сред Уральского отделения Российской академии наук; 614013, Россия, г. Пермь, ул. Академика Королева, дом 1; тел. +7(342) 2198318; e-mail: glot@icmm.ru

Igor N. Shardakov, Doctor of Physical and Mathematical Sciences, Head of Laboratory of Smart Monitoring, Institute of Continuous Media Mechanics of the Ural Branch of Russian Academy of Science; 614013, Academician Korolev Street, 1, Perm, Russian Federation;

tel. +7(342) 23783181; e-mail: shardakov@icmm.ru

Alexey P. Shesnakov, Research Engineer of Laboratory of Smart Monitoring, Institute of Continuous Media Mechanics of the Ural Branch of Russian Academy of Science; 614013, Academician Korolev Street, 1, Perm, Russian Federation; tel. +7(342) 2378308; e-mail: shap@icmm.ru

Georgiy N. Gusev, Ph.D., Head of Laboratory of Structural Mechanics, Institute of Continuous Media Mechanics of the Ural Branch of Russian Academy of Science; 
Экспериментальный стенд для исследования особенностей предкритических и критических деформационных процессов в железобетонных конструкциях (математическое моделирование, структурная схема)

614013, Academician Korolev Street, 1, Perm, Russian Federation; tel. +7(342) 2378384,

e-mail: gusev.g@icmm.ru

Maria L. Bartolomey, Ph.D., Associate Professor of Department of Computational Mathematics and Mechanics of Perm National Research Polytechnic University; 29 Komsomolsky prospekt, Perm, Perm krai, Russia, 614990; tel. +7 (342) 2391564

e-mail: mbartolomey@mail.ru

Galina G. Kashevarova, Adviser of the Russian Academy of Architecture and Construction Sciences, Professor, Doctor of Technical Sciences; Head of Department of Construction Structures and Structural Mechanics of Perm National Research Polytechnic University; 29 Komsomolsky prospekt, Perm, Perm krai, Russia, 614990; tel. +7(342) 2198361, e-mail: ggkash@mail.ru

Roman V. Tsvetkov, Ph.D., Research Assistant of Laboratory of Smart Monitoring, Institute of Continuous Media Mechanics of the Ural Branch of Russian Academy of Science; 614013, Academician Korolev Street, 1, Perm, Russian Federation; tel. +7(342) 2378308,

e-mail: flower@icmm.ru

Irina O. Glot, Ph.D., Senior Research Assistant of Laboratory of Smart Monitoring, Institute of Continuous Media Mechanics of the Ural Branch of Russian Academy of Science; 614013, Academician Korolev Street, 1, Perm, Russian Federation; tel. +7(342) 2198318;

e-mail: glot@icmm.ru 


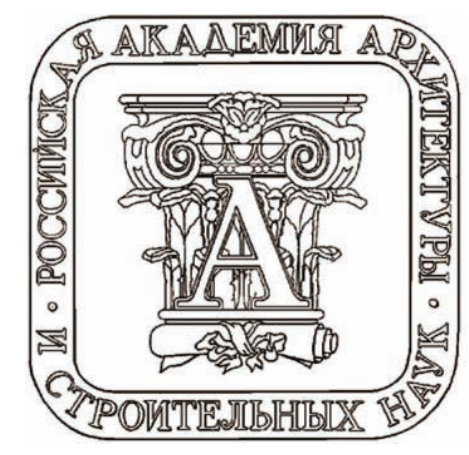

Утверждено постановлением Президиума Российской академии архитектуры и строительных наук от 7 декабря 2016 г. № 12

\section{РОССИЙСКАЯ АКАДЕМИЯ АРХИТЕКТУРЫ И СТРОИТЕЛЬНЫХ НАУК (РААСН) \\ ОБЪЯВЛЯЕТ ОЧЕРЕДНЫЕ ВЫБОРЫ АКАДЕМИКОВ РААСН И ЧЛЕНОВ-КОРРЕСПОНДЕНТОВ РААСН}

В соответствии с пунктом 24 Устава федерального государственного бюджетного учреждения «Российская академия архитектуры и строительных наук», утвержденного постановлением Правительства Российской Федерации от 28 мая 2014 г. № 488 (далее - Устав РАACH), президиум Российской академии архитектуры и строительных наук (РАACН) сообщает о проведении 20 и 21 апреля 2017 года очередных выборов академиков РААСН и членов-корреспондентов РААСН по отделениям и научным направлениям (специальностям).

1. Распределение вакансий членов РААСН (академиков РААСН и членовкорреспондентов РААСН) по отделениям и научным направлениям (специальностям) на выборах в РААСН в 2017 году.

\begin{tabular}{|c|c|c|}
\hline \multirow{2}{*}{$\begin{array}{c}\text { Научное направление } \\
\text { (специальность) }\end{array}$} & \multicolumn{2}{|c|}{ Число вакансий } \\
\cline { 2 - 3 } Отделение архитектуры РААСН \\
\hline \multicolumn{2}{|c|}{ РААСемиков } & $\begin{array}{c}\text { Членов-корреспондентов } \\
\text { РААСН }\end{array}$ \\
\hline Наука и образование & 1 & $2+2^{*}$ \\
\hline Архитектурная практика & 3 & $2+1^{*}$ \\
\hline \multicolumn{3}{|c|}{ Отение градостроительства РААСН } \\
\hline Градостроительная наука & 2 & $1+1^{*}$ \\
\hline Градостроительная практика & 1 & $1+1^{*}$ \\
\hline \multicolumn{3}{|c|}{ Отделение строительных наук РААСН } \\
\hline Теоретические основы строительных наук & 2 & $4+4^{*}$ \\
\hline
\end{tabular}

Примечание: Символ * означает, что на основании постановления президиума РААСН от 25 ноября 2016 г. № 11 данная вакансия объявляется с ограничением возраста кандидата на момент избрания в члены-корреспонденты РААСН - до 61 года включительно. 
Сообщение о проведении выборов членов Российской академии архитектуры и строительных наук (РАAСН) в 2017 году

\section{2. Порядок и условия избрания членов РААСН.}

Порядок и условия избрания членов РААСН, перечень документов, представляемых кандидатами в члены РАACH, а также порядок проведения голосования и подсчета голосов на выборах членов РААСН определяются соответственно Уставом РААСН и Регламентом выдвижения кандидатов, проведения голосования и подсчета голосов на выборах членов Российской академии архитектуры и строительных наук, утвержденным постановлением президиума РААСН от 25 ноября 2016 г. № 11.

Академиками РААСН избираются ученые из числа членов-корреспондентов РАACH, а также ведущие мастера архитектуры и градостроительства, обогатившие архитектуру, градостроительство и строительные науки трудами первостепенного научного и творческого значения.

Членами-корреспондентами РААСН избираются граждане Российской Федерации, обогатившие современные архитектуру, градостроительство и строительные науки выдающимися трудами и (или) значимыми практическими достижениями.

\section{Кандидатами в члены РААСН могут быть:}

\section{- по Отделению архитектуры РААСН}

- Ученые в области архитектурной науки, научно-педагогические работники образовательных организаций высшего образования (образовательных организаций высшего образования архитектурного профиля, структурных подразделений архитектурного профиля образовательных организаций высшего образования), имеющие опубликованные научные и учебно-методические труды, получившие широкое профессиональное и общественное признание, ученую степень доктора наук или ученое звание профессора, присвоенное Высшей аттестационной комиссией при Министерстве образования и науки Российской Федерации;

- Дипломированные архитекторы, являющиеся основными авторами выдающихся проектов и построек, получивших широкое общественное признание, творческая деятельность которых отмечена почетными званиями, государственными и профессиональными премиями и наградами;

- по Отделению градостроительства РААСН

- Ученые в области градостроительной науки, научно-педагогические работники образовательных организаций высшего образования (образовательных организаций высшего образования архитектурного профиля, структурных подразделений архитектурного профиля образовательных организаций высшего образования), имеющие опубликованные научные и учебно-методические труды, получившие широкое профессиональное и общественное признание, ученую степень доктора наук или ученое звание профессора, присвоенное Высшей аттестационной комиссией при Министерстве образования и науки Российской Федерации;

- Дипломированные градостроители, являющиеся основными авторами выдающихся проектов и построек, получивших широкое общественное признание, творческая деятельность которых отмечена почетными званиями, государственными и профессиональными премиями и наградами;

- по Отделению строительных наук РААСН

- Ученые строительных специальностей и научно-педагогические работники образовательных организаций высшего образования (образовательных организа- 
Сообщение о проведении выборов членов Российской академии архитектуры и строительных наук (РААСН) в 2017 году

ций высшего образования строительного профиля, структурных подразделений строительного профиля образовательных организаций высшего образования), имеющие опубликованные научные труды, получившие широкое профессиональное признание, ученую степень доктора наук.

\section{Право выдвижения кандидатов (без ограничения количества выдвижений) в члены РААСН предоставляется:}

- Членам РААСН (академикам РААСН и членам-корреспондентам РААСН)

- Право выдвижения кандидата в академики РААСН предоставляется академику РАACH, кандидата в члены-корреспонденты РААСН - члену РААСН.

- Президент РААСН, первый вице-президент РААСН, вице-президенты РААСН, главный ученый секретарь РААСН, академики-секретари отделений РАACH от рекомендаций по выдвижению кандидатов в члены РААСН воздерживаются.

- Государственным органам, осуществляющим управление в сфере архитектуры, градостроительства и строительных наук

- Выдвижение кандидата в члены РААСН проводится на заседании коллегиального органа государственного органа, осуществляющего управление в сфере архитектуры, градостроительства и строительных наук путем тайного голосования простым большинством голосов при наличии кворума.

- Научным организациям, действующим в сфере архитектуры, градостроительства и строительных наук

- Выдвижение кандидата в члены РААСН проводится на заседании ученого или научно-технического совета путем тайного голосования простым большинством голосов при наличии кворума.

- Общественным организациям ${ }^{1}$, действующим в сфере архитектуры, градостроительства и строительных наук, причем устав общественной организации должен содержать указание на научную и творческую деятельность как направление деятельности общественной организации и в структуре общественной организации должен присутствовать ученый совет или научно-технический совет (или иной орган), осуществляющий экспертную деятельность в области архитектуры, градостроительства и строительных наук.

- Выдвижение кандидата в члены РААСН проводится на заседании ученого совета или научно-технического совета, или иного органа, осуществляющего экспертную деятельность в области архитектуры, градостроительства и строительных наук путем тайного голосования простым большинством голосов при наличии кворума.

\section{3. Перечень документов для регистрации кандидата в члены РААСН.}

- Письменное заявление с согласием баллотироваться в состав иленов РААСН с личной подписью кандидата и с указанием категории членства (академик РАACH,

\footnotetext{
${ }^{1}$ В соответствии с Федеральным законом от 19 мая 1995 года №82-Ф3 (действующая редакция, 2016) «Об общественных объединениях» общественной организацией является основанное на членстве общественное объединение, созданное на основе совместной деятельности для защиты общих интересов и достижения уставных целей объединившихся граждан (Статья 8. Общественная организация).
} 
Сообщение о проведении выборов членов Российской академии архитектуры и строительных наук (РАAСН) в 2017 году

член-корреспондент РААСН), отделения РАACH, научного направления (специальности), соответствующего одному из объявленных; отметкой об участии в выборах с установленным ограничением возраста кандидата на момент избрания (в случае изъявления желания участвовать в выборах с установленным ограничением возраста кандидата на момент избрания);

- Представление кандидата в члены РААСН (допускается не более 1 (одного) представления):

- при выдвижении кандидата в члены РААСН академиком РААСН или членомкорреспондентом РААСН предоставляется письменное представление, содержащее соответствующее обоснование, подписанное соответственно академиком РААСН или членом-корреспондентом РААСН и заверенное;

- при выдвижении кандидата в члены РААСН государственным органом, осуществляющим управление в сфере архитектуры, градостроительства и строительных наук, предоставляется решение заседания коллегиального органа государственного органа, осуществляющего управление в сфере архитектуры, градостроительства и строительных наук, содержащее соответствующее обоснование, с выпиской из протокола с результатами тайного голосования (на решении заседания коллегиального органа и выписке из протокола обязательны подписи руководителя коллегиального органа (руководителя государственного органа), гербовая печать государственного органа);

- при выдвижении кандидата в члены РААСН научной организацией, действующей в сфере архитектуры, градостроительства и строительных наук, предоставляется решение заседания ученого совета или научно-технического совета, содержащее соответствующее обоснование, с выпиской из протокола с результатами тайного голосования (на решении совета и выписке из протокола обязательны подписи председателя ученого совета или научно-технического совета и ученого секретаря ученого совета или научно-технического совета, гербовая печать организации (печать организации, приравненная к гербовой печати));

- при выдвижении кандидата в члены РААСН общественной организацией, действующей в сфере архитектуры, градостроительства и строительных наук, предоставляется решение заседания ученого совета или научно-технического совета, или иного органа, осуществляющего экспертную деятельность в области архитектуры, градостроительства и строительных наук, содержащее соответствующее обоснование, с выпиской из протокола с результатами тайного голосования (на решении совета и выписке из протокола обязательны подписи председателя ученого совета или научно-технического совета, или иного органа, осуществляющего экспертную деятельность в области архитектуры, градостроительства и строительных наук, и ученого секретаря (секретаря) ученого совета или научно-технического совета, или иного органа, осуществляющего экспертную деятельность в области архитектуры, градостроительства и строительных наук, гербовая печать организации (печать организации, приравненная к гербовой печати));

- Научная и творческая характеристика кандидата в члены РААСН, заверенная по основному месту работы (подпись руководителя или заместителя руководителя и гербовая печать организации);

- Список научных и учебно-методических трудов в хронологическом порядке со сквозной нумерацией, заверенные организацией, выдвинувшей кандидата или по месту работы кандидата (на последней странице - личная подпись кандидата, под- 
Сообщение о проведении выборов членов Российской академии архитектуры и строительных наук (РААСН) в 2017 году

пись ученого секретаря (руководителя) и гербовая печать организации (печать организации, приравненная к гербовой печати));

- Список проектов и построек, выполненных кандидатом в члены РААСН в качестве основного автора, заверенные организацией, выдвинувшей кандидата или по месту работы кандидата (на последней странице - личная подпись кандидата, подпись ученого секретаря (руководителя) и гербовая печать организации (печать организации, приравненная к гербовой печати)) - только для кандидатов в члены PAACH, выдвинутых как дипломированные архитекторы и градостроители, являющиеся основными авторами выдающихся проектов и построек, получивших широкое общественное признание, творческая деятельность которых отмечена почетными званиями, государственными и профессиональными премиями и наградами;

- Доклад о наиболее важных сторонах научной, практической, педагогической деятельности по объявленному научному направлению (специальности) объемом до 15 (пятнадцати) страниц текста ${ }^{2}$ или альбом творческих работ (проекты, постройки) с аннотациями форматом А-3 или А-4 (для архитекторов и градостроителей, занимающихся творческой практикой) с личной подписью кандидата;

- Заверенный личный листок по учету кадров с приклеенной фотографией;

- Автобиография в произвольной форме с личной подписью кандидата;

- Краткая научная и творческая характеристика кандидата в члены РААСН объемом до 1 (одной) страницы текста ${ }^{2}$ с личной подписью кандидата;

- Заверенная справка о месте работы;

- Заверенные копии дипломов (аттестатов) об окончании образовательной организации высшего образования, о присвоении ученых степеней, о присвоении ученых званий, удостоверений и грамот о присвоении почетных званий, лауреатов премий, членстве в других государственных академиях;

- Цветные фотографии кандидата в члень РААСН размером 4.5 см х 6 см. (3 штуки);

- Заверенная копия устава общественной организации (при выдвижении кандидата в члены РААСН общественной организацией, действующей в сфере архитектуры, градостроительства и строительных наук).

Указанный комплект документов представляется в 1 (одном) экземпляре на бумажном носителе и в 1 (одном) экземпляре на электронном носителе (на CD-диске или DVD-диске). Электронные версии автобиографии кандидата в члены РАACH и краткой научной и творческой характеристики кандидата в члены PAACH должны быть представлены в формате DOC (текстовый процессор Microsoft Word).

Кандидат в члены РААСН может выдвигаться только по одной из объявленных вакансий, в соответствии с указанным им в письменном заявлении с согласием баллотироваться в состав членов РААСН, категорией членства, отделением РААСН, научным направлением (специальностью).

Даты необходим проставить на всех предоставляемых документах. Действительны документы, датированные только в период с 13 января 2017 года по 01 марта 2017 года.

\footnotetext{
2 Формат страницы - A4 (ширина - 21 см, высота - 29.7 см).

Поля: верхнее -2 см, нижнее -2 см, левое -3 см, правое -1.5 см.

Шрифт - Times New Roman, размер 14.

Межстрочный интервал - одинарный.
} 
Сообщение о проведении выборов членов Российской академии архитектуры и строительных наук (РАAСН) в 2017 году

Отсутствие или представление незаверенным какого-либо из документов по указанному перечню может служить основанием для отклонения данного кандидата от участия в выборах.

Комплект указанных документов должен быть представлен в соответствующее отделение РААСН не позднее 17 часов 00 минут 01 марта 2017 года.

Документы, отправленные по почте и не полученные в указанный срок, или представленные позднее указанной даты и часа, не рассматриваются.

Документы, представленные кандидатами в члены РААСН, рассматриваются на заседании бюро отделения РААСН на предмет соответствия представленных документов объявленным условиям выборов.

Кандидаты, выдвигаемые в члены-корреспонденты РААСН, могут заслушиваться на заседании бюро отделения РААСН.

По результатам заседания бюро отделения РААСН отделение РААСН подготавливает экспертные заключения в отношении выдвинутых по соответствующему отделению кандидатов и рекомендует кандидатуры для утверждения президиумом РААСН кандидатами в члены РААСН.

Президиум РААСН утверждает кандидатов в члены РААСН.

Списки утвержденных президиумом РААСН кандидатов в члены РААСН, подготовленные в соответствии с Распределением вакансий будут опубликованы на официальном интернет-сайте РАACH (http://raasn.ru/) в срок до 20 марта 2017 года.

Утвержденные президиумом РААСН кандидаты в члены РАACH, официально приглашаются на Общее собрание членов РААСН-2017, на котором 20 и 21 апреля 2017 года состоятся выборы членов РААСН.

Выборы членов РААСН проводятся путем тайного голосования на Общем собрании членов РААСН и на основе тайных голосований на общих собраниях отделений РААСН, проводимых в рамках Общего собрания членов РААСН.

В случае избрания члена-корреспондента РААСН академиком РААСН, образовавшаяся таким образом вакансия члена-корреспондента РААСН остается вакантной до следующих выборов членов РААСН.

\section{4. Прием документов кандидатов в члены РААСН.}

Прием документов осуществляется в здании Российской академии архитектуры и строительных наук по адресу: 107031, Российская Федерация, г. Москва, ул. Большая Дмитровка, д. 24, стр. 1, ежседневно с 10-00 до 17-00 часов, кроме выходных и праздничных дней, в период с 13 января 2017 года по 01 марта 2017 года.

\section{5. Контактная информация.}

\begin{tabular}{|c|c|c|}
\hline $\begin{array}{c}\text { Отделение } \\
\text { архитектуры PAACH }\end{array}$ & $\begin{array}{c}\text { Отделение } \\
\text { градостроительства РААСН }\end{array}$ & $\begin{array}{c}\text { Отделение } \\
\text { строительных наук PAACH }\end{array}$ \\
\hline $\begin{array}{c}\text { Телефоньл: } \\
+7 \text { (495) 629-14-95 } \\
\text { +7 (495) 625-76-84 } \\
\text { Адрес электронной почтыл: } \\
\text { oarch@ raasn.ru }\end{array}$ & $\begin{array}{c}\text { Телефоньл: } \\
+7 \text { (495) 625-79-75 } \\
\text { +7 (495) 629-19-91 } \\
\text { Адрес электронной почтьл: } \\
\text { grado@ raasn.ru }\end{array}$ & $\begin{array}{c}\text { Телефонь: } \\
+7 \text { (495) 625-73-16 } \\
\text { +7 (495) 625-76-80 } \\
\text { Адрес электронной почтыл: } \\
\text { osn@ raasn.ru }\end{array}$ \\
\hline
\end{tabular}




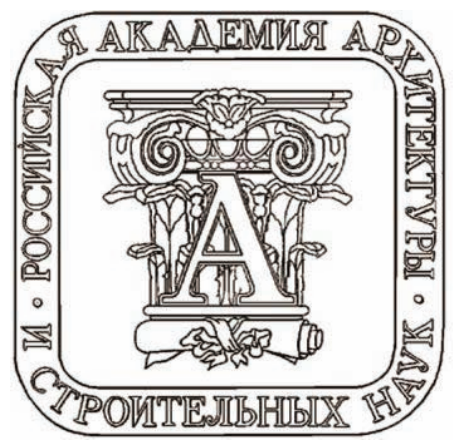

\section{КОНКУРС НА МЕДАЛИ И ДИПЛОМЫ РОССИЙСКОЙ АКАДЕМИИ АРХИТЕКТУРЫ И СТРОИТЕЛЬНЫХ НАУК (РААСН) ЗА 2016 ГОД}

В соответствии В соответствии с «Положением о Конкурсе на медали и дипломы Российской академии архитектуры и строительных наук за лучшие научные и творческие работы», утвержденным постановлением Общего собрания членов Российской академии архитектуры и строительных наук (РАACH) от 22 апреля 2016 г. Президиум федерального государственного бюджетного учреждения «Российская академии архитектуры и строительных наук» (РАACH) объявляет о проведении XX Конкурса на медали и дипломы РААСН на лучшие научные и творческие работы в области архитектуры, градостроительства и строительных наук за 2016 год.

Медали и дипломы РААСН присуждаются на конкурсной основе архитекторам, градостроителям, учёным архитектурно-градостроительных и строительных специальностей гражданам Российской Федерации и или иностранных государств - в следующих номинациях:

- $\quad$ за лучший реализованный проект, срок эксплуатации которого составляет не менее 1 (одного) года;

- $\quad$ за лучший проект, находящиеся в стадии реализации с подтвержденными документами об утверждении проекта (для градостроительных работ);

- за лучшую научно-исследовательскую и/или проектно-конструкторскую разработку, в результате которой получен значительный экономический или социальный эффект, подтвержденный конкретными расчетами и рекомендациями;

- $\quad$ за лучшие опубликованные научные труды, монографии, имеющие опубликованные положительные рецензии;

- $\quad$ за лучшее учебное издание (учебник, учебное пособие), и имеющее опубликованную положительную рецензию.

- за лучший неосуществленный архитектурный проект;

- $\quad$ за лучший концептуальный градостроительный проект.

На Конкурс представляются:

- $\quad$ реализованные (по архитектуре) или реализуемые (по градостроительству) проекты, а также опубликованные научные работы, выполненные в течение последних 5 (пяти) лет.

На Конкурс не представляются:

- $\quad$ работы, удостоенные Государственных премий Российской Федерации, премий Правительства Российской Федерации в области науки и техники, премии Правительства Российской федерации в области образования, премий Правительства Российской Фе- 


\section{Сообщение о проведении конкурса на медали и дипломы \\ Российской академии архитектуры и строительных наук (РАACH) за 2016 год}

дерации в области науки и техники для молодых ученый, Медалей и дипломов Российской академии наук, других государственных академий;

- работы, выдвинутые в 2016 году на соискание премии Правительства Российской Федерации в области науки и техники, премии Правительства Российской Федерации в области образования, премии Правительства Российской Федерации в области науки и техники для молодых ученых, медалей и дипломов Российской академии наук, других государственных академий наук;

- работы, участвовавшие в заказных и открытых архитектурных и градостроительных конкурсах;

- работы, ранее удостоенные медалей и дипломов РААСН;

- коллективные сборники научных трудов, за исключением коллективных монографий.

В рамках конкурса на соискание медалей и дипломов РААСН проводится отдельный конкурс «За лучшие труды и творческие работы в области архитектуры, градостроительства и строительных наук для молодых учёных и специалистов», в возрасте до 35 лет включительно. Конкурс проводится на основе «Положения о конкурсе для молодых учёных и специалистов», утверждённого президиумом РААСН. Работа, не прошедшая по конкурсу, может быть выдвинута на соискание медалей и дипломов РААСН еще один раз, при этом оформление документов производится заново.

\section{Решение о присуждении медалей и дипломов принимает президиум РААСН.}

\section{Установить следующие Медали \\ Российской академии архитектуры и строительных наук:}

\section{1 золотая и 1 серебряная медали}

\section{в области архитектуры}

- за лучший реализованный архитектурный проект;

- за лучшую опубликованную научную работу в области архитектурной науки.

1 золотая и 1 серебряная медали

\section{в области градостроительства}

- за лучшую проектную работу в области градостроительства, полностью или частично реализованную;

- за лучшую опубликованную научную работу в области градостроительной науки.

1 золотая и 1 серебряная медали

\section{в области строительных наук}

- за лучший реализованный инженерный проект или за лучшее научное сопровождение реализованного инженерного проекта или за лучшую разработанную и реализованную технологию;

- за лучшую опубликованную научную работу в области строительной науки.

Примечание:

- Золотая и серебряная медали РААСН присуждаются автору или каждому члену авторского коллектива на основе конкурса между работами, представленными в отделения РААСН в объявленных номинациях.

- Дополнительно к золотой и серебряной медали РААСН автору или каждому члену авторского коллектива вручается диплом РААСН. 
Сообщение о проведении конкурса на медали и дипломы

Российской академии архитектуры и строительных наук (РАACH) за 2016 год

\section{Установить следующие Дипломы \\ Российской академии архитектуры и строительных наук:}

12 дипломов (без разделения на номинации), в том числе:

- 4 диплома в области архитектуры,

- 4 диплома в области градостроительства,

- 4 диплома в области строительной науки.

1 диплом за «архитектурный проект» для работ, неосуществленных в строительстве (конкурсные проекты, эскизные проекты, проекты, принятые, но не реализованные в натуре и др.). 1 диплом за «за концептуальный градостроительный проект» (градостроительные концепции, инициативные проекты и другие разработки, не требующие утверждения).

1 диплом за «лучший изданный учебник, учебное пособие в области архитектуры».

1 диплом за «лучший изданный учебник, учебное пособие в области градостроительства».

1 диплом за «лучший изданный учебник, учебное пособие в области строительной науки».

2 диплома в области архитектуры для молодых ученых и специалистов.

2 диплома в области градостроительства для молодых ученых и специалистов.

2 диплома в области строительных наук для молодых ученых и специалистов.

Установить порядок выдвижения кандидатов на соискание медалей и дипломов:

1. На соискание наград РААСН представляются авторы или авторские коллективы в составе основных авторов, но не более 6 человек. Медали и дипломы РААСН присуждаются каждому члену представленного авторского коллектива. Не допускается включение в состав соискателей лиц:

- осуществлявших в процессе выполнения работы только административные и (или) организационные функции;

- включенных в авторский коллектив, выполнявший другую работу, выдвинутую в 2016 году на соискание медалей и дипломов РААСН, премии Правительства Российской Федерации в области науки и техники, премии Правительства Российской Федерации в области образования, премии Правительства Российской Федерации в области науки и техники для молодых ученых.

2. Архитекторы, градостроители, ученые архитектурно-градостроительных и строительных специальностей, награжденные медалями РААСН, имеют право повторно участвовать в конкурсе на медали и дипломы РААСН за лучшие научные и творческие работы не ранее чем через 5 (пять) лет после утверждения президиумом РАACH решения о присуждении медали РААСН.

3. Архитекторы, градостроители, ученые архитектурно-градостроительных и строительных специальностей, награжденные дипломами РАACH, имеют право повторно участвовать в конкурсе на медали и дипломы РАACH за лучшие научные и творческие работы не ранее чем через 3 (три) года после утверждения президиумом РАACH решения о присуждении диплома РААСН.

Выдвижение кандидатов на соискание медалей и дипломов РААСН проводится:

- профессиональными организациями в области архитектуры, градостроительства и строительных наук (союзами архитекторов и строителей, проектными и научно-исследовательскими организациями, образовательными организациями высшего образования и др.);

- академиками и членами-корреспондентами РААСН, бюро отделений РААСН и президиумами территориальных отделений РААСН, советом молодых ученых и специалистов РАACH; 
- государственными органами в области архитектуры, градостроительства и строительства.

На конкурс представляются следующие материалы:

- представление выдвигающей организации или члена $\mathrm{PAACH}$, содержащее основания для выдвижения;

- один экземпляр альбома (буклета) с описанием (текстовый и графический материал) архитектурного проекта, проектной работы в области градостроительства, инженерного проекта, научного сопровождения инженерного проекта, разработанной и реализованной технологии (в цветном разрешении, размер A3 или A4), в том числе электронная версия (на CD и/или DVD-диске) предоставляемых материалов;

- один комплект дополнительных материалов (копии статей, патентов на изобретения, свидетельств на полезные модели, патентов на промышленные образцы, свидетельств о государственной регистрации программ для ЭВМ и др.), раскрывающих сущность и поясняющих изложенные в указанном альбоме (буклете) сведения;

- один экземпляр опубликованной научной работы, учебного издания (учебника, учебного пособия) с опубликованной рецензией.

4. К материалам прилагаются краткие биографические и профессиональные сведения об авторе и членах авторского коллектива с указанием авторского вклада каждого члена коллектива.

5. Материалы, отправленные по почте, должны прибыть в РААСН не позднее установленного срока.

6. Работы, полученные позднее установленного срока, к рассмотрению не принимаются.

7. Материалы, представленные на конкурс (в том числе научные работы, монографии и учебные издания (учебники, учебные пособия), альбомы (буклеты) и электронные носители с проектами (с постройками)), не возвращаются.

8. Опубликованные научные труды, монографии, учебники, учебные пособия и альбомы (буклеты) проектов передаются в библиотеку РААСН.

Установить срок представления материалов на Конкурс не позднее 1 февраля 2017 года (среда) включительно, до 17 часов 00 минут.

Материалы представляются в РААСН по адресу:

Российская Федерация, 107 031, Москва, ул. Большая Дмитровка, дом 24, стр. 1, в соответствующее отделение РААСН

(архитектура, градостроительство и строительные науки).

\section{Контактная информация:}

Отделение архитектуры РААСН

Телефоны: 8(495) 629-14-95, 8(495) 625-76-84 (т/факс); e-mail: oarch@ raasn.ru

Отделение градостроительства РААСН

Телефоны: 8(495) 629-19-91, 625-79-75 (т/факс), e-mail: grado@ raasn.ru

Отделение строительных наук РААСН

Телефоны: 8(495) 625-76-80 (т/ф), 8(495) 625-73-16 (т/факс) e-mail: osn@ raasn.ru 


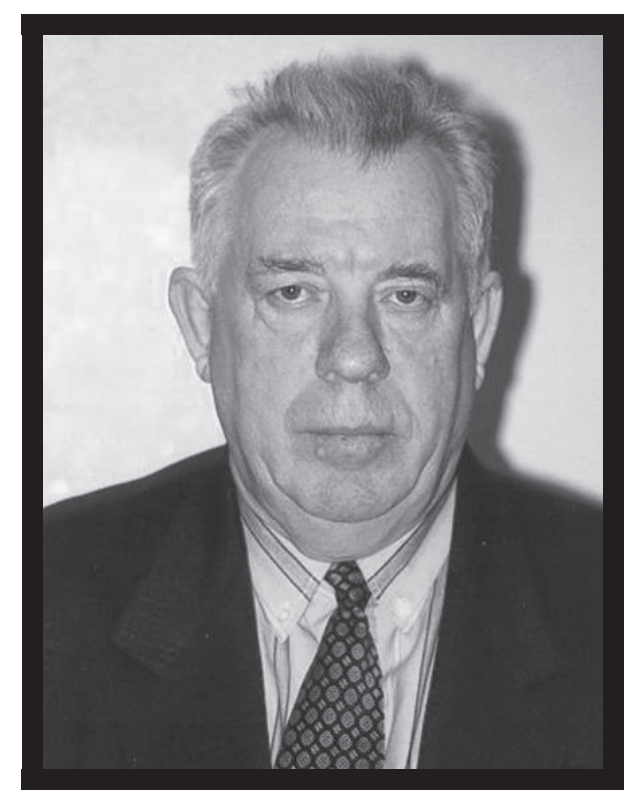

\section{УШЕЛ ИЗ ЖИЗНИ ЛЕВ ВАСИЛЬЕВИЧ ЕНДЖИЕВСКИЙ}

11 декабря 2016 года на 79 году ушел из жизни член-корреспондент РААСН, профессорконсультант кафедры строительных конструкций и управляемых систем Сибирского федерального университета, профессор, доктор технических наук Лев Васильевич Енджиевский.

Вся жизнь Л.В. Енджиевского была неразрывно была связана с развитием строительного образования Красноярского края. Лев Васильевич проявил себя как крупный организатор науки и образования, объединив под своим началом деятельность многих кафедр, создав новую научно-материальную базу и условия для повышения квалификации кадров.

Профессиональная деятельность Л.В. Енджиевского была отмечена государственными и отраслевыми наградами и почетными званиями, среди которых Заслуженный деятель науки и техники Российской Федерации, Почетный строитель Российской Федерации, Почетный работник высшего профессионального образования России, Академик Международной Академии наук Высшей школы, Лауреат профессорской премии главы г. Красноярска, медаль «Ветеран труда», медаль «За доблестный труд в ознаменование 100-летия со дня рождения В.И. Ленина», знак «Заслуженный деятель науки Российской Федерации».

Научные работы в области пространственных металлических конструкций, научнообразовательный комплекс «Управляемые конструкции», а также учебник и учебные пособия, соавтором которых является Лев Васильевич, отмечены многими наградами, в том числе премией им. Академика Галеркина, дипломом РААСН, первым местом в конкурсе 2004 года среди вузов и научных учреждений страны и др.

Уход из жизни Льва Васильевича Енджиевского - видного ученого и обаятельного человека - невосполнимая потеря для строительной науки, а также и для каждого, кто знал его лично. Благодарная память о нем навсегда сохранится в сердцах его друзей и коллег.

Редакиионный Совет международного научного журнала "International Journal for Computational Civil and Structural Engineering" 